\title{
Asymmetric Total Synthesis and Formal Total Synthesis of the Antitumor Sesquiterpenoid (+)- Eremantholide A
}

\author{
Yi Li and Karl J. Hale* \\ The UCL Center for Chemical Genomics, \\ The Christopher Ingold Laboratories, \\ The Chemistry Department, \\ University College London, \\ 20 Gordon Street, \\ London WC1H OAJ, UK.
}

\section{Supporting Information}

(303 Pages Inclusive of Title Pages and Index) Index

Pages 2-26. Part 1. Experimental Procedures.

Pages 27-297. Part 2. Copies of the 1D and 2D $500 \mathrm{MHz}{ }^{1} \mathrm{H}$ and $125 \mathrm{MHz}$ ${ }^{13} \mathrm{C}$ NMR Spectra, the HR Mass Spectra, and the IR Spectra for the Intermediates Used in the Asymmetric Total Synthesis and Formal Total Synthesis of (+)-Eremantholide A.

Pages 298-303. Part 3. Individual $500 \mathrm{MHz}{ }^{1} \mathrm{H}$ NMR Spectra of 32 and 33 in $\mathrm{CDCl}_{3}$. The $500 \mathrm{MHz}{ }^{1} \mathrm{H}$ NMR Spectra of the Crude Unseparated Mixtures of $\mathbf{3 2}$ and $\mathbf{3 3}$ Obtained From the Alkylations of 2 with 3 Using Lithium $\operatorname{Bis}[(S)-\alpha-$ Methylbenzylamide] and Its $(R)$-Enantiomer in PhMe $\left(-78^{\circ} \mathrm{C}\right.$ to $0{ }^{\circ} \mathrm{C}$ over $2 \mathrm{~h}$ ). The $400 \mathrm{MHz}{ }^{1} \mathrm{H}$ NMR Spectrum of 43. 


\title{
Asymmetric Total Synthesis and Formal Total Synthesis of the Antitumor Sesquiterpenoid (+)- Eremantholide A
}

\author{
Yi Li and Karl J. Hale* \\ The UCL Center for Chemical Genomics, \\ The Christopher Ingold Laboratories, \\ The Chemistry Department, \\ University College London, \\ 20 Gordon Street, \\ London WC1HOAJ, UK.
}

\section{Supporting Information}

\section{Part 1. Experimental Procedures}

\begin{abstract}
General Methods
Reaction solvents (THF, PhMe, $\mathrm{C}_{6} \mathrm{H}_{6}, \mathrm{CH}_{2} \mathrm{Cl}_{2}$ ) were freshly distilled from freshly activated $4 \AA$ molecular sieve beads under a $\mathrm{N}_{2}$ atmosphere prior to use. Cyclohexanone, EtOAc and Petrol 40$60{ }^{\circ} \mathrm{C}$ were distilled prior to use. $\mathrm{MeOH}$ was distilled from $\mathrm{Mg}$ metal under a $\mathrm{N}_{2}$ atmosphere prior to use. All $500 \mathrm{MHz}{ }^{1} \mathrm{H}$ NMR spectral data that are reported in $\mathrm{CDCl}_{3}$ are referenced relative to the residual $\mathrm{CHCl}_{3}$ peak at $\delta 7.24$, and all data that are reported in $\mathrm{C}_{6} \mathrm{D}_{6}$ are referenced with respect to the residual $\mathrm{C}_{6} \mathrm{H}_{6}$ peak at $\delta 7.15$. For the $125 \mathrm{MHz}{ }^{13} \mathrm{C}$ NMR data that are reported in $\mathrm{CDCl}_{3}$, the triplet at $\delta 77.00$ is used as the reference peak, whilst for spectra recorded in $\mathrm{C}_{6} \mathrm{D}_{6}$, the triplet centred at $\delta 128.00$ is the reference peak. Unless otherwise specified, all IR spectra were recorded as neat films on $\mathrm{NaCl}$ plates.
\end{abstract}




\section{Experimental Procedures for the Asymmetric Total Synthesis of (+)-Eremantholide A}

Please Note: For our NMR assignments, we have used (+)-eremantholide A numbering throughout.

\section{Oxazolidinone 13 (II-YL-105)}

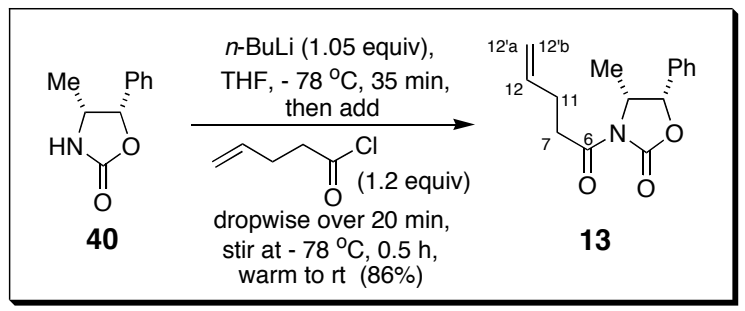

To a stirred solution of oxazolidinone 40 (35.5 g, $0.2 \mathrm{~mol})$ in dry THF (250 mL) at $-78{ }^{\circ} \mathrm{C}$ under $\mathrm{N}_{2}$ was added $n$-BuLi (2.5 M solution in hexanes, $84 \mathrm{~mL}, 0.21 \mathrm{~mol}, 1.05$ equiv) dropwise over $0.5 \mathrm{~h}$. The resulting bright orange solution was stirred at $-78^{\circ} \mathrm{C}$ for a further $5 \mathrm{~min}$, whereafter 4-pentenoyl chloride $(26.5 \mathrm{~mL}, 0.24 \mathrm{~mol})$ was added dropwise over $20 \mathrm{~min}$. The reactants were stirred at $-78{ }^{\circ} \mathrm{C}$ for $0.5 \mathrm{~h}$ and the cooling bath was removed. After warming the reaction mixture to rt, it was quenched with saturated aqueous $\mathrm{NH}_{4} \mathrm{Cl}(500 \mathrm{~mL})$. After separation of the organic layer, the aqueous phase was extracted with $\mathrm{Et}_{2} \mathrm{O}(3 \times 1 \mathrm{~L})$. The combined organic layers were washed with $\mathrm{H}_{2} \mathrm{O}(500 \mathrm{~mL})$, brine $(200 \mathrm{~mL})$, dried over $\mathrm{MgSO}_{4}$, filtered, and concentrated in vacuo. Recrystallisation of the crude residue from petrol $\left(40-60{ }^{\circ} \mathrm{C}\right)$ gave oxazolidinone 13 $(44.5 \mathrm{~g}, 86 \%)$ as white crystals.

Data for 13: $[\alpha]_{\mathrm{D}}+38.6^{\circ}\left(c\right.$ 0.684, $\left.\mathrm{CH}_{2} \mathrm{Cl}_{2}\right)$; IR (KBr) 3071 (w), 2989 (w), 2939 (w), 1776 (s), 1699 (s), 1643 (w), 1454 (m), 1352 (s), $1277(\mathrm{~m}), 1196$ (s), $1155(\mathrm{~m}), 1124(\mathrm{~m}), 1039(\mathrm{~m}), 991$ (m), $914(\mathrm{~m}), 766(\mathrm{~m}), 698$ (m) $\mathrm{cm}^{-1} ;{ }^{1} \mathrm{H}$ NMR $\left(500 \mathrm{MHz}, \mathrm{CDCl}_{3}, 298 \mathrm{~K}\right) \delta$ 7.44-7.24 (m, 5H, Ph), $5.82(\mathrm{~m}, 1 \mathrm{H}, \mathrm{H}-12), 5.63(\mathrm{~d}, 1 \mathrm{H}, J=7.3$

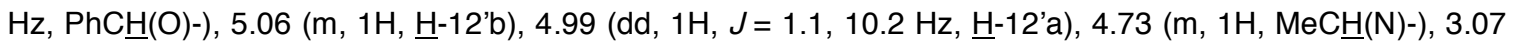

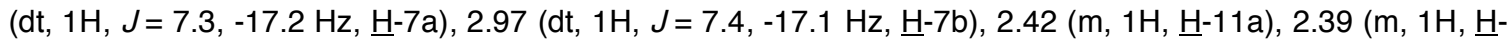
$11 b), 0.86(\mathrm{~d}, 3 \mathrm{H}, J=6.6 \mathrm{~Hz}, \underline{\mathrm{Me}} \mathrm{CH}(\mathrm{N})-)$ ppm; ${ }^{13} \mathrm{C} \mathrm{NMR}\left(125 \mathrm{MHz}, \mathrm{CDCl}_{3}, 298 \mathrm{~K}\right) \delta 172.18$ (ㅁ-6 C=O),

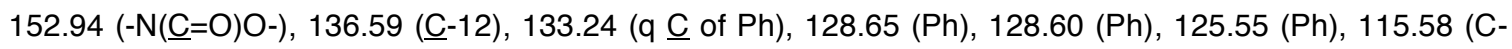

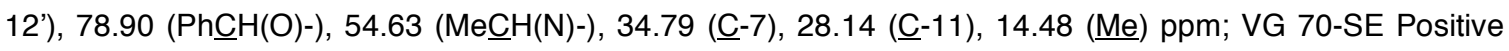
ion FAB HRMS: Calcd. for $\mathrm{C}_{15} \mathrm{H}_{17} \mathrm{NNaO}_{3}(\mathrm{M}+\mathrm{Na})^{+}:$282.11061. Found: 282.11104.

\section{Aldol Adduct 12 (II-YL-101)}

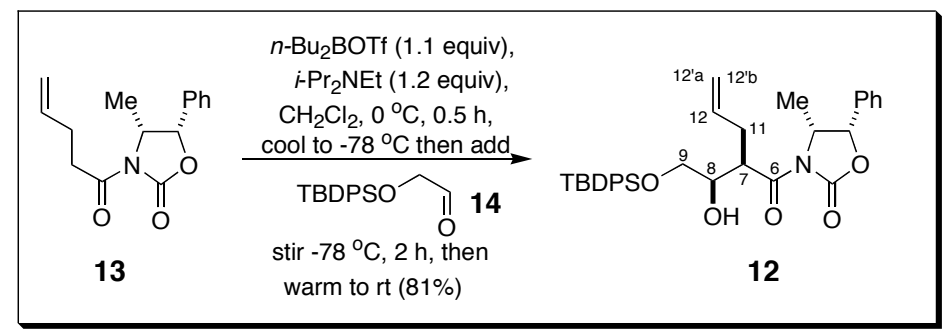


To a stirred solution of $13(32.0 \mathrm{~g}, 0.123 \mathrm{~mol})$ in dry $\mathrm{CH}_{2} \mathrm{Cl}_{2}(500 \mathrm{~mL})$ under $\mathrm{N}_{2}$ at $0{ }^{\circ} \mathrm{C}$ was added $n$-Bu $\mathrm{BOTf}_{2}$ (Aldrich $1 \mathrm{M}$ solution in $\mathrm{CH}_{2} \mathrm{Cl}_{2}, 136 \mathrm{~mL}, 0.136 \mathrm{~mol}$ ) dropwise over 5 min followed by $N, N$ diisopropylethylamine $(25.8 \mathrm{~mL}, 0.148 \mathrm{~mol})$ dropwise over $10 \mathrm{~min}$. The mixture was stirred at $0{ }^{\circ} \mathrm{C}$ for $0.5 \mathrm{~h}$ and cooled to $-78^{\circ} \mathrm{C}$ whereafter a solution of aldehyde $14^{9}(48.5 \mathrm{~g}, 0.163 \mathrm{~mol})$ in $\mathrm{CH}_{2} \mathrm{Cl}_{2}(50 \mathrm{~mL})$ was added dropwise over $10 \mathrm{~min}$. The reactants were stirred at $-78^{\circ} \mathrm{C}$ for $2 \mathrm{~h}$ and then warmed to rt. The reaction was quenched by the addition of $\mathrm{pH} 7$ aqueous $\mathrm{KH}_{2} \mathrm{PO}_{4}$ buffer solution $(50 \mathrm{~mL}$ ) (see General Methods section for details of the preparation of this buffer). The organic solvents were removed in vacuo and the concentrated residue was dissolved in $\mathrm{MeOH}(360 \mathrm{~mL})$. After cooling to $0{ }^{\circ} \mathrm{C}$, aqueous $\mathrm{H}_{2} \mathrm{O}_{2}(30 \% \mathrm{wt}, 120 \mathrm{~mL})$ was added and the reactants were stirred for $2 \mathrm{~h}$. The solvents were removed in vacuo and the crude residue was dissolved in EtOAc $(500 \mathrm{~mL})$, washed with $\mathrm{H}_{2} \mathrm{O}(100 \mathrm{~mL})$, dried over $\mathrm{MgSO}_{4}$, and filtered. The crude residue was purified by $\mathrm{SiO}_{2}$ flash chromatography (gradient elution, $\mathrm{Hex}_{\mathrm{Et}} \mathrm{O}$ 10:1 to 2:1). Aldol adduct 12 was isolated as colorless oil $(55.4 \mathrm{~g}, 81 \%)$.

Data for 12: $[\alpha]_{\mathrm{D}}-1.7^{\circ}\left(\mathrm{c} 0.648, \mathrm{CH}_{2} \mathrm{Cl}_{2}\right)$; IR 3512 (b r w), $3071(\mathrm{w}), 2934(\mathrm{~m}), 2860(\mathrm{w}), 1782(\mathrm{~s}), 1695(\mathrm{~m})$, 1639 (w), 1462 (w), 1425 (w), 1344 (s), 1194 (s), 1115 (s), 1069 (m), 1036 (w), 997 (w), 920 (w), 889 (w), 820 (w), 768 (w), 702 (s), 613 (w) cm ${ }^{-1} ;{ }^{1} \mathrm{H}$ NMR (500 MHz, $\left.\mathrm{CDCl}_{3}, 298 \mathrm{~K}\right) \delta$ 7.71-7.66 (m, 4H, Ph), 7.47-7.33 (m, 9H, Ph), 7.29-7.25 (m, 2H, Ph), 5.85 (m, 1H, H-12), 5.44 (d, 1H, J=7.2 Hz, PhCㅌ(O)-), 5.03 (m, $1 \mathrm{H}, \underline{H}-$ 12'b), 4.98 (m, 1H, $\underline{\mathrm{H}}-12 \mathrm{a}), 4.67$ (m, 1H, MeC$(\mathrm{N})-), 4.33$ (m, 1H, $\underline{\mathrm{H}}-7), 4.10$ (br dd, 1H, $\underline{\mathrm{H}}-8), 3.79$ (dd, $1 \mathrm{H}, J$ = 4.6, $10.4 \mathrm{~Hz}, \underline{\mathrm{H}}-9 \mathrm{a}), 3.75$ (dd, $1 \mathrm{H}, \mathrm{J}=6.0,10.4 \mathrm{~Hz}, \mathrm{H}-9 \mathrm{~b}), 2.79$ (br s, $1 \mathrm{H}, \mathrm{OH}), 2.55$ (m, 2H, $\underline{\mathrm{H}}-11 \mathrm{a}, \underline{\mathrm{H}}-11 \mathrm{~b})$, $1.10\left(\mathrm{~s}, 9 \mathrm{H},-\mathrm{C}(\underline{\mathrm{Me}})_{3}\right), 0.82(\mathrm{~d}, 3 \mathrm{H}, J=6.6 \mathrm{~Hz}, \underline{\mathrm{MeCH}}(\mathrm{N})-)$ ppm; ${ }^{13} \mathrm{C}$ NMR $\left(125 \mathrm{MHz}, \mathrm{CDCl}_{3}, 298 \mathrm{~K}\right) \delta 174.07$

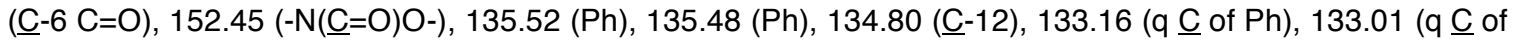
$\mathrm{Ph}), 132.92$ (q $\underline{\mathrm{C}}$ of $\mathrm{Ph}), 129.79(\mathrm{Ph}), 129.78(\mathrm{Ph}), 128.66(\mathrm{Ph}), 128.61(\mathrm{Ph}), 127.73(\mathrm{Ph}), 125.50(\mathrm{Ph})$,

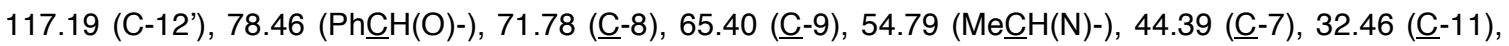
$26.79\left(-\mathrm{C}(\underline{\mathrm{Me}})_{3}\right), 19.14\left(-\underline{\mathrm{C}}(\mathrm{Me})_{3}\right), 14.44$ ( $\left.\underline{\mathrm{Me}}\right)$ ppm; VG 70-SE Positive Ion FAB HRMS: Calcd. for $\mathrm{C}_{33} \mathrm{H}_{39} \mathrm{NNaO}_{5} \mathrm{Si}(\mathrm{M}+\mathrm{Na})^{+}: 580.24951$. Found: 580.24990 .

\section{Diol 15 (II-YL-46)}

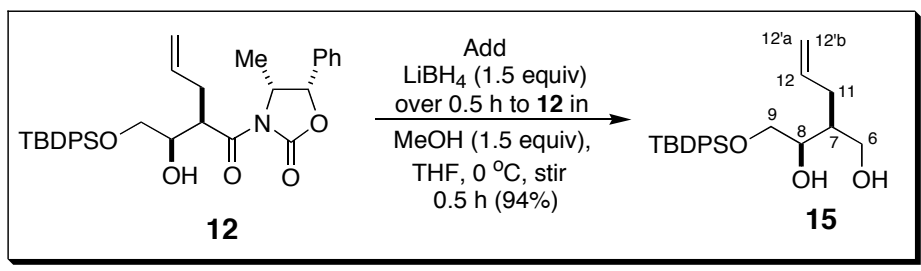

To a stirred solution of aldol adduct $12(60.8 \mathrm{~g}, 0.109 \mathrm{~mol})$ in THF (500 mL) under $\mathrm{N}_{2}$ was added $\mathrm{MeOH}$ $(6.66 \mathrm{~mL}, 0.164 \mathrm{~mol})$ and the contents of the reaction vessel cooled to $0{ }^{\circ} \mathrm{C}$. $\mathrm{LiBH}_{4}(3.58 \mathrm{~g} .0 .164 \mathrm{~mol})$ was then added portionwise over $0.5 \mathrm{~h}$. When the addition was complete, the reaction mixture was allowed to stir for a further $0.5 \mathrm{~h}$ whereafter TLC analysis indicated that the reaction was complete. The reaction was carefully quenched by adding saturated aqueous $\mathrm{NH}_{4} \mathrm{Cl}$ solution $(100 \mathrm{~mL})$, whereupon a thick white precipitate was observed. EtOAc $(500 \mathrm{~mL})$ was added and the mixed organic layer washed with $\mathrm{H}_{2} \mathrm{O}(100$ 
$\mathrm{mL}$ ), separated, and dried over $\mathrm{MgSO}_{4}$. After filtration, and concentration in vacuo, the crude residue was purified by $\mathrm{SiO}_{2}$ flash chromatography (gradient elution 10:1 to 2:1 petrol:EtOAc) to give 15 (39.2 g, $94 \%$ ) as an oil.

Data for 15: $[\alpha]_{D}-2.65^{\circ}\left(c 0.642, \mathrm{CH}_{2} \mathrm{Cl}_{2}\right)$; IR 3385 (br w), $3071(w), 3932(w), 2860(w), 1468(w), 1427(w)$, 1389 (w), 1362 (w), 1109 (s), 1040 (w), 1003 (w), 914 (w), 793 (w), 702 (s), 741 (w), 613 (m) cm ${ }^{-1} ;{ }^{1} \mathrm{H} \mathrm{NMR}$ $\left(500 \mathrm{MHz} \mathrm{CDCl}_{3}, 298 \mathrm{~K}\right) \delta$ 7.73-7.62 (m, 4H, Ph), 7.48-7.33 (m, 6H, Ph), $5.75(\mathrm{~m}, 1 \mathrm{H}, \mathrm{H}-12), 5.00(\mathrm{~m}, 1 \mathrm{H}, \underline{\mathrm{H}}-$ 12'b), 4.98 (m, 1H, ㅍ-12'a), 3.97 (m, 1H, $\underline{H}-8), 3.74$ (dd, 1H, J = 7.4, 10.4 Hz, 브-9a), 3.71 (m, 1H, $\underline{H}-9 b)$, 3.69 (dd, $1 \mathrm{H}, J=6.1,10.9 \mathrm{~Hz}, \underline{\mathrm{H}}-6 \mathrm{a}), 3.64$ (dd, $1 \mathrm{H}, J=4.0,11.0 \mathrm{~Hz}, \underline{\mathrm{H}}-6 \mathrm{~b}), 2.85$ (br s, 2H, O브-6 and O브-8), $2.11(\mathrm{~m}, 2 \mathrm{H}, \underline{\mathrm{H}}-11 \mathrm{a}, \underline{\mathrm{H}}-11 \mathrm{~b}), 1.84(\mathrm{~m}, 1 \mathrm{H}, \underline{\mathrm{H}}-7), 1.09$ (s, 9H, $\left.-\mathrm{C}(\underline{\mathrm{Me}})_{3}\right) \mathrm{ppm} ;{ }^{3} \mathrm{C} \mathrm{NMR}\left(125 \mathrm{MHz}, \mathrm{CDCl}_{3}, 298 \mathrm{~K}\right)$

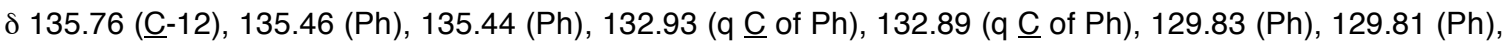

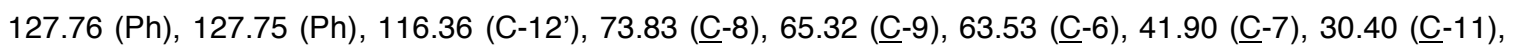
$26.78\left(-\mathrm{C}(\underline{\mathrm{Me}})_{3}\right), 19.13\left(-\underline{\mathrm{C}}(\mathrm{Me})_{3}\right)$ ppm; VG 70-SE Positive Ion FAB HRMS: Calcd. for $\mathrm{C}_{23} \mathrm{H}_{32} \mathrm{NaO}_{3} \mathrm{Si}(\mathrm{M}+\mathrm{Na})^{+}$: 407.20183. Found: 407.20203.

\section{Triol 16 (II-YL-47)}

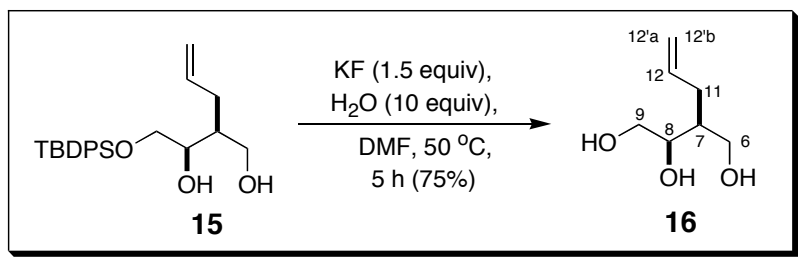

To diol 15 (40.1 g, $0.104 \mathrm{~mol})$ and KF $(9.1 \mathrm{~g}, 0.157 \mathrm{~mol})$ was successively added DMF $(200 \mathrm{~mL})$ and $\mathrm{H}_{2} \mathrm{O}$ $(18.7 \mathrm{~mL}, 1.04 \mathrm{~mol})$ and the mixture was heated at $50{ }^{\circ} \mathrm{C}$ for $5 \mathrm{~h}$. TLC analysis thereafter indicated that no starting material remained. The solvents were removed in vacuo and the crude residue was suspended in $\mathrm{CH}_{2} \mathrm{Cl}_{2}$ and the suspension loaded onto a $\mathrm{SiO}_{2}$ flash chromatographic column. Elution of the column with neat $\mathrm{CH}_{2} \mathrm{Cl}_{2}$ followed by $\mathrm{CH}_{2} \mathrm{Cl}_{2}: \mathrm{MeOH}(1: 2)$ gave triol $16(11.4 \mathrm{~g}, 75 \%)$ as a thick oil that solidified upon standing.

Data for 16: $[\alpha]_{D}+6.16^{\circ}(c 0.406, \mathrm{MeOH})$; IR (KBr) 3352 (very br s), $3078(\mathrm{~m}), 2930(\mathrm{~s}), 2889(\mathrm{~s}), 1641(\mathrm{~m})$, 1441 (s), 1418 (s), 1340 (br), 1213 (w), 1034 (s), 916 (s), 866 (w), 829 (w), 635 (m) cm-1; ${ }^{1} \mathrm{H}$ NMR (500 MHz, $\left.\mathrm{CDCl}_{3}, 298 \mathrm{~K}\right) \delta 5.74(\mathrm{~m}, 1 \mathrm{H}, \mathrm{H}-12), 5.03$ (m, 1H, $\underline{\mathrm{H}}-12$ 'b), 5.00 (m, 1H, $\underline{\mathrm{H}}-12$ 'a), 4.29 (s, 3H, C-9-OH, C-8$\mathrm{OH}, \mathrm{C}-6-\mathrm{OH}), 3.80(\mathrm{~m}, 1 \mathrm{H}, \underline{\mathrm{H}}-8), 3.64$ (dd, $1 \mathrm{H}, J=3.8,11.0 \mathrm{~Hz}, \underline{\mathrm{H}}-6 \mathrm{a}), 3.61$ (m, 2H, H-9a, H-9b), 3.56 (dd, $1 \mathrm{H}, J=7.2,11.1 \mathrm{~Hz}, \underline{\mathrm{H}}-6 \mathrm{~b}), 2.12$ (m, 1H, $\underline{\mathrm{H}}-11 \mathrm{a}), 2.02$ (m, 1H, $\underline{\mathrm{H}}-11 \mathrm{~b}), 1.73$ (m, 1H, $\underline{\mathrm{H}}-7) \mathrm{ppm} ;{ }^{13} \mathrm{C}$ NMR $(125$

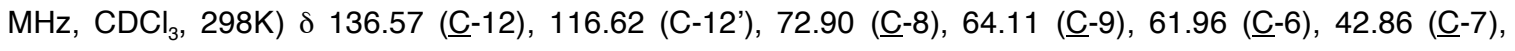
31.05 (-11) ppm; Electrospray HRMS: Calcd. for $\mathrm{C}_{7} \mathrm{H}_{15} \mathrm{O}_{3}(\mathrm{M}+\mathrm{H})^{+}:$147.10212. Found: 147.10194. 


\section{Cyclohexylidene Acetal 17 (II-YL-53)}

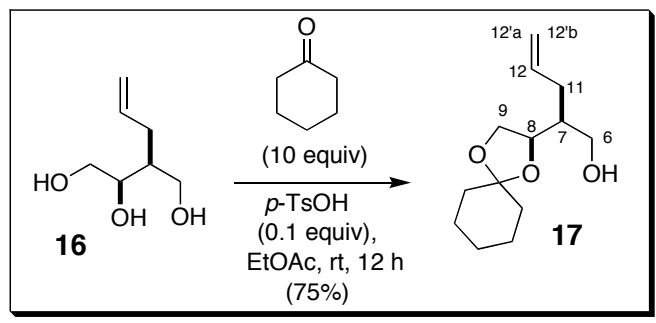

Triol 16 (13.5 g, $92.35 \mathrm{mmol}$ ), cyclohexanone (95.7 mL, $0.9235 \mathrm{~mol}, 10$ equiv), and $p-\mathrm{TsOH}$ (1.75 g, 9.2 $\mathrm{mmol}$ ) were mixed together at it under $\mathrm{N}_{2}$ and EtOAc $(500 \mathrm{~mL})$ was added. The reactants were stirred at $\mathrm{rt}$ for $12 \mathrm{~h}$ and thereafter quenched with $0.1 \mathrm{~N}$ aqueous $\mathrm{NaOH}$ solution $(50 \mathrm{~mL})$. The organic layer was separated, washed with $\mathrm{H}_{2} \mathrm{O}(50 \mathrm{~mL})$, dried $\left(\mathrm{MgSO}_{4}\right)$, filtered, and concentrated in vacuo (the majority of excess cyclohexanone was best removed under high vacuum conditions). The residue was purified by $\mathrm{SiO}_{2}$ flash chromatography (gradient elution from 10:1 to $3: 1$ petrol:EtOAc) to give alcohol 17 (15.6 g, 75\%) as a colorless oil.

Data for 17: $[\alpha]_{D}+1.17^{\circ}\left(c 1.026, \mathrm{CH}_{2} \mathrm{Cl}_{2}\right)$; IR $3445(\mathrm{br} \mathrm{m}), 3074(\mathrm{w}), 2936(\mathrm{~s}), 2860(\mathrm{~m}), 1638(\mathrm{w}), 1443$ (w), 1366 (w), $1337(w), 1281(w), 1232(w), 1163(m), 1103(s), 1040(s), 1001(w), 928(m), 845(w) c^{-1}$;

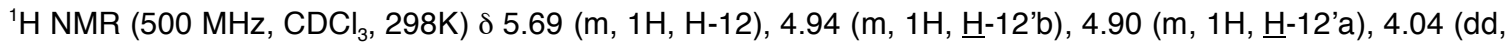
$1 \mathrm{H}, J=6.3,13.9 \mathrm{~Hz}, \underline{\mathrm{H}}-8), 3.89$ (dd, $1 \mathrm{H}, J=6.2,8.2 \mathrm{~Hz}, \underline{\mathrm{H}}-9 \mathrm{a}), 3.59$ (dd, $1 \mathrm{H}, J=7.9,8.0 \mathrm{~Hz}, \mathrm{H}-9 \mathrm{~b}), 3.47$

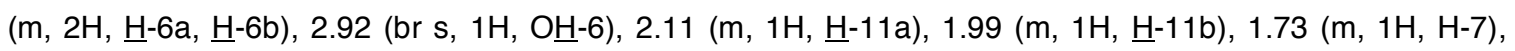
1.56-1.36 (m, $8 \mathrm{H}$, cyclohex), 1.35-1.19 (m, 2H, cyclohex) ppm; ${ }^{13} \mathrm{C}$ NMR (125 MHz, $\left.\mathrm{CDCl}_{3}, 298 \mathrm{~K}\right) \delta 136.09$ (-12), 116.29 (C-12'), 108.69 (-O-C-O-), 76.69 (C-8), 66.49 (ㄷ-9), 62.22 (C-6), 42.64 (C-7), 35.92 (cyclohex), 34.55 (cyclohex), 31.64 (-11), 24.90 (cyclohex), 23.73 (cyclohex), 23.54 (cyclohex) ppm; VG 70-SE Positive Ion FAB HRMS: Calcd. for $\mathrm{C}_{13} \mathrm{H}_{22} \mathrm{NaO}_{3}(\mathrm{M}+\mathrm{Na})^{+}: 249.14666$. Found: 249.14687.

\section{O-Benzoate 18 (II-YL-61)}

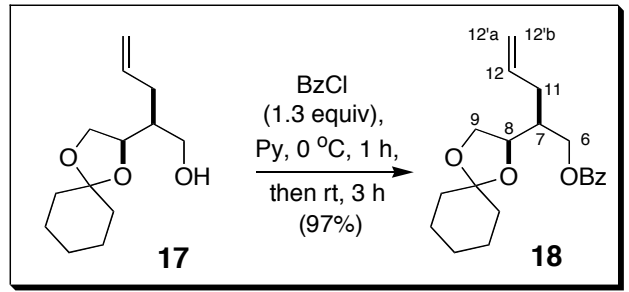

To a $0{ }^{\circ} \mathrm{C}$ solution of $17(12.1 \mathrm{~g}, 53.5 \mathrm{mmol})$ in dry pyridine $(200 \mathrm{~mL})$ under $\mathrm{N}_{2}$ was added benzoyl chloride $(8.1 \mathrm{~mL}, 69.78 \mathrm{mmol})$ dropwise over $10 \mathrm{~min}$. The mixture was stirred at $0{ }^{\circ} \mathrm{C}$ for $1 \mathrm{~h}$ and allowed to warm to rt where it was stirred for a further $3 \mathrm{~h}$. After quenching with $\mathrm{MeOH}(2 \mathrm{~mL})$ concentration in vacuo furnished a residue that was co-evaporated with $\mathrm{PhMe}(40 \mathrm{~mL})$ several times to remove residual pyridine. The residue was purified by $\mathrm{SiO}_{2}$ flash chromatography with petrol:Et $\mathrm{O}_{2}(7: 1)$ to give 18 as a colorless oil (17.2 g, 97\%). 
Data for 18: $[\alpha]_{D}+5.1^{\circ}\left(c 1.05, \mathrm{CH}_{2} \mathrm{Cl}_{2}\right)$; IR $3071(\mathrm{w}), 2937(\mathrm{~s}), 2860(\mathrm{w}), 1722(\mathrm{~s}), 1641(\mathrm{w}), 1603(\mathrm{w})$, 1448 (w), 1367 (w), 1313 (w), 1273 (s), 1167 (w), 1107 (s), 1070 (w), 1034 (w), $995(w), 930(w), 712(\mathrm{~s}) \mathrm{cm}^{-}$

1; ${ }^{1} \mathrm{H}$ NMR (500 MHz, $\left.\mathrm{CDCl}_{3}, 298 \mathrm{~K}\right) \delta 7.99(\mathrm{~m}, 2 \mathrm{H}, \mathrm{Ph}), 7.54(\mathrm{~m}, 1 \mathrm{H}, \mathrm{Ph}), 7.42(\mathrm{~m}, 2 \mathrm{H}, \mathrm{Ph}), 5.84(\mathrm{~m}, 1 \mathrm{H}, \mathrm{H}-$ 12), 5.09 (m, 1H, ㅂ-12'b), 5.05 (m, 1H, ㅂ-12'a), 4.35 (dd, 1H, J=4.7, 11.4 Hz, 브-6a), 4.20 (dd, 1H, J=6.7, $11.4 \mathrm{~Hz}, \underline{\mathrm{H}}-6 \mathrm{~b}), 4.10$ (dd, $1 \mathrm{H}, J=7.0,13.3 \mathrm{~Hz}, \mathrm{H}-8), 4.08(\mathrm{dd}, 1 \mathrm{H}, J=6.0,13.3 \mathrm{~Hz}, \underline{\mathrm{H}}-9 \mathrm{a}), 3.73(\mathrm{dd}, 1 \mathrm{H}, J$ = 7.1, $7.3 \mathrm{~Hz}, \underline{\mathrm{H}}-9 \mathrm{~b}), 2.47(\mathrm{~m}, 1 \mathrm{H}, \underline{\mathrm{H}}-11 \mathrm{a}), 2.24(\mathrm{~m}, 1 \mathrm{H}, \underline{\mathrm{H}}-11 \mathrm{~b}), 2.07(\mathrm{~m}, 1 \mathrm{H}, \underline{\mathrm{H}}-7), 1.70-1.46(\mathrm{~m}, 8 \mathrm{H}$,

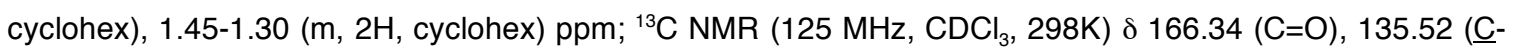
12), $133.02(\mathrm{Ph}), 130.00(\mathrm{Ph}), 129.50(\mathrm{Ph}), 128.40(\mathrm{Ph}), 117.23$ (C-12'), 109.11 (-O-C-O-), 76.21 (ㅁ-8),

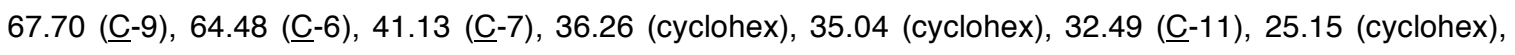
23.97 (cyclohex), 23.84 (cyclohex) ppm; VG 70-SE Positive lon FAB HRMS: Calcd. for $\mathrm{C}_{20} \mathrm{H}_{26} \mathrm{NaO}_{4}(\mathrm{M}+\mathrm{Na})^{+}$: 353.17287. Found: 353.17187.

\section{Alcohol 19 (II-YL-158)}

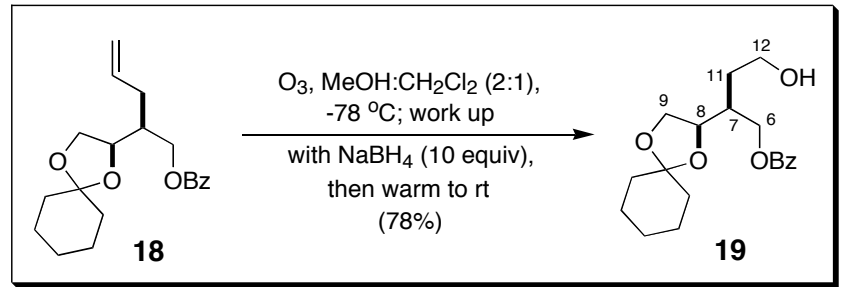

Alkene 18 (13.1 g, $39.65 \mathrm{mmol})$ was dissolved in a mixture of $\mathrm{MeOH}: \mathrm{CH}_{2} \mathrm{Cl}_{2}(2: 1,900 \mathrm{~mL})$ in a $2 \mathrm{~L}$ roundbottomed flask, and the solution was cooled to $-78{ }^{\circ} \mathrm{C}$. Ozone was continuously bubbled through the initially colorless reaction mixture until the color of the solution became blue. After this time, whilst maintaining the reaction flask at $-78{ }^{\circ} \mathrm{C}, \mathrm{NaBH}_{4}(7.5 \mathrm{~g}, 198.26 \mathrm{mmol})$ was added carefully in portions via spatula over 2-3 min. The reaction mixture was thereafter allowed to warm to $0{ }^{\circ} \mathrm{C}$ whereupon a second batch of $\mathrm{NaBH}_{4}(7.5$ $\mathrm{g}, 198.26 \mathrm{mmol}$ ) was added in one portion. After $10 \mathrm{~min}$ at $0^{\circ} \mathrm{C}, \mathrm{TLC}$ analysis revealed that a slower-moving major product had formed and that no starting alkene 18 remained. The reaction mixture was carefully quenched by the cautious dropwise addition of $3 \mathrm{~N}$ aqueous $\mathrm{AcOH}$ until $\mathrm{pH} 7$ was attained. The solvents were removed in vacuo. The residue was taken up in EtOAc $(500 \mathrm{~mL})$, washed with $\mathrm{H}_{2} \mathrm{O}(100 \mathrm{~mL})$ and brine $(100 \mathrm{~mL})$, dried $\left(\mathrm{MgSO}_{4}\right)$, filtered and concentrated in vacuo. The residue was purified by $\mathrm{SiO}_{2} \mathrm{flash}$ chromatography with petrol:EtOAc (gradient elution 5:1 to 2:1) to give the title alcohol 19 (10.3 g, 78\%).

Data for 19: $[\alpha]_{D}+6.48{ }^{\circ}\left(c 0.648, \mathrm{CH}_{2} \mathrm{Cl}_{2}\right)$; IR $3429(\mathrm{br}), 2936(\mathrm{~m}), 1719(\mathrm{~s}), 1450(\mathrm{w}), 1275(\mathrm{~s}), 1167(\mathrm{w})$, 1103 (s), 1167 (w), 1030 (w), 932 (w), 714 (m) cm ${ }^{-1} ;{ }^{1} \mathrm{H}$ NMR (500 MHz, CDCl, $\left.298 \mathrm{~K}\right) \delta 8.02-7.90(\mathrm{~m}, 2 \mathrm{H}$, $\mathrm{Ph})$, 7.57-7.47 (m, 1H, Ph), 7.45-7.33 (m, 2H, Ph), 4.30 (dd, 1H, J=4.7, 11.4 Hz, H-6a), 4.20 (dd, $1 \mathrm{H}, J=$ 6.4, $11.4 \mathrm{~Hz}, \underline{\mathrm{H}}-6 \mathrm{~b}), 4.08$ (m, 2H, H-9a, H-8), 3.77 (m, 1H, $\underline{\mathrm{H}}-12 \mathrm{a}), 3.72$ (m, 1H, $\underline{\mathrm{H}}-12 \mathrm{~b}), 3.69(\mathrm{~m}, 1 \mathrm{H}, \mathrm{H}-9 \mathrm{~b})$, 2.89 (br s, 1H, O프-12), 2.09 (m, 1H, $\underline{\mathrm{H}}-7), 1.86$ (m, 1H, H-11a), 1.74 (m, 1H, H-11b), 1.63-1.46 (m, $8 \mathrm{H}$, cyclohex), 1.40-1.28 (m, 2H, cyclohex) ppm; ${ }^{13} \mathrm{C}$ NMR (125 MHz, $\left.\mathrm{CDCl}_{3}, 298 \mathrm{~K}\right) \delta 166.29(\mathrm{C}=\mathrm{O}), 133.04$

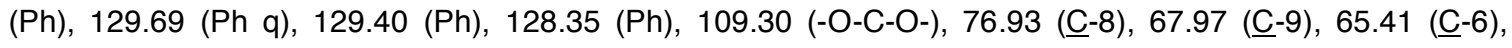




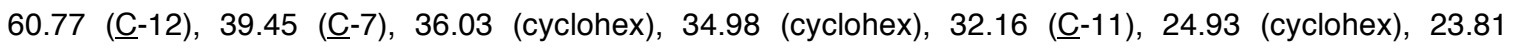
(cyclohex), 23.74 (cyclohex) ppm; VG 70-SE Positive Ion FAB HRMS: Calcd. for $\mathrm{C}_{19} \mathrm{H}_{26} \mathrm{NaO}_{5}(\mathrm{M}+\mathrm{Na})^{+}$: 357.16778. Found: 357.16823.

\section{Silyl Ether 20 (II-YL-122)}

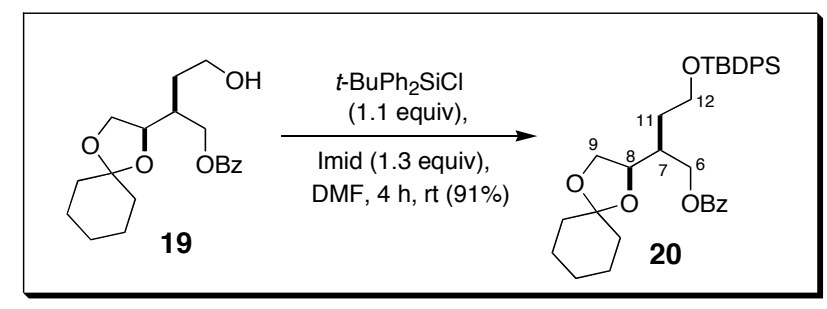

To a stirred rt solution of alcohol $19(25.4 \mathrm{~g}, 75.96 \mathrm{mmol})$ in dry DMF (200 mL) under $\mathrm{N}_{2}$ was added $t$ $\mathrm{BuPh}_{2} \mathrm{SiCl}(21.76 \mathrm{~mL}, 83.7 \mathrm{mmol})$ followed by imidazole $(6.72 \mathrm{~g}, 98.7 \mathrm{mmol})$. The reactants were stirred at rt for $4 \mathrm{~h}$ whereupon TLC analysis indicated that no starting alcohol 19 remained. $\mathrm{MeOH}(2 \mathrm{~mL}) \mathrm{was}$ added and the solvents were removed in vacuo. The oily residue was dissolved in $\mathrm{Et}_{2} \mathrm{O}(500 \mathrm{~mL})$, successively washed with $\mathrm{H}_{2} \mathrm{O}(50 \mathrm{~mL})$ and brine $(50 \mathrm{~mL})$, and then dried over $\mathrm{MgSO}_{4}$. After filtration and concentration in vacuo, the residue was purified by $\mathrm{SiO}_{2}$ flash chromatography using petrol: $\mathrm{Et}_{2} \mathrm{O}$ (gradient elution $20: 1$ to $10: 1)$ to give $20(39.7 \mathrm{~g}, 91 \%)$ as a colorless oil.

Data for 20: $[\alpha]_{D}+7.2^{\circ}\left(c 0.708, \mathrm{CH}_{2} \mathrm{Cl}_{2}\right)$; IR $2936(\mathrm{~m}), 2858(\mathrm{w}), 1720(\mathrm{~s}), 1429(\mathrm{w}), 1366(\mathrm{w}), 1273(\mathrm{~s})$, 1165 (w), 1105 (s), 1032 (w), 932 (w), 824 (w), 739 (w), 706 (s), 613 (w) cm ${ }^{-1} ;{ }^{1} \mathrm{H}$ NMR (500 MHz, CDCl

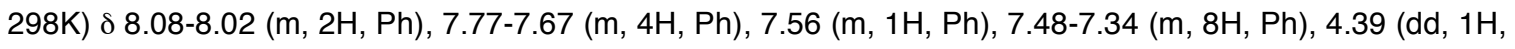
$J=4.7,11.3 \mathrm{~Hz}, \mathrm{H}-6 \mathrm{a}), 4.32$ (dd, $1 \mathrm{H}, J=6.4,11.4 \mathrm{~Hz}, \underline{\mathrm{H}}-6 \mathrm{~b}), 4.19(\mathrm{~m}, 1 \mathrm{H}, \mathrm{H}-8), 4.11$ (dd, $1 \mathrm{H}, J=6.2,8.1$ $\mathrm{Hz}, \mathrm{H}-9 \mathrm{a}), 3.88$ (m, 2H, $\underline{\mathrm{H}}-12 \mathrm{a}, \underline{\mathrm{H}}-12 \mathrm{~b}), 3.78$ (dd, 1H, J = 7.5, $7.8 \mathrm{~Hz}, \mathrm{H}-9 \mathrm{~b}), 2.25$ (m, 1H, 브-7), 2.04 (m, 1H, $\mathrm{H}-11 \mathrm{a}), 1.74(\mathrm{~m}, 1 \mathrm{H}, \mathrm{H}-11 \mathrm{~b}), 1.70-1.53(\mathrm{~m}, 8 \mathrm{H}$, cyclohex), 1.46-1.35 (m, 2H, cyclohex), $1.10(\mathrm{~s}, 9 \mathrm{H}, t-\mathrm{Bu})$ ppm; ${ }^{13} \mathrm{C}$ NMR (125 MHz, $\left.\mathrm{CDCl}_{3}, 298 \mathrm{~K}\right) \delta 166.22$ (C=O), $135.45(\mathrm{Ph}), 135.44(\mathrm{Ph}), 133.64(\mathrm{Ph}), 133.62$ (Ph), $132.87(\mathrm{Ph}), 130.01(\mathrm{Ph}), 129.54(\mathrm{Ph}), 129.44(\mathrm{Ph}), 128.30(\mathrm{Ph}), 127.57(\mathrm{Ph}), 127.56(\mathrm{Ph}), 109.07(-$

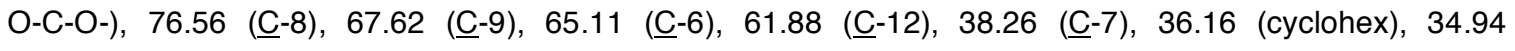
(cyclohex), 30.92 (ㅁ-11), $26.79\left(-\mathrm{C}(\underline{\mathrm{Me}})_{3}\right), 25.11$ (cyclohex), 23.88 (cyclohex), 23.77 (cyclohex), 19.08 ($\left.\underline{\mathrm{C}}(\mathrm{Me})_{3}\right)$ ppm; VG 70-SE Positive Ion FAB HRMS: Calcd. for $\mathrm{C}_{35} \mathrm{H}_{44} \mathrm{NaO}_{5} \mathrm{Si}(\mathrm{M}+\mathrm{Na})^{+}$: 595.28556. Found: 595.28414 .

\section{Alcohol 21 (II-YL-125)}

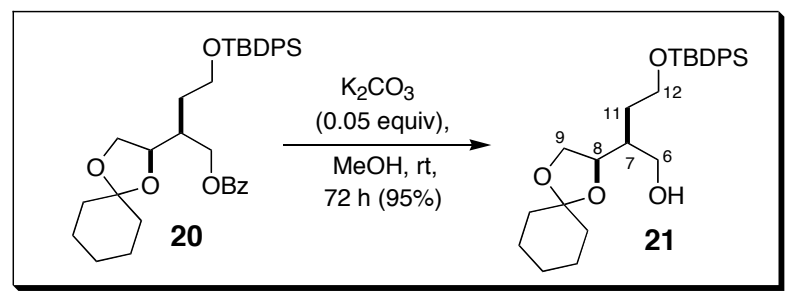


To a rt solution of $O$-benzoate $20(20.0 \mathrm{~g}, 34.9 \mathrm{mmol})$ in $\mathrm{MeOH}(50 \mathrm{~mL})$ was added $\mathrm{K}_{2} \mathrm{CO}_{3}(0.24 \mathrm{~g}, 1.74$ $\mathrm{mmol})$. The reaction mixture was stirred for $72 \mathrm{~h}$, whereafter it was concentrated in vacuo. The residue was taken up in $\mathrm{Et}_{2} \mathrm{O}(500 \mathrm{~mL})$, washed successively with $\mathrm{H}_{2} \mathrm{O}(50 \mathrm{~mL})$, brine $(50 \mathrm{~mL})$, and dried over $\mathrm{MgSO}_{4}$. After filtration, concentration in vacuo furnished a residue that was purified by $\mathrm{SiO}_{2}$ flash chromatography with petrol: $\mathrm{Et}_{2} \mathrm{O}$ (gradient elution 6:1 to 2:1). Alcohol 21 (15.6 g, 95\%) was isolated as a colorless oil.

Data for 21: $[\alpha]_{\mathrm{D}}+1.57^{\circ}\left(c 0.382, \mathrm{CH}_{2} \mathrm{Cl}_{2}\right)$; IR $3456(\mathrm{br} \mathrm{w}), 2936(\mathrm{~s}), 2860(\mathrm{~m}), 1429(\mathrm{w}), 1363(\mathrm{w}), 1279$ (w), 1161 (w), 1107 (s), 1038 (m), 934 (w), 824 (w), 739 (w), 702 (s), 613 (w), 502 (m) cm ; $^{1}$ H NMR (500 $\left.\mathrm{MHz}_{\mathrm{CDCl}}, 298 \mathrm{~K}\right) \delta$ 7.70-7.64 (m, 4H, Ph), 7.45-7.36 (m, 6H, Ph), $4.19(\mathrm{dd}, 1 \mathrm{H}, \mathrm{J}=6.3,12.7 \mathrm{~Hz}, \mathrm{H}-8)$, 4.01 (dd, $1 \mathrm{H}, J=6.3,8.2 \mathrm{~Hz}, \mathrm{H}-9 \mathrm{a}), 3.80$ (m, 1H, $\underline{\mathrm{H}}-12 \mathrm{a}), 3.74(\mathrm{~m}, 1 \mathrm{H}, \underline{\mathrm{H}}-12 \mathrm{~b}), 3.70$ (dd, $1 \mathrm{H}, J=7.8,7.9 \mathrm{~Hz}$, H-9b), 3.67 (dd, 1H, J=5.7, $11.3 \mathrm{~Hz}, \underline{\mathrm{H}}-6 \mathrm{a}), 3.60$ (dd, 1H, J=4.6, $11.3 \mathrm{~Hz}, \underline{\mathrm{H}}-6 \mathrm{~b}), 3.01$ (br s, $1 \mathrm{H}, \mathrm{OH}), 1.96$ (m, 1H, $\underline{\mathrm{H}}-7), 1.76(\mathrm{~m}, 1 \mathrm{H}, \mathrm{H}-11 \mathrm{a}), 1.64(\mathrm{~m}, 1 \mathrm{H}, \mathrm{H}-11 \mathrm{~b}), 1.64-1.47$ (m, $8 \mathrm{H}$, cyclohex), 1.43-1.33 (m, 2H, cyclohex), 1.07 (s, 9H, $t$-Bu) ppm; ${ }^{13} \mathrm{C}$ NMR (125 MHz, $\left.\mathrm{CDCl}_{3}, 298 \mathrm{~K}\right) \delta 135.45(\mathrm{Ph}), 133.23(\mathrm{Ph}), 133.21$

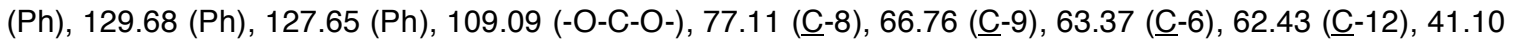

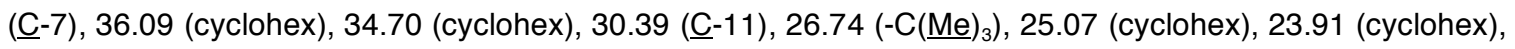
23.73 (cyclohex), 19.02 (- $\left.\underline{\mathrm{C}}(\mathrm{Me})_{3}\right)$ ppm; VG 70-SE Positive Ion FAB HRMS: Calcd. for $\mathrm{C}_{28} \mathrm{H}_{40} \mathrm{NaO}_{4} \mathrm{Si}$ $(\mathrm{M}+\mathrm{Na})^{+}:$491.25934. Found: 491.25812 .

\section{Aldehyde 23 (II-YL-166)}

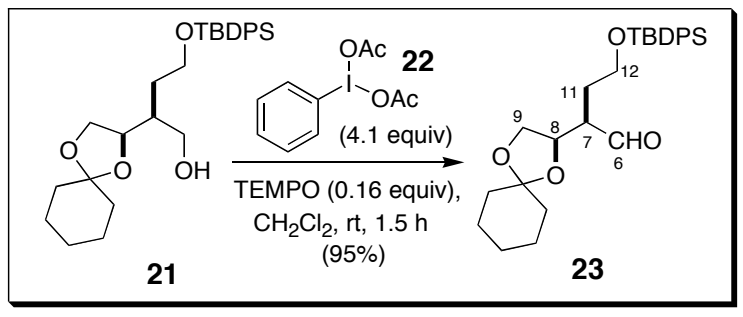

To a rt solution of alcohol 21 (13.2 g, $28.2 \mathrm{mmol}$ ) in dry $\mathrm{CH}_{2} \mathrm{Cl}_{2}(200 \mathrm{~mL})$ under $\mathrm{N}_{2}$ was added [bis(acetoxy)iodo]benzene 22 (37.2 g, $0.116 \mathrm{~mol}, 4.1$ equiv) in one portion followed by TEMPO (0.72 g, 4.61 mmol, 0.16 equiv), also in one portion. Stirring was continued for $1.5 \mathrm{~h}$ whereafter TLC analysis revealed that all of the starting alcohol $\mathbf{2 1}$ had been consumed and that a single faster-moving major product had formed. The reaction mixture was concentrated in vacuo, $\mathrm{Et}_{2} \mathrm{O}(50 \mathrm{~mL})$ was added, precipitated solids were filtered off, and the filtrate was concentrated in vacuo. The residue was purified by $\mathrm{SiO}_{2} \mathrm{flash}$ chromatography using petrol: $\mathrm{Et}_{2} \mathrm{O}$ as eluent (3:1). Aldehyde 23 (12.5 g, 95\%) was obtained as a colorless oil.

Data for 23: $[\alpha]_{D}+9.86^{\circ}\left(c 0.284, \mathrm{CH}_{2} \mathrm{Cl}_{2}\right)$; IR $2936(\mathrm{~s}), 2858(\mathrm{~m}), 1722(\mathrm{~m}), 1429(\mathrm{w}), 1364(\mathrm{w}), 1163(\mathrm{w})$, 1107 (s), 1038 (w), 932 (w), 824 (w), 739 (wm), 704 (s), 613 (w) cm-1; ${ }^{1} \mathrm{H}$ NMR (500 MHz, C $\left.6 \mathrm{D}_{6}, 298 \mathrm{~K}\right) \delta$ 9.52 (s, 1H, 트-6), 7.76-7.64 (m, 4H, Ph), 7.30-7.16 (m, 6H, Ph), 4.14 (dd, 1H, J = 6.5, $13.1 \mathrm{~Hz}, \underline{\mathrm{H}}-8), 3.99$

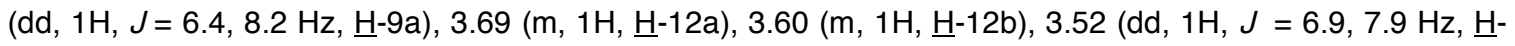


9b), 2.32 (m, 1H, $\underline{H}-7), 1.91$ (m, 1H, $\underline{H}-11 \mathrm{a}), 1.77$ (m, 1H, $\underline{H}-11 \mathrm{~b}), 1.65-1.42$ (m, $8 \mathrm{H}$, cyclohex), 1.30-1.15 (m, 2H, cyclohex), 1.12 (s, 9H, $t$-Bu) ppm; ${ }^{13} \mathrm{C}$ NMR (125 MHz, $\left.\mathrm{C}_{6} \mathrm{D}_{6}, 298 \mathrm{~K}\right) \delta 201.74$ (C-6), $135.90(\mathrm{Ph})$,

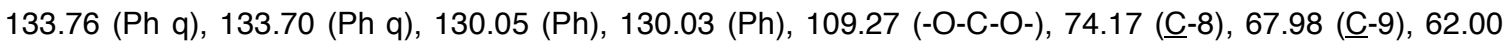

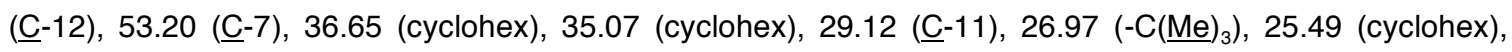
24.28 (cyclohex), 24.10 (cyclohex), $19.26\left(-\underline{\mathrm{C}}(\mathrm{Me})_{3}\right)$ ppm; VG 70-SE Positive lon FAB HRMS: Calcd. for $\mathrm{C}_{28} \mathrm{H}_{38} \mathrm{NaO}_{4} \mathrm{Si}(\mathrm{M}+\mathrm{Na})^{+}:$489.24369. Found: 489.24277.

\section{Enoate 24 (II-YL-167)}

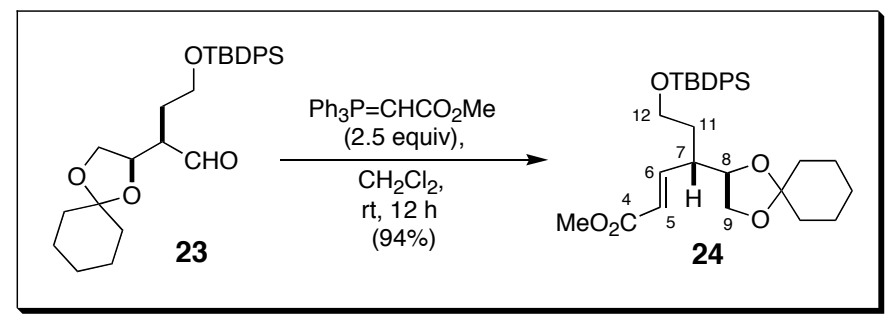

To a stirred solution of aldehyde $23(12.5 \mathrm{~g}, 26.8 \mathrm{mmol})$ in dry $\mathrm{CH}_{2} \mathrm{Cl}_{2}(200 \mathrm{~mL})$ under $\mathrm{N}_{2}$ was added (carbomethoxymethylene)triphenylphosphorane $(22.4 \mathrm{~g}, 67.0 \mathrm{mmol}, 2.5$ equiv) in one portion. The reactants were stirred at $\mathrm{rt}$ for $12 \mathrm{~h}$ before $\mathrm{Et}_{2} \mathrm{O}(250 \mathrm{~mL})$ was added. After removal of the precipitated $\mathrm{Ph}_{3} \mathrm{P}=\mathrm{O}$ by filtration, the solvents were removed in vacuo, and the residue was purified by $\mathrm{SiO}_{2}$ flash chromatography using petrol: $\mathrm{Et}_{2} \mathrm{O}(7: 1)$ as eluent. Enoate 24 (13.2 g, 94\%) was obtained as a colorless oil.

Data for 24: $[\alpha]_{D}+7.65^{\circ}\left(c 0.614, \mathrm{CH}_{2} \mathrm{Cl}_{2}\right)$; IR $2936(\mathrm{~s}), 2858(\mathrm{~m}), 1726(\mathrm{~s}), 1655(\mathrm{w}), 1431(\mathrm{w}), 1273(\mathrm{~m})$, $1217(\mathrm{w}), 1165(\mathrm{~m}), 1107(\mathrm{~s}), 1038(\mathrm{~m}), 932(\mathrm{w}), 824(\mathrm{w}), 739(\mathrm{w}), 704(\mathrm{~s}) \mathrm{cm}^{-1} ;{ }^{1} \mathrm{H}$ NMR (500 MHz, $\mathrm{C}_{6} \mathrm{D}_{6}$, 298K) ठ 7.78-7.68 (m, 4H, Ph), 7.30-7.18 (m, 6H, Ph), $6.75(\mathrm{dd}, 1 \mathrm{H}, J=9.5,15.7 \mathrm{~Hz}, \underline{\mathrm{H}}-6), 5.85(\mathrm{dd}, 1 \mathrm{H}, J=$ 0.6, $15.7 \mathrm{~Hz}, \underline{\mathrm{H}}-5), 3.71(\mathrm{~m}, 1 \mathrm{H}, \underline{\mathrm{H}}-8), 3.63(\mathrm{dd}, 1 \mathrm{H}, J=6.2,8.3 \mathrm{~Hz}, \underline{\mathrm{H}}-9 \mathrm{a}), 3.59(\mathrm{~m}, 1 \mathrm{H}, \underline{\mathrm{H}}-12 \mathrm{a}), 3.55(\mathrm{~m}, 1 \mathrm{H}$, $\underline{H}-12 b), 3.43(\mathrm{dd}, 1 \mathrm{H}, J=6.5,8.2 \mathrm{~Hz}, \mathrm{H}-9 \mathrm{~b}), 3.40(\mathrm{~s}, 3 \mathrm{H}, \mathrm{OMe}), 2.49(\mathrm{~m}, 1 \mathrm{H}, \underline{\mathrm{H}}-7), 2.06(\mathrm{~m}, 1 \mathrm{H}, \mathrm{H}-11 \mathrm{a})$, 1.70-1.48 (m, $8 \mathrm{H}$, cyclohex), 1.34 (m, 1H, H-11b), 1.30-1.18 (m, 2H, cyclohex), 1.13 (s, 9H, $t$-Bu) ppm; ${ }^{13} \mathrm{C}$

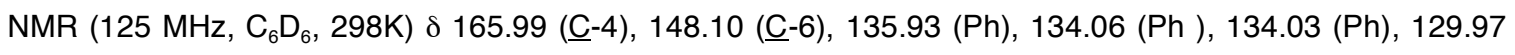

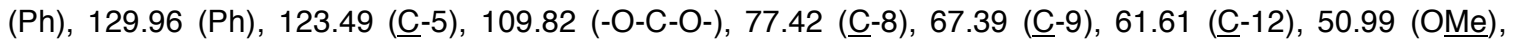

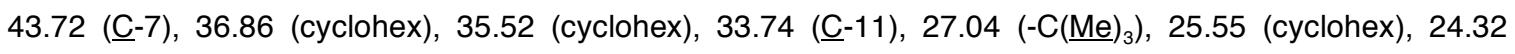
(cyclohex), 24.17 (cyclohex), 19.36 (- $\left.\underline{-}(\mathrm{Me})_{3}\right)$ ppm; VG 70-SE Positive Ion FAB HRMS: Calcd. for $\mathrm{C}_{31} \mathrm{H}_{42} \mathrm{NaO}_{5} \mathrm{Si}(\mathrm{M}+\mathrm{Na})^{+}:$545.26991. Found: 545.26894.

\section{Allylic Alcohol 11 (II-YL-168)}

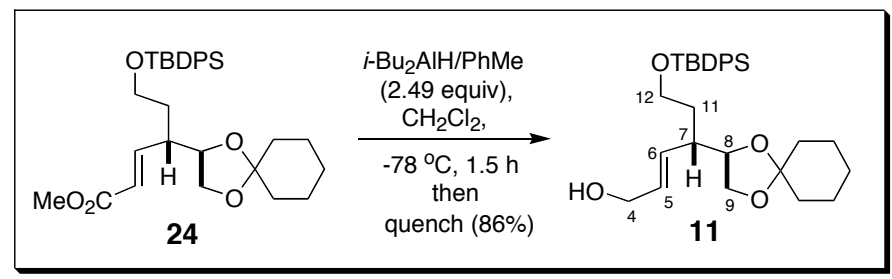


To a stirred $-78{ }^{\circ} \mathrm{C}$ solution of enoate $24(13.5 \mathrm{~g}, 25.83 \mathrm{mmol})$ in dry $\mathrm{CH}_{2} \mathrm{Cl}_{2}(200 \mathrm{~mL})$ under $\mathrm{N}_{2}$ was added $i-$ $\mathrm{Bu}_{2} \mathrm{AlH}$ (1.5 M solution in PhMe, $42.8 \mathrm{~mL}, 64.2 \mathrm{mmol}, 2.49$ equiv) dropwise over $30 \mathrm{~min}$. The mixture was stirred at $-78^{\circ} \mathrm{C}$ for $1.5 \mathrm{~h}$ whereafter TLC analysis revealed that a single slower-moving product had formed and no starting enoate 24 remained. Saturated aqueous Rochelle salt solution $(30 \mathrm{~mL})$ was cautiously added dropwise (NB effervescence occurs) followed by EtOAc (500 mL). The reaction mixture was warmed to rt and stirred for $40 \mathrm{~min}$, before being transferred to a large separatory funnel. After partitioning had occurred, the organic layer was separated and the aqueous layer was further extracted with EtOAc $(2 \times 200$ $\mathrm{mL})$. The combined organic layers were washed with $\mathrm{H}_{2} \mathrm{O}(200 \mathrm{~mL})$, dried $\left(\mathrm{MgSO}_{4}\right)$, filtered and concentrated in vacuo. The residue was purified by $\mathrm{SiO}_{2}$ flash chromatography with petrol:EtOAc (gradient elution $7: 1$ to $4: 1)$ to give allylic alcohol $11(11.0 \mathrm{~g}, 86 \%)$ as a colorless oil.

Data for 11: $[\alpha]_{D}+5.32^{\circ}\left(c\right.$ 1.17, $\left.\mathrm{CH}_{2} \mathrm{Cl}_{2}\right)$; IR 3420 (br w), $2936(\mathrm{~s}), 2860(\mathrm{~s}), 1427(\mathrm{w}), 1364(\mathrm{w}), 1279(\mathrm{w})$, 1163 (w), 1105 (s), 976 (w), 932 (w), 824 (w), 739 (w), 702 (s), 613 (w) cm ${ }^{-1} ;{ }^{1} \mathrm{H}$ NMR (500 MHz, CDCl ${ }_{3}$,

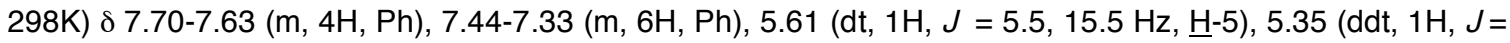
9.1, $15.5 \mathrm{~Hz}, \underline{\mathrm{H}}-6), 4.00$ (d, 1H, J = 1.3 Hz, ㅂ-4a), 3.99 (d, 1H, J = 1.3 Hz, ㅂ-4b), 3.96-3.87 (m, 2H, H-8, 브9a), $3.71(\mathrm{~m}, 1 \mathrm{H}, \underline{\mathrm{H}}-12 \mathrm{a}), 3.65(\mathrm{~m}, 1 \mathrm{H}, \underline{\mathrm{H}}-12 \mathrm{~b}), 3.64(\mathrm{~m}, 1 \mathrm{H}, \mathrm{H}-9 \mathrm{~b}), 2.42(\mathrm{~m}, 1 \mathrm{H}, \underline{\mathrm{H}}-7), 2.08(\mathrm{~m}, 1 \mathrm{H}, \mathrm{H}-11 \mathrm{a})$, 1.74 (br s, 1H, OH), 1.68-1.49 (m, $8 \mathrm{H}$, cyclohex), 1.45 (m, 1H, H-11b), 1.42-1.32 (m, 2H, cyclohex), 1.05 (s, $9 \mathrm{H}, t$-Bu) ppm; ${ }^{13} \mathrm{C}$ NMR (125 MHz, $\left.\mathrm{CDCl}_{3}, 298 \mathrm{~K}\right) \delta 135.50(\mathrm{Ph}), 133.85(\mathrm{Ph} \mathrm{q}), 133.83$ (Ph q), $131.94(\underline{\mathrm{C}}-5)$,

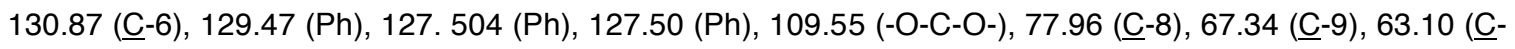

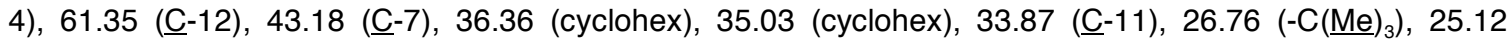
(cyclohex), 23.90 (cyclohex), 23.78 (cyclohex), 19.09 (- $\left.\underline{\mathrm{C}}(\mathrm{Me})_{3}\right)$ ppm; VG 70-SE Positive lon FAB HRMS: Calcd. for $\mathrm{C}_{30} \mathrm{H}_{42} \mathrm{NaO}_{4} \mathrm{Si}(\mathrm{M}+\mathrm{Na})^{+}:$517.27499. Found: 517.27390.

\section{Epoxy Alcohol 25 (II-YL-134)}

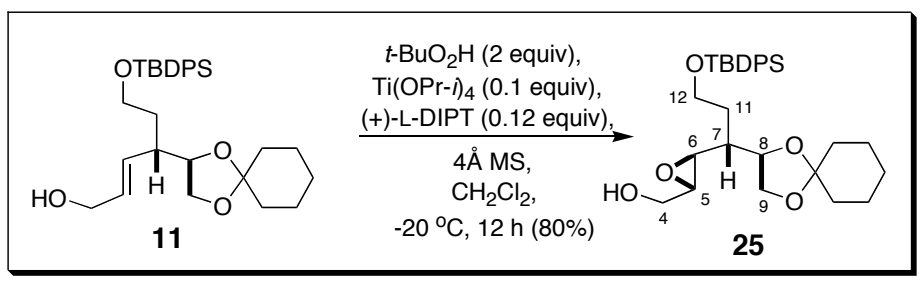

To a stirred suspension of $4 \AA$ molecular sieves $(10 \mathrm{~g})$ in dry $\mathrm{CH}_{2} \mathrm{Cl}_{2}(250 \mathrm{~mL})$ under $\mathrm{N}_{2}$ was added L-(+)diisopropyl tartrate $\left(0.637 \mathrm{~mL}, 3.03 \mathrm{mmol}, 0.12\right.$ equiv) in one portion. The mixture was cooled to $-20^{\circ} \mathrm{C}$ and $\mathrm{Ti}\left(\mathrm{OPr}-I_{4}\left(0.74 \mathrm{~mL}, 2.51 \mathrm{mmol}, 0.1\right.\right.$ equiv) was added in one portion. After $10 \mathrm{~min}, t-\mathrm{BuO}_{2} \mathrm{H}(8.69 \mathrm{M}$ solution in $\mathrm{CH}_{2} \mathrm{Cl}_{2}, 5.8 \mathrm{~mL}, 50.2 \mathrm{mmol}, 2$ equiv) was added dropwise over $5 \mathrm{~min}$. The mixture was stirred at $-20^{\circ} \mathrm{C}$ for $30 \mathrm{~min}$ whereafter a solution of allylic alcohol 11 (12.5 g, $25.27 \mathrm{mmol})$ in dry $\mathrm{CH}_{2} \mathrm{Cl}_{2}(50 \mathrm{~mL})$ was added dropwise over $30 \mathrm{~min}$. The reactants were stirred at $-20{ }^{\circ} \mathrm{C}$ for $12 \mathrm{~h}$ and then quenched with $\mathrm{H}_{2} \mathrm{O}(50 \mathrm{~mL})$. EtOAc $(500 \mathrm{~mL})$ was added and the reaction mixture was allowed to warm to $\mathrm{rt}$. The organic layer was separated and washed with $\mathrm{H}_{2} \mathrm{O}(200 \mathrm{~mL})$, dried $\left(\mathrm{MgSO}_{4}\right)$, and filtered. Following evaporation of the solvent 
in vacuo, the residue was purified by $\mathrm{SiO}_{2}$ flash chromatography with petrol:EtOAc (4:1) as eluent to give 25 $(10.3 \mathrm{~g}, 80 \%)$ as a colorless oil.

Data for 25: $[\alpha]_{D}-6.95^{\circ}\left(c 0.82, \mathrm{CH}_{2} \mathrm{Cl}_{2}\right)$; IR 3462 (br w), $2936(\mathrm{~s}), 2859(\mathrm{~m}), 1427(\mathrm{w}), 1364(\mathrm{w}), 1279(\mathrm{w})$,

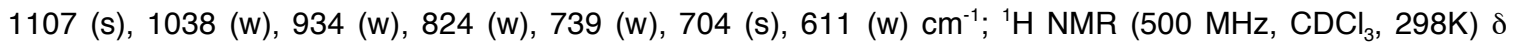
7.68-7.63 (m, 4H, Ph), 7.43-7.34 (m, 6H, Ph), $4.09(\mathrm{~m}, 1 \mathrm{H}, \underline{\mathrm{H}}-8), 3.95(\mathrm{dd}, 1 \mathrm{H}, J=6.4,8.2 \mathrm{~Hz}, \mathrm{H}-9 \mathrm{a}), 3.83$ (dd, $1 \mathrm{H}, J=2.5,12.6 \mathrm{~Hz}, \mathrm{H}-4 \mathrm{a}), 3.79$ (m, 2H, $\underline{\mathrm{H}}-12 \mathrm{a}, \underline{\mathrm{H}}-12 \mathrm{~b}), 3.64$ (dd, $1 \mathrm{H}, J=7.8,8.0 \mathrm{~Hz}, \mathrm{H}-9 \mathrm{~b}), 3.54$ (dd, $1 \mathrm{H}, J=4.4,12.6 \mathrm{~Hz}, \mathrm{H}-4 \mathrm{~b}), 2.96$ (dt, $1 \mathrm{H}, J=2.5,4.6 \mathrm{~Hz}, \underline{\mathrm{H}}-5), 2.89$ (dd, $1 \mathrm{H}, J=2.3,6.7 \mathrm{~Hz}, \underline{\mathrm{H}}-6), 1.90$ (br $\mathrm{s}, 1 \mathrm{H}, \mathrm{O} \underline{\mathrm{H}}), 1.80$ (m, 1H, $\underline{\mathrm{H}}-7), 1.77$ (m, 1H, H-11a), 1.71 (m, 1H, $\underline{\mathrm{H}}-11 \mathrm{~b}), 1.62-1.47$ (m, 8 H, cyclohex), 1.43$1.33\left(\mathrm{~m}, 2 \mathrm{H}\right.$, cyclohex), 1.04 (s, 9H, t-Bu) ppm; ${ }^{13} \mathrm{C} \mathrm{NMR}\left(125 \mathrm{MHz}, \mathrm{CDCl}_{3}, 298 \mathrm{~K}\right) \delta 135.52(\mathrm{Ph}), 135.51$

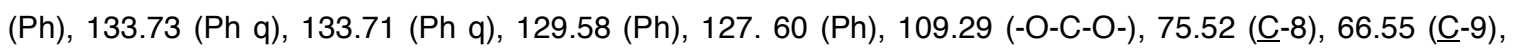

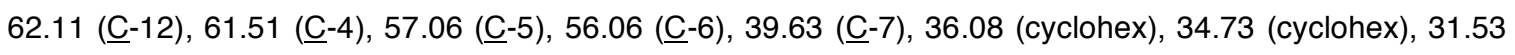

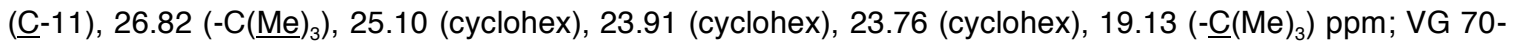
SE Positive Ion FAB HRMS: Calcd. for $\mathrm{C}_{30} \mathrm{H}_{42} \mathrm{NaO}_{5} \mathrm{Si}(\mathrm{M}+\mathrm{Na})^{+}$: 533.26991. Found: 533.27059.

\section{lodo Epoxide 10 (II-YL-136)}

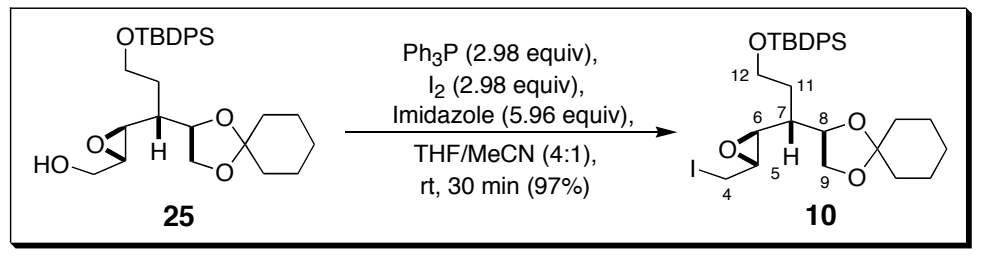

To a vigorously stirred rt solution of epoxy alcohol 25 (4.4 g, $8.62 \mathrm{mmol})$ in dry THF:MeCN $(4: 1,100 \mathrm{~mL})$ under $\mathrm{N}_{2}$ was added imidazole $(3.5 \mathrm{~g}, 51.41 \mathrm{mmol}), \mathrm{Ph}_{3} \mathrm{P}(6.73 \mathrm{~g}, 25.66 \mathrm{mmol})$ and $\mathrm{I}_{2}(6.52 \mathrm{~g}, 25.69 \mathrm{mmol})$ successively in single portions. Stirring was continued at rt for $0.5 \mathrm{~h}$ whereupon $\mathrm{Et}_{2} \mathrm{O}(200 \mathrm{~mL})$ was added to precipitate out $\mathrm{Ph}_{3} \mathrm{P}=\mathrm{O}$. The solids were filtered through a short pad of $\mathrm{SiO}_{2}$ gel, and the filter pad was washed with $\mathrm{Et}_{2} \mathrm{O}$ until TLC analysis revealed that no more of 10 was emerging in the filtrate. The filtrate was concentrated in vacuo and the residue was purified by $\mathrm{SiO}_{2}$ flash chromatography using petrol: $\mathrm{Et}_{2} \mathrm{O}$ (7:1) as eluent. lodide 10 (5.2 g, 97\%) was obtained as colorless oil.

Data for 10: $[\alpha]_{D}+0.95^{\circ}\left(c 0.632, \mathrm{CH}_{2} \mathrm{Cl}_{2}\right)$; IR $2934(\mathrm{~s}), 2858(\mathrm{~m}), 1429(\mathrm{w}), 1364(\mathrm{w}), 1163(\mathrm{w}), 1105(\mathrm{~s})$, 1038 (w), 934 (w), 897 (w), $822(w), 739$ (w), 702 (s), 611 (w) cm ${ }^{-1} ;{ }^{1} \mathrm{H}$ NMR (500 MHz, $\left.\mathrm{C}_{6} \mathrm{D}_{6}, 298 \mathrm{~K}\right) \delta 7.82-$ 7.75 (m, 4H, Ph), 7.24-7.20 (m, 6H, Ph), 3.85-3.80 (m, 4H, H-8, H-12a, H-12b, H-9a), $3.49(\mathrm{~m}, 1 \mathrm{H}, \mathrm{H}-9 \mathrm{~b})$, $2.63(\mathrm{~m}, 1 \mathrm{H}, \mathrm{H}-5), 2.62$ (m, 1H, $\underline{\mathrm{H}}-4 \mathrm{a}), 2.42$ (m, 1H, $\underline{\mathrm{H}}-4 \mathrm{~b}), 2.33$ (dd, 1H, J = 1.6, $7.2 \mathrm{~Hz}, \underline{\mathrm{H}}-6), 1.94$ (m, $1 \mathrm{H}$, ㅂ-11a), 1.72 (m, 1H, $\underline{\mathrm{H}}-11 \mathrm{~b}), 1.67-1.54$ (m, $8 \mathrm{H}$, cyclohex), 1.53 (m, 1H, H-7), 1.32-1.22 (m, 2H, cyclohex), $1.16(\mathrm{~s}, 9 \mathrm{H}, t-\mathrm{Bu}) \mathrm{ppm} ;{ }^{13} \mathrm{C}$ NMR (125 MHz, $\left.\mathrm{C}_{6} \mathrm{D}_{6}, 298 \mathrm{~K}\right) \delta 136.01(\mathrm{Ph}), 135.98(\mathrm{Ph}), 134.25(\mathrm{Ph} \mathrm{q}), 134.22$

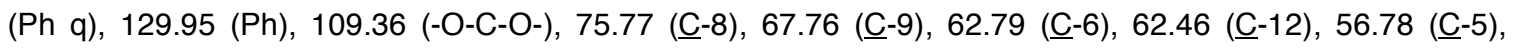

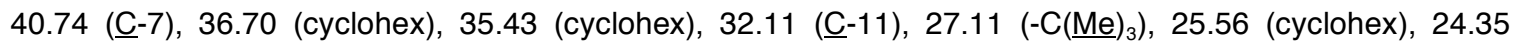

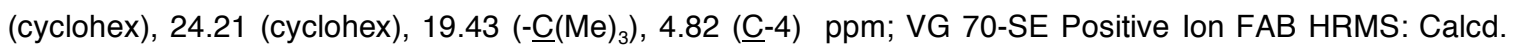
for $\mathrm{C}_{30} \mathrm{H}_{41} \mathrm{INaO}_{4} \mathrm{Si}(\mathrm{M}+\mathrm{Na})^{+}:$643.17164. Found: 643.17024. 
Allylic Alcohol 26 (II-YL-137)

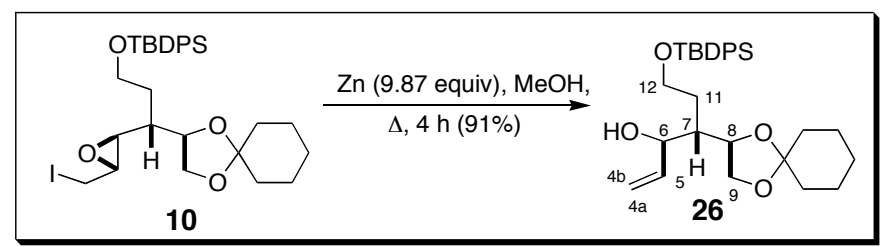

To a vigorously stirred solution of iodide 10 (5.0 g, $8.06 \mathrm{mmol})$ in $\mathrm{MeOH}(100 \mathrm{~mL})$ was added $\mathrm{Zn}$ dust (5.2 g, $79.52 \mathrm{mmol}$ ) and the reaction mixture was heated at reflux for $4 \mathrm{~h}$. After cooling to $\mathrm{rt}$, it was filtered through a short pad of $\mathrm{SiO}_{2}$, which was washed well with $\mathrm{MeOH}$. Concentration of the filtrate in vacuo and purification of the residue by $\mathrm{SiO}_{2}$ flash chromatography with petrol:EtOAc (7:1) as eluent furnished allylic alcohol 26 $(3.61 \mathrm{~g}, 91 \%)$ as a colorless oil.

Data for 26: $[\alpha]_{D}+17.3^{\circ}\left(c 0.902, \mathrm{CH}_{2} \mathrm{Cl}_{2}\right)$; IR 3452 (br w), $2936(\mathrm{~s}), 2858(\mathrm{~m}), 1429(\mathrm{w}), 1364(\mathrm{w}), 1281(\mathrm{w})$, 1161 (w), 1107 (s), 1038 (m), 999 (m), 932 (m), 824 (w), 739 (w), 704 (s), 613 (w) cm ${ }^{-1} ;{ }^{1} \mathrm{H}$ NMR (500 MHz, $\left.\mathrm{CDCl}_{3}, 298 \mathrm{~K}\right) \delta$ 7.70-7.64 (m, 4H, Ph), 7.45-7.34 (m, 6H, Ph), 5.83 (ddd, $\left.1 \mathrm{H}, J=5.2,10.5,17.1 \mathrm{~Hz}, \underline{\mathrm{H}}-5\right)$, 5.28 (ddd, $J=1.6,1.6,17.2 \mathrm{~Hz}, \underline{\mathrm{H}}-4 \mathrm{~b}), 5.14$ (ddd, $1 \mathrm{H}, J=1.6,1.6,10.5 \mathrm{~Hz}, \mathrm{H}-4 \mathrm{a}), 4.28$ (m, 1H, H-8), 4.25 $(\mathrm{m}, 1 \mathrm{H}, \underline{\mathrm{H}}-6), 4.03$ (dd, $1 \mathrm{H}, J=6.3,8.2 \mathrm{~Hz}, \underline{\mathrm{H}}-9 \mathrm{a}), 3.77(\mathrm{~m}, 1 \mathrm{H}, \mathrm{H}-12 \mathrm{a}), 3.73(\mathrm{dd}, 1 \mathrm{H}, J=7.7,7.9 \mathrm{~Hz}, \underline{\mathrm{H}}-9 \mathrm{~b})$, 3.69 (m, 1H, H-12b), 3.16 (br s, 1H, Oㅡㅡ), 1.86 (m, 1H, 브-7), 1.82-1.76 (m, 2H, 브-11a, $\underline{\mathrm{H}}-11 \mathrm{~b}), 1.63-1.45$ (m, $8 \mathrm{H}$, cyclohex), 1.43-1.30 (m, 2H, cyclohex), $1.07(\mathrm{~s}, 9 \mathrm{H}, t-\mathrm{Bu}) \mathrm{ppm} ;{ }^{13} \mathrm{C} \mathrm{NMR}\left(125 \mathrm{MHz}, \mathrm{CDCl}_{3}, 298 \mathrm{~K}\right) \delta$ 139.34 (C-5), 135.51 (Ph), 133.31 (Ph q), $129.64(\mathrm{Ph}), 129.62(\mathrm{Ph}), 127.62(\mathrm{Ph}), 115.03$ (C-4), 109.04 (-O-

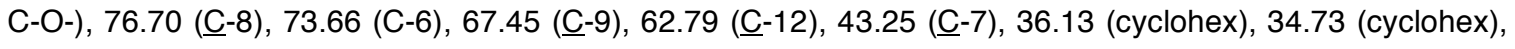
27.68 (ㅁ-11), $26.78\left(-\mathrm{C}(\underline{\mathrm{Me}})_{3}\right), 25.11$ (cyclohex), 23.93 (cyclohex), 23.79 (cyclohex), $19.06\left(-\underline{\mathrm{C}}(\mathrm{Me})_{3}\right) \mathrm{ppm}$; VG 70-SE Positive Ion FAB HRMS: Calcd. for $\mathrm{C}_{30} \mathrm{H}_{42} \mathrm{NaO}_{4} \mathrm{Si}(\mathrm{M}+\mathrm{Na})^{+}:$517.27499. Found: 517.27438.

\section{Diol 9 (II-YL-138)}

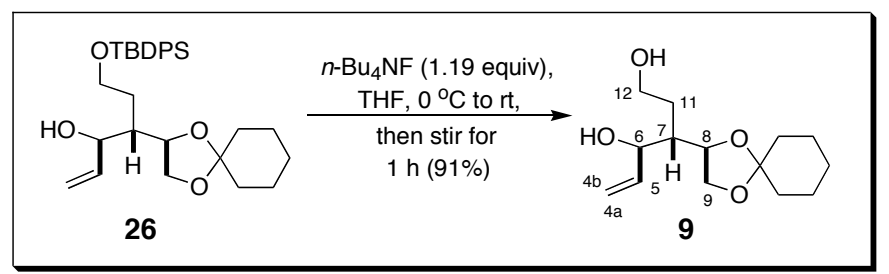

To a stirred solution of $O$-silyl ether $26(8.7 \mathrm{~g}, 17.59 \mathrm{mmol})$ in dry THF $(150 \mathrm{~mL})$ under $\mathrm{N}_{2}$ at $0{ }^{\circ} \mathrm{C}$ was added tetra- $n$-butylammonium fluoride (1 M solution in THF, $20.9 \mathrm{~mL}, 20.9 \mathrm{mmol}, 1.19$ equiv) in one portion. The cooling bath was removed and the reaction mixture was allowed to stir at $\mathrm{rt}$ for $1 \mathrm{~h}$, whereafter it was concentrated in vacuo. The residue was purified by $\mathrm{SiO}_{2}$ flash chromatography with petrol:EtOAc (1:2) as eluent; diol 9 (4.1 g, 91\%) was obtained as a colorless oil. 
Data for 9: $[\alpha]_{D}+25.5^{\circ}\left(c 0.318, \mathrm{CH}_{2} \mathrm{Cl}_{2}\right)$; IR $3389(\mathrm{br} \mathrm{m}), 2935(\mathrm{~s}), 2860(\mathrm{~m}), 1645(\mathrm{w}), 1447(\mathrm{w}), 1366(\mathrm{w})$, 1279 (w), 1232 (m), 1103 (s), 1038 (m), 995 (m), 930 (s) cm ${ }^{-1} ;{ }^{1} \mathrm{H}$ NMR (500 MHz, CDCl $\left.3,298 \mathrm{~K}\right) \delta 5.81$ (ddd, $1 \mathrm{H}, J=5.3,10.5,17.2 \mathrm{~Hz}, \underline{\mathrm{H}}-5$ ), 5.23 (ddd, $J=1.6,1.6,17.2 \mathrm{~Hz}, \underline{\mathrm{H}}-4 \mathrm{~b}$ ), 5.14 (ddd, $1 \mathrm{H}, J=1.5,1.6$, $10.6 \mathrm{~Hz}, \mathrm{H}-4 \mathrm{a}), 4.17(\mathrm{~m}, 1 \mathrm{H}, \mathrm{H}-8), 4.11(\mathrm{~m}, 1 \mathrm{H}, \underline{\mathrm{H}}-6), 4.07$ (dd, 1H, J=6.0, 8.1 Hz, H-9a), 3.66 (br m, 2H, 브12a, and $\underline{\mathrm{H}}-12 \mathrm{~b})$, superimposed upon 3.65 (dd, $1 \mathrm{H}, J=8.0,8.0 \mathrm{~Hz}, \underline{\mathrm{H}}-9 \mathrm{~b}), 3.45$ (br t, $1 \mathrm{H}, \mathrm{OH}-12), 3.41$ (br d, $1 \mathrm{H}, J=4.5 \mathrm{~Hz}, \mathrm{O} \underline{\mathrm{H}}-6), 1.80-1.67$ (m, 3H, ㅂ-11a, 브-11b, $\underline{\mathrm{H}}-7), 1.62-1.42$ (m, $8 \mathrm{H}$, cyclohex), 1.41-1.26 (m,

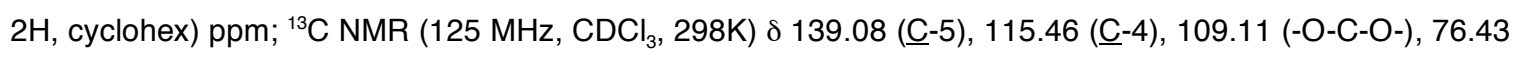

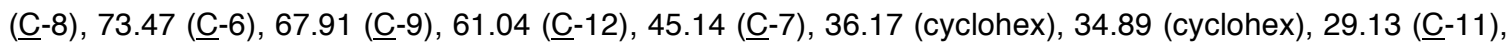
25.02 (cyclohex), 23.91 (cyclohex), 23.80 (cyclohex) ppm; VG 70-SE Positive lon FAB HRMS: Calcd. for $\mathrm{C}_{14} \mathrm{H}_{24} \mathrm{NaO}_{4}(\mathrm{M}+\mathrm{Na})^{+}:$279.15722. Found: 279.15731 .

\section{$\boldsymbol{\gamma}$-Butyrolactone 7 (II-YL-140)}

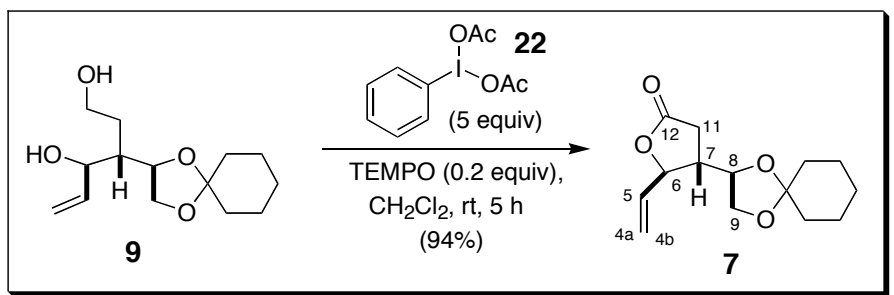

To a stirred rt solution of diol $9(1.07 \mathrm{~g}, 4.17 \mathrm{mmol})$ in dry $\mathrm{CH}_{2} \mathrm{Cl}_{2}(40 \mathrm{~mL})$ under $\mathrm{N}_{2}$ was sequentially added [bis(acetoxy)iodo]benzene 22 (6.73 g, $20.89 \mathrm{mmol}, 5$ equiv) and TEMPO (131 mg, $0.838 \mathrm{mmol}, 0.2$ equiv) in single portions. The reactants were stirred at $\mathrm{rt}$ for $5 \mathrm{~h}$ whereafter TLC analysis indicated that a single fastermoving product had formed. Saturated aqueous sodium thiosulfate $(50 \mathrm{~mL})$ and $\mathrm{Et}_{2} \mathrm{O}(100 \mathrm{~mL})$ were added, and the organic layer was separated and washed successively with saturated aqueous $\mathrm{NaHCO}_{3}(10 \mathrm{~mL})$ and $\mathrm{H}_{2} \mathrm{O}(10 \mathrm{~mL})$ before being dried $\left(\mathrm{MgSO}_{4}\right)$, filtered, and concentrated in vacuo. Purification of the residue by $\mathrm{SiO}_{2}$ flash chromatography gave the lactone $7(0.99 \mathrm{~g}, 94 \%)$ as a colorless oil.

Data for 7: $[\alpha]_{D}+48.6^{\circ}\left(c 0.42, \mathrm{CH}_{2} \mathrm{Cl}_{2}\right)$; IR 2937 (s), 2860 (w), 1782 (s), 1425 (w), 1366 (w), 1273 (w), 1169 (m), 1105 (s), 991 (w), $934(\mathrm{~m}), 853(\mathrm{w}) \mathrm{cm}^{-1} ;{ }^{1} \mathrm{H}$ NMR (500 MHz, $\left.\mathrm{CDCl}_{3}, 298 \mathrm{~K}\right) \delta 5.80$ (ddd, 1H, J=6.8, 10.4, 17.2 Hz, ㅂ-5), 5.35 (ddd, J=1.1, 1.1, $17.1 \mathrm{~Hz}, \underline{\mathrm{H}}-4 \mathrm{~b}), 5.25$ (ddd, $1 \mathrm{H}, J=1.0,1.0,10.4 \mathrm{~Hz}, \underline{\mathrm{H}}-4 \mathrm{a}), 4.70$ (dd, $1 \mathrm{H}, J=7.0,7.0 \mathrm{~Hz}$, ㅌ-6), 4.12 (ddd, $1 \mathrm{H}, J=4.3,6.6,10.8 \mathrm{~Hz}, \underline{\mathrm{H}}-8$ ), 4.01 (dd, $1 \mathrm{H}, J=6.5,8.3 \mathrm{~Hz}, \mathrm{H}-$ 9a), 3.50 (dd, $1 \mathrm{H}, J=6.7,8.3 \mathrm{~Hz}, \underline{\mathrm{H}}-9 \mathrm{~b}), 2.61$ (dd, $1 \mathrm{H}, J=8.6,17.5 \mathrm{~Hz}, \underline{\mathrm{H}}-11 \mathrm{a}), 2.50(1 \mathrm{H}, J=8.7,17.5 \mathrm{~Hz}$, $\underline{\mathrm{H}}-11 \mathrm{~b}), 2.35(\mathrm{~m}, 1 \mathrm{H}, \underline{\mathrm{H}}-7), 1.62-1.42$ (m, $8 \mathrm{H}$, cyclohex), 1.41-1.28 (m, 2H, cyclohex) ppm; ${ }^{13} \mathrm{C} \mathrm{NMR}(125$

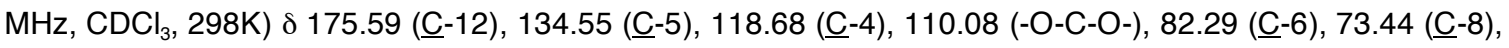

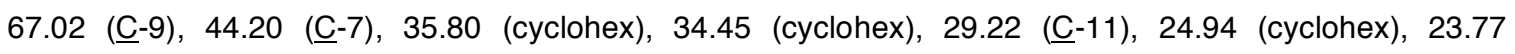
(cyclohex), 23.59 (cyclohex) ppm; VG 70-SE Positive Ion FAB HRMS: Calcd. for $\mathrm{C}_{14} \mathrm{H}_{20} \mathrm{NaO}_{4}(\mathrm{M}+\mathrm{Na})^{+}$: 275.12592. Found: 275.12622 . 


\section{$\gamma$-Butyrolactone O-Pivaloate 41 (II-YL-174)}

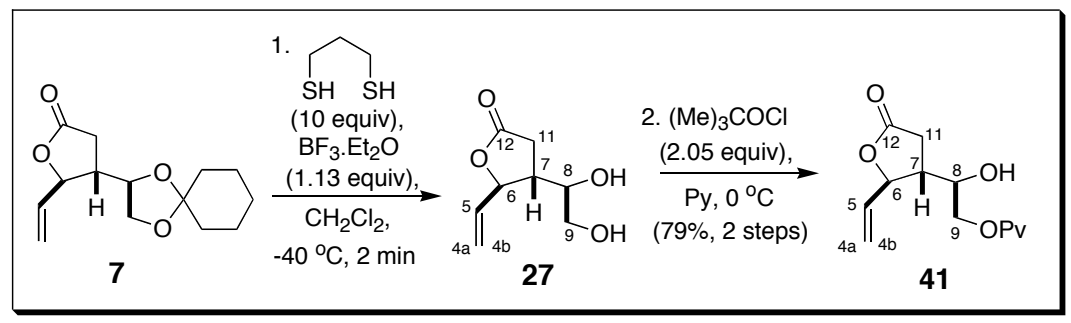

\section{Part 1. Preparation and Partial Purification of Diol 27 (II-YL-182)}

To a stirred $-40{ }^{\circ} \mathrm{C}$ solution of cyclohexylidene acetal $7(0.3 \mathrm{~g}, 1.19 \mathrm{mmol})$ in dry $\mathrm{CH}_{2} \mathrm{Cl}_{2}(6 \mathrm{~mL})$ under $\mathrm{N}_{2}$ was added 1,3-propanedithiol (1.2 mL, $11.95 \mathrm{mmol}, 10$ equiv) in one portion followed by boron trifluoride etherate ( $0.17 \mathrm{~mL}, 1.34 \mathrm{mmol}, 1.13$ equiv). After $2 \mathrm{~min}$ at $-40{ }^{\circ} \mathrm{C}$, TLC analysis of the reaction mixture indicated that no starting acetal 7 remained, and that a slower-moving major product had formed. The reaction was quenched by adding $\mathrm{Et}_{3} \mathrm{~N}(2 \mathrm{~mL})$. The reaction mixture was then applied directly to the top of a $\mathrm{SiO}_{2}$ flash chromatography column which was eluted initially with $\mathrm{CH}_{2} \mathrm{Cl}_{2}$ to remove the excess thiol and 1,3-dithiane by-product. Further elution with 10:1 $\mathrm{CH}_{2} \mathrm{Cl}_{2}: \mathrm{MeOH}$ eventually provided the desired diol $27(0.205 \mathrm{~g})$ as a slightly yellow oil after concentration in vacuo. It was used directly for the next step without further chromatographic purification to minimise the risk of lactone migration to the $C(8)$-hydroxyl. In this regard, we recommend that the $C(8)$ and $C(9)$-hydroxyls of $\mathbf{2 7}$ be immediately protected and that the compound be used directly for the next reaction without being stored at this stage.

Data for 27: $[\alpha]_{D}+55.25^{\circ}(c 0.724, \mathrm{MeOH})$; IR 3385 (br s), $2932(w), 1767(\mathrm{~s}), 1639(\mathrm{w}), 1556(\mathrm{w}), 1421(\mathrm{w})$, $1271(\mathrm{w}), 1204(\mathrm{~s}), 1080(\mathrm{~m}), 991(\mathrm{~m}), 945(\mathrm{~m}), 887(\mathrm{w}) \mathrm{cm}^{-1} ;{ }^{1} \mathrm{H}$ NMR (500 MHz, CDCl, $\left.298 \mathrm{~K}\right) \delta 5.83$ (ddd, $1 \mathrm{H}, J=7.0,10.4,17.2 \mathrm{~Hz}, \underline{\mathrm{H}}-5$ ), 5.39 (ddd, $J=1.0,1.0,17.1 \mathrm{~Hz}, \underline{\mathrm{H}}-4 \mathrm{~b}$ ), 5.30 (ddd, $1 \mathrm{H}, J=1.0,1.0,10.4 \mathrm{~Hz}$, $\underline{H}-4 a), 4.82$ (br m, 1H, $\underline{H}-6$ ), 3.79 (ddd, $1 \mathrm{H}, J=3.5,3.8,7.3 \mathrm{~Hz}, \underline{\mathrm{H}}-8$ ), 3.62 (dd, $1 \mathrm{H}, J=3.5,11.0 \mathrm{~Hz}, \mathrm{H}-9 \mathrm{a}$ ), $3.46(\mathrm{dd}, 1 \mathrm{H}, J=7.7,11.0 \mathrm{~Hz}, \underline{\mathrm{H}}-9 \mathrm{~b}), 2.73(\mathrm{dd}, 1 \mathrm{H}, J=9.3,17.6 \mathrm{~Hz}, \underline{\mathrm{H}}-11 \mathrm{a})$ superimposed upon $2.73(\mathrm{br} \mathrm{s}$, $2 \mathrm{H}, \mathrm{OH}$ ), 2.47 (dd, $1 \mathrm{H}, J=8.9,17.6 \mathrm{~Hz}, \underline{\mathrm{H}}-11 \mathrm{~b}), 2.31$ (m, $1 \mathrm{H}, \underline{\mathrm{H}}-7$ ), ppm; ${ }^{13} \mathrm{C} \mathrm{NMR}\left(125 \mathrm{MHz}, \mathrm{CDCl}_{3}, 298 \mathrm{~K}\right)$

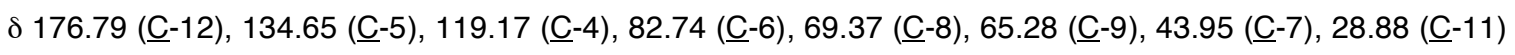
ppm; Cl HRMS: Calcd. for $\mathrm{C}_{8} \mathrm{H}_{13} \mathrm{O}_{4}(\mathrm{M}+\mathrm{H})^{+}:$173.08138. Found: 173.08184 .

\section{Part 2. Preparation of O-Pivaloate 41 (II-YL-174)}

To diol 27 (0.205 g, ca $1.19 \mathrm{mmol})$ in dry pyridine $(3 \mathrm{~mL})$ at $0{ }^{\circ} \mathrm{C}$ was added pivaloyl chloride $(0.3 \mathrm{~mL}, 2.44$ mmol, 2.05 equiv) over $2 \mathrm{~min}$. The reactants were stirred under $\mathrm{N}_{2}$ at $0{ }^{\circ} \mathrm{C}$ for $1 \mathrm{~h}$ and then allowed to warm to $\mathrm{rt}$, where they were kept for $12 \mathrm{~h}$. $\mathrm{MeOH}(1 \mathrm{~mL})$ was added, and the reaction mixture was diluted with EtOAc $(25 \mathrm{~mL})$, filtered through a short pad of $\mathrm{SiO}_{2}$ and the filtrate evaporated in vacuo. The residue was purified by $\mathrm{SiO}_{2}$ flash chromatography with petrol:EtOAc (2:1) as eluent to give O-pivaloate $41(0.24 \mathrm{~g}, 79 \%)$ as slightly yellow oil.

(Again, due to the potential for lactone migration involving the $\mathrm{C}(8)-\mathrm{OH}$, we recommend that $\mathbf{4 1}$ be used directly for the next step.) 
Data for 41: $[\alpha]_{\mathrm{D}}+36.14^{\circ}\left(\mathrm{c} 0.498, \mathrm{CH}_{2} \mathrm{Cl}_{2}\right)$; IR $3468(\mathrm{br} \mathrm{m}), 2972(\mathrm{~m}), 2937(\mathrm{w}), 2878(\mathrm{w}), 1773(\mathrm{~s}), 1728$

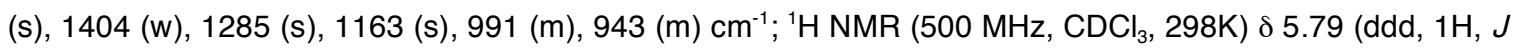
$=7.1,10.4,17.3 \mathrm{~Hz}, \underline{\mathrm{H}}-5$ ), 5.36 (d of narrow t, $J=17.1 \mathrm{~Hz}, \underline{\mathrm{H}}-4 \mathrm{~b}$ ), 5.27 (d, $1 \mathrm{H}, J=10.4 \mathrm{~Hz}, \underline{\mathrm{H}}-4 \mathrm{a}$ ), 4.79 (dd, $1 \mathrm{H}, J=7.2,8.0 \mathrm{~Hz}, \underline{\mathrm{H}}-6), 4.04$ (dd, $1 \mathrm{H}, J=4.4,11.5 \mathrm{~Hz}, \underline{\mathrm{H}}-9 \mathrm{a}), 3.95$ (dd, $1 \mathrm{H}, J=6.5,11.5 \mathrm{~Hz}, \underline{\mathrm{H}}-9 \mathrm{~b}), 3.89$ (br m, 1H, ㄱ-8), 3.13 (br d, J=4.2 Hz, C-8-OH), 2.74 (dd, 1H, J=9.7, $17.5 \mathrm{~Hz}, \underline{\mathrm{H}}-11 \mathrm{a}), 2.45$ (dd, $1 \mathrm{H}, J=$ 8.8, $17.5 \mathrm{~Hz}, \underline{\mathrm{H}}-11 \mathrm{~b}), 2.31$ (br ddd, $1 \mathrm{H}, J=3.1,8.8,17.8 \mathrm{~Hz}, \underline{\mathrm{H}}-7), 1.16$ (s, 9H, OC(O)CMe $\left.{ }_{3}\right) \mathrm{ppm} ;{ }^{13} \mathrm{C} \mathrm{NMR}$

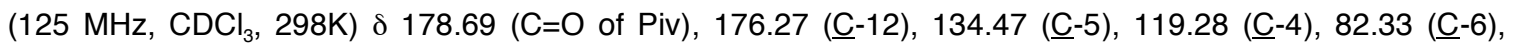

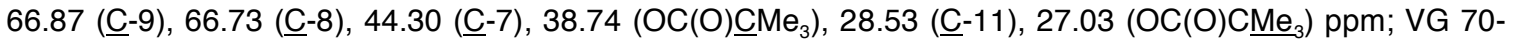
SE Positive Ion FAB HRMS: Calcd. for $\mathrm{C}_{13} \mathrm{H}_{20} \mathrm{NaO}_{5}(\mathrm{M}+\mathrm{Na})^{+}:$279.12084. Found: 279.12075.

\section{O-Silylated Butyrolactone 28 (II-YL-176)}

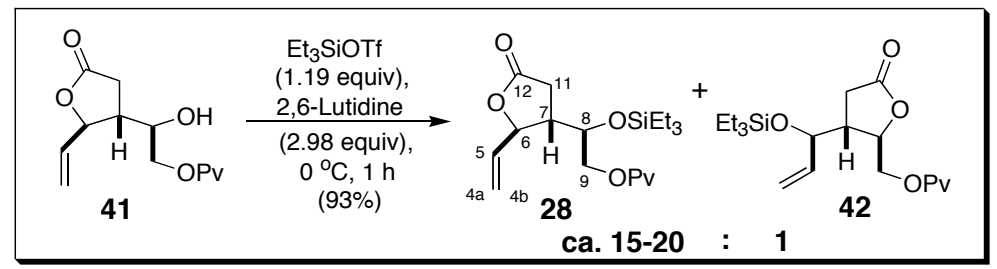

To a stirred $0{ }^{\circ} \mathrm{C}$ solution of $41(0.59 \mathrm{~g}, 2.30 \mathrm{mmol})$ in dry $\mathrm{CH}_{2} \mathrm{Cl}_{2}(10 \mathrm{~mL})$ under $\mathrm{N}_{2}$ was successively added 2,6-lutidine (0.80 mL, $6.87 \mathrm{mmol}, 2.98$ equiv) and $\mathrm{Et}_{3} \mathrm{SiOTf}(0.62 \mathrm{~mL}, 2.74 \mathrm{mmol}, 1.19$ equiv) in single portions. Stirring was continued at $0{ }^{\circ} \mathrm{C}$ for $1 \mathrm{~h}$ whereafter TLC analysis revealed that all of the starting alcohol 41 had been consumed. Excess solid $\mathrm{NaHCO}_{3}$ was added followed by $\mathrm{Et}_{2} \mathrm{O}(80 \mathrm{~mL})$ and saturated aqueous sodium bicarbonate $(20 \mathrm{~mL})$. The organic layer was separated and washed successively with $\mathrm{H}_{2} \mathrm{O}$ $(20 \mathrm{~mL})$ and brine $(20 \mathrm{~mL})$, before being dried over $\mathrm{MgSO}_{4}$, filtered, and concentrated in vacuo. Purification of the crude residue by $\mathrm{SiO}_{2}$ flash chromatography with petrol:EtOAc (8:1) furnished the O-silyl ether 28 $(0.79 \mathrm{~g}, 93 \%)$ as a colorless oil. $500 \mathrm{MHz}{ }^{1} \mathrm{H}$ NMR analysis of 28 typically revealed that it was typically a 15:1-20:1 mixture of $\mathbf{2 8}$ (as the major component) and a molecule we have tentatively assigned as the $\mathrm{C}(8)$ O-lactonised product 42.

Data for 28: $[\alpha]_{D}+49.3^{\circ}\left(c 0.430, \mathrm{CH}_{2} \mathrm{Cl}_{2}\right)$; IR $2959(\mathrm{~s}), 2912(\mathrm{w}), 2880(\mathrm{~m}), 1786(\mathrm{~s}), 1732(\mathrm{~s}), 1460(\mathrm{w})$, 1418 (w), 1369 (w), 1283 (w), 1238 (w), 1151 (s), 1080 (m), 1005 (s), 935 (w), 739 (s) cm ${ }^{-1}$; ${ }^{1} \mathrm{H}$ NMR (500

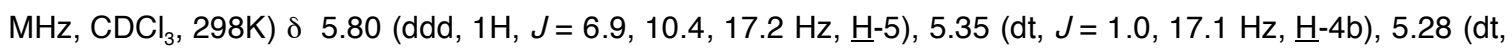
$1 \mathrm{H}, J=1.0,10.4 \mathrm{~Hz}, \underline{\mathrm{H}}-4 \mathrm{a}), 4.66(\mathrm{~m}, 1 \mathrm{H}, \underline{\mathrm{H}}-6), 4.03$ (dd, 1H, J=4.1, $11.0 \mathrm{~Hz}, \underline{\mathrm{H}}-9 \mathrm{a}), 3.91$ (ddd, $1 \mathrm{H}, J=2.2$, 4.1, 6.4 Hz, $\underline{\mathrm{H}}-8$ ), 3.84 (dd, $1 \mathrm{H}, J=6.9,11.0 \mathrm{~Hz}, \underline{\mathrm{H}}-9 \mathrm{~b}), 2.77$ (dd, $1 \mathrm{H}, J=9.2,17.2 \mathrm{~Hz}, \underline{\mathrm{H}}-11 \mathrm{a}), 2.44$ (dd, $1 \mathrm{H}$, $J=8.8,17.2 \mathrm{~Hz}, \underline{\mathrm{H}}-11 \mathrm{~b}), 2.37(\mathrm{~m}, 1 \mathrm{H}, \underline{\mathrm{H}}-7), 1.16\left(\mathrm{~s}, 9 \mathrm{H}, \mathrm{OC}(\mathrm{O}) \mathrm{CMe}_{3}\right), 0.93(\mathrm{t}, 9 \mathrm{H}, J=8.0 \mathrm{~Hz}, \mathrm{Me}$ of TES), 0.61 (2 x q superimposed upon one another, $6 \mathrm{H},-\mathrm{CH}_{2}-$ of TES) ppm; ${ }^{13} \mathrm{C} \mathrm{NMR}\left(125 \mathrm{MHz}, \mathrm{CDCl}_{3}, 298 \mathrm{~K}\right) \delta$

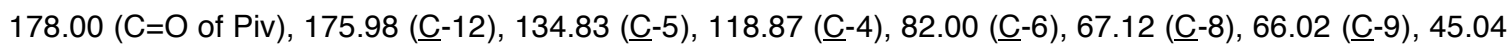

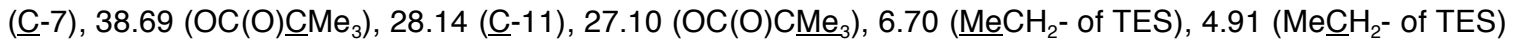
ppm; VG 70-SE Positive Ion FAB HRMS: Calcd. for $\mathrm{C}_{19} \mathrm{H}_{34} \mathrm{NaO}_{5} \mathrm{Si}(\mathrm{M}+\mathrm{Na})^{+}$: 393.20731. Found: 393.20688. 


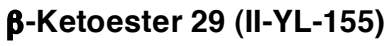

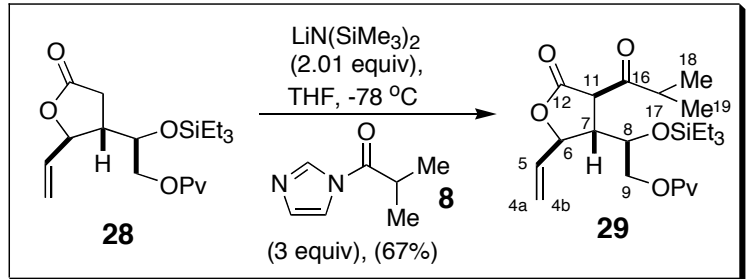

To a $-78{ }^{\circ} \mathrm{C}$ solution of lactone $28(1.12 \mathrm{~g}, 3.02 \mathrm{mmol})$ in dry THF $(20 \mathrm{~mL})$ under $\mathrm{N}_{2}$ was added $\mathrm{LiN}\left(\mathrm{SiMe}_{3}\right)_{2}$ (1 $\mathrm{M}$ solution in THF, $6.7 \mathrm{~mL}, 6.7 \mathrm{mmol}, 2.01$ equiv) dropwise over $5 \mathrm{~min}$. Stirring was continued for a further 30 min before a solution of isobutyryl imidazole $8(1.25 \mathrm{~g}, 9.05 \mathrm{mmol}, 3$ equiv) in dry THF (1 mL) was added dropwise over $5 \mathrm{~min}$. After stirring at $-78{ }^{\circ} \mathrm{C}$ for $1.5 \mathrm{~h}$, the reaction was quenched at $-78{ }^{\circ} \mathrm{C}$ with saturated aqueous $\mathrm{NH}_{4} \mathrm{Cl}(20 \mathrm{~mL})$, and diluted with $\mathrm{Et}_{2} \mathrm{O}(100 \mathrm{~mL})$. The organic layer was separated and washed successively with $\mathrm{H}_{2} \mathrm{O}(2 \times 20 \mathrm{~mL})$ and brine $(20 \mathrm{~mL})$, dried over $\mathrm{MgSO}_{4}$ and filtered. The filtrate was concentrated in vacuo and the residue was purified by $\mathrm{SiO}_{2}$ flash chromatography with petrol:EtOAc (20:1) to give 29 (0.89 g, 67\%).

Data for 29: $[\alpha]_{D}+21.8^{\circ}\left(c 0.924, \mathrm{CH}_{2} \mathrm{Cl}_{2}\right)$; IR $2964(\mathrm{~s}), 2882(\mathrm{~m}), 1774(\mathrm{~s}), 1728(\mathrm{~s}), 1468(\mathrm{w}), 1375(\mathrm{w})$, $1283(\mathrm{~m}), 1150$ (s), 1005 (s), $941(\mathrm{~m}), 739(\mathrm{~s}) \mathrm{cm}^{-1} ;{ }^{1} \mathrm{H}$ NMR $\left(500 \mathrm{MHz}, \mathrm{C}_{6} \mathrm{D}_{6}, 298 \mathrm{~K}\right) \delta 5.67$ (ddd, $1 \mathrm{H}, J=$ 7.3, 10.3, 17.4 Hz, $\underline{\mathrm{H}}-5$ ), 5.17 (br d, $J=17.1 \mathrm{~Hz}, \underline{\mathrm{H}}-4 \mathrm{~b}$ ), 4.93 (d, $1 \mathrm{H}, J=10.3 \mathrm{~Hz}, \underline{\mathrm{H}}-4 \mathrm{a}), 4.60$ (dd, $1 \mathrm{H}, J=$ 7.5, 7.6 Hz, $\underline{\mathrm{H}}-6), 4.24$ (dd, $1 \mathrm{H}, J=8.4 \mathrm{~Hz}, \underline{\mathrm{H}}-11), 4.02(\mathrm{dd}, 1 \mathrm{H}, J=5.3,11.1 \mathrm{~Hz}, \underline{\mathrm{H}}-9 \mathrm{a}), 3.93(\mathrm{~m}, 1 \mathrm{H}, \mathrm{H}-8)$, 3.69 (dd, $1 \mathrm{H}, J=8.2,11.1 \mathrm{~Hz}, \underline{\mathrm{H}}-9 \mathrm{~b}$ ), 3.25 (ddd, $1 \mathrm{H}, J=1.9,8.1,8.1 \mathrm{~Hz}, \underline{\mathrm{H}}-7$ ), 3.21 (septet, $1 \mathrm{H}, J=6.9 \mathrm{~Hz}$, $\underline{\mathrm{H}}-17), 1.18\left(\mathrm{~s}, 9 \mathrm{H}, \mathrm{OC}(\mathrm{O}) \mathrm{CMe}_{3}\right.$ ), 1.09 (d, 3H, $J=6.6 \mathrm{~Hz}, \mathrm{Me}-18$ or Me-19), 1.01 (d, 3H, $J=7.2 \mathrm{~Hz}, \mathrm{Me}-18$ or Me-19), $0.86(\mathrm{t}, 9 \mathrm{H}, J=8.0 \mathrm{~Hz}$, Me of TES), 0.52-0.44 (2 x q superimposed upon one another, $6 \mathrm{H}, J=7.8$, $7.9 \mathrm{~Hz},-\mathrm{CH}_{2}$ - of TES) ppm; ${ }^{13} \mathrm{C} \mathrm{NMR}\left(125 \mathrm{MHz}, \mathrm{C}_{6} \mathrm{D}_{6}, 298 \mathrm{~K}\right.$ ) $\delta 206.14$ ( $\left.\underline{\mathrm{C}}-16\right), 177.33$ (C=O of Piv), 171.21

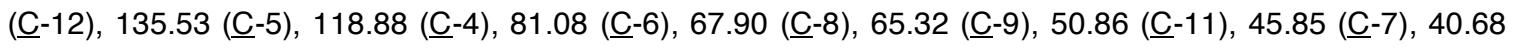
(-17), $38.77\left(\mathrm{OC}(\mathrm{O}) \underline{\mathrm{CMe}}_{3}\right), 27.21\left(\mathrm{OC}(\mathrm{O}) \mathrm{CMe}_{3}\right), 18.73$ (Me-18 or Me-19), 17.34 (Me-18 or Me-19), 6.89 ( $\mathrm{MeCH}_{2}$ - of TES), 5.17 ( $\mathrm{Me}^{\mathrm{C}} \mathrm{H}_{2}$ - of TES) ppm; VG 70-SE Positive Ion FAB HRMS: Calcd. for $\mathrm{C}_{23} \mathrm{H}_{40} \mathrm{NaO}_{6} \mathrm{Si}$ $(\mathrm{M}+\mathrm{Na})^{+}:$463.24917. Found: 463.24980 .

\section{$\beta$-Ketoester 30 (II-YL-159)}

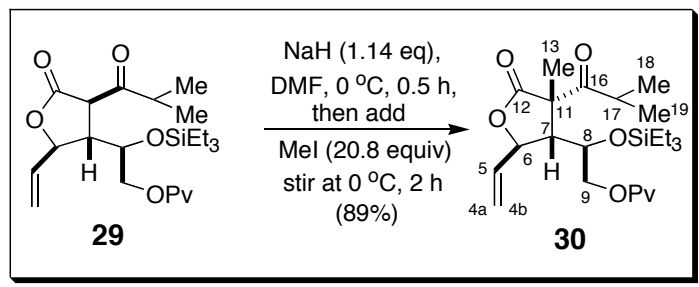

To a stirred $0{ }^{\circ} \mathrm{C}$ solution of $29(0.34 \mathrm{~g}, 0.772 \mathrm{mmol})$ in dry DMF $(6 \mathrm{~mL})$ under $\mathrm{N}_{2}$ was added $\mathrm{NaH}(60 \%$ dispersion in mineral oil, $35.3 \mathrm{mg}, 0.883 \mathrm{mmol}, 1.14$ equiv) in one portion. The mixture was stirred at $0{ }^{\circ} \mathrm{C}$ for 
$30 \mathrm{~min}$ and $\mathrm{Mel}\left(1 \mathrm{~mL}, 16.06 \mathrm{mmol}, 20.8\right.$ equiv) was added in one portion. Stirring was continued at $0{ }^{\circ} \mathrm{C}$ for $2 \mathrm{~h}$, whereafter TLC analysis indicated that a single slightly-slower-moving major product had formed. Saturated aqueous $\mathrm{NH}_{4} \mathrm{Cl}(10 \mathrm{~mL})$ was added to the reaction mixture followed by $\mathrm{Et}_{2} \mathrm{O}(50 \mathrm{~mL})$ and the organic layer was separated. The aqueous layer was further extracted with $\mathrm{Et}_{2} \mathrm{O}(10 \mathrm{~mL})$ and the combined organic layers were washed with $\mathrm{H}_{2} \mathrm{O}(10 \mathrm{~mL})$, dried over $\mathrm{MgSO}_{4}$, filtered, and concentrated in vacuo. Purification of the residue by $\mathrm{SiO}_{2}$ flash chromatography with petrol: $\mathrm{Et}_{2} \mathrm{O}(10: 1)$ gave $30(0.31 \mathrm{~g}, 89 \%)$ as a colorless oil.

Data for 30: $[\alpha]_{D}+29.5^{\circ}\left(c 0.234, \mathrm{CH}_{2} \mathrm{Cl}_{2}\right)$; IR $2963(\mathrm{~s}), 2912(\mathrm{~m}), 2880(\mathrm{~m}), 1773(\mathrm{~s}), 1732(\mathrm{~s}), 1703(\mathrm{~m})$, 1462 (m), 1377 (w), 1323 (w), 1283 (m), 1244 (m), 1204 (m), 1157 (s), 1128 (s), 1009 (s), 945 (m), 739 (s) $\mathrm{cm}^{-1} ;{ }^{1} \mathrm{H}$ NMR $\left(500 \mathrm{MHz}, \mathrm{C}_{6} \mathrm{D}_{6}, 298 \mathrm{~K}\right) \delta 5.50$ (ddd, $\left.1 \mathrm{H}, J=8.0,10.2,18.2 \mathrm{~Hz}, \underline{\mathrm{H}}-5\right), 5.13$ (dt, $1 \mathrm{H}, J=17.1$ $\mathrm{Hz}, \underline{\mathrm{H}}-4 \mathrm{~b}), 4.96$ (br dt, $1 \mathrm{H}, J=10.3 \mathrm{~Hz}, \underline{\mathrm{H}}-4 \mathrm{a}), 4.85$ (dd, $1 \mathrm{H}, J=8.0,10.0 \mathrm{~Hz}, \underline{\mathrm{H}}-6), 4.31$ (m, $1 \mathrm{H}, \underline{\mathrm{H}}-8), 4.13$ (dd, $1 \mathrm{H}, J=4.5,12.4 \mathrm{~Hz}, \underline{\mathrm{H}}-9 \mathrm{a}), 3.97$ (dd, J=3.5, $12.4 \mathrm{~Hz}, \underline{\mathrm{H}}-9 \mathrm{~b}), 2.94$ (septet, $1 \mathrm{H}, J=6.7, \mathrm{~Hz}, \underline{\mathrm{H}}-17$ ), 2.35

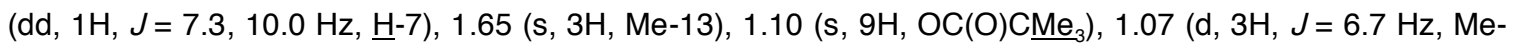
18 or Me-19), 1.04 (d, 3H, J=6.6 Hz, Me-18 or Me-19), 0.89 (t, 9H, J=8.0 Hz, Me of TES), 0.53 (2 x q superimposed upon one another, $6 \mathrm{H}, J=8.0 \mathrm{~Hz},-\mathrm{CH}_{2}-$ of TES) ppm; ${ }^{13} \mathrm{C}$ NMR $\left(125 \mathrm{MHz}, \mathrm{C}_{6} \mathrm{D}_{6}, 298 \mathrm{~K}\right) \delta$

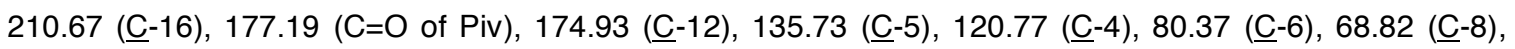

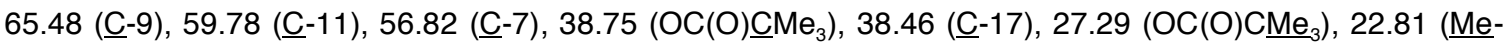

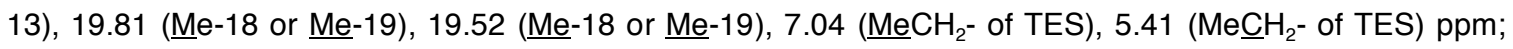
VG 70-SE Positive Ion FAB HRMS: Calcd. for $\mathrm{C}_{24} \mathrm{H}_{42} \mathrm{NaO}_{6} \mathrm{Si}(\mathrm{M}+\mathrm{Na})^{+}:$477.26482. Found: 477.26381.

\section{Bicyclic Furanoside 31 (II-YL-161)}

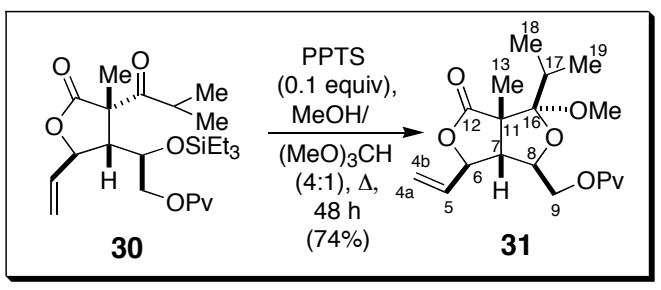

A mixture of $\beta$-ketoester 30 (200 mg, $0.44 \mathrm{mmol})$, pyridine $p$-toluenesulfonate (11 $\mathrm{mg}, 0.044 \mathrm{mmol}, 0.1$ equiv), trimethyl orthoformate $(2 \mathrm{~mL})$ and $\mathrm{MeOH}(8 \mathrm{~mL})$ were heated at reflux for $48 \mathrm{~h}$ whereupon TLC analysis revealed that a single slower-moving major product had formed and unreacted $\mathbf{3 0}$ was also present. The solvents were evaporated under reduced pressure and the residue was purified by $\mathrm{SiO}_{2}$ flash chromatography with petrol: $\mathrm{Et}_{2} \mathrm{O}(7: 1)$ as eluent. Initially, 30 (46 mg, 23\%) was isolated followed by methyl furanoside 31 (0.116 g, 74\%); both were colorless oils.

Data for 31: $[\alpha]_{D}-50.99^{\circ}\left(c 0.404, \mathrm{CH}_{2} \mathrm{Cl}_{2}\right)$; IR (KBr) $2976(\mathrm{~s}), 2936(\mathrm{~m}), 1774(\mathrm{~s}), 1732(\mathrm{~s}), 1464(\mathrm{w}), 1391$ (w), 1366 (w), 1281 (m), 1223 (w), 1155 (s), 1092 (s), 1049 (m), 1016 (s), 935 (w) cm c-1 ; H NMR (500 MHz, $\left.\mathrm{C}_{6} \mathrm{D}_{6}, 298 \mathrm{~K}\right) \delta 5.56$ (ddd, $\left.1 \mathrm{H}, J=6.6,10.4,17.1 \mathrm{~Hz}, \underline{\mathrm{H}}-5\right), 5.11$ (ddd, $\left.1 \mathrm{H}, J=1.2,1.2,17.1 \mathrm{~Hz}, \underline{\mathrm{H}}-4 \mathrm{~b}\right), 4.90$ (ddd, $1 \mathrm{H}, J=1.1,10.4 \mathrm{~Hz}, \underline{\mathrm{H}}-4 \mathrm{a}), 4.50(\mathrm{~m}, 1 \mathrm{H}, \underline{\mathrm{H}}-6), 4.02$ (dd, $1 \mathrm{H}, J=5.4,11.5 \mathrm{~Hz}, \underline{\mathrm{H}}-9 \mathrm{a}), 3.88$ (dd, $1 \mathrm{H}, J=$ 5.5, $11.5 \mathrm{~Hz}, \underline{\mathrm{H}}-9 \mathrm{~b}), 3.80$ (m, 1H, $\underline{\mathrm{H}}-8), 2.99$ (s, 3H, OMe), 2.13 (dd, 1H, J=3.7, 7.6 Hz, $\underline{\mathrm{H}}-7$ ), 2.00 (septet, 
$1 \mathrm{H}, J=7.2 \mathrm{~Hz}, \underline{\mathrm{H}}-17), 1.17$ (d, 3H, J=7.0 Hz, Me-18 or $\underline{\mathrm{Me}}-19), 1.12$ (d, 3H, J= $7.1 \mathrm{~Hz}$, $\underline{\mathrm{Me}}-18$ or $\underline{\mathrm{Me}}-19)$ superimposed upon 1.12 (s, 9H, OC(O)CMe $\left.\mathrm{CM}_{3}\right), 1.119$ (s, 3H, $\left.\underline{\mathrm{Me}}-13\right)$ ppm; ${ }^{13} \mathrm{C} \mathrm{NMR}\left(125 \mathrm{MHz}, \mathrm{C}_{6} \mathrm{D}_{6}, 298 \mathrm{~K}\right)$

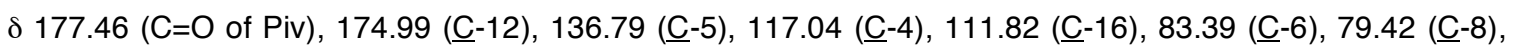

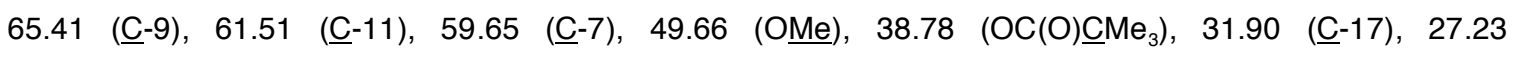
(OC(O)CMe ${ }_{3}$ ), 21.63 (ㄷ-13), 18.53 ( $\underline{\mathrm{Me}}-18$ or $\left.\underline{\mathrm{Me}}-19\right), 17.09$ ( $\underline{\mathrm{Me}}-18$ or $\left.\underline{\mathrm{Me}}-19\right)$ ppm; VG 70-SE Positive Ion FAB HRMS: Calcd. for $\mathrm{C}_{19} \mathrm{H}_{30} \mathrm{NaO}_{6}(\mathrm{M}+\mathrm{Na})^{+}: 377.19400$. Found: 377.19432.

\section{Alcohol 6 (III-YL151)}

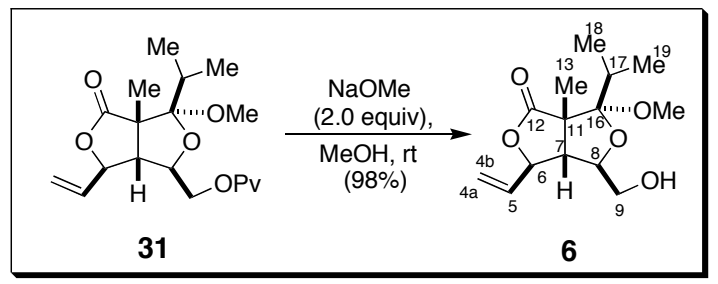

A pre-prepared solution of $\mathrm{Na}\left(26 \mathrm{mg}, 1.13 \mathrm{mmol}, 2\right.$ equiv) in dry $\mathrm{MeOH}(5 \mathrm{~mL})$ under $\mathrm{N}_{2}$ was added to a round-bottomed flask containing the O-pivaloate $31(0.2 \mathrm{~g}, 0.564 \mathrm{mmol})$ at rt. The reactants were stirred for $24 \mathrm{~h}$ before being diluted with $\mathrm{CH}_{2} \mathrm{Cl}_{2}(30 \mathrm{~mL})$ and filtered through a short plug of $\mathrm{SiO}_{2}$ gel. After concentration of the filtrate in vacuo, the residue was purified by $\mathrm{SiO}_{2}$ flash chromatography with petrol:EtOAc (3:1) to give alcohol $6(0.153 \mathrm{~g}, 98 \%)$ as a white amorphous solid.

Data for 6: $[\alpha]_{\mathrm{D}}-71.6^{\circ}\left(c 0.282, \mathrm{CH}_{2} \mathrm{Cl}_{2}\right)$; IR (KBr) $3416(\mathrm{~s}), 2966(\mathrm{~m}), 2930(\mathrm{~m}), 1747(\mathrm{~s}), 1466(\mathrm{w}), 1412$ (w), $1281(\mathrm{~m}), 1240$ (m), 1146 (m), 1090 (s), 1057 (s), 1013 (s), 935 (m) cm ${ }^{-1} ;{ }^{1} \mathrm{H}$ NMR (500 MHz, C $\mathrm{D}_{6}, 298$ K) $\delta 5.57$ (ddd, $1 \mathrm{H}, J=6.3,10.5,17.0 \mathrm{~Hz}, \underline{\mathrm{H}}-5), 5.12$ (ddd, $1 \mathrm{H}, J=1.3,1.3,17.2 \mathrm{~Hz}, \underline{\mathrm{H}}-4 \mathrm{~b}), 4.89$ (ddd, $1 \mathrm{H}$, $J=1.2,10.5 \mathrm{~Hz}, \underline{\mathrm{H}}-4 \mathrm{a}), 4.51$ (m, 1H, $\underline{\mathrm{H}}-6), 3.58$ (dd, $1 \mathrm{H}, J=4.3,8.5 \mathrm{~Hz}, \underline{\mathrm{H}}-8), 3.28$ (br m, 1H, $\underline{\mathrm{H}}-9 \mathrm{a}), 3.18$ (br m, 1H, $\underline{H}-9 b), 3.01$ (s, 3H, OMe), 2.23 (dd, 1H, J=3.8, 7.6 Hz, 브-7), 1.99 (septet, 1H, J=7.1 Hz, 브-17),

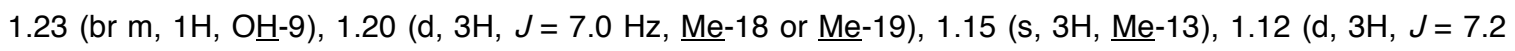

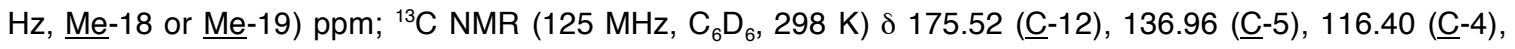

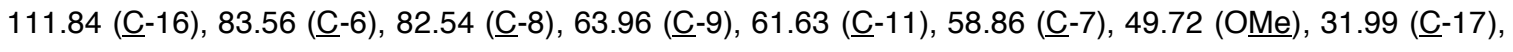

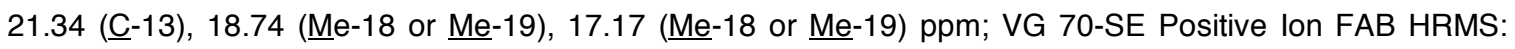
Calcd. for $\mathrm{C}_{14} \mathrm{H}_{22} \mathrm{NaO}_{5}(\mathrm{M}+\mathrm{Na})^{+}:$293.13649. Found: 293.13724.

Preparation of O-Triflate Ester 3 and Its Alkylative Union with $3(2 \mathrm{H})$-Dihydrofuranone $2^{5}$ to Obtain 32 (III-YL-17a) and 33 (III-YL-17b)

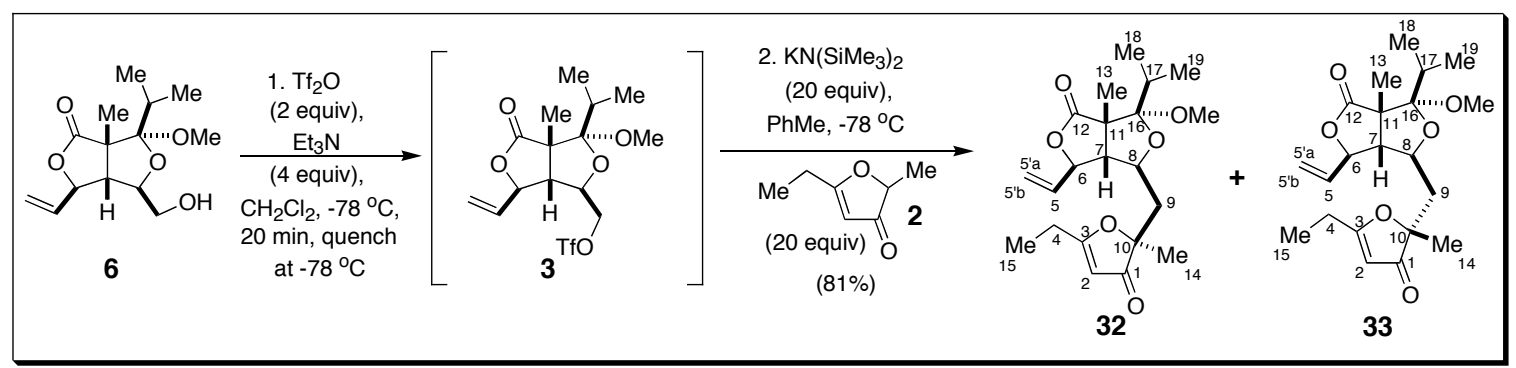


Part (i). Formation of O-Triflate Ester 3. To a stirred $-78^{\circ} \mathrm{C}$ solution of alcohol 6 (150 mg, $\left.0.555 \mathrm{mmol}\right)$ in dry $\mathrm{CH}_{2} \mathrm{Cl}_{2}(5 \mathrm{~mL})$ under $\mathrm{N}_{2}$ was added $\mathrm{Et}_{3} \mathrm{~N}(0.31 \mathrm{~mL}, 2.22 \mathrm{mmol})$. Triflic anhydride $(0.187 \mathrm{~mL}, 1.11 \mathrm{mmol})$ was then added dropwise over $5 \mathrm{~min}$, and the reactants were stirred at $-78^{\circ} \mathrm{C}$ for $15 \mathrm{~min} . \mathrm{H}_{2} \mathrm{O}(2 \mathrm{~mL})$ was added followed by $\mathrm{Et}_{2} \mathrm{O}(15 \mathrm{~mL})$. The organic layer was separated, dried over $\mathrm{MgSO}_{4}$, filtered and evaporated. The residue was purified by $\mathrm{SiO}_{2}$ flash chromatography with petrol/EtOAc (3:1) to give the unstable $O$-triflate $3(230 \mathrm{mg})$ as a colorless oil that was used immediately for the next step.

Part (ii). Alkylation of O-Triflate Ester 3 with $3(2 \mathrm{H})$-Dihydrofuranone $2^{5}$ to Obtain 32 and 33 . To a stirred $-78{ }^{\circ} \mathrm{C}$ solution of $2^{5}\left(1.40 \mathrm{~g}, 11.1 \mathrm{mmol}, 20\right.$ equiv) in dry PhMe $(5 \mathrm{~mL})$ under $\mathrm{N}_{2}$ was added $\mathrm{KN}\left(\mathrm{SiMe}_{3}\right)_{2}(0.5 \mathrm{M}$ solution in PhMe, $22.2 \mathrm{~mL}, 11.1 \mathrm{mmol}, 20$ equiv) dropwise over $20 \mathrm{~min}$. The resulting solution was stirred at $-78{ }^{\circ} \mathrm{C}$ for $0.5 \mathrm{~h}$ whereafter a solution of $3(230 \mathrm{mg}, 0.555 \mathrm{mmol})$ in dry PhMe $(2 \mathrm{~mL})$ was added dropwise over $2 \mathrm{~min}$. The reactants were gradually warmed to $0{ }^{\circ} \mathrm{C}$ over $2 \mathrm{~h}$ whereupon sat. aq. $\mathrm{NH}_{4} \mathrm{Cl}(1 \mathrm{~mL})$ was added. Following dilution with EtOAc $(15 \mathrm{~mL})$, the organic layer was separated, washed with $\mathrm{H}_{2} \mathrm{O}(3 \mathrm{~mL})$, brine $(3 \mathrm{~mL})$, and then dried over $\mathrm{MgSO}_{4}$. After filtration and evaporation of the solvents in vacuo, purification of the residue by $\mathrm{SiO}_{2}$ flash chromatography (4:1 petrol:EtOAc) followed by preparative TLC (4:1 petrol:EtOAc) gave 32 and $\mathbf{3 3}(171 \mathrm{mg}, 81 \%)$ as essentially a 1:1 mixture of C(10)-epimers that appeared chromatographically homogenous according to single-elution TLC analysis.

The two epimers could only be separated by multiple-elution preparative TLC (petrol/EtOAc 4:1) on several TLC plates with recycling of the separated mixed fractions. By this method, after further final purification of the residue by $\mathrm{SiO}_{2}$ flash chromatography (4:1 petrol:EtOAc) the faster-moving product 32 (71 $\mathrm{mg}, 34 \%)$ was isolated as an oil; the slower-moving product $33(79 \mathrm{mg}, 38 \%)$ was also obtained as a colorless oil. The characterisation data for these two molecules is listed below.

Data for 32: $[\alpha]_{D}-135.5^{\circ}\left(c 0.42, \mathrm{CH}_{2} \mathrm{Cl}_{2}\right)$; IR $2964(\mathrm{~m}), 1773(\mathrm{~m}), 1699(\mathrm{~m}), 1593(\mathrm{~m}), 1556(\mathrm{w}), 1385(\mathrm{w})$, 1261 (s), 1090 (s), 1018 (s), 800 (s) cm ${ }^{-1}$; ${ }^{1} \mathrm{H}$ NMR (500 MHz, $\mathrm{C}_{6} \mathrm{D}_{6}, 298 \mathrm{~K}$ ) $\delta 5.61$ (ddd, $1 \mathrm{H}, J=6.6,10.4$, $17.1 \mathrm{~Hz}, \mathrm{H}-5$ ), 5.16 (s, $1 \mathrm{H}, \mathrm{H}-2$ ), 5.15 (ddd, $1 \mathrm{H}, J=1.2,1.2,17.1 \mathrm{~Hz}, \mathrm{H}-5$ 'a), 4.89 (ddd, $1 \mathrm{H}, J=1.1,1.1,10.4$ $\mathrm{Hz}, \mathrm{H}-5$ 'b), 4.58 (dd, 1H, J=6.9, 7.0, H-6), 3.98 (ddd, 1H, J=3.8, 3.8, 7.9 Hz, H-8), 2.97 (s, 3H, OMe), 2.031.90 (complex m, 5H, H-17, H-4a, H-4b, H-7, H-9a), 1.63 (dd, 1H, J=3.7, $14.9 \mathrm{~Hz}, \mathrm{H}-9 \mathrm{~b}$ ), 1.29 (s, 3H, Me14), 1.19 (d, 3H, $J=7.0 \mathrm{~Hz}, \mathrm{Me}-18$ or Me-19), 1.10 (d, 3H, $J=7.2 \mathrm{~Hz}, \mathrm{Me}-18$ or Me-19), 1.08 (s, 3H, Me13), 0.74 (t, 3H, $J=7.6 \mathrm{~Hz}, \mathrm{Me}-15) ;{ }^{13} \mathrm{C}$ NMR (125 MHz, $\mathrm{C}_{6} \mathrm{D}_{6}, 298 \mathrm{~K}$ ) $\delta 204.79$ (C-1), 192.46 (C-3), 175.16 (C-12), 137.14 (C-5), 116.72 (C-5), 111.83 (C-16), 101.09 (C-2), 89.13 (C-10), 83.43 (C-6), 78.33 (C-8), 63.68 (C-7), 61.23 (C-11), 49.53 (OMe), 42.86 (C-9), 31.98 (C-17), 24.03 (C-4), 21.92 (C-14), 21.88 (C-13), 18.65 (C-18 or C-19), 17.09 (C-18 or C-19), 10.04 (C-15); VG 70SE Positive Ion FAB HRMS: Calcd. for $\mathrm{C}_{21} \mathrm{H}_{30} \mathrm{NaO}_{6}(\mathrm{M}+\mathrm{Na})^{+}$: 401.19400. Found: 401.19457. The stereochemical integrity of $\mathbf{3 2}$ was proven by its chemical conversion into 43 (by successive treatment with $\mathrm{OsO}_{4} / \mathrm{NaIO}_{4}$ and $\mathrm{NaBH}_{4}$ ) and $400 \mathrm{MHz}{ }^{1} \mathrm{H} \mathrm{NMR}$ comparison with Tadano's spectrum for this compound. ${ }^{2 b}$

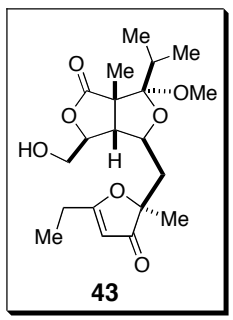


Data for 33: $[\alpha]_{D}-26.7^{\circ}\left(c 0.296, \mathrm{CH}_{2} \mathrm{Cl}_{2}\right)$; IR $2976(\mathrm{~m}), 2937(\mathrm{~m}), 1773(\mathrm{~s}), 1705(\mathrm{~s}), 1599(\mathrm{~s}), 1454(\mathrm{w})$, 1379 (m), 1258 (w), 1223 (w), 1155 (s), 1124 (m), 1088 (s), 1051 (s), 1015 (s), 926 (m), 810 (w) cm ; ${ }^{1} \mathrm{H}$ NMR (500 MHz, $\left.C_{6} \mathrm{D}_{6}, 298 \mathrm{~K}\right) \delta 5.50$ (ddd, $\left.1 \mathrm{H}, J=6.6,10.4,17.1 \mathrm{~Hz}, \mathrm{H}-5\right), 5.21(\mathrm{~s}, 1 \mathrm{H}, \mathrm{H}-2), 5.06$ (ddd, $1 \mathrm{H}$, $J=1.2,1.2,17.1 \mathrm{~Hz}, \mathrm{H}-5$ 'a), 4.86 (ddd, 1H, J=1.1, 1.1, $10.4 \mathrm{~Hz}, \mathrm{H}-5$ 'b), 4.55 (br dd, 1H, J= 7.0, 7.0, H-6), 3.96 (ddd, 1H, J=2.9, 2.8, $10.6 \mathrm{~Hz}, \mathrm{H}-8$ ), 3.01 (s, 3H, OMe), 2.02 (septet, $1 \mathrm{H}, J=7.2 \mathrm{~Hz}, \mathrm{H}-17$ ), 1.96 (dd, $1 \mathrm{H}, J=10.6,14.3 \mathrm{~Hz}, \mathrm{H}-9 \mathrm{a}), 1.94$ (qd, 2H, J=0.8, $7.5 \mathrm{~Hz}, \mathrm{H}-4 \mathrm{a}, \mathrm{H}-4 \mathrm{~b}), 1.85$ (dd, $1 \mathrm{H}, J=3.8,7.5 \mathrm{~Hz}, \mathrm{H}-7$ ), 1.59 (dd, 1H, 2.6, 14.4 Hz, H-9b), 1.25 (s, 3H, Me-14), 1.22 (d, 3H, J = 7.0 Hz, Me-18 or Me-19), 1.14 (d, $3 \mathrm{H}, J=7.2 \mathrm{~Hz}, \mathrm{Me}-18$ or Me-19), 1.12 (s, 3H, Me-13), 0.77 (t, 3H, J=7.5 Hz, Me-15); ${ }^{13} \mathrm{C} \mathrm{NMR}(125 \mathrm{MHz}$, $\mathrm{C}_{6} \mathrm{D}_{6}, 298$ K) $\delta 204.30$ (C-1), 190.62 (C-3), 175.18 (C-12), 137.13 (C-5), 116.66 (C-5'), 112.23 (C-16), 101.66 (C-2), 87.80 (C-10), 83.40 (C-6), 76.90 (C-8), 63.09 (C-7), 61.31 (C-11), 49.78 (OMe), 43.36 (C-9), 32.13 (C-17), 23.85 (C-4), 23.23 (C-14), 21.87 (C-13), 18.77 (C-18 or C-19), 17.00 (C-18 or C-19), 10.19 (C15); VG 70-SE Positive Ion FAB HRMS: Calcd. for $\mathrm{C}_{21} \mathrm{H}_{30} \mathrm{NaO}_{6}(\mathrm{M}+\mathrm{Na})^{+}:$401.19400. Found: 401.19455. The structural integrity of $\mathbf{3 3}$ was proven by its chemical conversion into $\mathbf{4}$ and (+)-eremantholide $A$ and our ${ }^{1} \mathrm{H} /{ }^{13} \mathrm{C}$ NMR correlations with Tadano's spectra and his reported spectral data for these two compounds.

Whilst the separation of $\mathbf{3 2}$ and $\mathbf{3 3}$ could successfully be effected at this stage, and the purified molecules individually taken forward to alkenes 35 and $\mathbf{1}$ respectively, we have generally found it much more convenient to take the purified but unseparated mixture of $\mathbf{3 2}$ and $\mathbf{3 3}$ forward to the alkene stage and effect isomer separation at that point. Accordingly, we now present the optimised procedures for securing 35 and 1 from the $1: 1$ mixture of $\mathbf{3 2 / 3 3}$.

Preparation of 1 (III-YL-37b) and 35 (III-YL-26) from the Mixture of 32 and 33

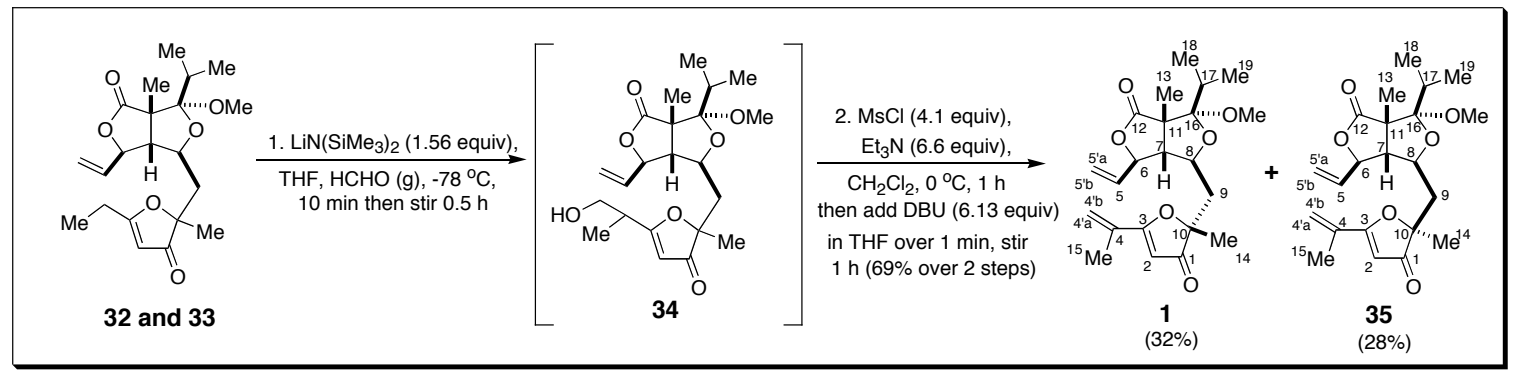

To a stirred $-78{ }^{\circ} \mathrm{C}$ solution of the purified but unseparated mixture of $\mathbf{3 2}$ and $\mathbf{3 3}(82.5 \mathrm{mg}, 0.218 \mathrm{mmol})$ in dry THF $(2 \mathrm{~mL})$ under $\mathrm{N}_{2}$ was added $\mathrm{LiN}\left(\mathrm{SiMe}_{3}\right)_{2}(1 \mathrm{M}$ solution in THF, $0.34 \mathrm{~mL}, 0.34 \mathrm{mmol}, 1.56$ equiv) dropwise over 2 min. Stirring was continued for 20 min whereupon a combined stream of gaseous $\mathrm{HCHO}$ and $\mathrm{N}_{2}$ was passed just above the surface of the $-78{ }^{\circ} \mathrm{C}$ reaction mixture for $10 \mathrm{~min}$. The gaseous $\mathrm{HCHO} / \mathrm{N}_{2}$ mixture was generated by heating solid paraformaldehyde in a two necked round bottom flask at $170{ }^{\circ} \mathrm{C}$ in an oil-bath and slowly carrying it over into the reaction flask via thermally-stable, wide-bore, silicone-type tubing through application of a positive pressure of $\mathrm{N}_{2}$ gas (Warning: Do NOT attempt to use a metal cannula for this gaseous $\mathrm{HCHO} / \mathrm{N}_{2}$ transfer, $\underline{\mathrm{NOR}}$ use a heat gun to crack the solid paraformadehyde, as thin metal cannulae are liable to get blocked very quickly due to rapid cooling and solidification of gaseous $\mathrm{HCHO}$; the rapid cracking of $(\mathrm{HCHO})_{n}$ with a heat gun is also liable to cause a dangerous pressure build-up 
within the generating apparatus, particularly when the metal cannula gets blocked with condensed HCHO, as often happens. By adhering to the experimental set-up we have described, we have not encountered any problems in generating gaseous HCHO safely). Following exposure of the reaction mixture to gaseous $\mathrm{HCHO}$ for $10 \mathrm{~min}$, the reactants were allowed to stir for $0.5 \mathrm{~h}$, and then quenched by adding saturated aqueous $\mathrm{NH}_{4} \mathrm{Cl}(0.5 \mathrm{~mL})$, EtOAc $(10 \mathrm{~mL})$ and $\mathrm{H}_{2} \mathrm{O}(2 \mathrm{~mL})$. The organic layer was separated, washed successively with $\mathrm{H}_{2} \mathrm{O}(2 \mathrm{~mL})$ and brine $(2 \mathrm{~mL})$, dried over $\mathrm{MgSO}_{4}$, filtered and concentrated in vacuo. The residue was purified by $\mathrm{SiO}_{2}$ flash chromatography (gradient elution with petrol/EtOAc from 2:1 to 1:2), the desired product mixture 34 (95 $\mathrm{mg},>100 \%)$ being isolated as an oil after drying in vacuo; it appeared as essentially a single slower-moving product according to TLC analysis.

To a $0{ }^{\circ} \mathrm{C}$ solution of the aforementioned sample of 34 (95 $\mathrm{mg}$, assumed to be $0.218 \mathrm{mmol}$ ) in dry THF ( $2 \mathrm{~mL})$ under $\mathrm{N}_{2}$ was added $\mathrm{Et}_{3} \mathrm{~N}(0.20 \mathrm{~mL}, 1.44 \mathrm{mmol}, 6.6$ equiv) in one portion. A solution of $\mathrm{MsCl}$ $(0.069 \mathrm{~mL}, 0.891 \mathrm{mmol}, 4.1$ equiv) in dry THF $(0.5 \mathrm{~mL})$ was then added dropwise over $1 \mathrm{~min}$. The reactants were stirred at $0{ }^{\circ} \mathrm{C}$ for $1 \mathrm{~h}$, whereupon a solution of 1,8-diazabicyclo[5.4.0]undece-7-ene (DBU) (0.2 mL, $1.34 \mathrm{mmol}, 6.13$ equiv) in dry $\operatorname{THF}(0.5 \mathrm{~mL})$ was added dropwise over $1 \mathrm{~min}$. Stirring was continued at $0^{\circ} \mathrm{C}$ for $1 \mathrm{~h}$, whereafter the reaction was quenched with saturated aqueous $\mathrm{NaHCO}_{3}(5 \mathrm{~mL})$. Following dilution with EtOAc $(20 \mathrm{~mL})$, the organic layer was separated and washed with $\mathrm{H}_{2} \mathrm{O}(5 \mathrm{~mL})$ and brine $(5 \mathrm{~mL})$, dried over $\mathrm{MgSO}_{4}$, filtered and concentrated in vacuo. Purification of the residue by $\mathrm{SiO}_{2}$ flash chromatography with petrol/EtOAc (3:1) furnished the desired alkenes 1 and 35 as mixture $(59.0 \mathrm{mg}, 69 \%)$ that moved as a single spot after single elution TLC in the aforementioned solvent system. The two components could be successfully separated by multiple-elution preparative TLC using petrol/EtOAc (4:1) as eluent. By this method, the faster moving product 35 (24 mg, 28\%) and the slower-moving product 1 (27.4 mg, 32\%) were both isolated as oils after further purification by $\mathrm{SiO}_{2}$ flash chromatography using petrol/EtOAc (3:1) as eluent. Their respective characterisation data are reported below:

Data for 35: $[\alpha]_{D}-119.1^{\circ}\left(c 0.262, \mathrm{CH}_{2} \mathrm{Cl}_{2}\right)$; IR $2936(\mathrm{~m}), 1774(\mathrm{~s}), 1697(\mathrm{~s}), 1634(\mathrm{~m}), 1560$ (s), $1452(\mathrm{w})$, $1373(\mathrm{~m}), 1263(\mathrm{~m}), 1123(\mathrm{~s}), 1088(\mathrm{~m}), 1051(\mathrm{~m}), 1016(\mathrm{~m}), 934(\mathrm{w}), 812(\mathrm{w}) \mathrm{cm}^{-1} ;{ }^{1} \mathrm{H}$ NMR (500 MHz, $\left.\mathrm{C}_{6} \mathrm{D}_{6}, 298 \mathrm{~K}\right) \delta 5.81$ (br s, 1H, H-4'a), 5.60 (ddd, 1H, J=6.5, 10.4, $\left.17.0 \mathrm{~Hz}, \mathrm{H}-5\right), 5.38$ (s, $\left.1 \mathrm{H}, \mathrm{H}-2\right), 5.15$ (ddd, $1 \mathrm{H}, J=1.2,1.2,17.1 \mathrm{~Hz}, \mathrm{H}-5$ 'a), 4.93 (m, 1H, H-4'b), 4.88 (ddd, 1H, J=1.1, 1.1, $10.4 \mathrm{~Hz}, \mathrm{H}-5$ 'b), 4.58 (br m, 1H, H-6), 3.97 (ddd, 1H, J=3.8, 4.0, 7.9, 8.8 Hz, H-8), 2.97 (s, 3H, OMe), 2.04-1.92 (complex m, 3H, $\mathrm{H}-7, \mathrm{H}-9 \mathrm{a}, \mathrm{H}-17$ ), 1.71 (dd, $1 \mathrm{H}, J=3.7,14.8 \mathrm{~Hz}, \mathrm{H}-9 \mathrm{~b}$ ), 1.51 (narrow dd resembling a singlet, $3 \mathrm{H}, J=0.9$, $1.3 \mathrm{~Hz}, \mathrm{Me}-15), 1.29$ (s, 3H, Me-14), 1.19 (d, 3H, J = 7.0 Hz, Me-18 or Me-19), 1.08 (d, 3H, J=7.2 Hz, Me18 or Me-19) superimposed upon 1.078 (s, 3H, Me-13); ${ }^{13} \mathrm{C}$ NMR (125 MHz, $\left.\mathrm{C}_{6} \mathrm{D}_{6}, 298 \mathrm{~K}\right) \delta 204.98(\mathrm{C}-1)$, 183.17 (C-3), 175.16 (C-12), 137.10 (C-5), 134.04 (C-4), 120.76 (C-4'), 116.70 (C-5'), 111.82 (C-16), 101.24 (C-2), 89.22 (C-10), 83.44 (C-6), 78.42 (C-8), 63.60 (C-7), 61.25 (C-11), 49.54 (OMe), 43.09 (C-9), 32.01 (C17), 22.24 (C-14), 21.92 (C-13), 18.66 (C-18 or C-19), 18.48 (C-15), 17.12 (C-18 or C-19),; VG 70-SE Positive Ion FAB HRMS: Calcd. for $\mathrm{C}_{22} \mathrm{H}_{30} \mathrm{NaO}_{6}(\mathrm{M}+\mathrm{Na})^{+}:$413.19400. Found: 413.19337.

Data for 1: $[\alpha]_{D}-22.9^{\circ}\left(c 0.35, \mathrm{CH}_{2} \mathrm{Cl}_{2}\right)$; IR $2970(\mathrm{~m}), 2928(\mathrm{~m}), 1773(\mathrm{~s}), 1701(\mathrm{~s}), 1634(\mathrm{~m}), 1562(\mathrm{~s}), 1454$ (w), $1371(\mathrm{~m}), 1329$ (w), 1259 (m), 1108 (s), 1013 (s), 957 (m), $934(\mathrm{~m}) \mathrm{cm}^{-1} ;{ }^{1} \mathrm{H}$ NMR (500 MHz, $\mathrm{C}_{6} \mathrm{D}_{6}, 298$ K) $\delta 5.80$ (br s, 1H, H-4'a), 5.48 (ddd, 1H, J=6.7, 10.4, $17.1 \mathrm{~Hz}, \mathrm{H}-5), 5.45$ (s, 1H, H-2), 5.04 (ddd, $1 \mathrm{H}, J=$ 
1.2, 1.2, $17.1 \mathrm{~Hz}, \mathrm{H}-5$ 'a), 4.91 (m, 1H, H-4'b), 4.84 (ddd, 1H, J=1.1, 1.1, $10.4 \mathrm{~Hz}, \mathrm{H}-5$ 'b), 4.52 (dd, $1 \mathrm{H}, J=$ 7.0, 7.0 Hz, H-6), 3.99 (ddd, 1H, H-8), 2.99 (s, 3H, OMe), 2.02 (septet, 1H, J=7.2 Hz, H-17) superimposed upon 2.00 (dd, $1 \mathrm{H}, J=14.3,14.4 \mathrm{~Hz}, \mathrm{H}-9 \mathrm{a}), 1.85$ (dd, $1 \mathrm{H}, J=3.9,7.4 \mathrm{~Hz}, \mathrm{H}-7$ ), 1.64 (dd, $1 \mathrm{H}, J=2.7,14.3$ $\mathrm{Hz}, \mathrm{H}-9 \mathrm{~b}), 1.52$ (large narrow dd that resembles a s, 3H, J=0.9, $1.3 \mathrm{~Hz}, \mathrm{Me}-15), 1.26$ (s, 3H, Me-14), 1.22 $\left(\mathrm{d}, 3 \mathrm{H}, J=7.0 \mathrm{~Hz}, \mathrm{Me}-18\right.$ or Me-19), 1.13 (d, 3H, J=7.2 Hz, Me-18 or Me-19), 1.11 (s, 3H, Me-13); ${ }^{13} \mathrm{C}$ NMR (125 MHz, C $\mathrm{D}_{6}, 298$ K) $\delta 204.54$ (C-1), 181.48 (C-3), 175.16 (C-12), 137.14 (C-5), 134.08 (C-4), 119.77 (C-4'), 116.61 (C-5'), 112.19 (C-16), 101.97 (C-2), 87.83 (C-10), 83.41 (C-6), 77.06 (C-8), 62.96 (C7), 61.38 (C-11), 49.66 (OMe), 43.64 (C-9), 32.08 (C-17), 23.31 (C-14), 21.92 (C-13), 18.77 (C-18 or C-19), 18.53 (C-15), 16.99 (C-18 or C-19); VG 70-SE Positive lon FAB HRMS: Calcd. for $\mathrm{C}_{22} \mathrm{H}_{30} \mathrm{NaO}_{6}(\mathrm{M}+\mathrm{Na})^{+}$: 413.19400. Found: 413.19298.

\section{(+)-16-Methoxy-Eremantholide A (III-YL-38)}
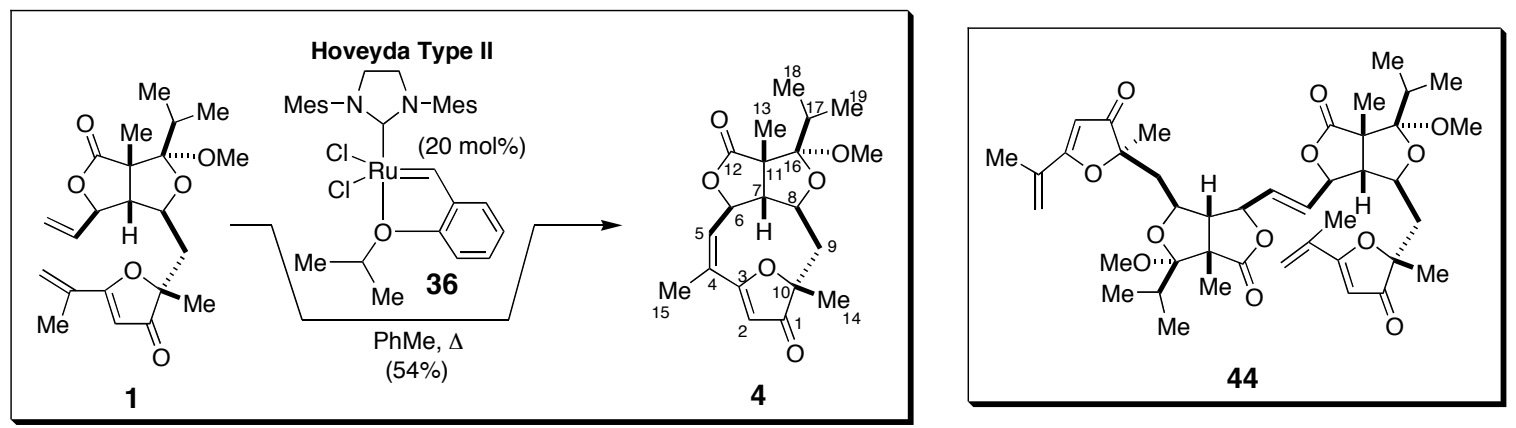

A solution of 1 (20 mg, $0.0512 \mathrm{mmol})$ and the Hoveyda type II RCM catalyst 36 (6.4 mg, 0.0102 mmol, 0.2 equiv) in dry PhMe $(50 \mathrm{~mL})$ was heated at reflux for $14 \mathrm{~h}$. After this time, TLC analysis (petrol/EtOAc 2:1) revealed that a slightly faster-moving product 4 had formed together with a much slower-moving cross metathesis product 44. At this juncture, the solvent was removed in vacuo and the crude residue was purified by multiple-elution preparative TLC using petrol/EtOAc (3:1) as eluent. The desired product 4 (10 $\mathrm{mg}, 54 \%$ ) was isolated as a colorless oil following $\mathrm{SiO}_{2}$ flash chromatography of the concentrated residue using the same eluent. The cross-metathesis product $\mathbf{4 4}$ was typically isolated in about $15 \%$ yield on the various runs in which the yield of this undesired by-product was quantified.

Data for 4: $[\alpha]_{\mathrm{D}}+21.4^{\circ}\left(c 0.168, \mathrm{CHCl}_{3}\right)$; IR $2976(\mathrm{~m}), 2928(\mathrm{~m}), 1776(\mathrm{~s}), 1709(\mathrm{~s}), 1659(\mathrm{~m}), 1587(\mathrm{~s}), 1450$ (w), 1369 (m), 1288 (m), 1134 (s), 1099 (s), 1057 (s), 1013 (s), 910 (m) 735 (m) cm ; ; H NMR (500 MHz, $\left.\mathrm{CDCl}_{3}, 298 \mathrm{~K}\right) \delta 5.97(\mathrm{~m}, 1 \mathrm{H}, \mathrm{H}-5), 5.58(\mathrm{~s}, 1 \mathrm{H}, \mathrm{H}-2), 4.71(\mathrm{~m}, 1 \mathrm{H}, \mathrm{H}-6), 3.70$ (ddd, 1H, J = 2.6, 4.1, 11.7 $\mathrm{Hz}, \mathrm{H}-8$ ), 3.19 (s, 3H, OMe), 2.75 (dd, 1H, J=4.2, $7.2 \mathrm{~Hz}, \mathrm{H}-7$ ), 2.35 (dd, 1H, J = 2.5, $13.6 \mathrm{~Hz}, \mathrm{H}-9 \mathrm{a}), 2.08$ (septet, 1H, J=7.1 Hz, H-17), 2.02 (m, H-9b) superimposed upon 2.02 (s, 3H, Me-15), 1.47 (s, 3H, Me-14), 1.28 (s, 3H, Me-13), 1.09 (d, 3H, J = 7.3 Hz, Me-18 or Me-19), 1.07 (d, 3H, J=7.1 Hz, Me-18 or Me-19); ${ }^{13} \mathrm{C}$ NMR (125 MHz, CDCl, 298 K) $\delta 205.18$ (C-1), 186.85 (C-3), 175.86 (C-12), 134.74 (C-5), 129.96 (C-4), 109.97 (C-16), 104.41 (C-2), 89.90 (C-10), 81.49 (C-6), 78.43 (C-8), 63.85 (C-7), 62.28 (C-11), 50.01 (OMe), 43.95 (C-9), 31.15 (C-17), 22.25 (C-13), 20.54 (C-14), 20.38 (C-15), 18.46 (C-18 or C-19), 16.71 (C-18 or C- 
19); VG 70-SE Positive Ion FAB HRMS: Calcd. for $\mathrm{C}_{20} \mathrm{H}_{27} \mathrm{O}_{6}(\mathrm{M}+\mathrm{H})^{+}$: 363.18075. Found: 363.17937. Our spectra and spectral data for $\mathbf{4}$ correlated well with the spectra and data reported for $\mathbf{4}$ by Tadano et al. ${ }^{2 b}$

\section{(+)-Eremantholide A (III-YL-39)}

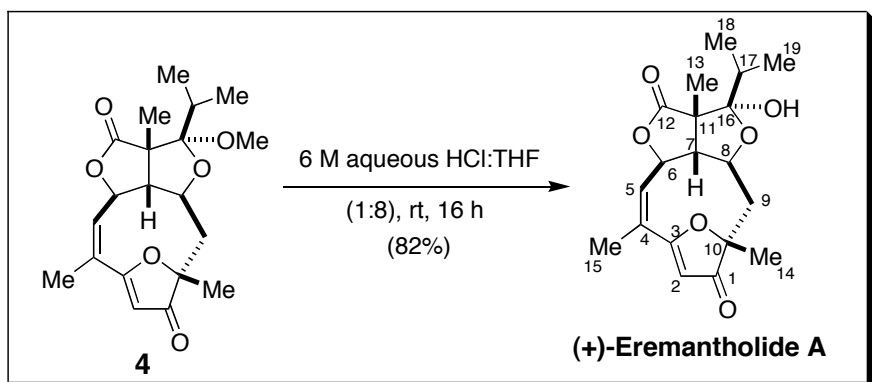

(+)-16-Methoxy-eremantholide A $4(5.1 \mathrm{mg}, 0.0141 \mathrm{mmol})$ was treated with an 8:1 v/v mixture of THF: $6 \mathrm{M}$ aqueous $\mathrm{HCl}(1 \mathrm{~mL})$ at $\mathrm{rt}$ for $16 \mathrm{~h}$. Solid $\mathrm{NaHCO}_{3}$ was then added to neutralise the excess aqueous $\mathrm{HCl}$, and EtOAc $(10 \mathrm{~mL})$ was added followed by $\mathrm{H}_{2} \mathrm{O}(5 \mathrm{~mL})$. The organic layer was separated and washed with $\mathrm{H}_{2} \mathrm{O}(5 \mathrm{~mL})$ and brine $(5 \mathrm{~mL})$, dried over $\mathrm{MgSO}_{4}$, filtered and concentrated in vacuo. The residue was purified by preparative TLC using EtOAc/petrol (1:3) as eluent. Further final purification was effected by $\mathrm{SiO}_{2}$ flash chromatography with the same eluent. Our synthetic (+)-eremantholide A (4.0 mg, 82\%) was obtained as an amorphous white solid; its spectra and spectral properties matched those reported by Tadano et al. ${ }^{2 b}$

Data for synthetic (+)-Eremantholide A: $[\alpha]_{D}+38.8^{\circ}\left(c 0.08\right.$, EtOH) $\left[\mathrm{Lit.}^{2 \mathrm{~b}}:[\alpha]_{\mathrm{D}}+68.8^{\circ}(c 0.18, \mathrm{EtOH})\right] ;{ }^{1} \mathrm{H}$ NMR $\left(500 \mathrm{MHz}, \mathrm{CDCl}_{3}, 298 \mathrm{~K}\right) \delta 5.99(\mathrm{~m}, 1 \mathrm{H}, \mathrm{H}-5), 5.57$ (s, 1H, H-2), $4.93(\mathrm{~m}, 1 \mathrm{H}, \mathrm{H}-6), 4.02$ (ddd, 1H, J= 2.6, 4.2, $11.8 \mathrm{~Hz}, \mathrm{H}-8$ ), 2.78 (dd, $1 \mathrm{H}, J=4.2,7.3 \mathrm{~Hz}, \mathrm{H}-7$ ), 2.28 (dd, 1H, J= 2.5, 13.6 Hz, H-9a) superimposed upon 2.27 (s, C-16 OH), 2.03 (dd, 3H, J=2.2, $1.8 \mathrm{~Hz}, \mathrm{Me}-15), 1.98$ (dd, 1H, J=13.6, 13.5 $\mathrm{Hz}, \mathrm{H}-9 \mathrm{~b}$ ) superimposed upon 1.99 (septet, 1H, H-17), 1.46 (s, 3H, Me-14), 1.30 (s, 3H, Me-13), 1.04 (d, 3H, $J=6.9 \mathrm{~Hz}, \mathrm{Me}-18$ or Me-19), $0.93\left(\mathrm{~d}, 3 \mathrm{H}, J=6.8 \mathrm{~Hz}, \mathrm{Me}-18\right.$ or Me-19); ${ }^{33} \mathrm{C} \mathrm{NMR}\left(125 \mathrm{MHz}, \mathrm{CDCl}_{3}, 298 \mathrm{~K}\right)$

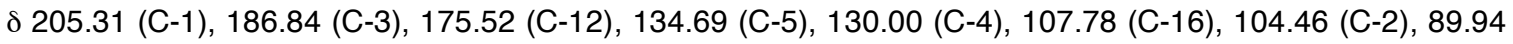
(C-10), 81.34 (C-6), 77.87 (C-8), 63.54 (C-7), 60.08 (C-11), 43.84 (C-9), 32.00 (C-17), 21.02 (C-13), 20.50 (C-14), 20.37 (C-15), 16.77 (C-18 or C-19), 16.56 (C-18 or C-19),; VG 70-SE Positive lon FAB HRMS: Calcd. for $\mathrm{C}_{19} \mathrm{H}_{25} \mathrm{O}_{6}(\mathrm{M}+\mathrm{H})^{+}:$349.16511. Found: 349.16412 .

Intersection with Tadano's Synthesis; A New Formal Asymmetric Total Synthesis of (+)Eremantholide A

Alcohol 37 (III-YL-45)

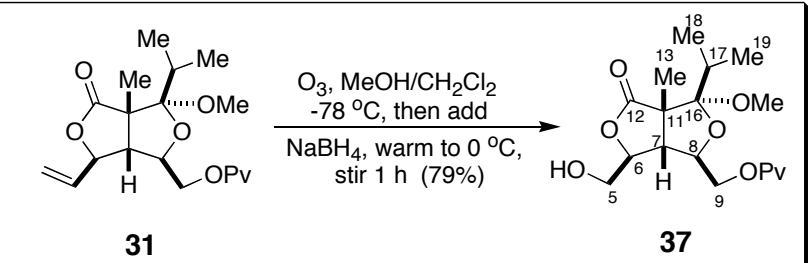


A stream of gaseous $\mathrm{O}_{3}$ was bubbled into a $-78{ }^{\circ} \mathrm{C}$ solution of alkene $31(20 \mathrm{mg}, 0.056 \mathrm{mmol})$ in $\mathrm{MeOH} / \mathrm{CH}_{2} \mathrm{Cl}_{2}(2: 1)(3 \mathrm{~mL})$ until a slightly blue color appeared. Whilst maintaining the reaction mixture at -78 ${ }^{\circ} \mathrm{C}, \mathrm{NaBH}_{4}(21 \mathrm{mg}, 0.56 \mathrm{mmol}$ ) was added in one portion and, after $5 \mathrm{~min}$, the reaction mixture was allowed to warm to $0{ }^{\circ} \mathrm{C}$. A second portion of $\mathrm{NaBH}_{4}(21 \mathrm{mg}, 0.56 \mathrm{mmol})$ was added and stirring was continued at 0 ${ }^{\circ} \mathrm{C}$ for $1 \mathrm{~h}$. EtOAc (30 mL) was then added and the mixture was passed through a short column of $\mathrm{SiO}_{2}$ gel which was later washed thoroughly with EtOAc. Following concentration of the solvents in vacuo, the residue was purified by $\mathrm{SiO}_{2}$ flash chromatography (petrol/EtOAc 3:1) whereafter 37 (16 mg, 79\%) was obtained as a colorless oil.

Data for 37: $[\alpha]_{D}-41.1^{\circ}\left(c 0.246, \mathrm{CH}_{2} \mathrm{Cl}_{2}\right)$; IR $3467(\mathrm{br} \mathrm{m}), 2941(\mathrm{~s}), 1771(\mathrm{~s}), 1734(\mathrm{~s}), 1460(\mathrm{~m}), 1371(\mathrm{~m})$,

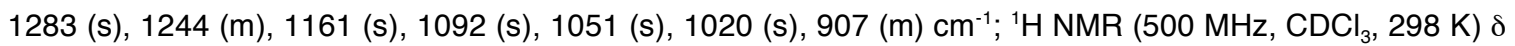
4.35 (ddd, $1 \mathrm{H}, J=3.5,3.8,7.3 \mathrm{~Hz}, \underline{\mathrm{H}}-6$ ), 4.19 (m, 1H, $\underline{\mathrm{H}}-9 \mathrm{a}$ ), 4.08 (complex m, 2H, H-8, H-9b), 3.83 (br m, $\underline{H}-5 \mathrm{a}), 3.57$ (m, 1H, ㅂ- -5b), 3.30 (s, 3H, OMe), 2.62 (dd, 1H, $J=3.9,7.0 \mathrm{~Hz}, \underline{\mathrm{H}}-7$ ), 2.16 (septet, $1 \mathrm{H}, J=7.2$ $\mathrm{Hz}, \underline{\mathrm{H}}-17), 2.06$ (br t, 1H, OH-5), 1.35 (s, 3H, Me-13), 1.20 (s, 9H, OC(O)CMe $\left.)_{3}\right), 1.13$ (d, 3H, J=7.2 Hz, Me18 or $\underline{\mathrm{Me}}-19), 1.09$ (d, 3H, J=7.0 Hz, Me-18 or $\underline{\mathrm{Me}}-19) \mathrm{ppm} ;{ }^{13} \mathrm{C}$ NMR $\left(125 \mathrm{MHz}, \mathrm{CDCl}_{3}, 298 \mathrm{~K}\right) \delta 178.31$

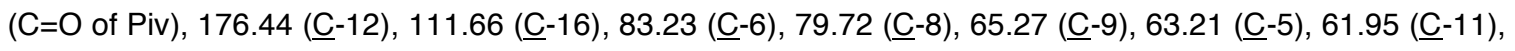

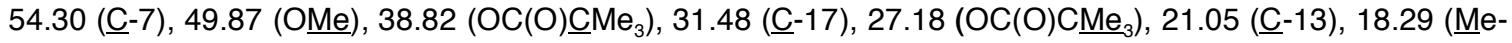
18 or $\underline{\mathrm{Me}}-19$ ), 16.83 (Me-18 or Me-19) ppm; VG 70-SE Positive Ion FAB HRMS: Calcd. for $\mathrm{C}_{18} \mathrm{H}_{30} \mathrm{NaO}_{7}$ $(\mathrm{M}+\mathrm{Na})^{+}:$381.18891. Found: 381.18794.

Pivaloate 38 (III-YL-46)

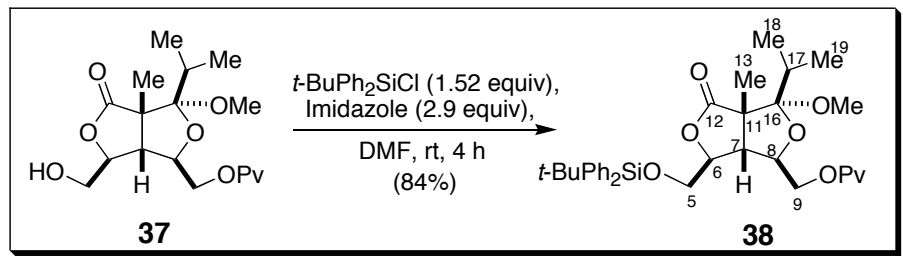

To a stirred rt solution of alcohol $37(20 \mathrm{mg}, 0.0558 \mathrm{mmol})$ in dry DMF (1 mL) under $\mathrm{N}_{2}$ was successively added imidazole $(11 \mathrm{mg}, 0.162 \mathrm{mmol})$ and $t-\mathrm{BuPh}_{2} \mathrm{SiCl}(0.022 \mathrm{~mL}, 0.0846 \mathrm{mmol})$ in single portions. The reaction mixture was stirred for $4 \mathrm{~h}$, whereafter it was diluted with $\mathrm{Et}_{2} \mathrm{O}(20 \mathrm{~mL})$ and washed with $\mathrm{H}_{2} \mathrm{O}(2 \times 2$ $\mathrm{mL})$, brine $(2 \mathrm{~mL})$ and dried over $\mathrm{MgSO}_{4}$. After filtration and concentration in vacuo, the residue was purified by $\mathrm{SiO}_{2}$ flash chromatography to give $38(28 \mathrm{mg}, 84 \%)$ as a colorless oil.

Data for 38: $[\alpha]_{D}-17.5^{\circ}\left(c 0.228, \mathrm{CH}_{2} \mathrm{Cl}_{2}\right)$; IR $2936(\mathrm{~s}), 2862(\mathrm{~m}), 1773(\mathrm{~s}), 1732(\mathrm{~s}), 1470(\mathrm{~m}), 1431(\mathrm{w})$, 1391 (w), 1367 (w), 1281 (w), 1157 (s), 1113 (s), 1057 (s), 1018 (m), 939 (w), 704 (s) cm ${ }^{-1}$; ${ }^{1} \mathrm{H}$ NMR (500 $\left.\mathrm{MHz}, \mathrm{CDCl}_{3}, 298 \mathrm{~K}\right) \delta$ 7.66-7.59 (m, 4H, Ph), 7.45-7.34 (m, 6H, Ph), 4.28 (partially overlapped ddd, 1H, J= 3.5, 3.4, 6.9 Hz, 브-6), 4.14-4.06 (m, 2H, 브-9a. H-9b), 4.01 (dd, 1H, J=4.4, 8.9 Hz, H-8), 3.82 (dd, 1H, J= 3.7, $11.4 \mathrm{~Hz}, \underline{\mathrm{H}}-5 \mathrm{a}), 3.63$ (dd, $1 \mathrm{H}, J=3.5,11.4 \mathrm{~Hz}, \underline{\mathrm{H}}-5 \mathrm{~b}), 3.28$ (s, 3H, OMe), 2.75 (dd, 1H, J=4.3, $6.8 \mathrm{~Hz}$, 
H-7), 2.17 (septet, $1 \mathrm{H}, J=7.2 \mathrm{~Hz}, \underline{\mathrm{H}}-17$ ), 1.38 (s, 3H, $\underline{\mathrm{Me}}-13$ ), 1.16 (s, 9H, OC(O)CMe $\left.{ }_{3}\right), 1.14$ (d, 3H, J= 7.2 $\mathrm{Hz}$, Me-18 or Me-19), 1.11 (d, 3H, $J=7.1 \mathrm{~Hz}$, Me-18 or Me-19), 1.03 (s, 9H, $t$-BuPh ${ }_{2} \mathrm{Si}$-) ppm; ${ }^{13} \mathrm{C}$ NMR (125

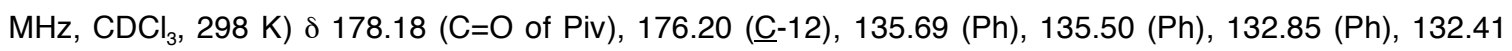

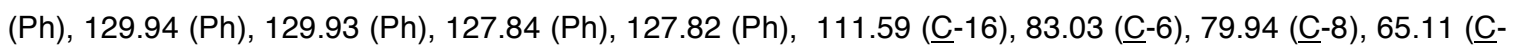

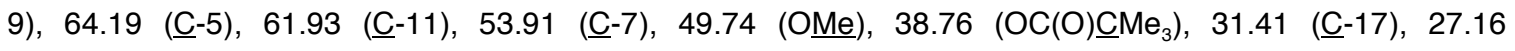

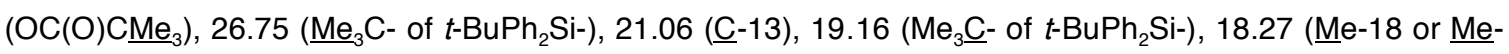
19), 16.86 (Me-18 or $\underline{\mathrm{Me}}-19)$ ppm; VG 70-SE Positive Ion FAB HRMS: Calcd. for $\mathrm{C}_{34} \mathrm{H}_{48} \mathrm{NaO}_{7} \mathrm{Si}(\mathrm{M}+\mathrm{Na})^{+}$: 619.30668. Found: 619.30708 .

\section{Tadano's Alcohol 39 (III-YL-48)}

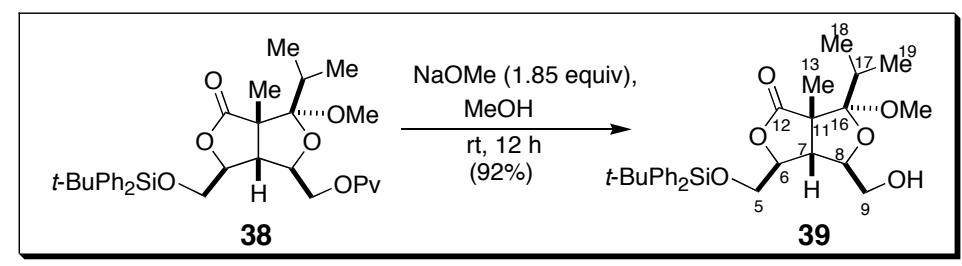

To a stirred rt solution of pivaloate $38(28 \mathrm{mg}, 0.047 \mathrm{mmol})$ in dry $\mathrm{MeOH}(1 \mathrm{~mL})$ was added a solution of $\mathrm{NaOMe}$ (prepared from $\mathrm{Na}(2 \mathrm{mg}, 0.087 \mathrm{mmol}, 1.85$ equiv) in $\mathrm{MeOH}(0.5 \mathrm{~mL}))$. The reactants were stirred for $12 \mathrm{~h}$, diluted with $\mathrm{CH}_{2} \mathrm{Cl}_{2}(15 \mathrm{~mL})$ and filtered through a short column of $\mathrm{SiO}_{2}$ gel that was washed thoroughly with $\mathrm{CH}_{2} \mathrm{Cl}_{2} / \mathrm{MeOH}(10: 1)$. The filtrate was concentrated in vacuo and the residue was purified by $\mathrm{SiO}_{2}$ flash chromatography with petrol/EtOAc (5:1) as eluent. The title compound $\mathbf{3 9}$ (22 $\left.\mathrm{mg}, 92 \%\right)$ was obtained as a white solid.

Data for 39: $[\alpha]_{\mathrm{D}}-32.4^{\circ}\left(\mathrm{c} \mathrm{0.364}, \mathrm{CHCl}_{3}\right)\left[\mathrm{Litt}^{2 \mathrm{~b}}:-35.1^{\circ}\left(\mathrm{c} .0 .92 \mathrm{CHCl}_{3}\right)\right]$; IR (KBr) 3555 (s br), 2934 (s), 2883 $(\mathrm{m}), 2858(\mathrm{~m}), 1745(\mathrm{~s}), 1466(\mathrm{w}), 1425(\mathrm{w}), 1381(\mathrm{w}), 1234(\mathrm{~m}), 1109$ (s), $1086(\mathrm{~s}), 1055(\mathrm{~s}), 974(\mathrm{w}), 949$ (w), 808 (w), 706 (s) cm ${ }^{-1} ;{ }^{1} \mathrm{H}$ NMR (500 MHz, $\left.\mathrm{CDCl}_{3}, 298 \mathrm{~K}\right) \delta$ 7.66-7.61 (m, 4H, Ph), 7.45-7.35 (m, 6H, Ph), 4.26 (ddd, $1 \mathrm{H}, J=4.1,6.9,8.3 \mathrm{~Hz}, \underline{\mathrm{H}}-6$ ), 3.90 (dd, $1 \mathrm{H}, J=4.1,8.6 \mathrm{~Hz}, \underline{\mathrm{H}}-8$ ), 3.76 (dd, $1 \mathrm{H}, J=4.6,11.2 \mathrm{~Hz}$, $\underline{\mathrm{H}}-5 \mathrm{a}), 3.68$ (dd, $1 \mathrm{H}, J=3.8,11.2 \mathrm{~Hz}, \underline{\mathrm{H}}-5 \mathrm{~b}$ ) superimposed upon 3.72-3.64 (very br s, $1 \mathrm{H}, \mathrm{H}-9 \mathrm{a}$ ), 3.55 (br m, $1 \mathrm{H}, \mathrm{H}-9 \mathrm{~b}$ ), 3.29 (s, 3H, OMe), 2.70 (dd, $1 \mathrm{H}, J=4.2,6.9 \mathrm{~Hz}, \underline{\mathrm{H}}-7$ ), 2.15 (septet, $1 \mathrm{H}, J=7.2 \mathrm{~Hz}, \underline{\mathrm{H}}-17$ ), 1.73 (br t, $1 \mathrm{H}, \mathrm{OH}-9$ ), 1.31 (s, 3H, Me-13), 1.15 (d, 3H, $J=7.2 \mathrm{~Hz}, \underline{\mathrm{Me}}-18$ or $\underline{\mathrm{Me}}-19), 1.11$ (d, 3H, J=7.0 Hz, Me-

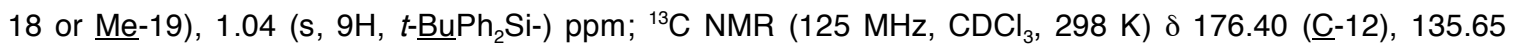
(Ph), $135.57(\mathrm{Ph}), 132.85(\mathrm{Ph}), 132.60(\mathrm{Ph}), 2 \times 129.94(\mathrm{Ph}), 127.83(\mathrm{Ph}), 127.82(\mathrm{Ph}), 111.71(\underline{\mathrm{C}}-16)$,

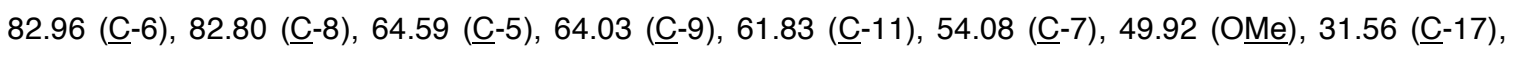
$26.81\left(\mathrm{Me}_{3} \mathrm{C}\right.$ - of $\left.t-\mathrm{BuPh}_{2} \mathrm{Si}-\right), 20.84$ ( $\left.\underline{\mathrm{C}}-13\right), 19.20\left(\mathrm{Me}_{3} \mathrm{C}-\right.$ of $\left.t-\mathrm{BuPh}_{2} \mathrm{Si}-\right), 18.50$ ( $\mathrm{Me}-18$ or $\left.\mathrm{Me}-19\right), 16.92$ (Me18 or $\mathrm{Me}-19)$ ppm; VG 70-SE Positive Ion FAB HRMS: Calcd. for $\mathrm{C}_{29} \mathrm{H}_{40} \mathrm{NaO}_{6} \mathrm{Si}(\mathrm{M}+\mathrm{Na})^{+}:$535.24917. Found: 535.25002. 


\title{
Asymmetric Total Synthesis and Formal Total Synthesis of the Antitumor Sesquiterpenoid (+)- Eremantholide A
}

\author{
Yi Li and Karl J. Hale* \\ The UCL Center for Chemical Genomics, \\ The Christopher Ingold Laboratories, \\ The Chemistry Department, \\ University College London, \\ 20 Gordon Street, \\ London WC1HOAJ, UK.
}

Supporting Information

Part 2. Copies of the 1D and 2D $500 \mathrm{MHz}{ }^{1} \mathrm{H}$ and $125 \mathrm{MHz}$ ${ }^{13} \mathrm{C}$ NMR Spectra, the HR Mass Spectra, and the IR Spectra for the Intermediates Used in the Asymmetric Total Synthesis and Formal Total Synthesis of (+)Eremantholide A. 


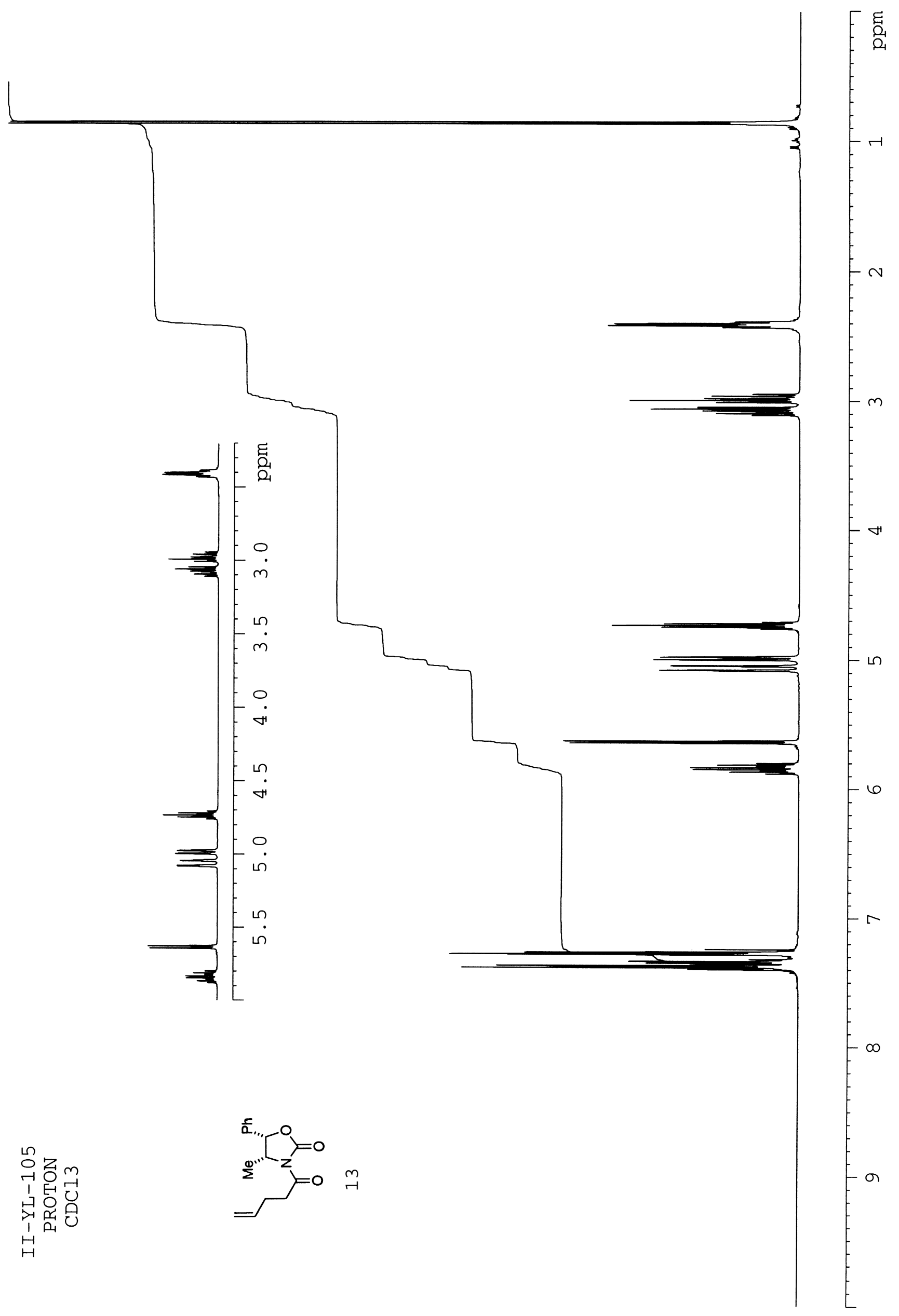




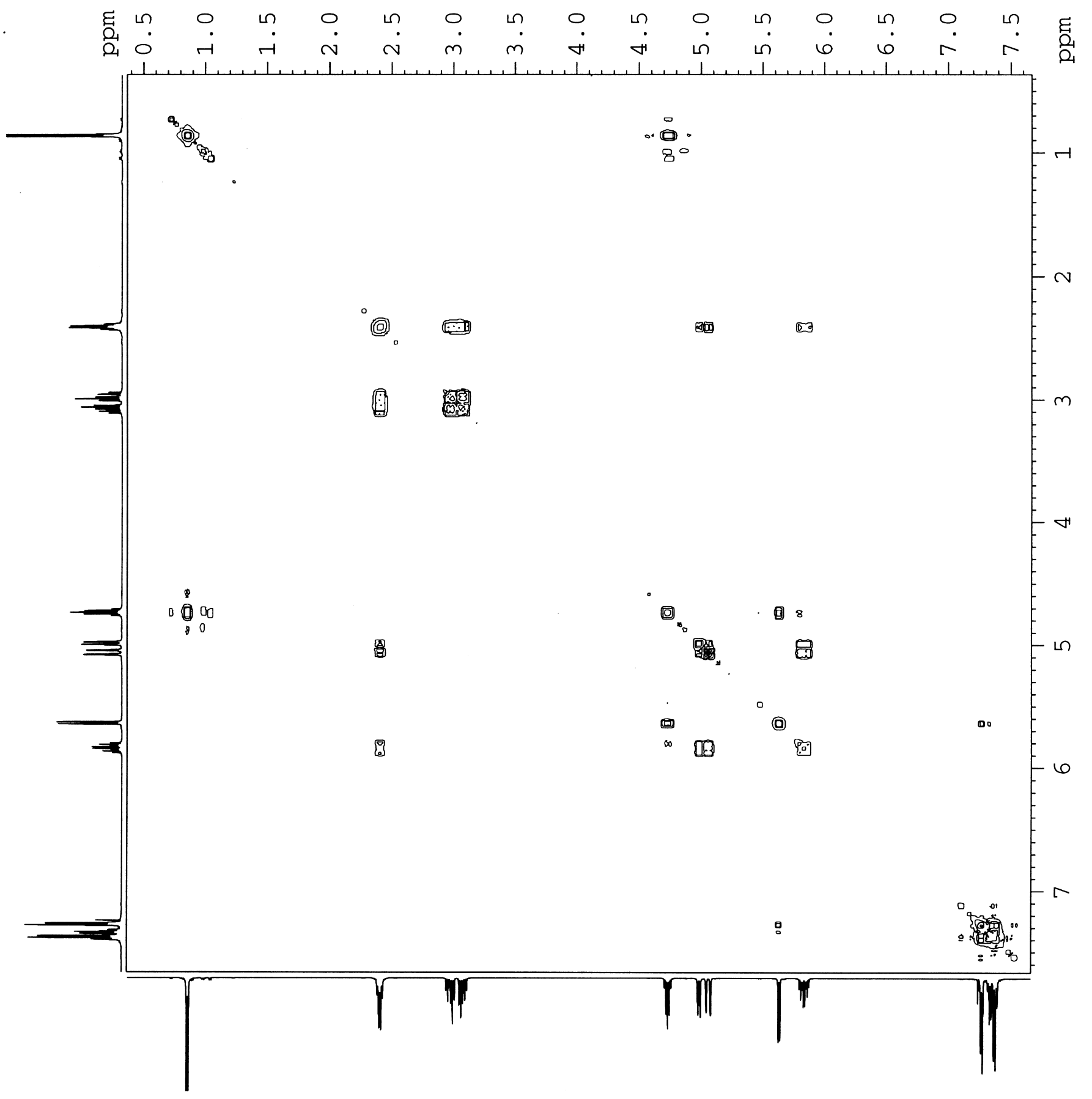

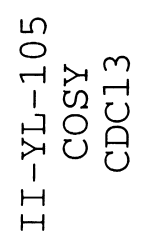

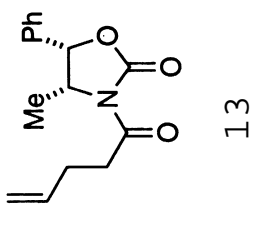




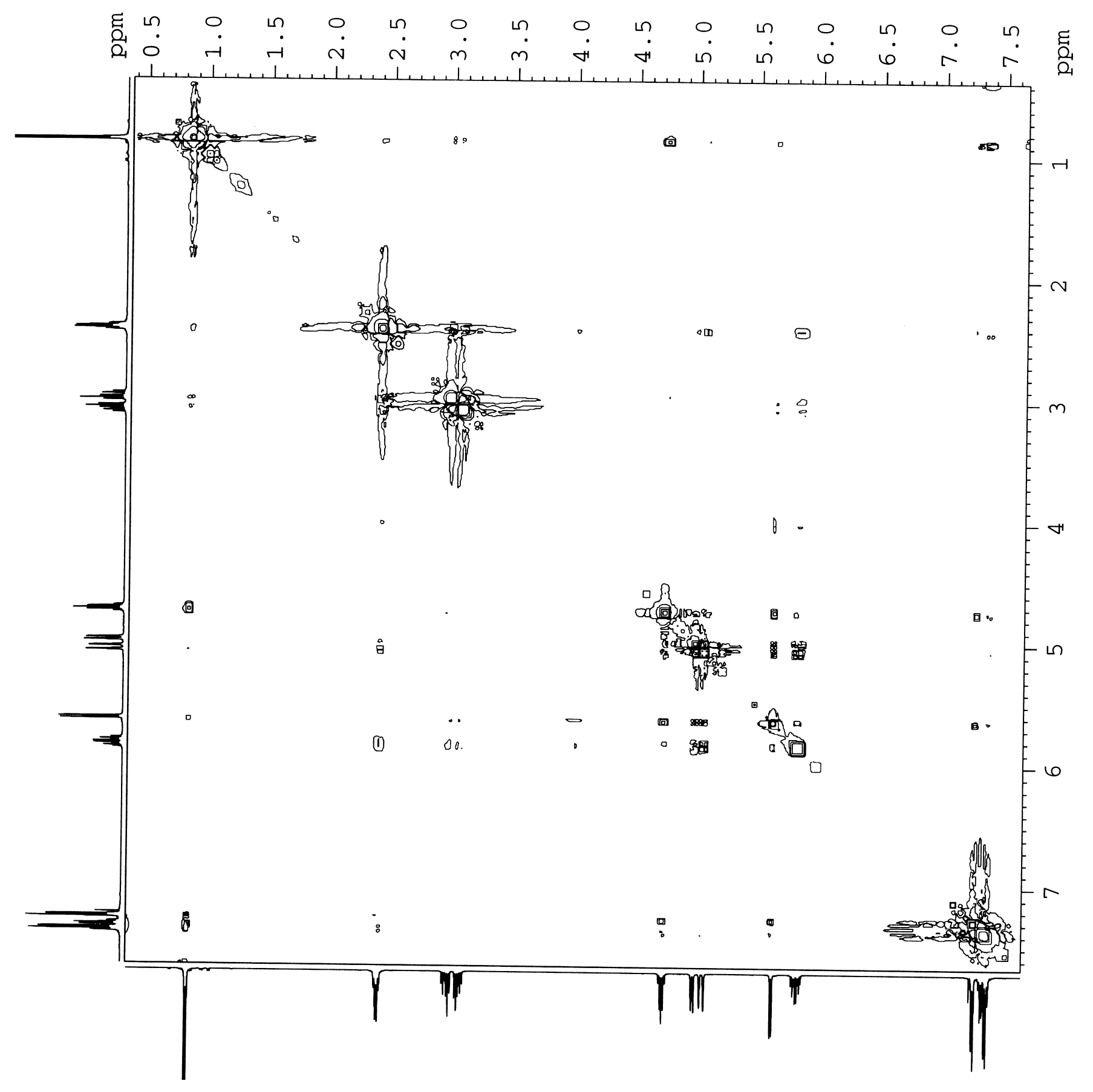

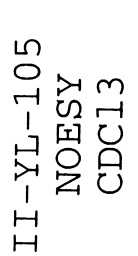

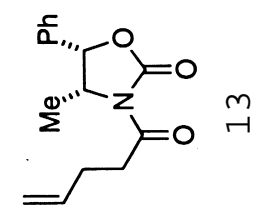




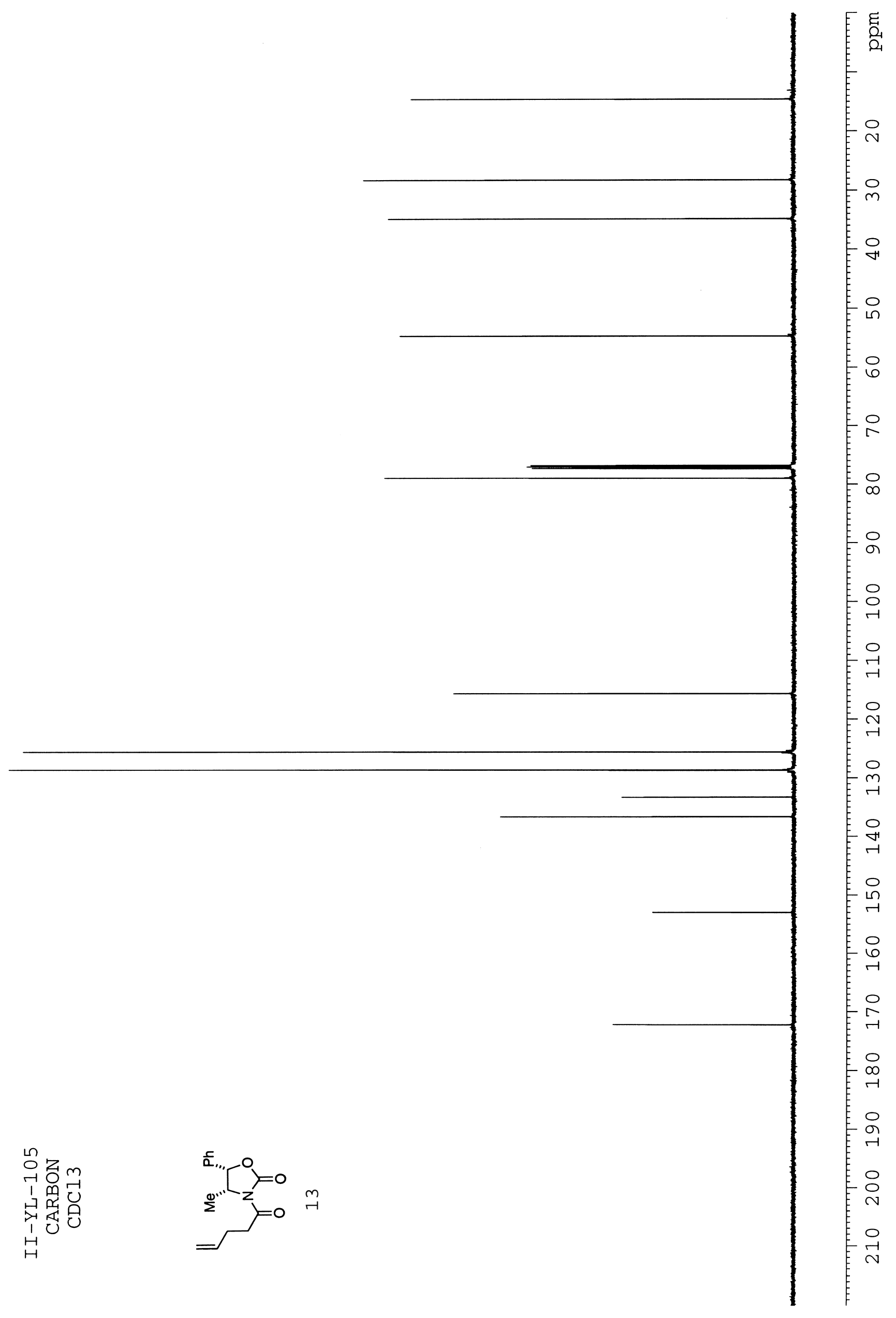




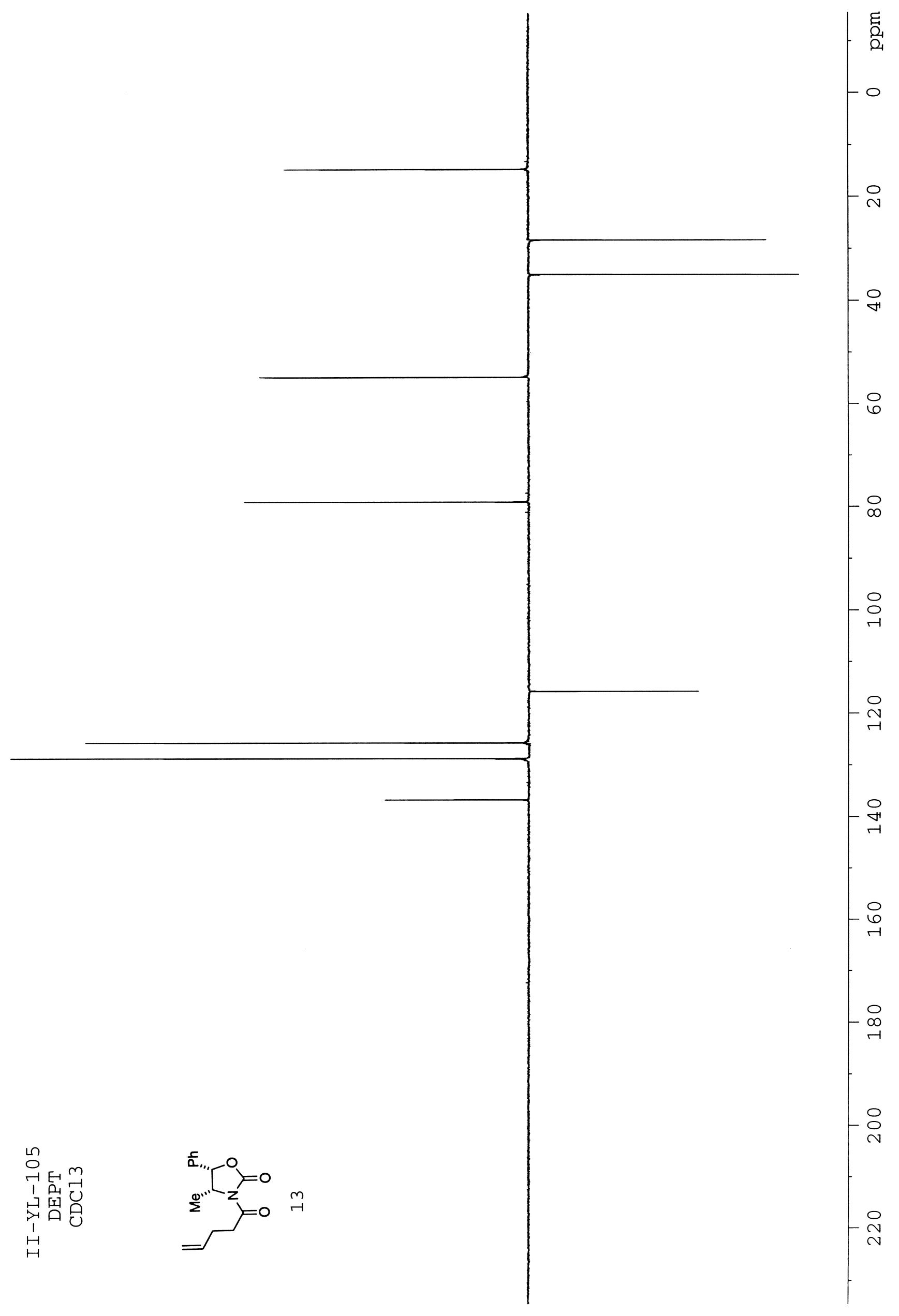




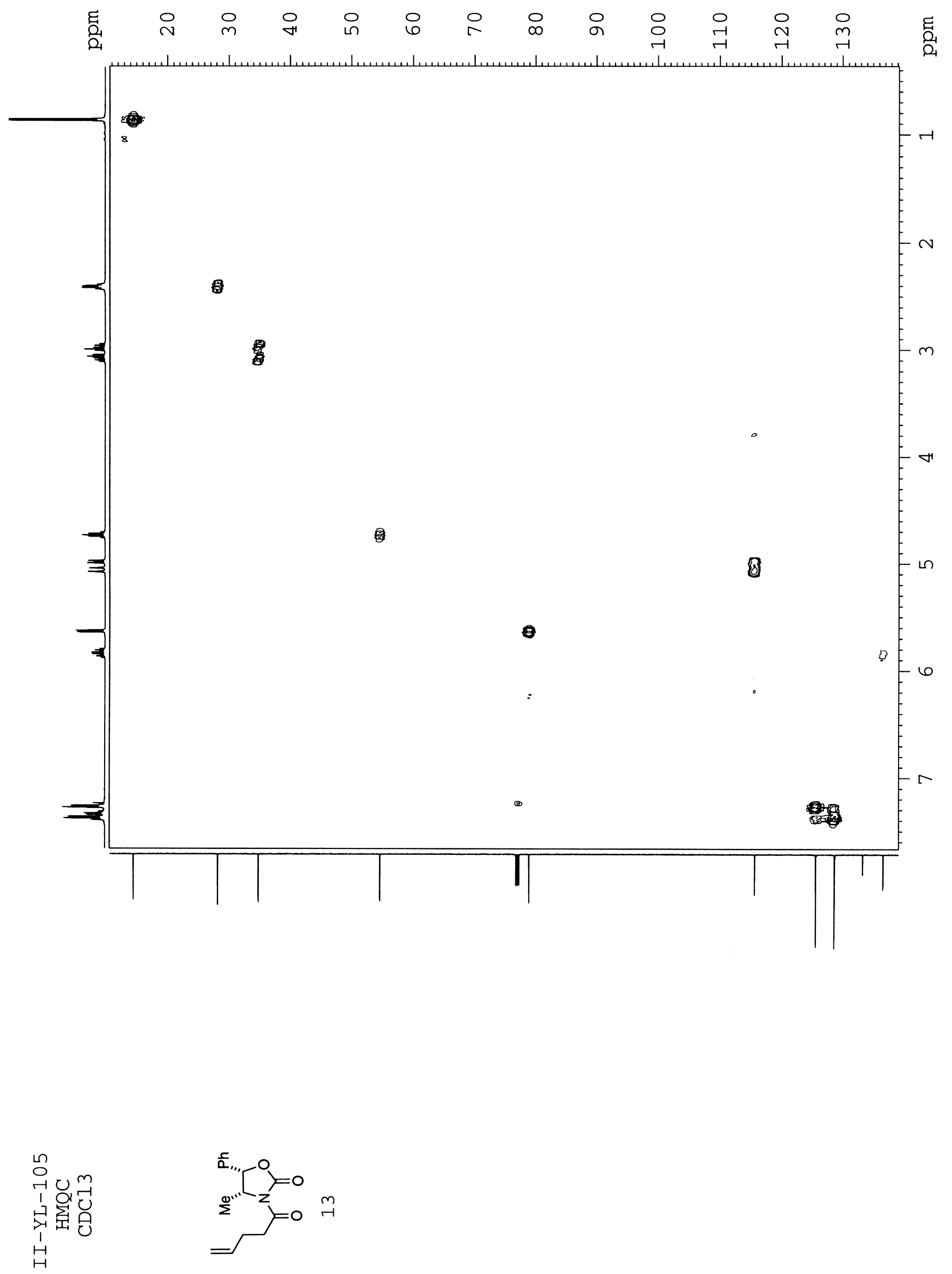



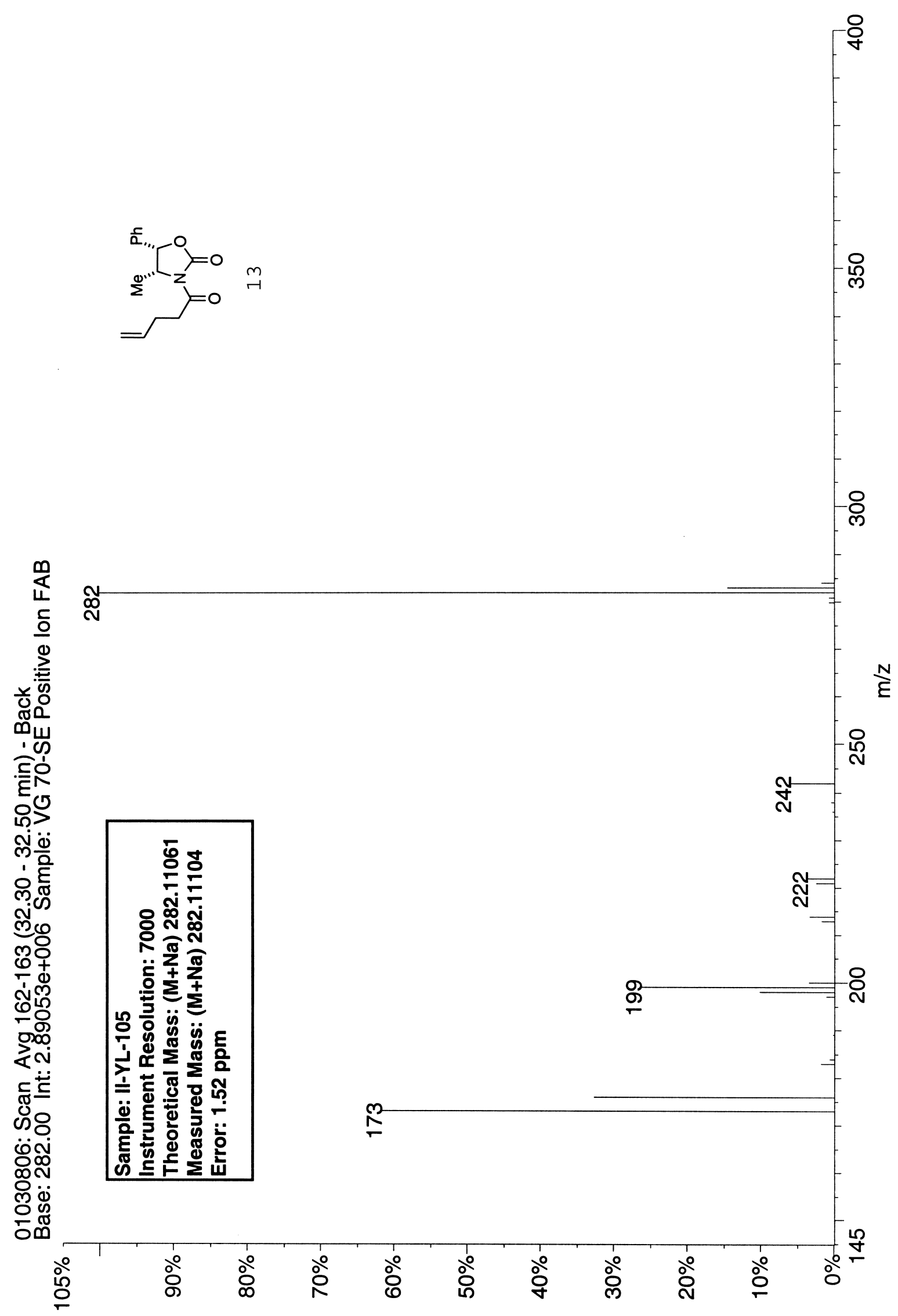


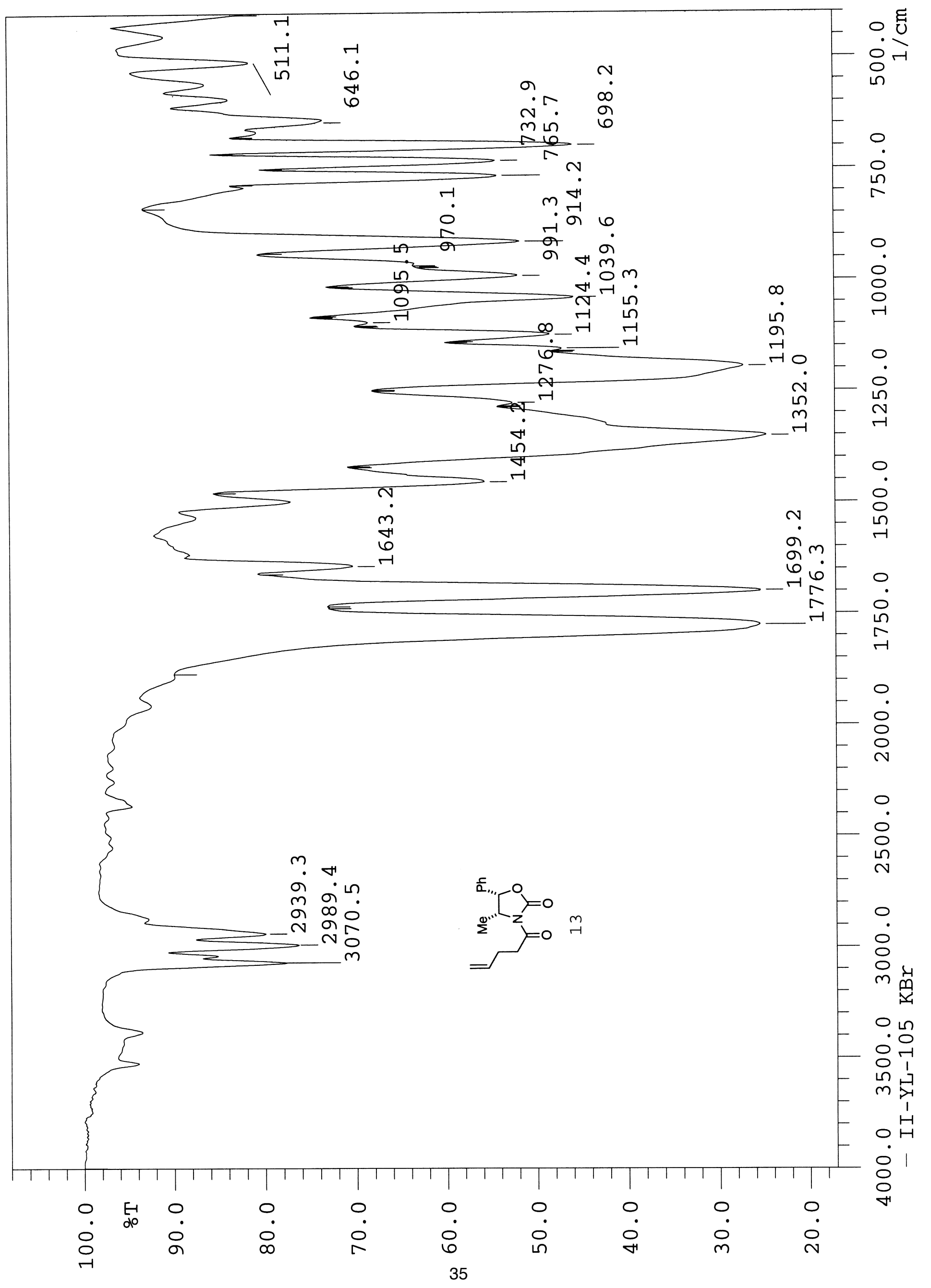




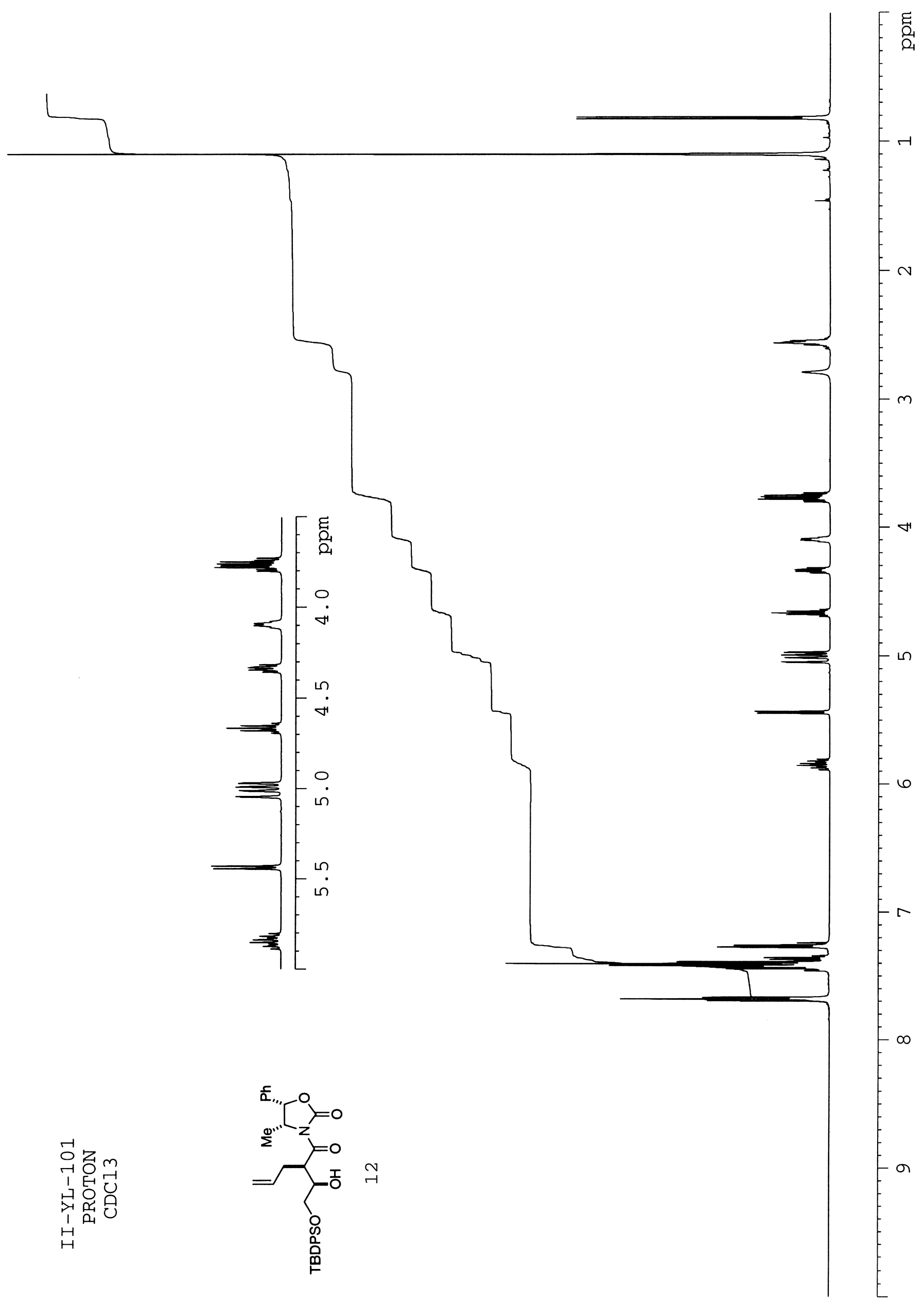



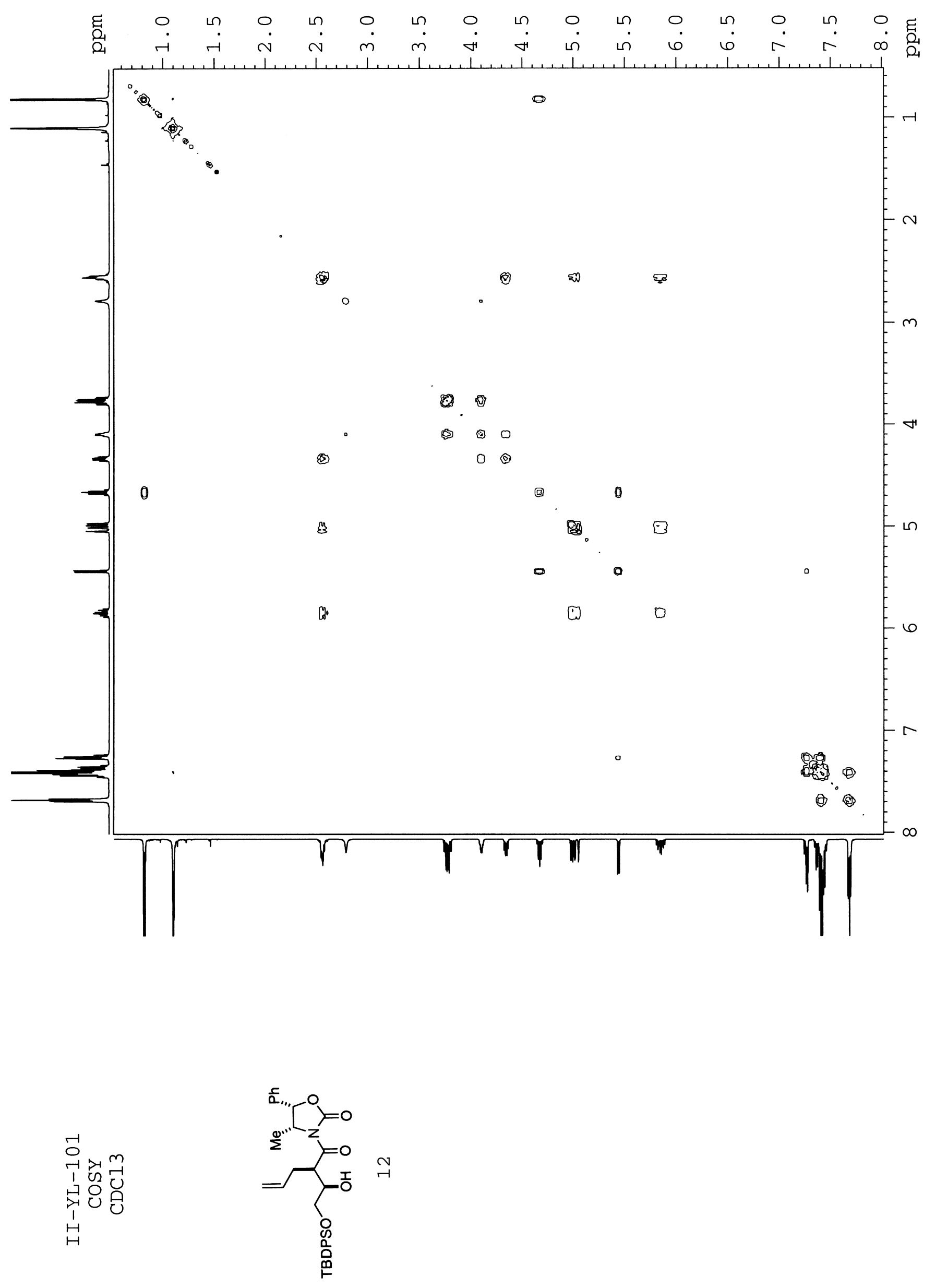


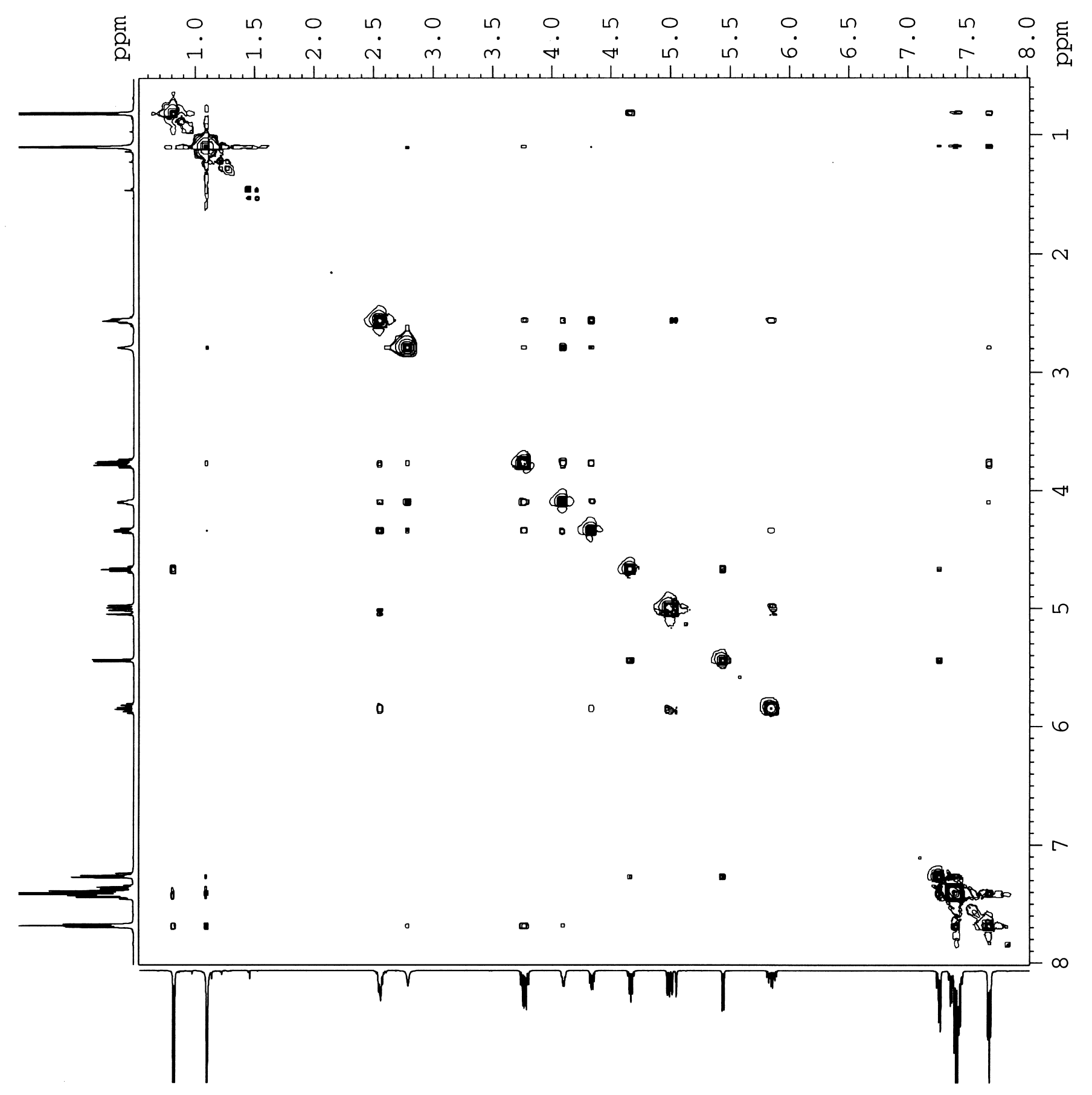

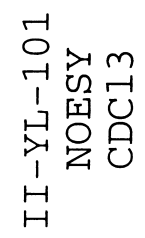

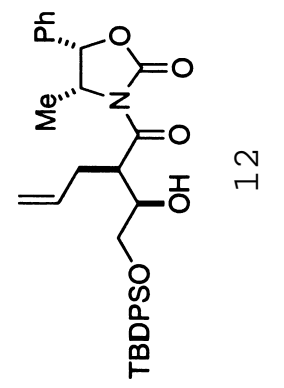




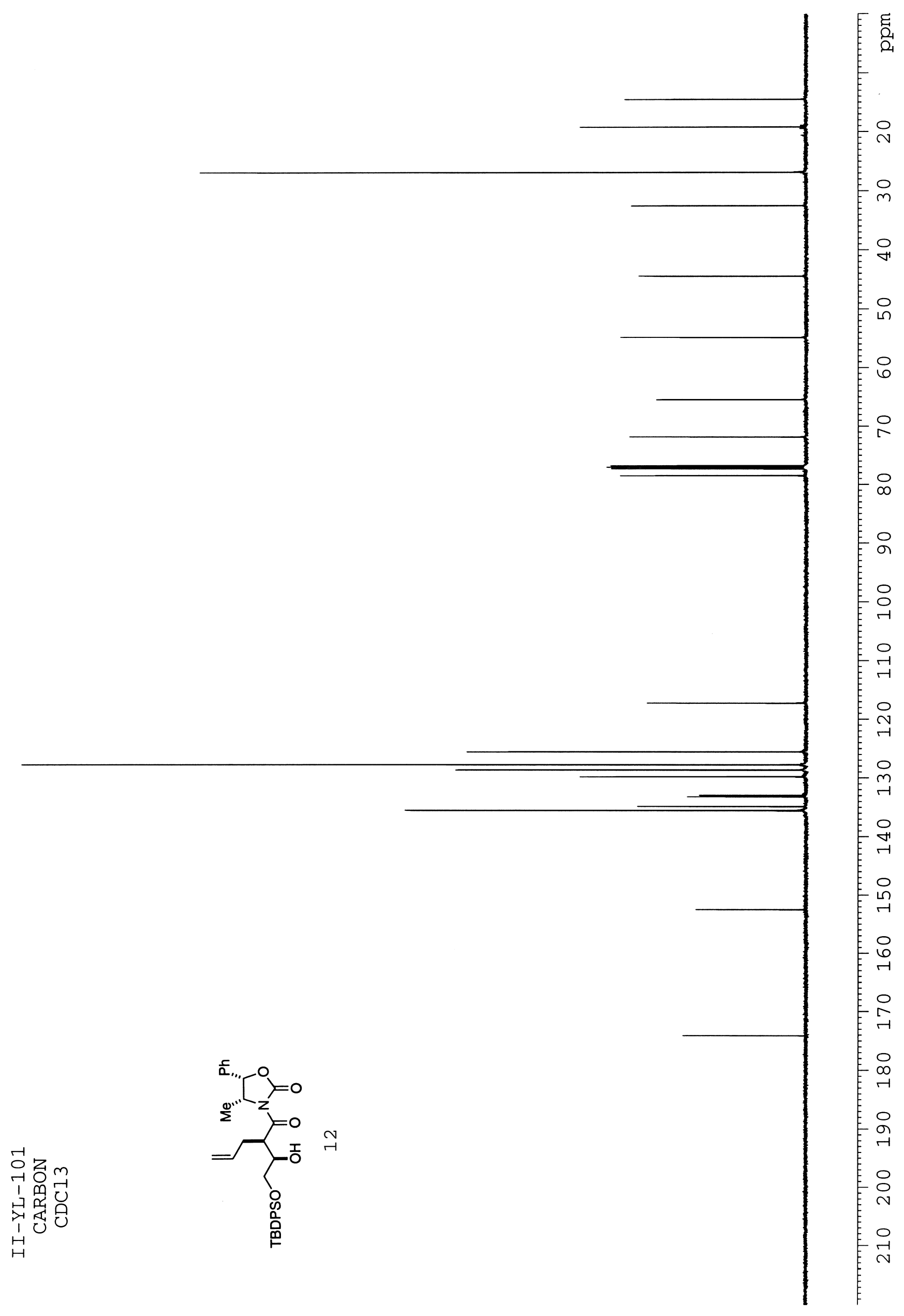




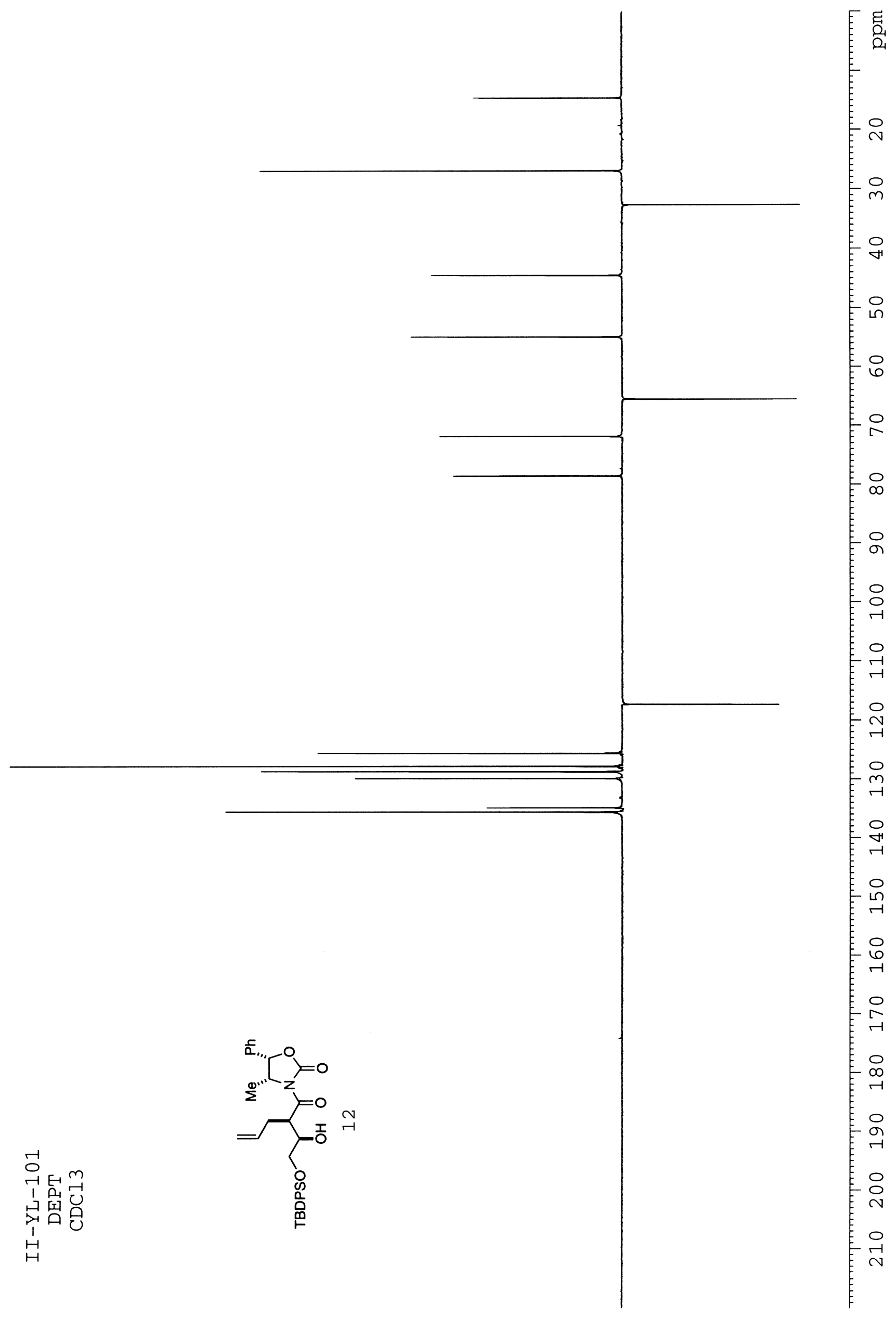




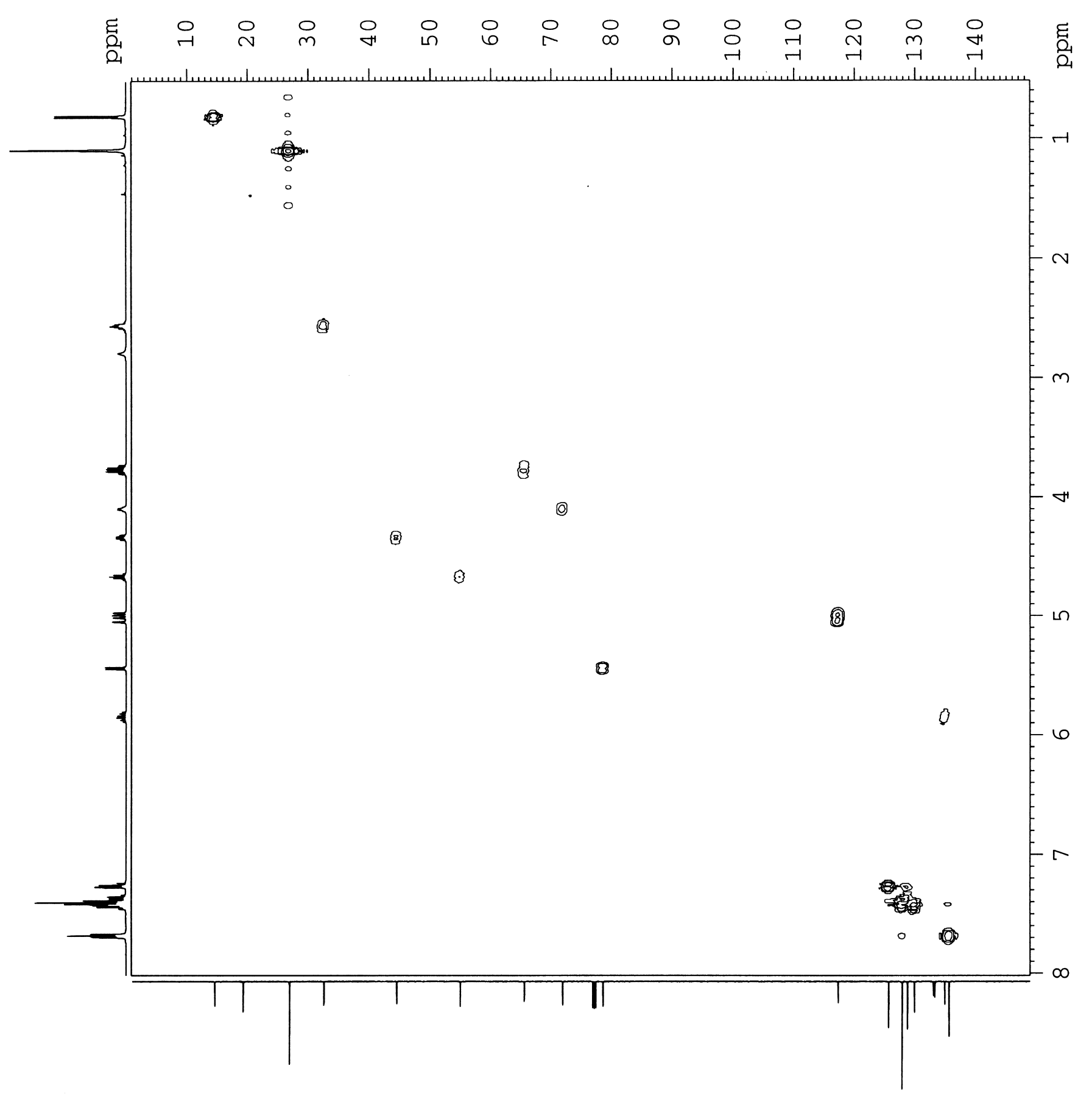

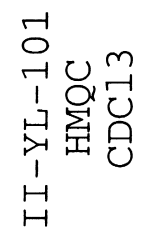

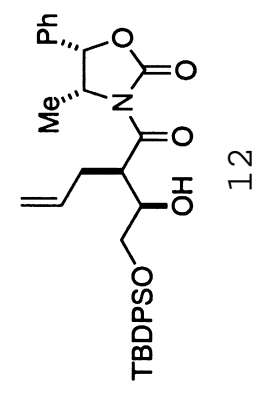



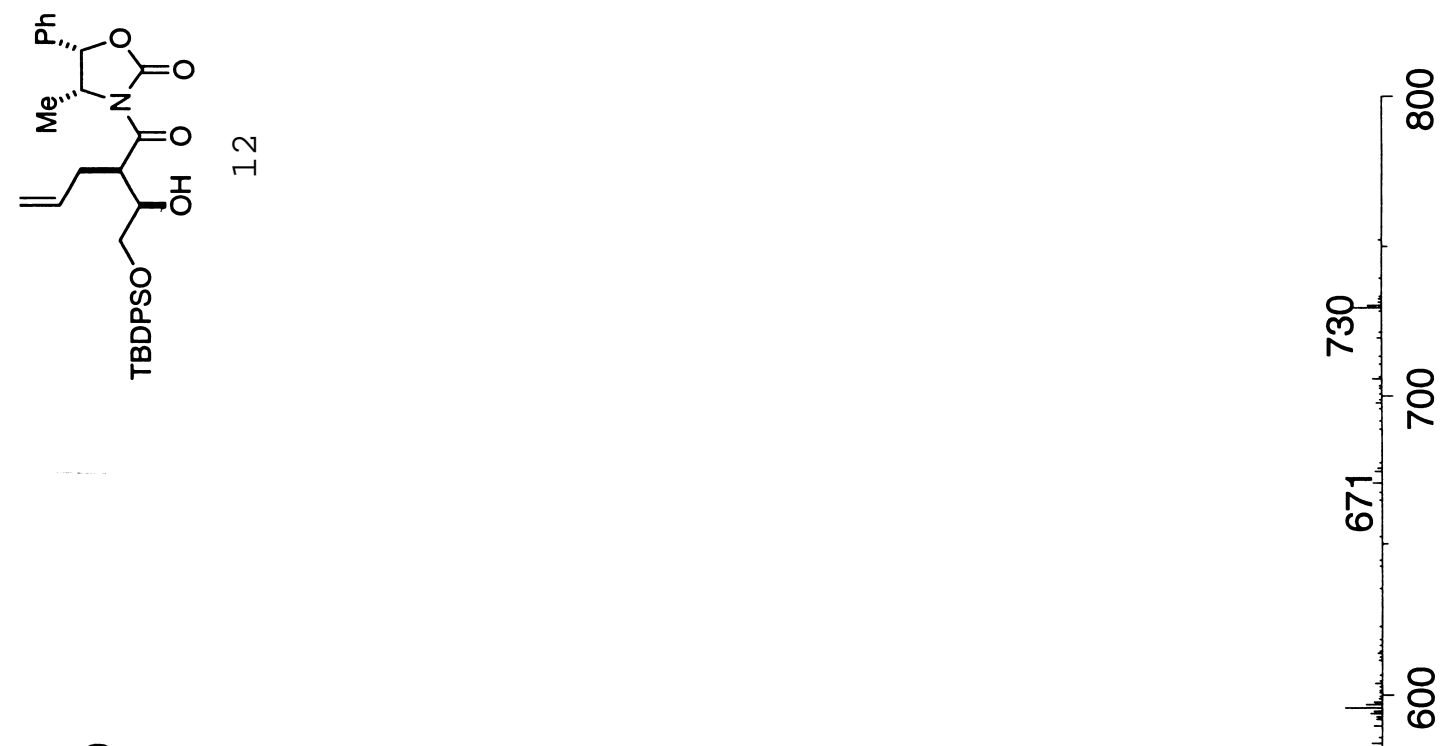

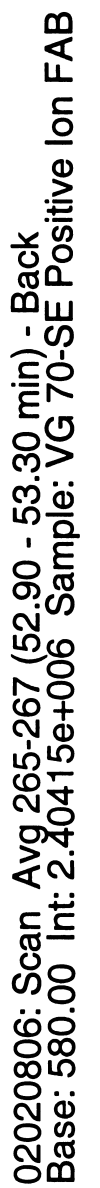
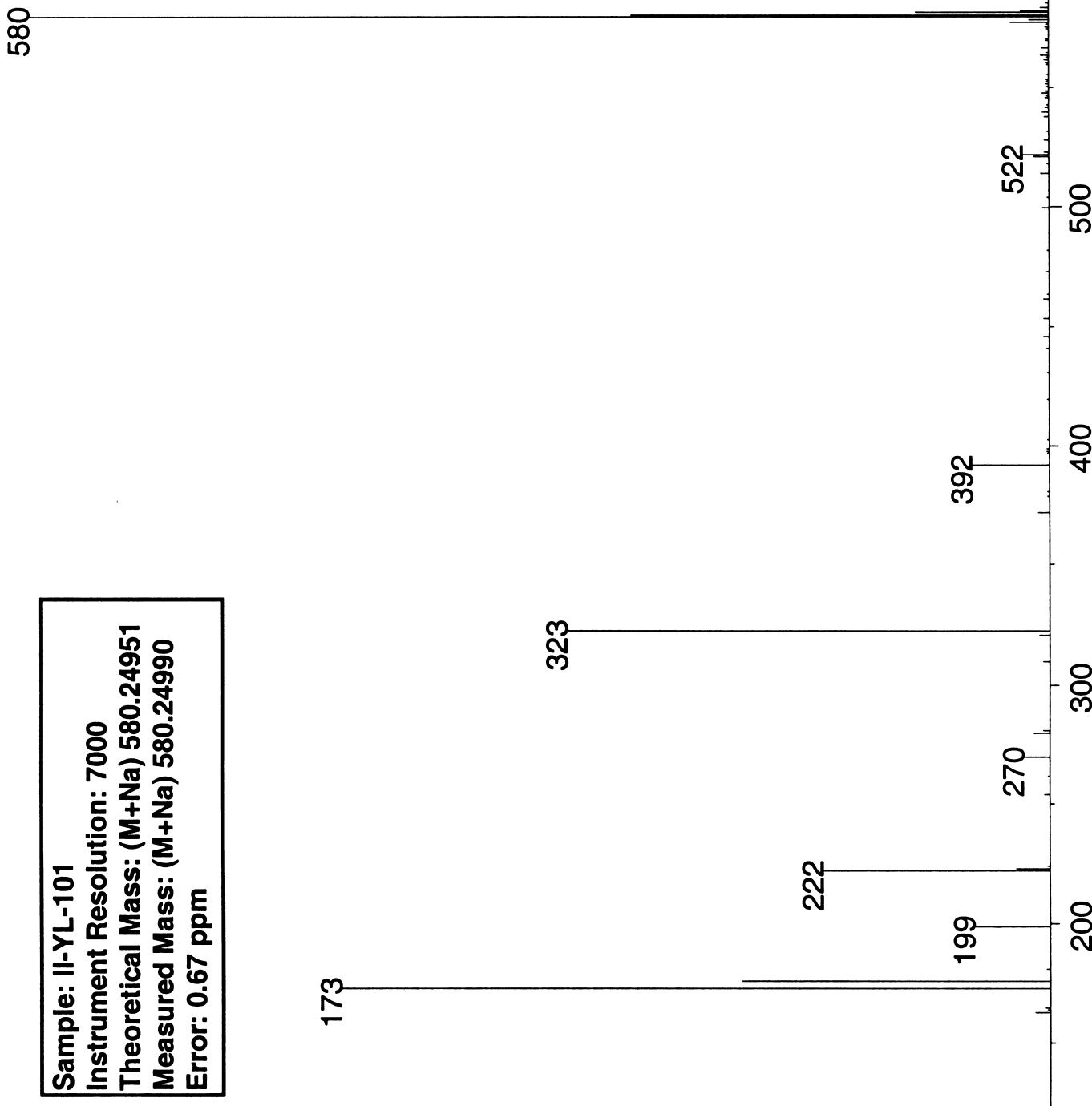

लై

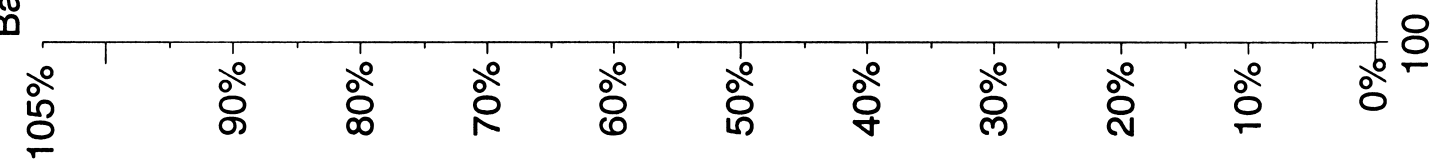




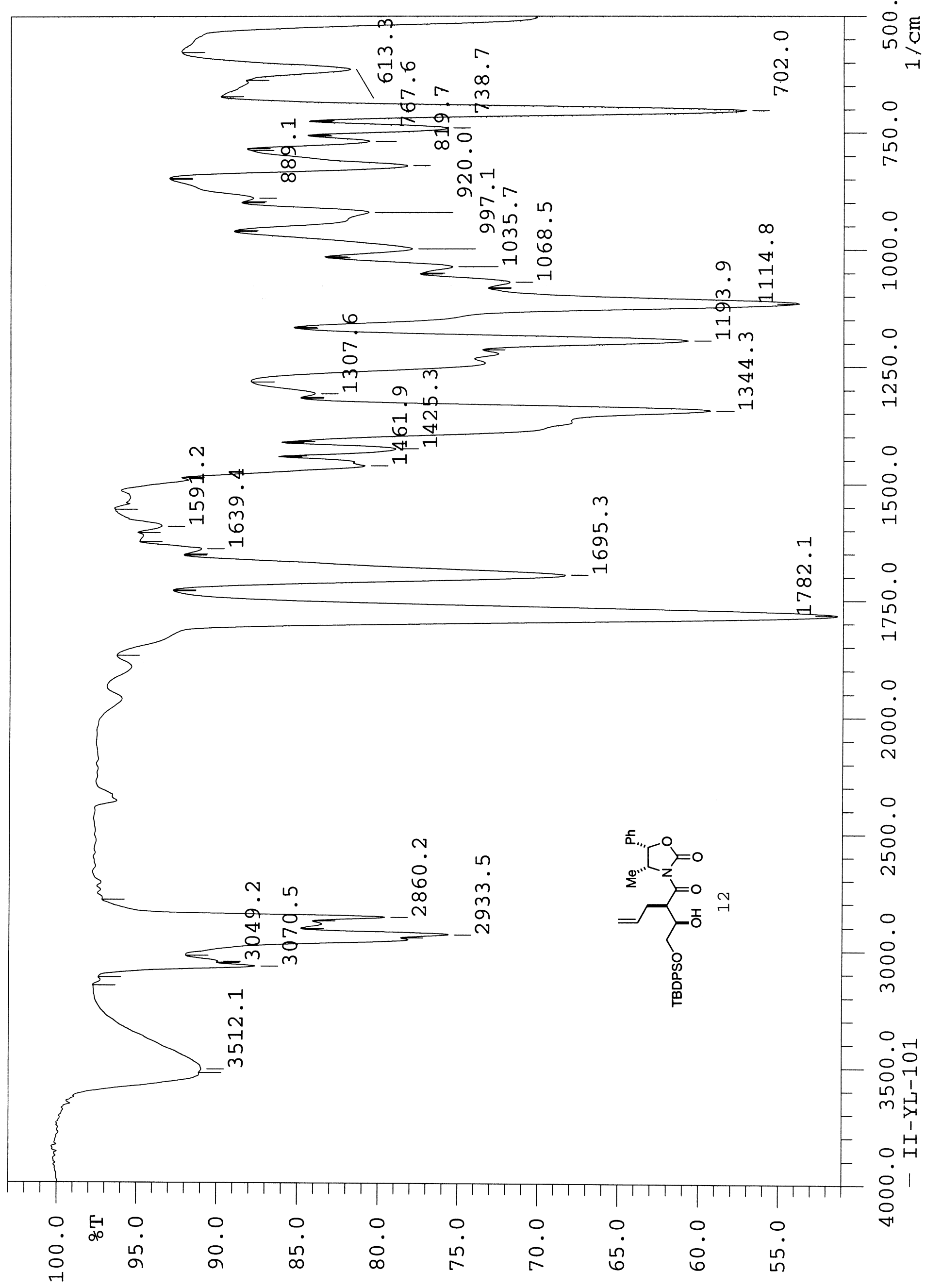




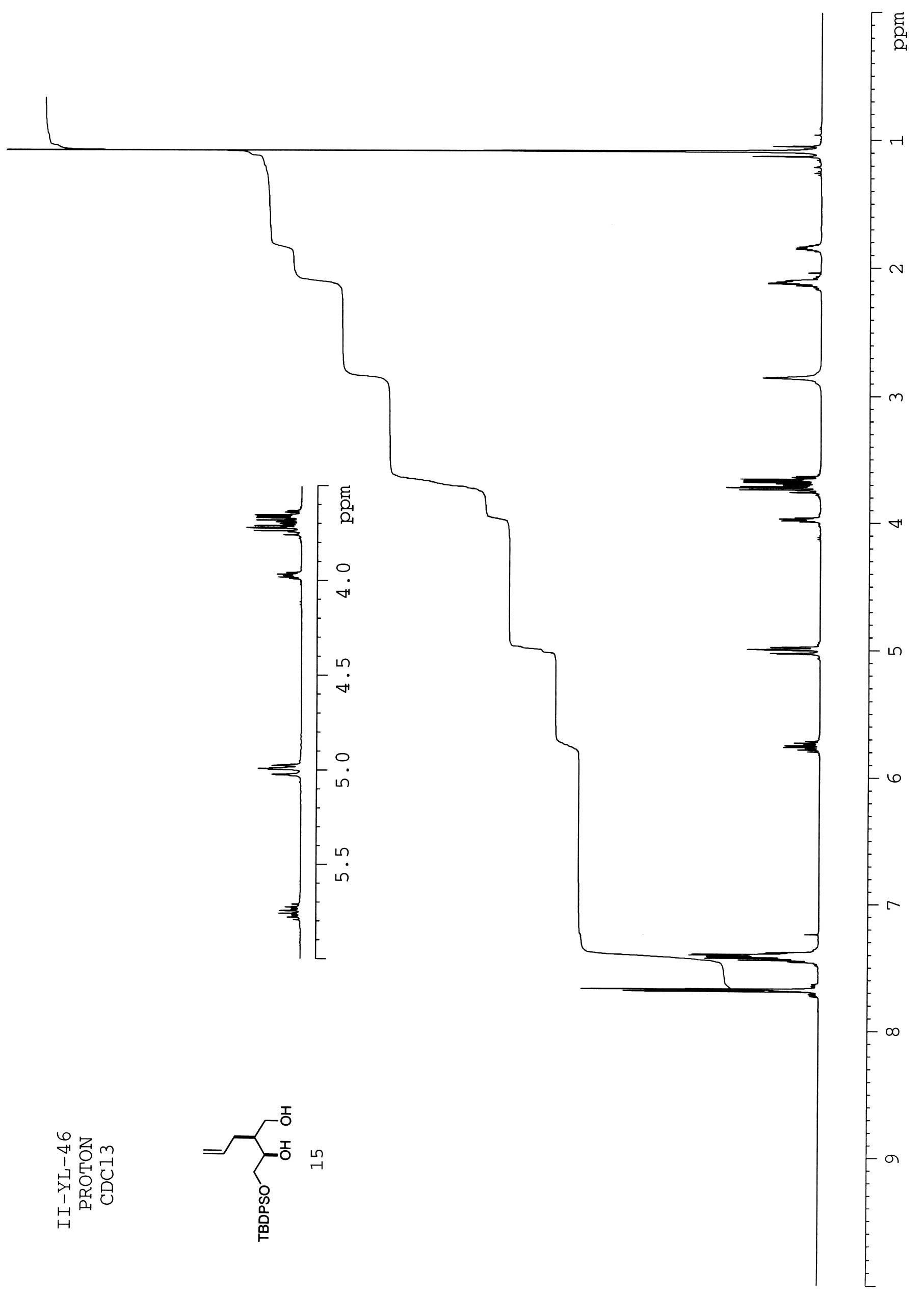




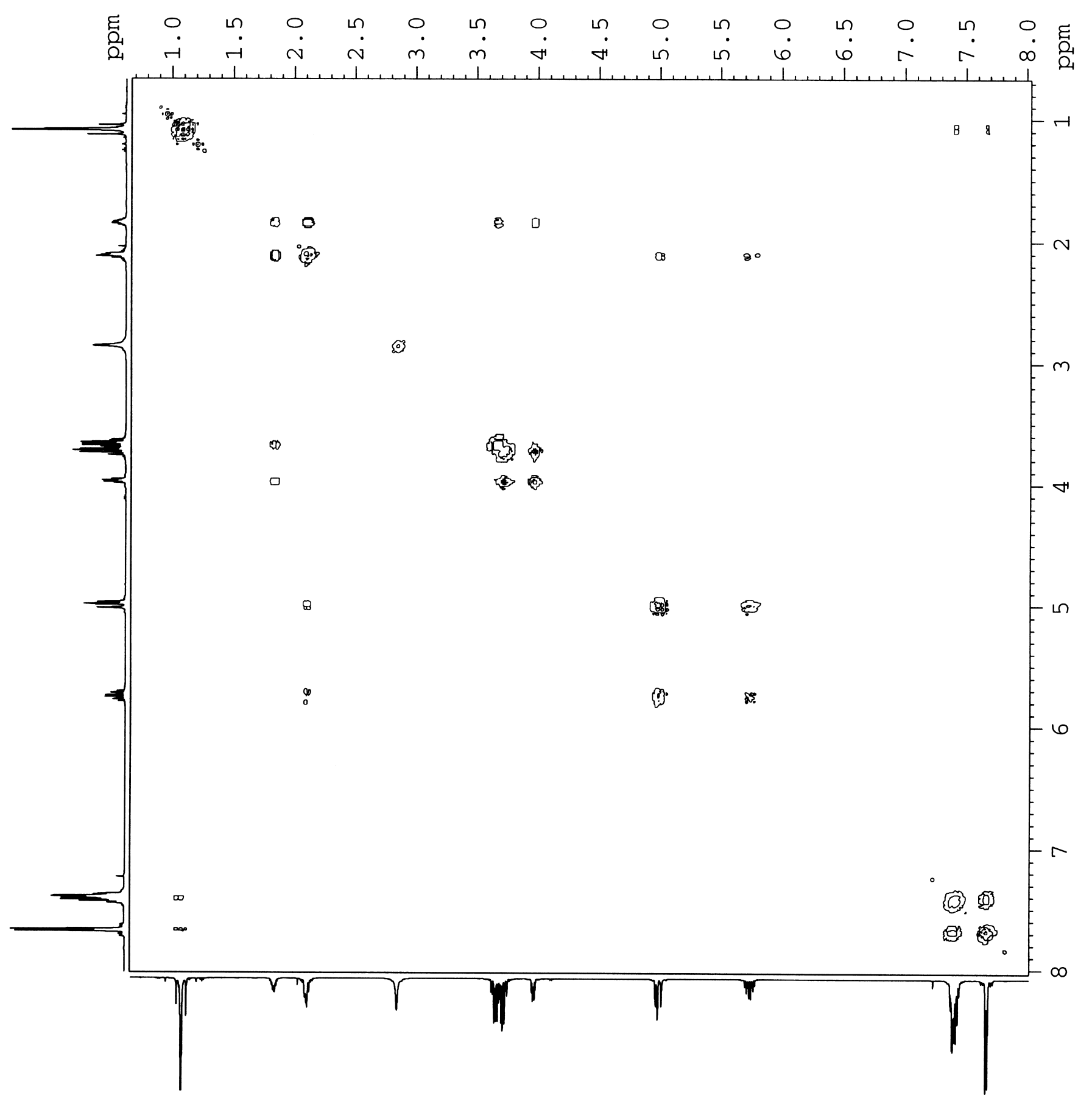

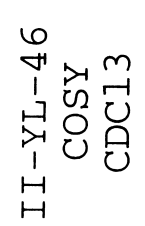

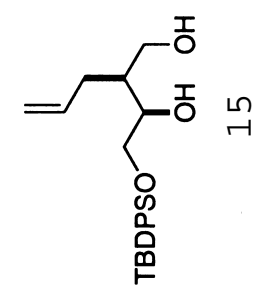




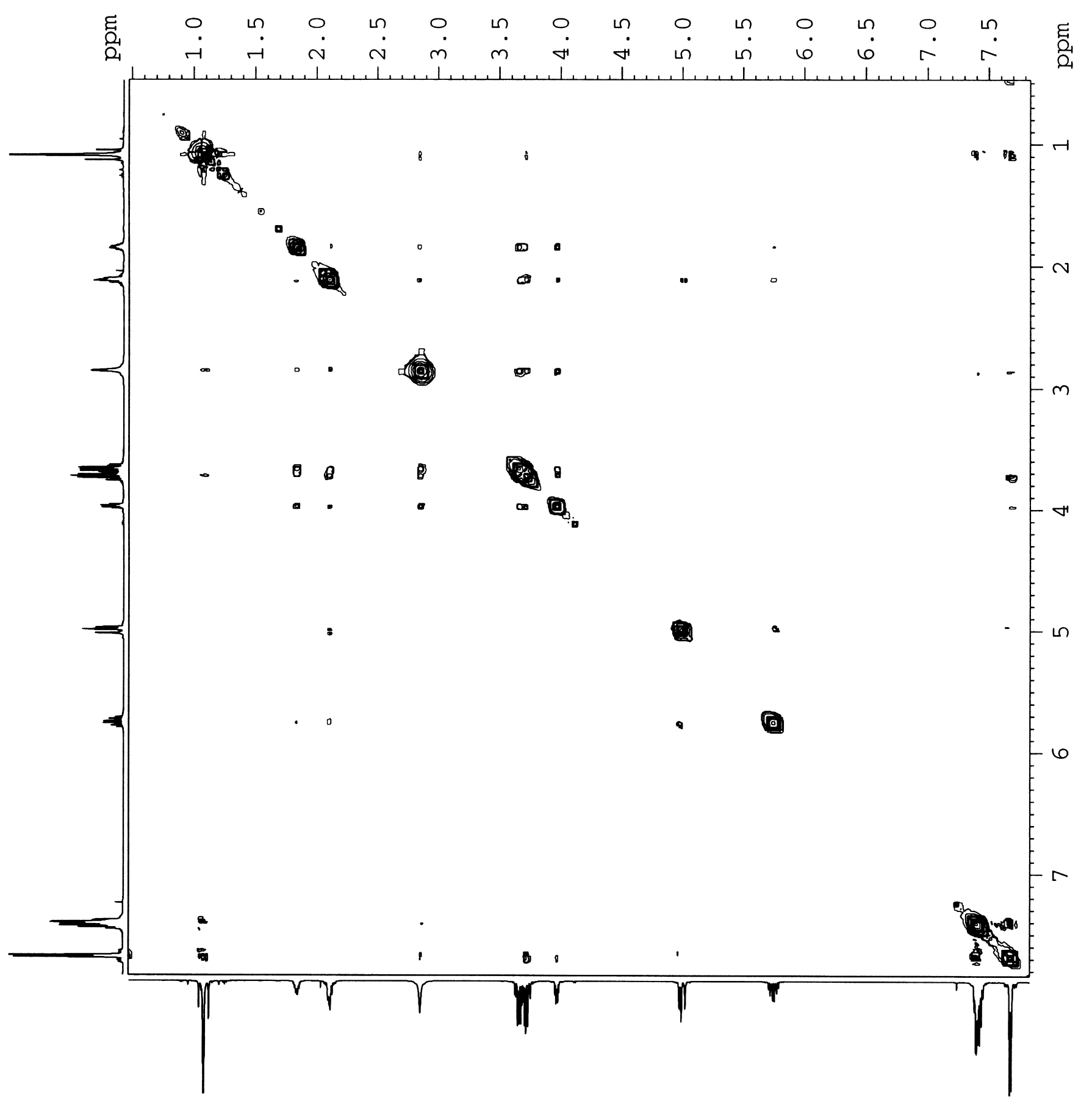

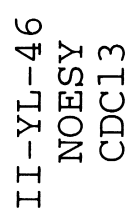

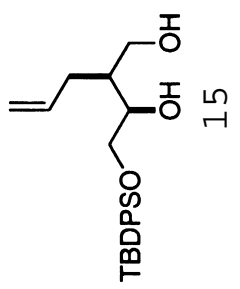




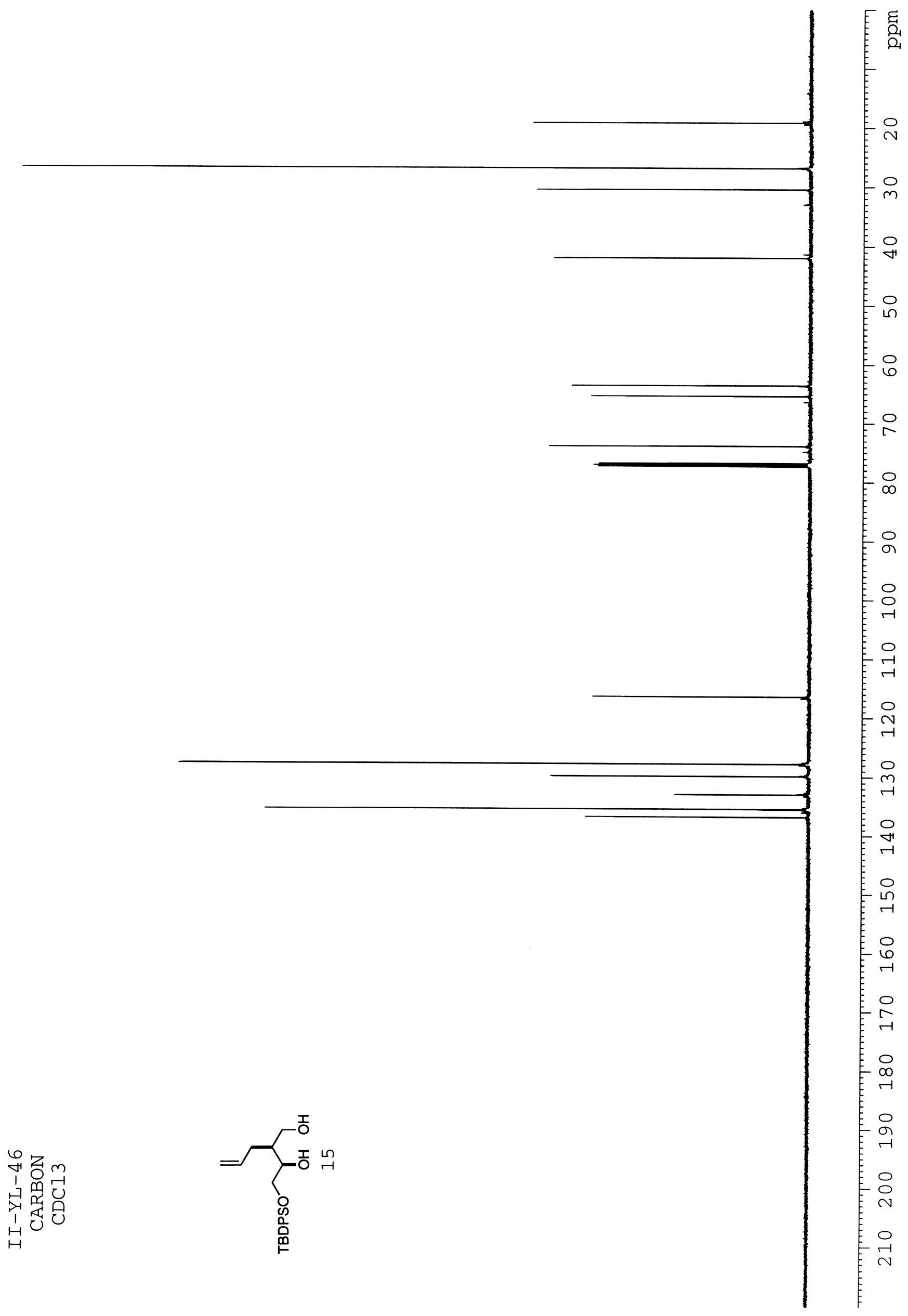




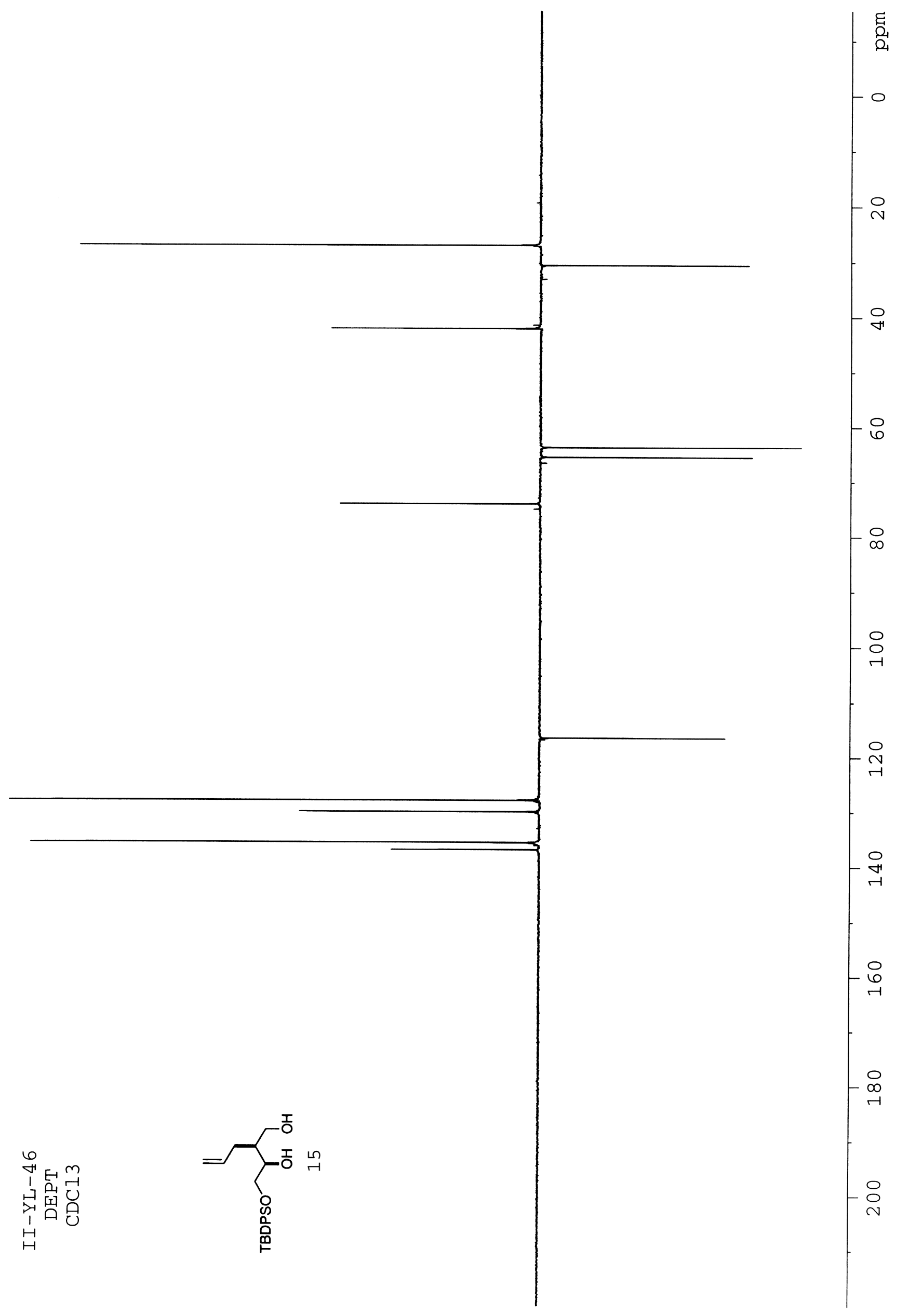



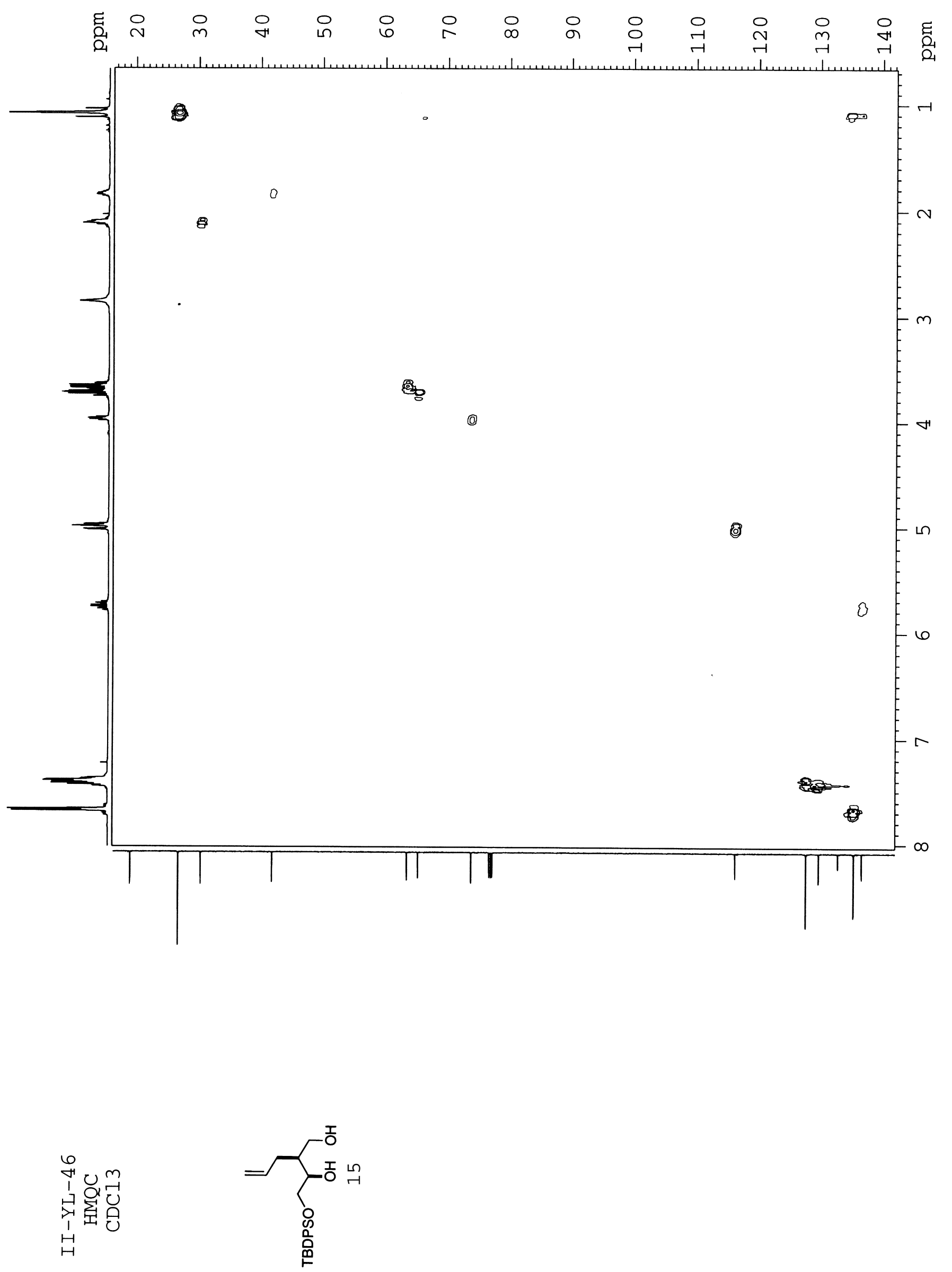


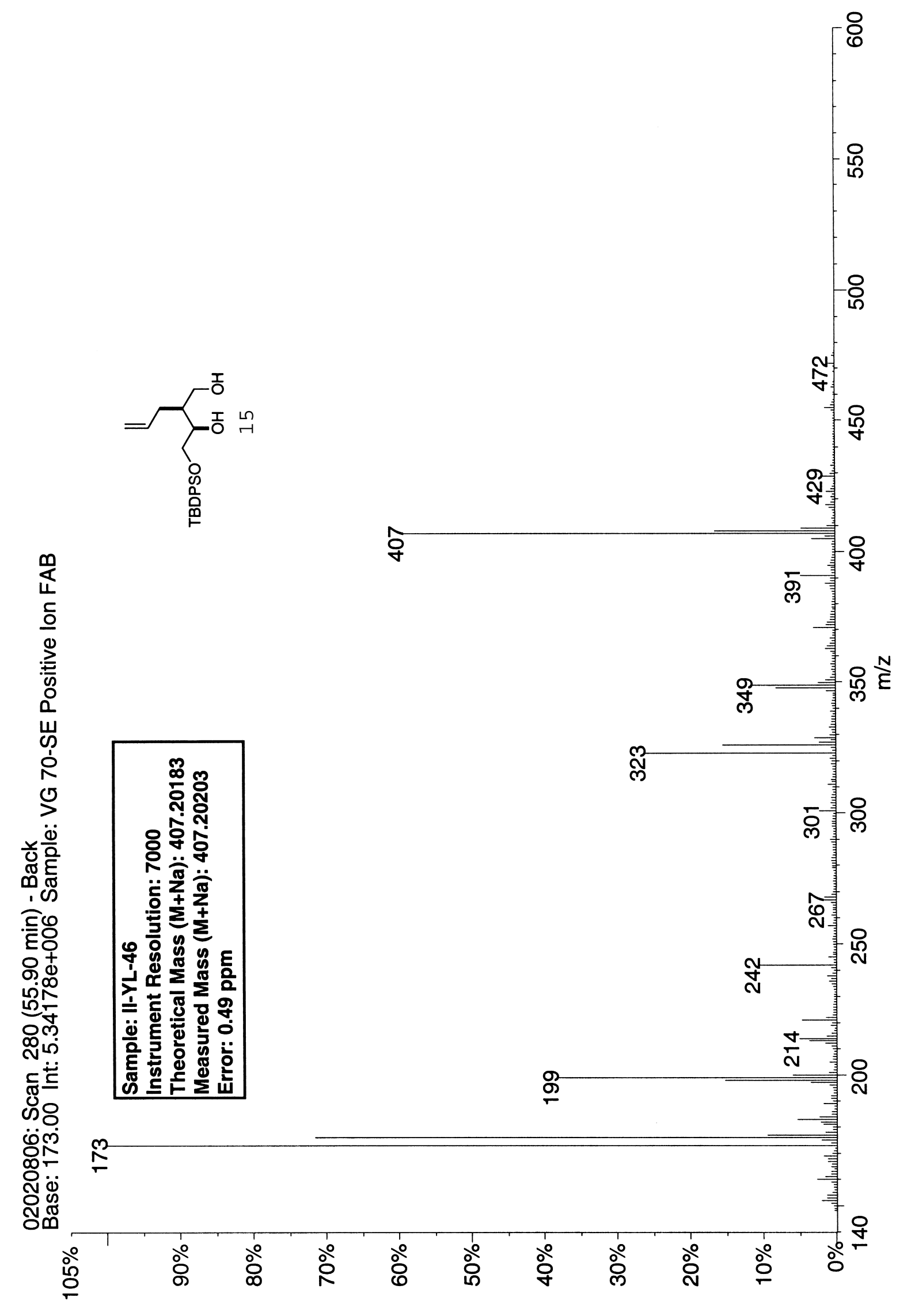




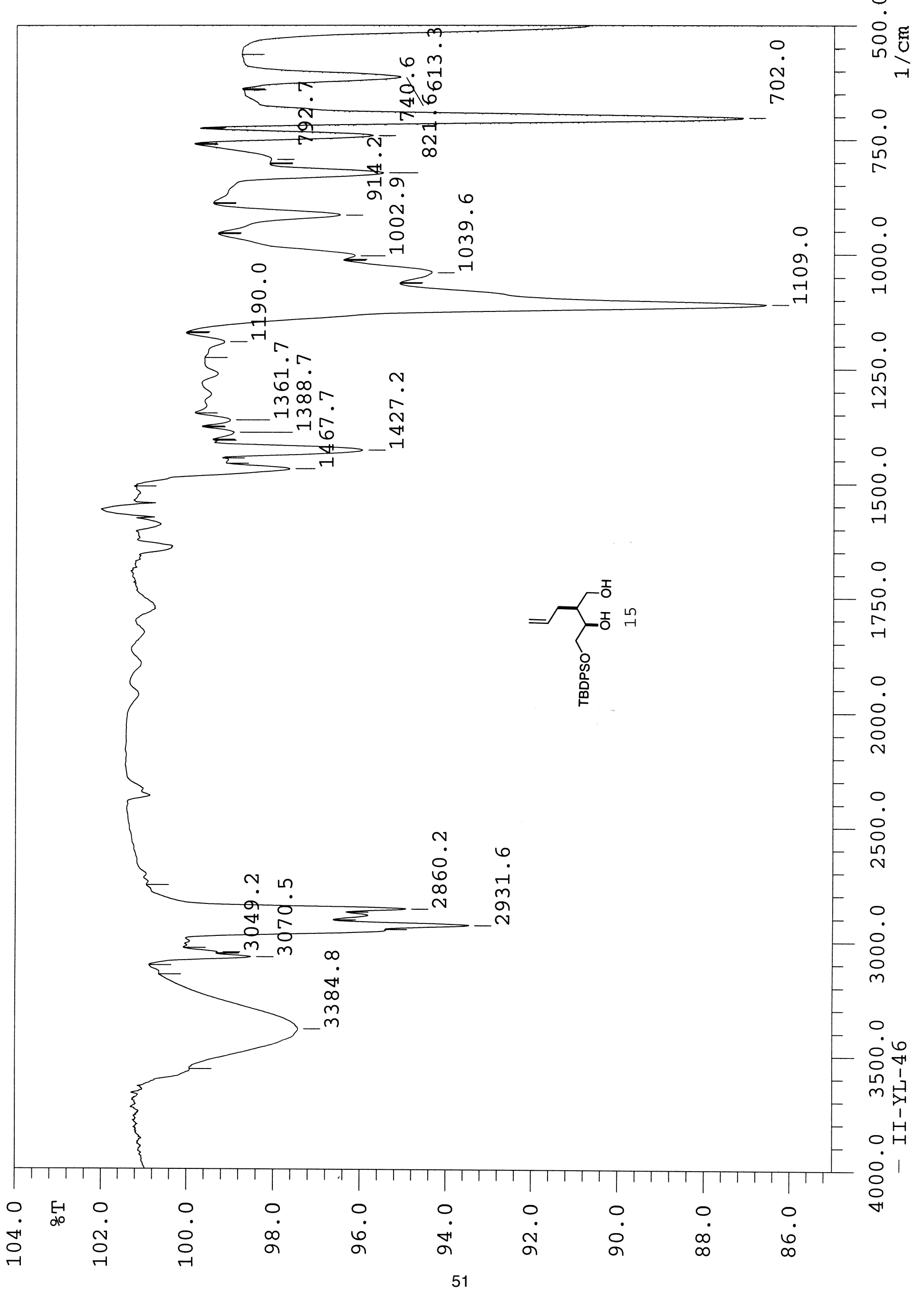




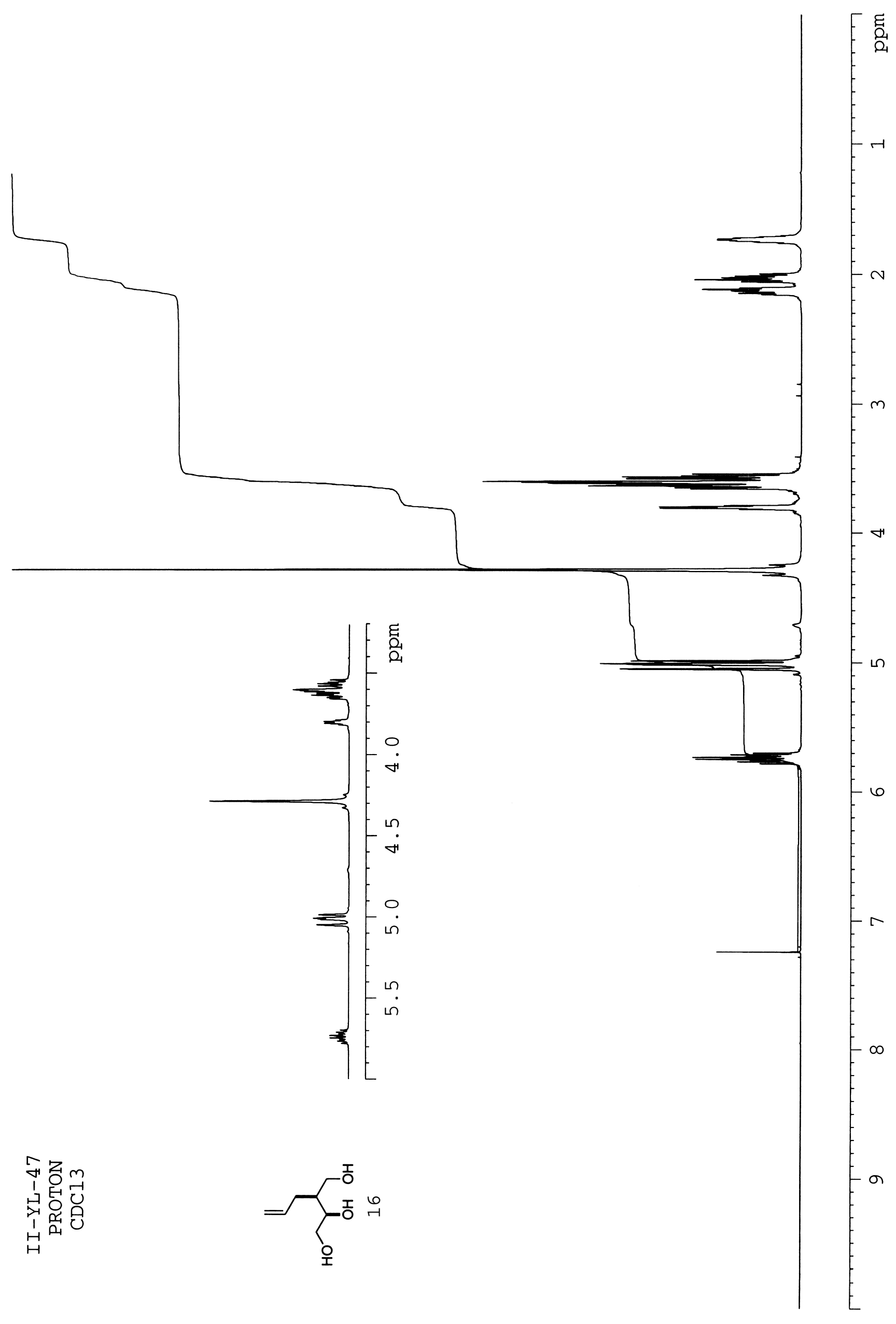




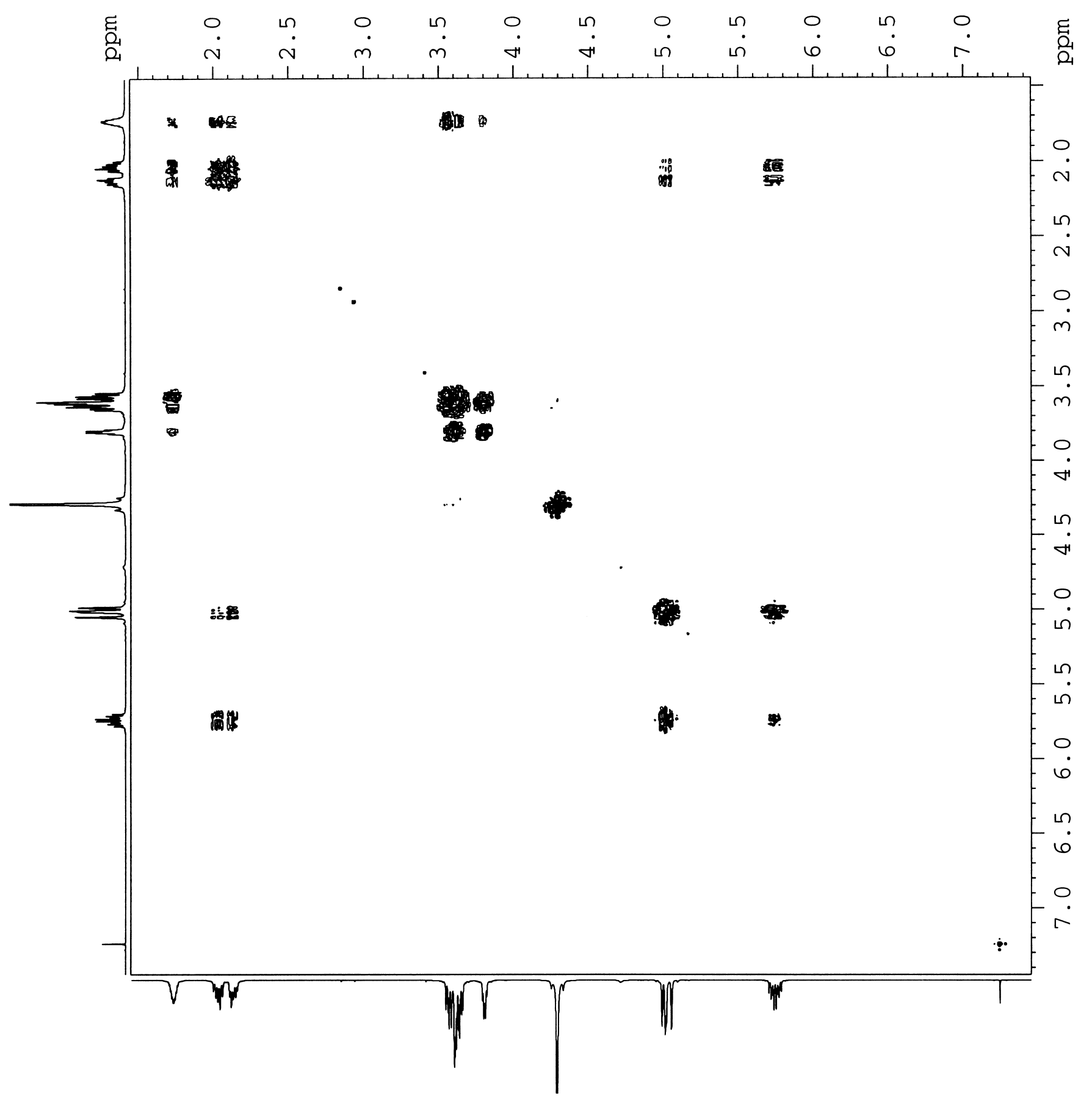

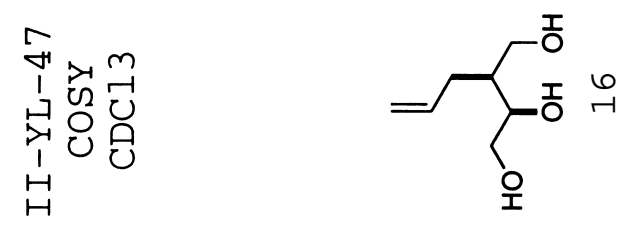




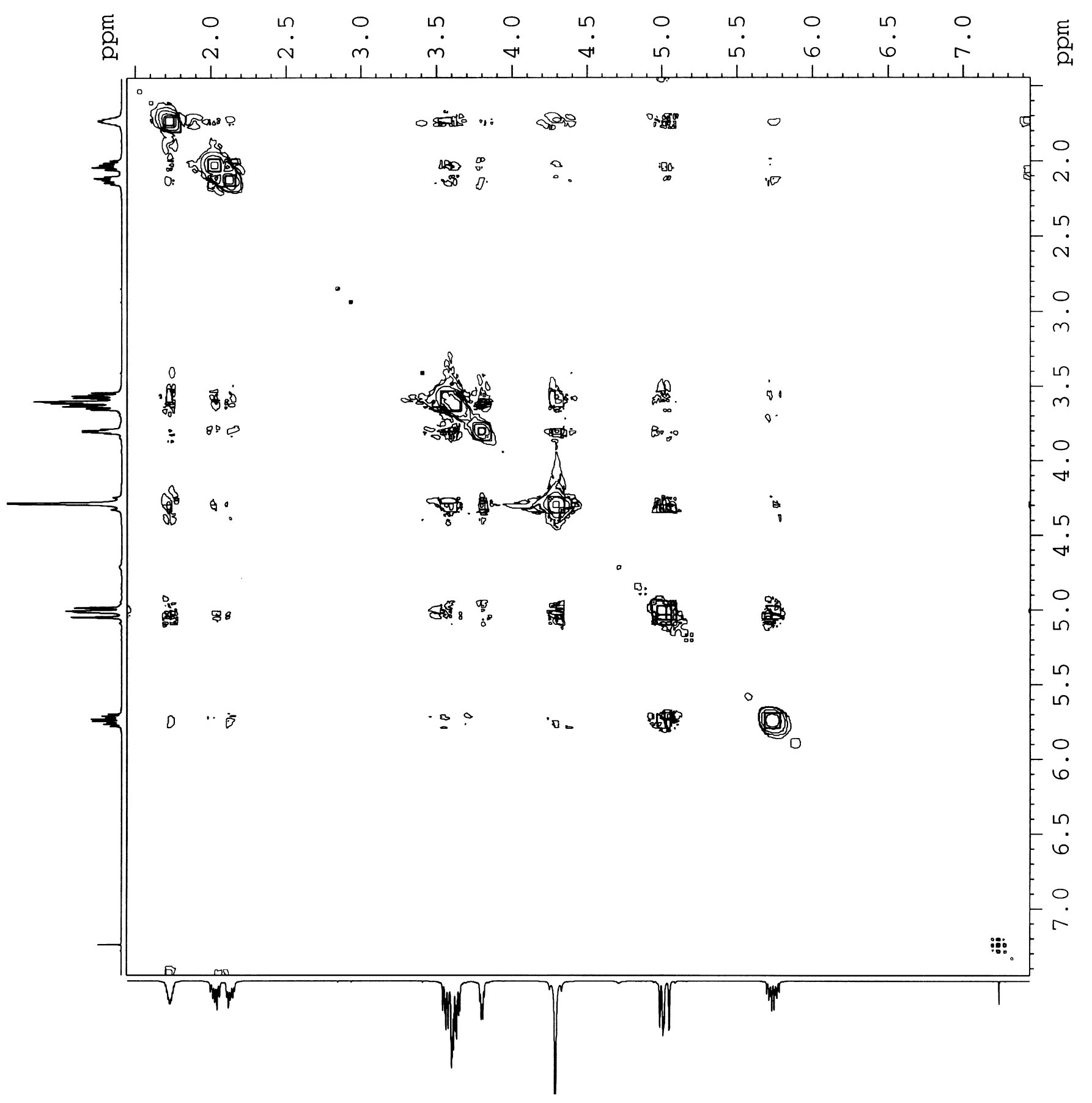

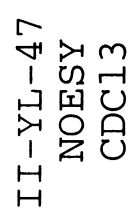<smiles>C=CCC(C[CH2-])C([O-])COC</smiles> 


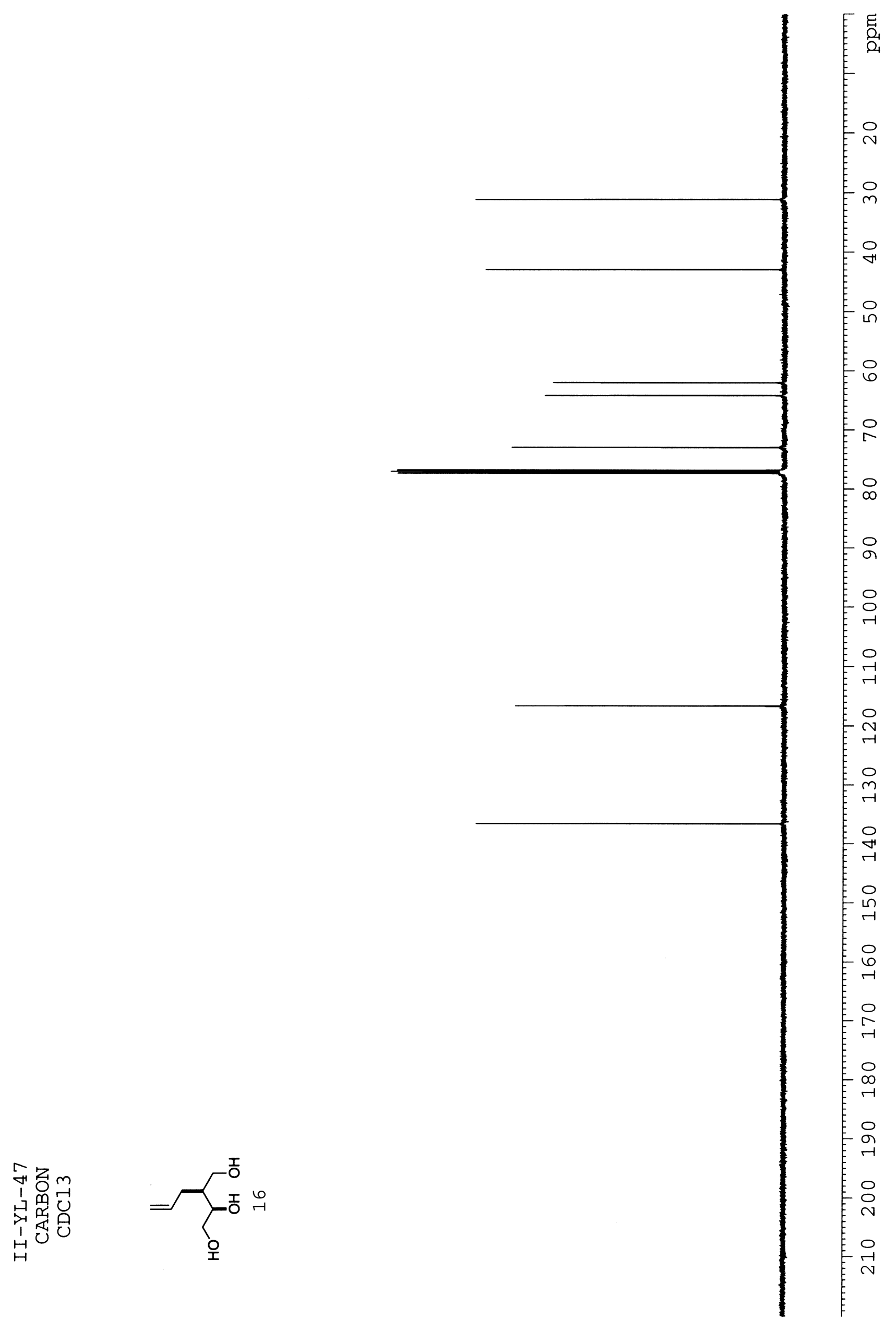




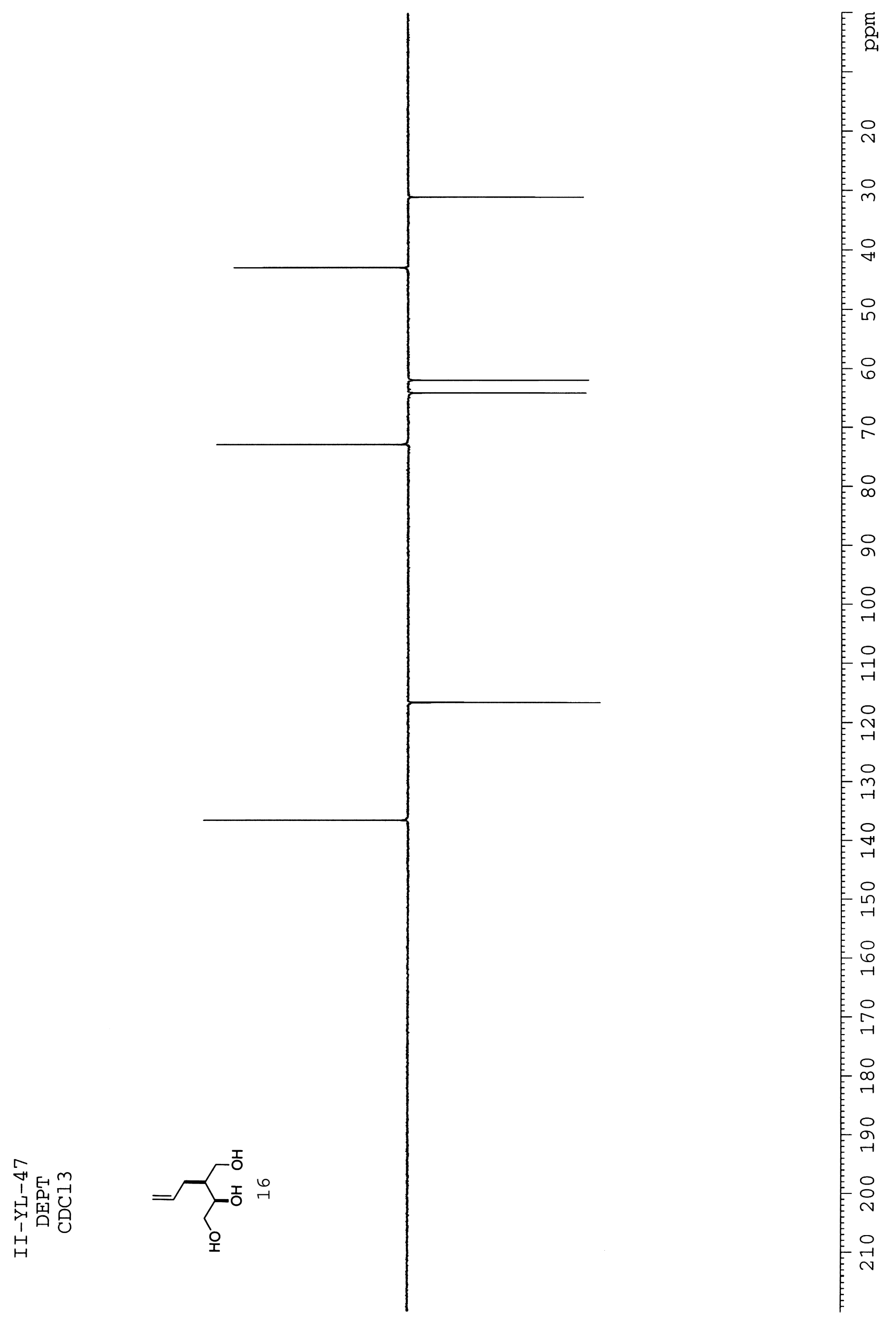




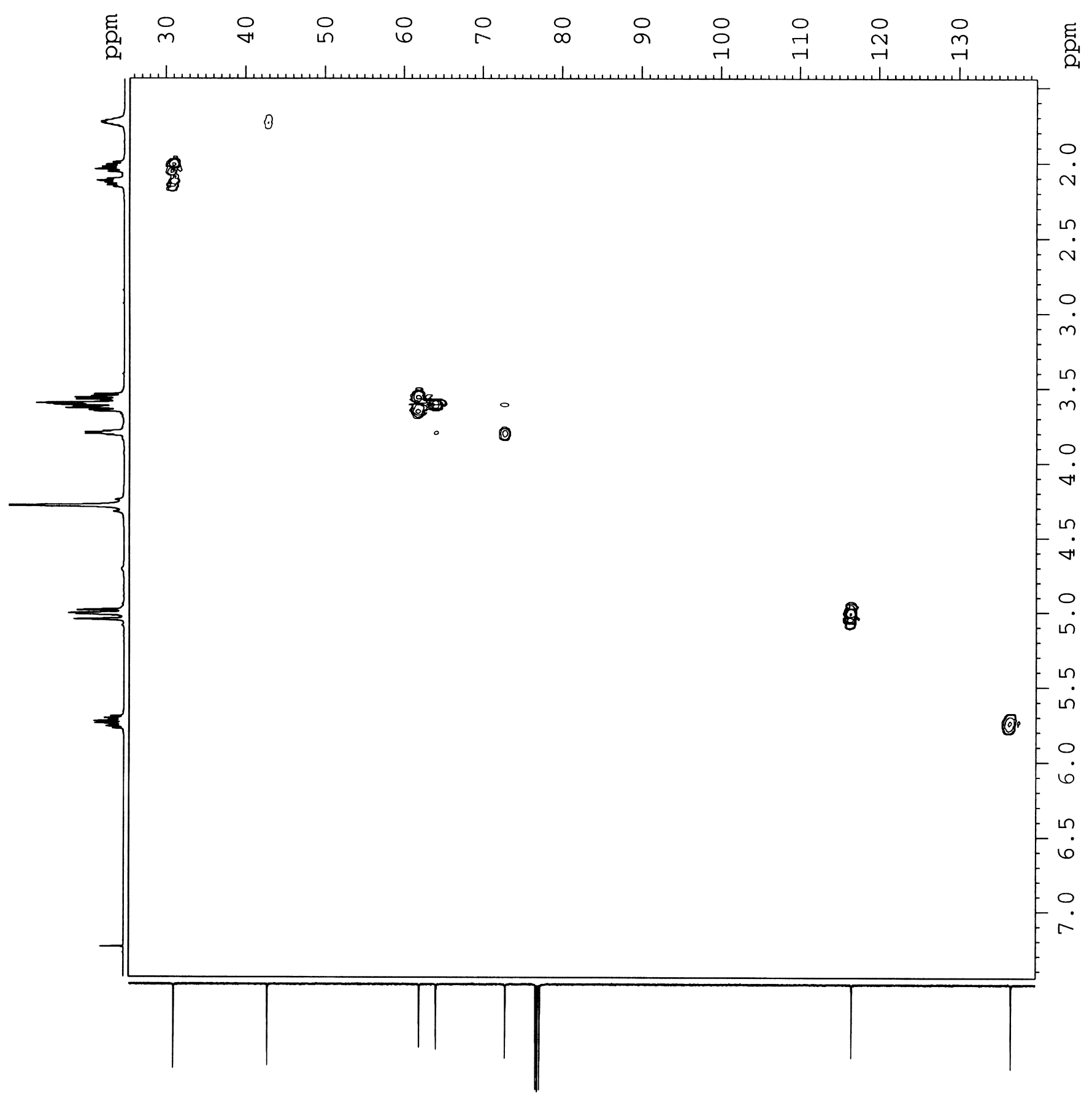

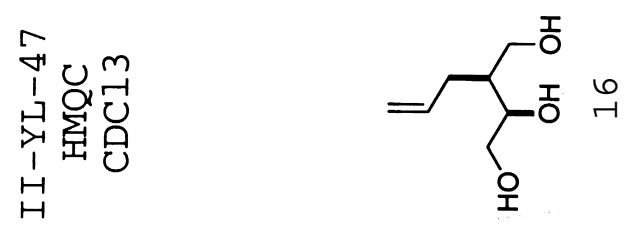




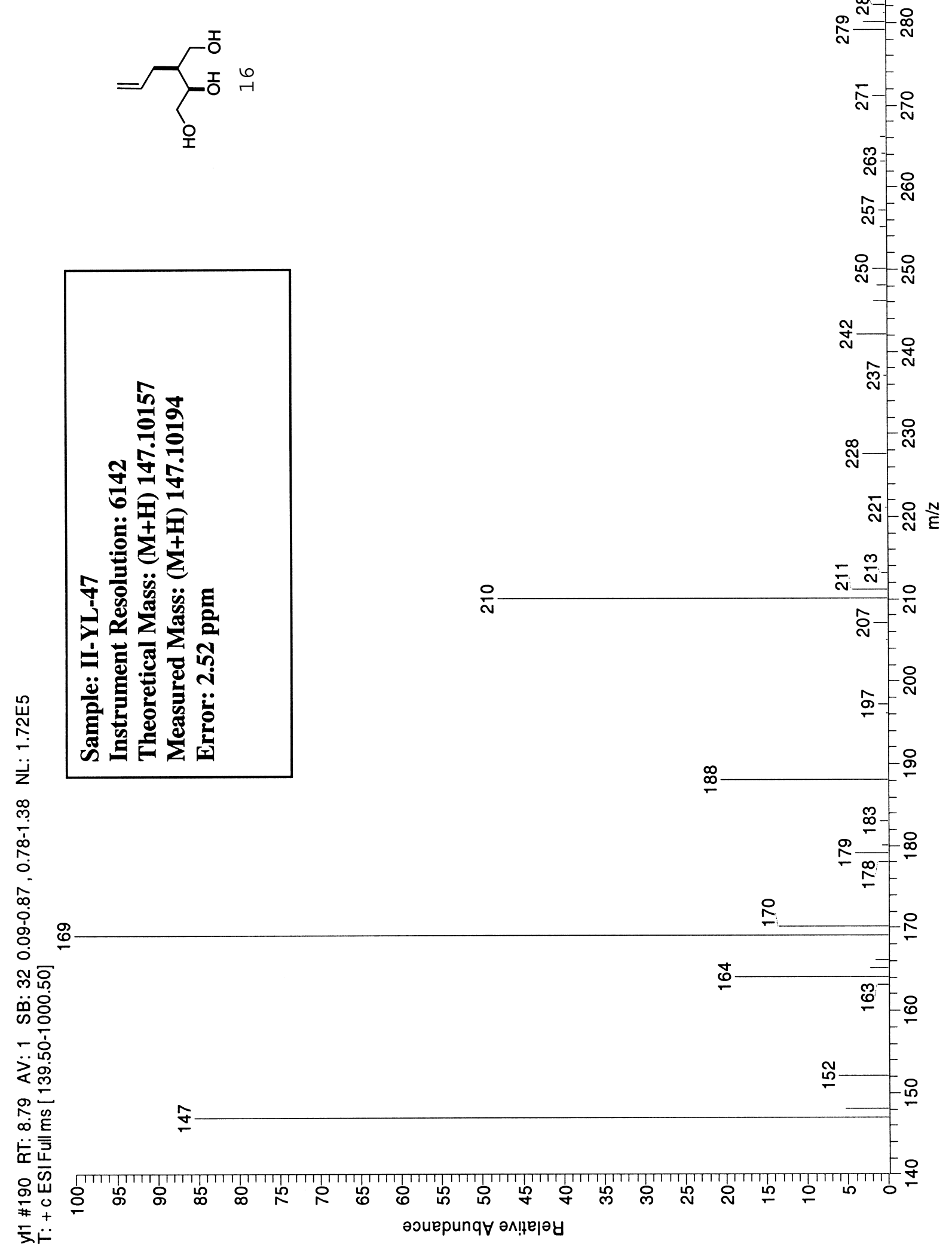




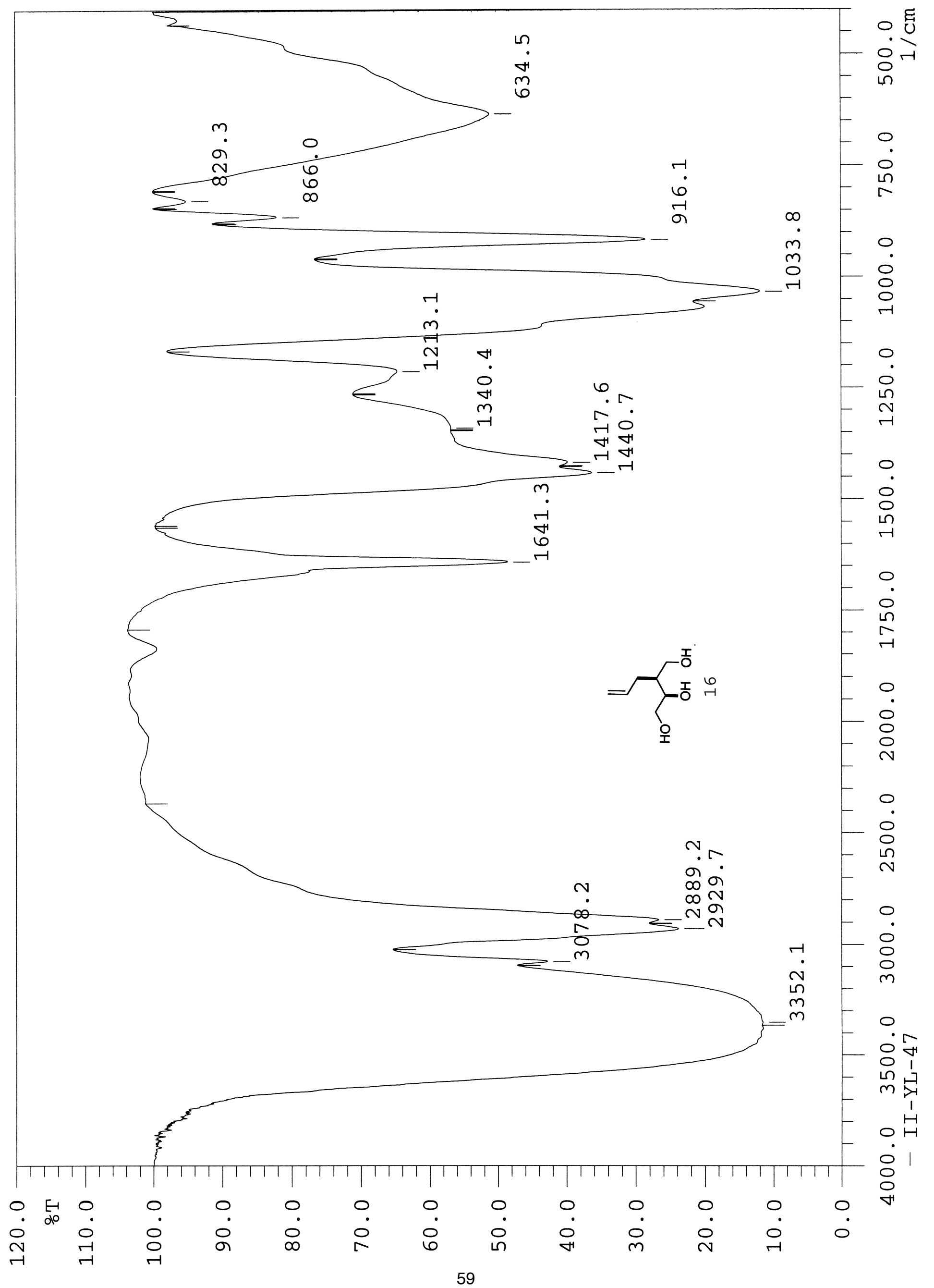




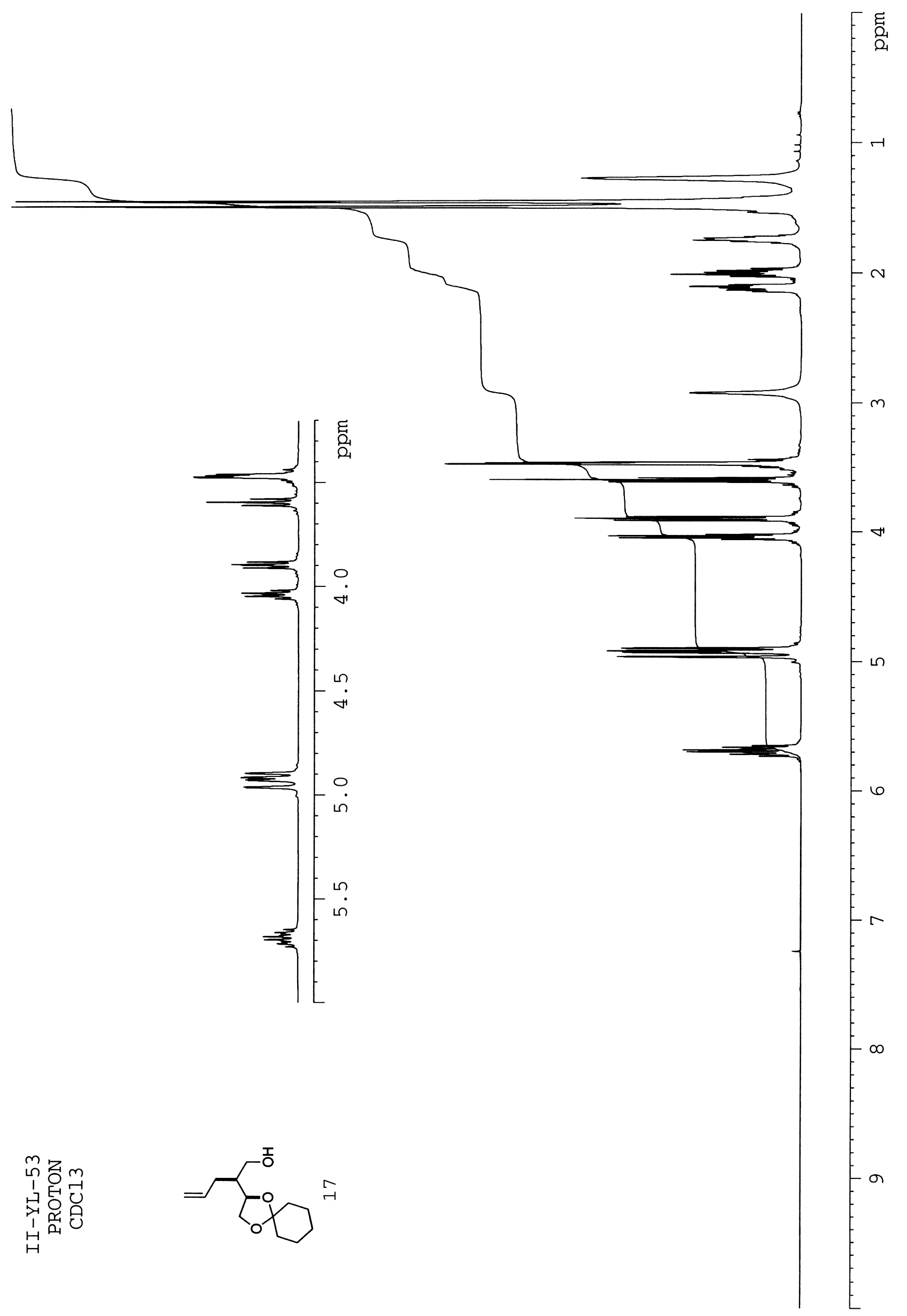




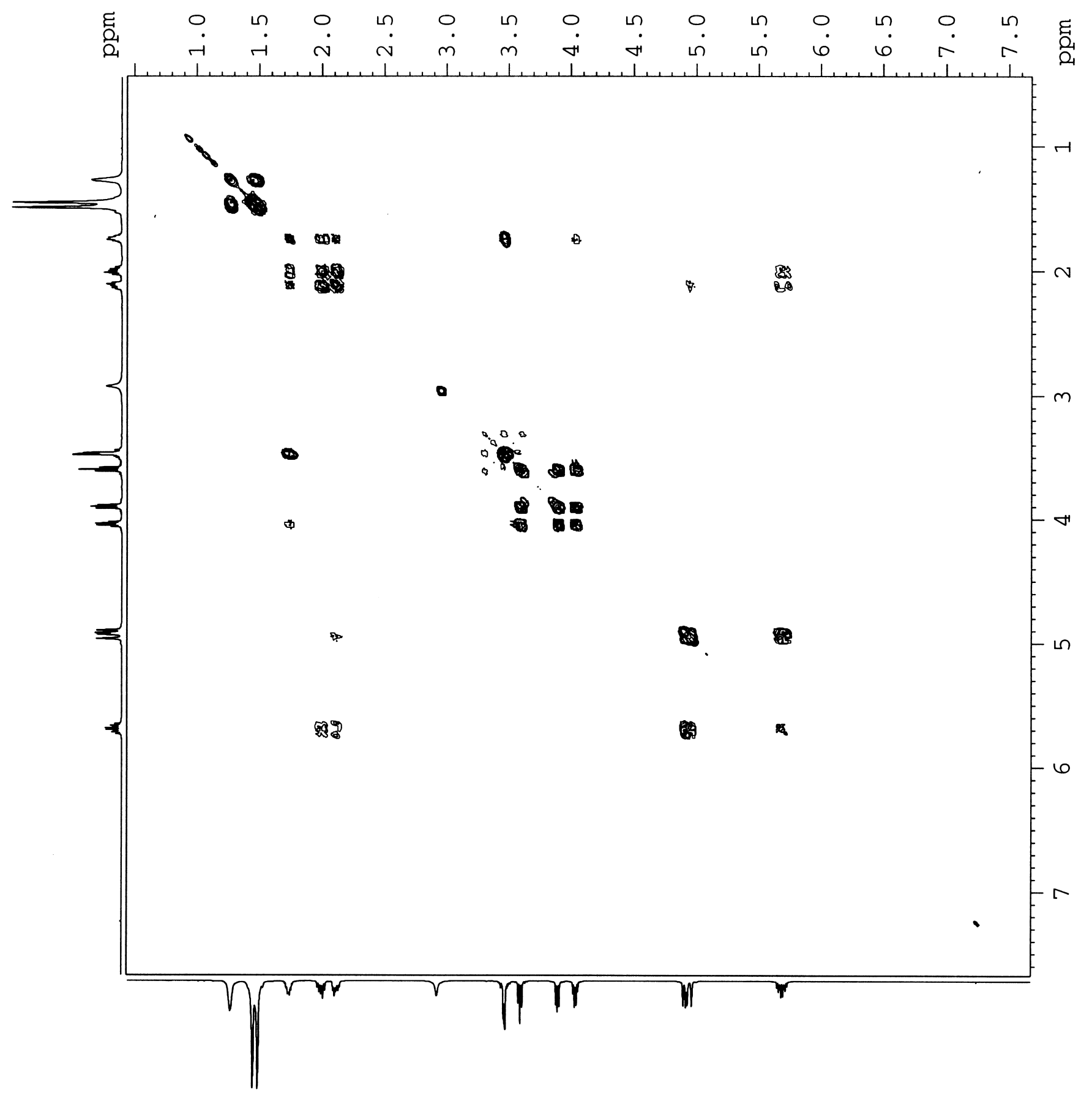

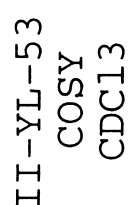

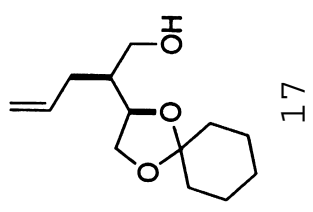




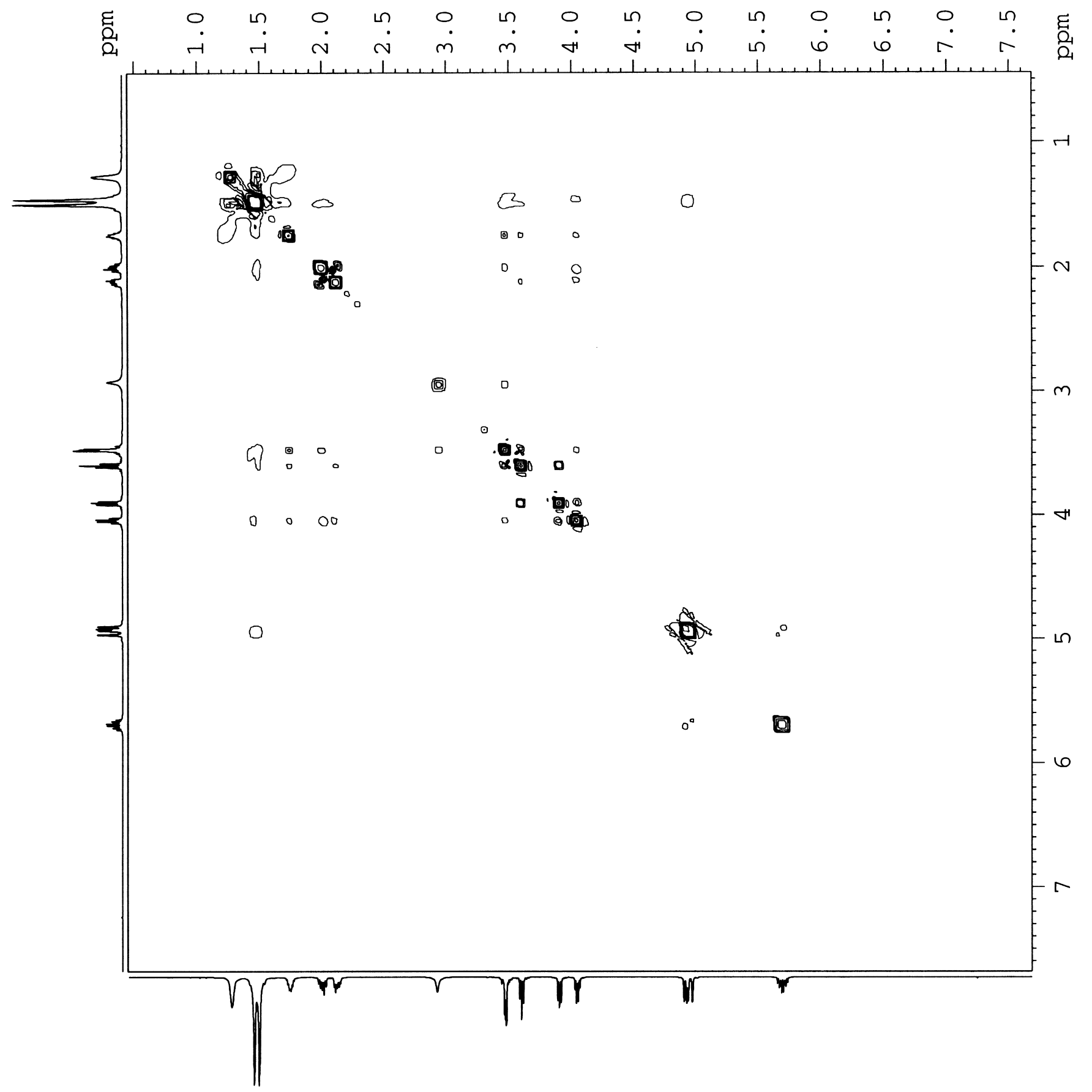

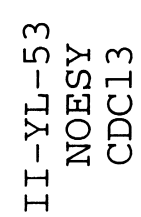<smiles>C=CCC(C[CH2-])C1COC2(CCCCC2)O1</smiles> 


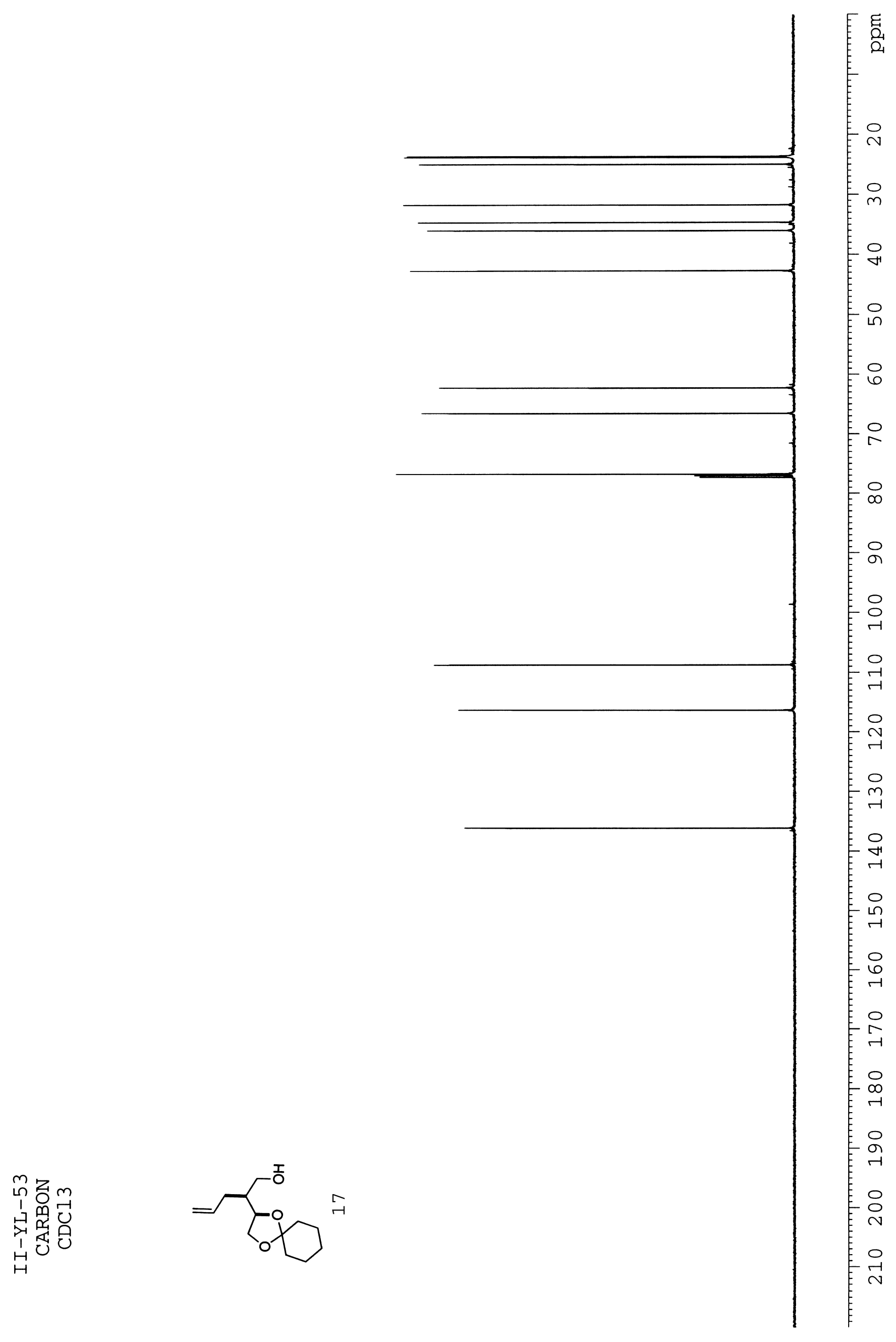




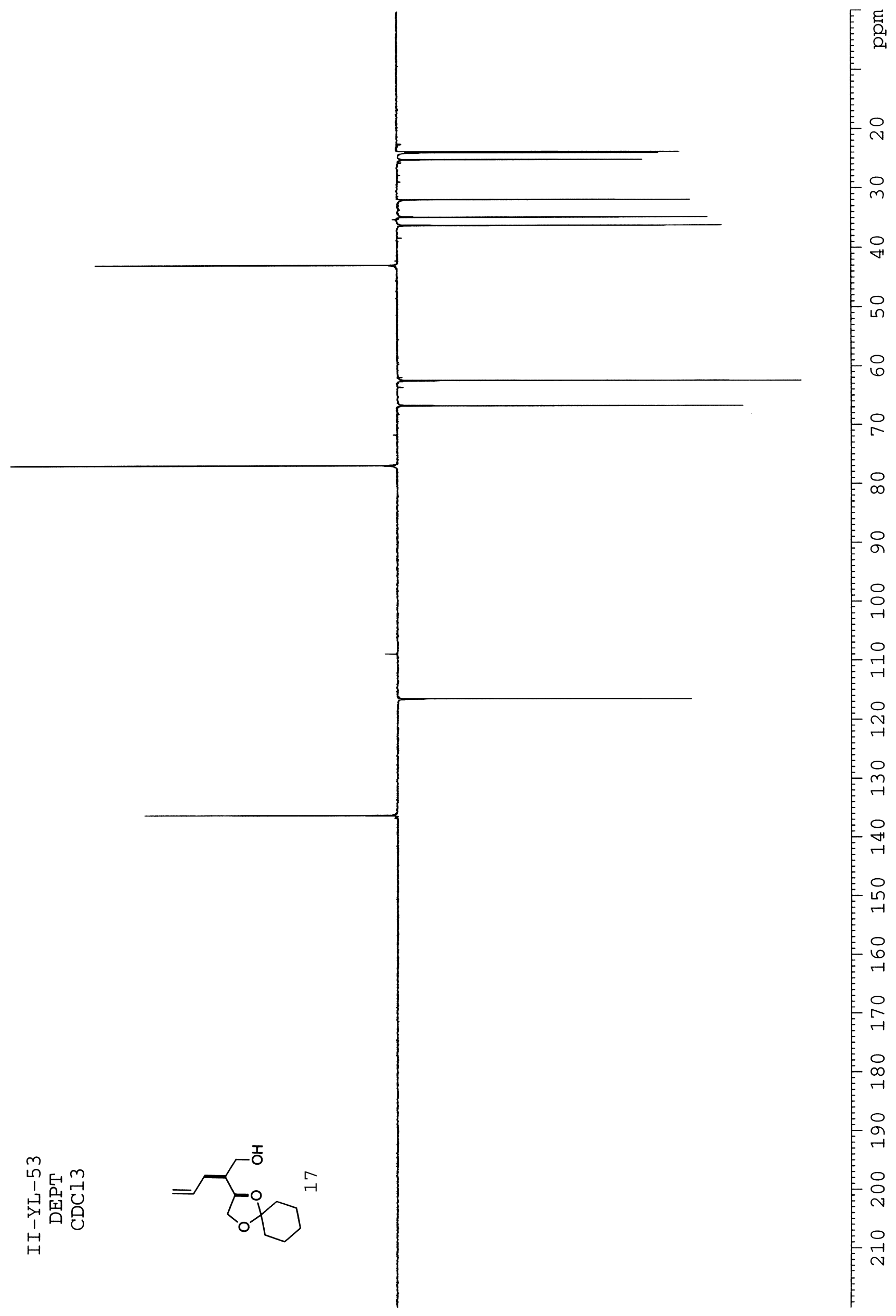



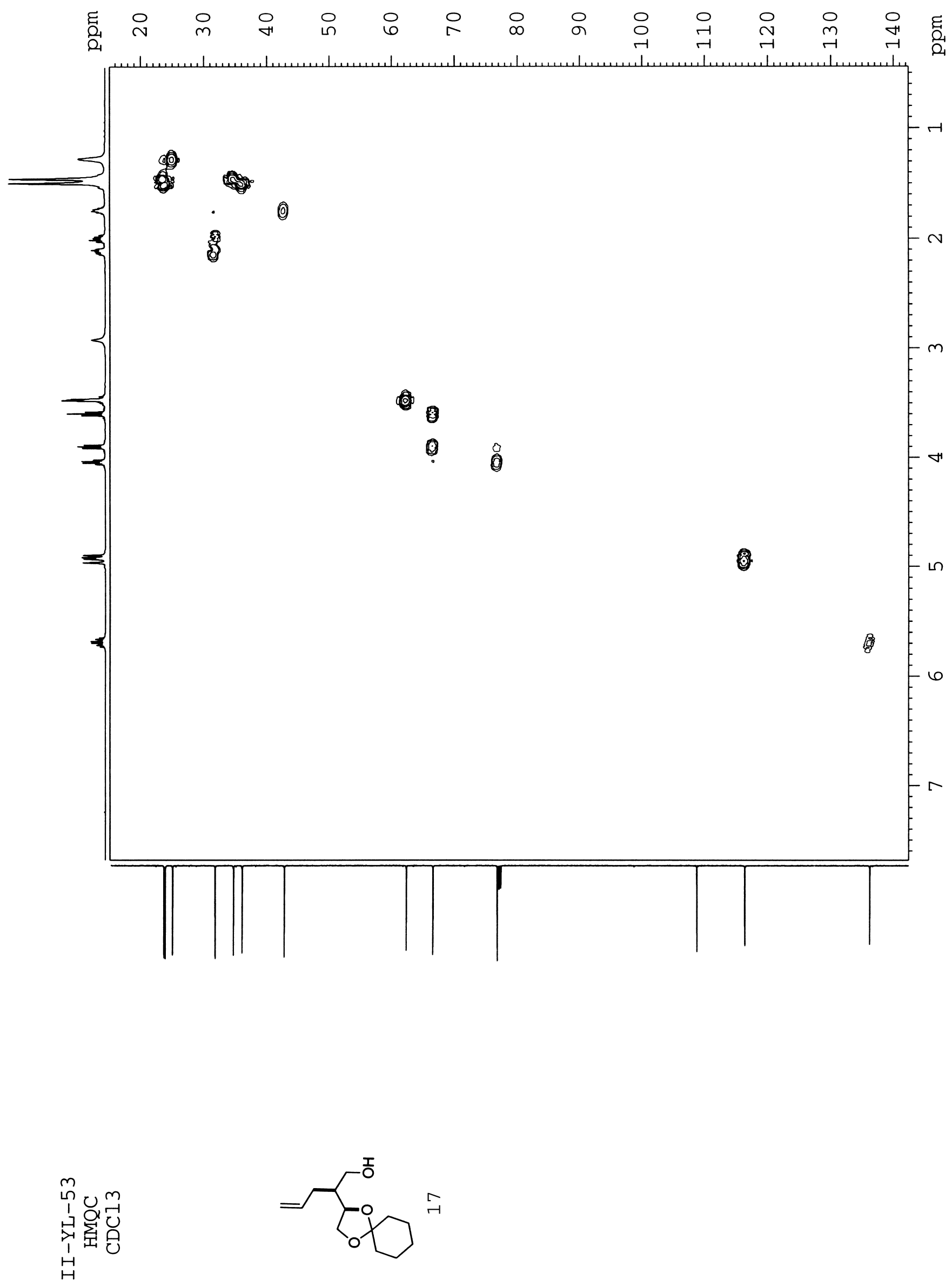

65 


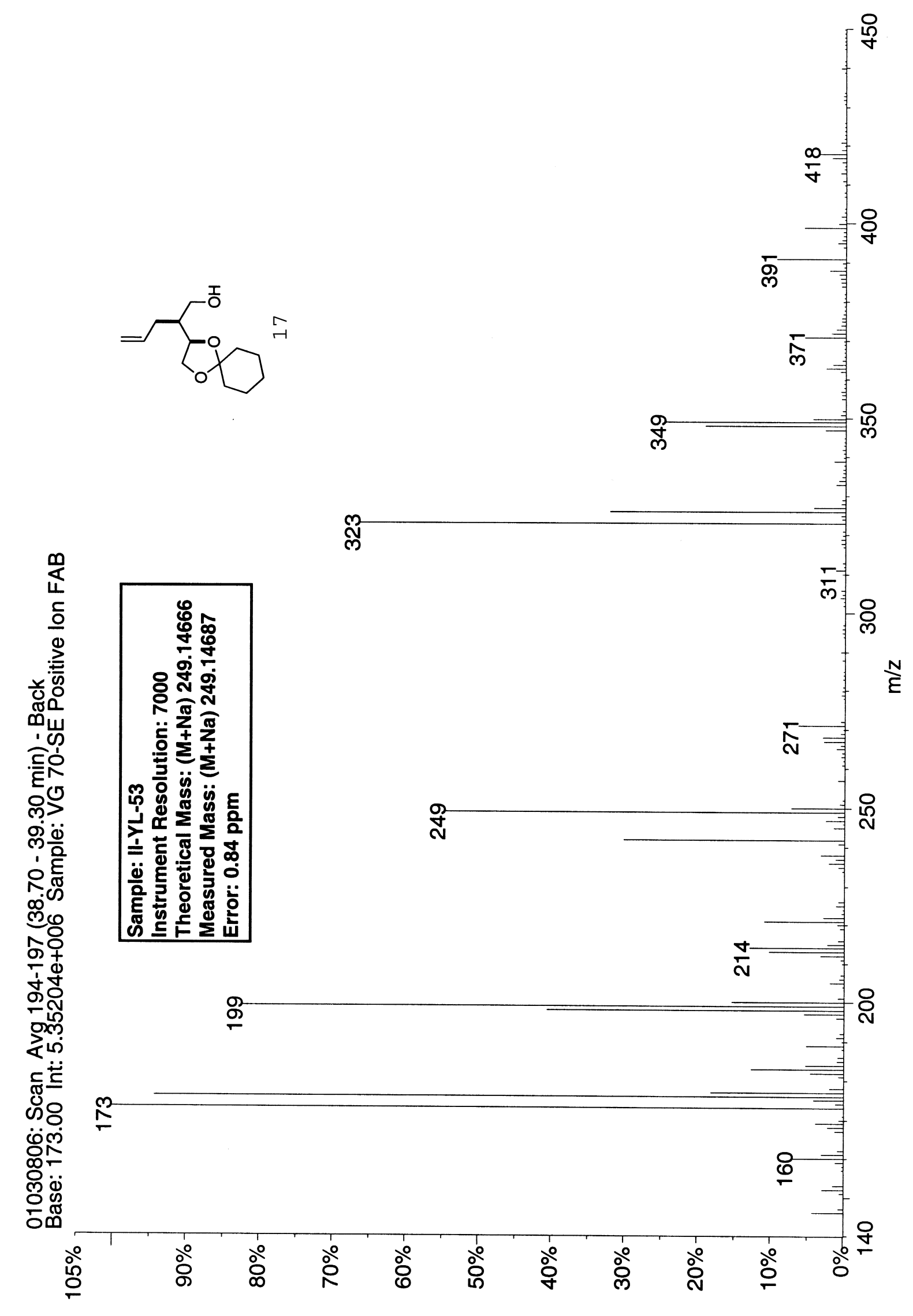




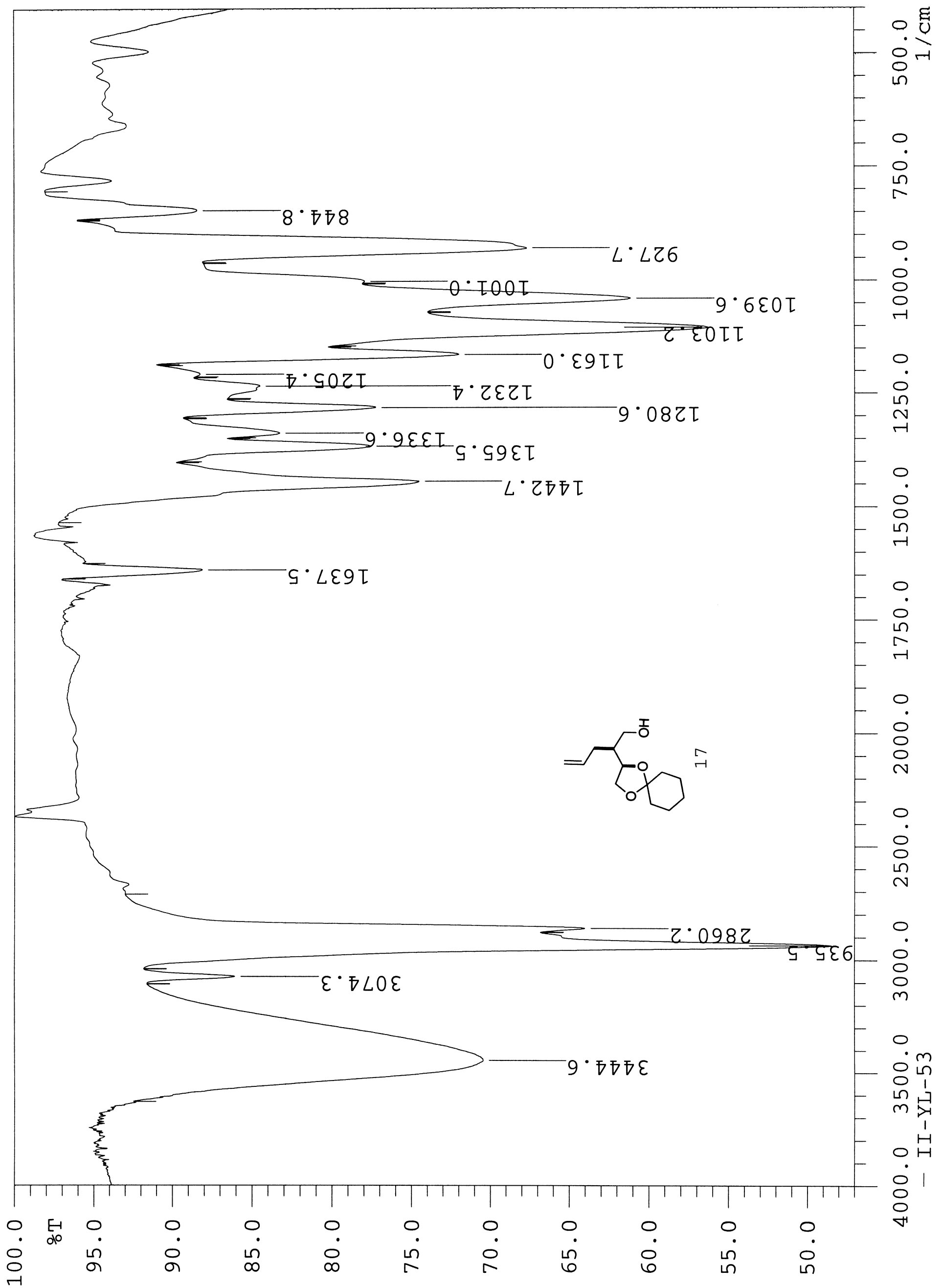




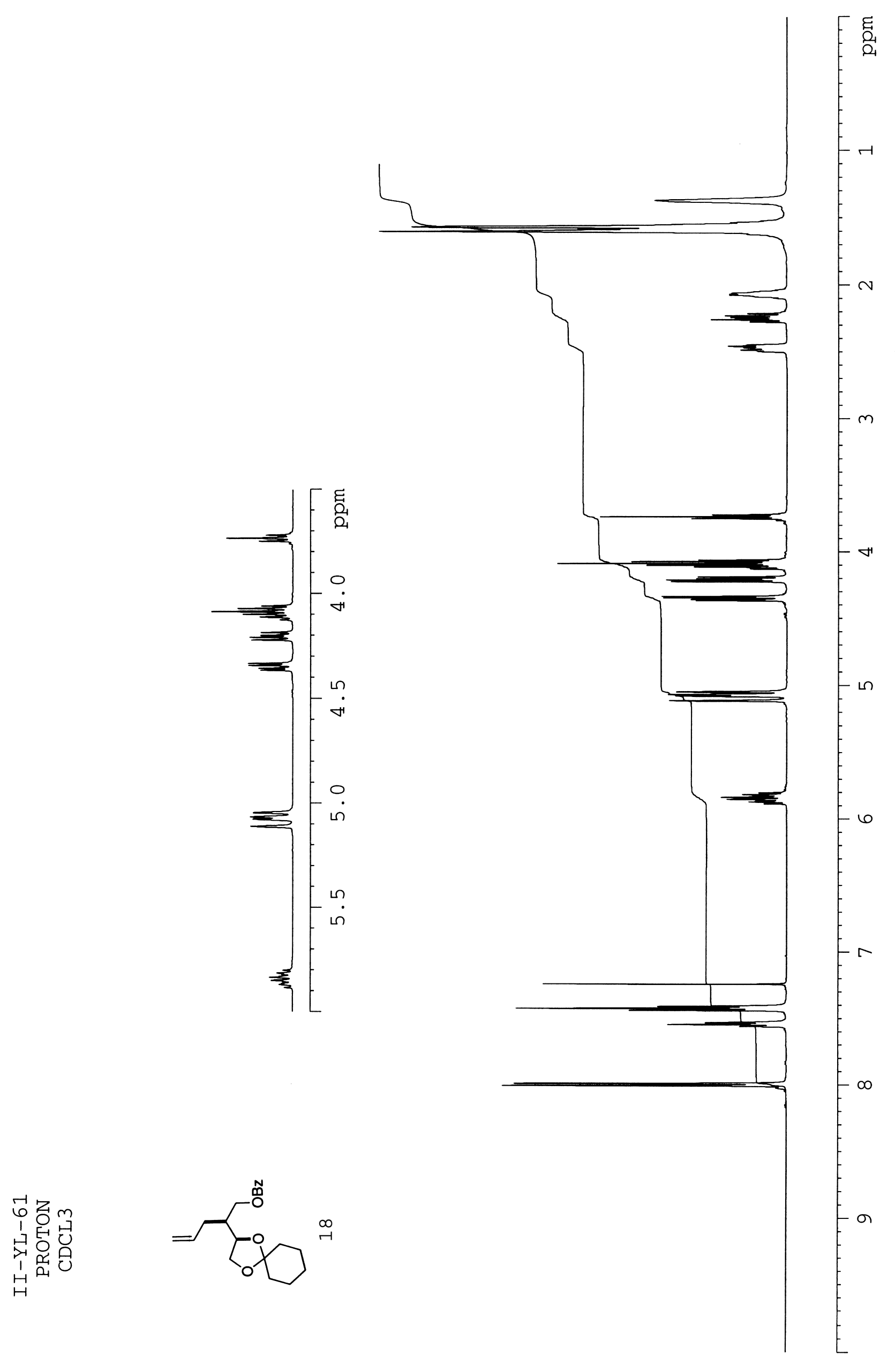




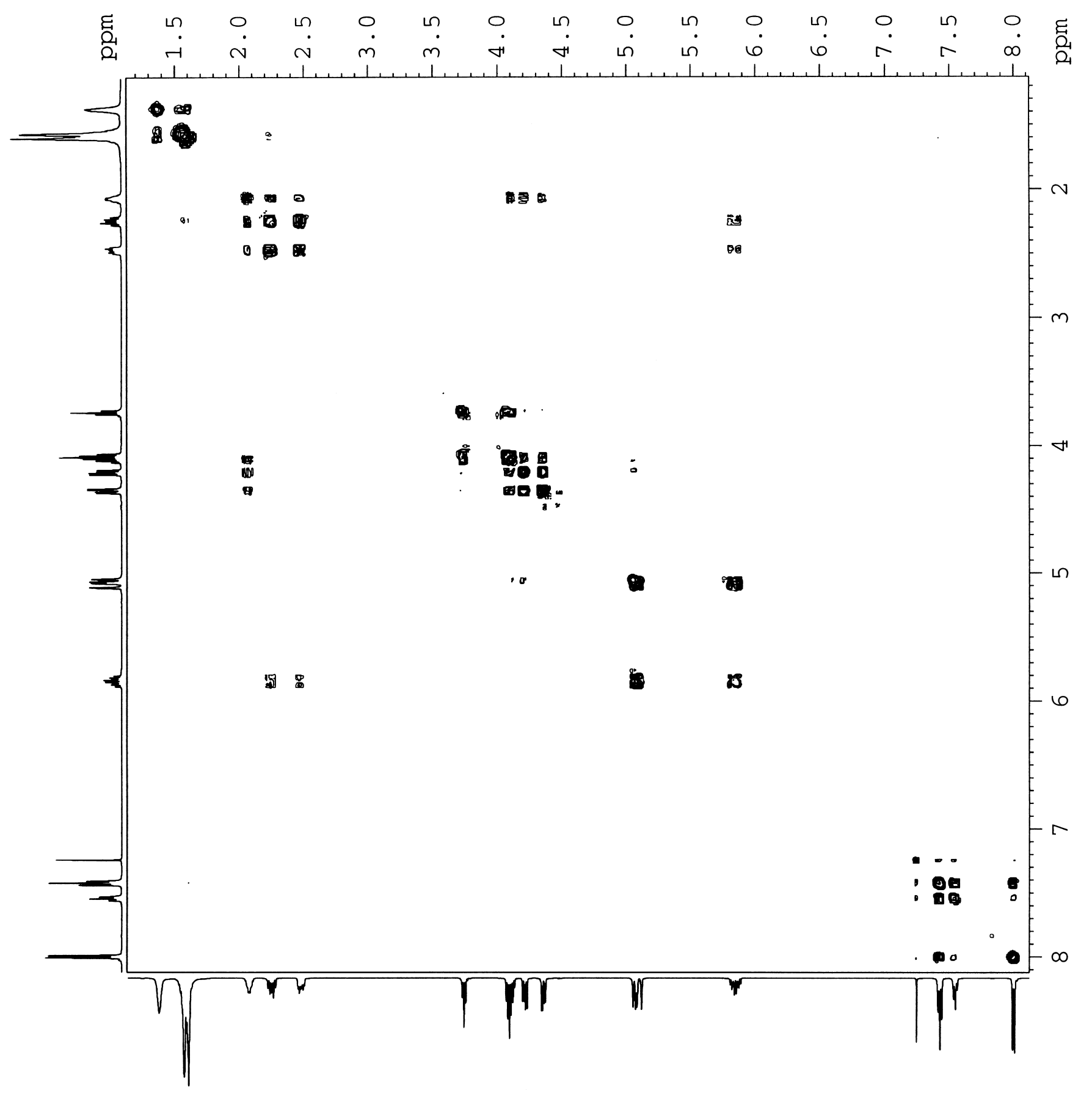

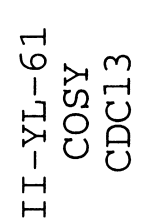

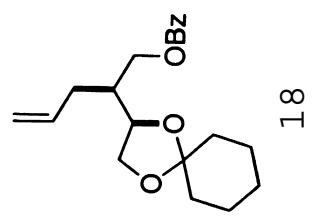




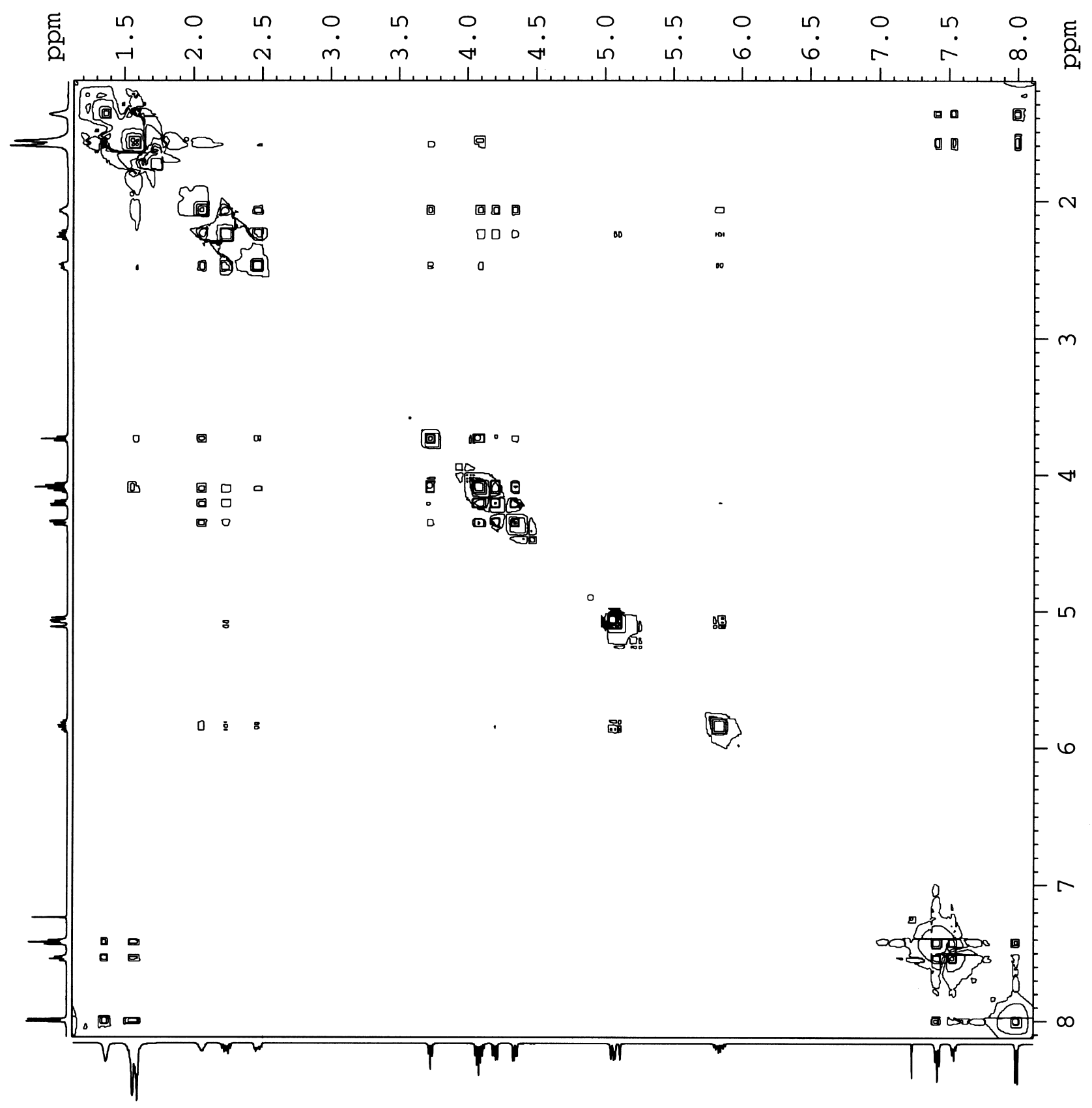

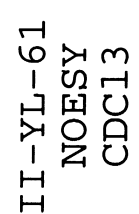<smiles>C=CCC(C[O])C1COC2(CCCCC2)O1</smiles> 


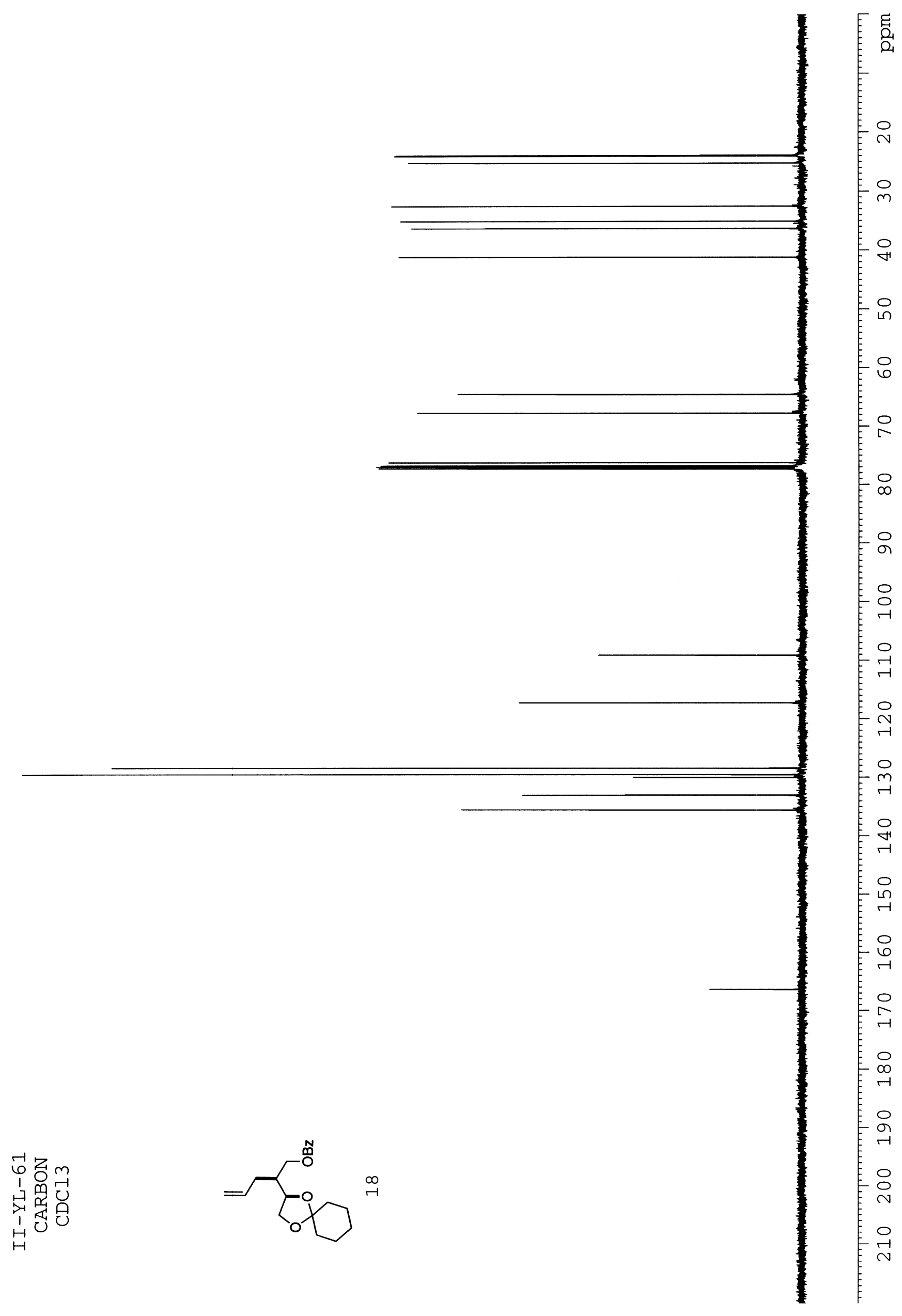




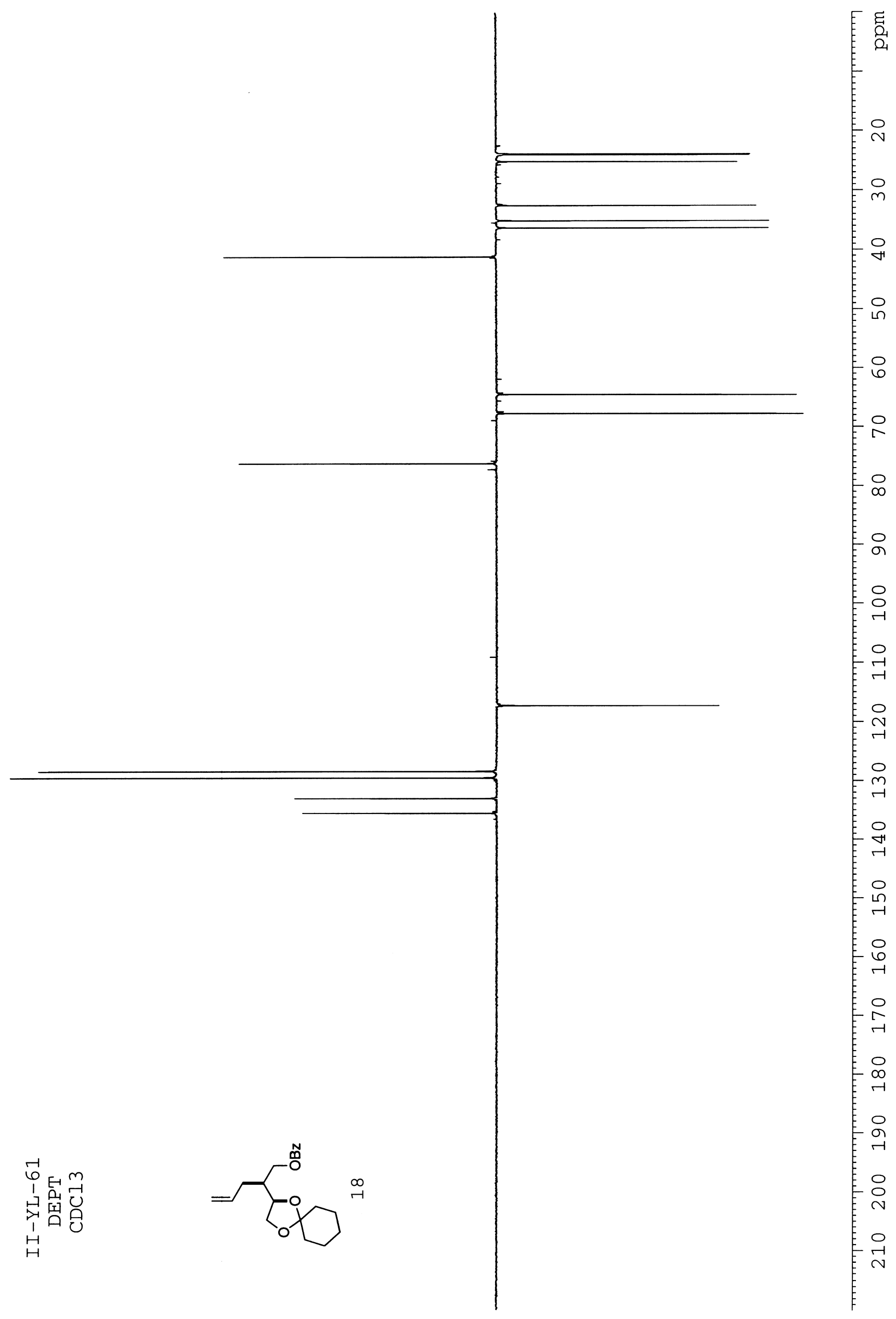




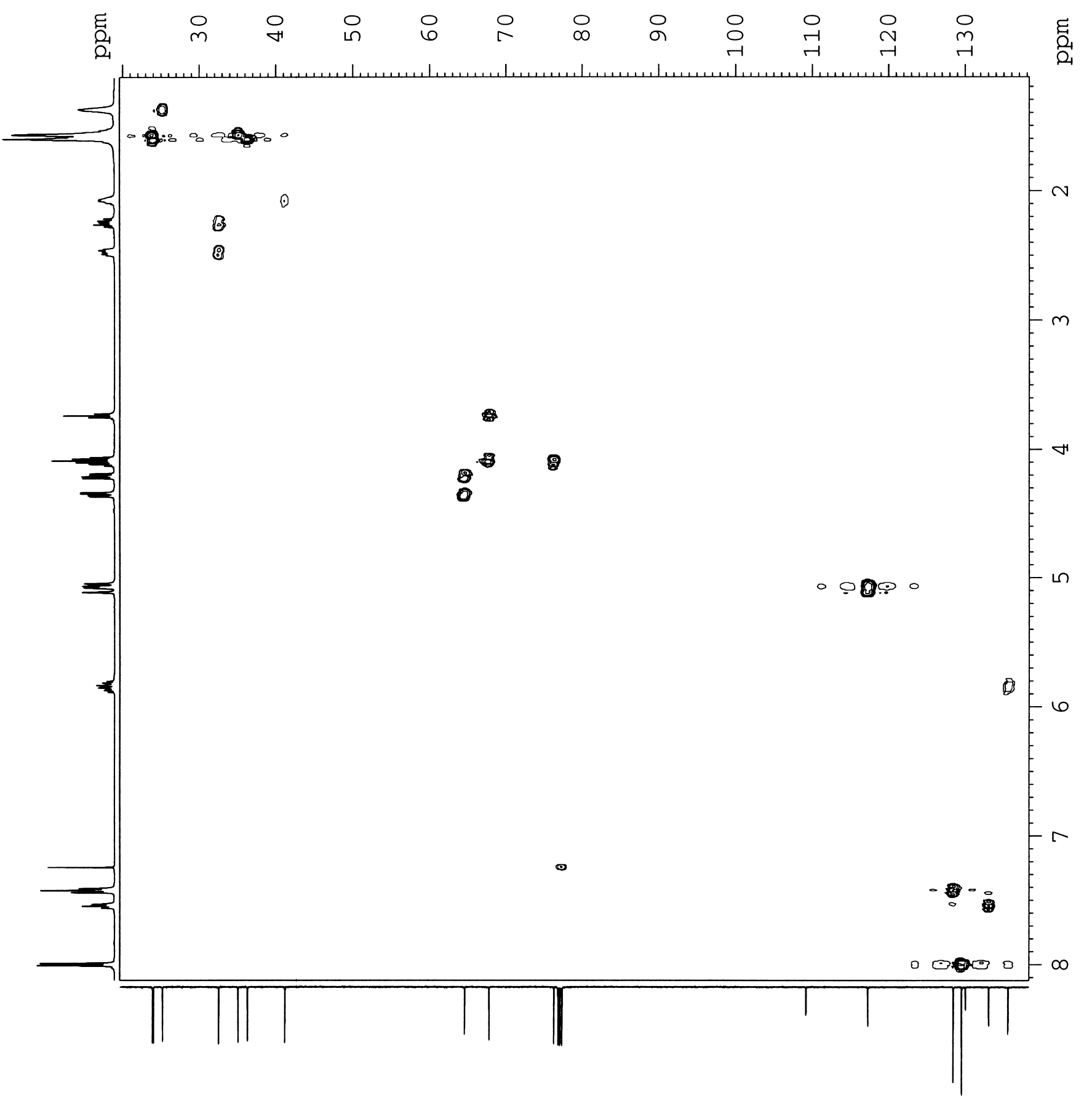

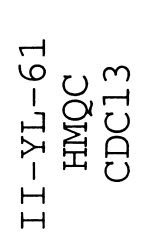

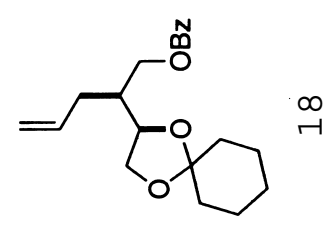




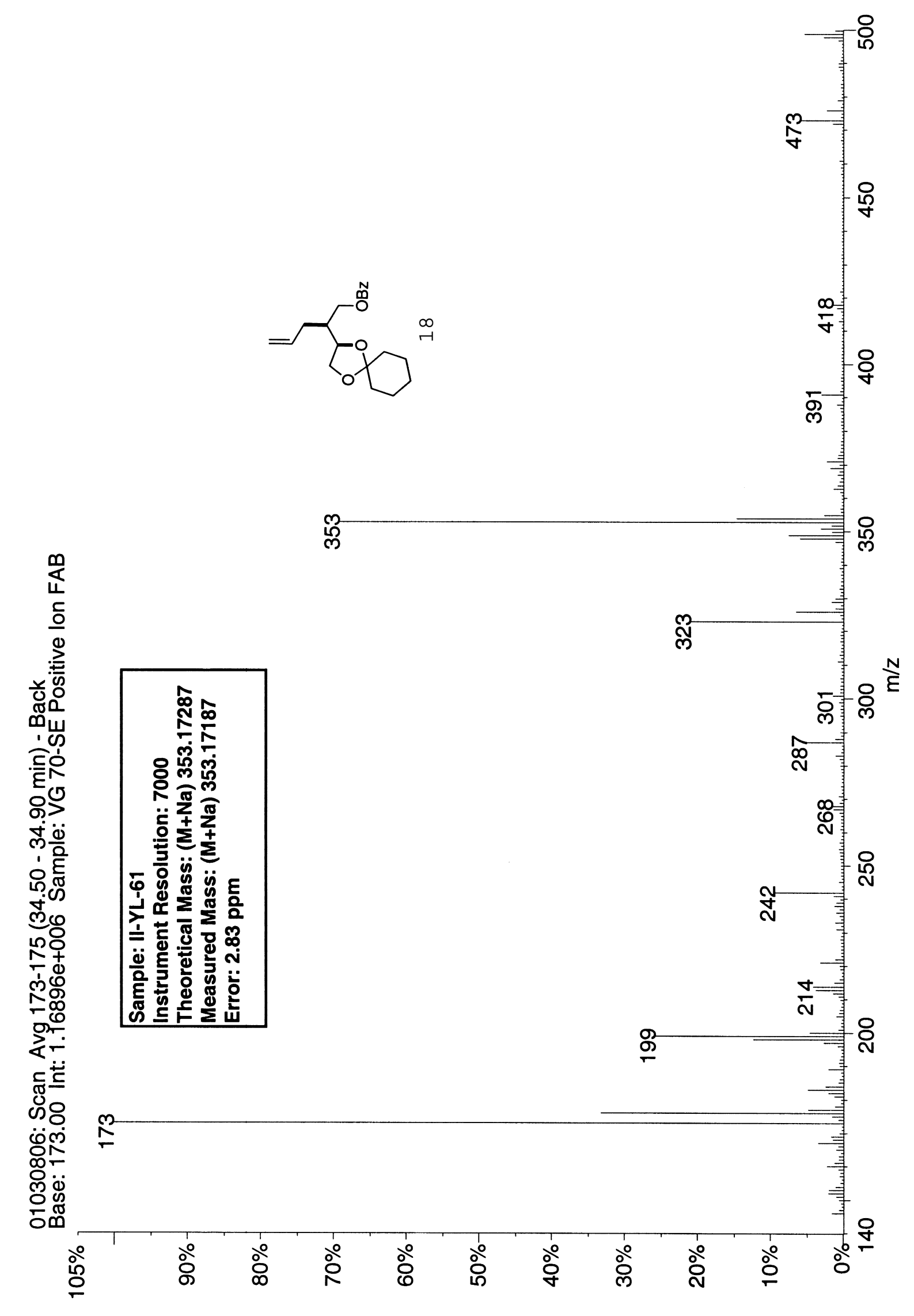




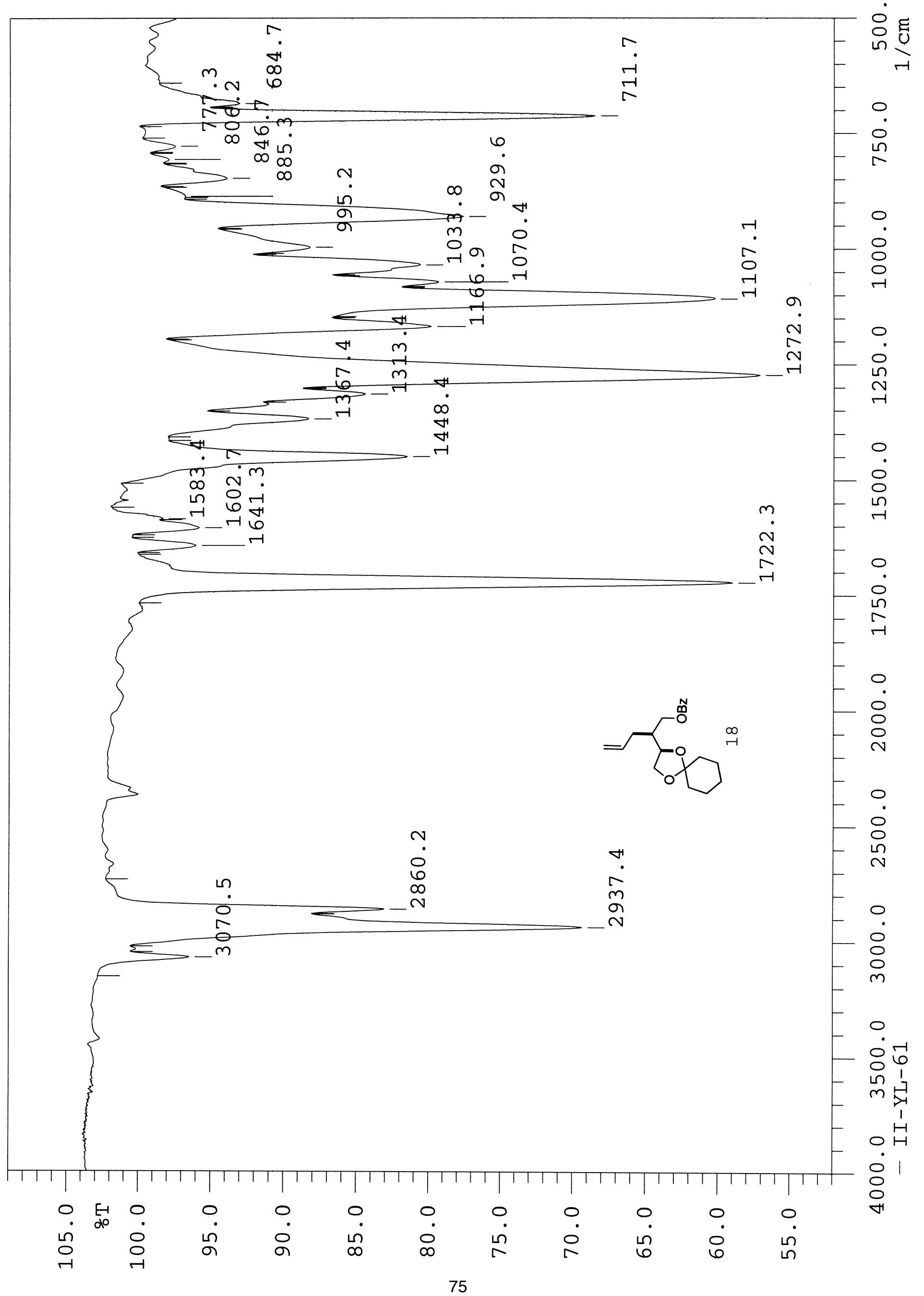




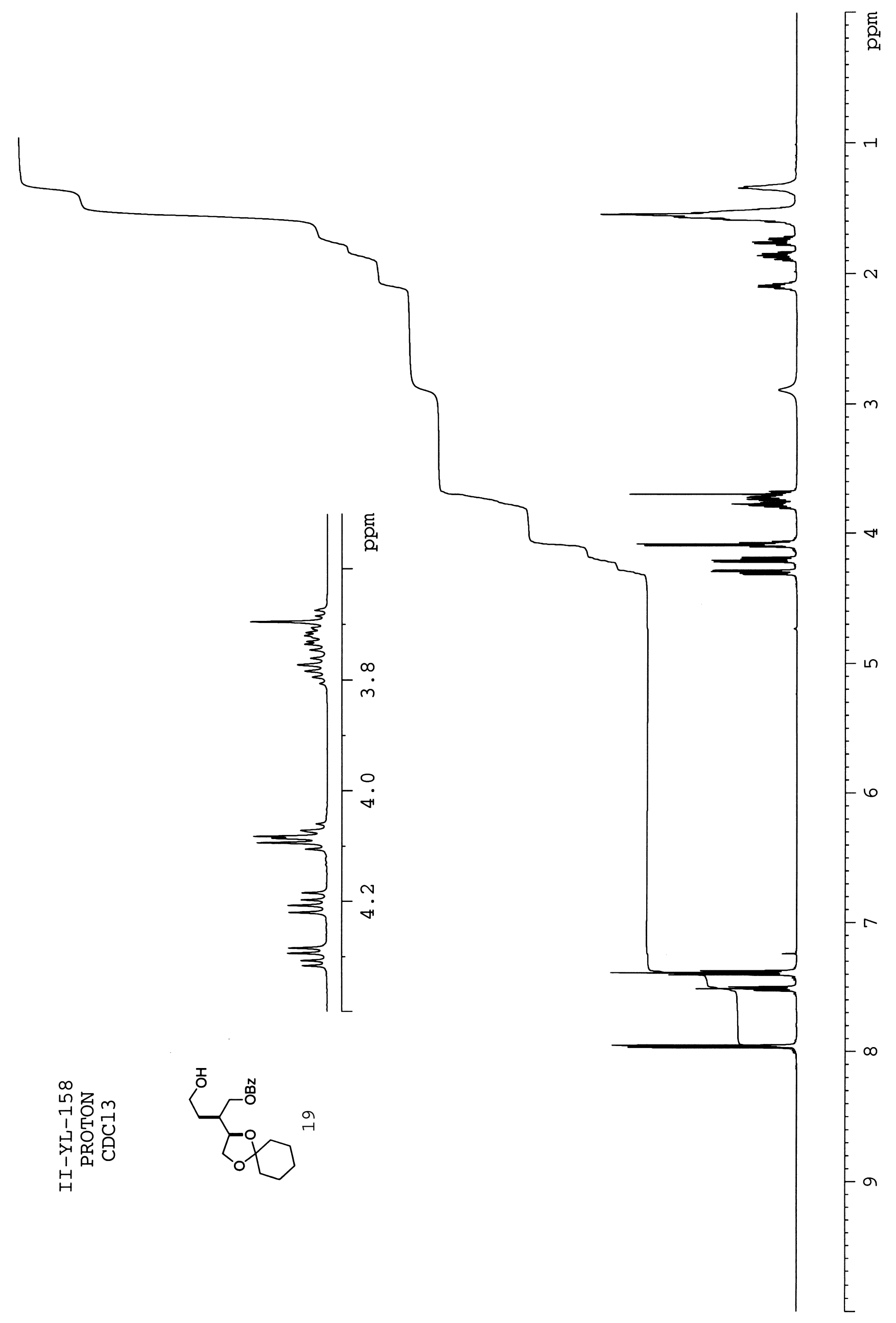




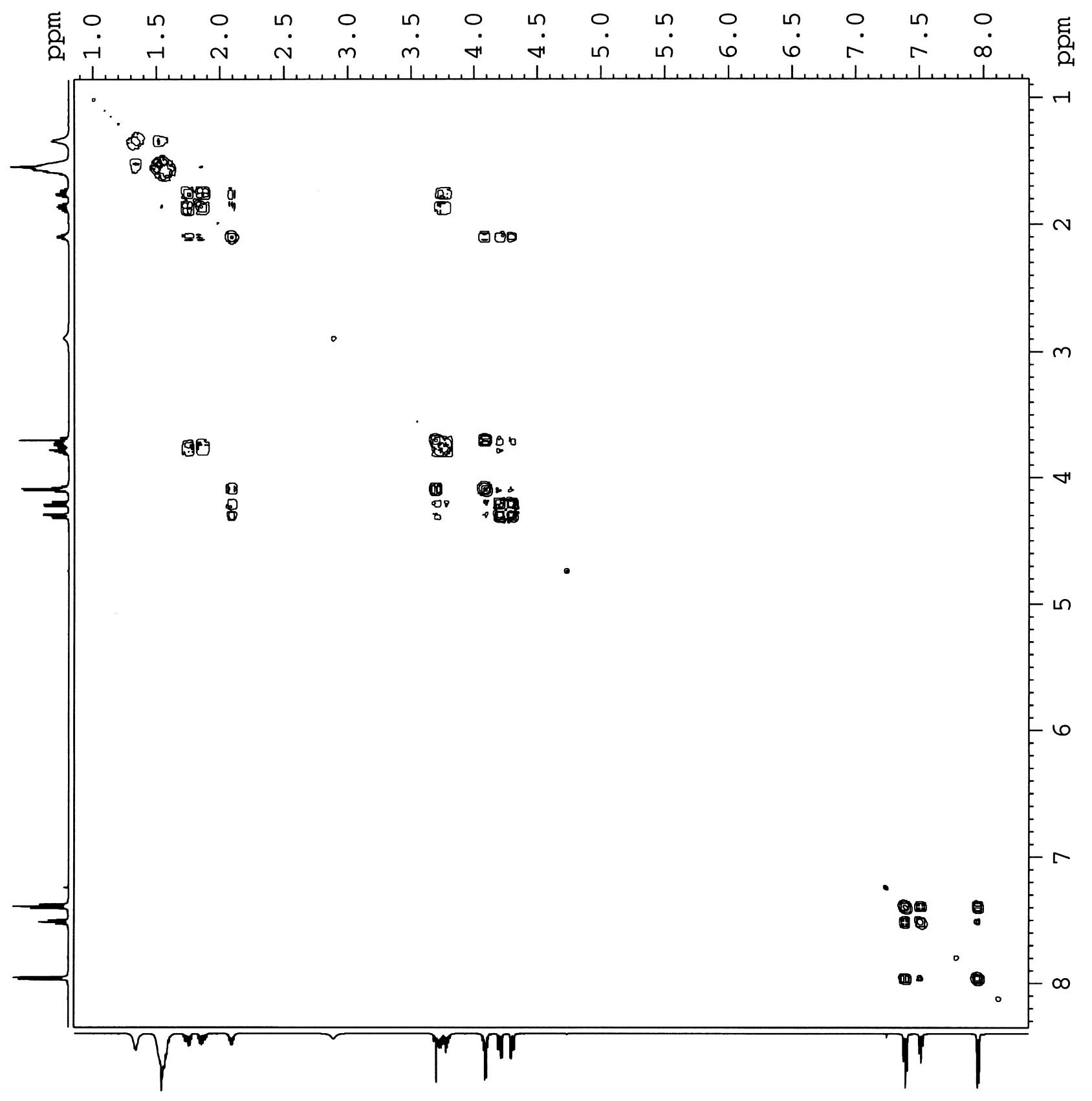

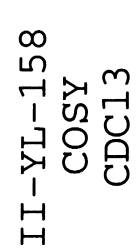

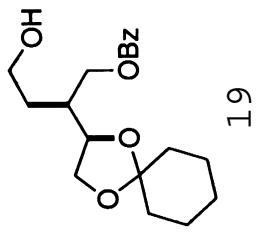



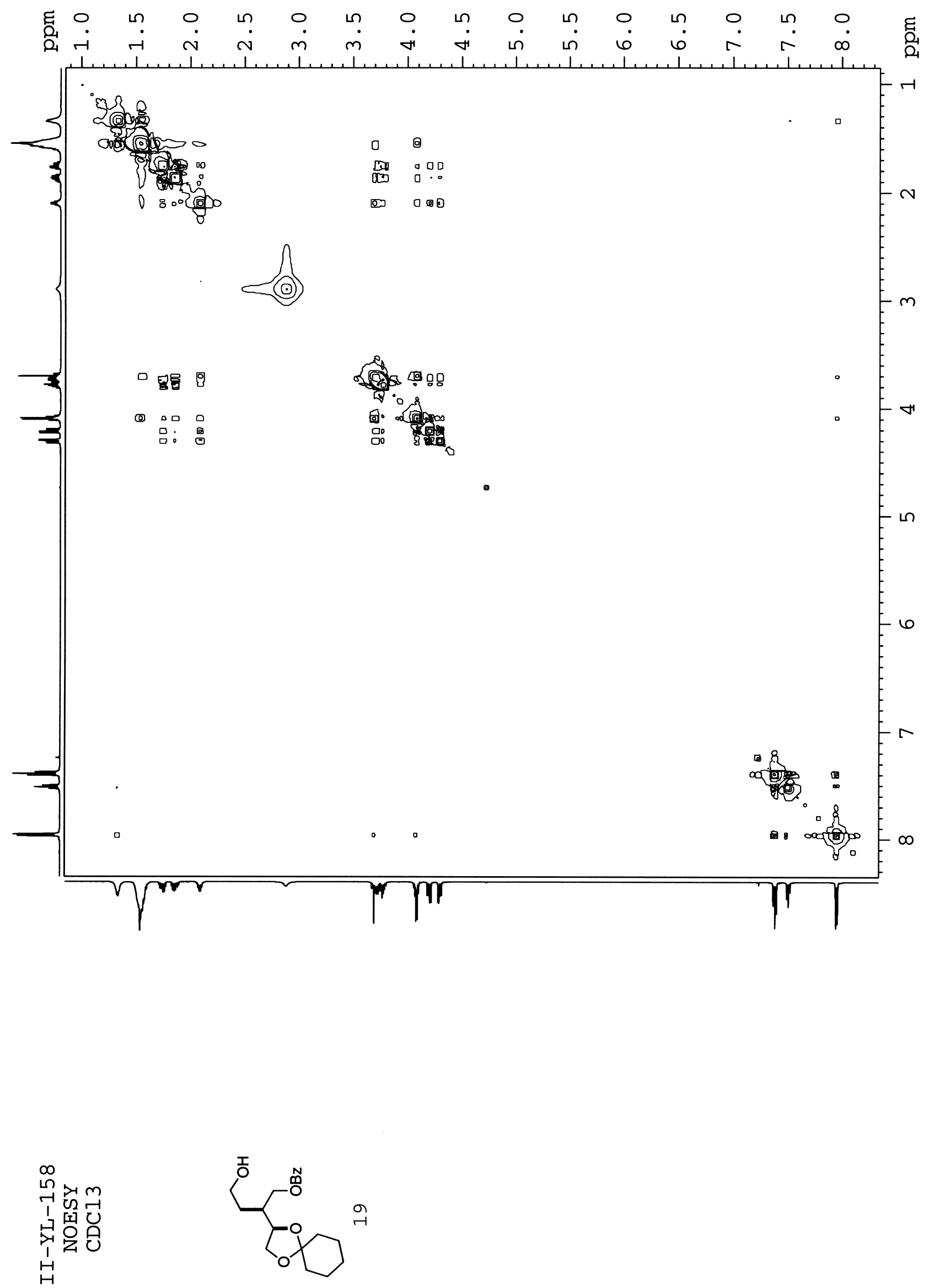


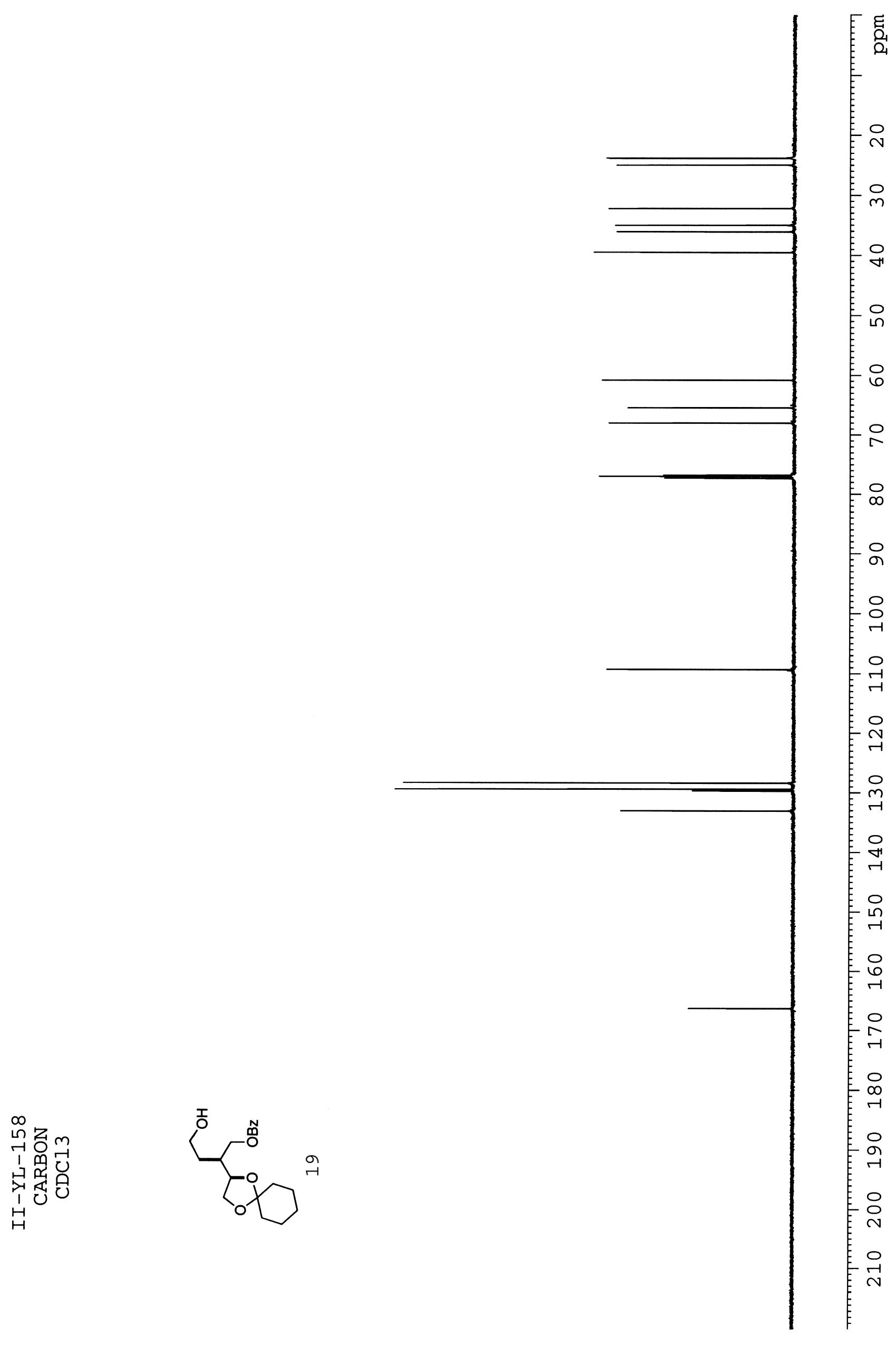




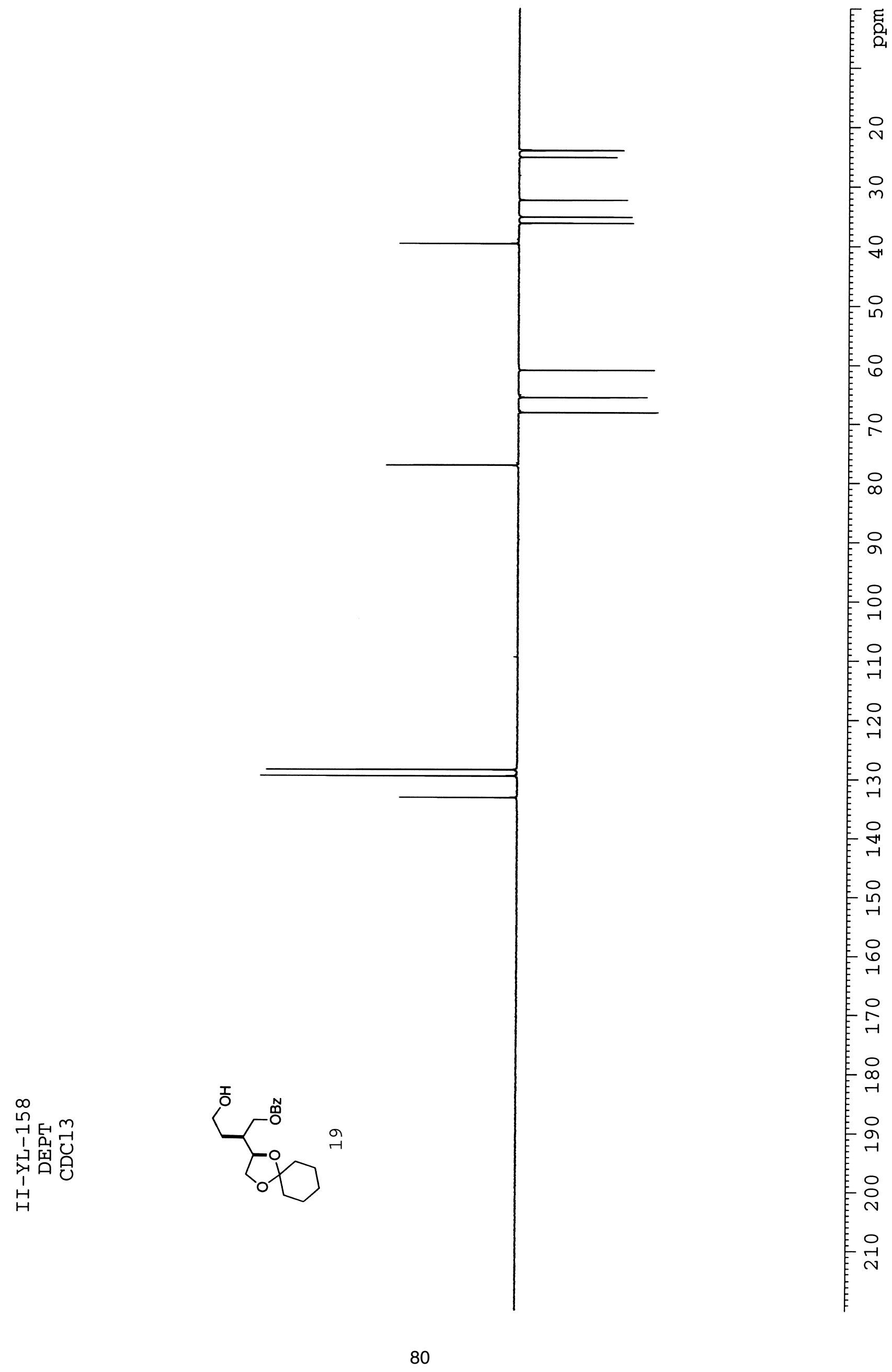




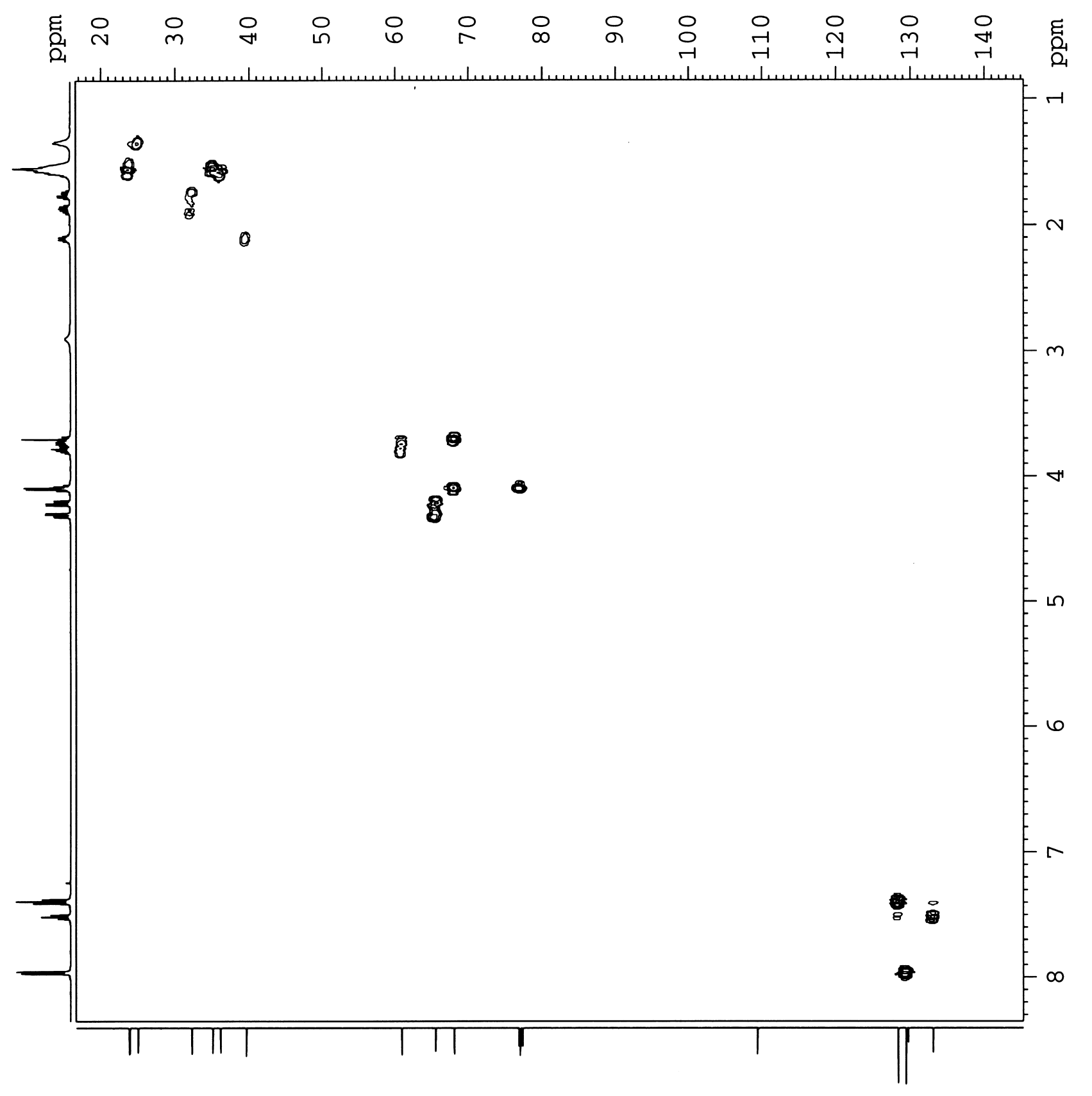

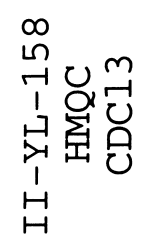<smiles>[CH2]CCC(C[18O])C1COC2(CCCCC2)O1</smiles> 

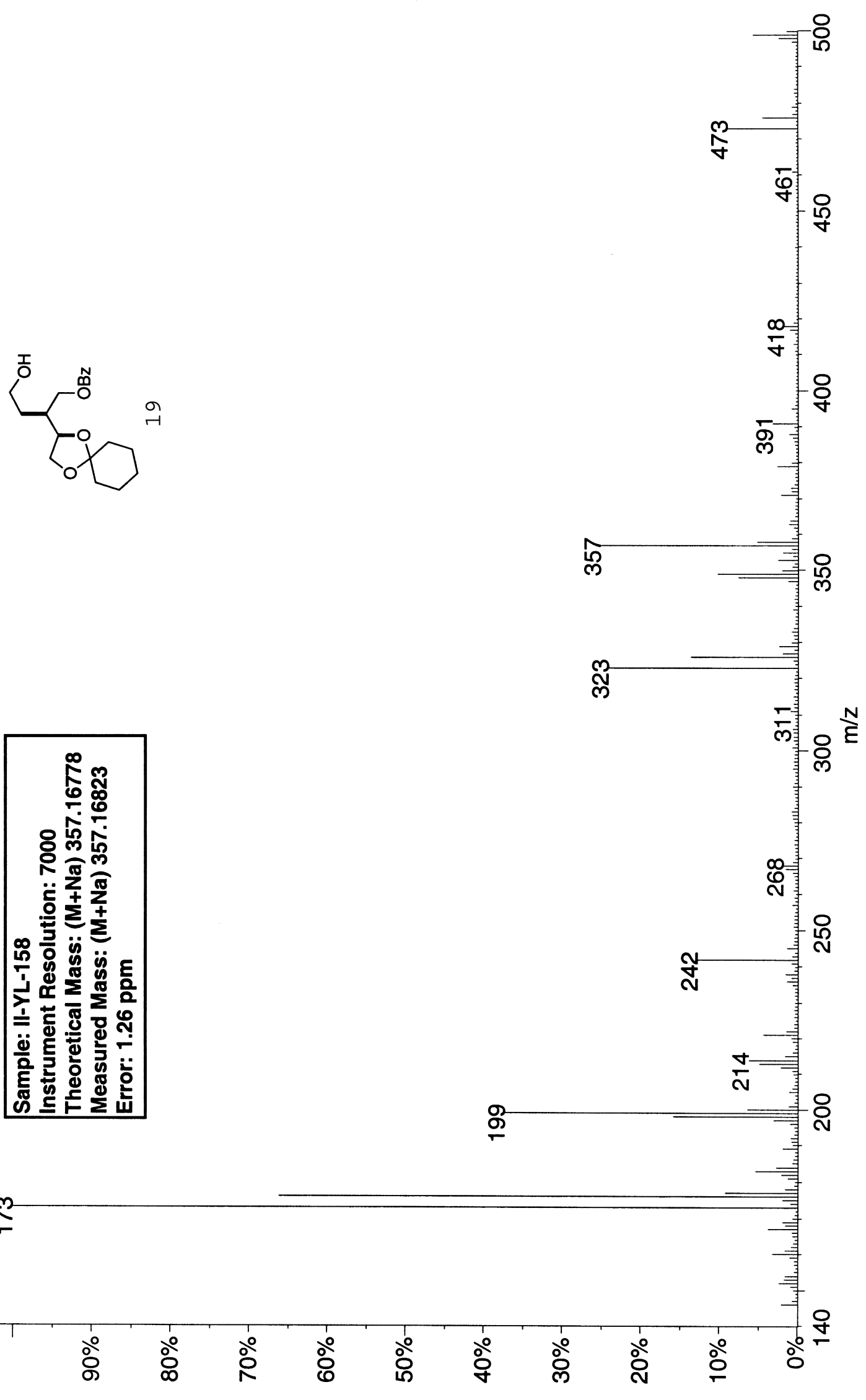


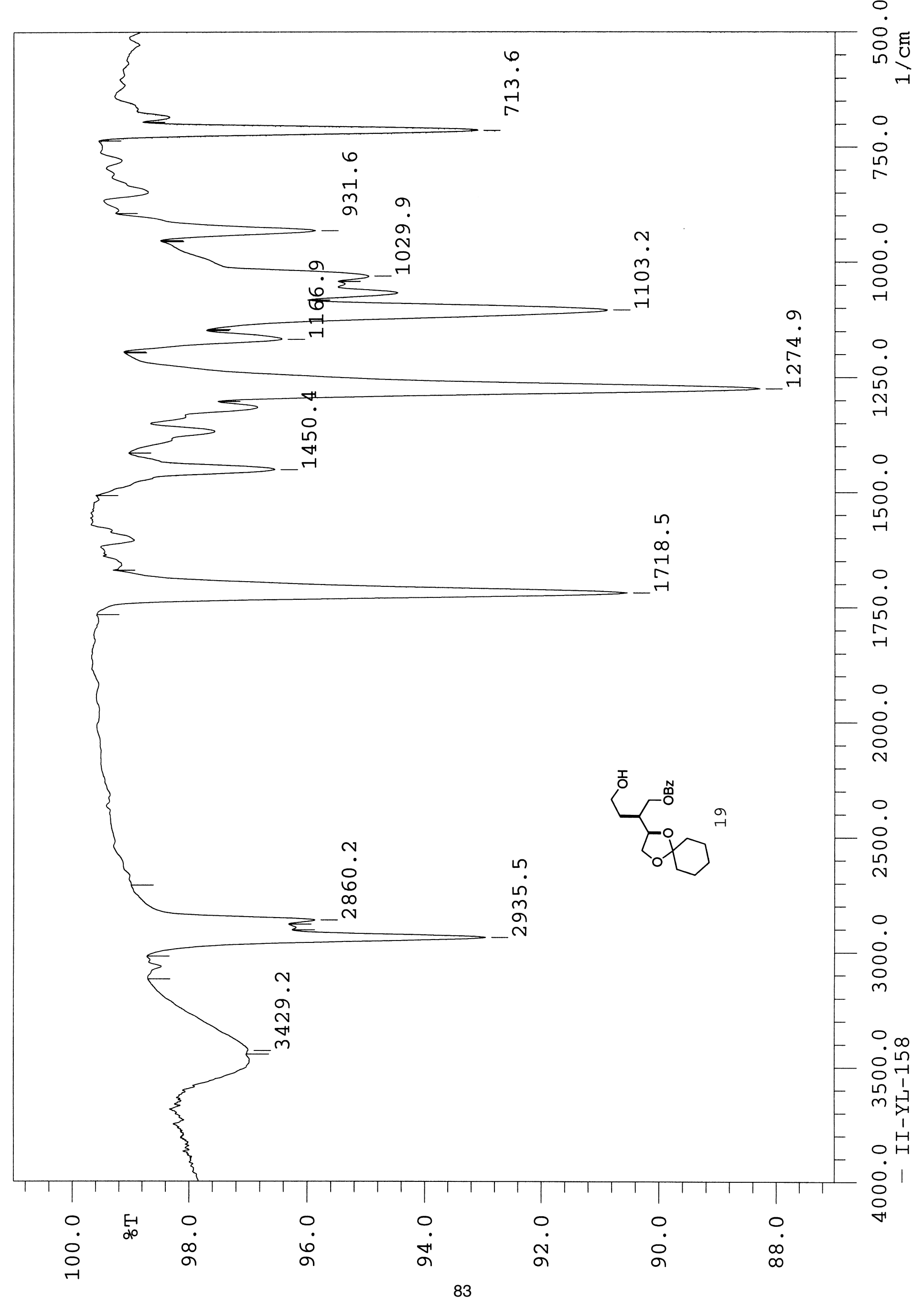




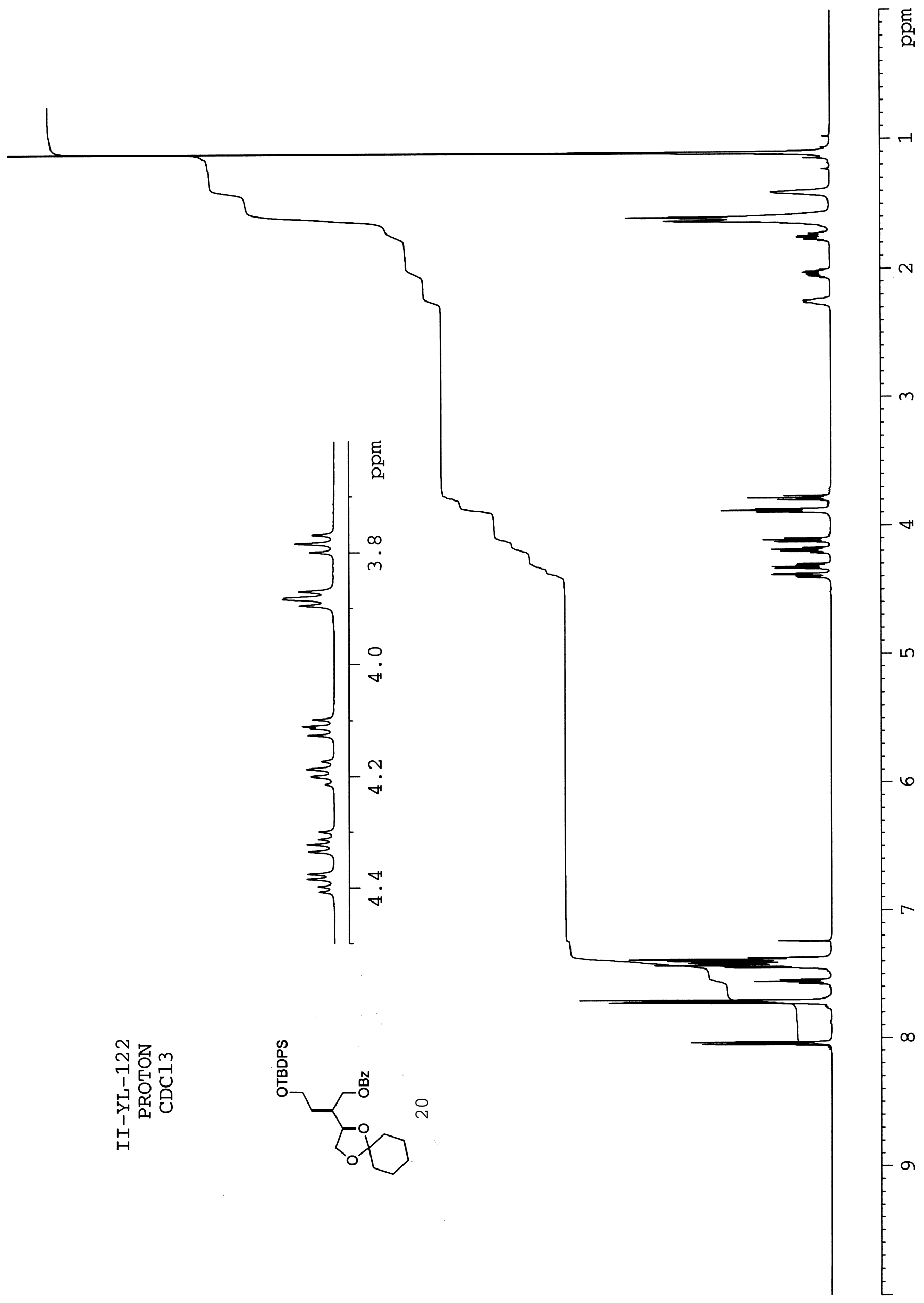



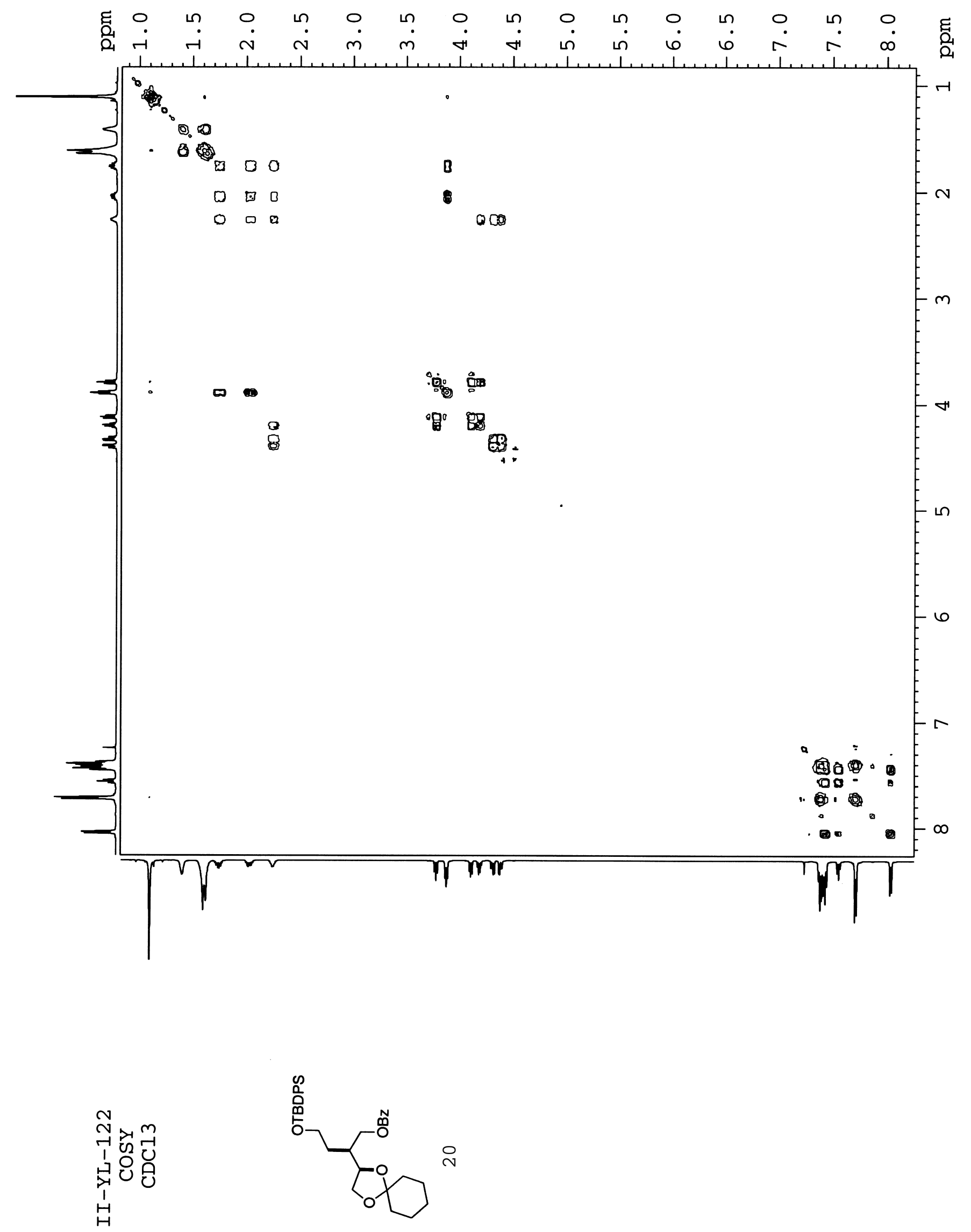

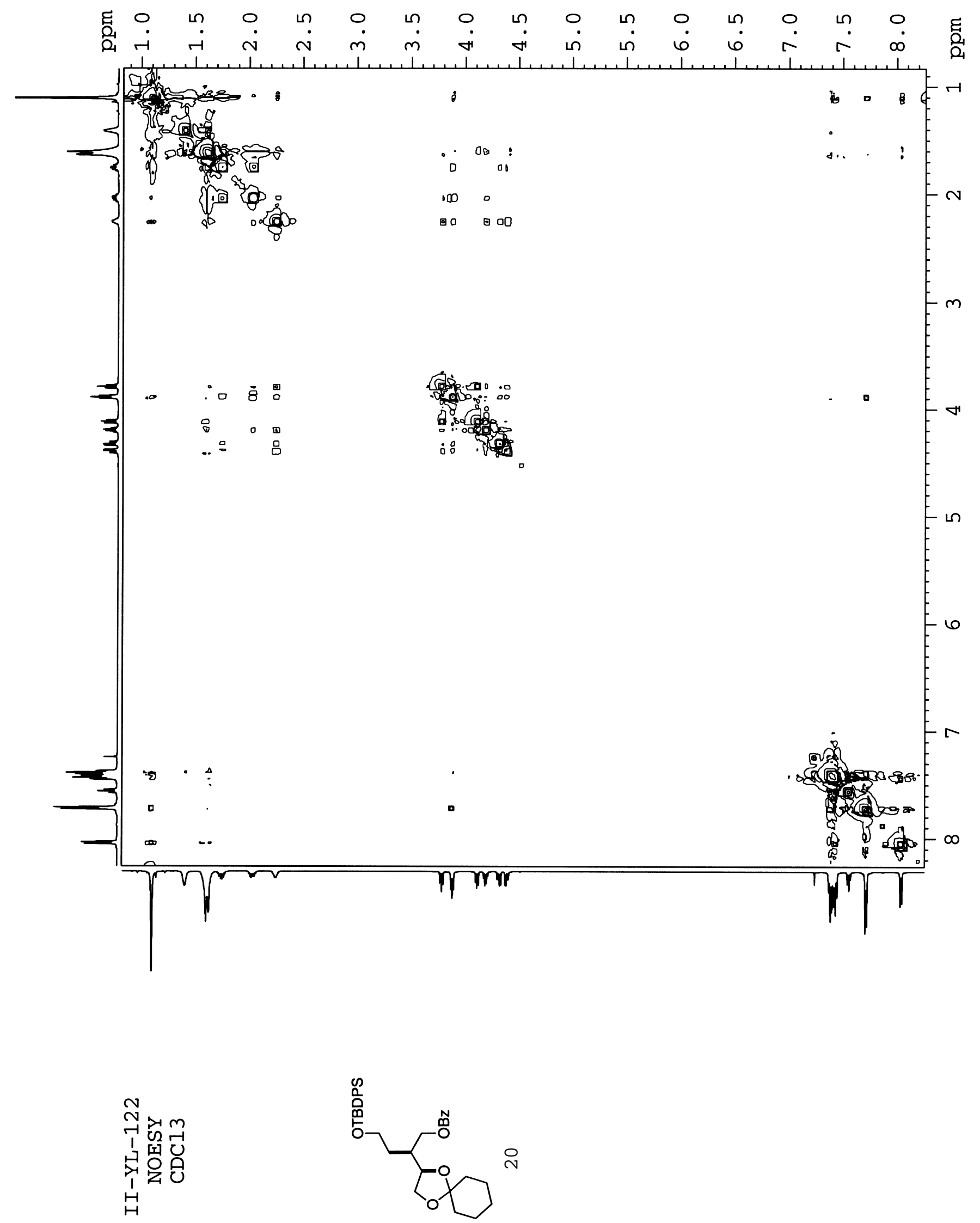


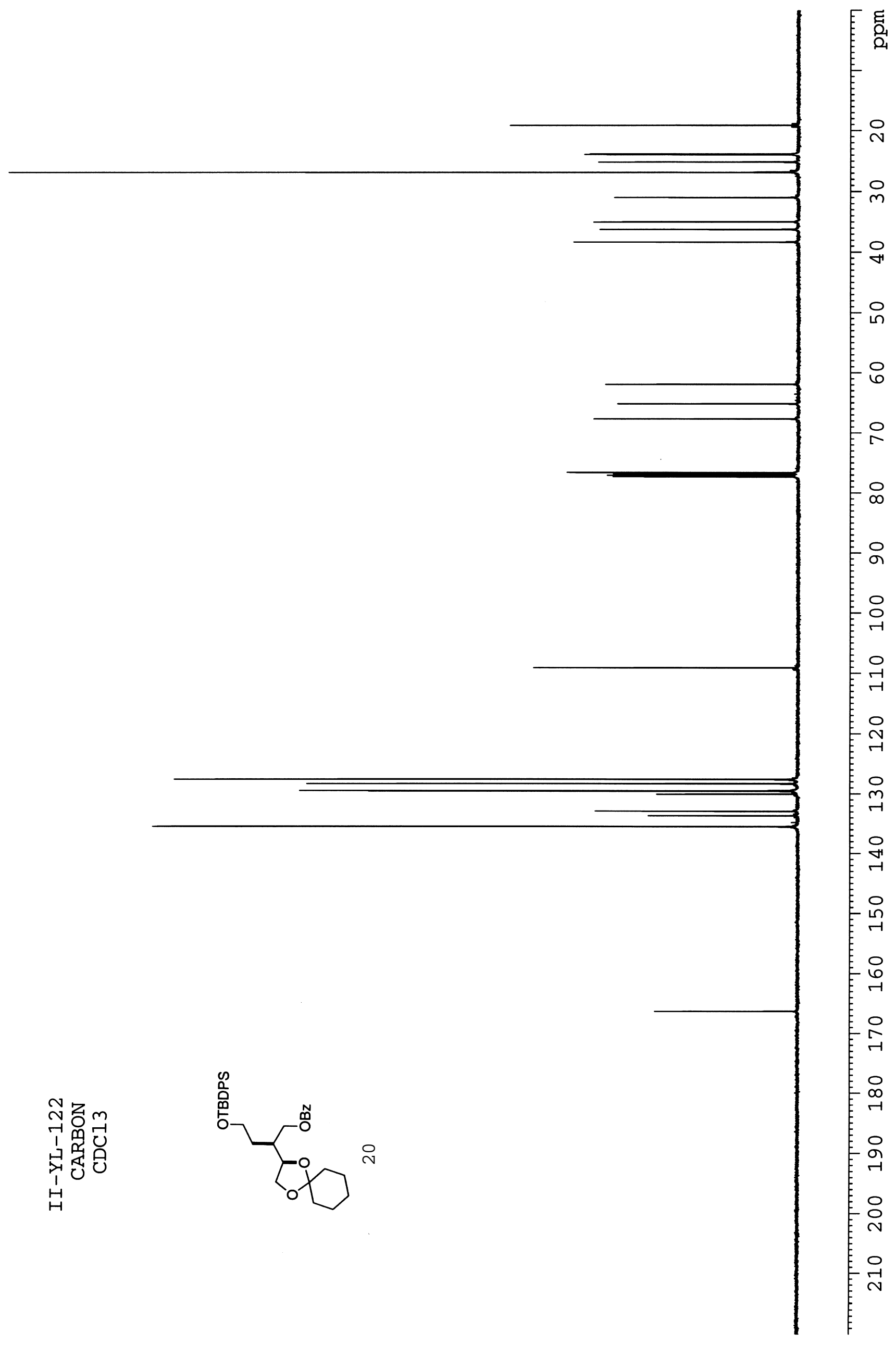




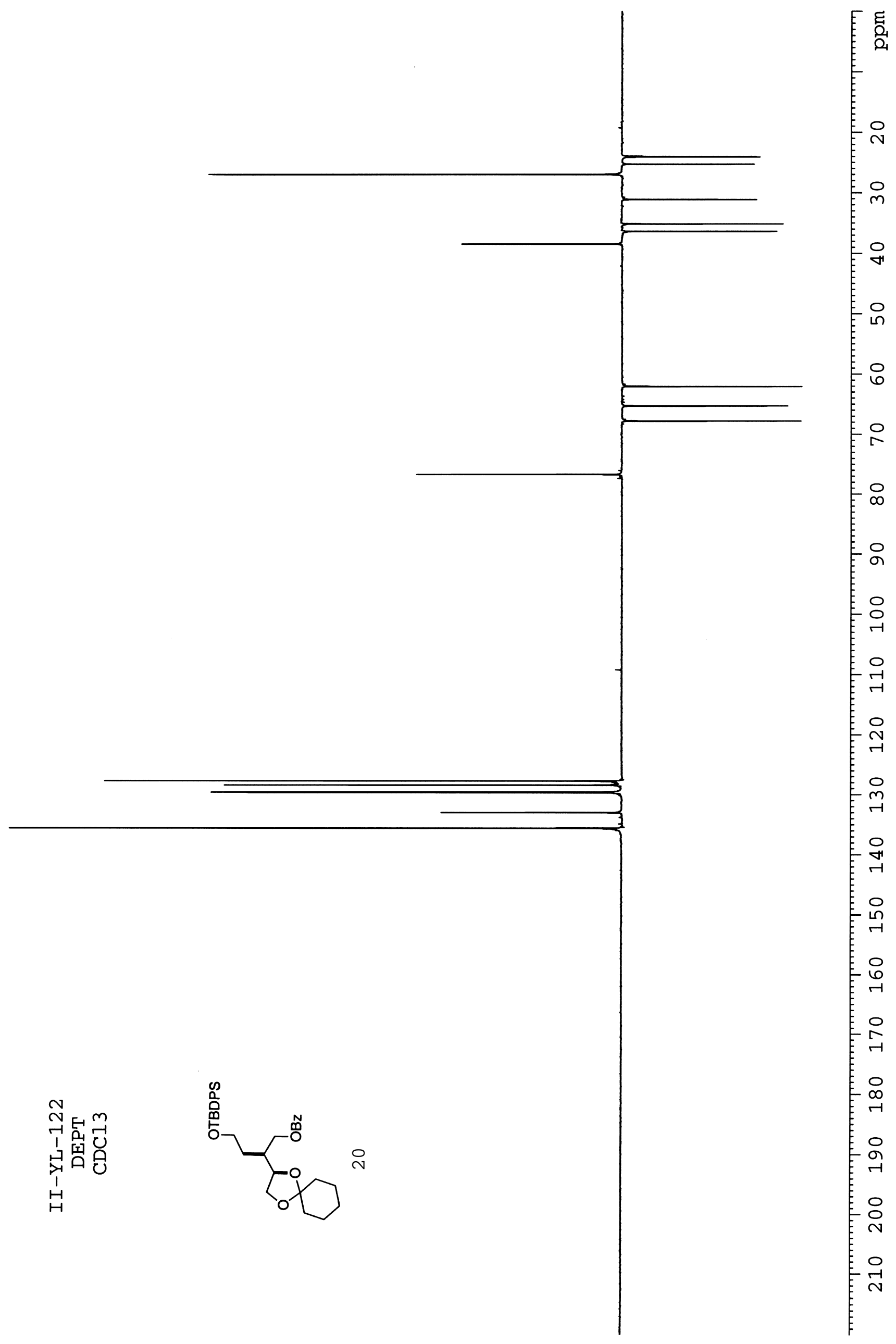



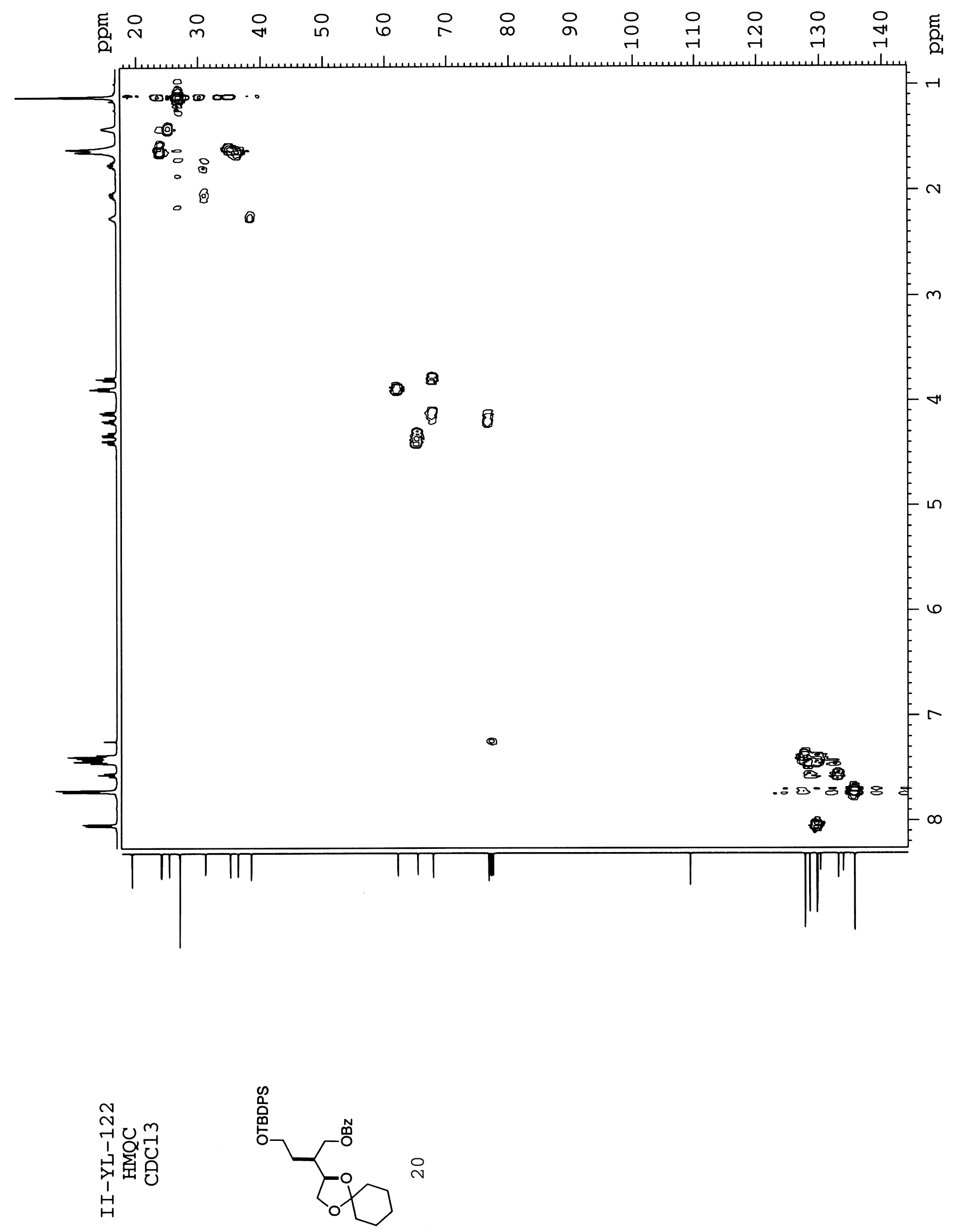


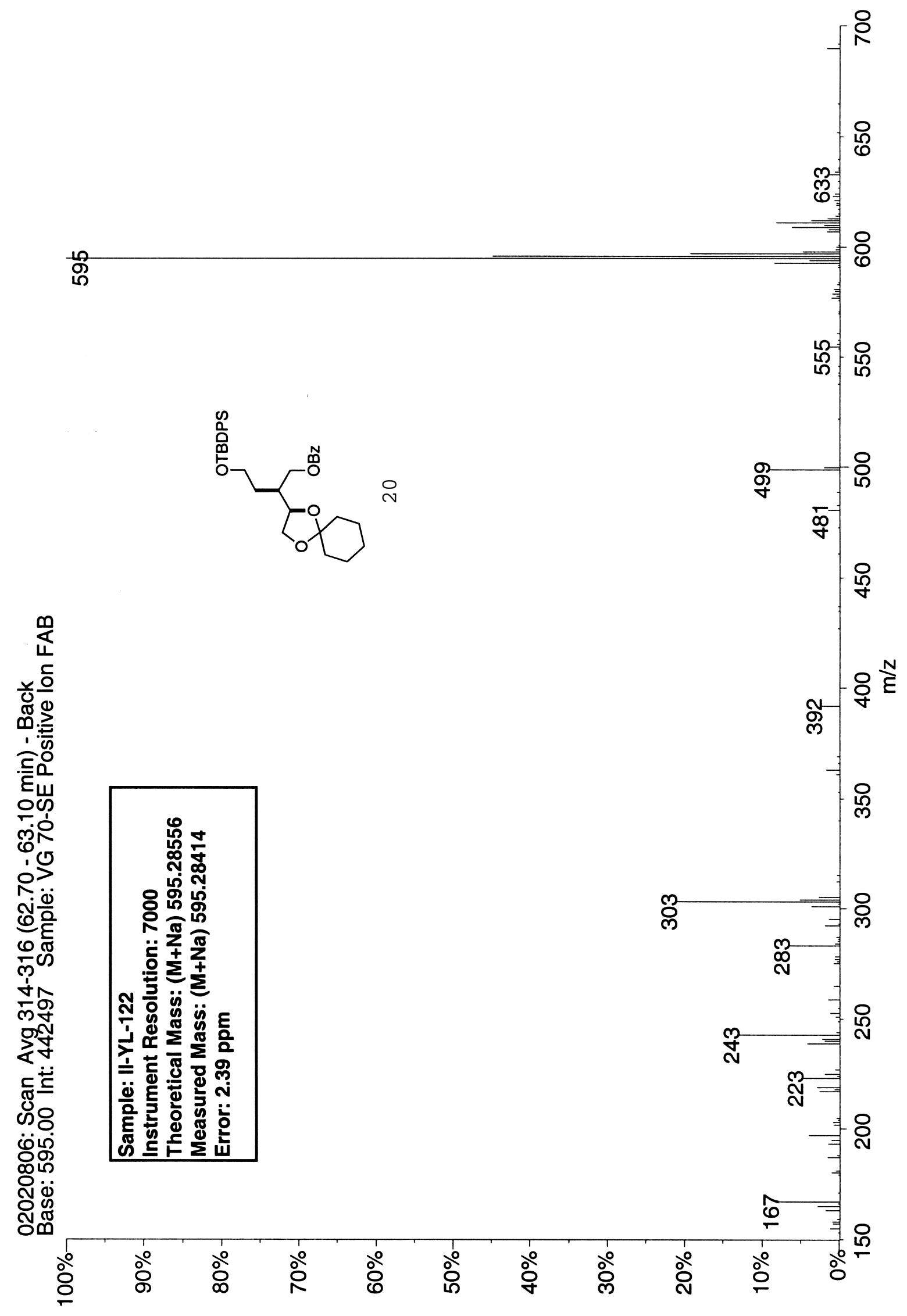




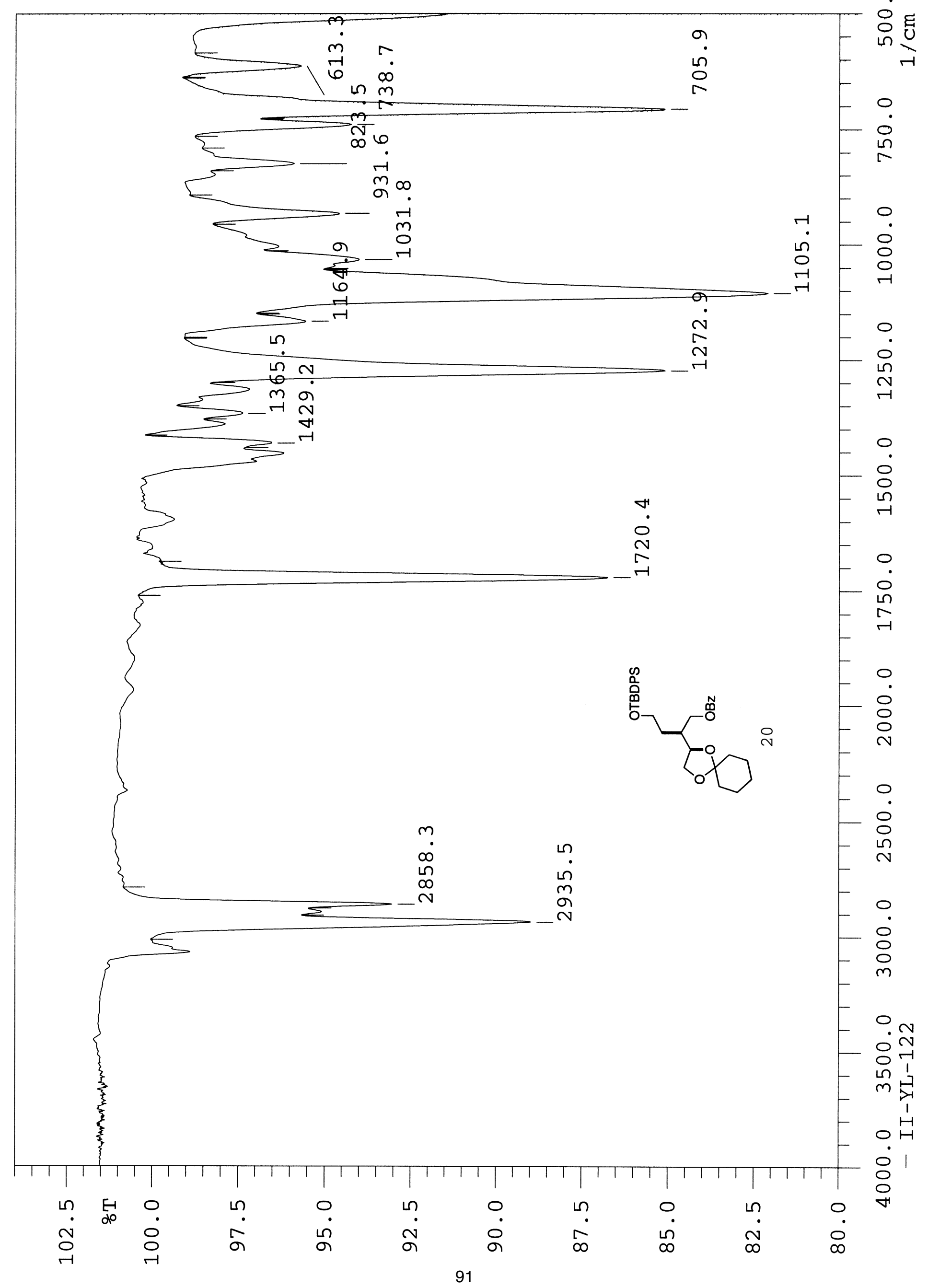




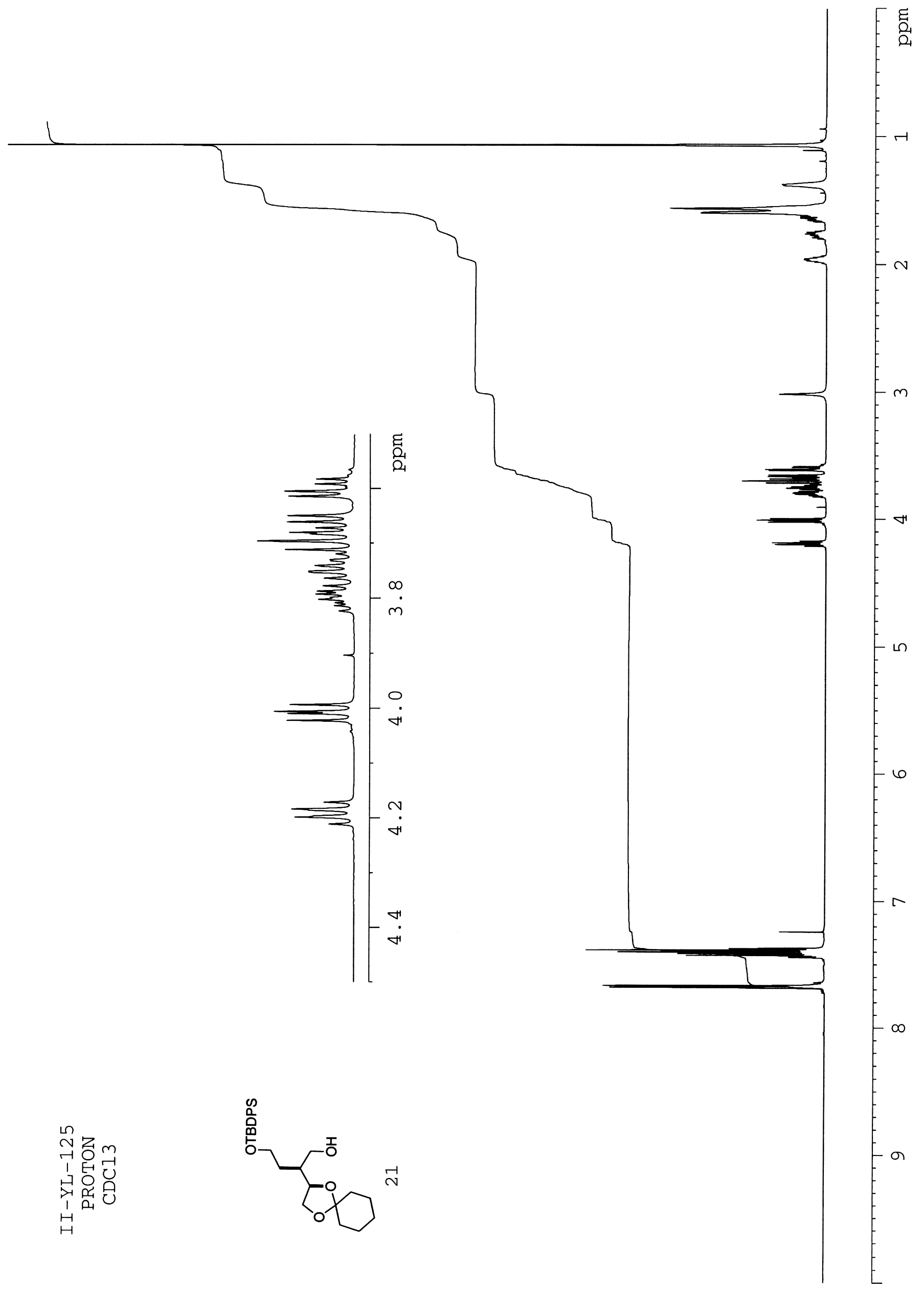




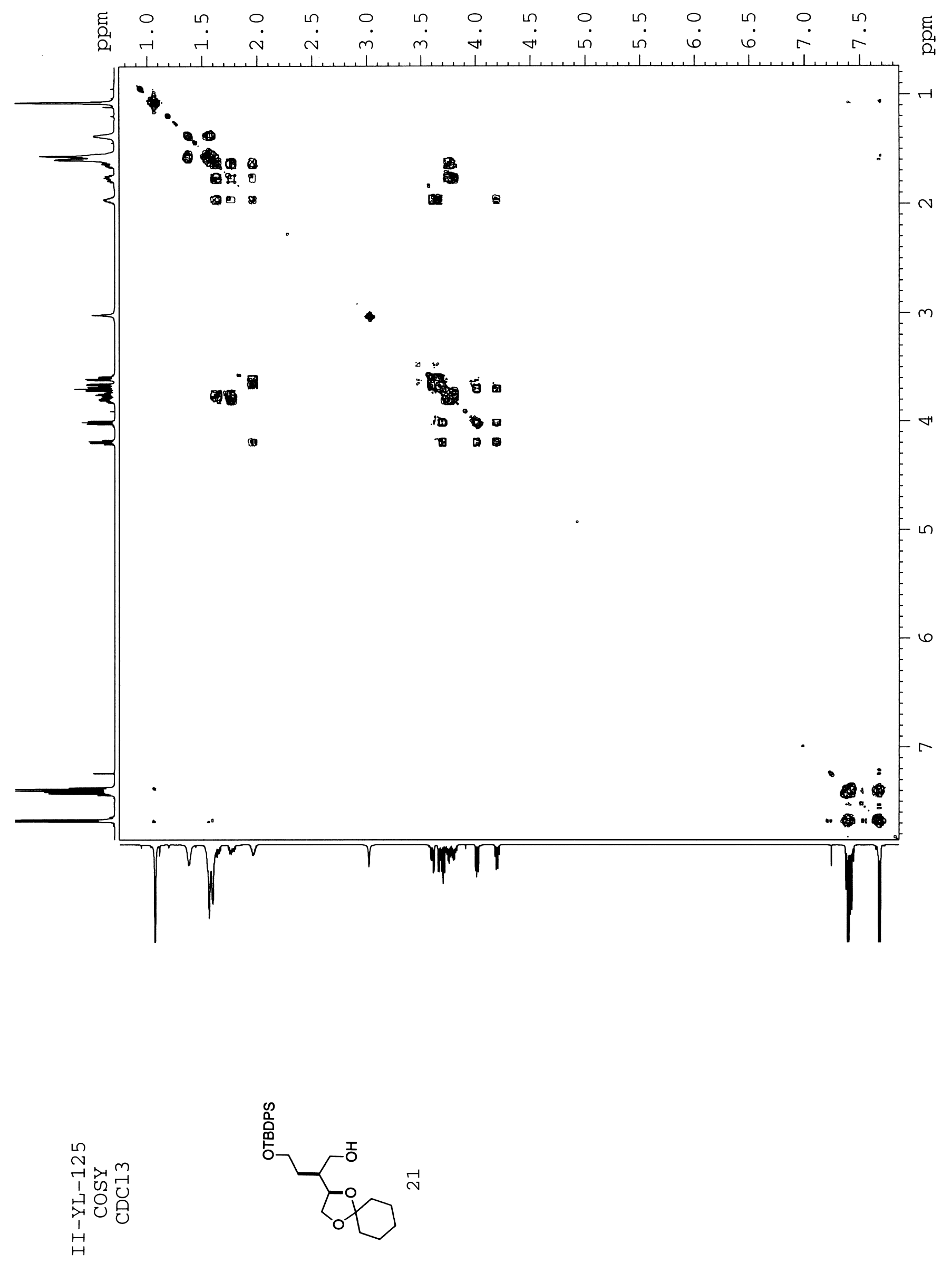



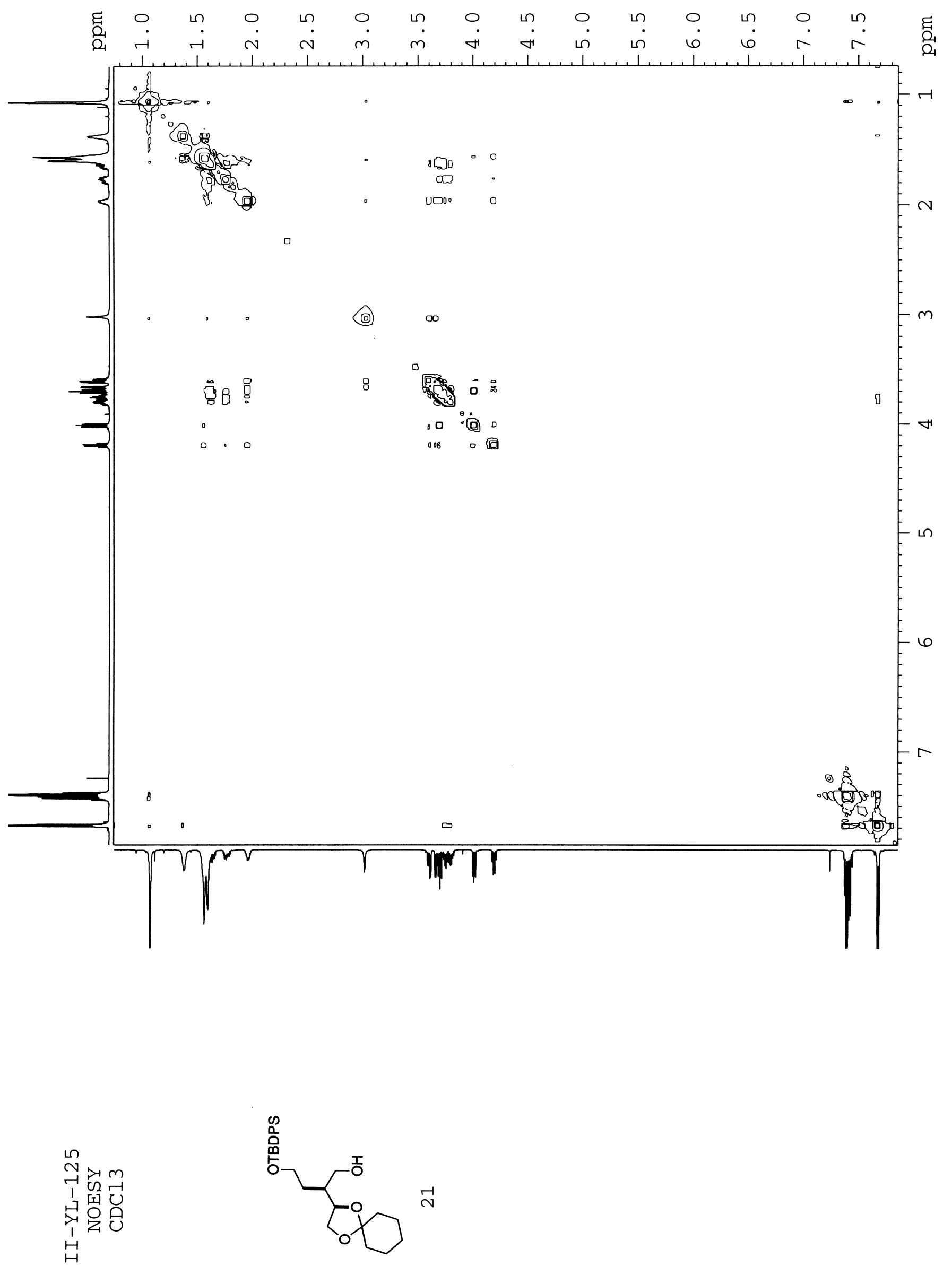


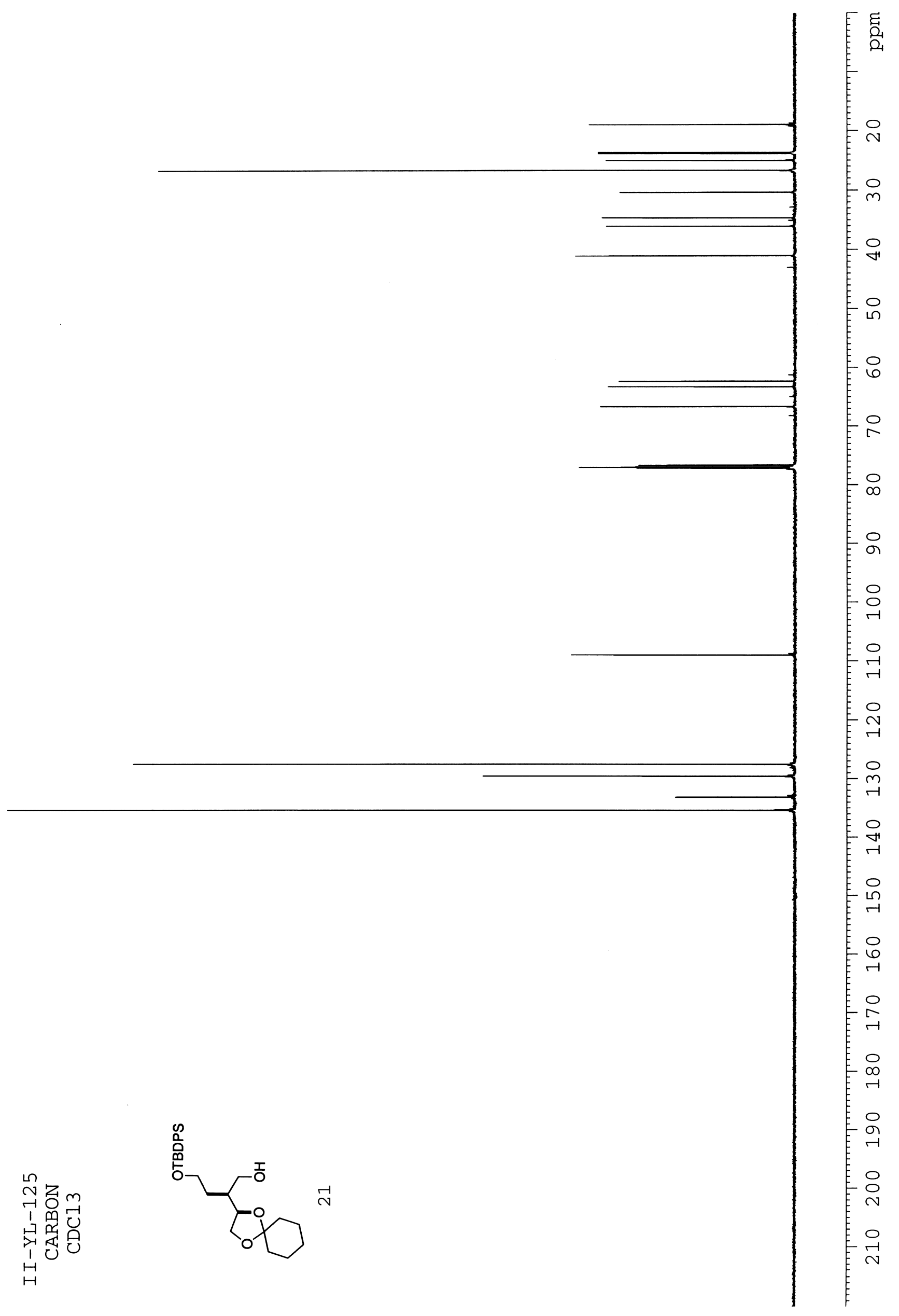




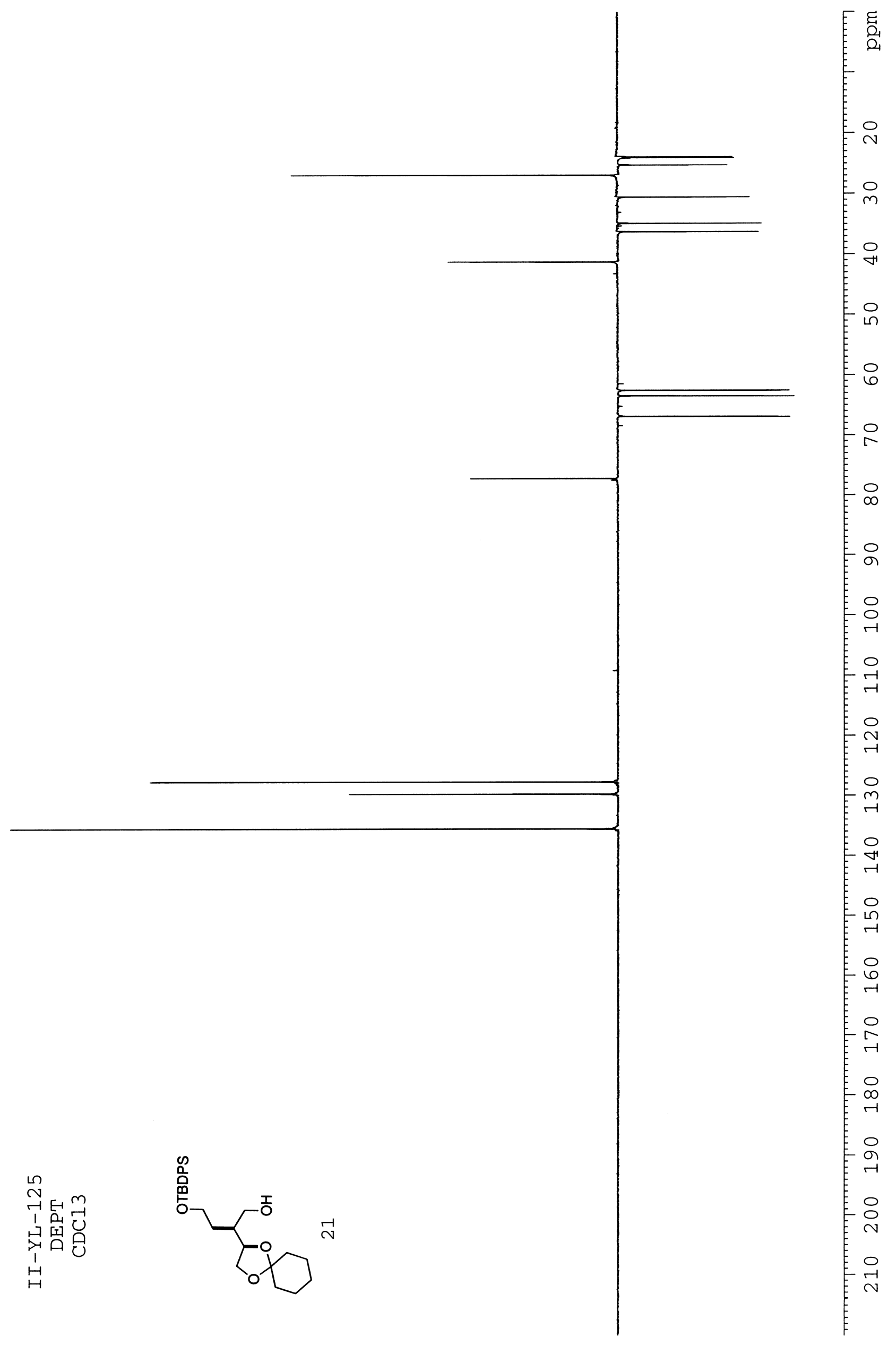




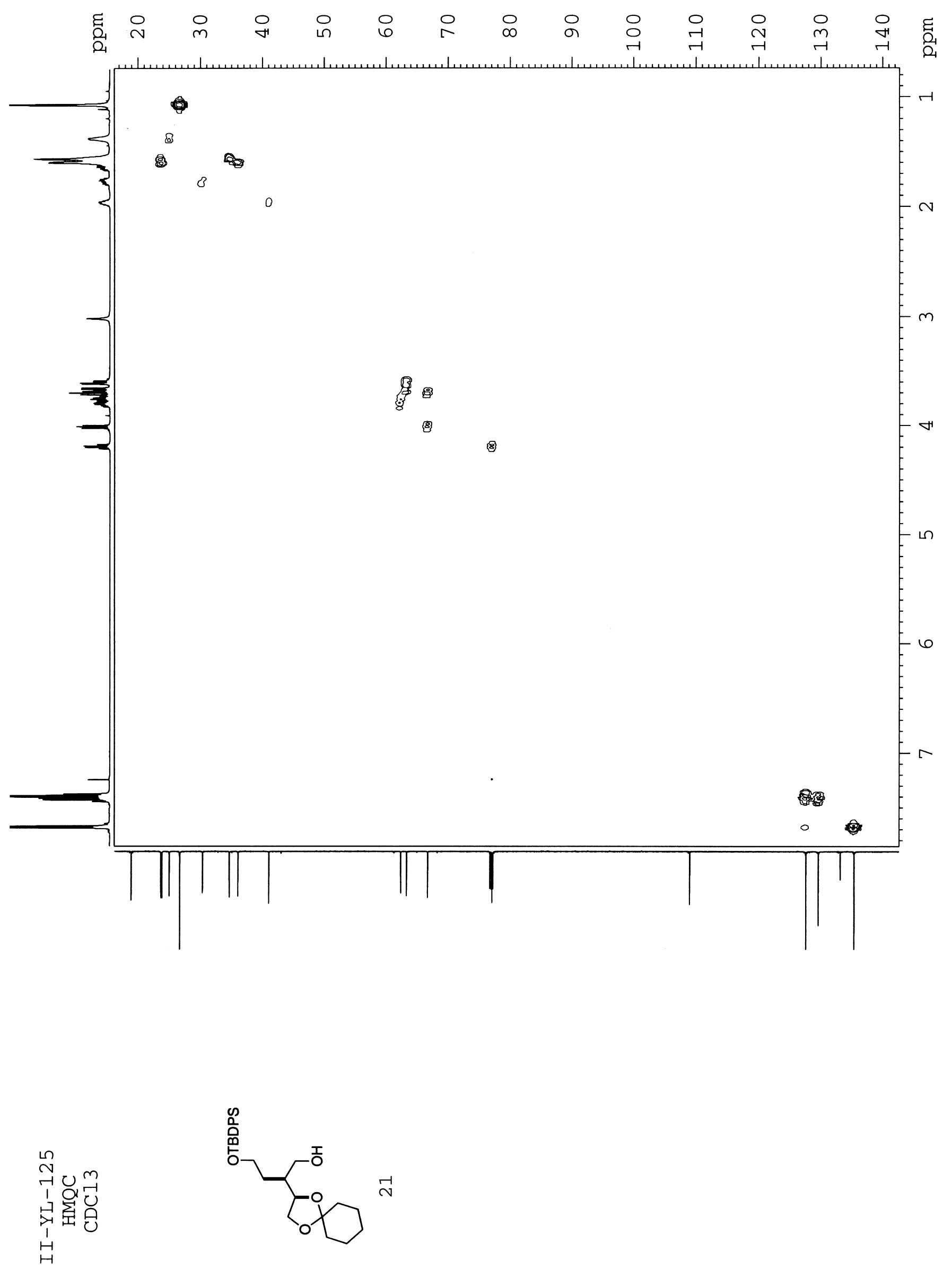



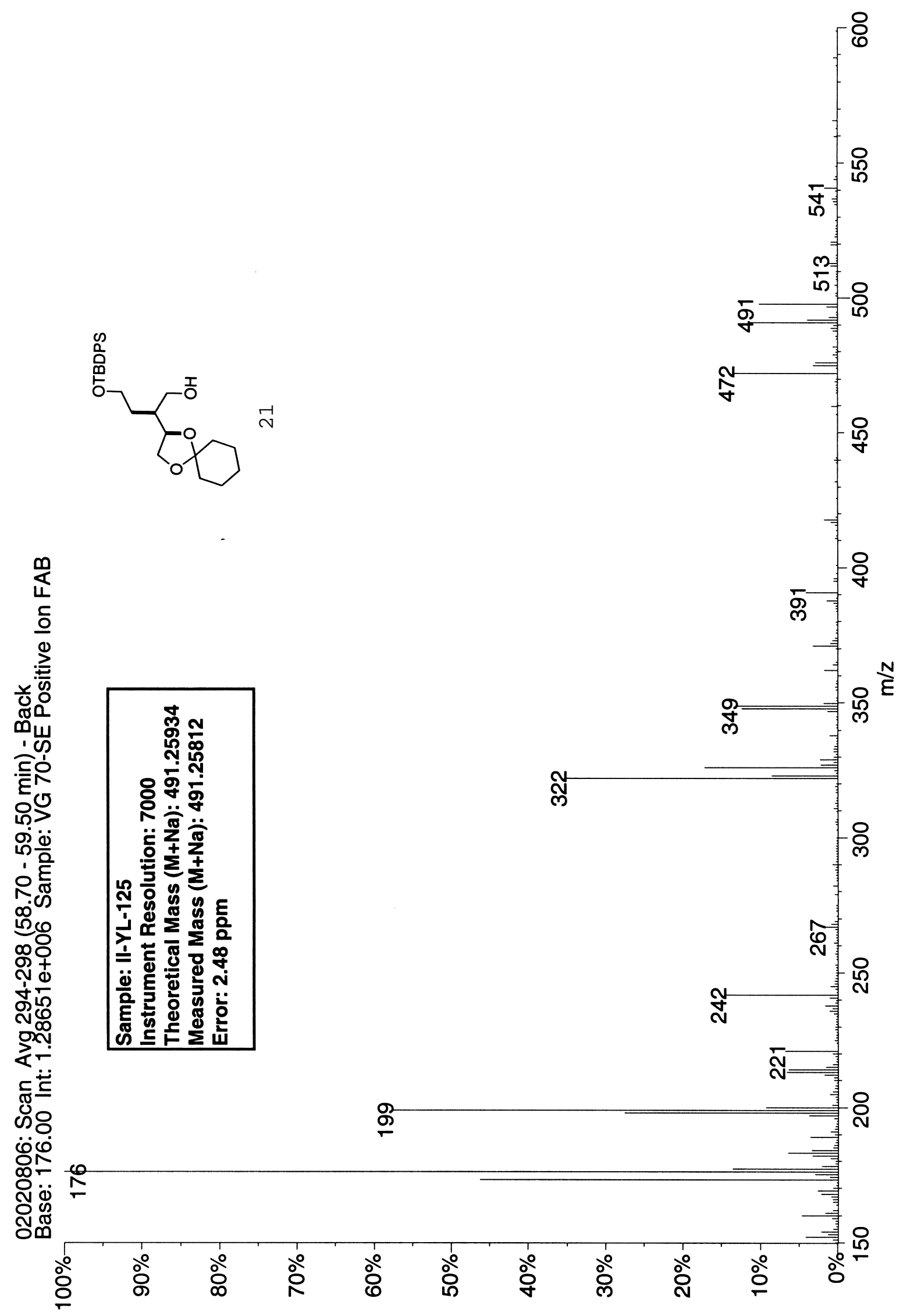

더으

응

ต்

유 뚱

సิ๊

○े 


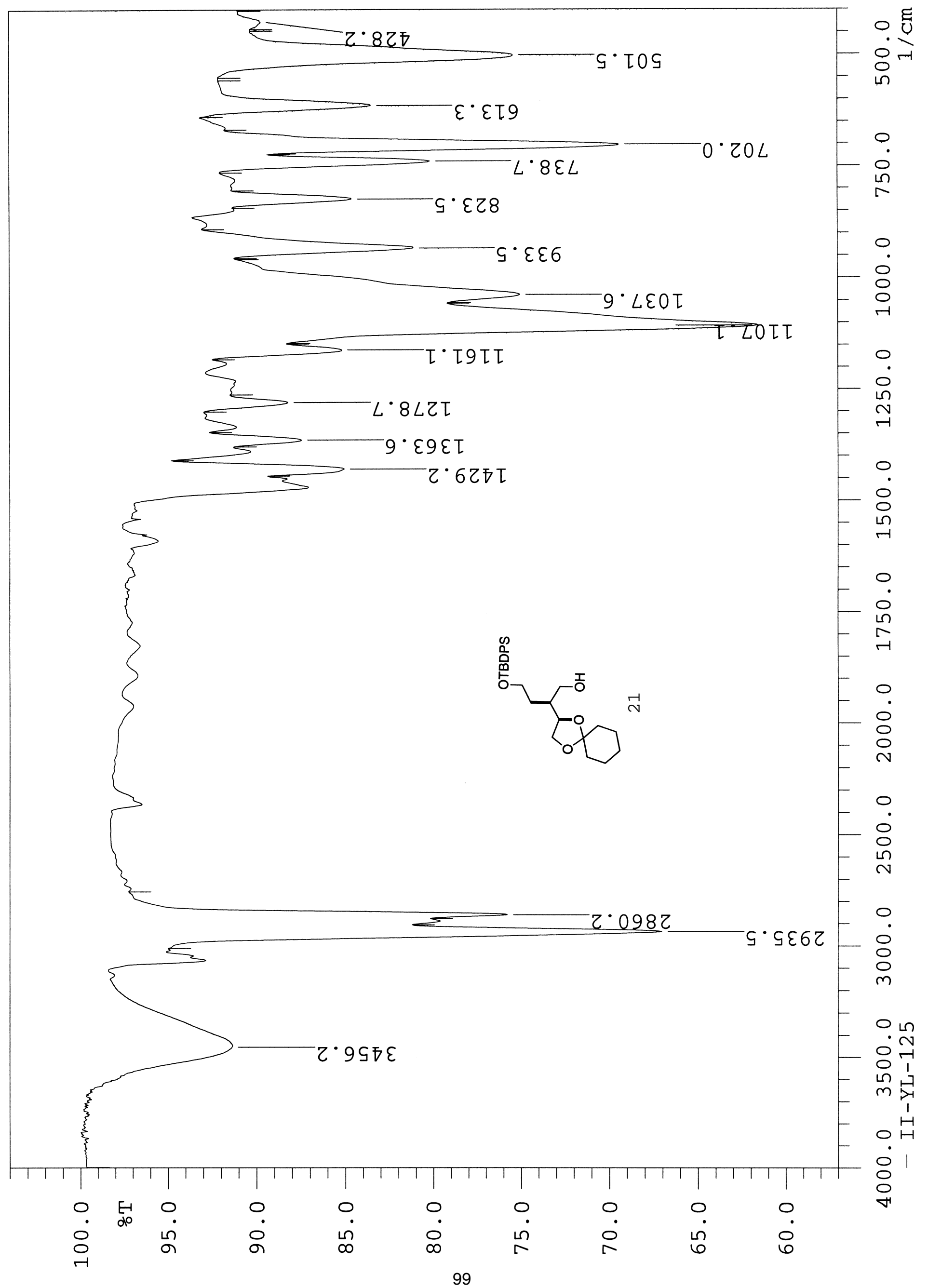




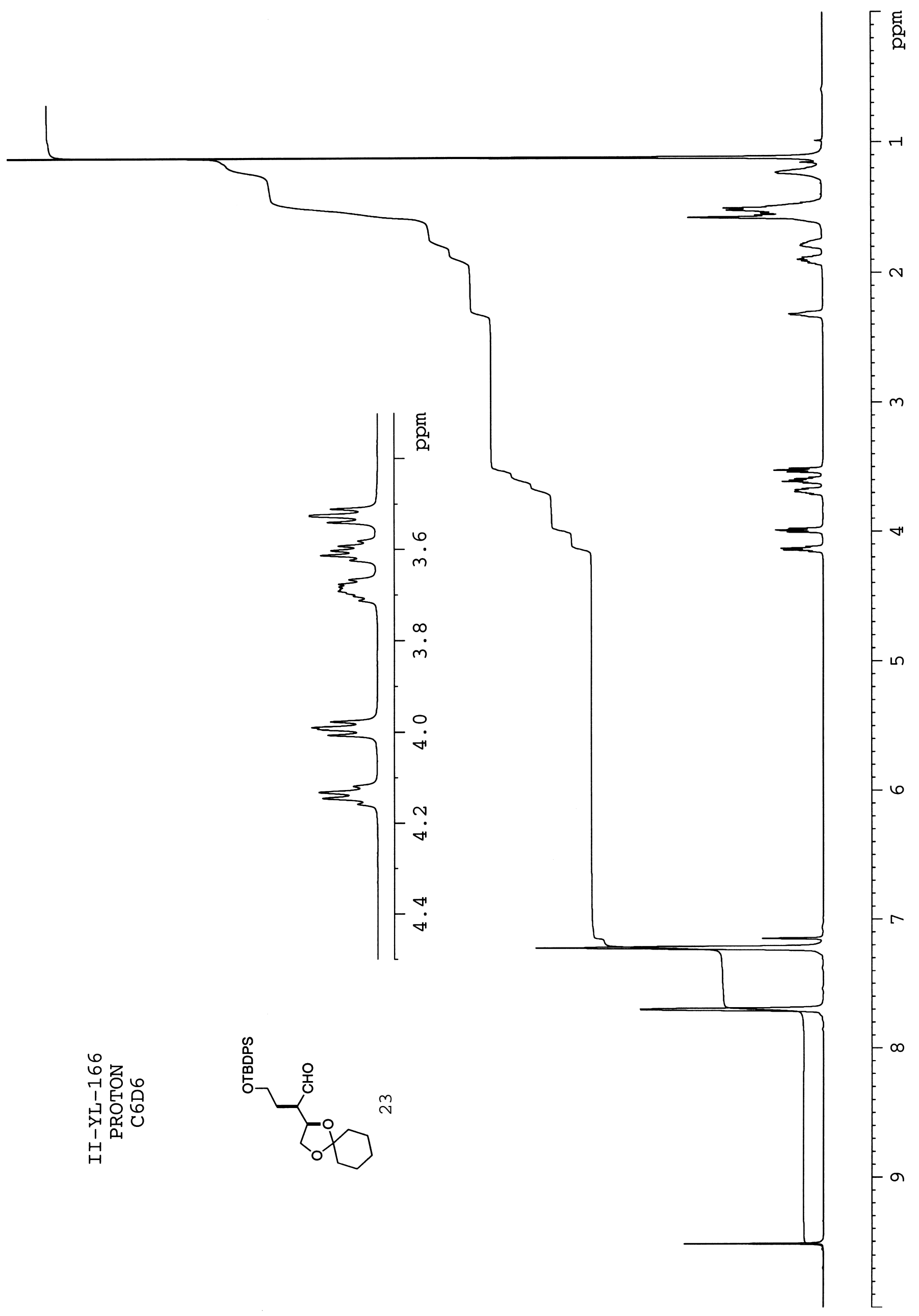



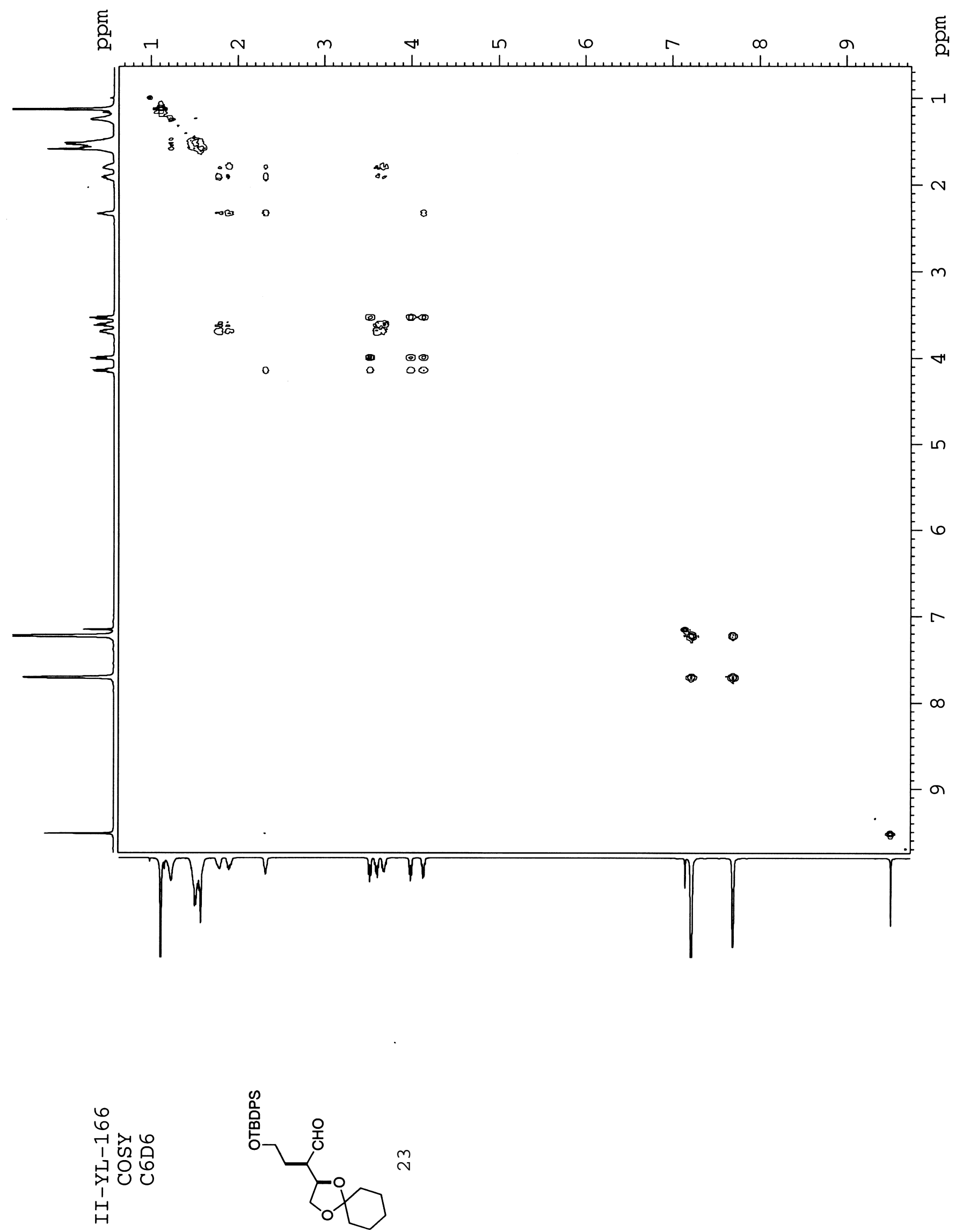

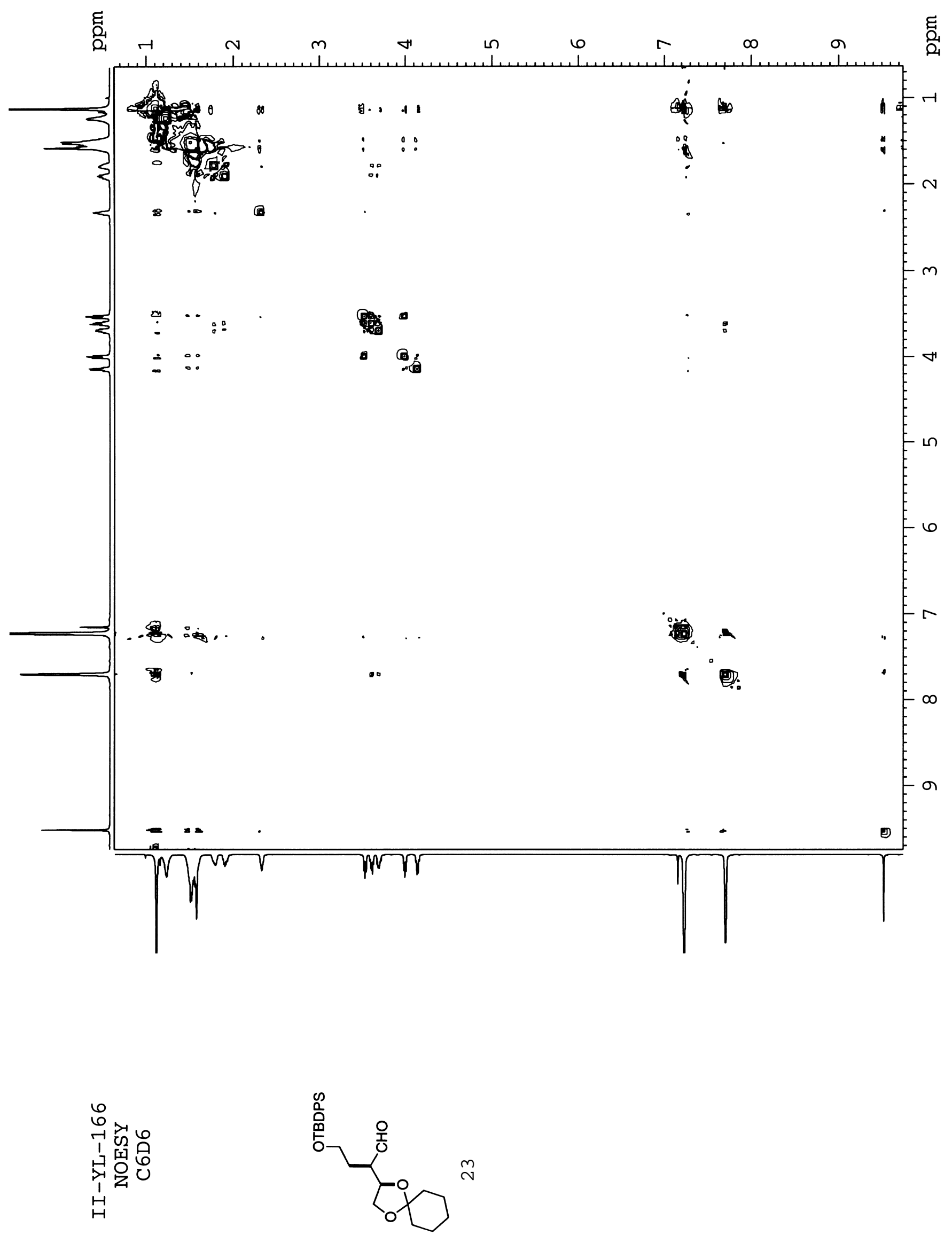


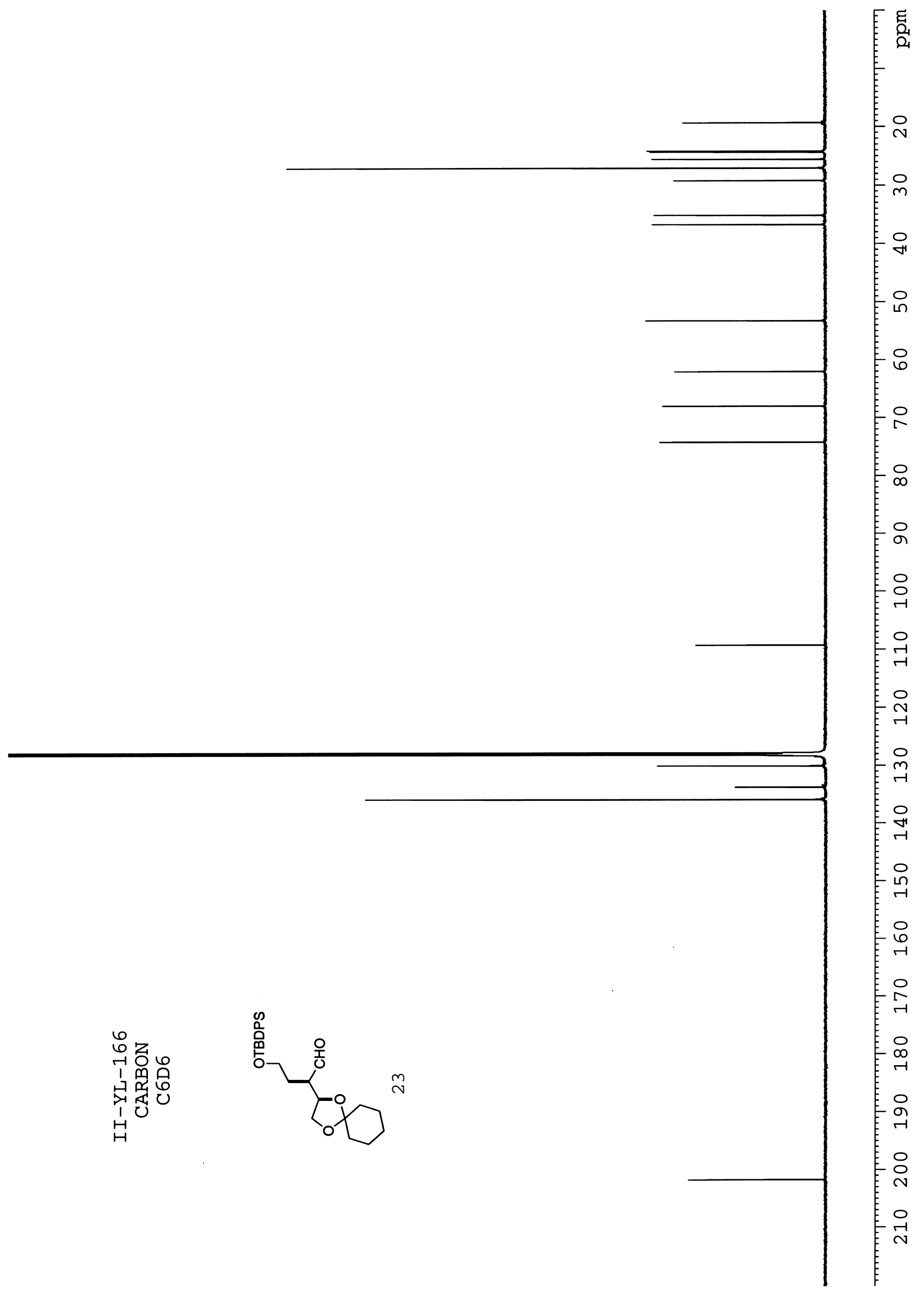




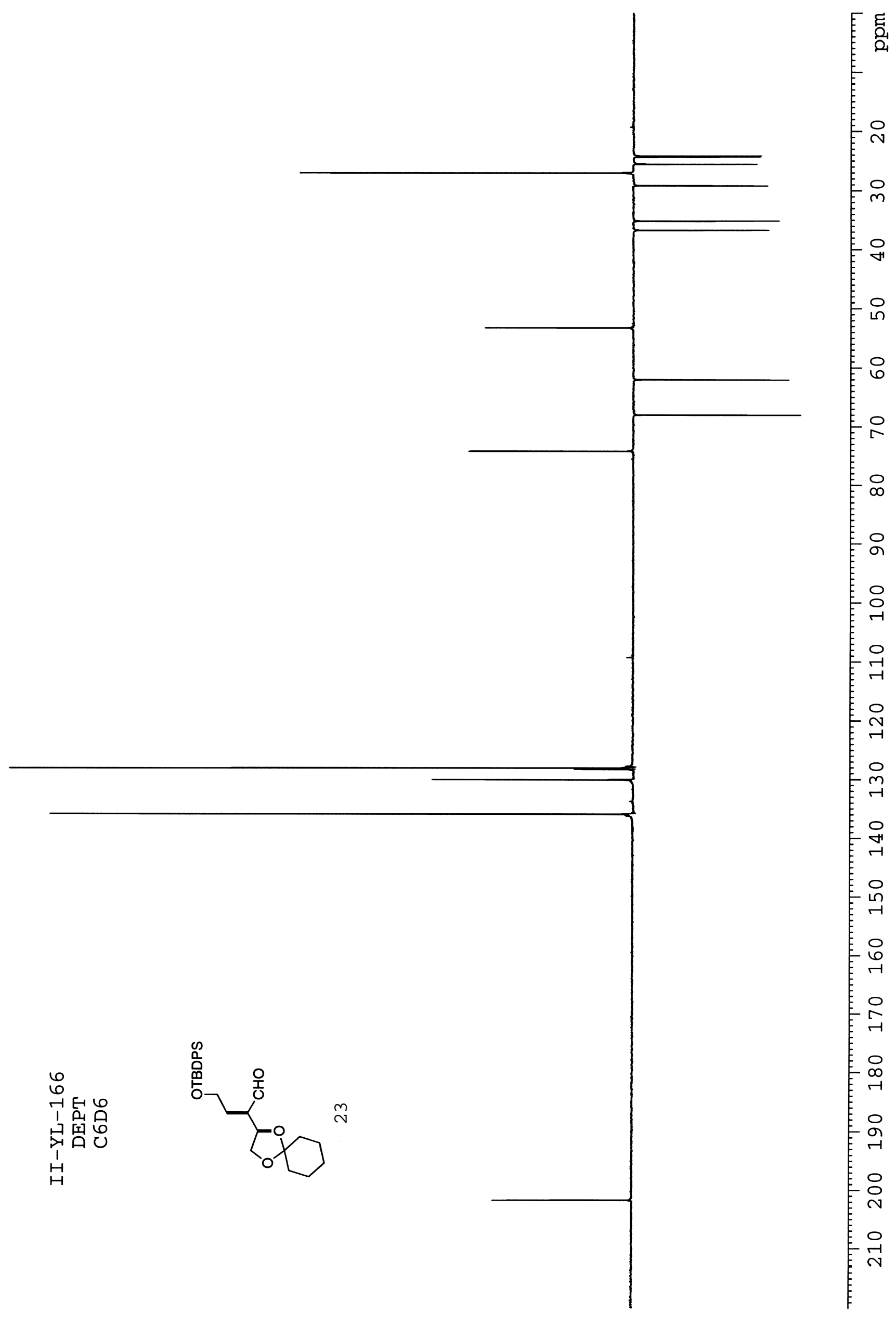

104 


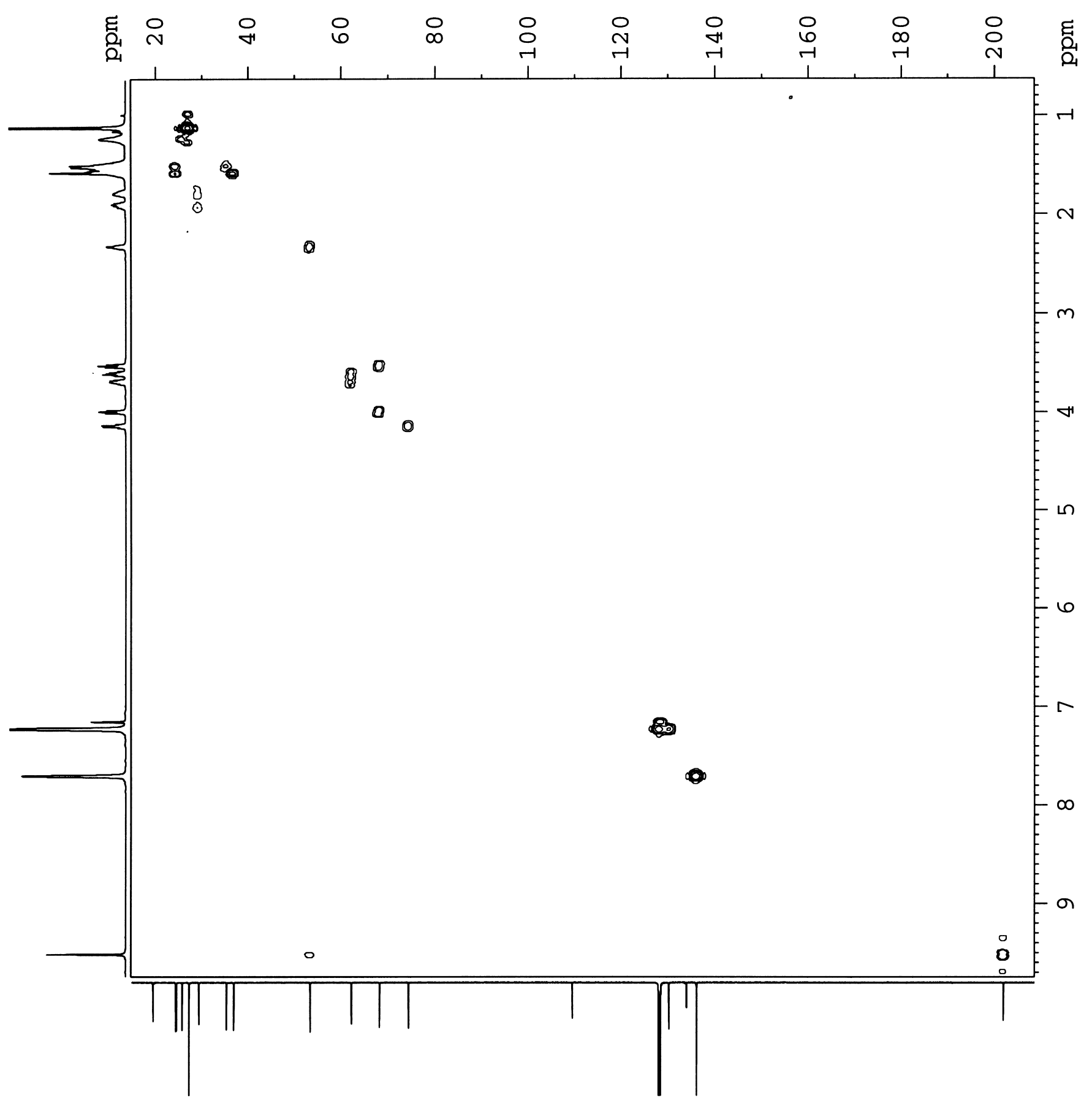

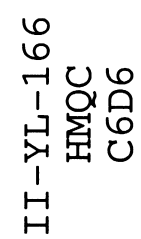

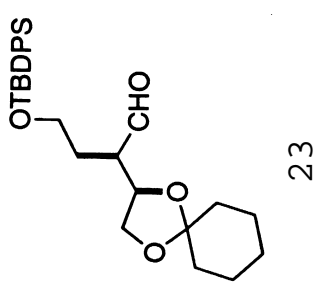




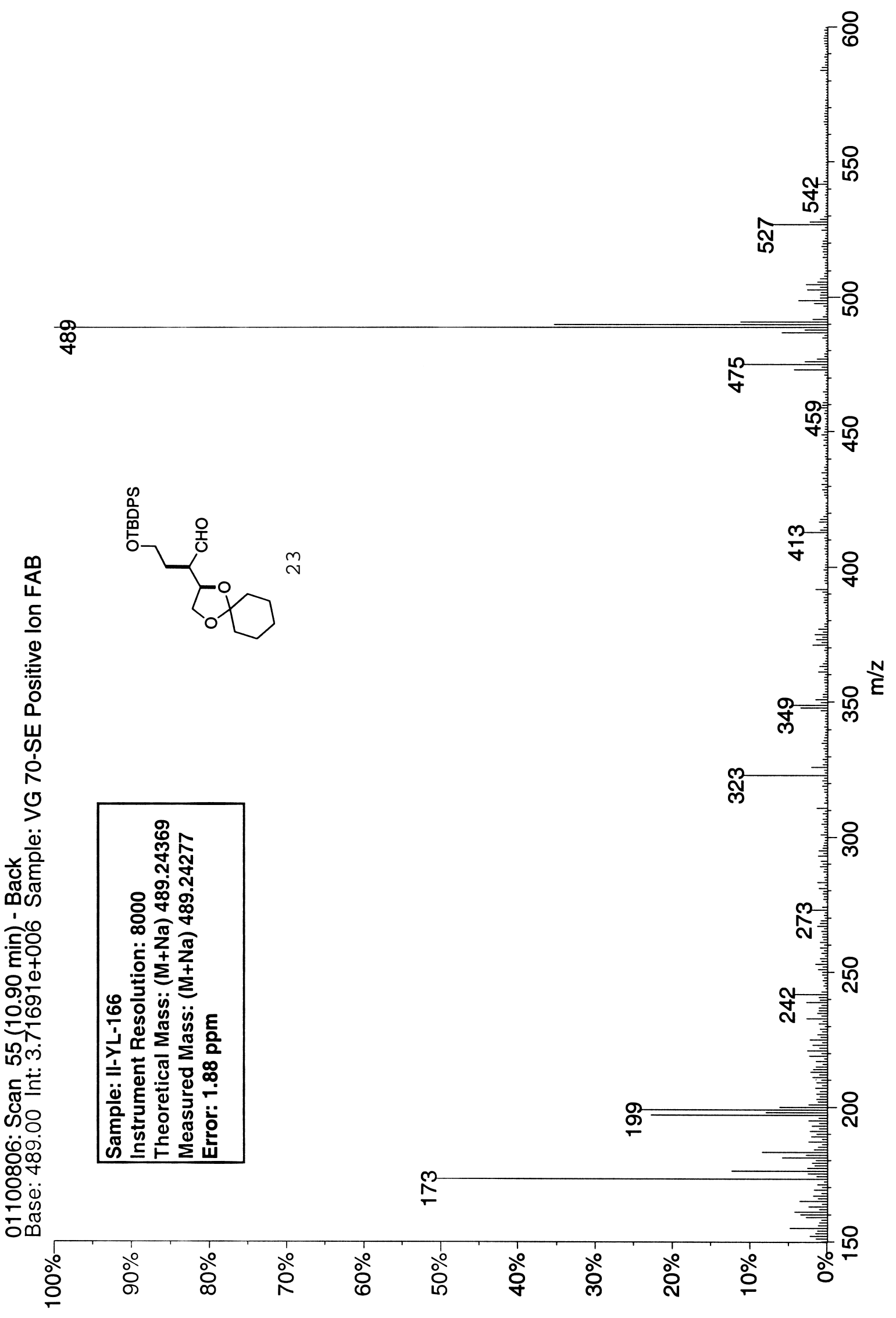




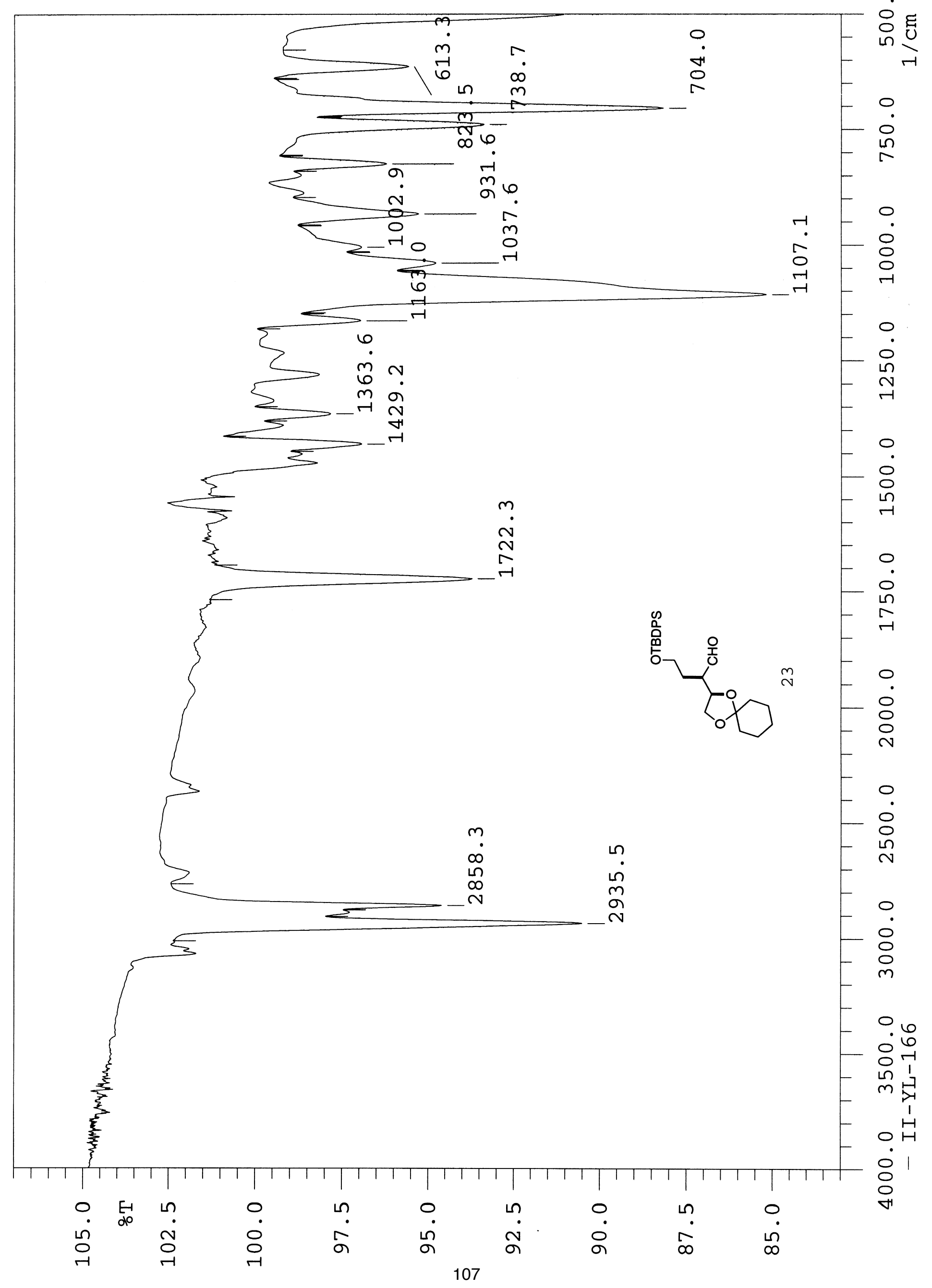




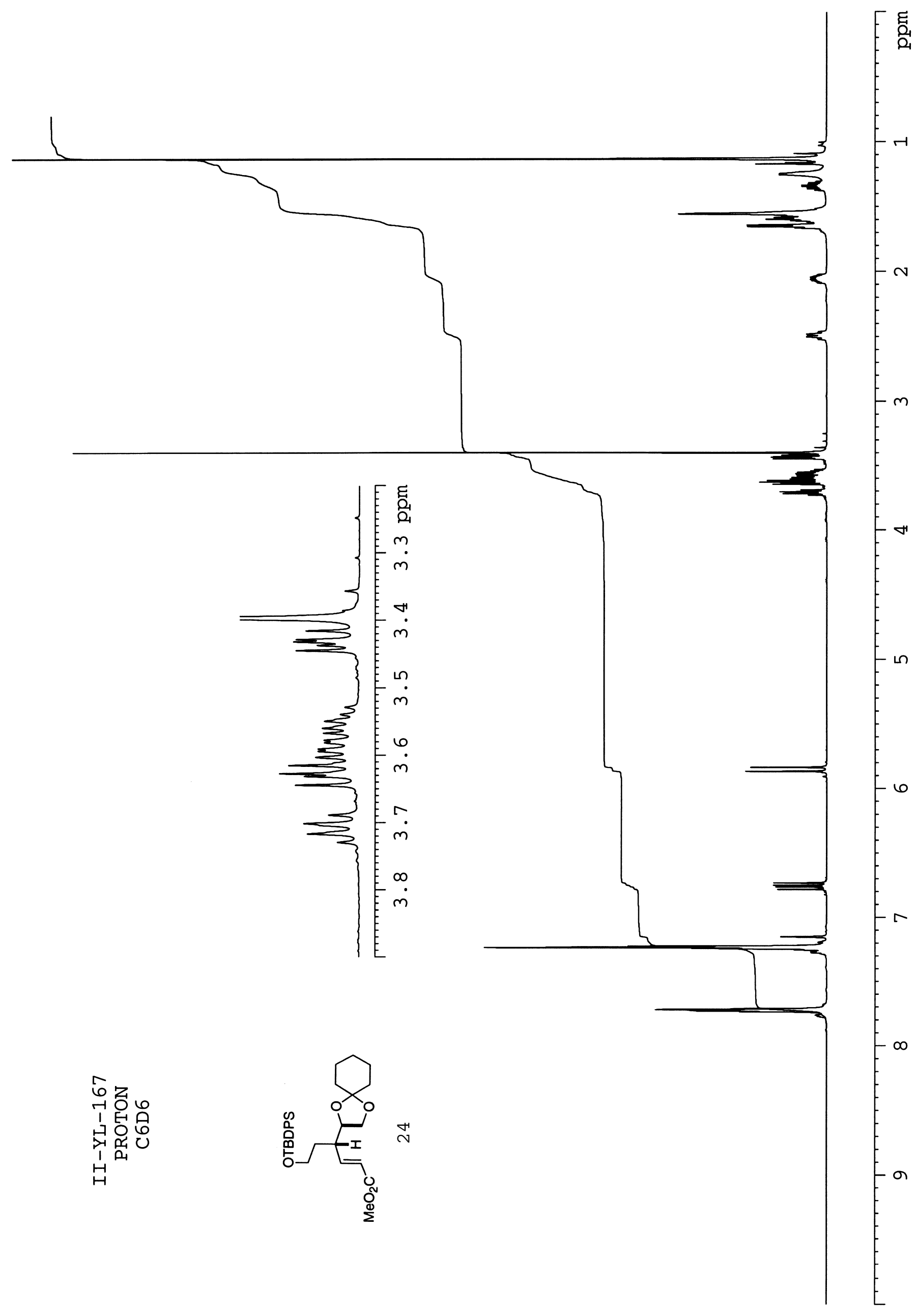




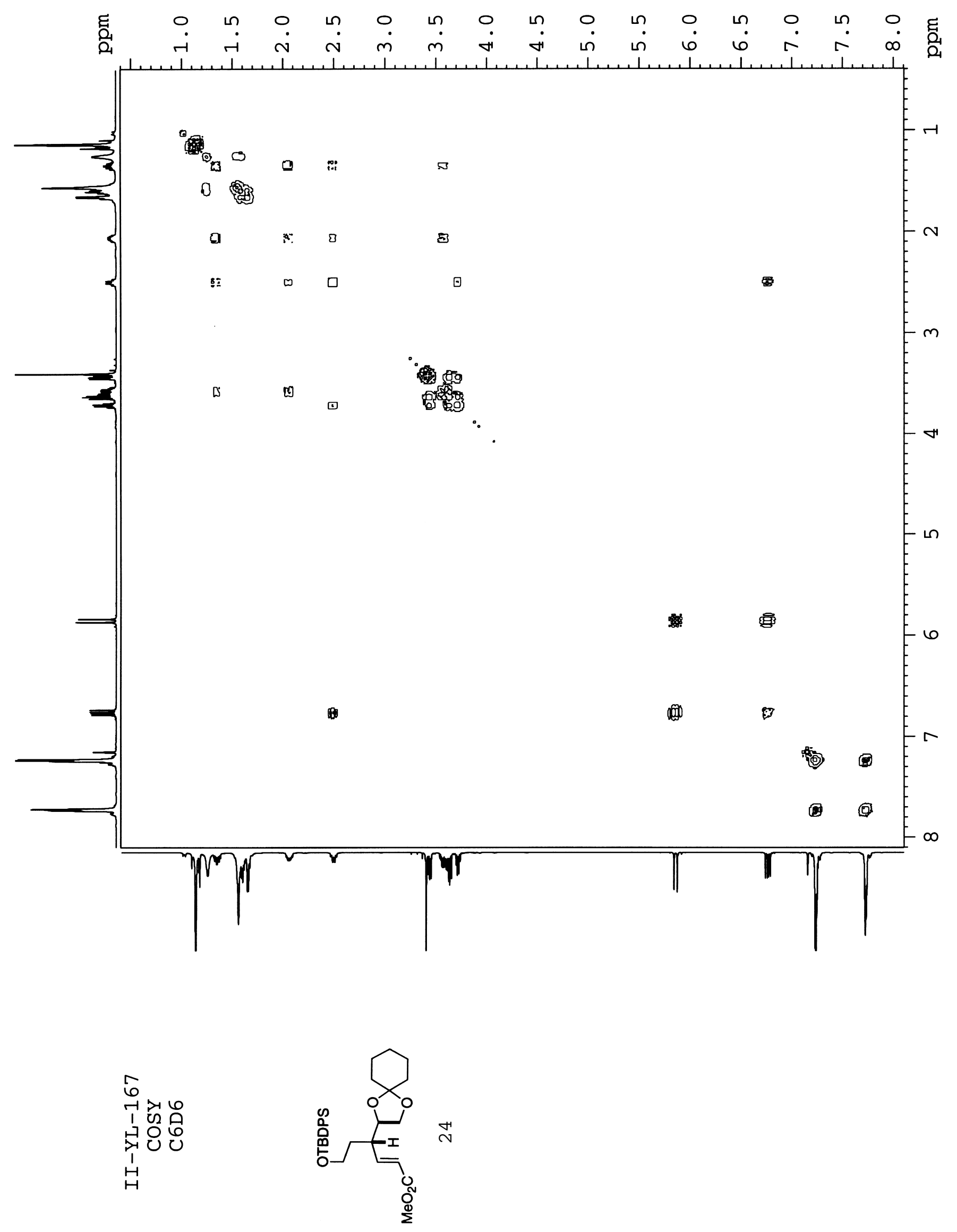



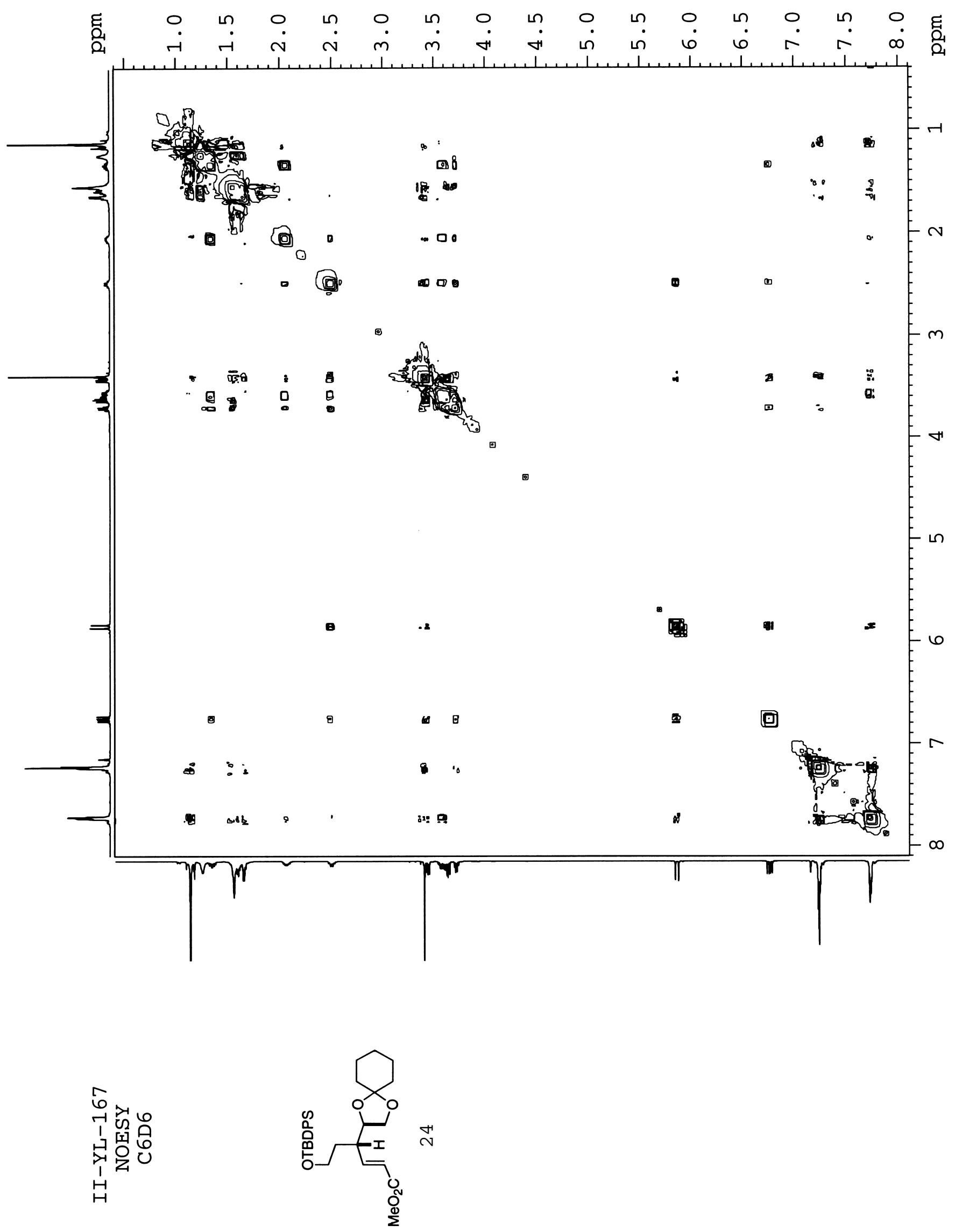


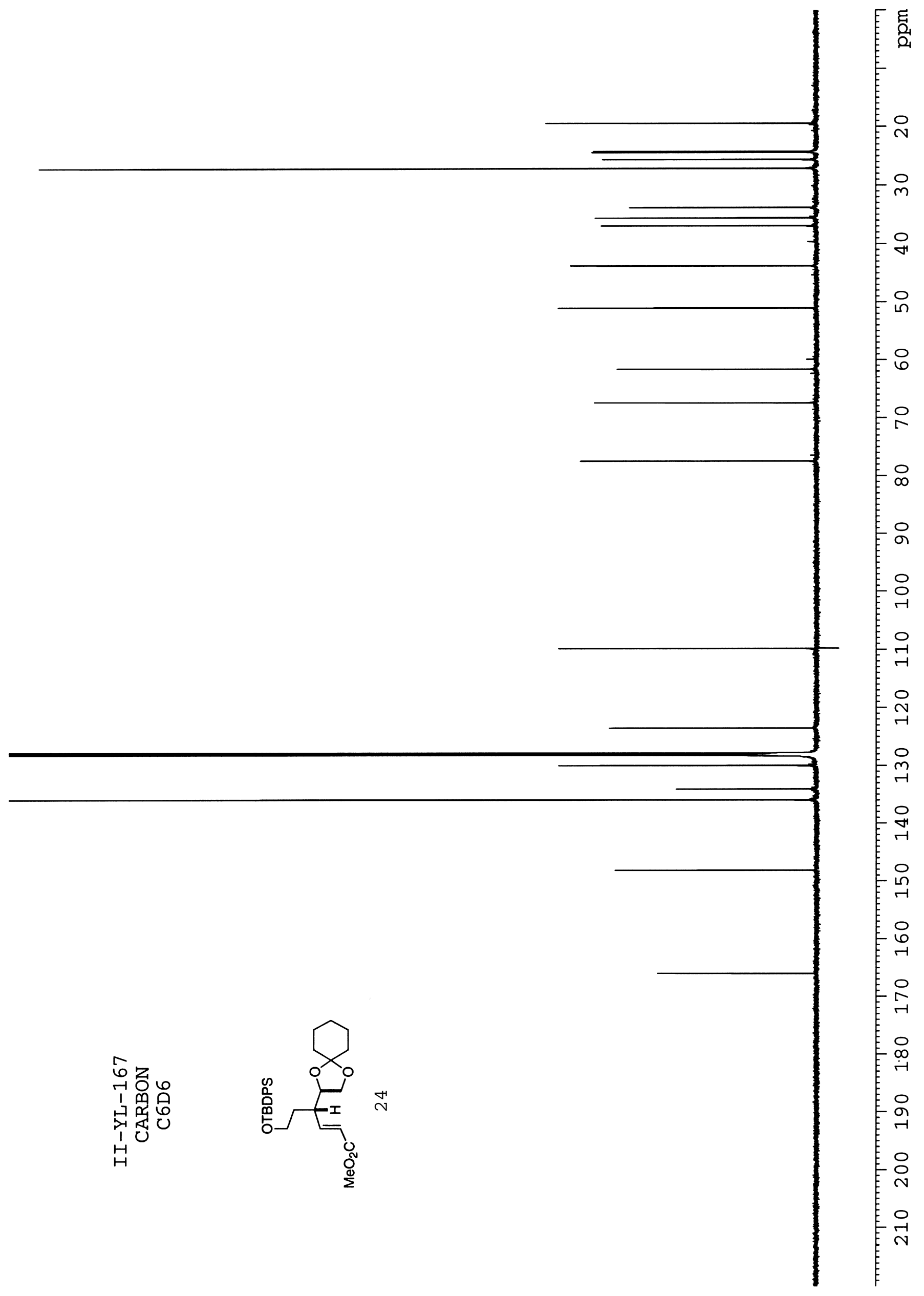




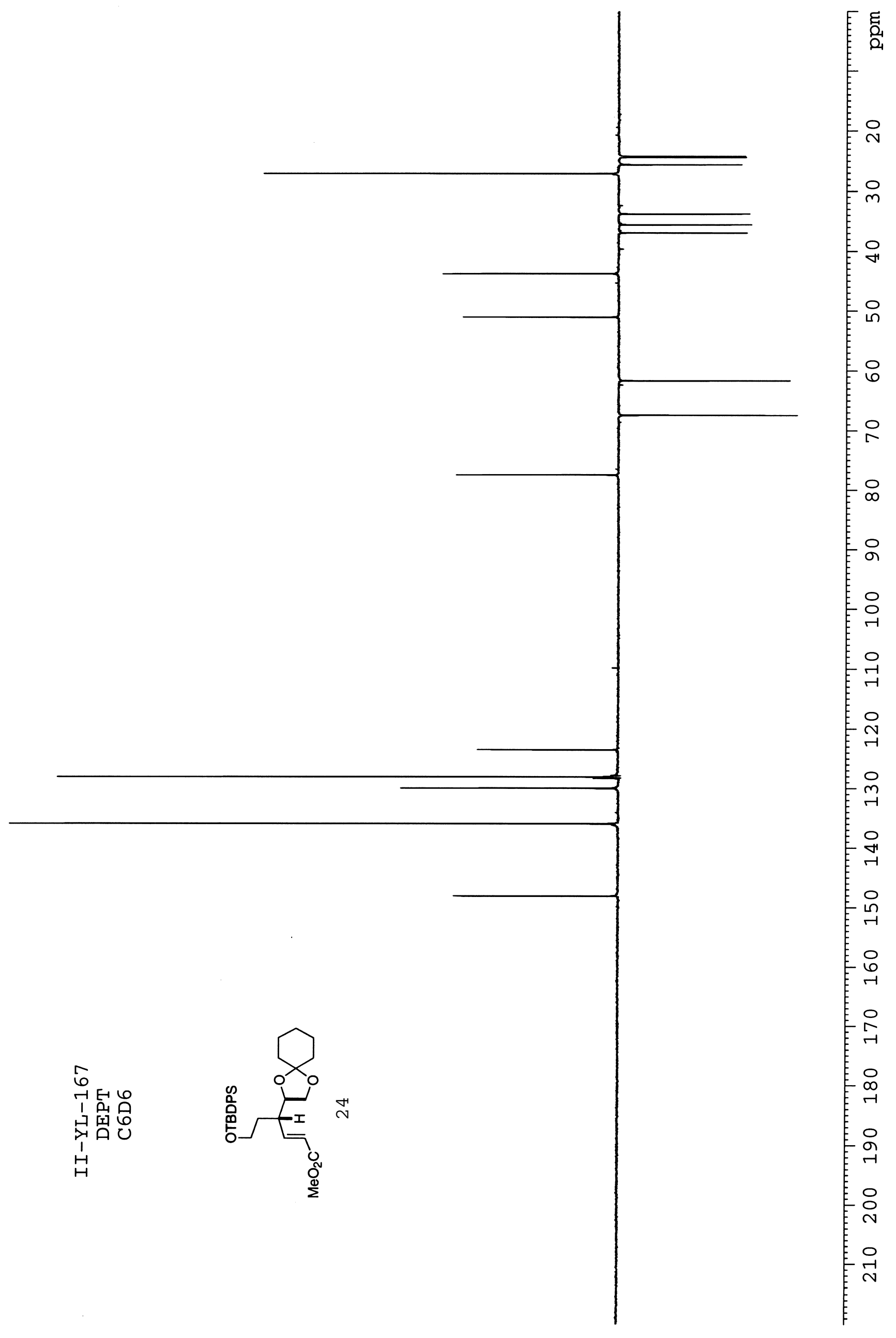




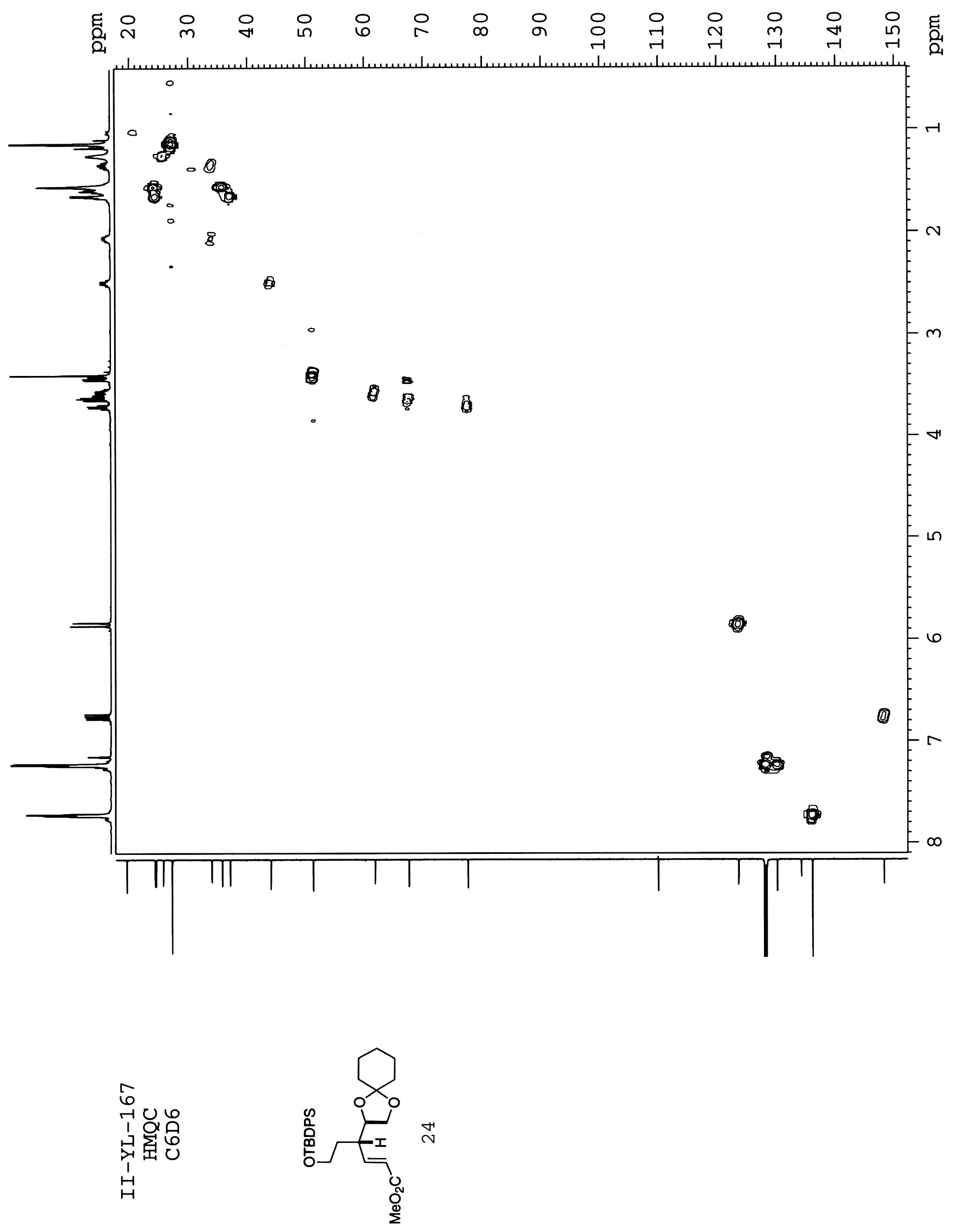




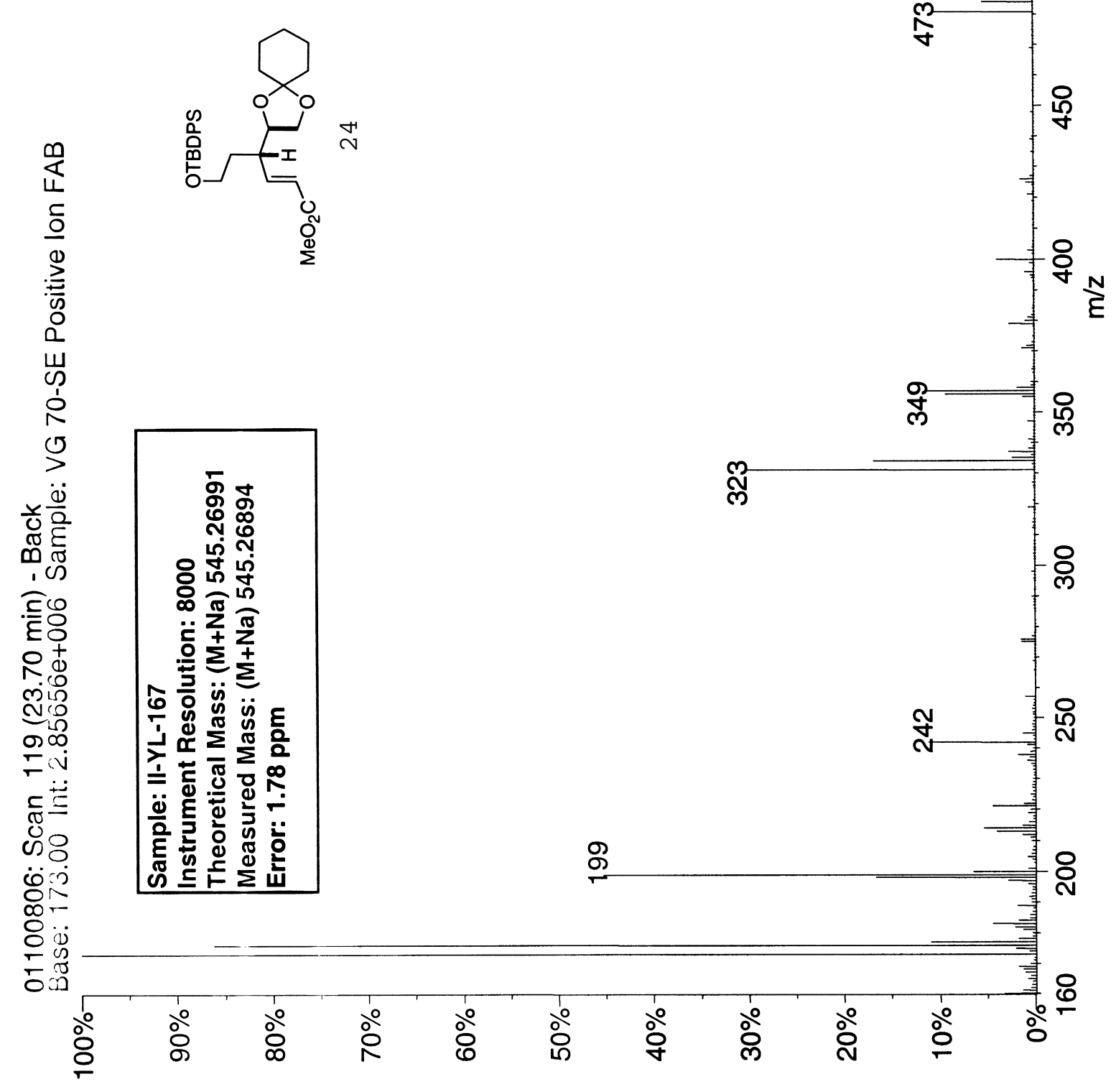




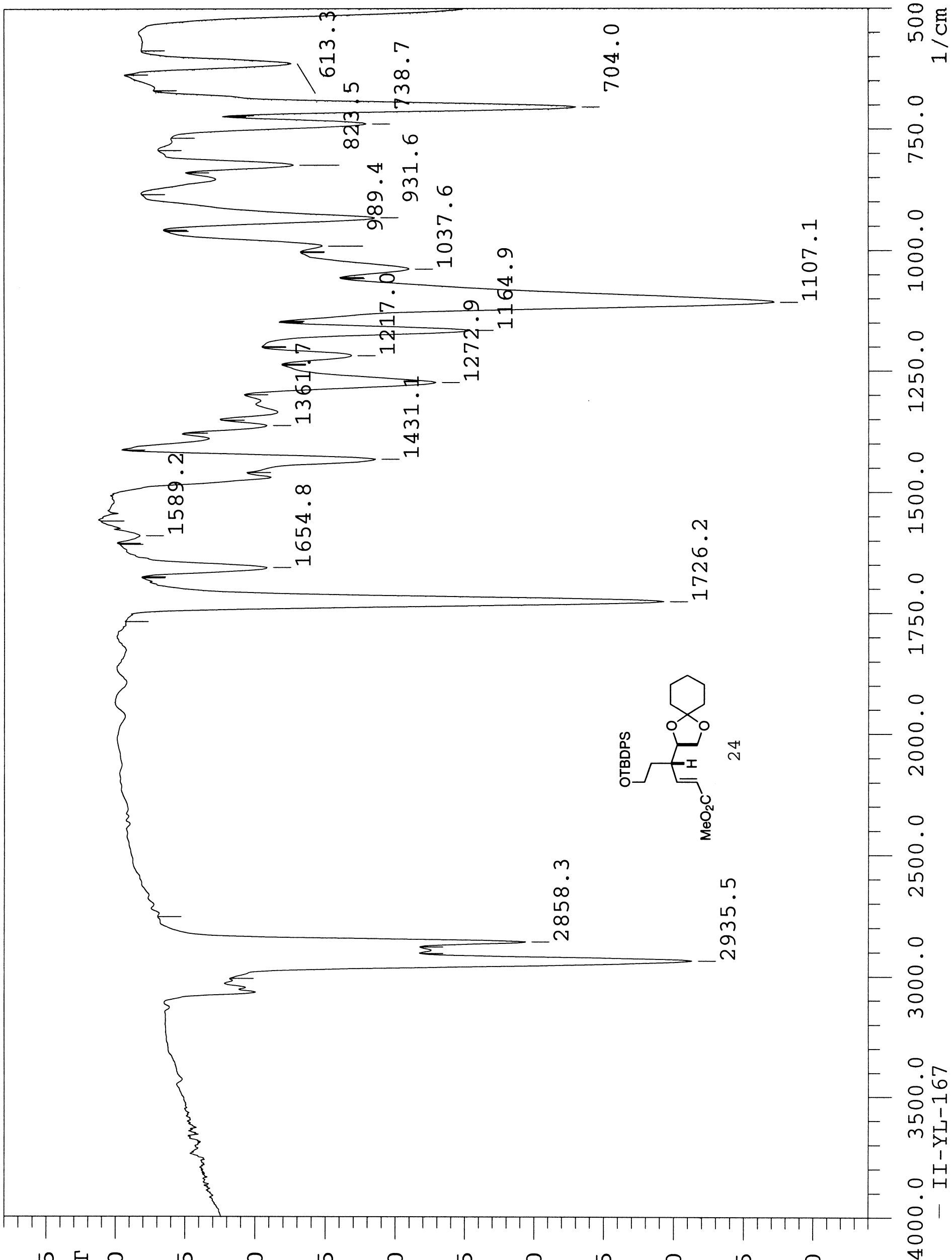

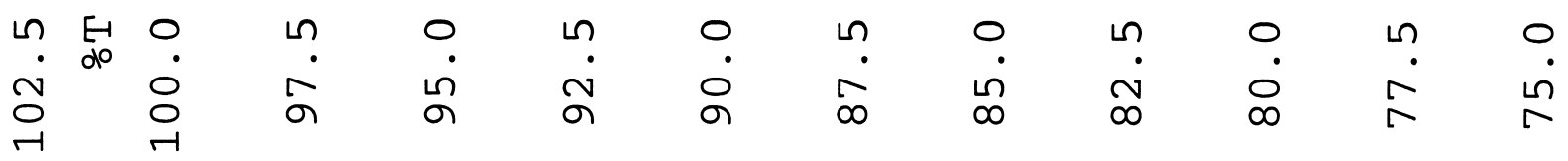




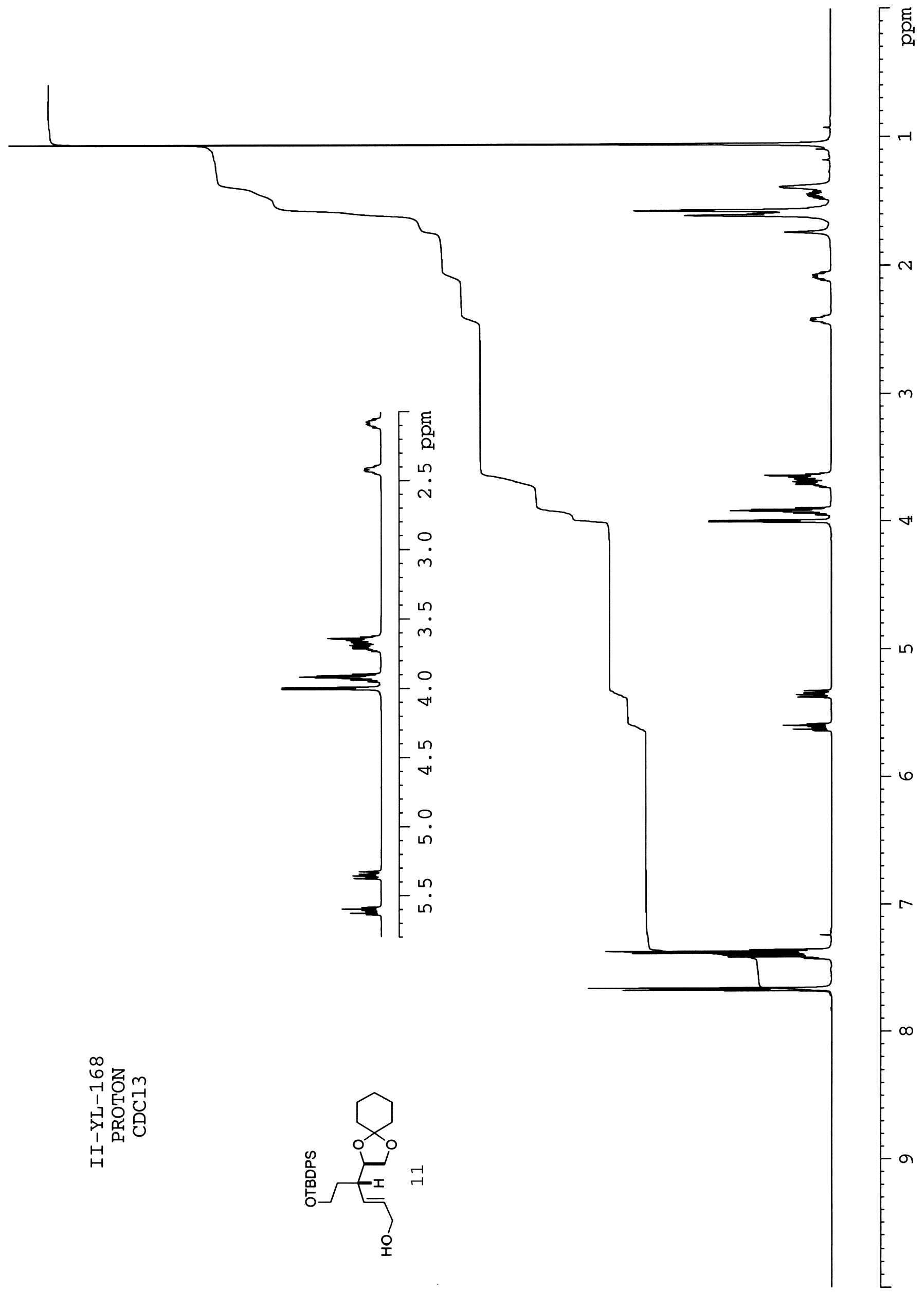




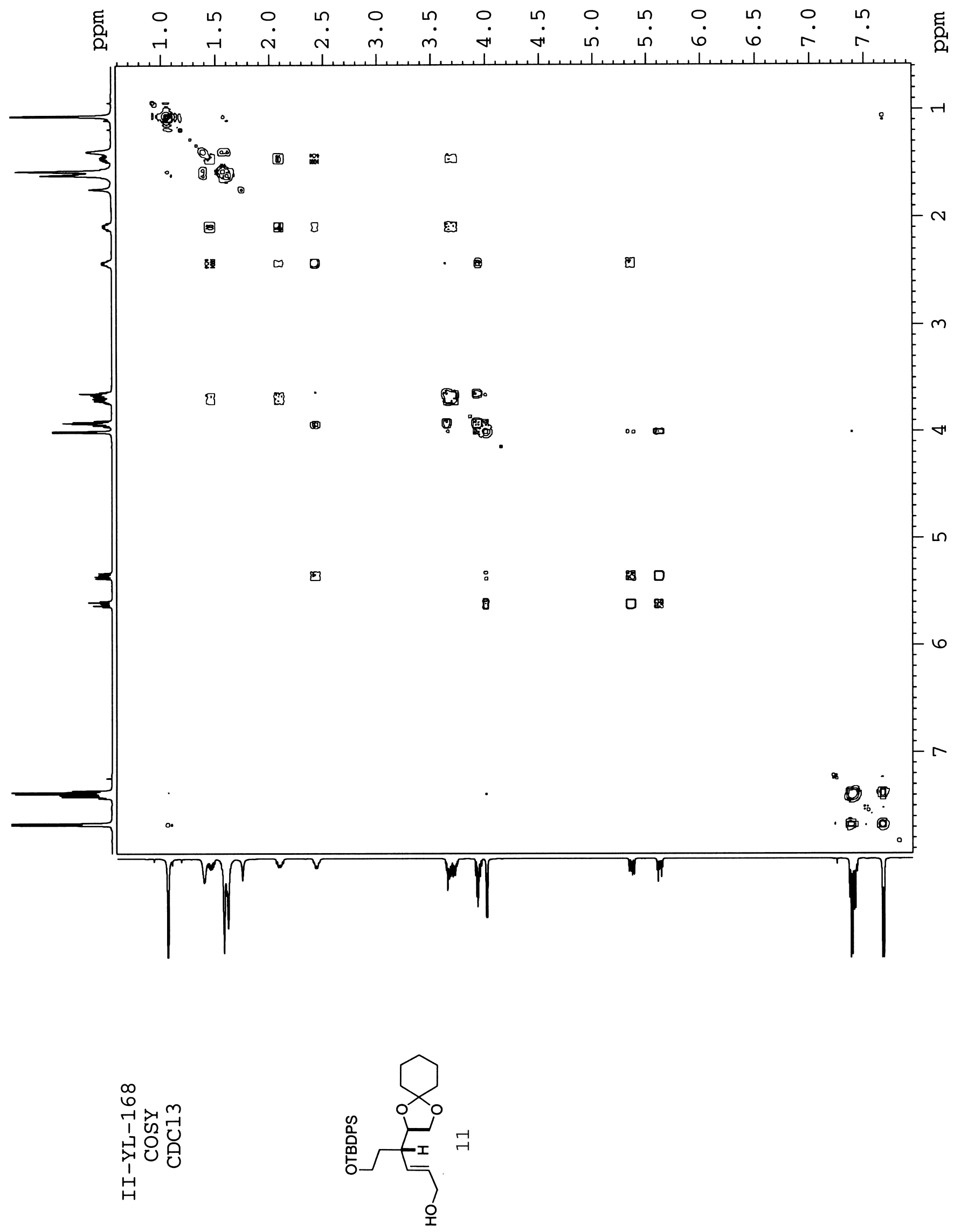



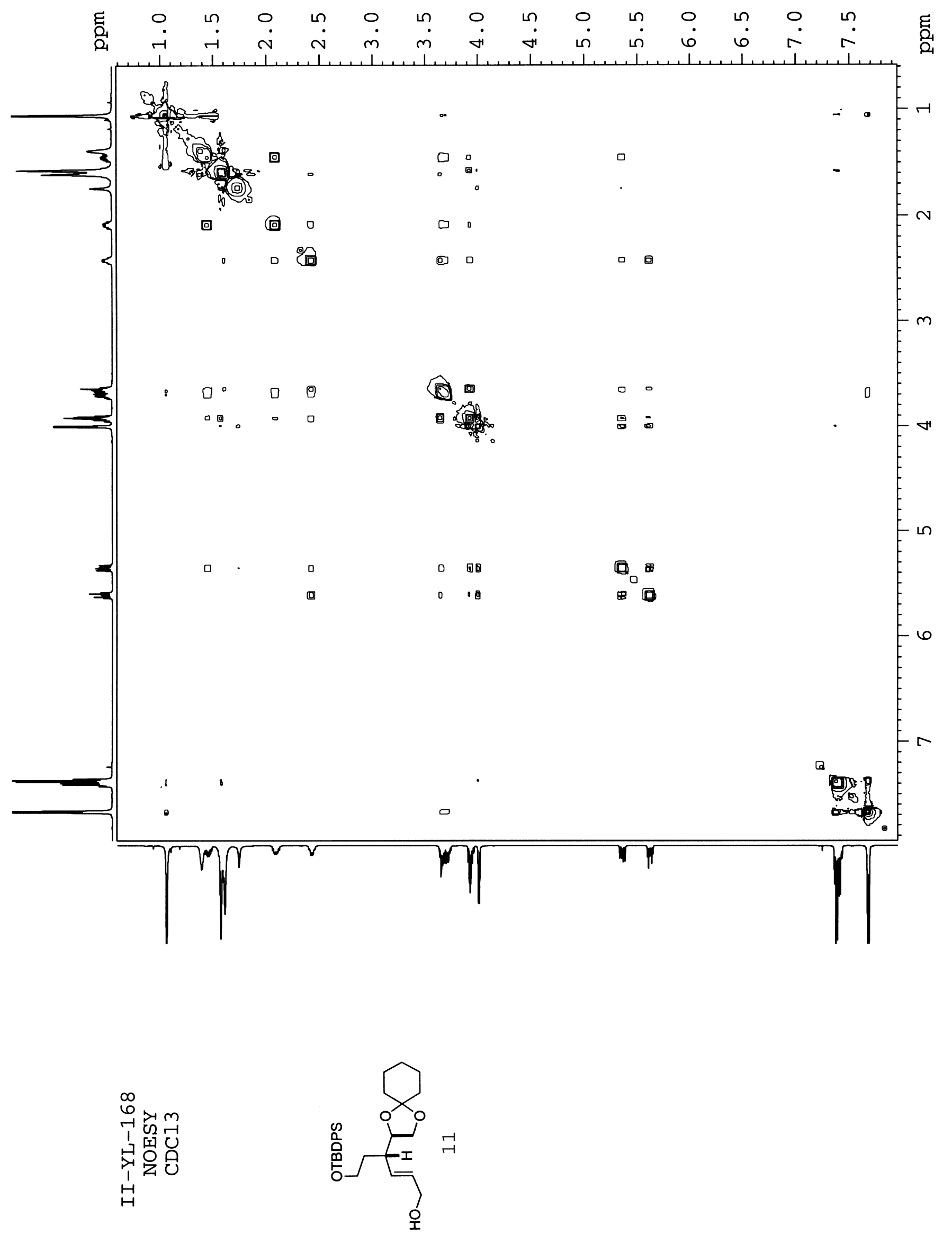


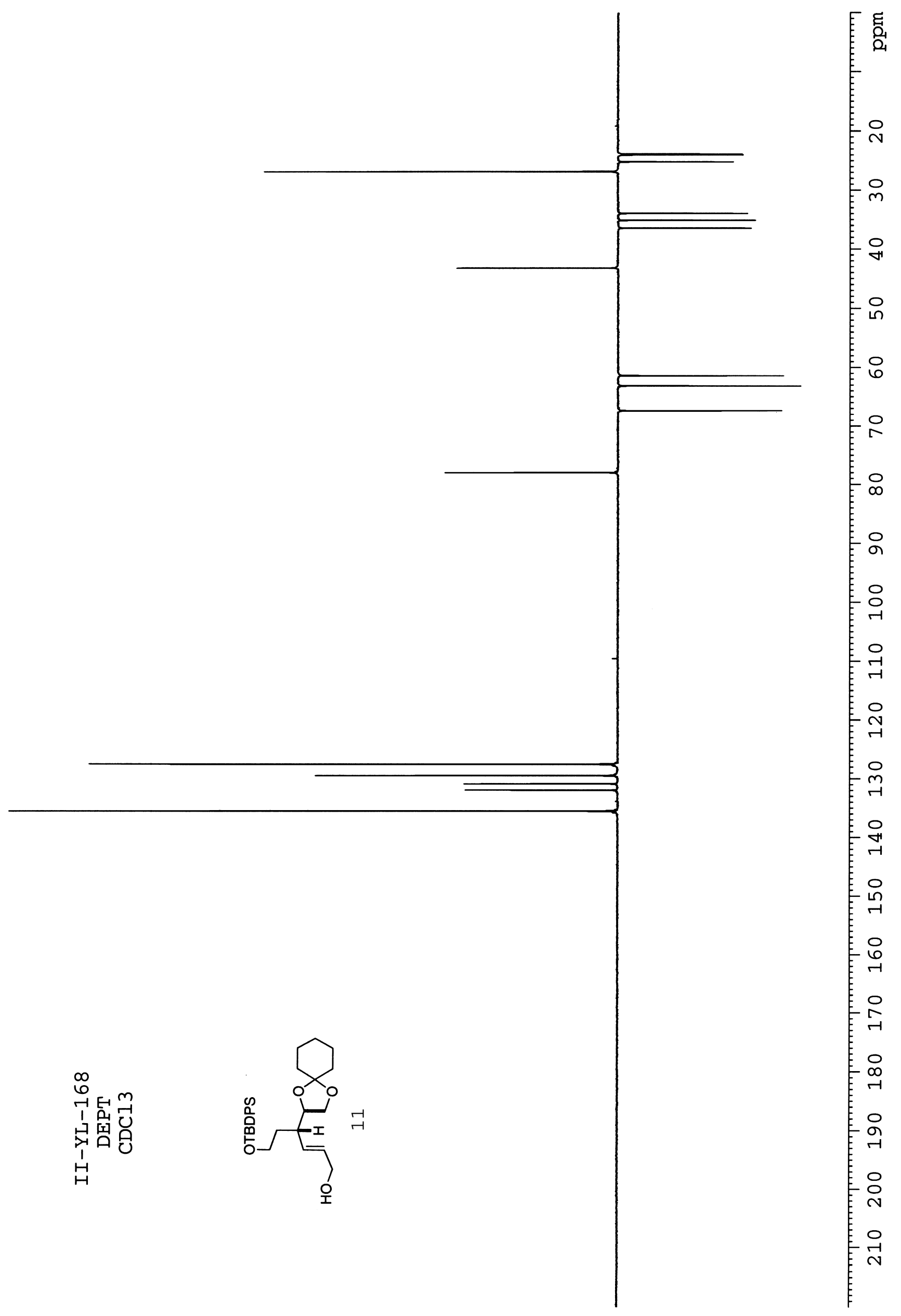




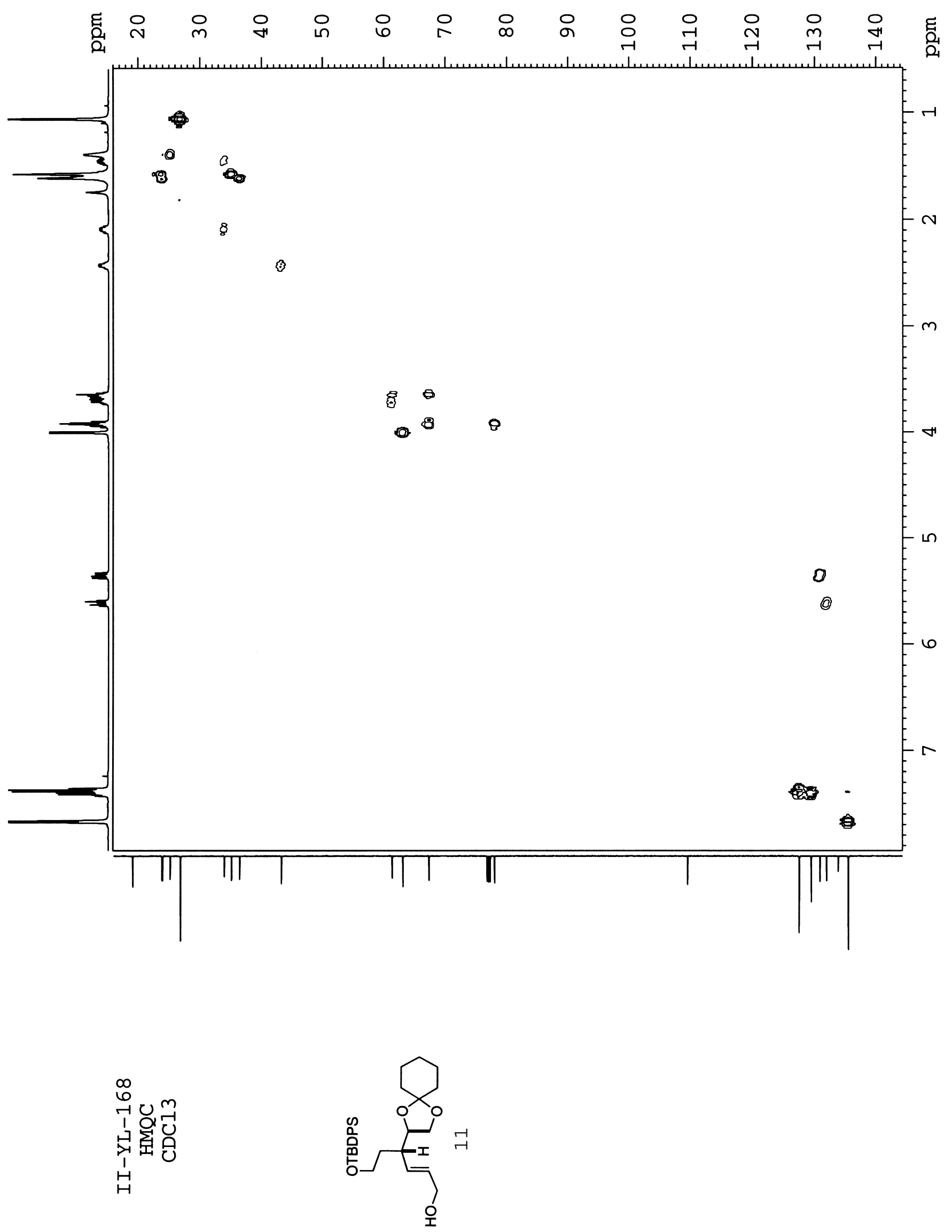




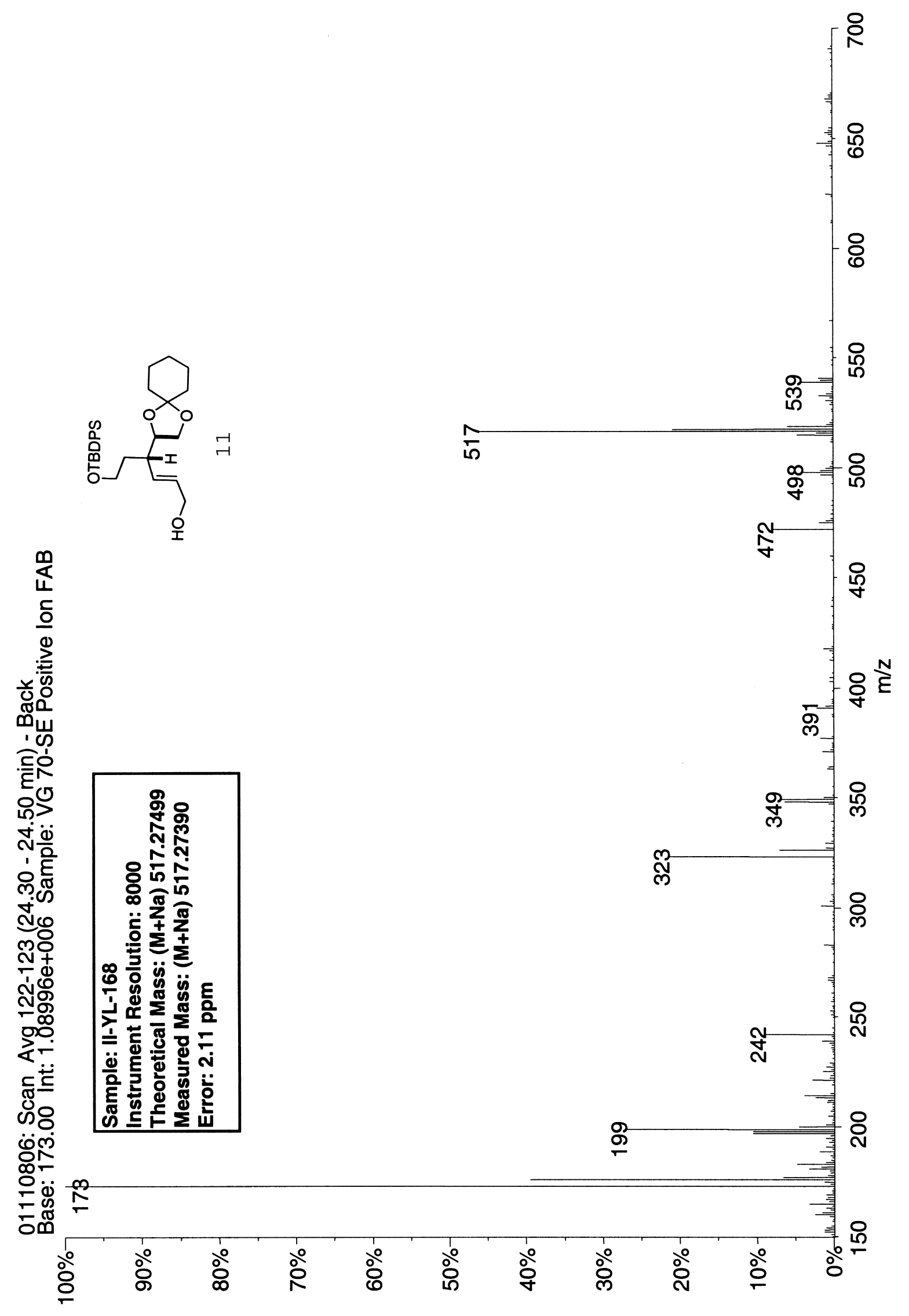




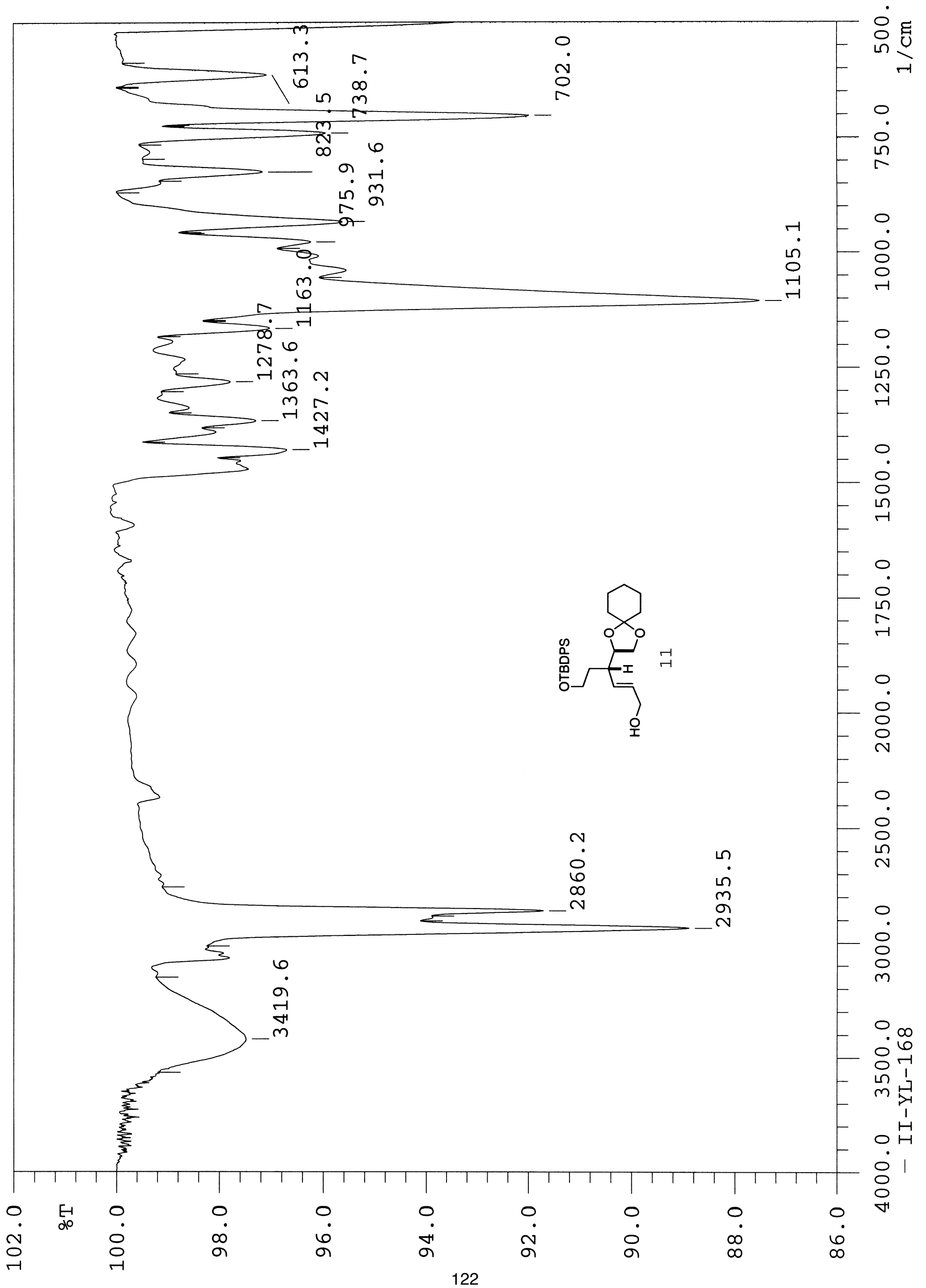




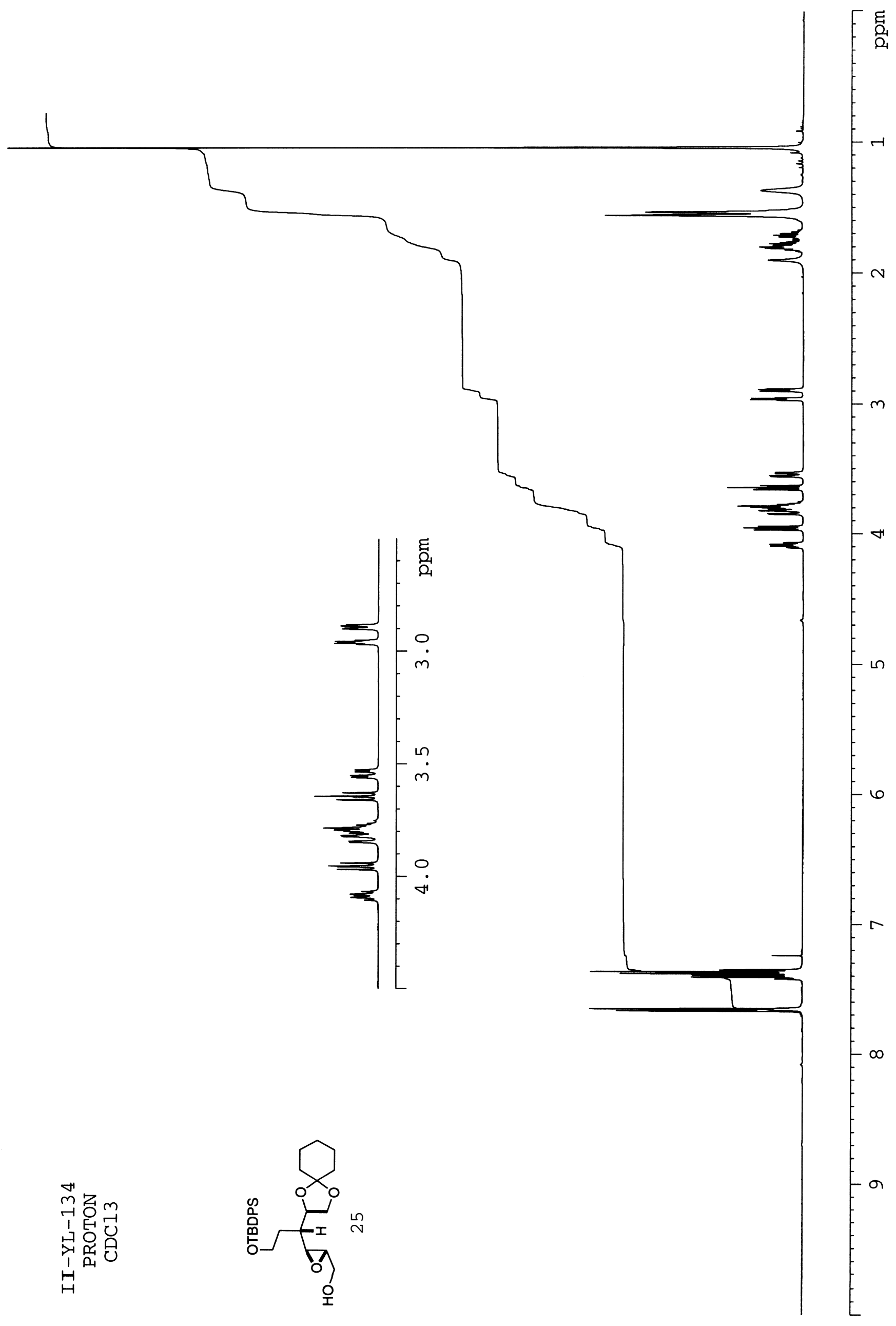



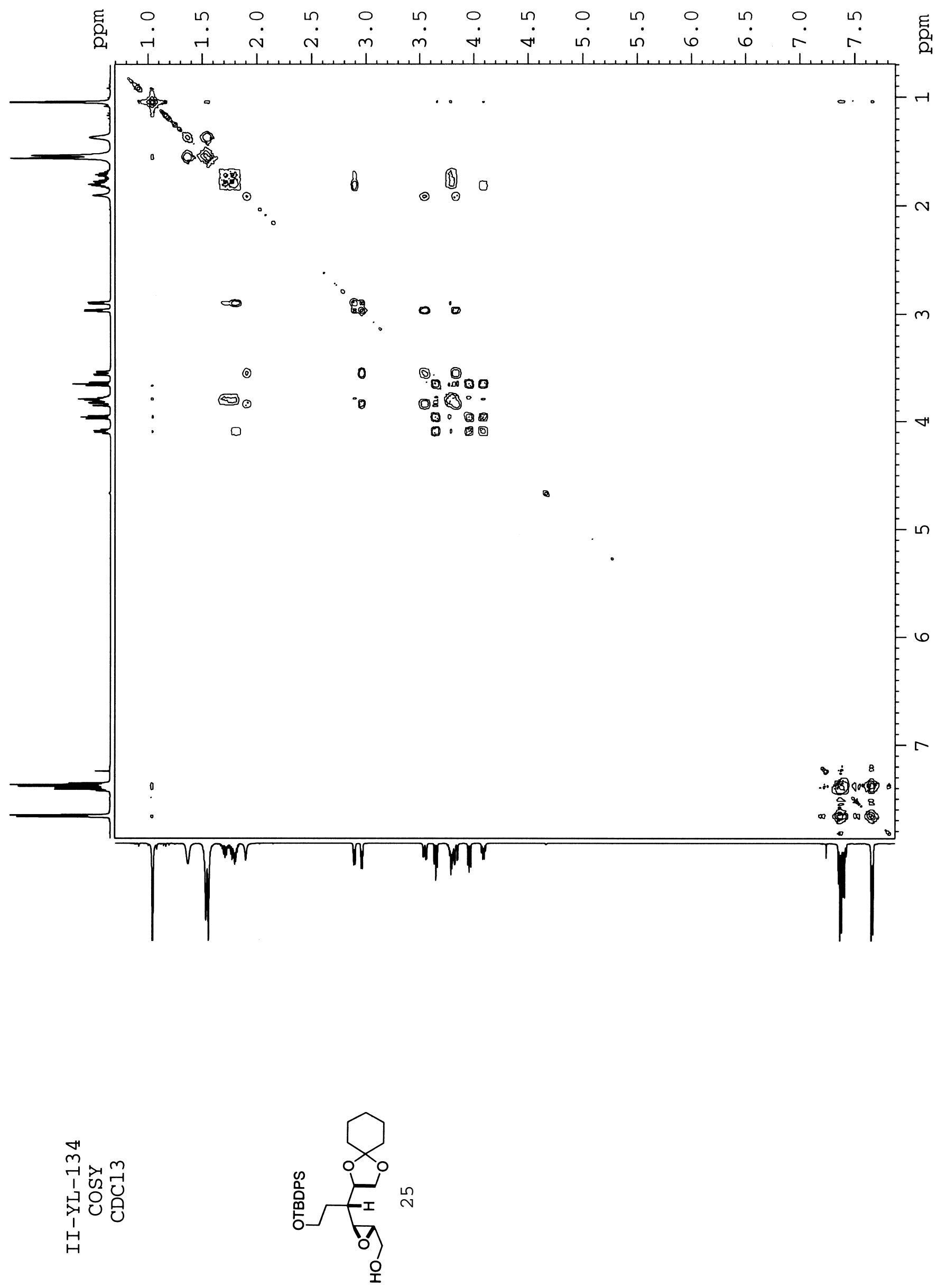

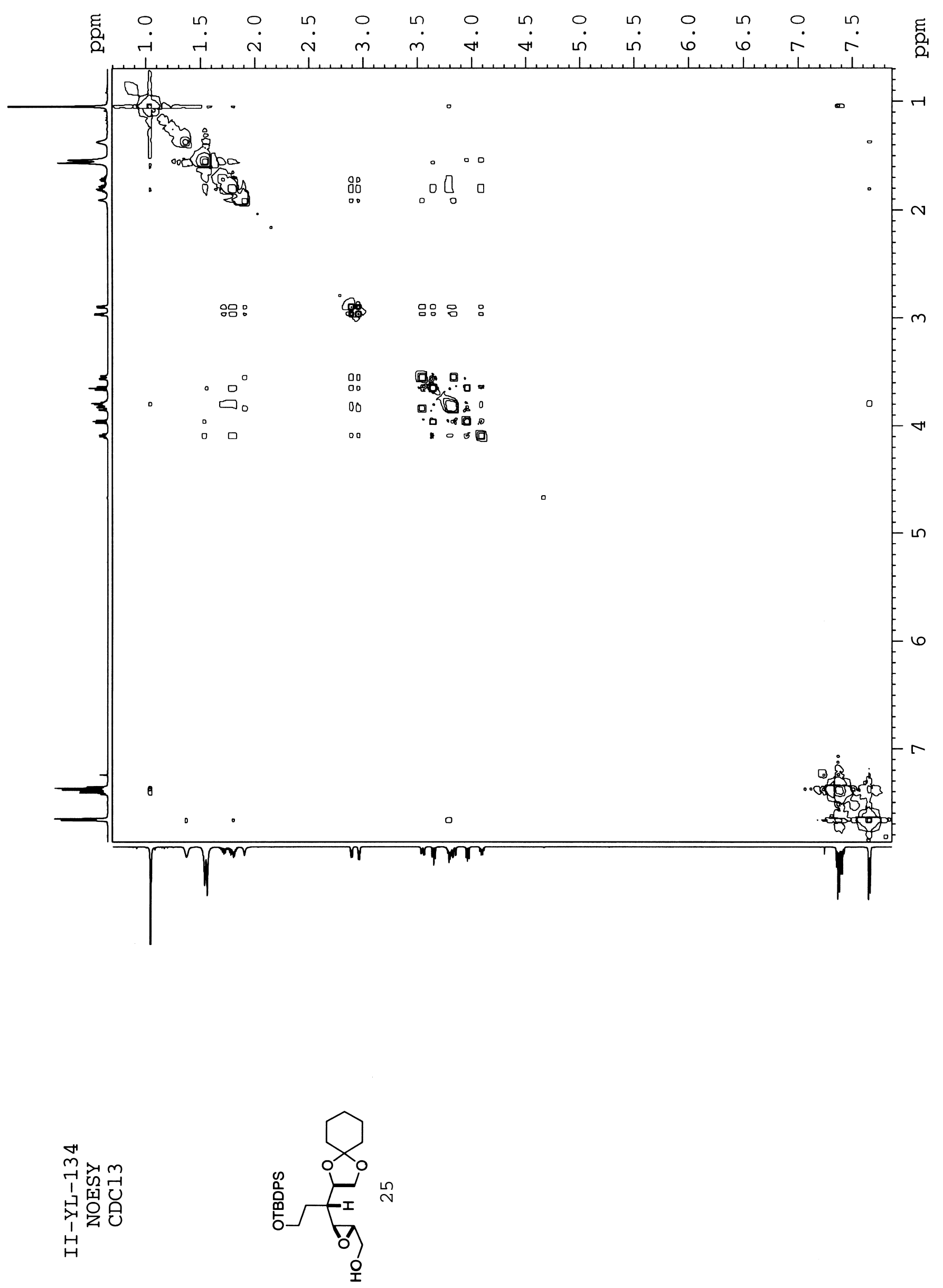


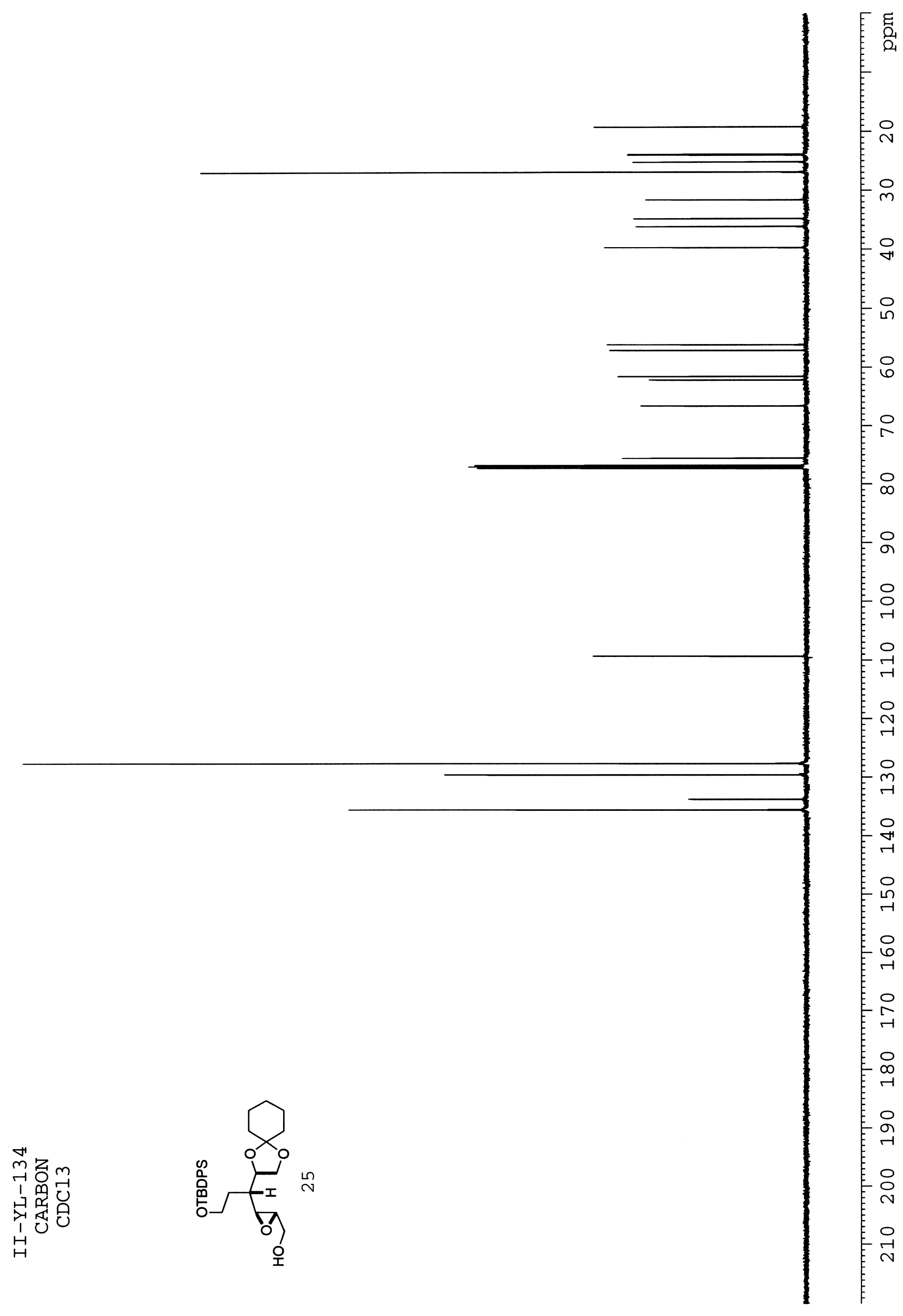




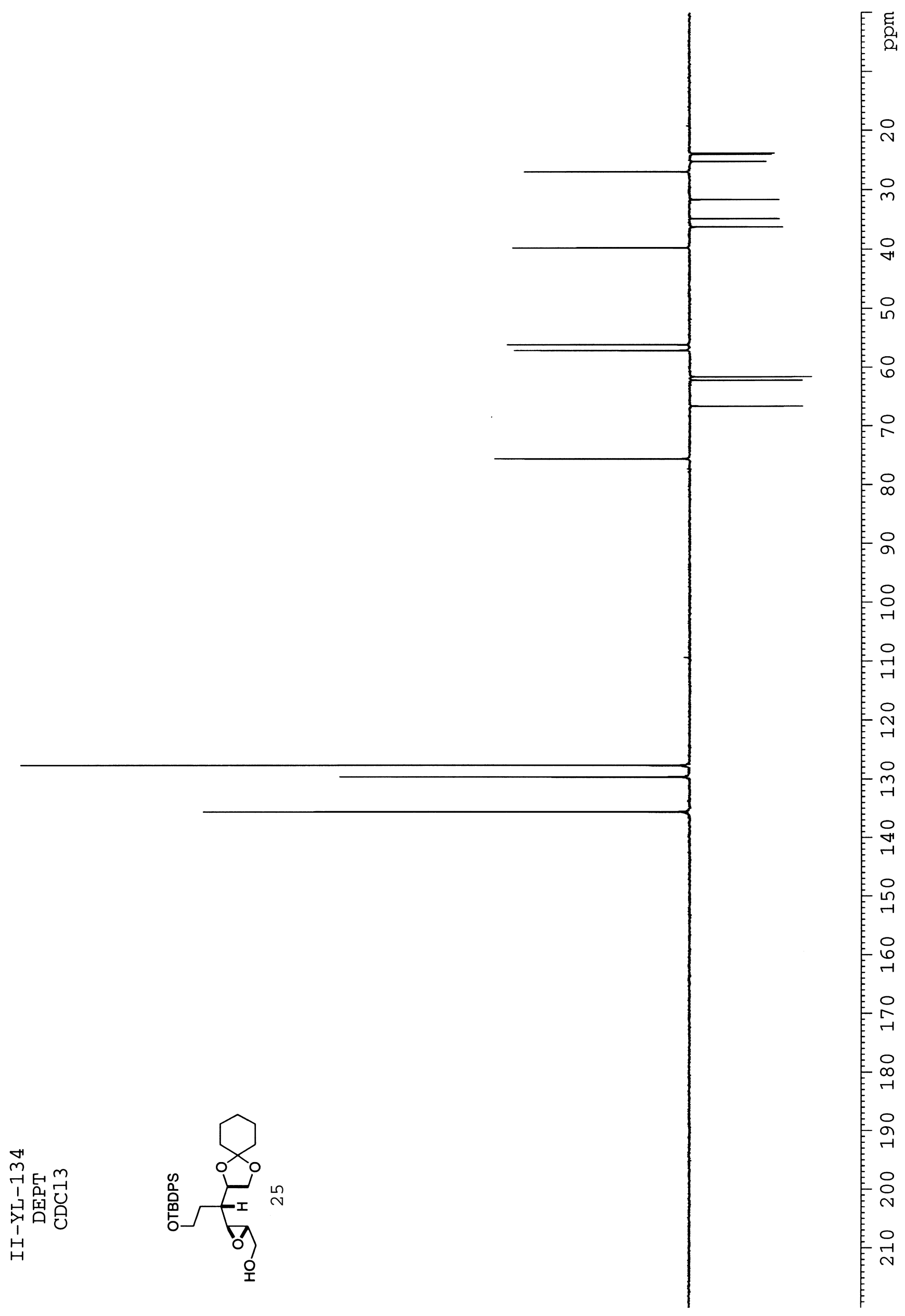




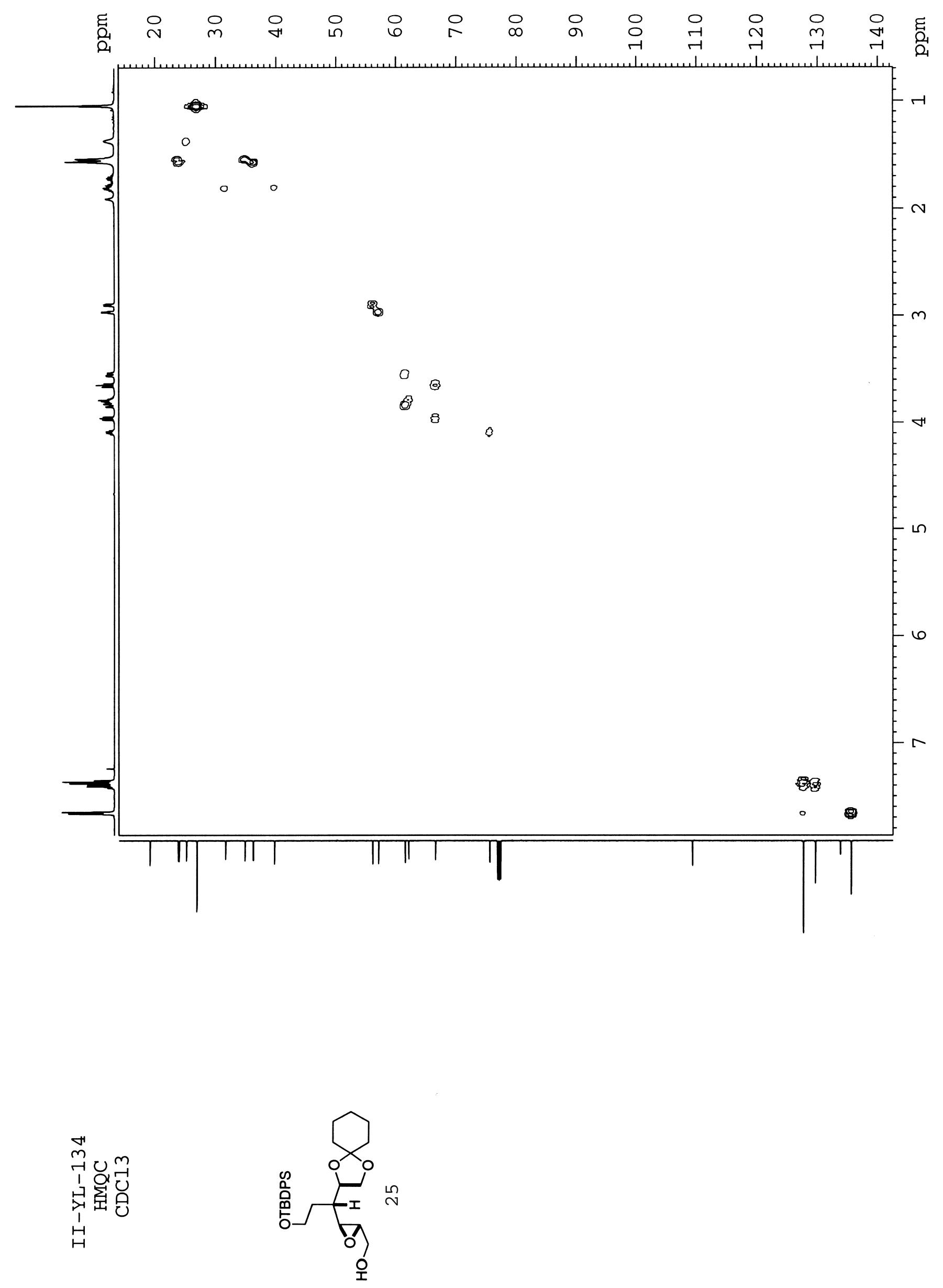




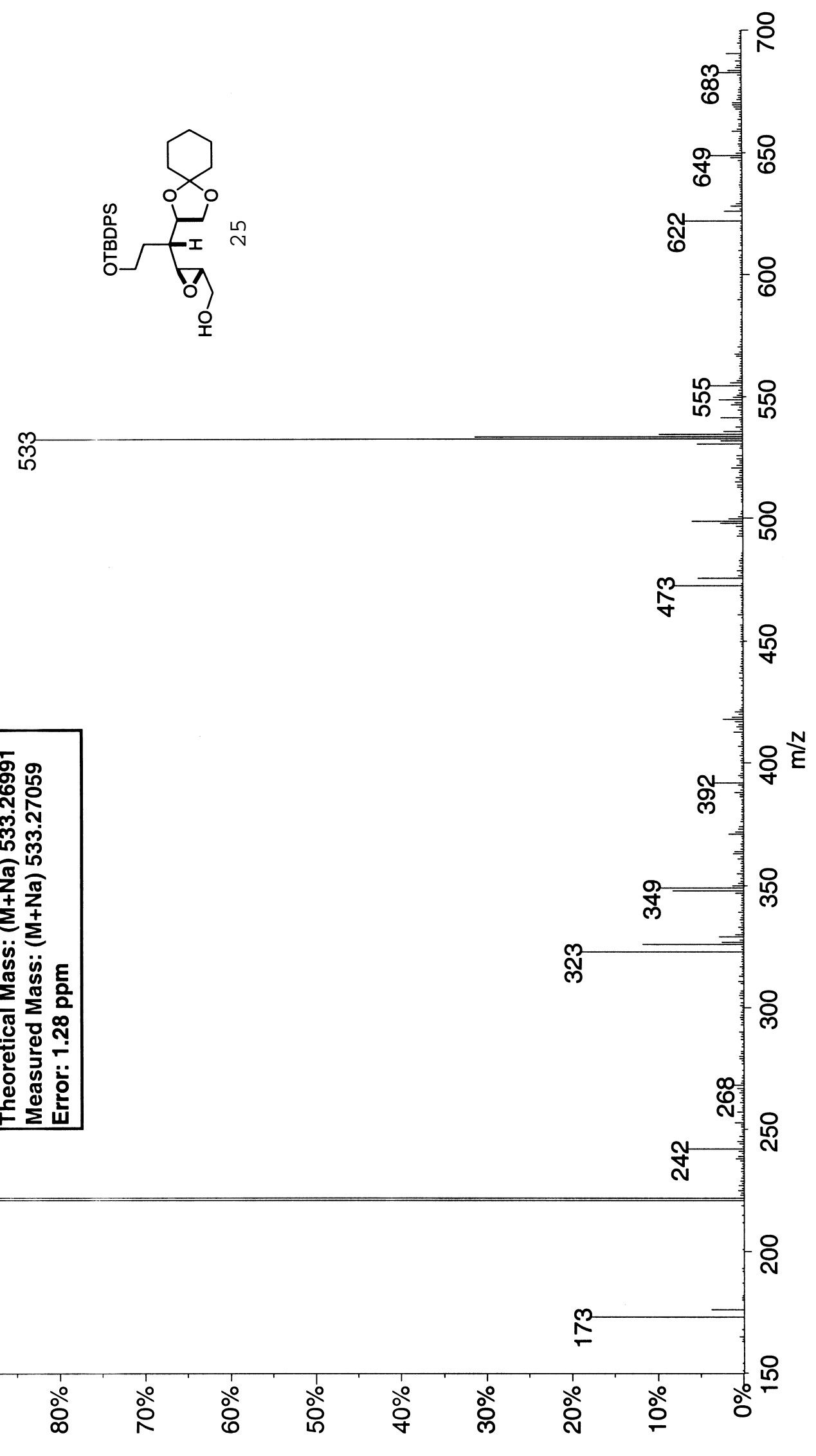




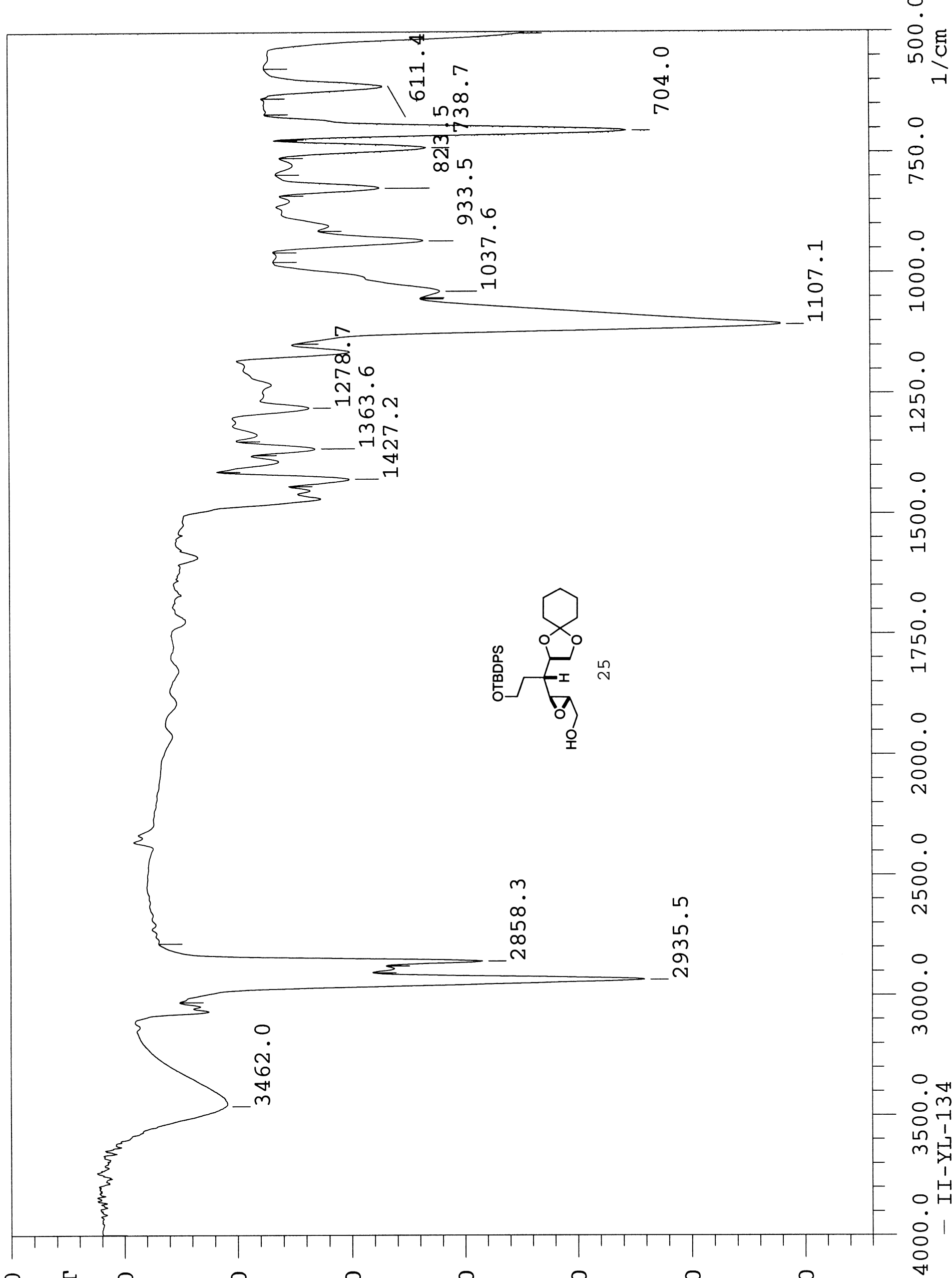

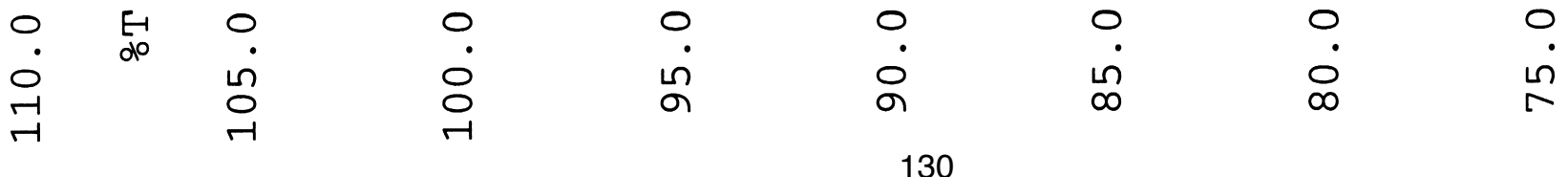




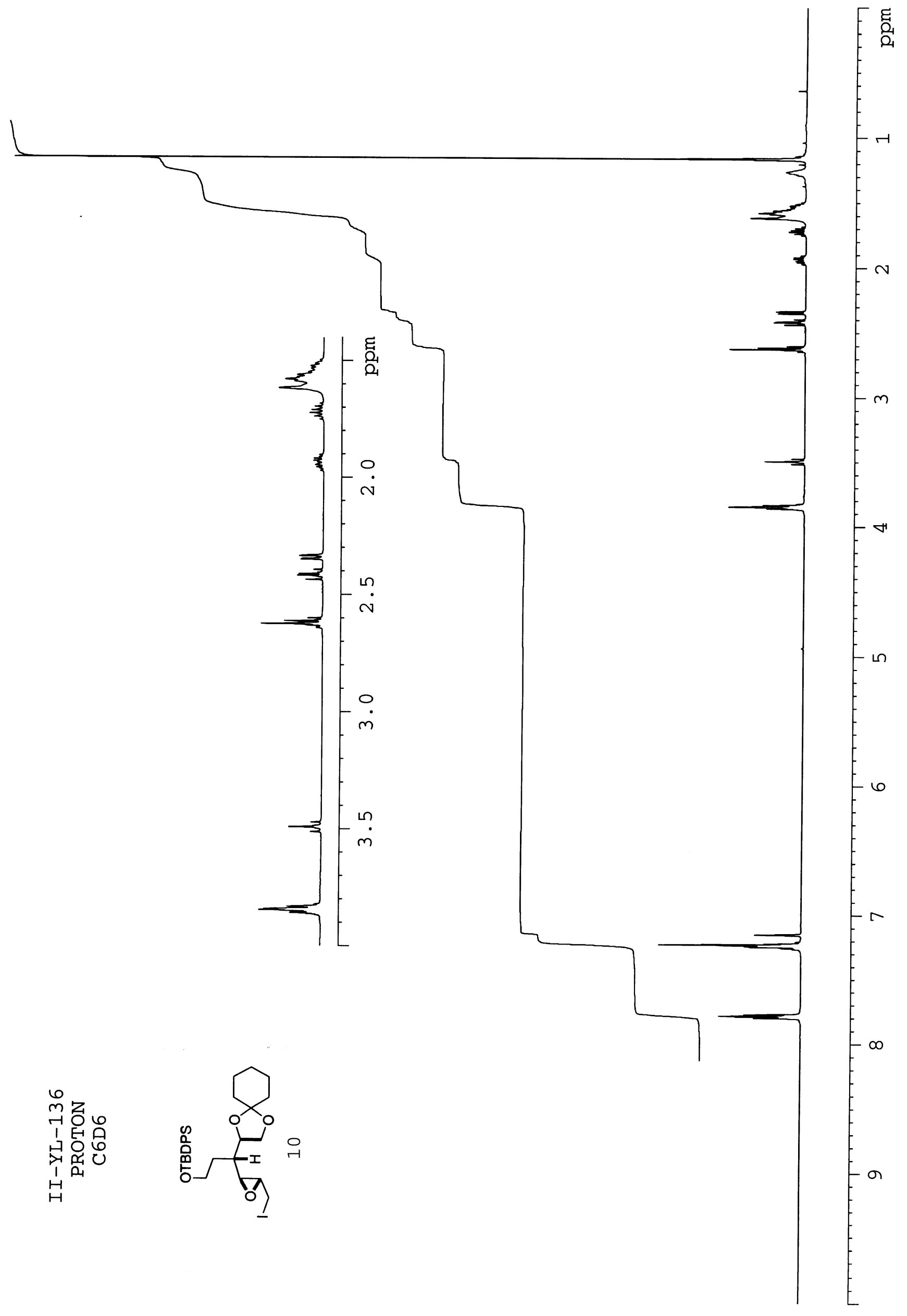



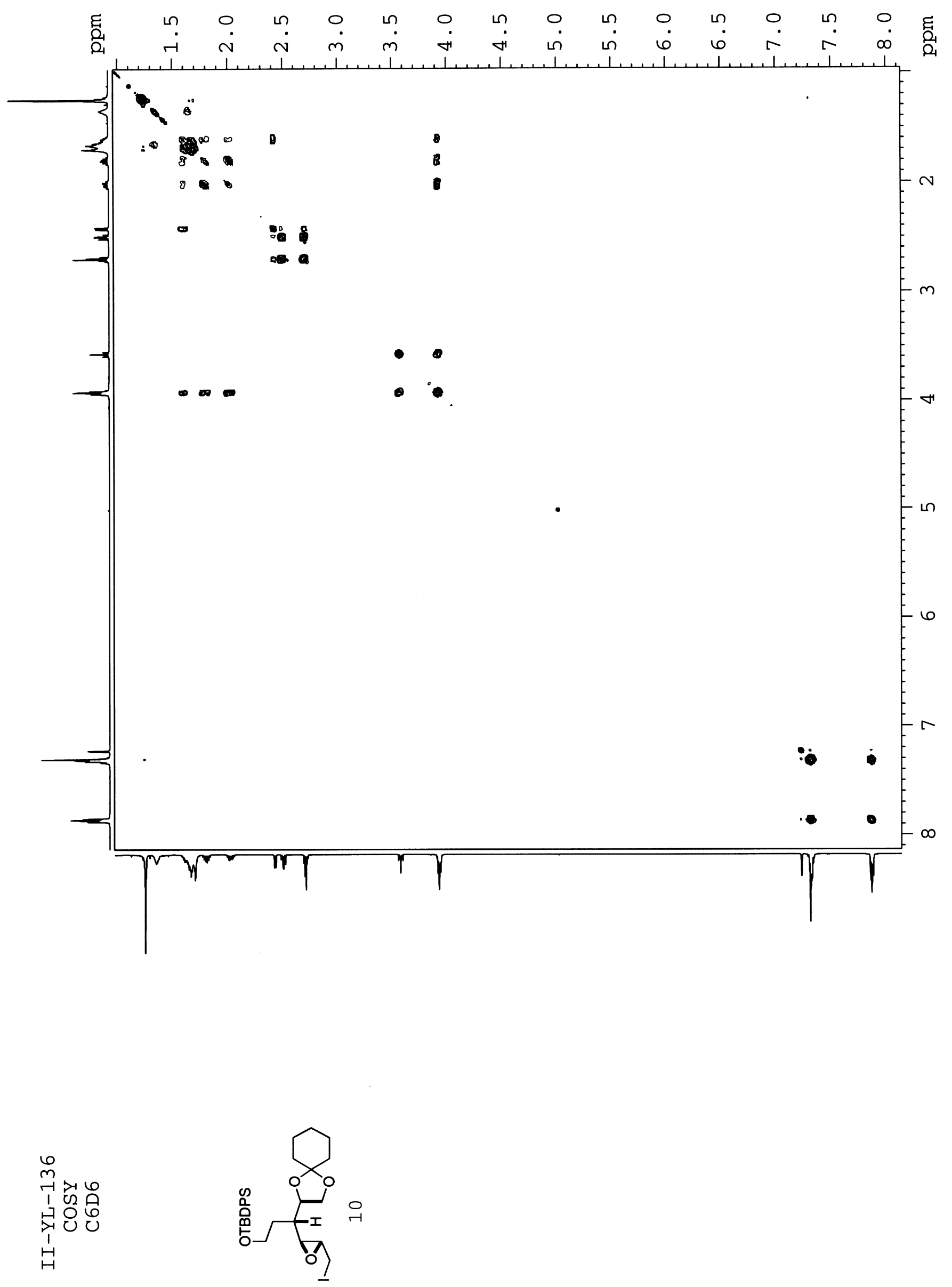

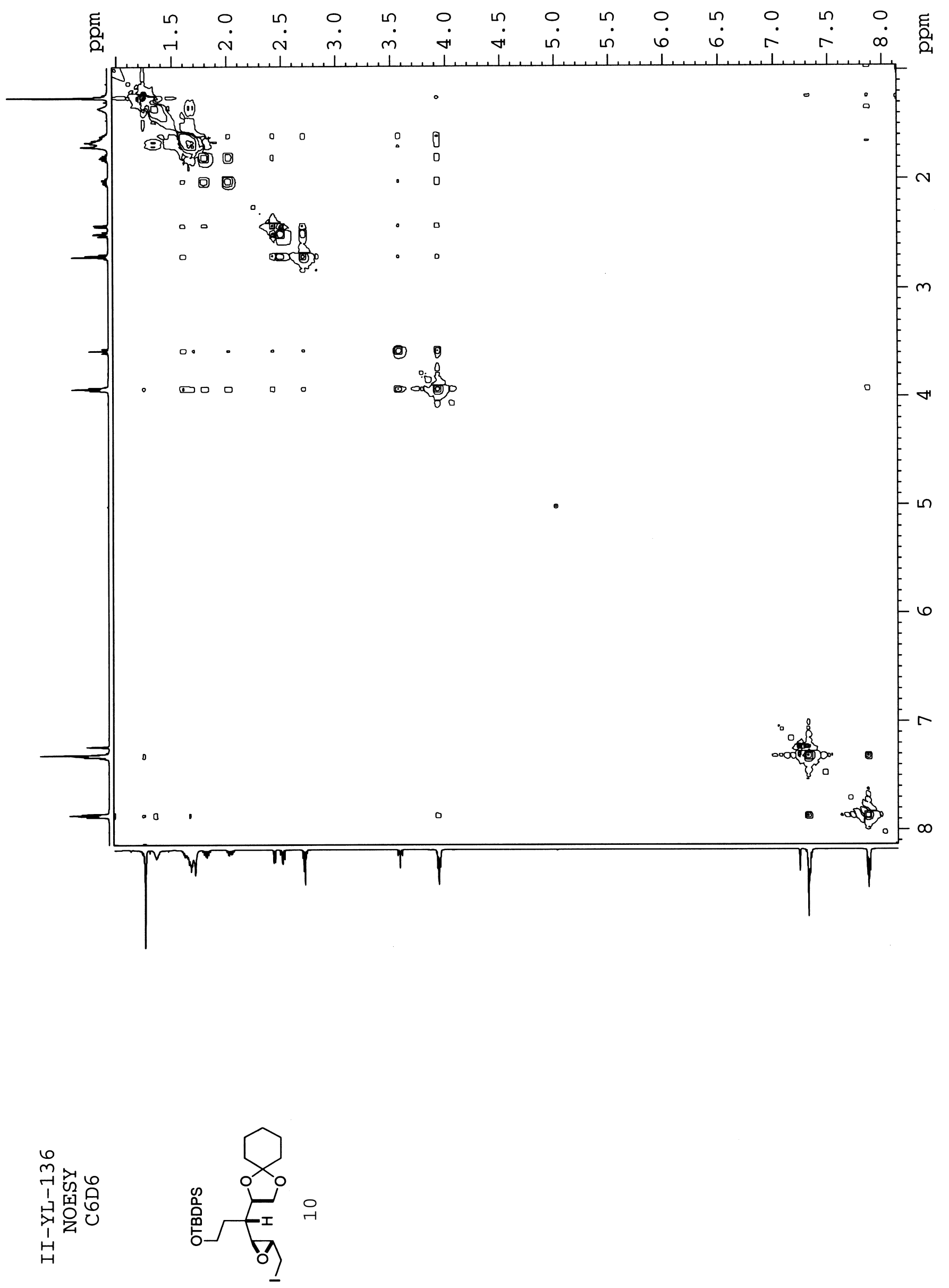


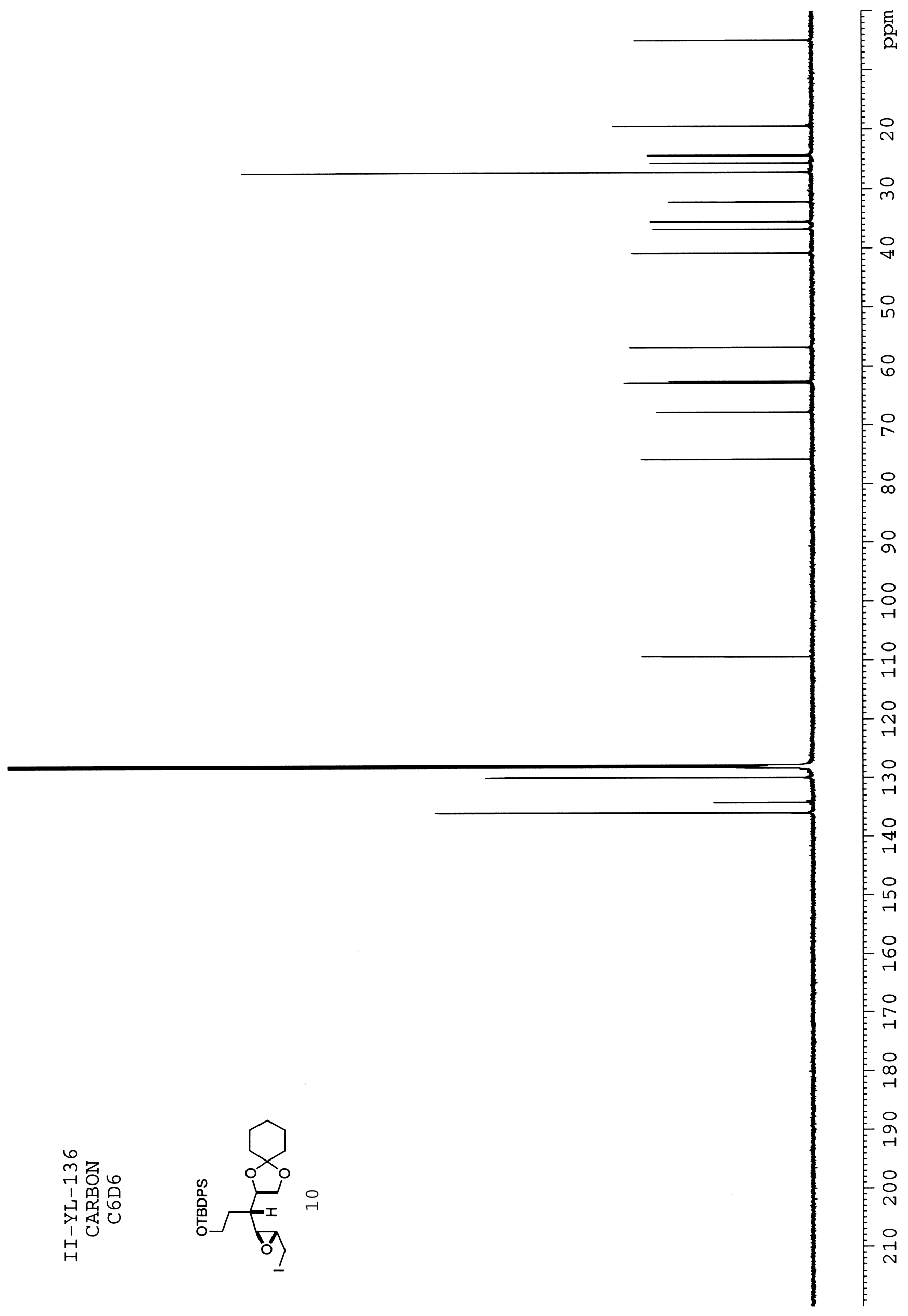




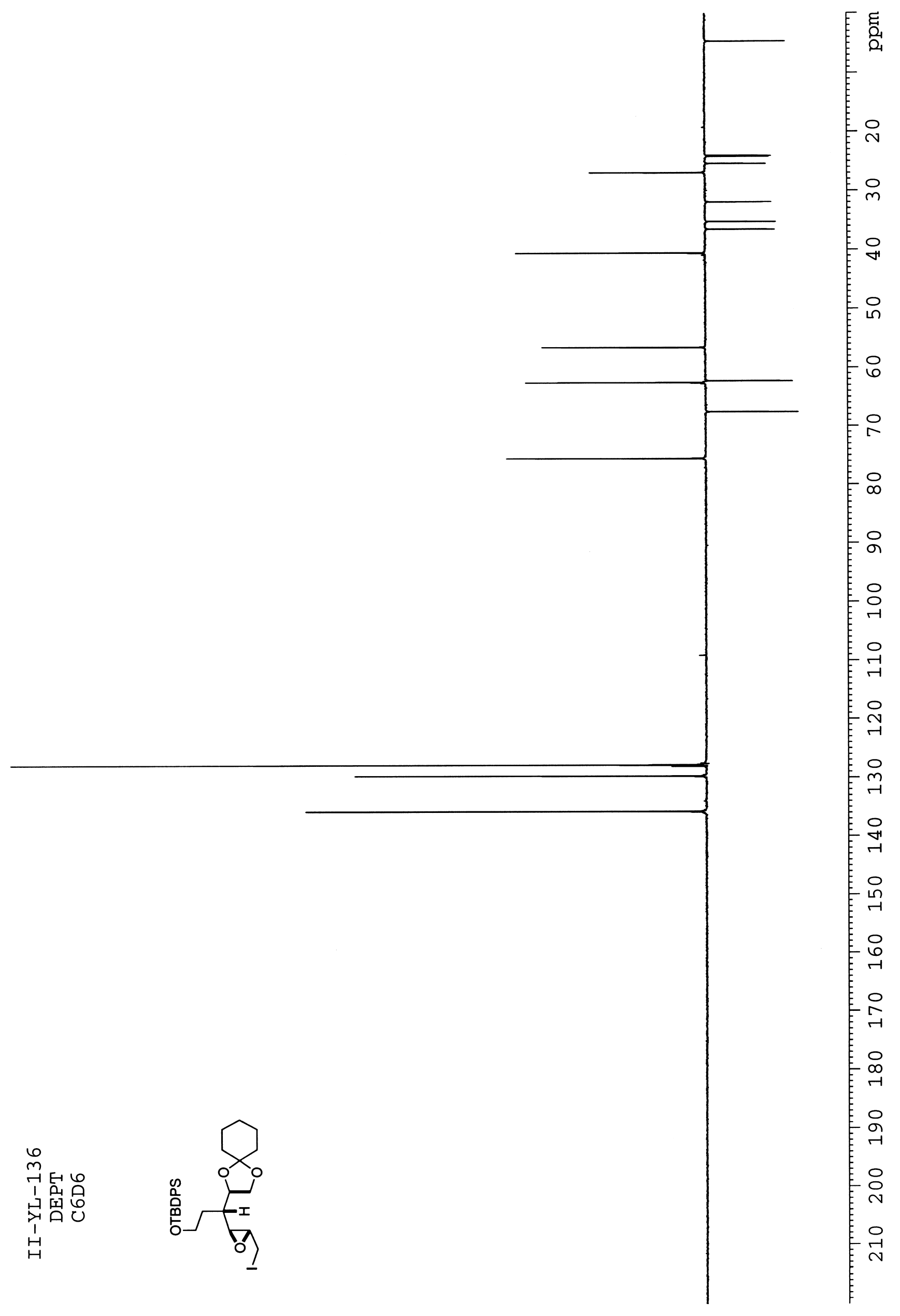




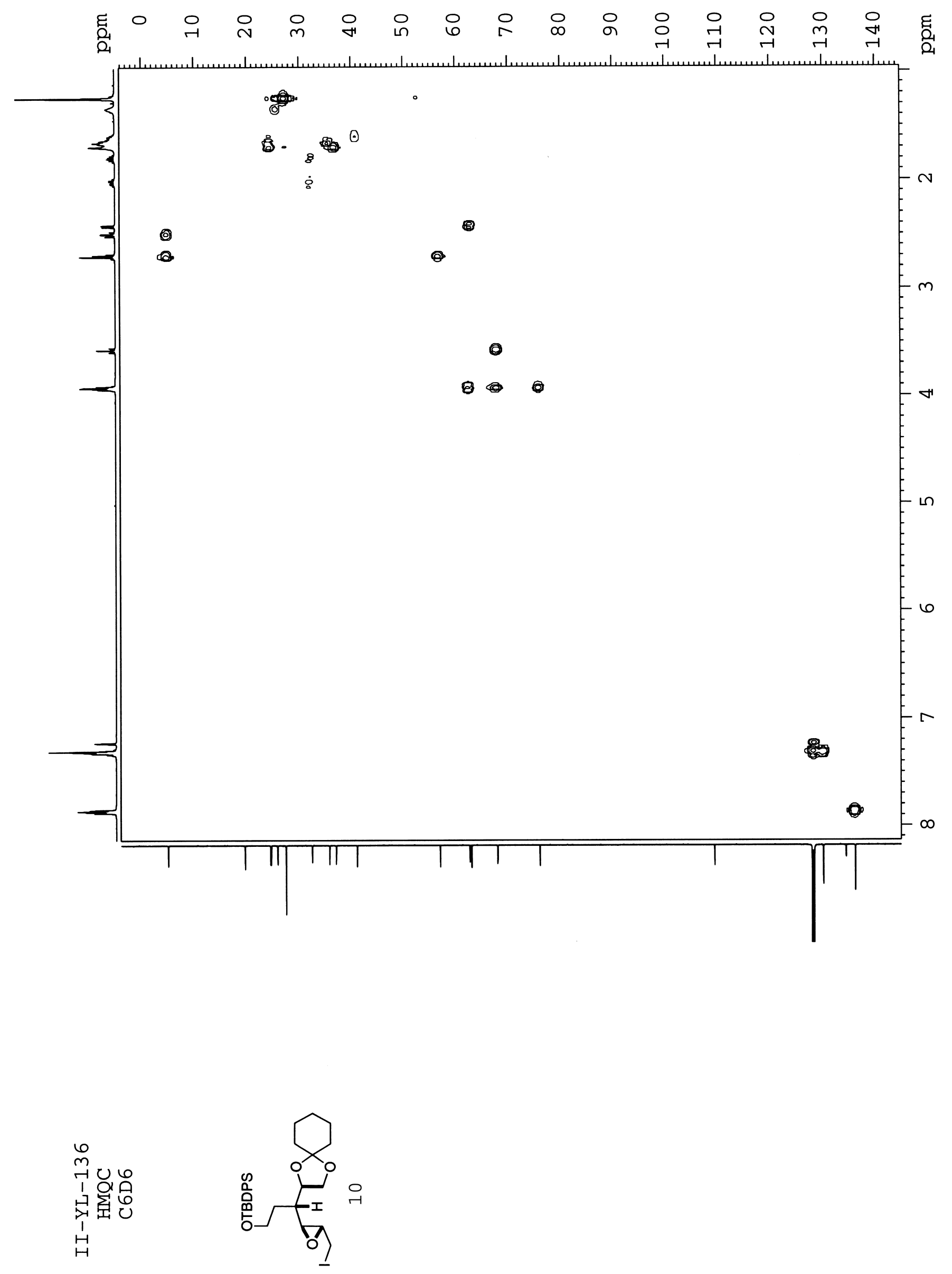




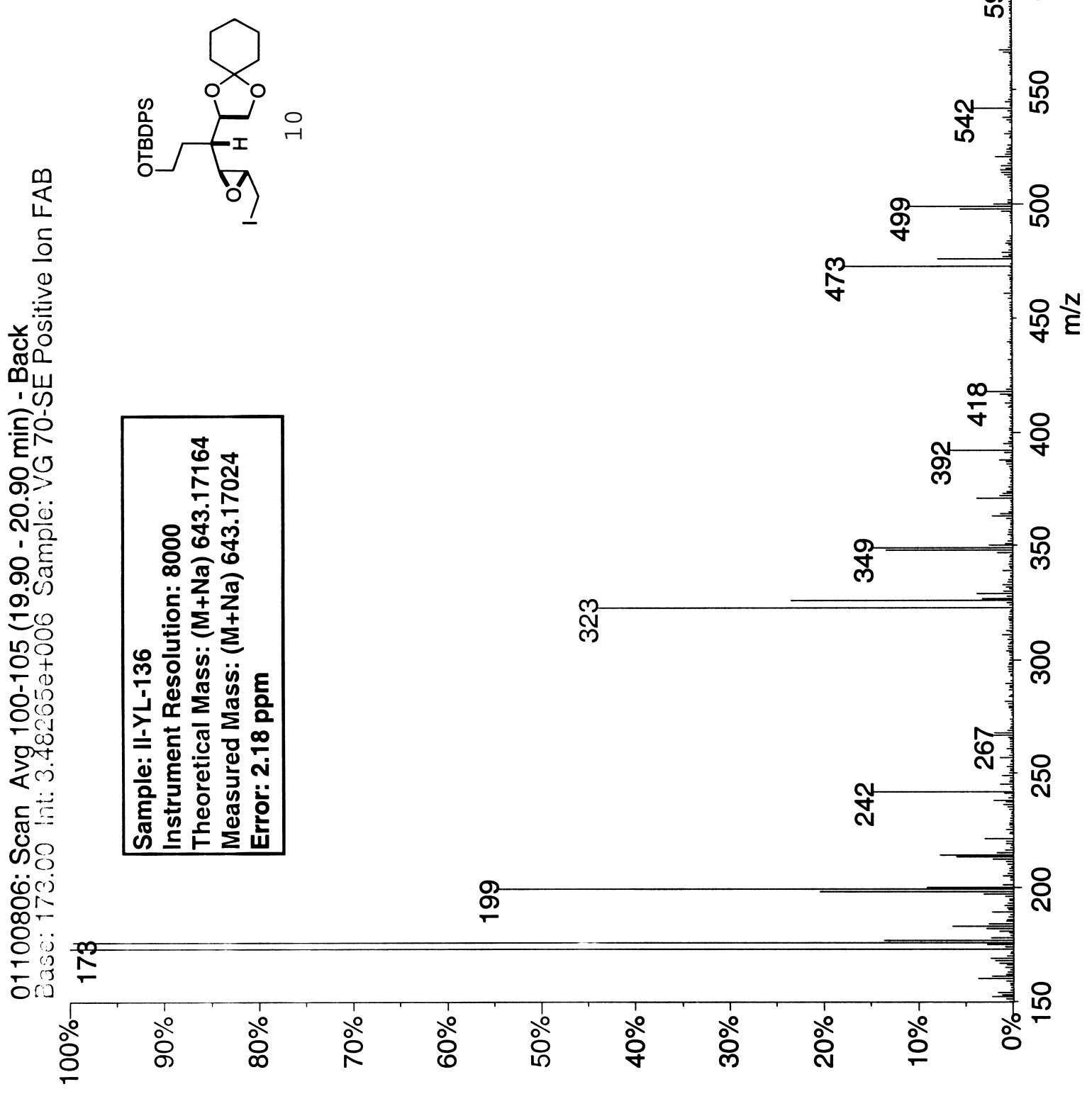




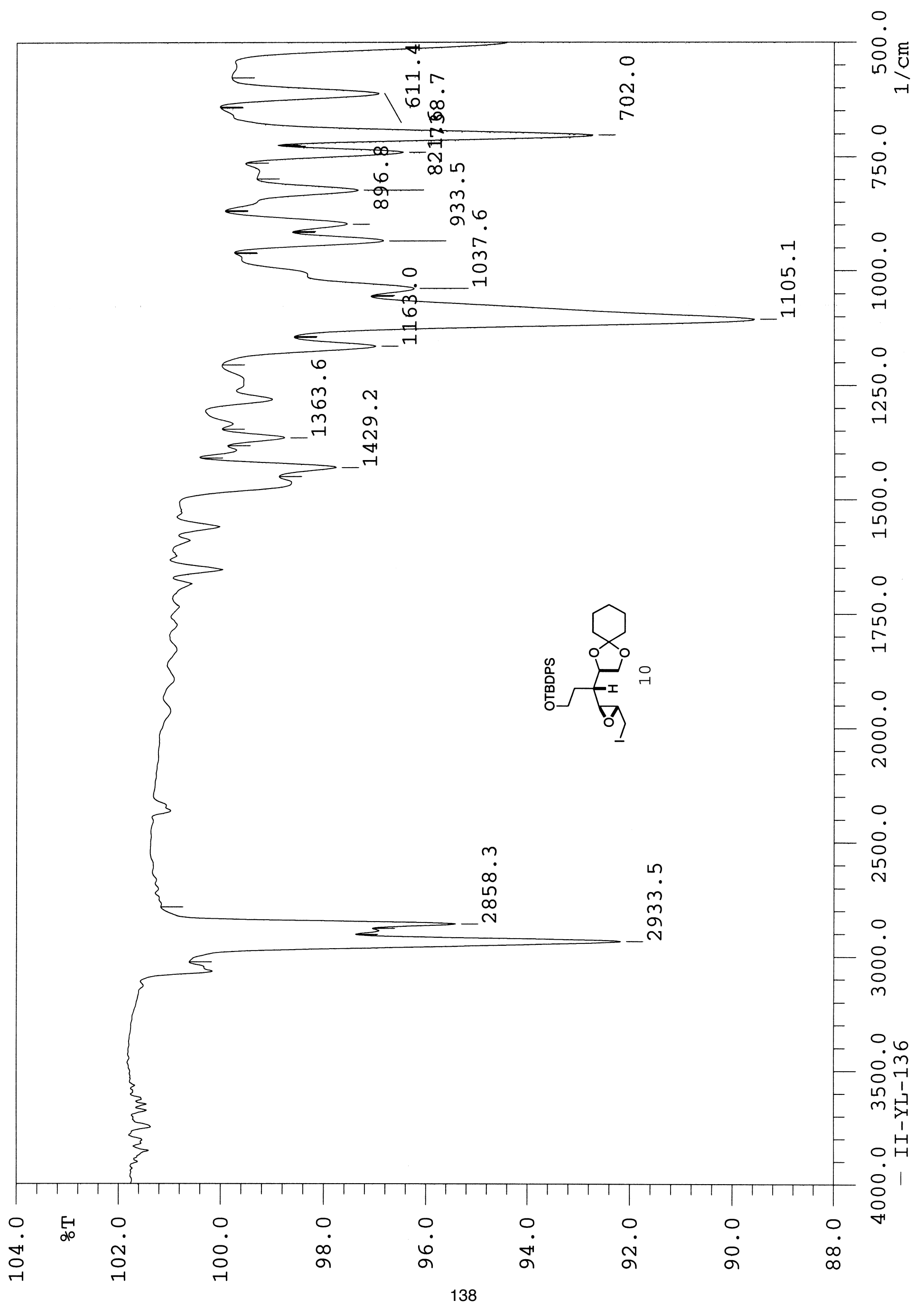




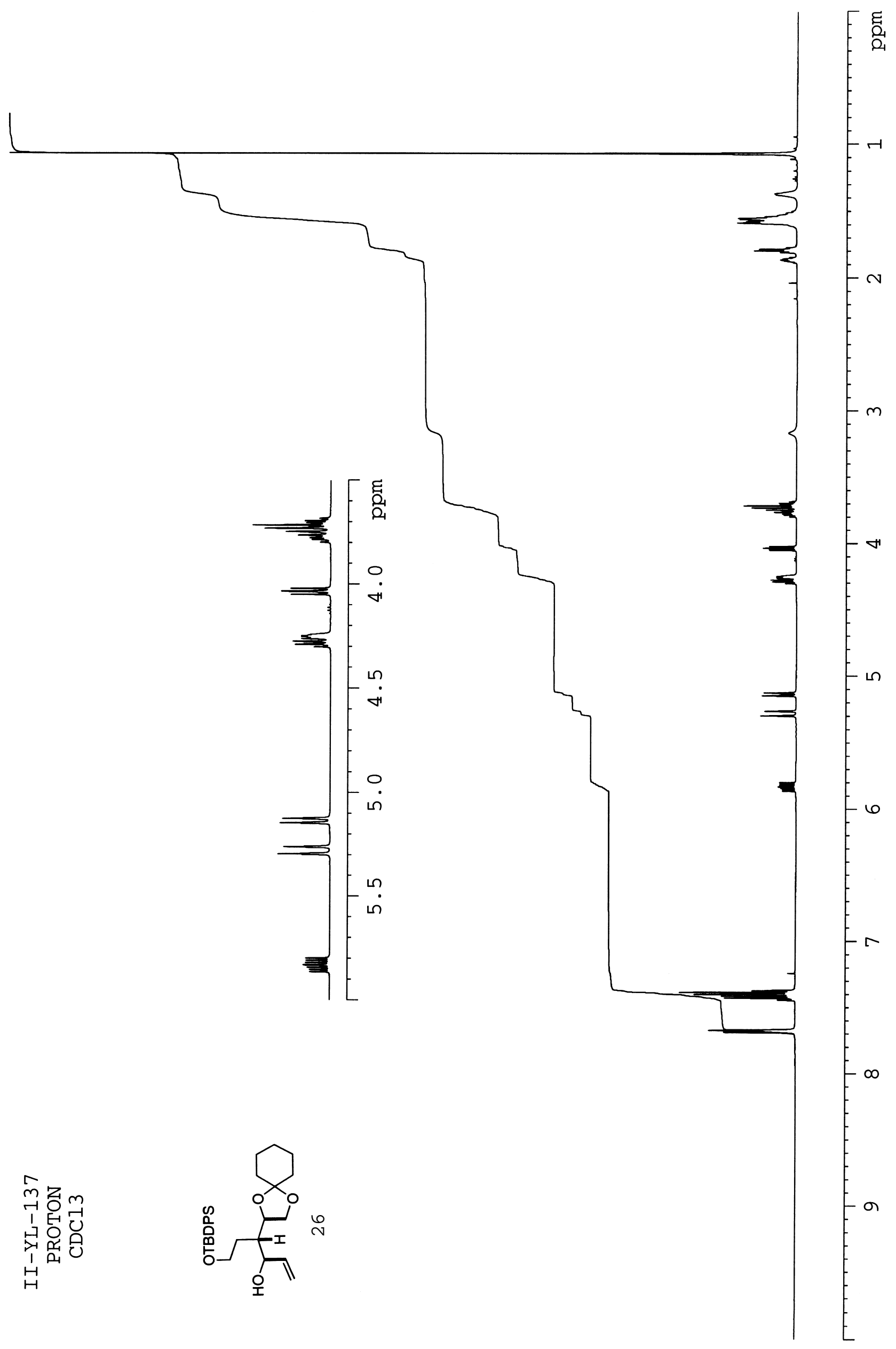




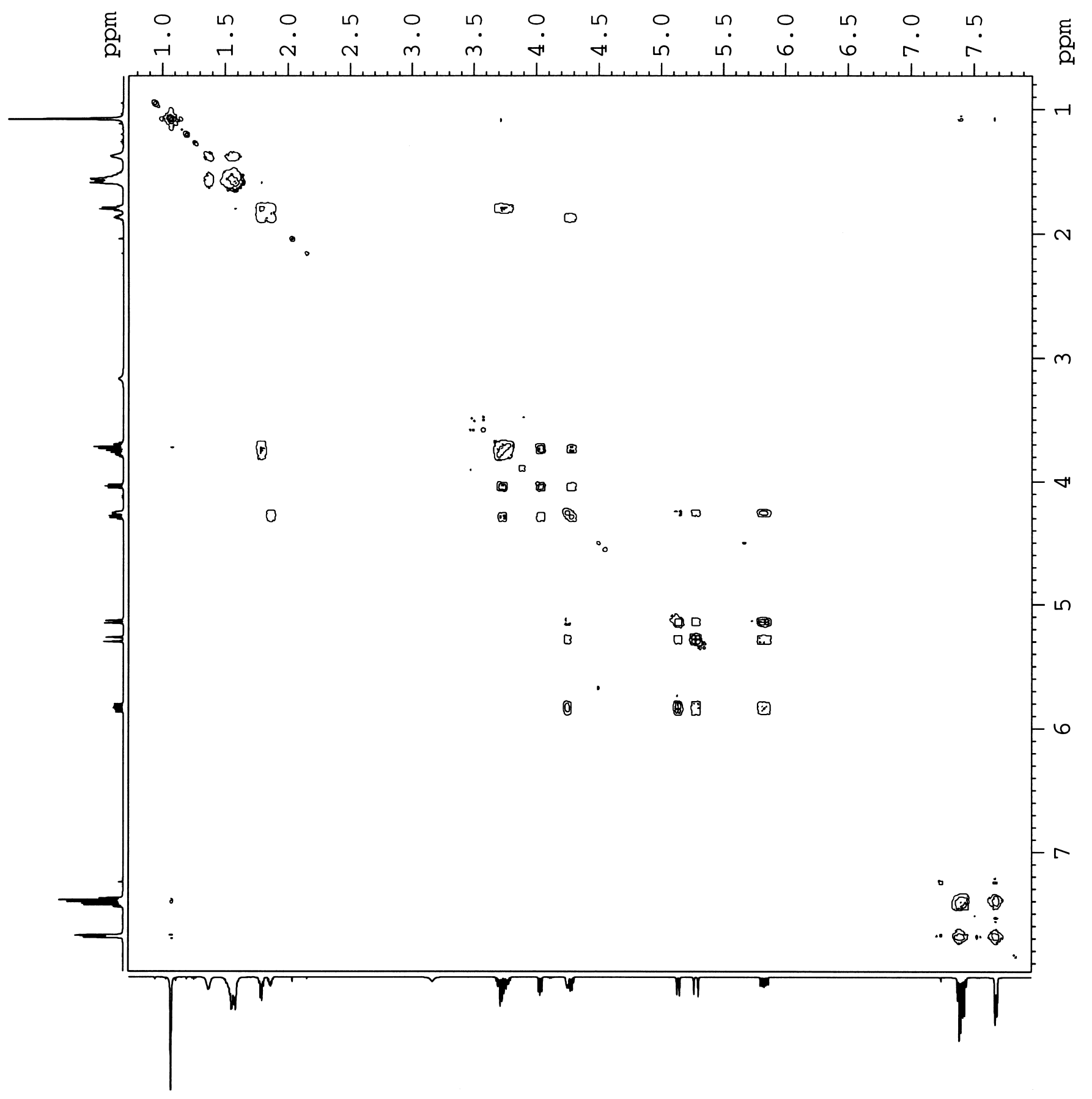

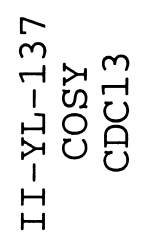

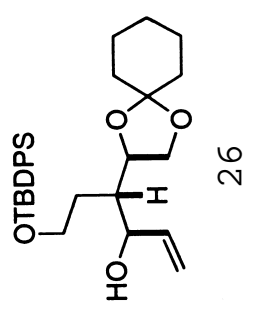



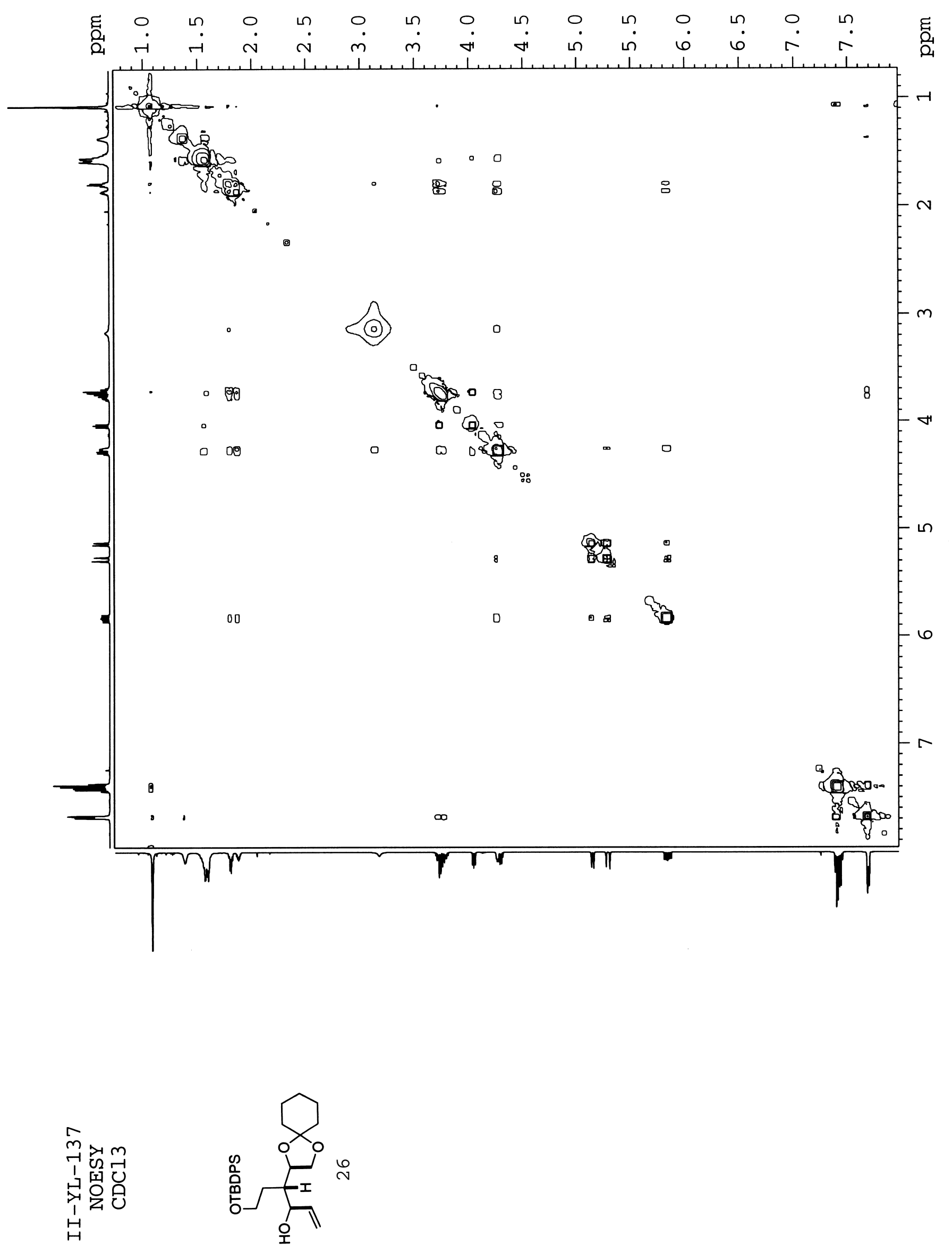


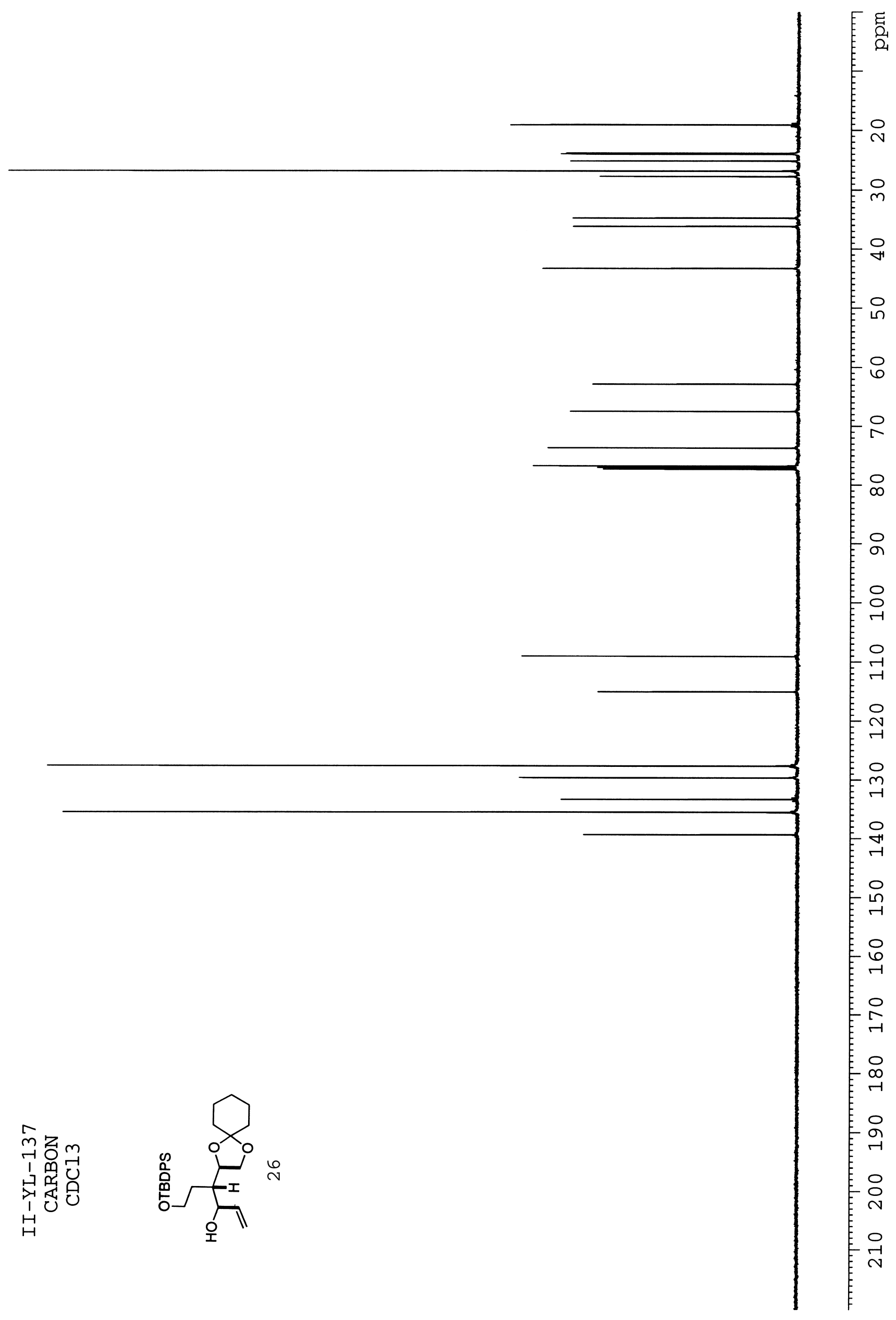




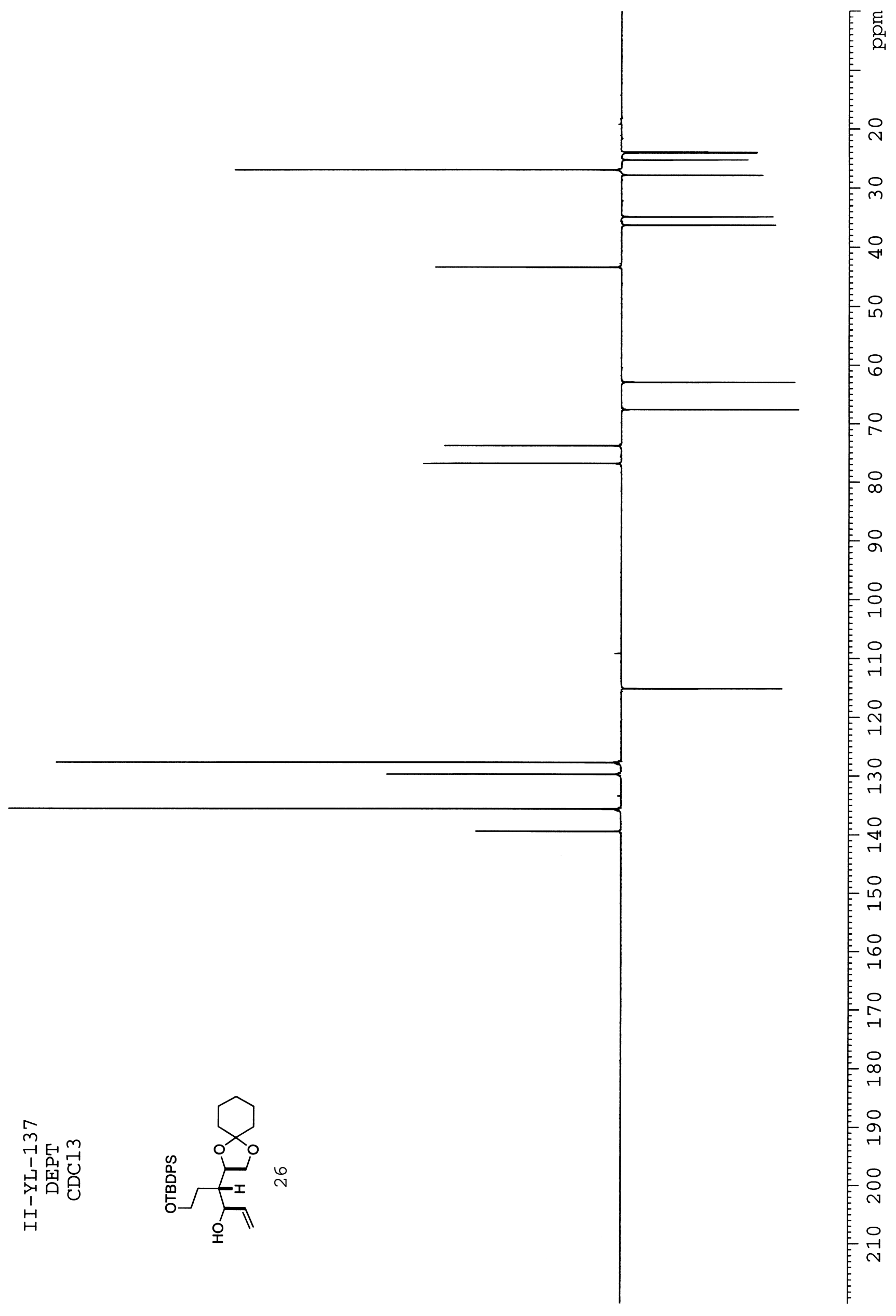




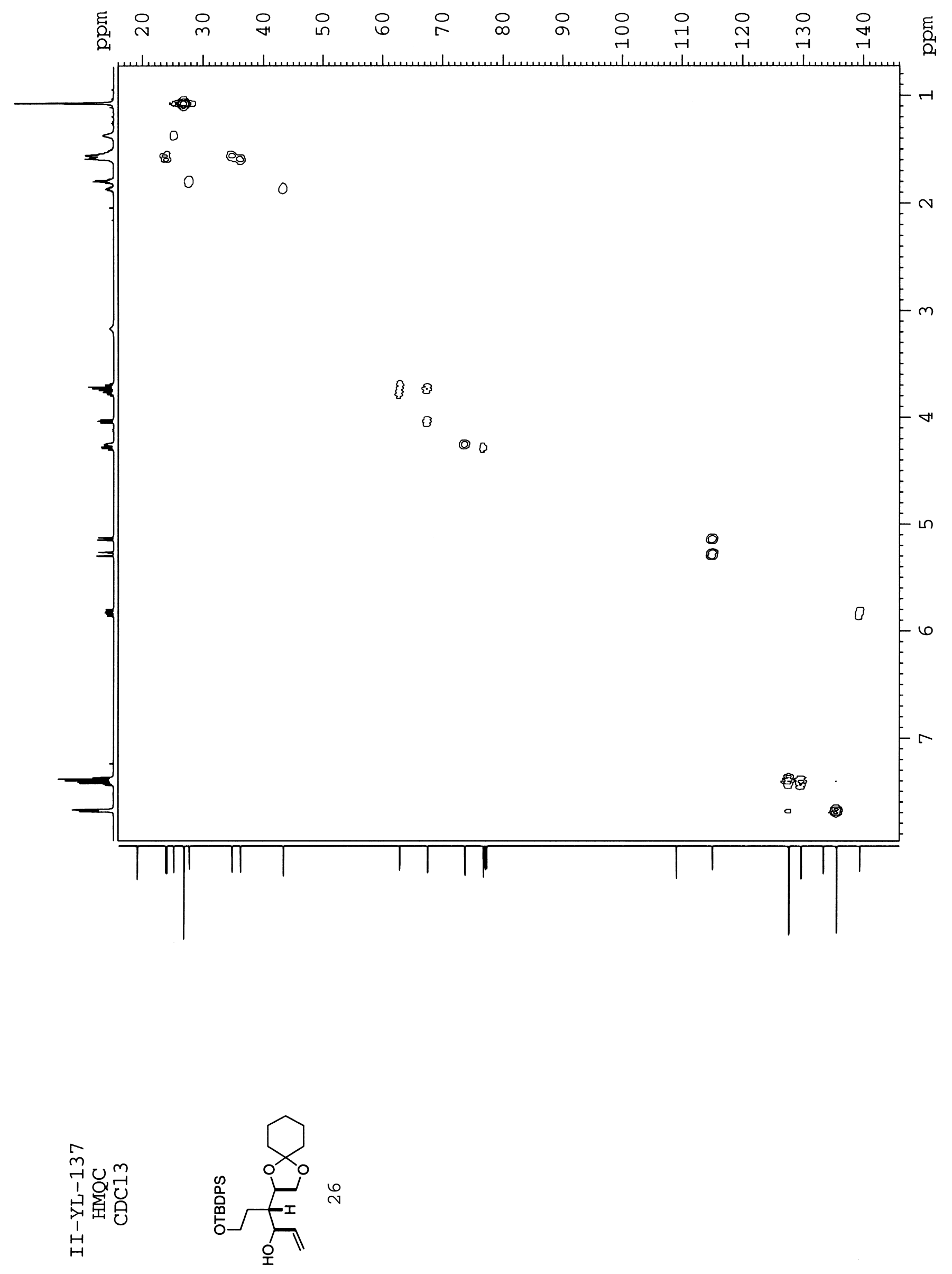




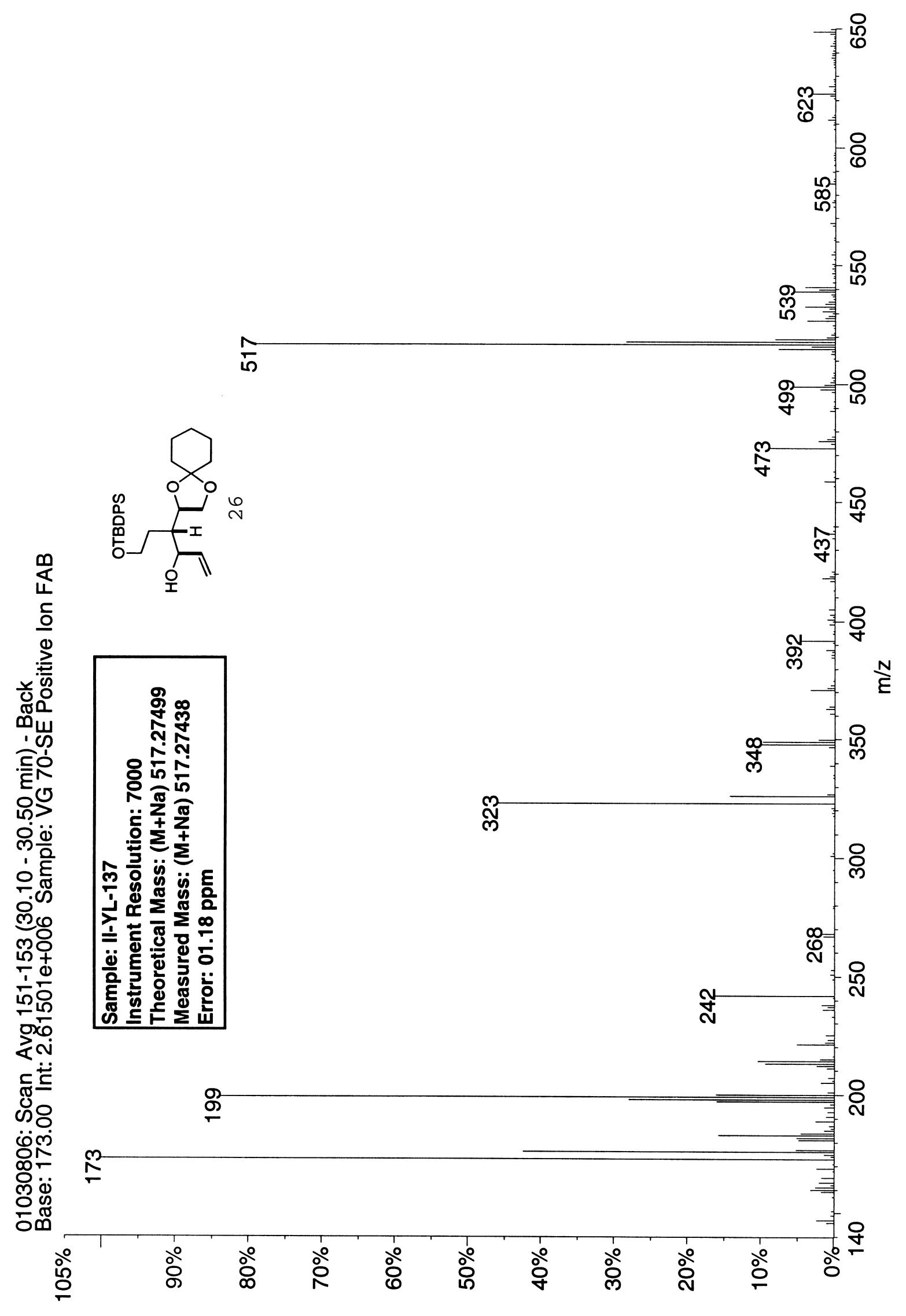




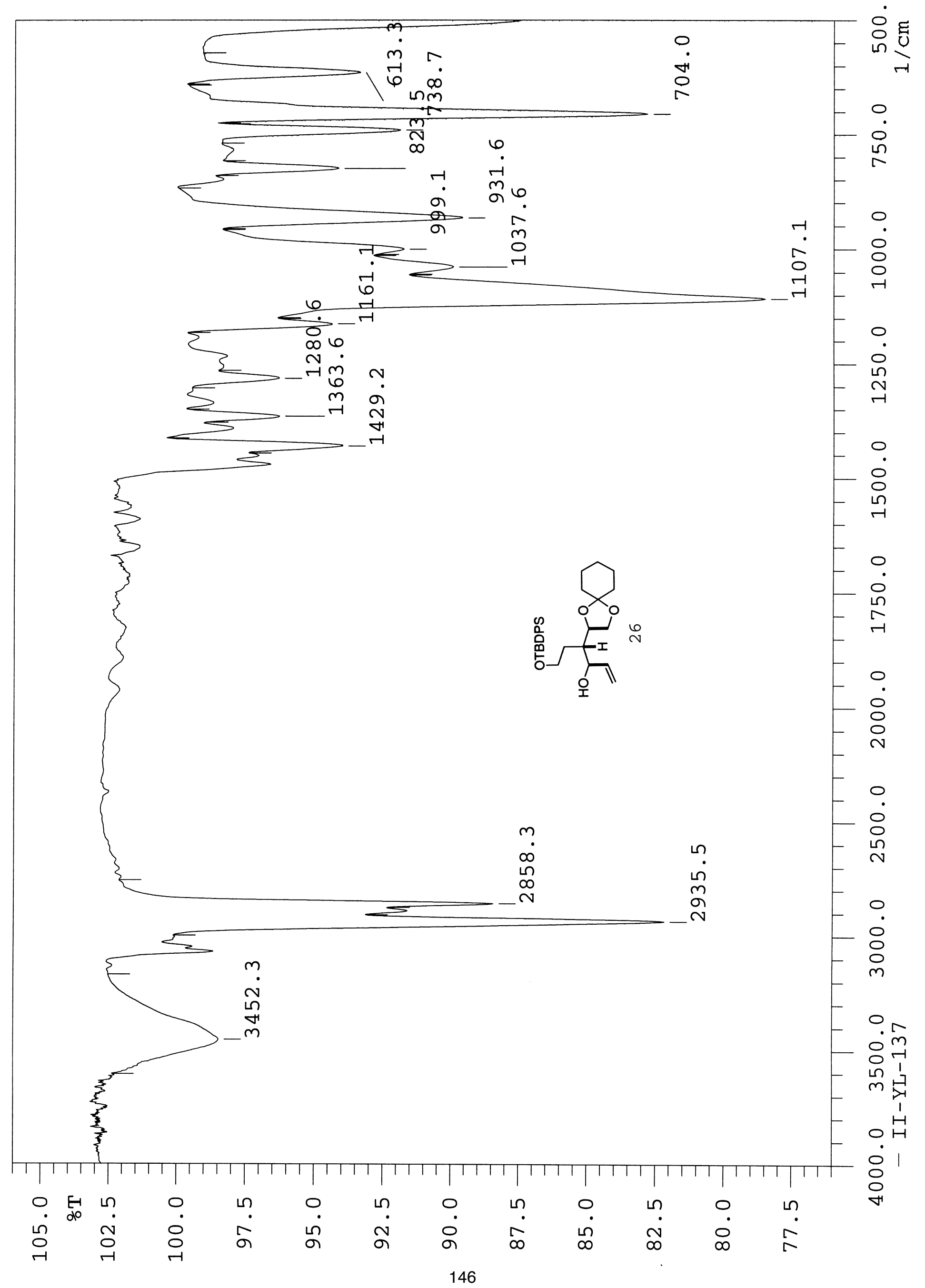




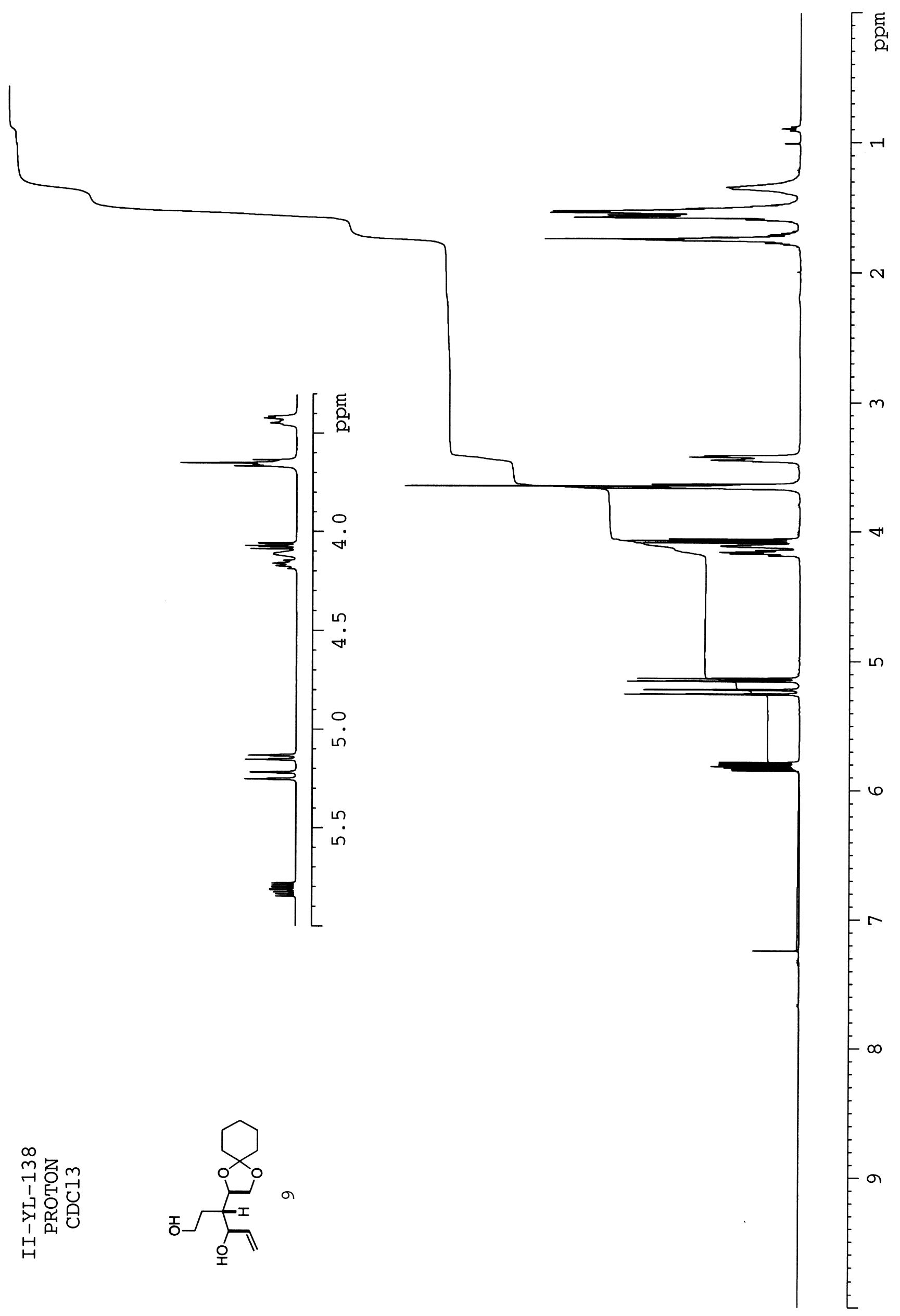




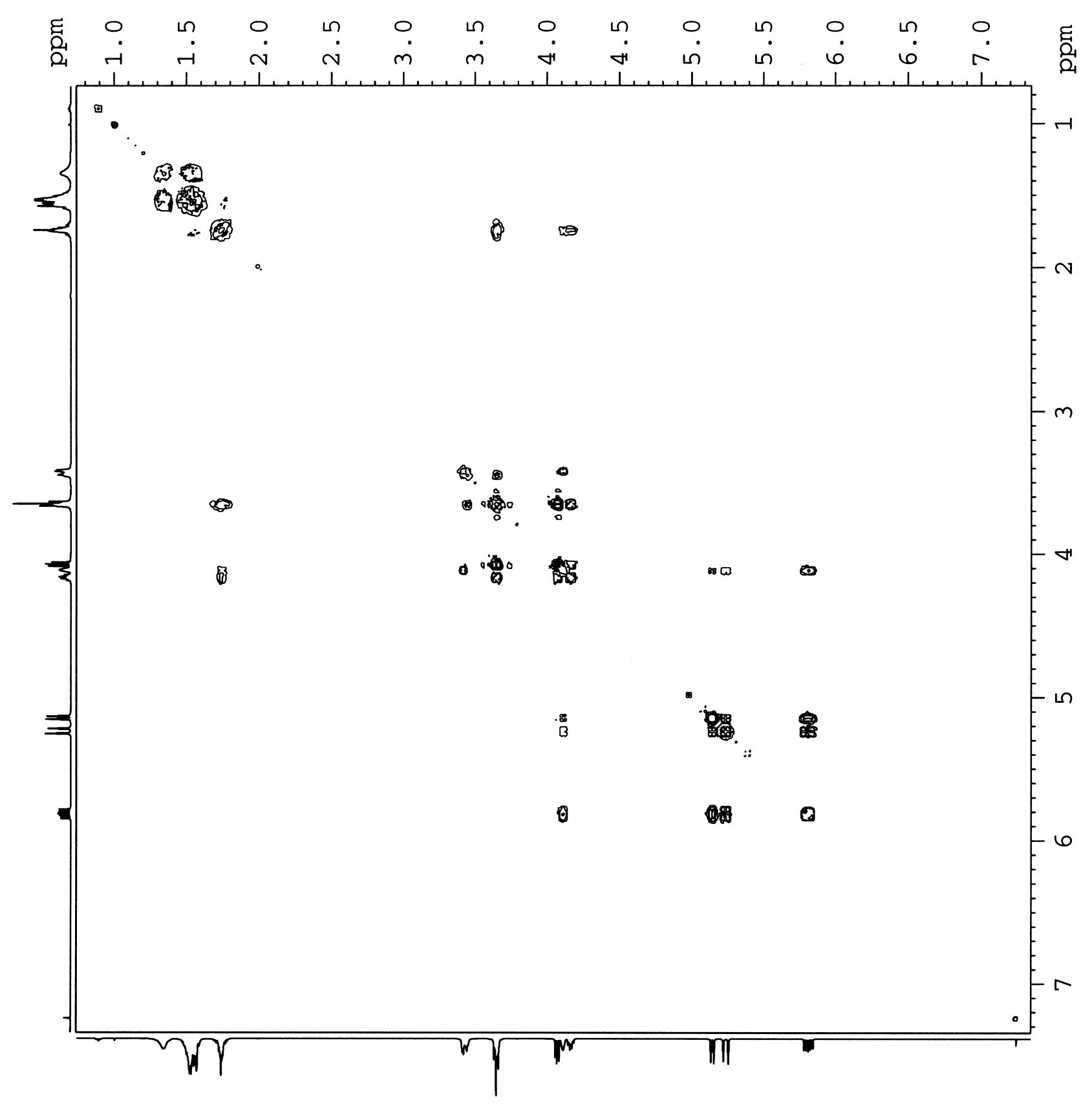

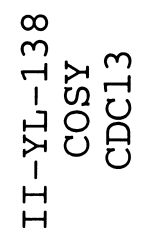

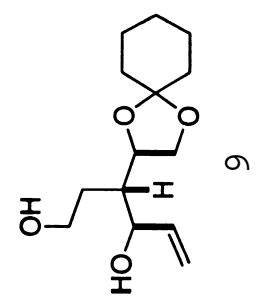



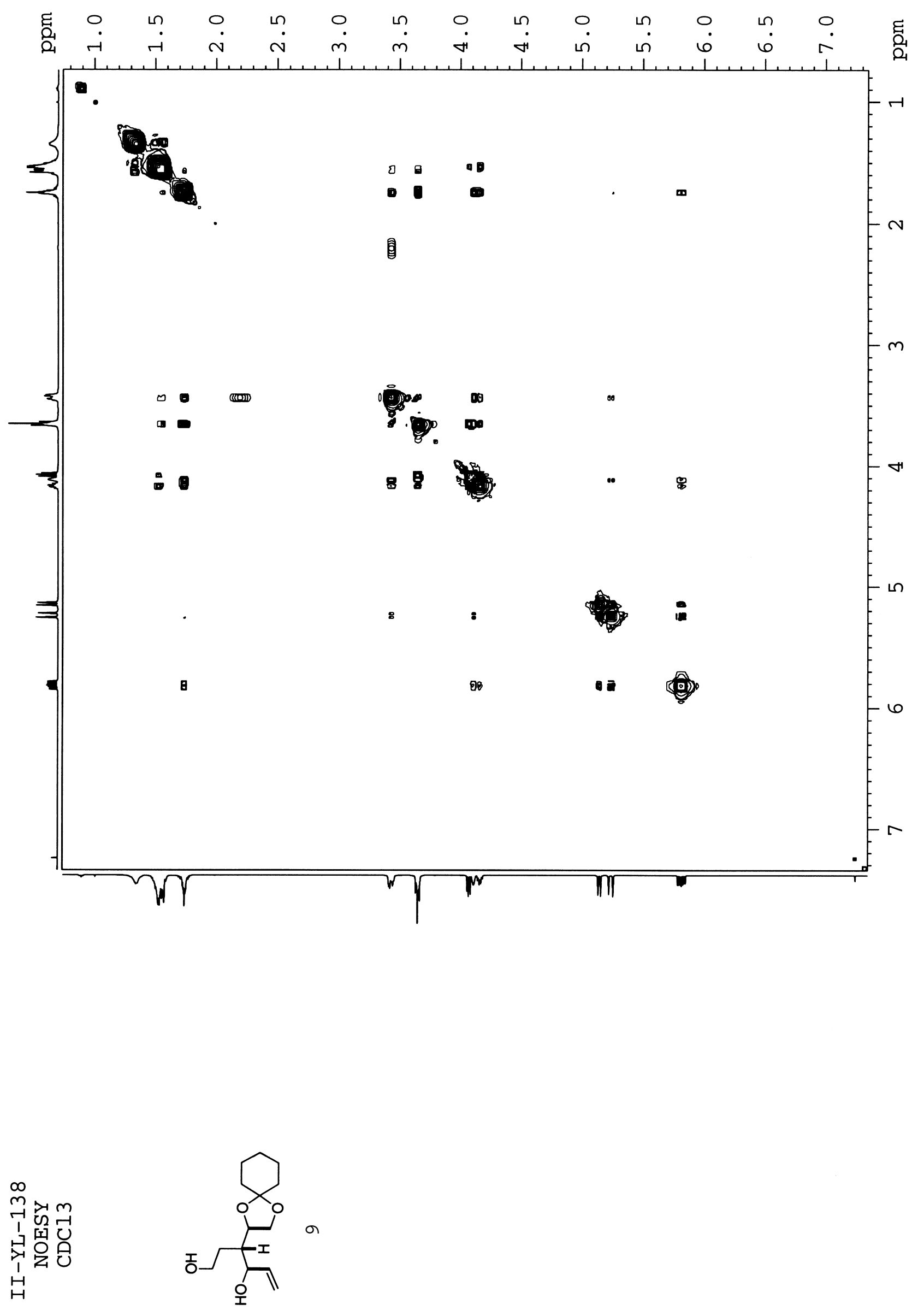


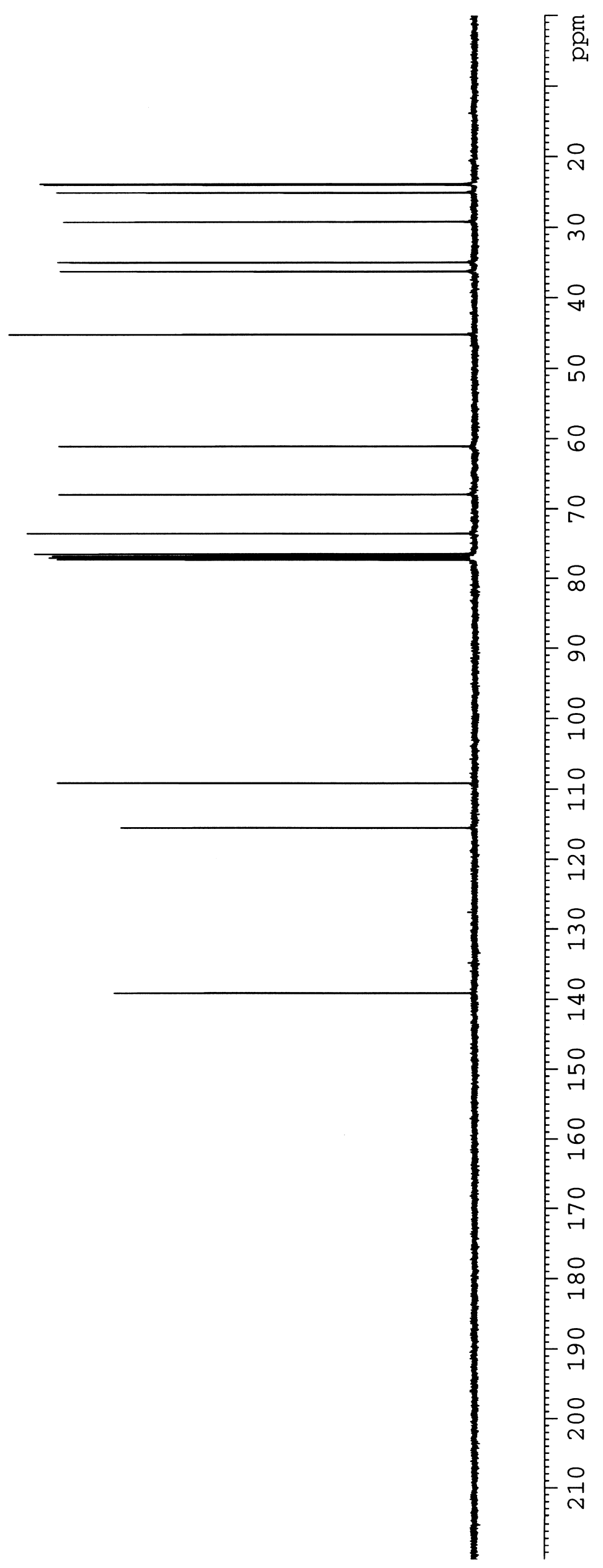




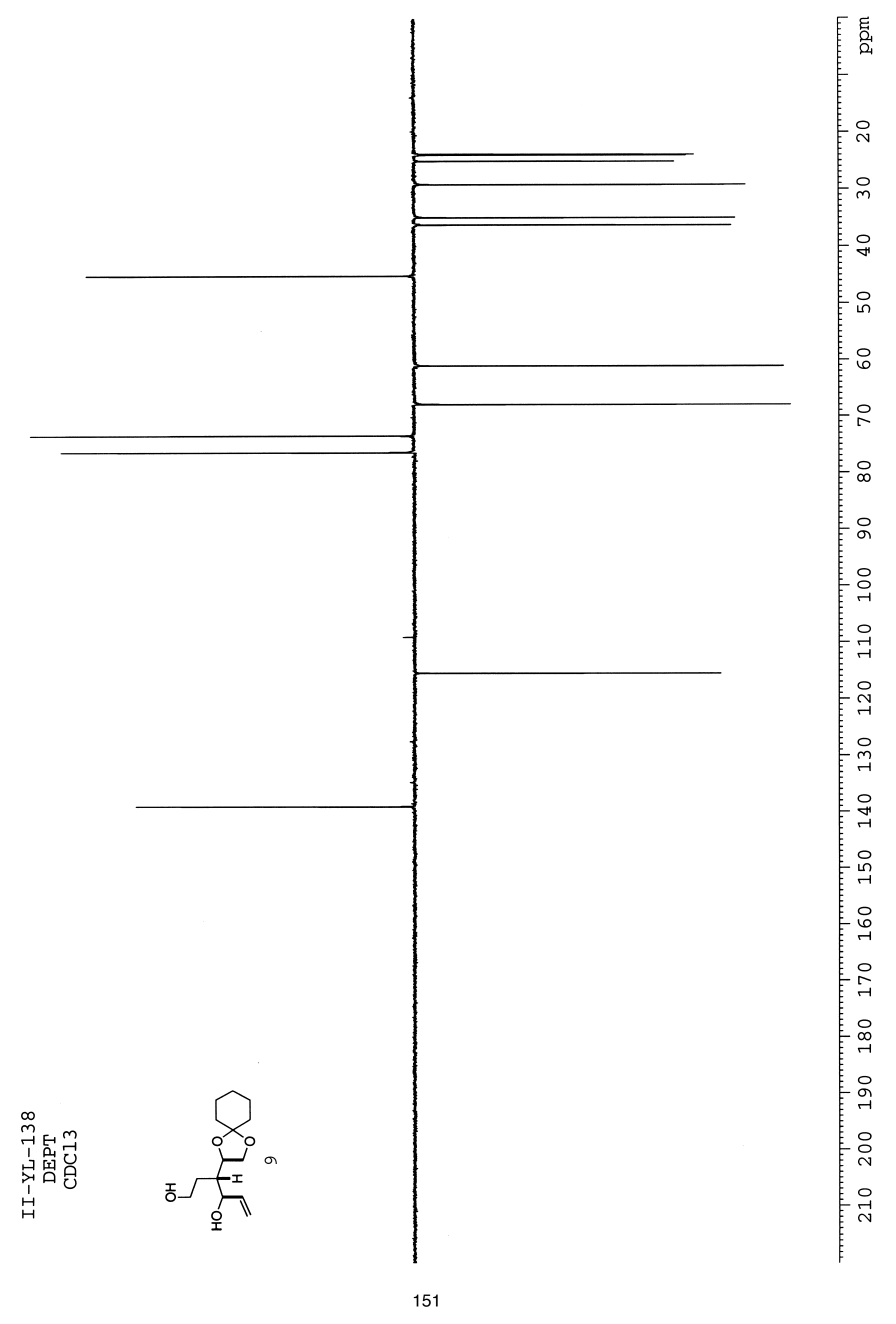



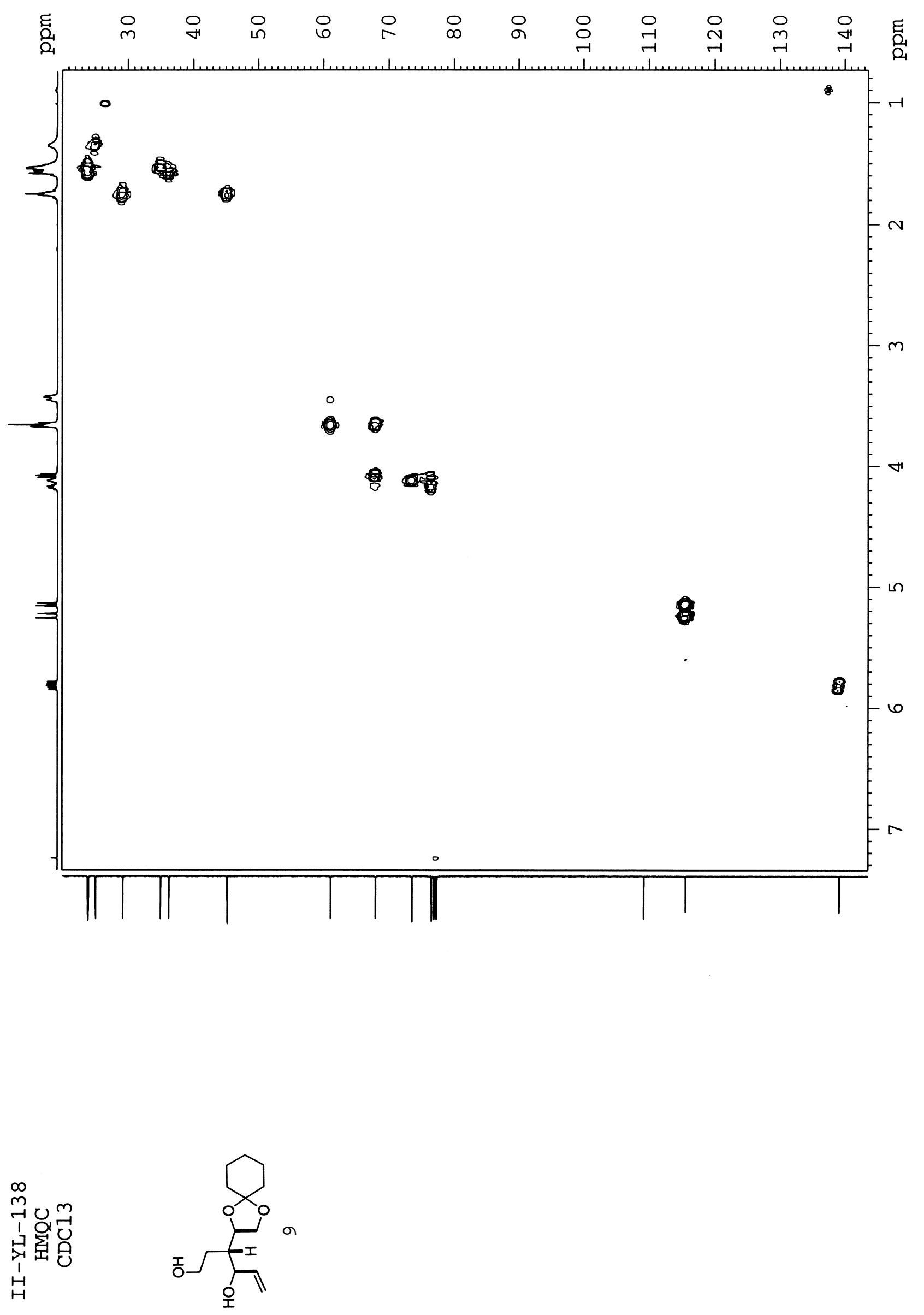


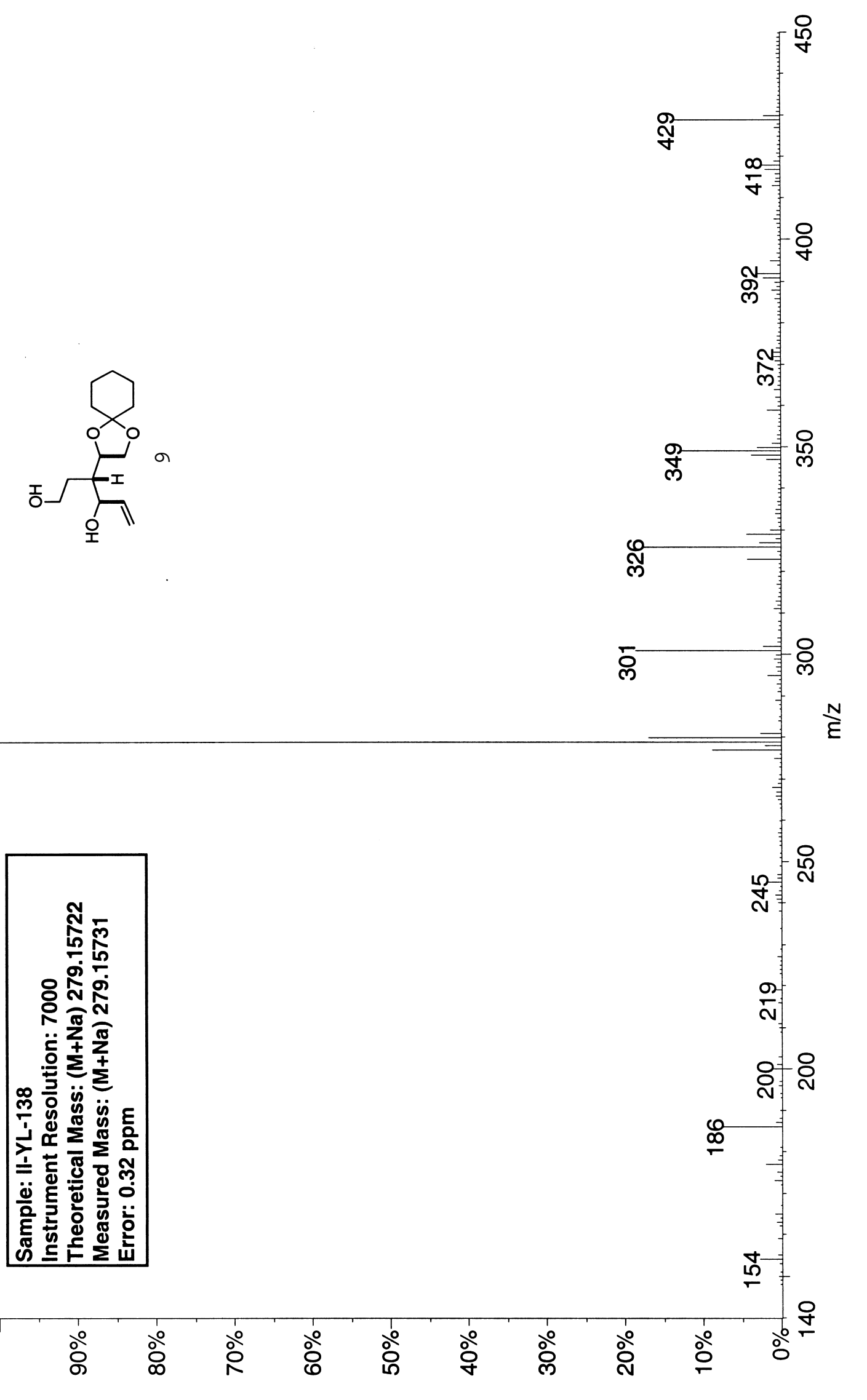




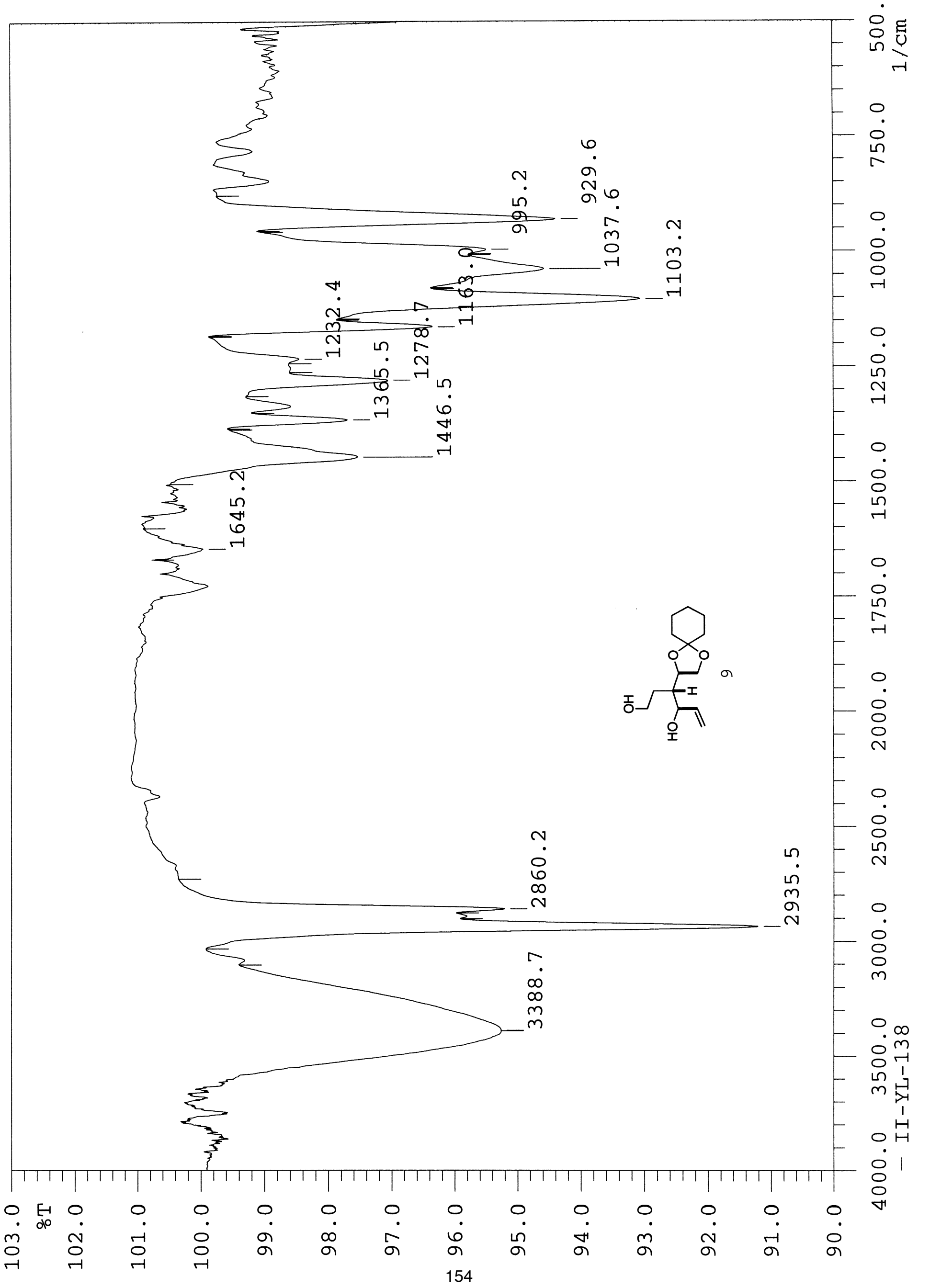




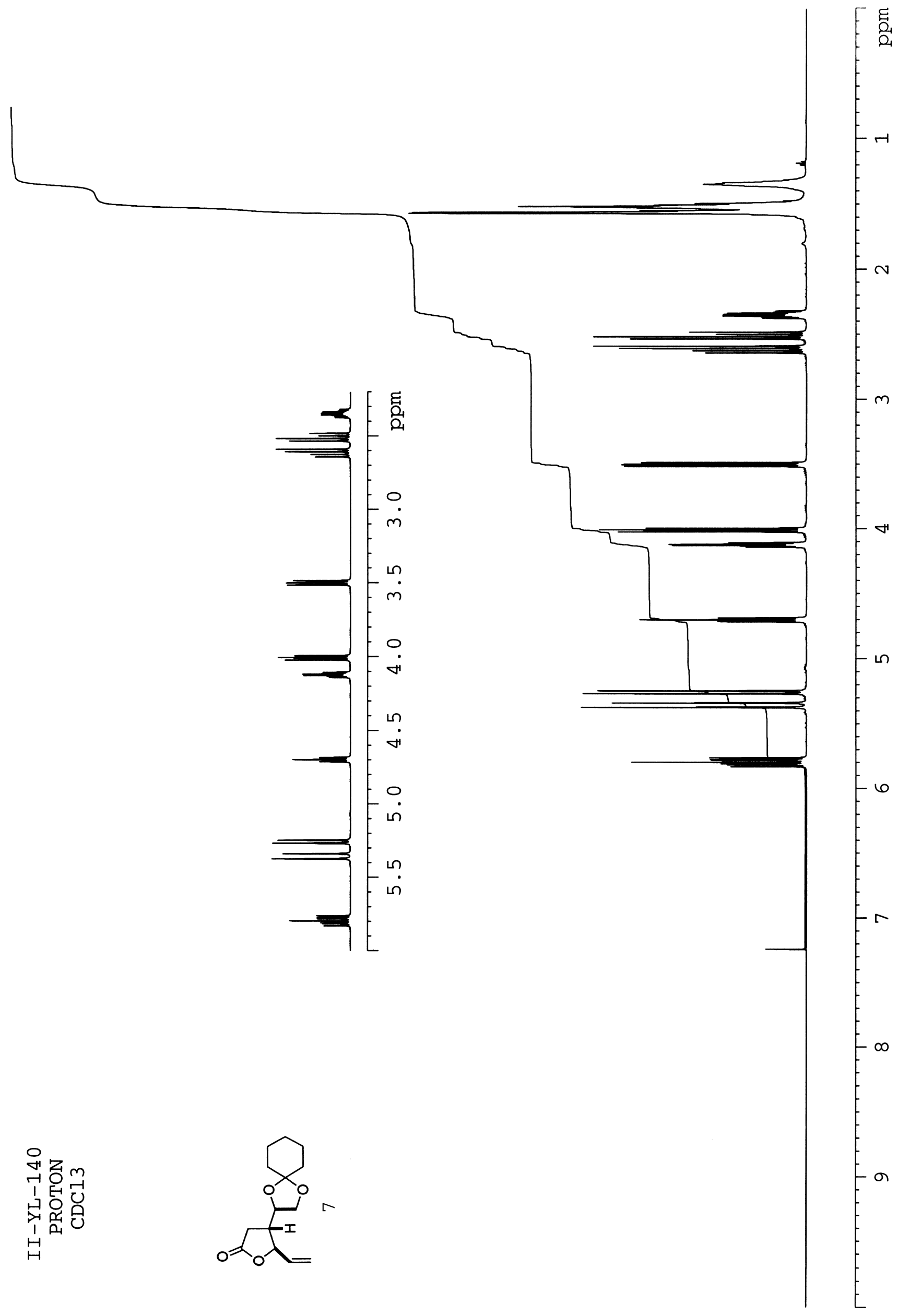



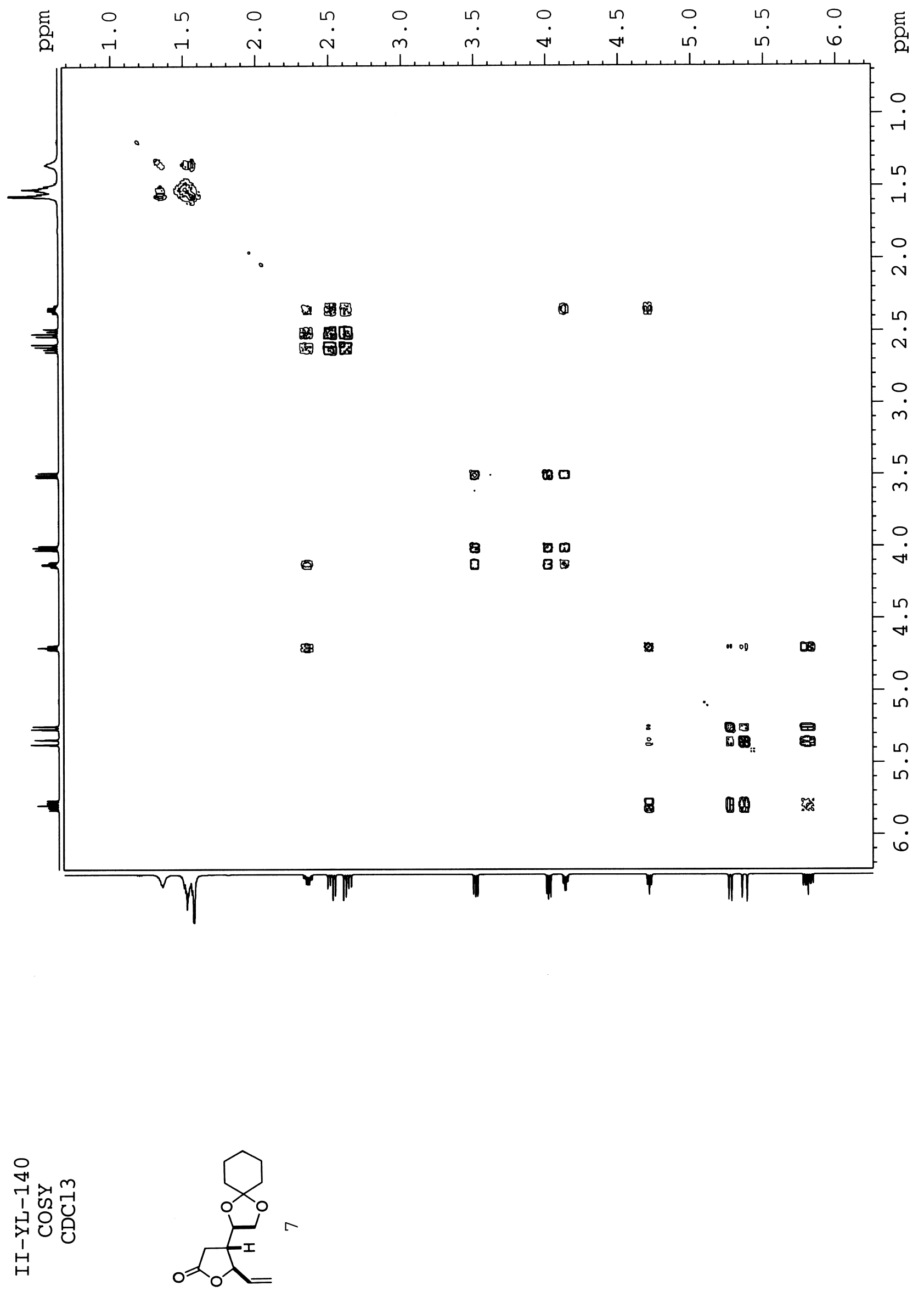

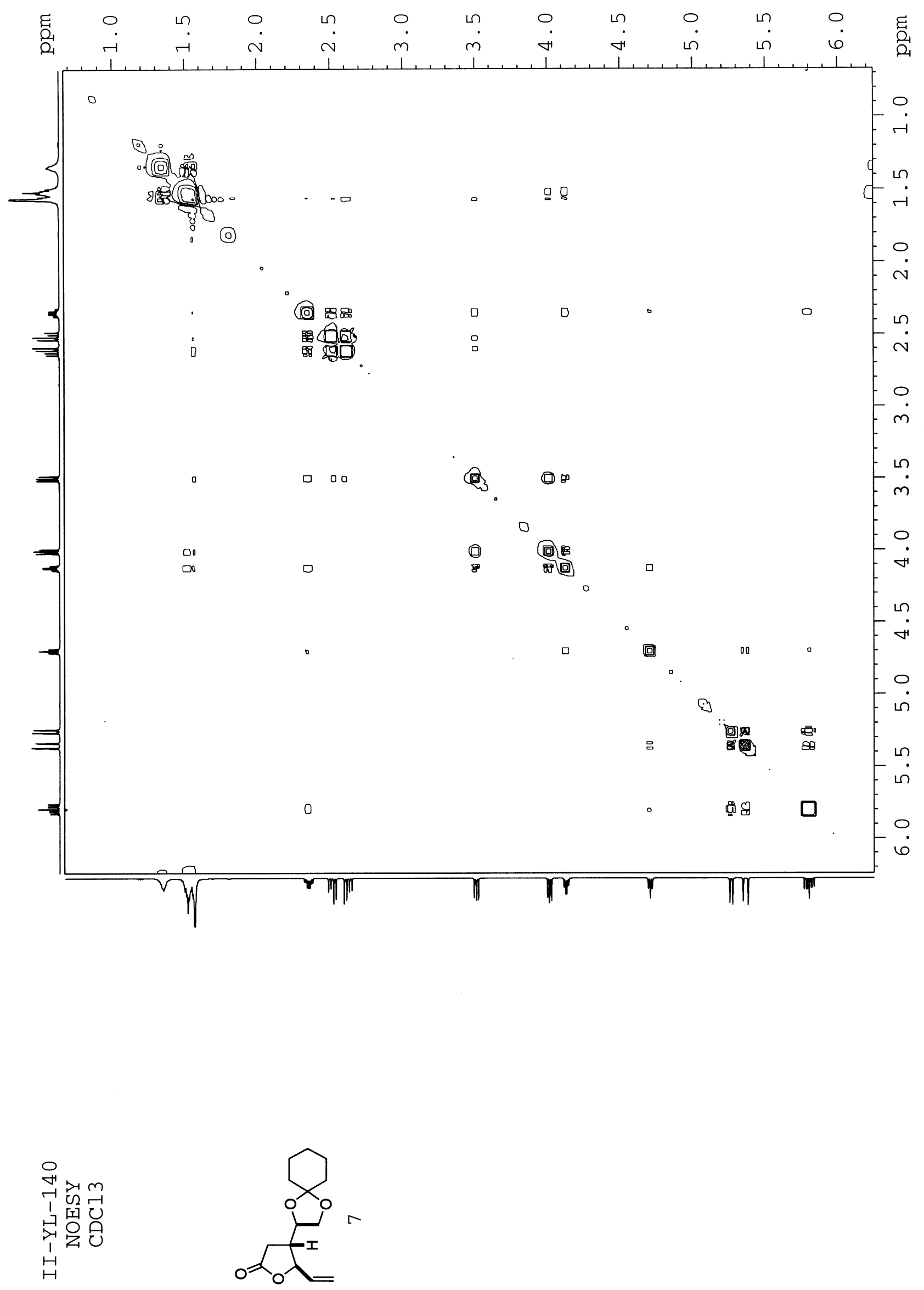


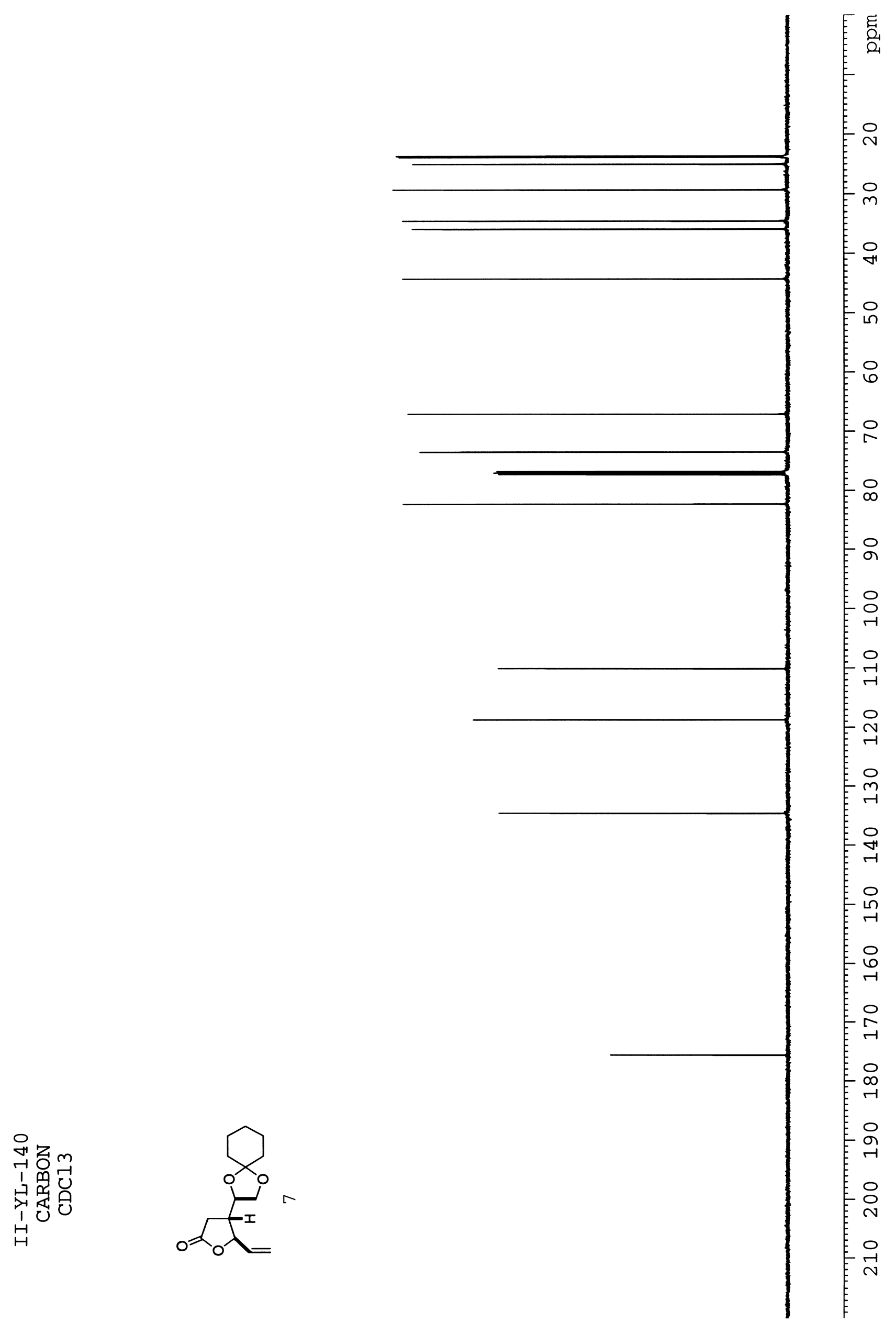




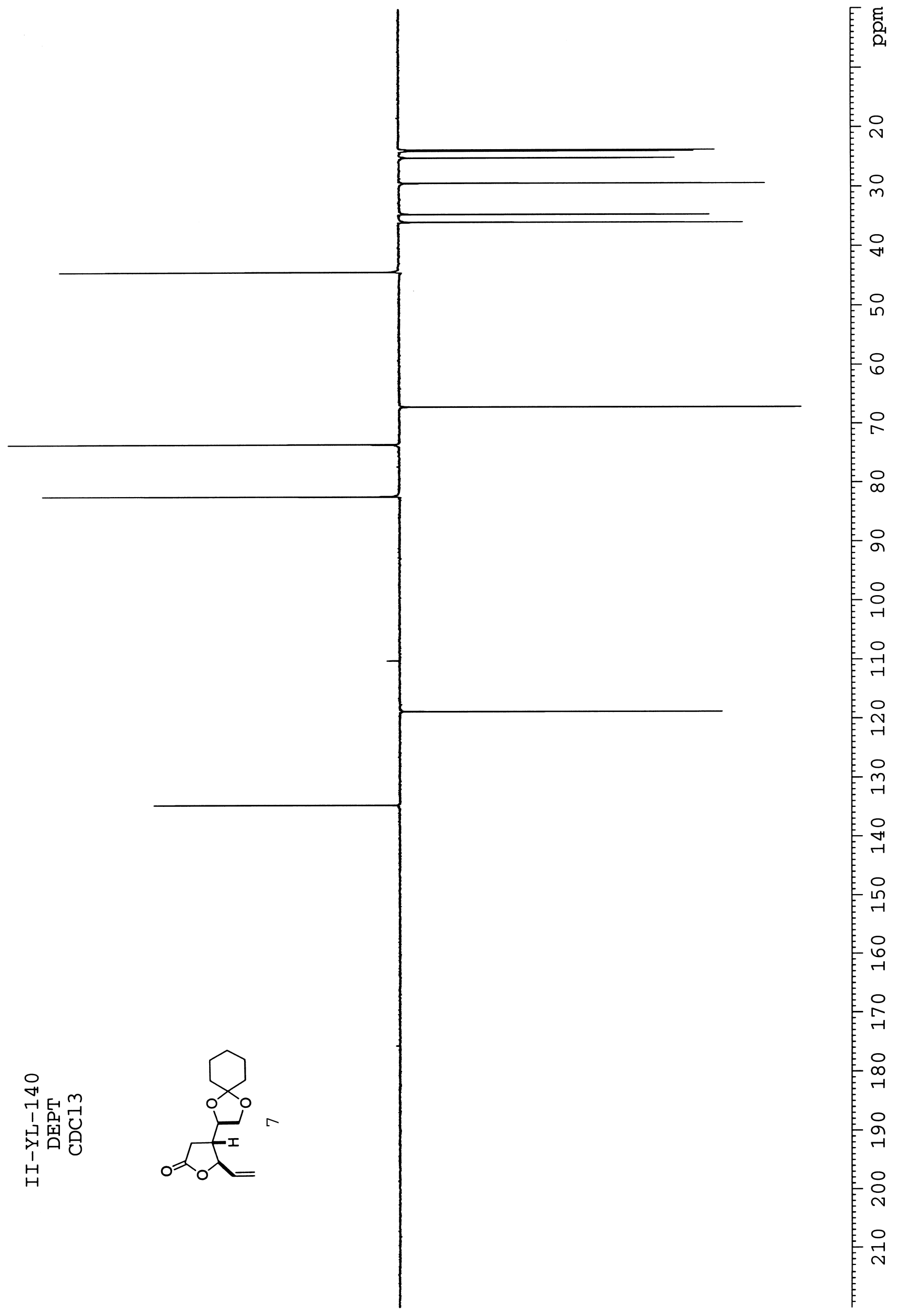



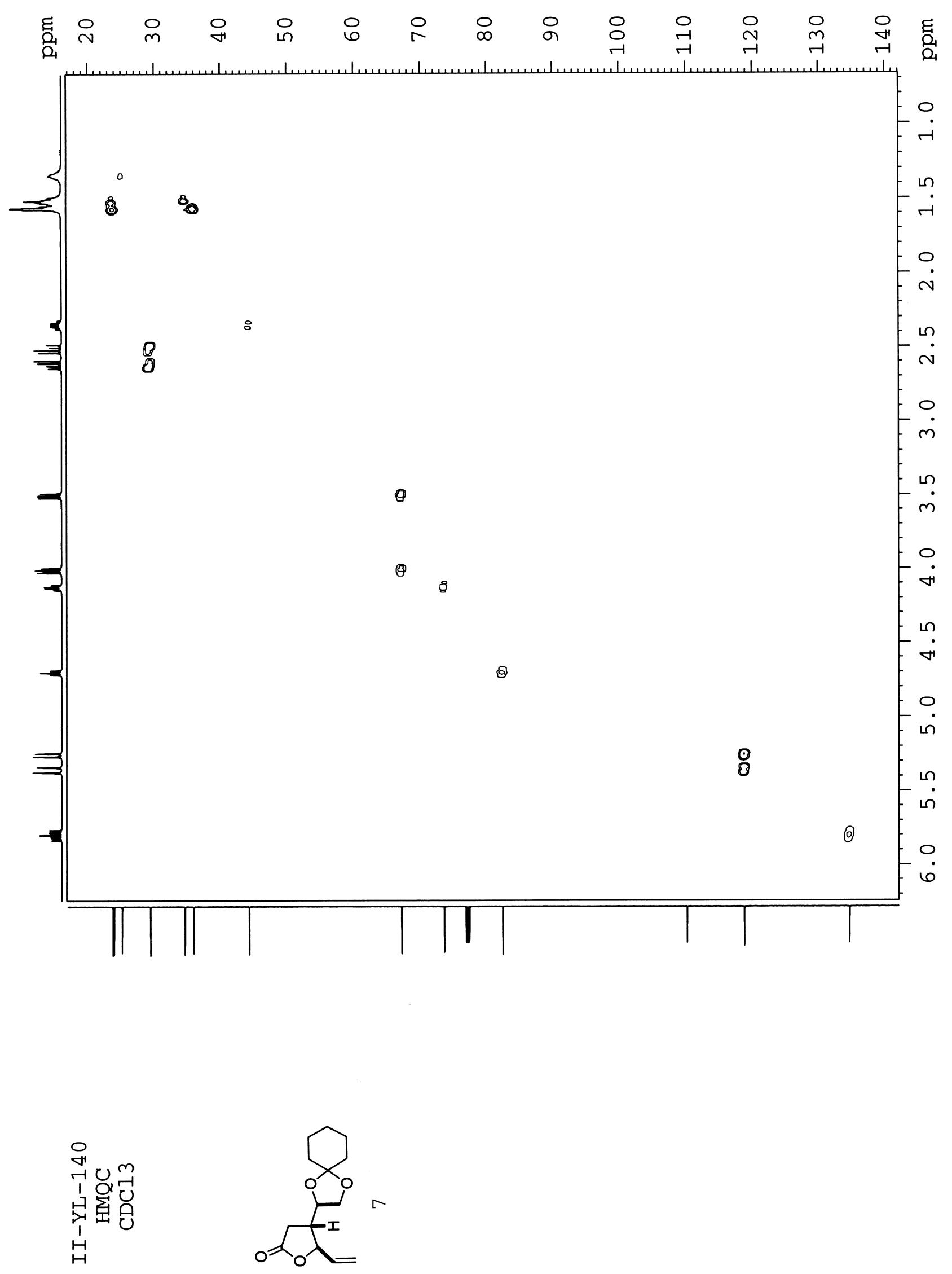


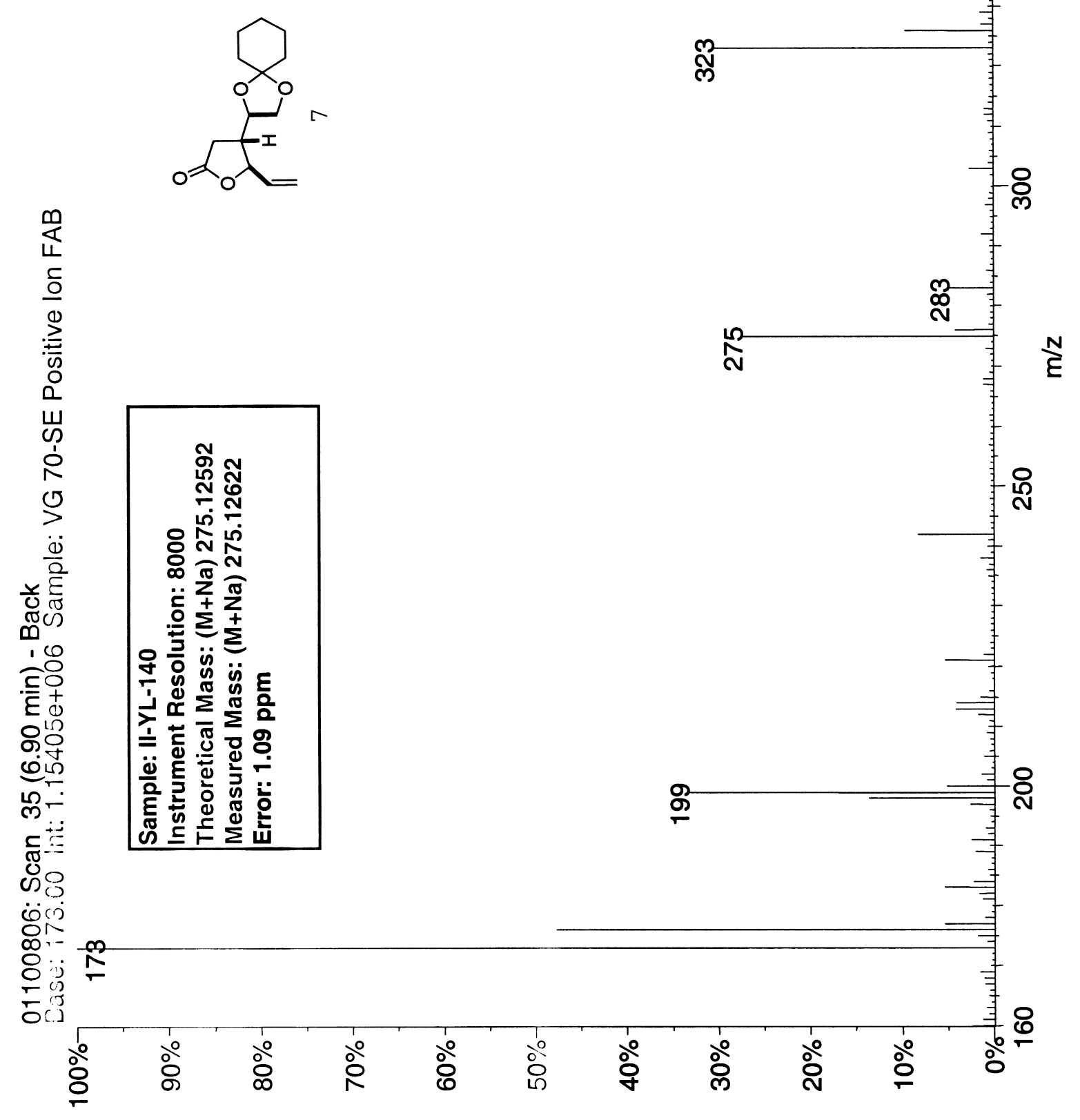




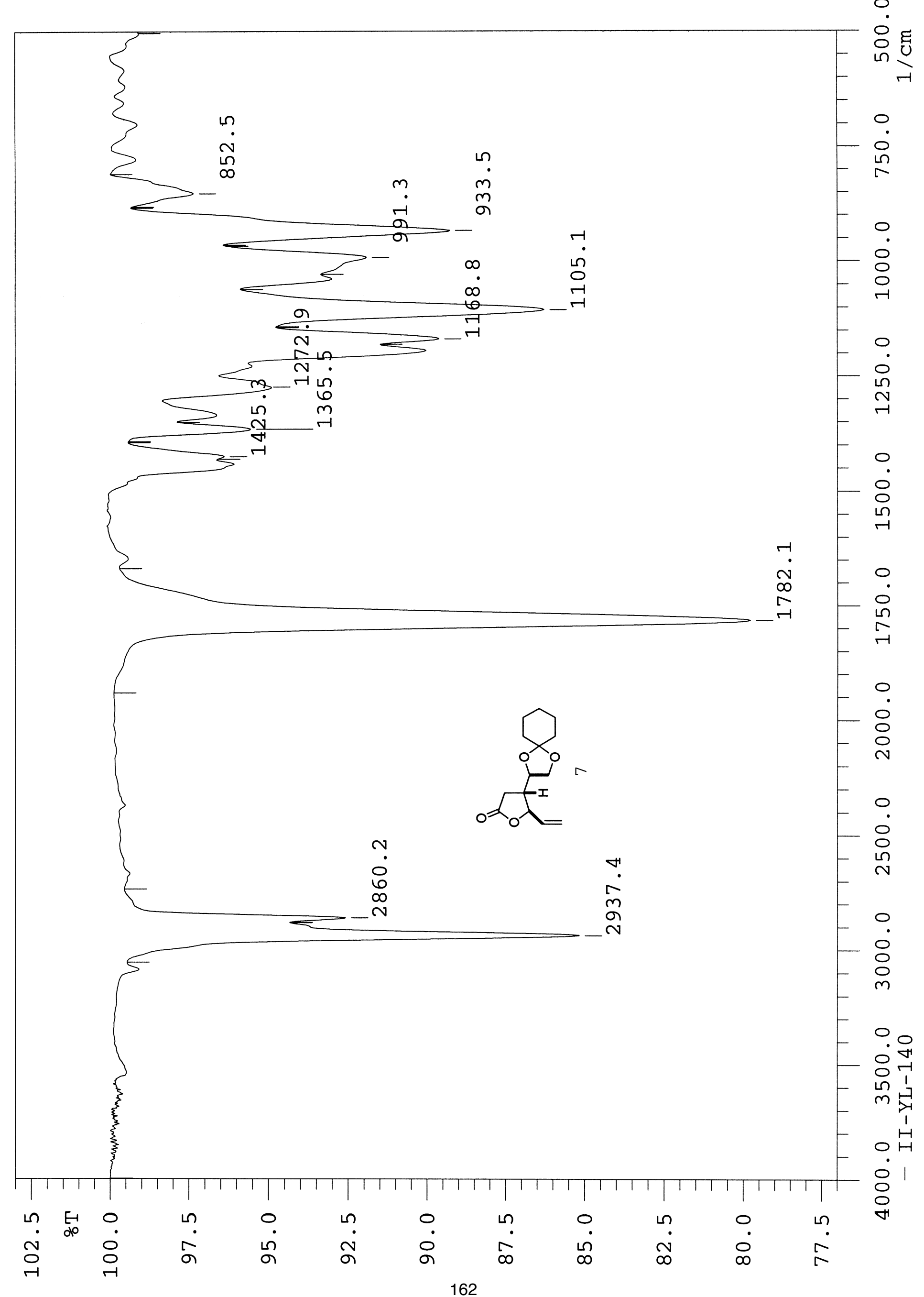




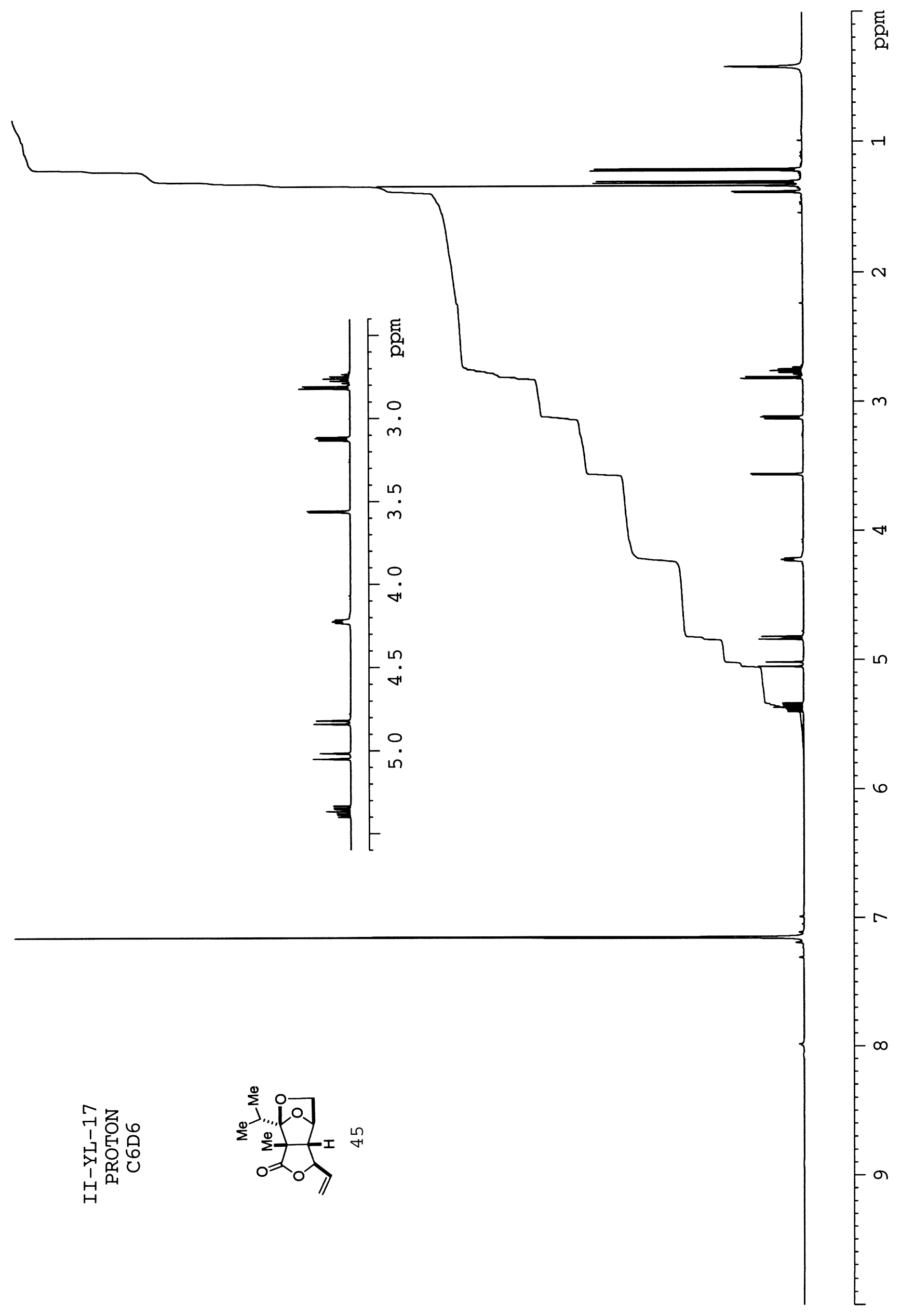




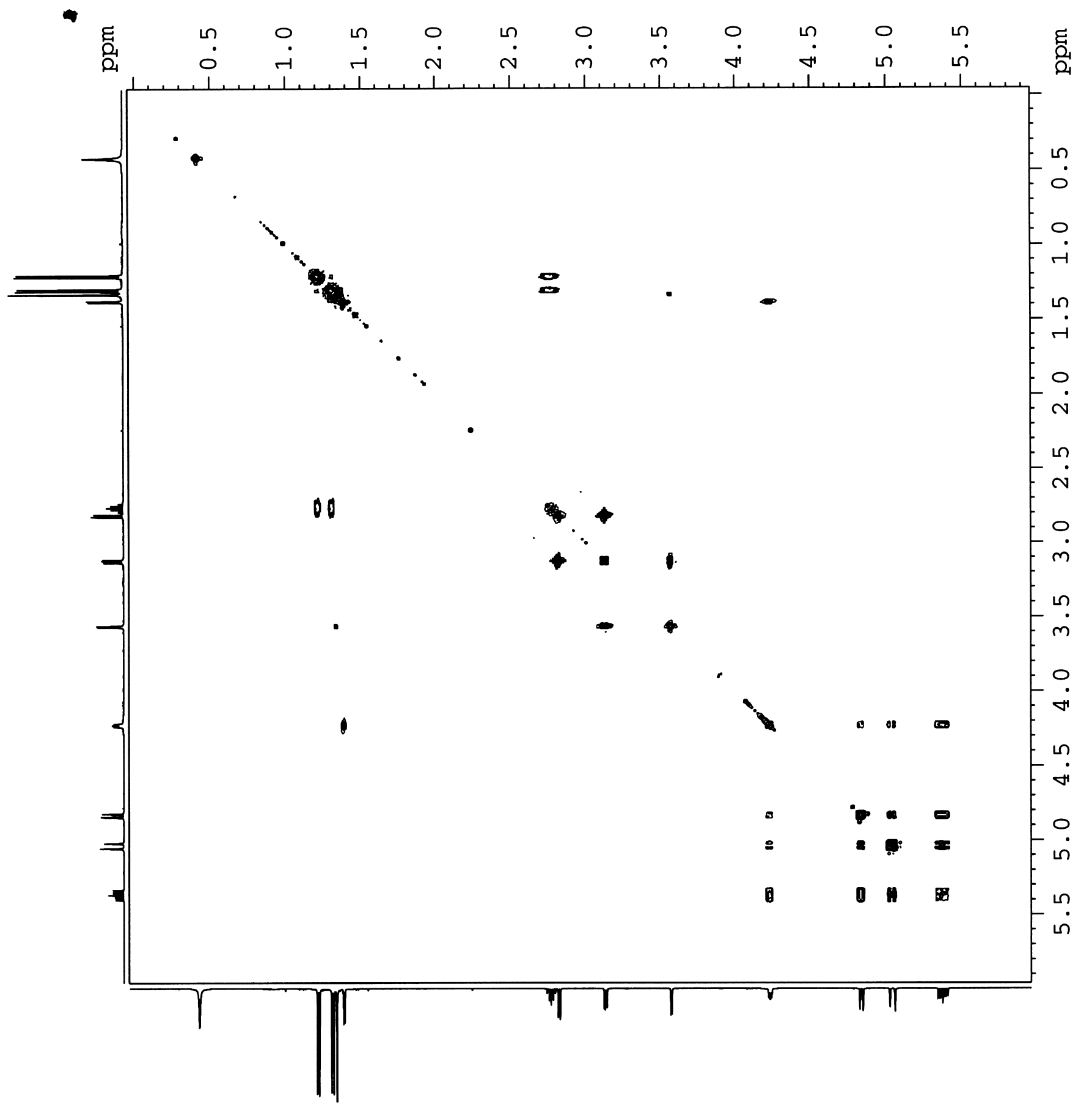

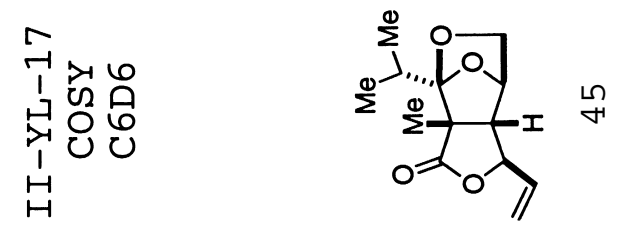




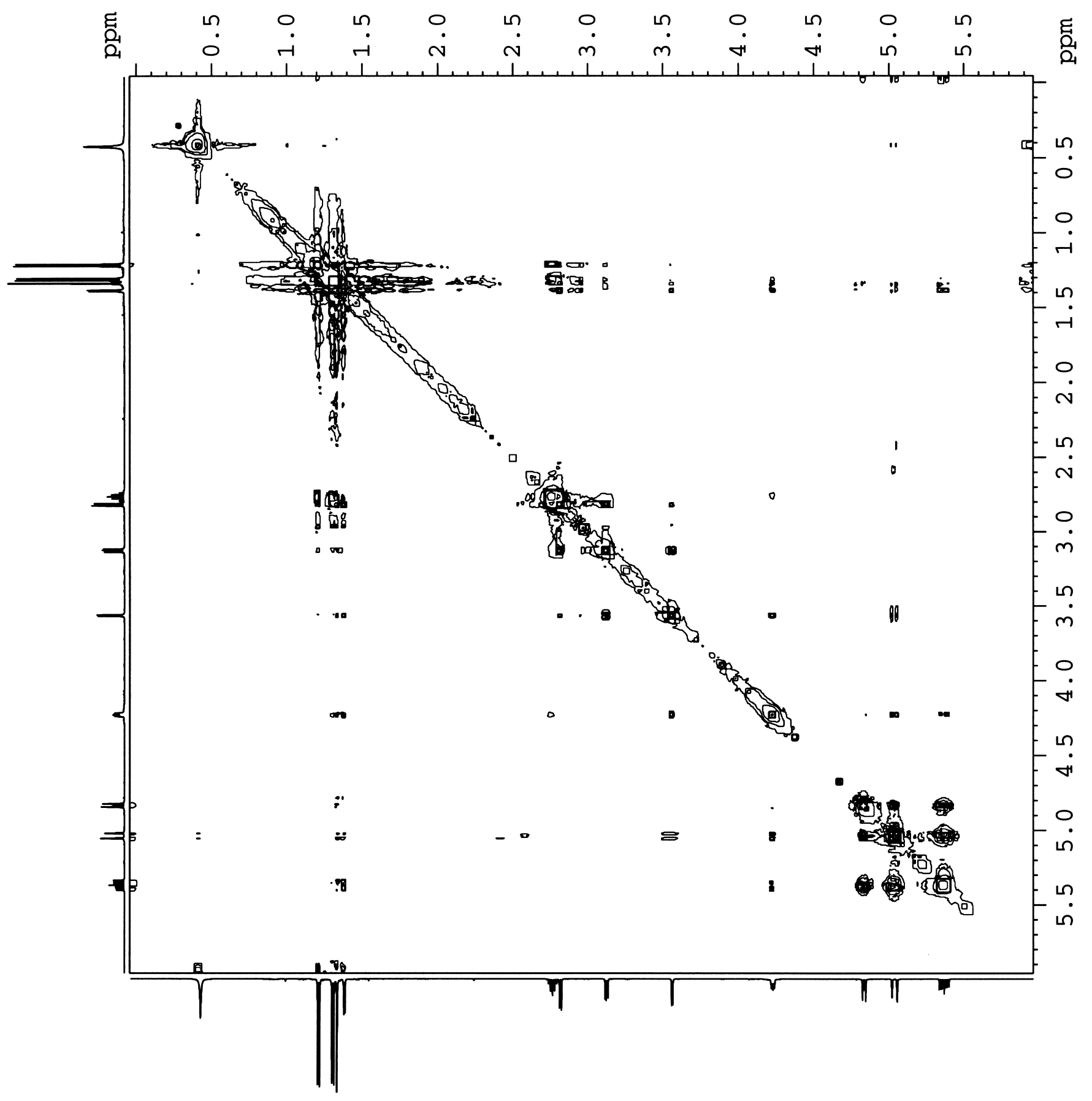

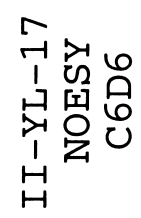

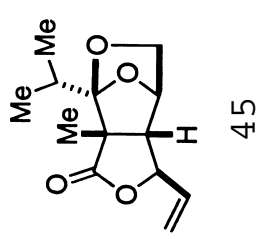




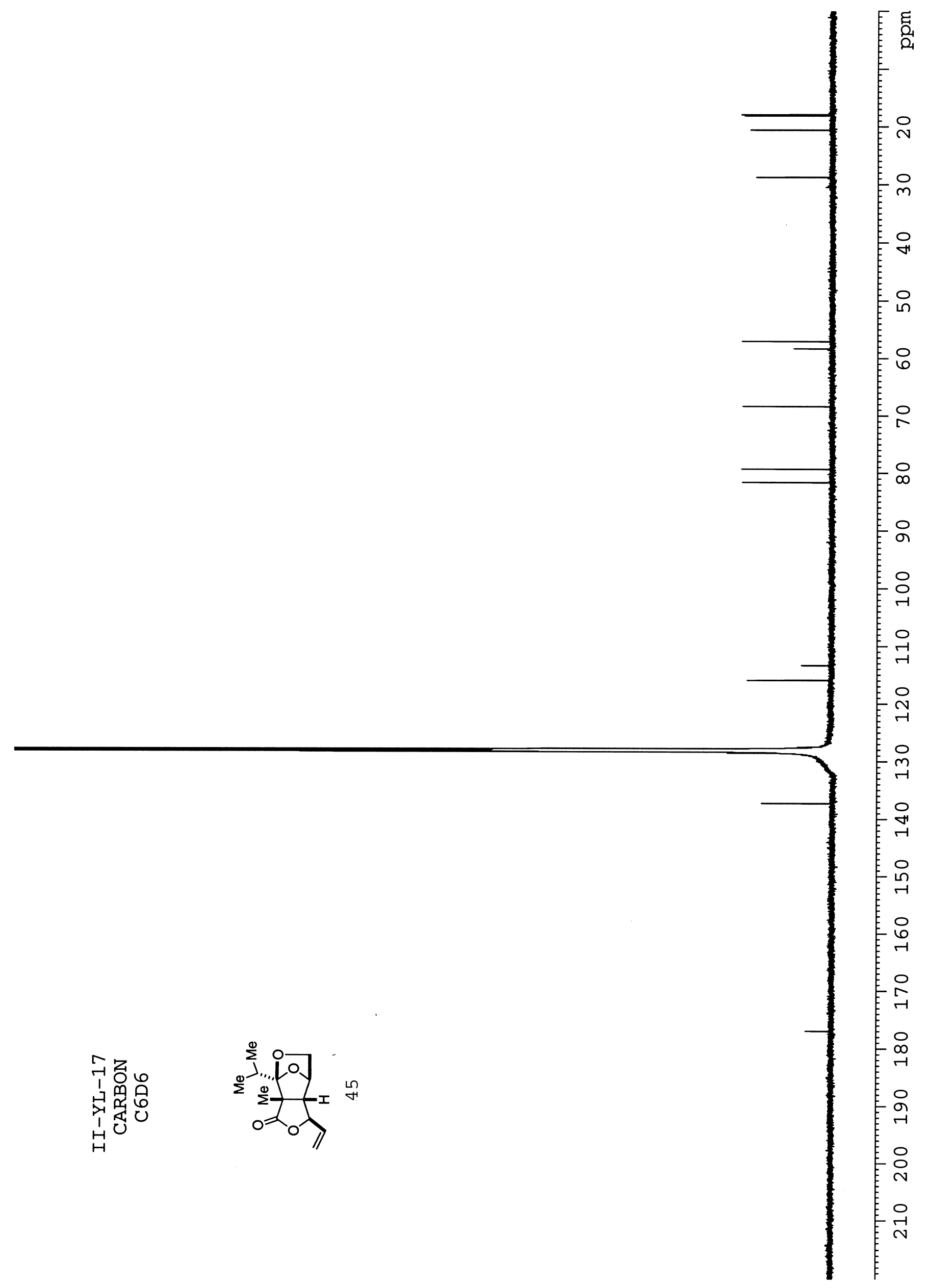




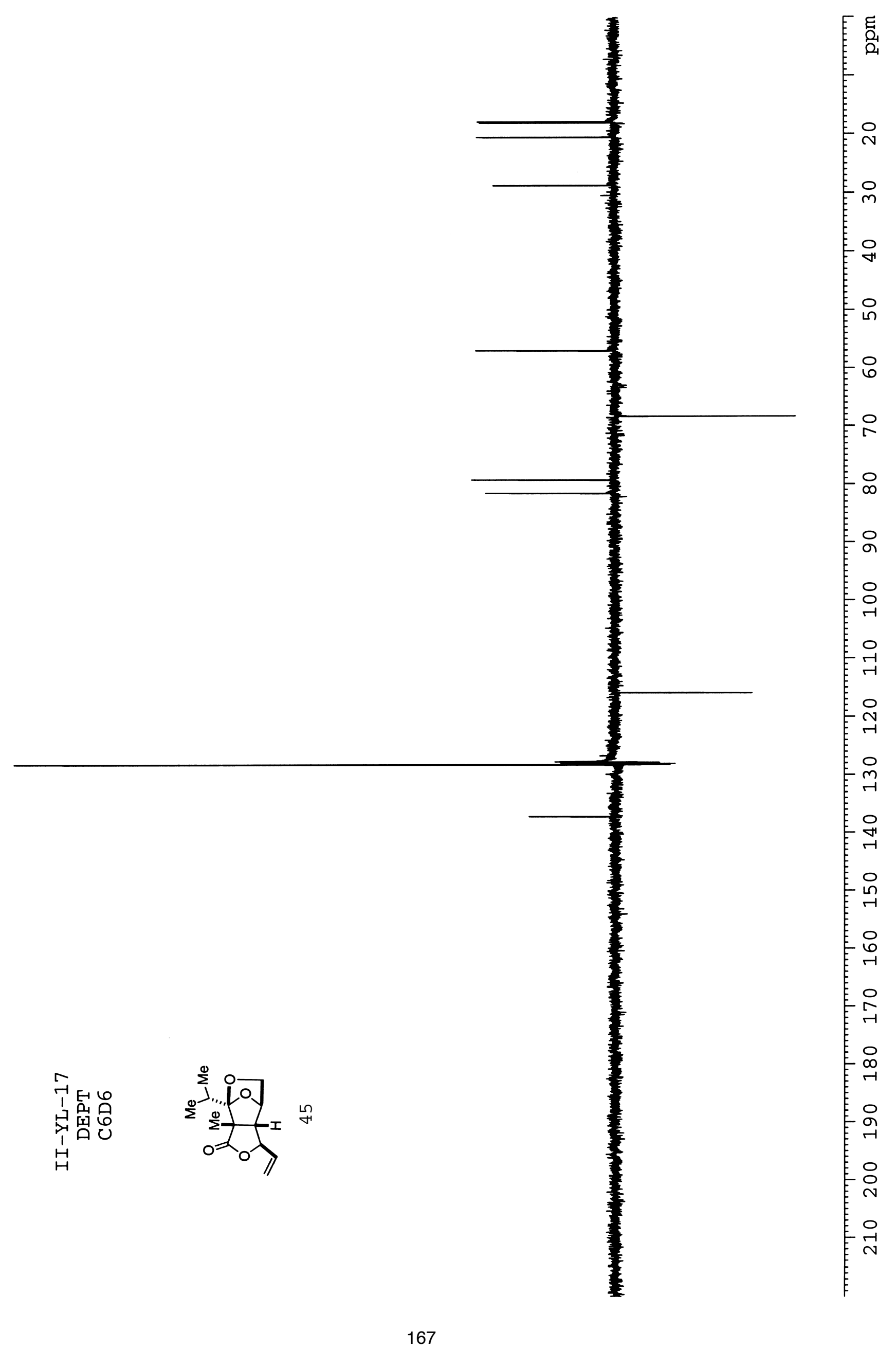



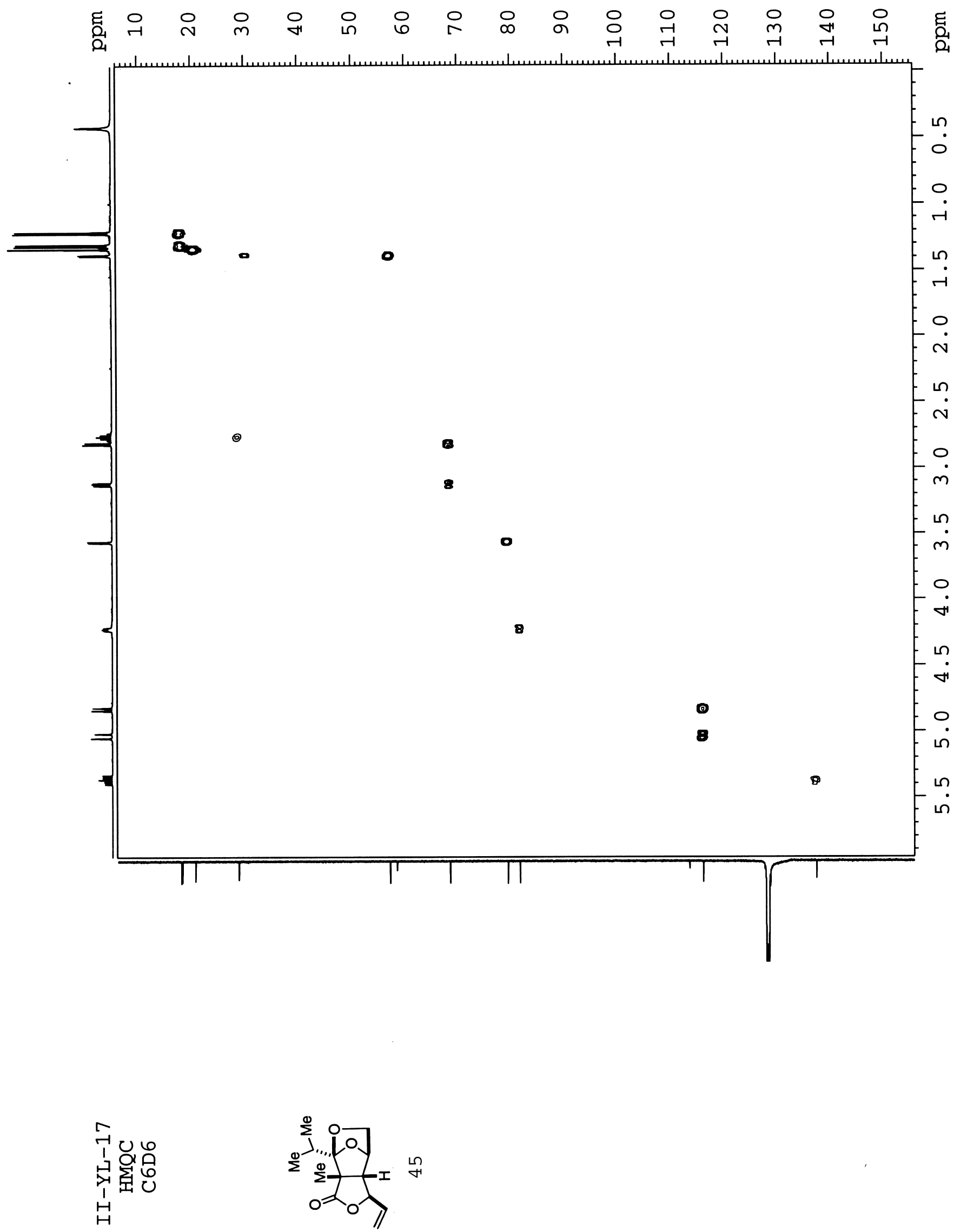


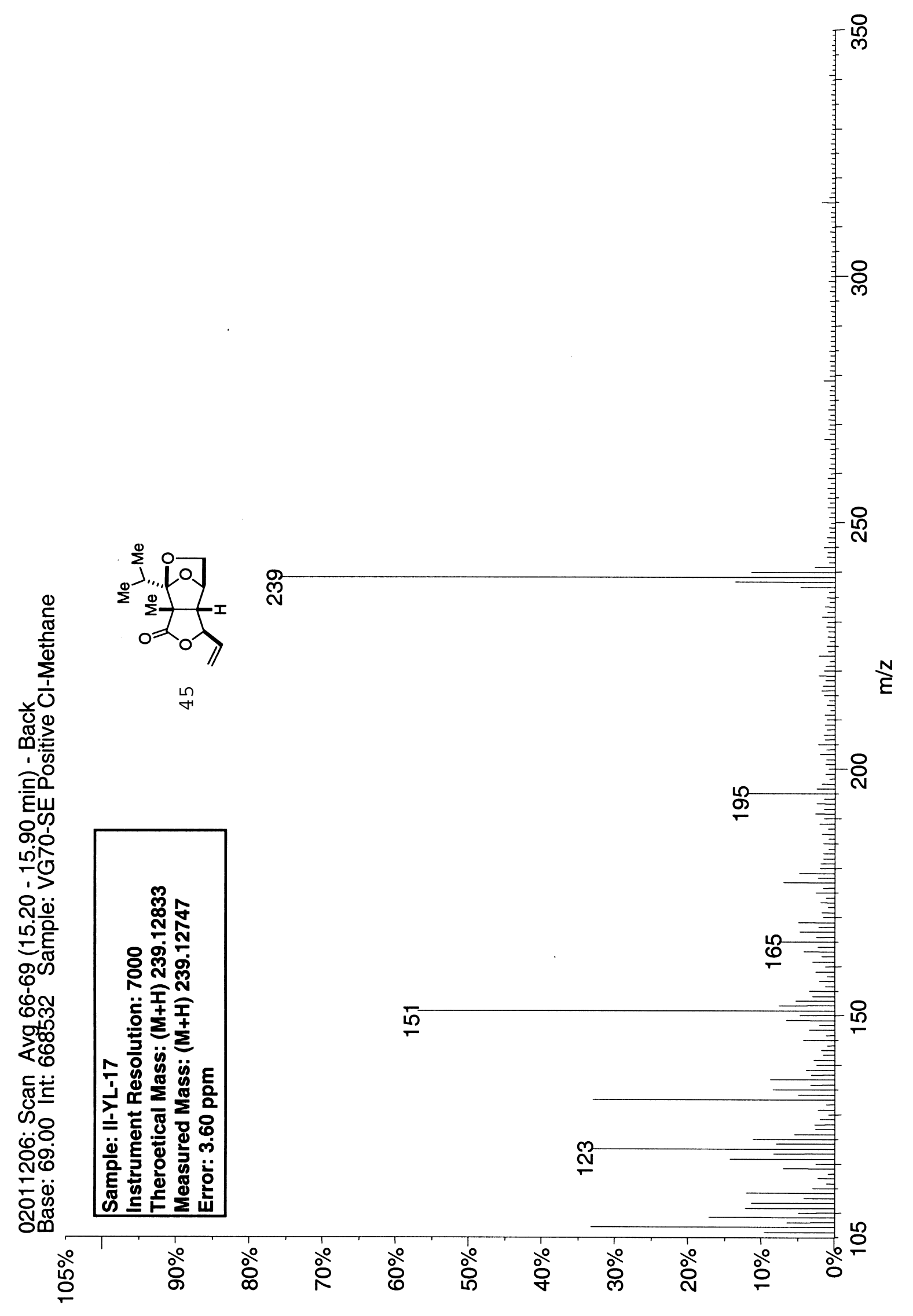




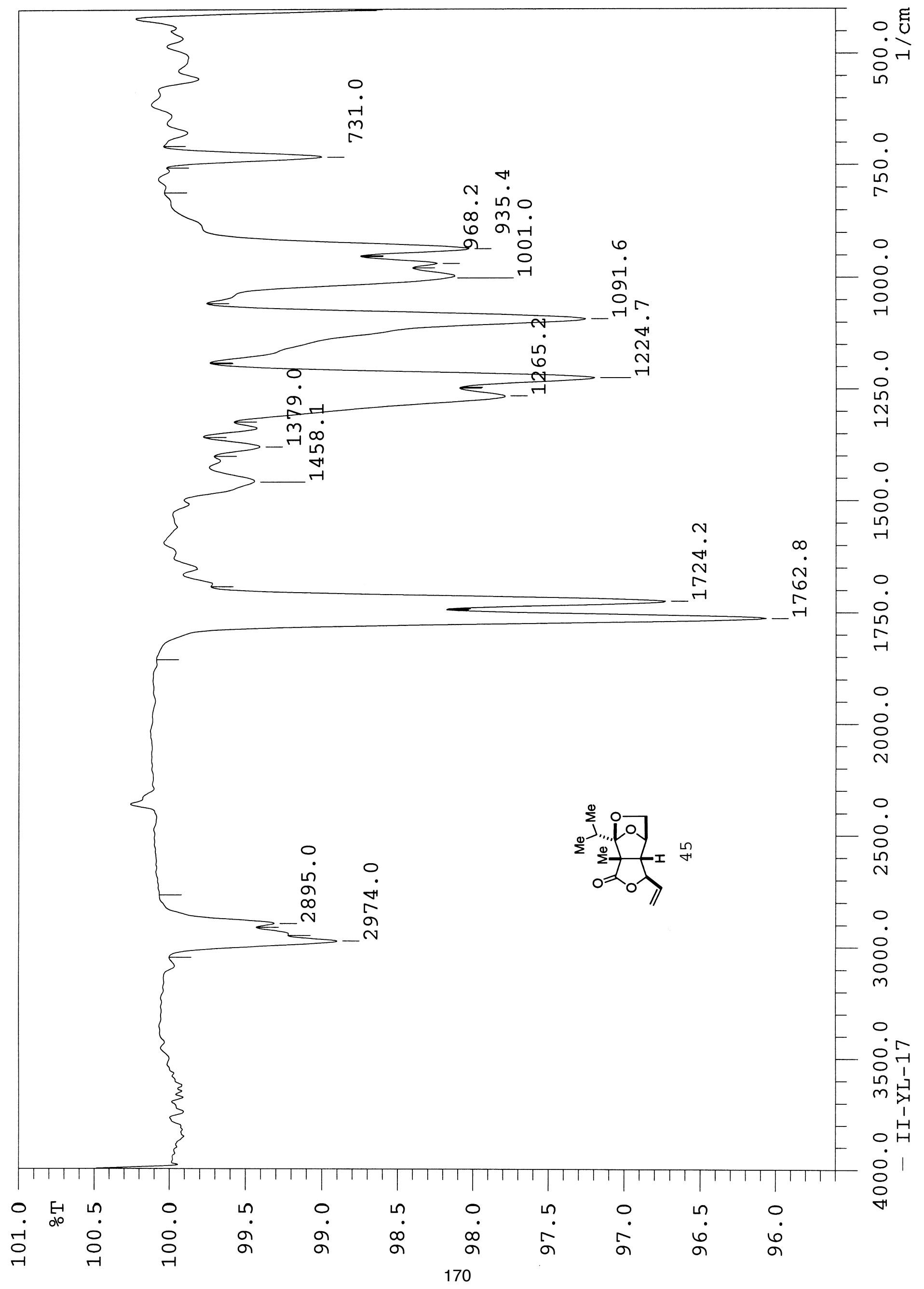




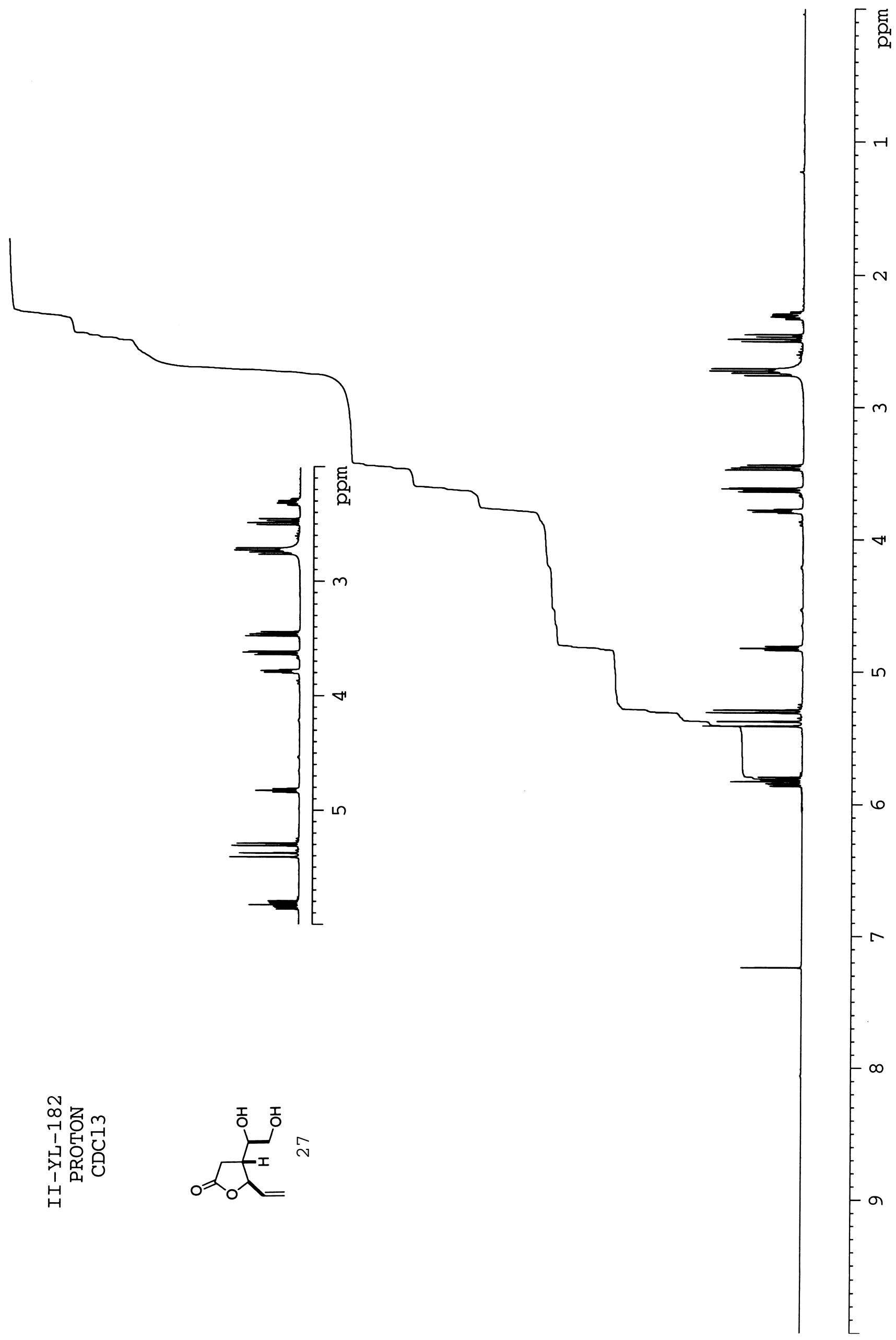




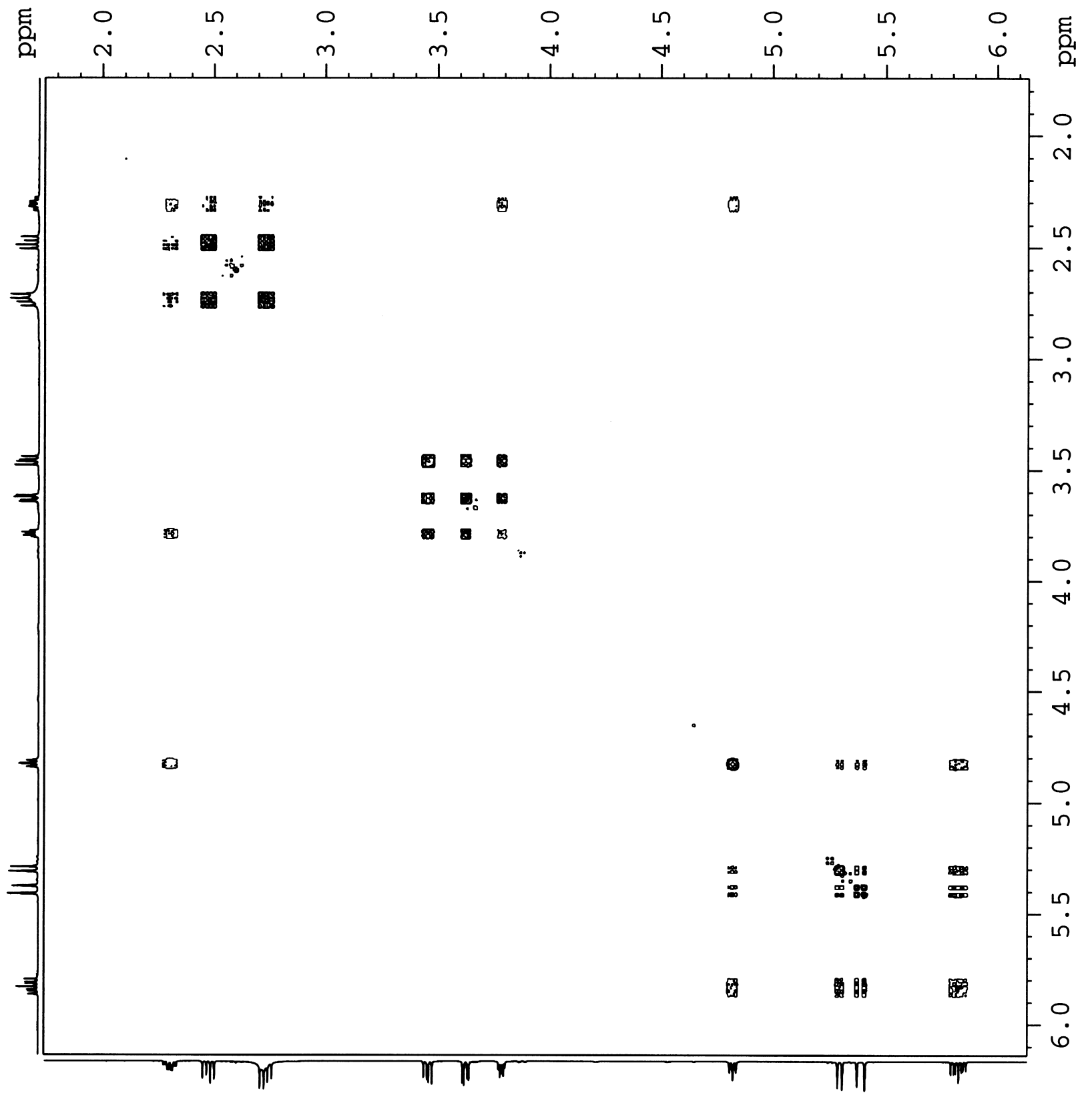

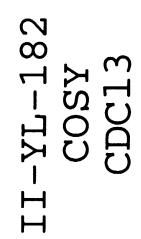

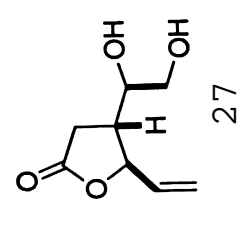




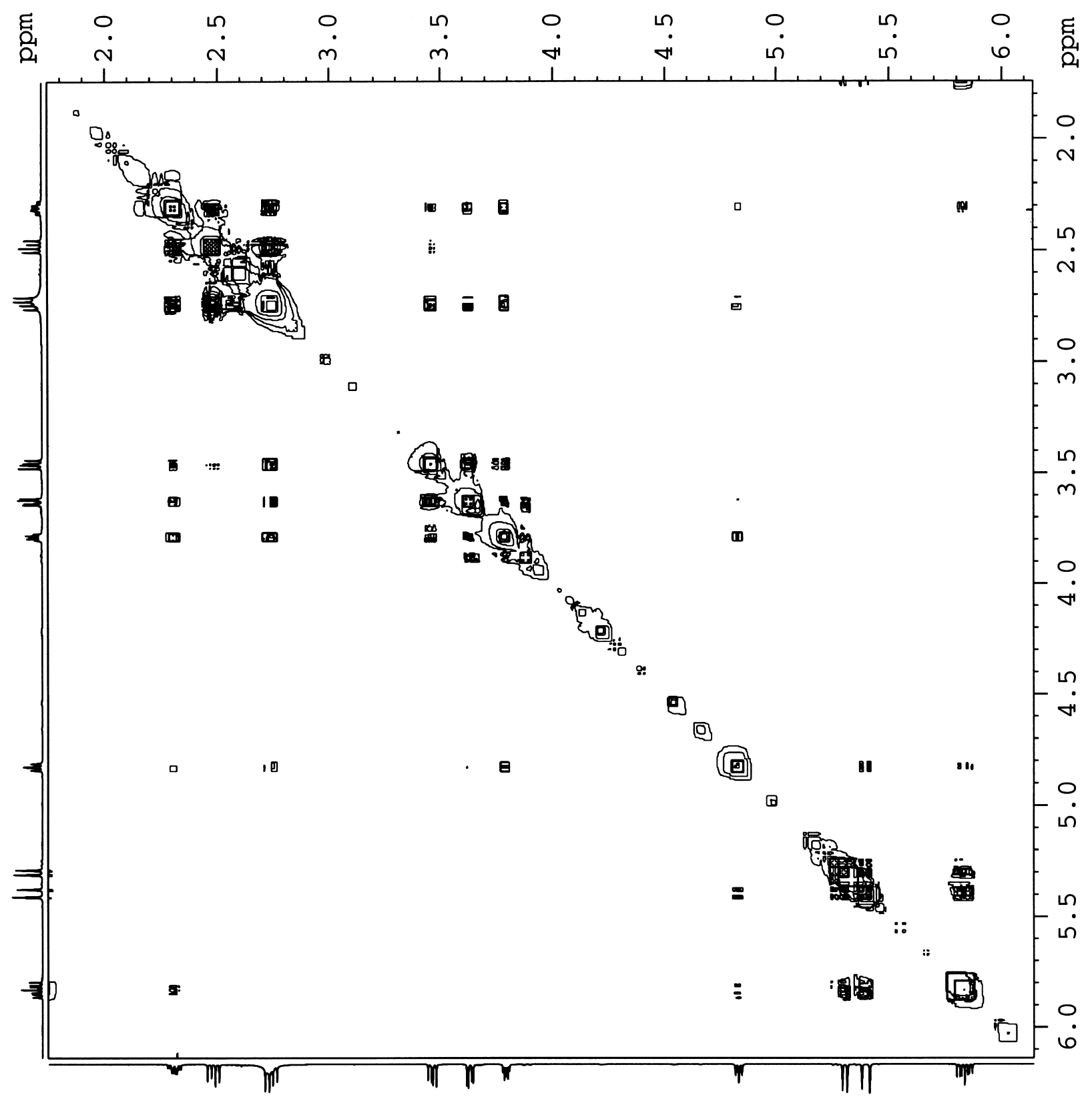

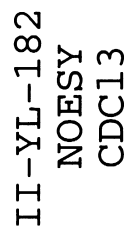

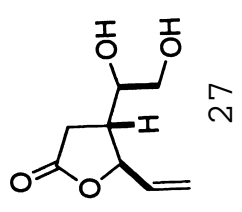




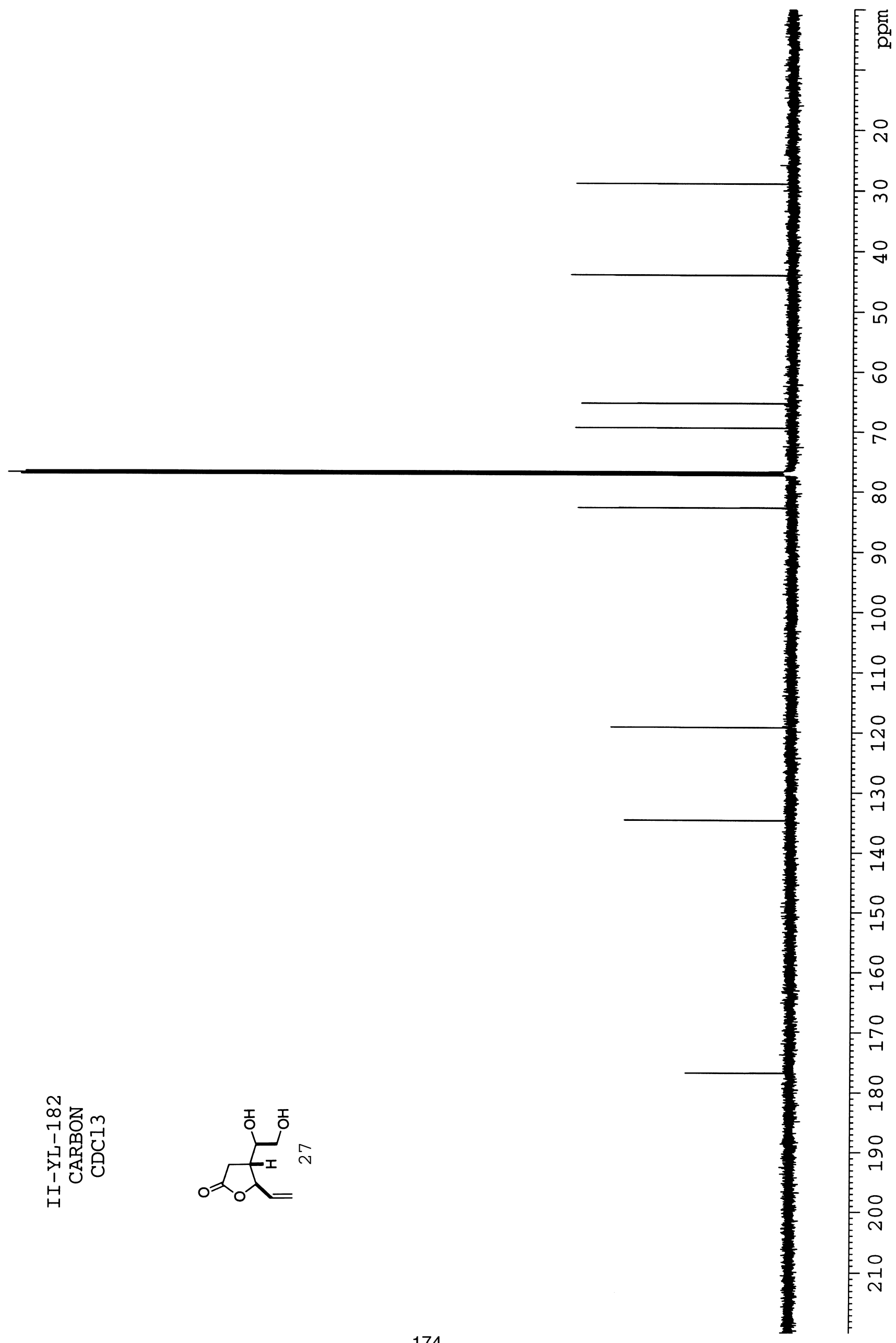




$$
\text { . }
$$



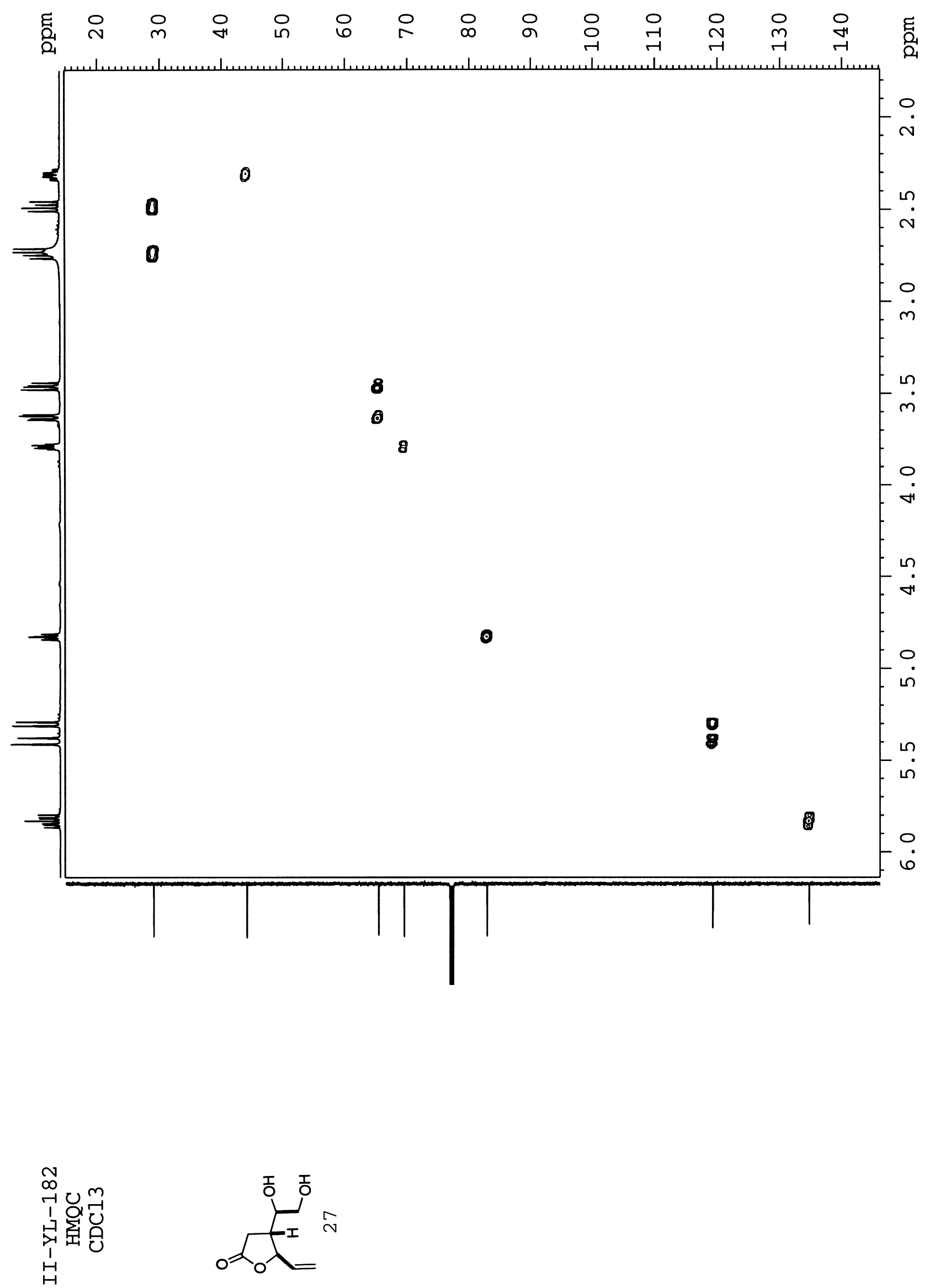


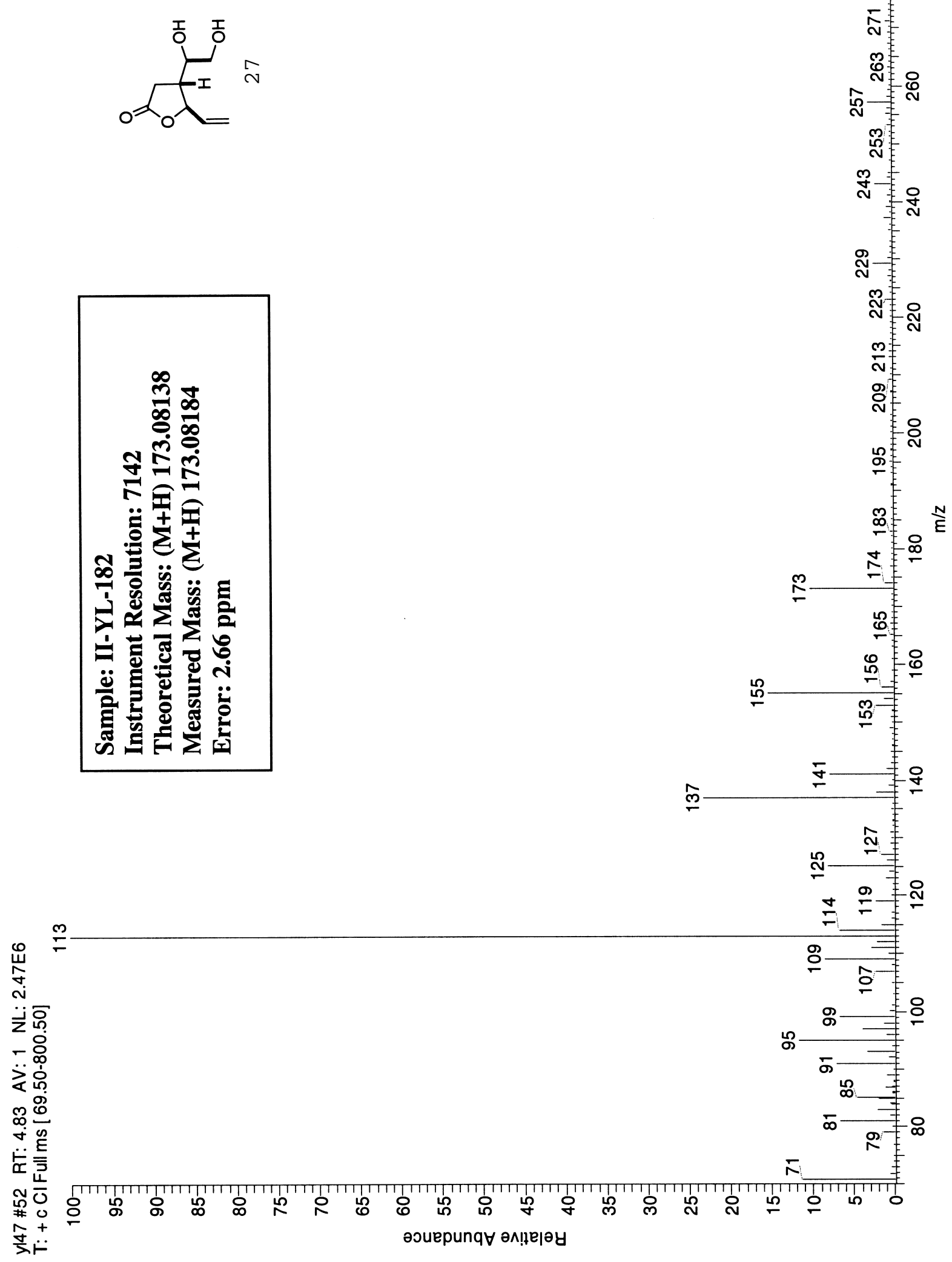




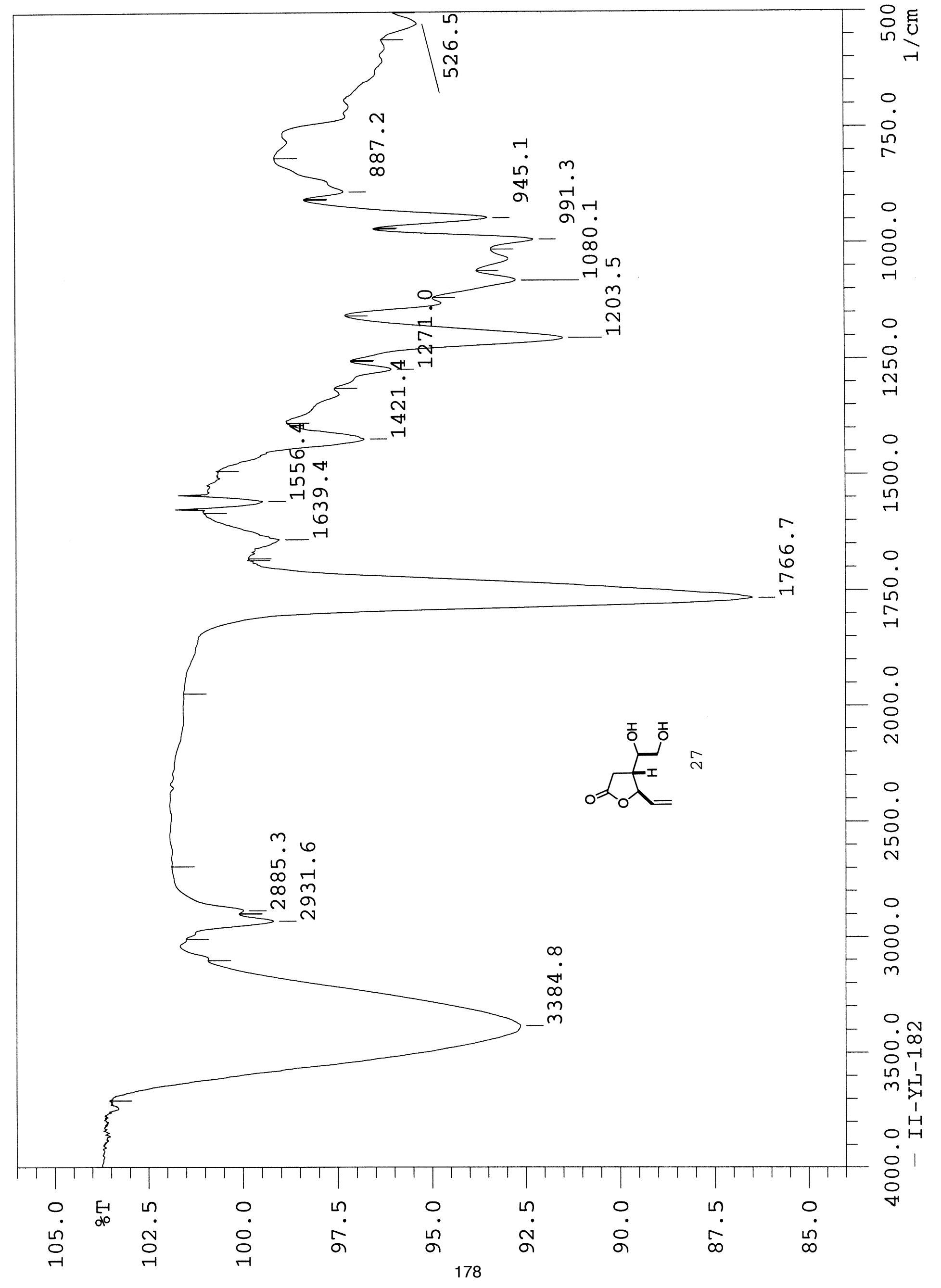




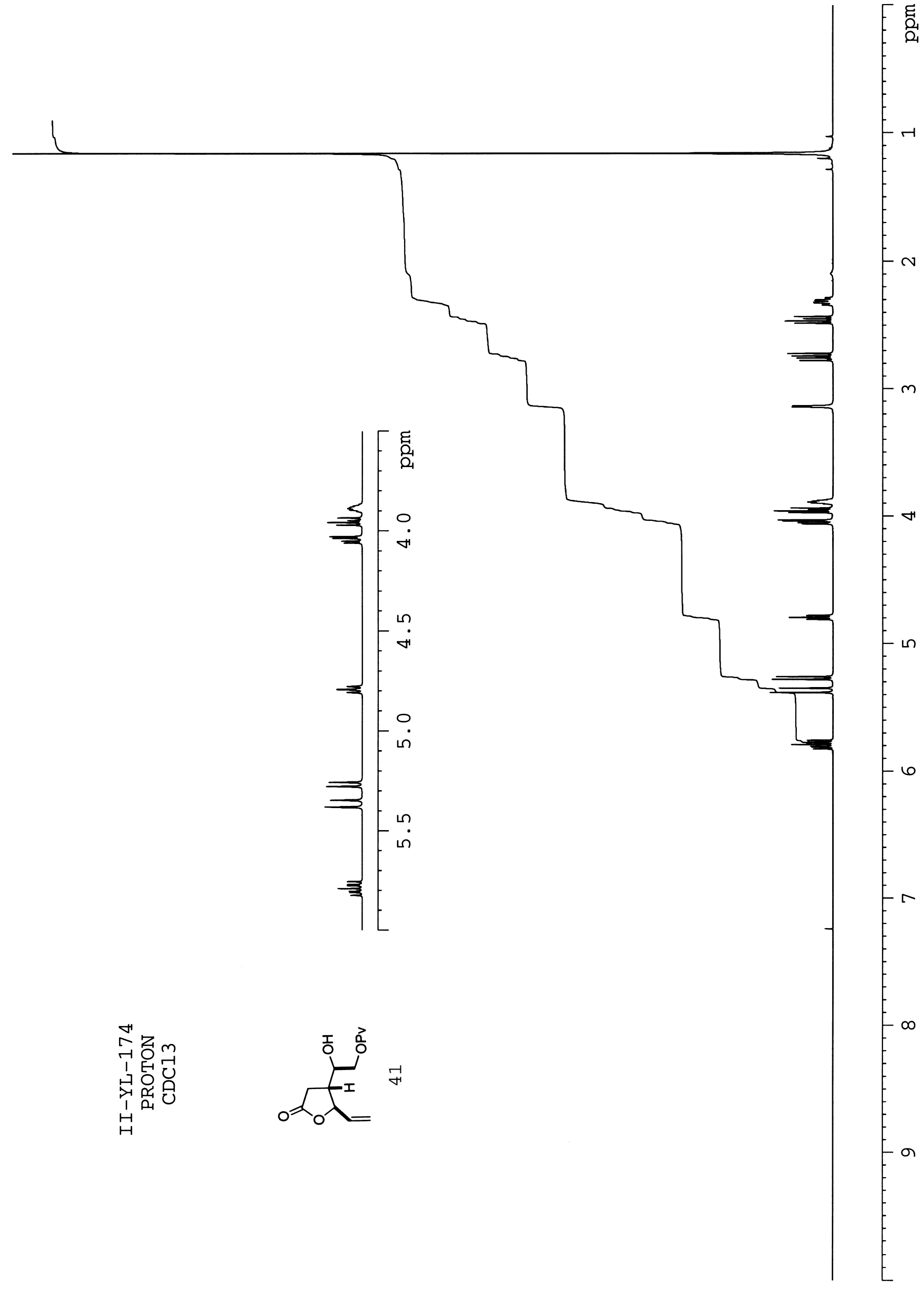




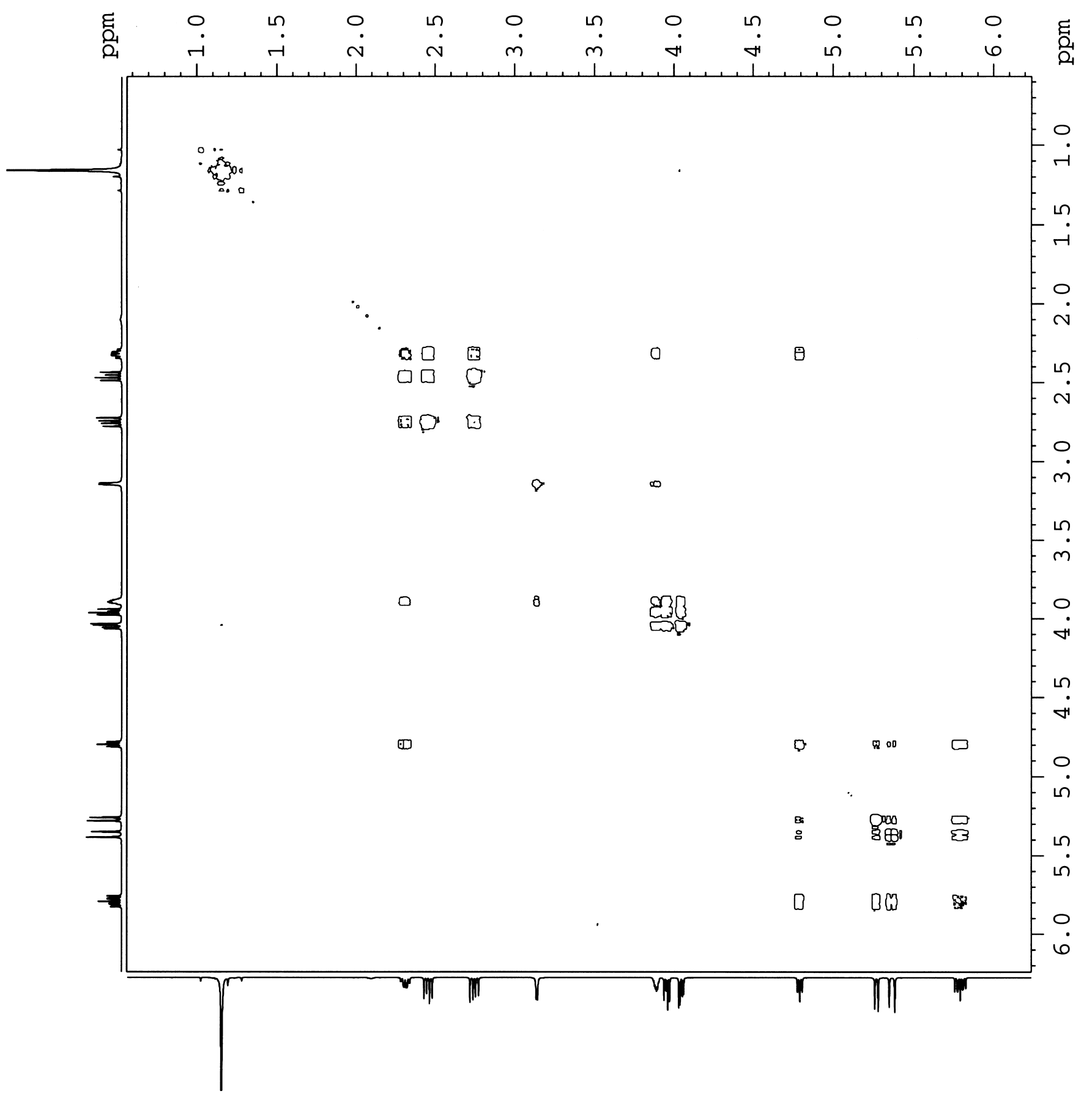

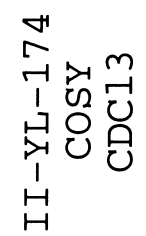

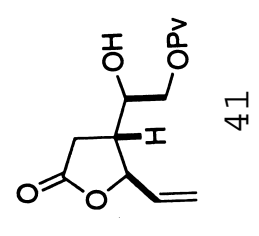



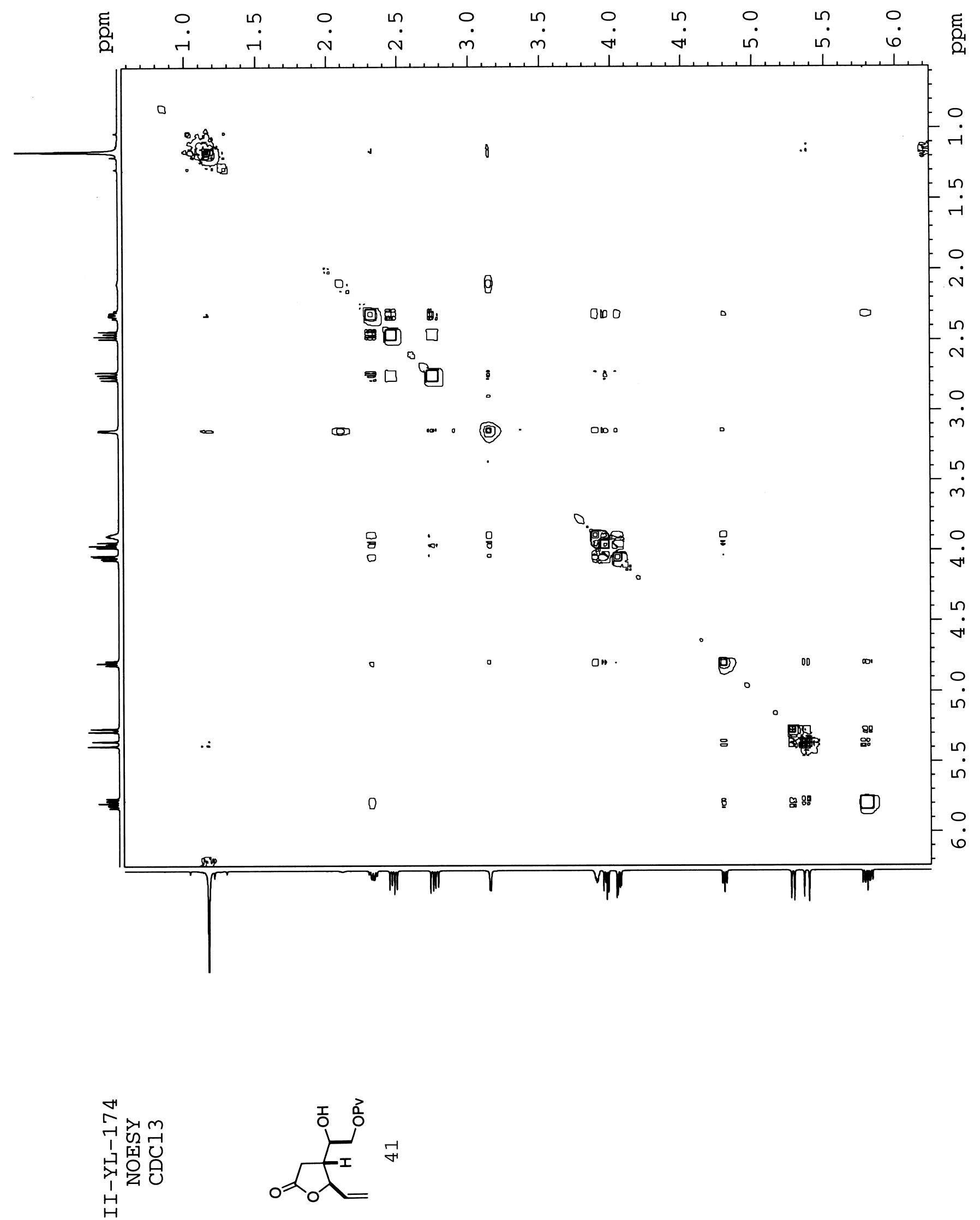


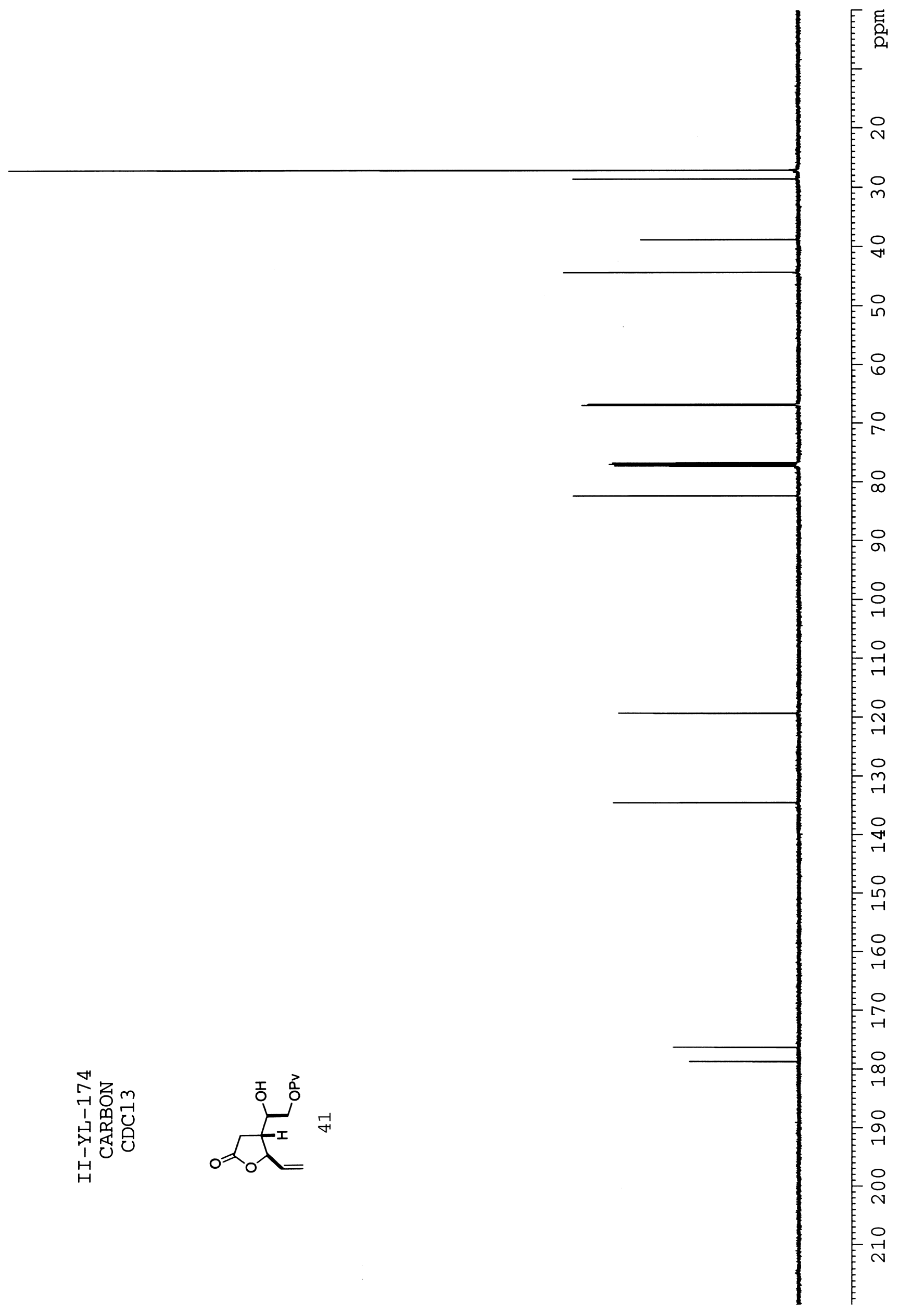




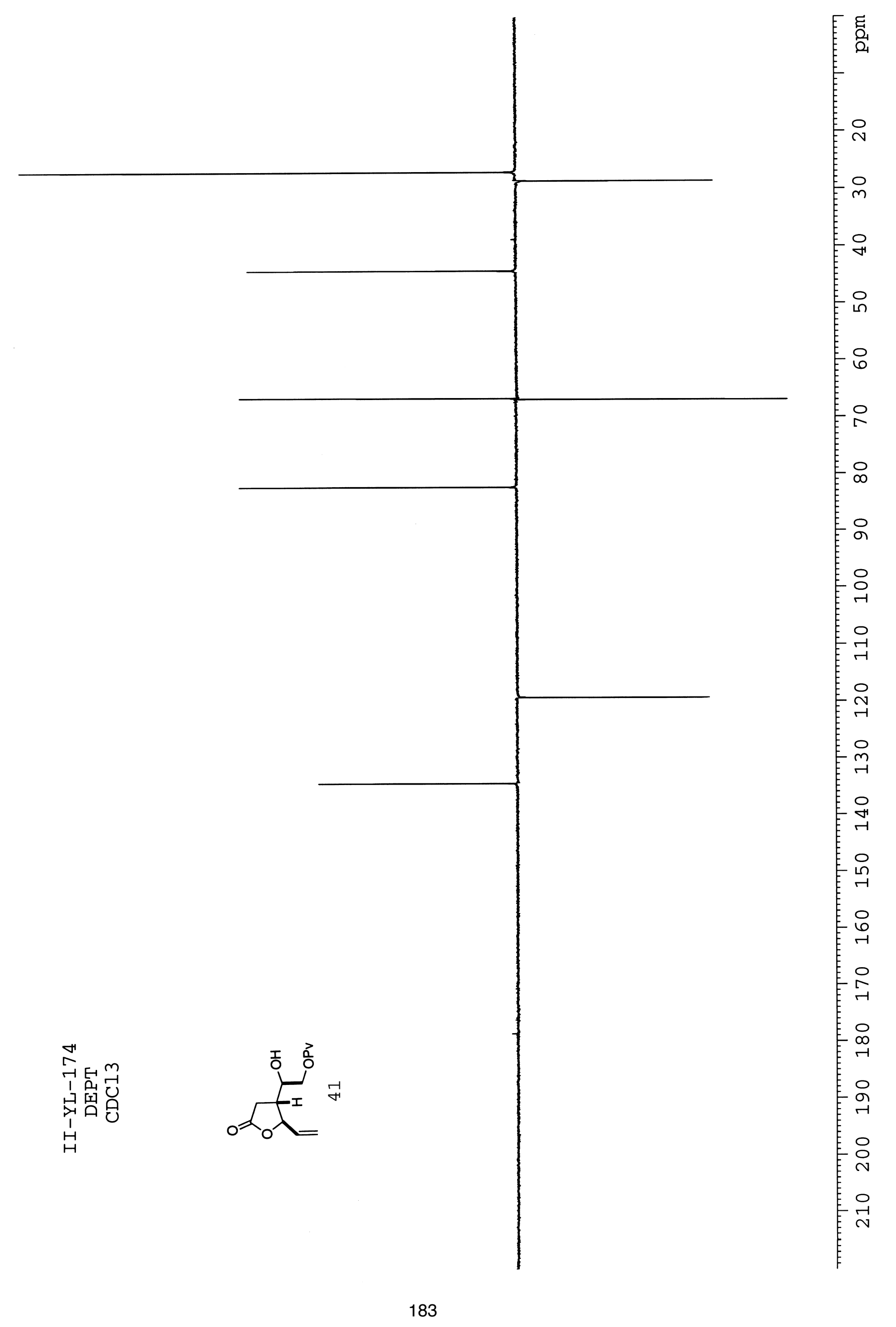




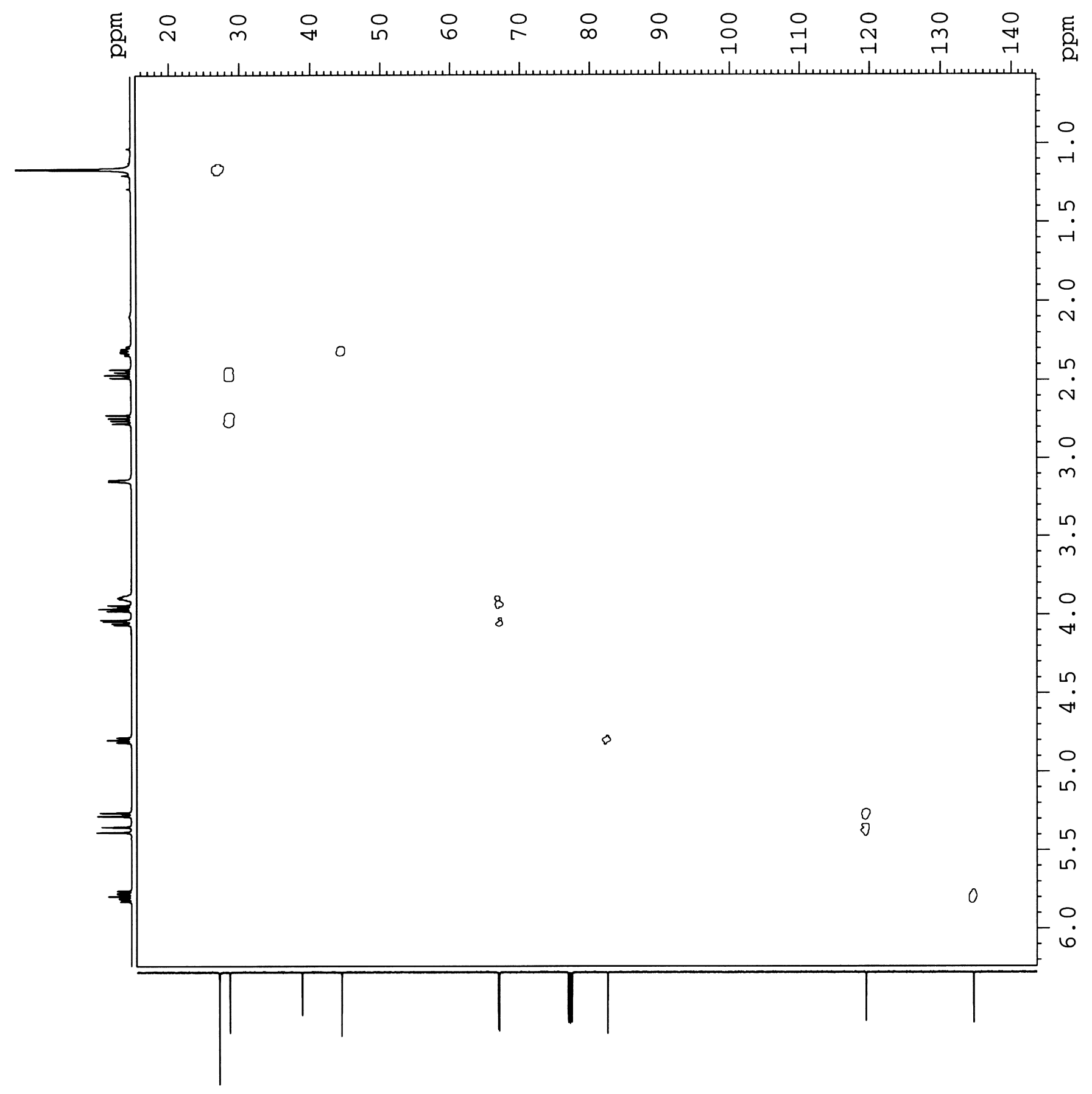

怘界 

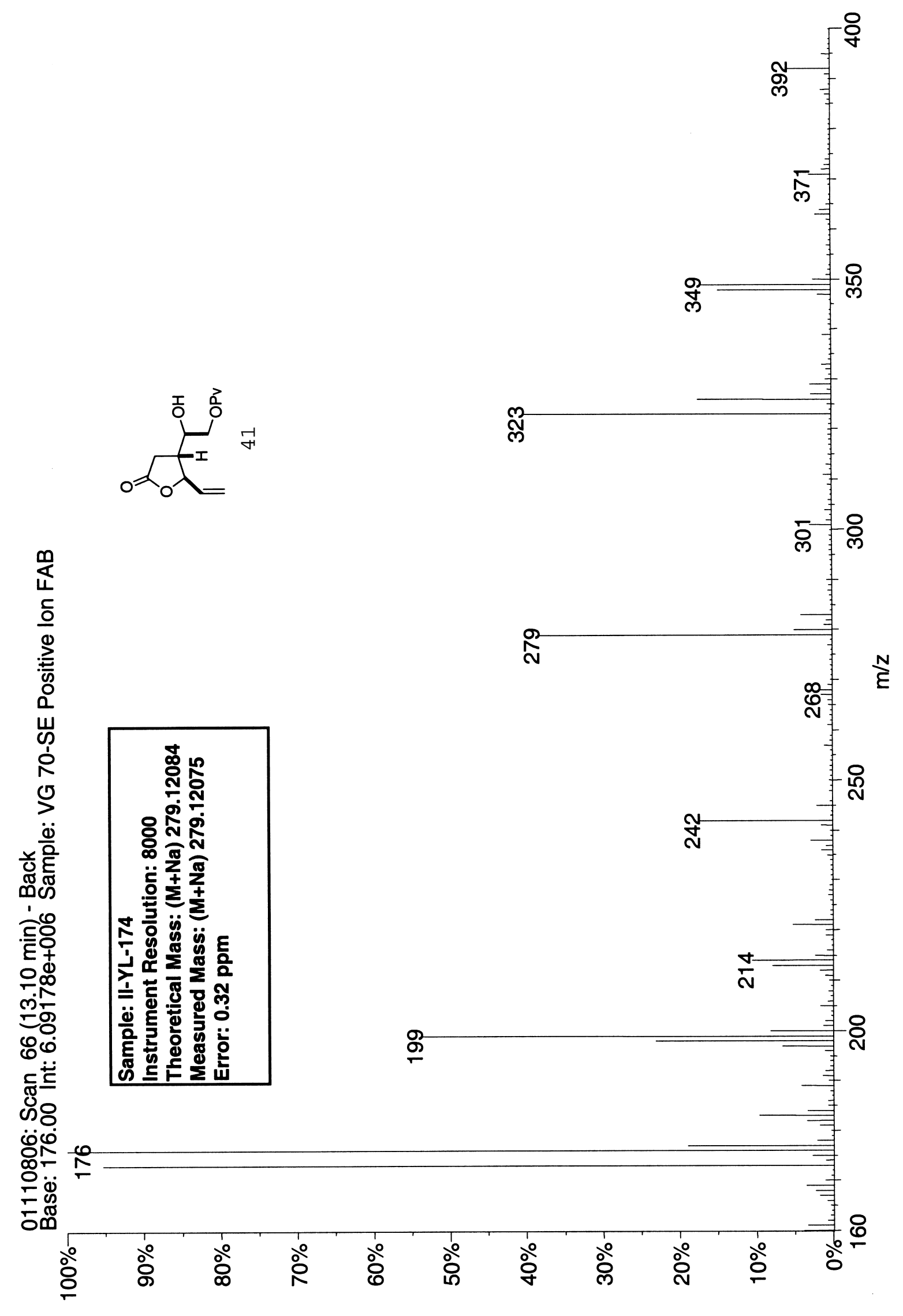


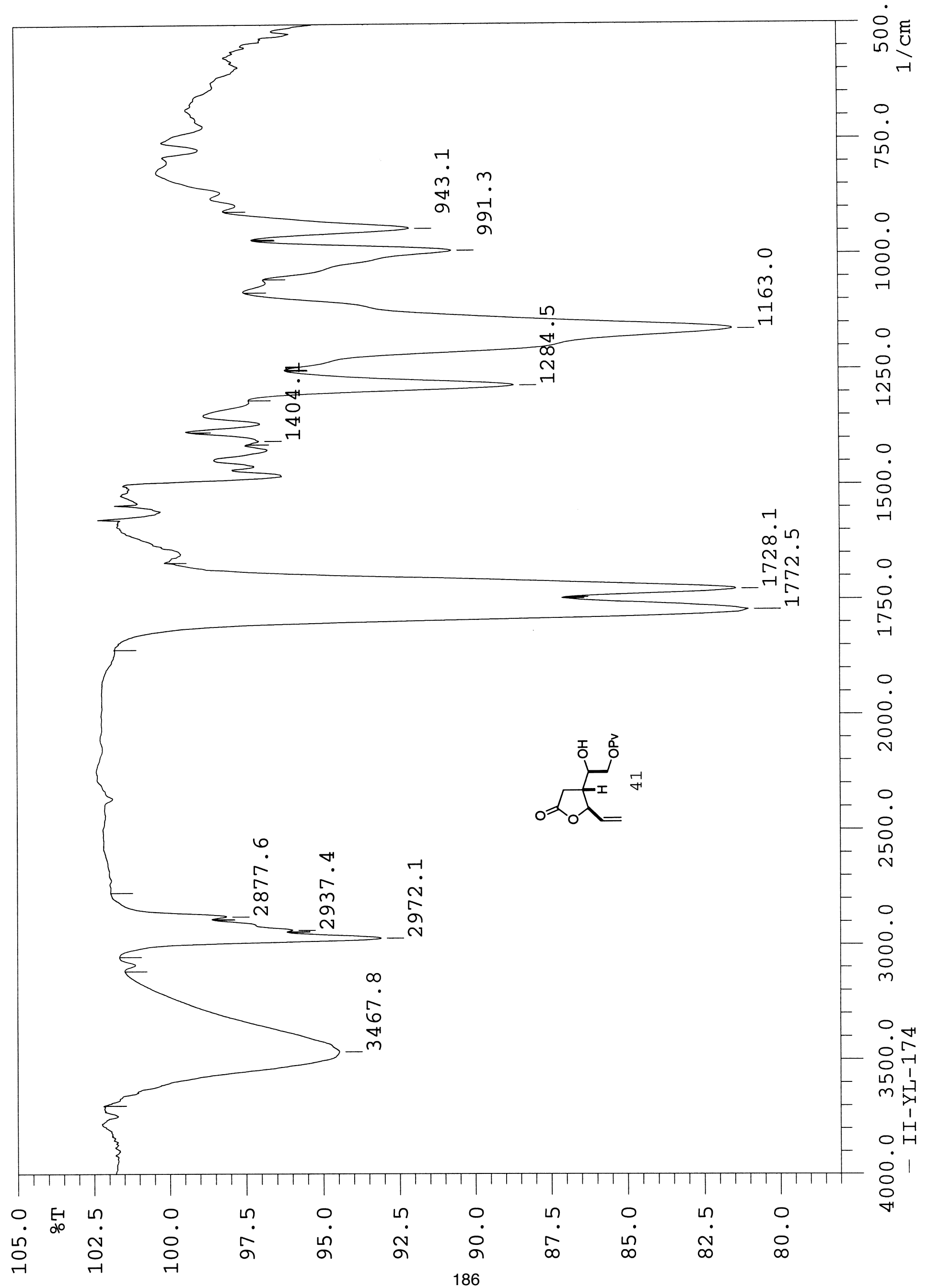




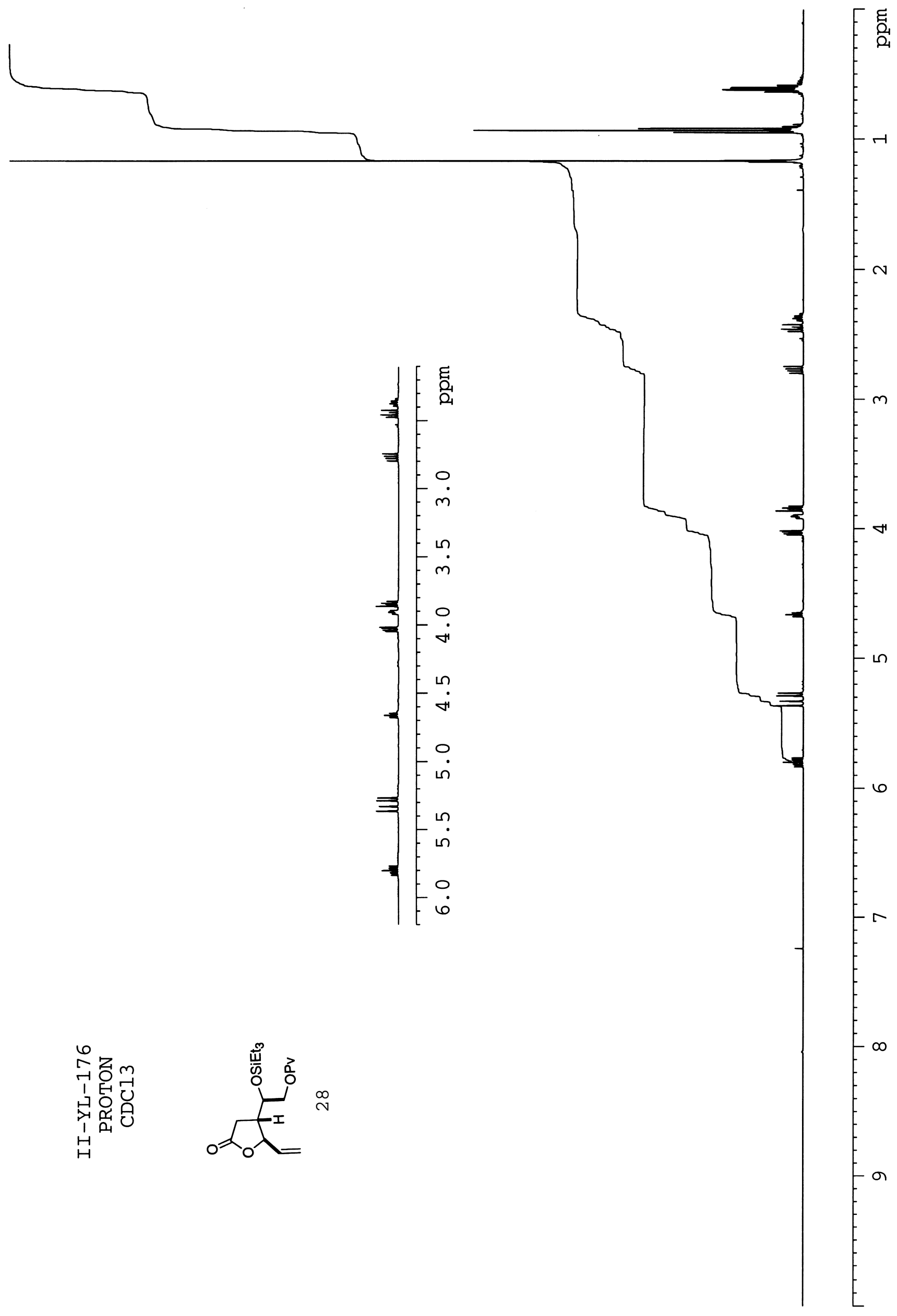



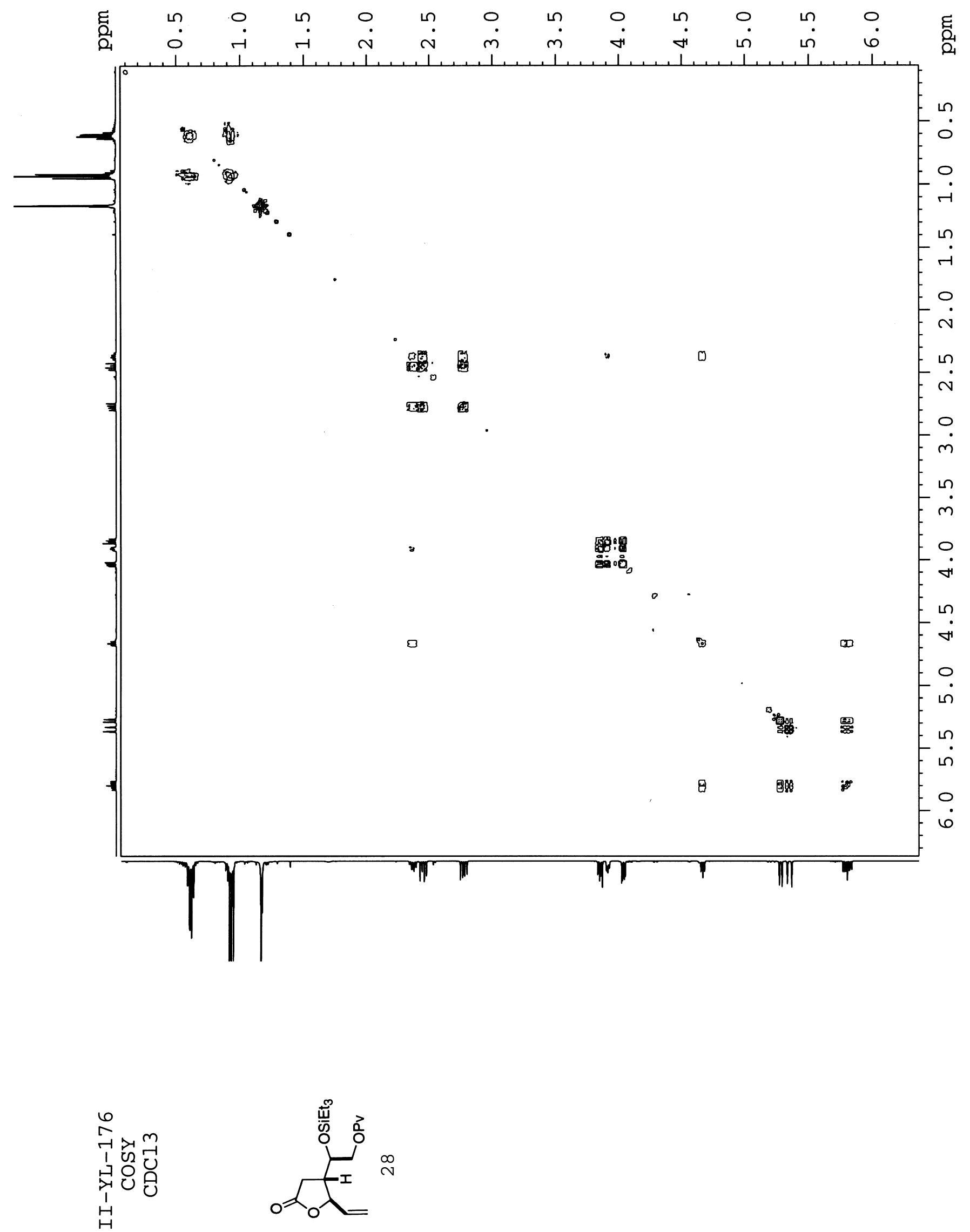


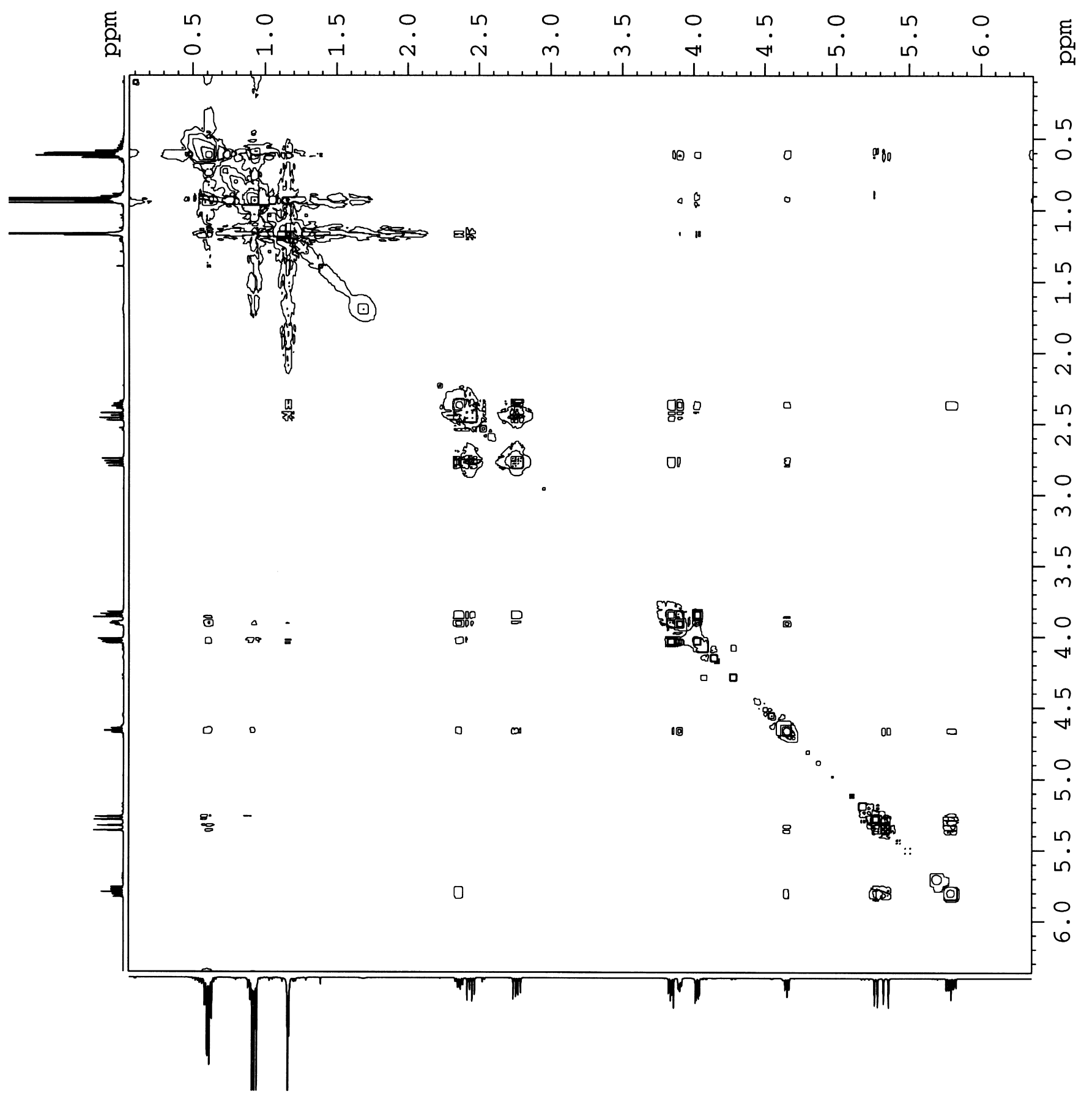

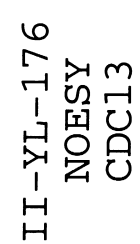

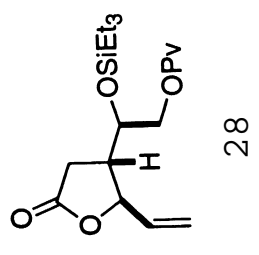




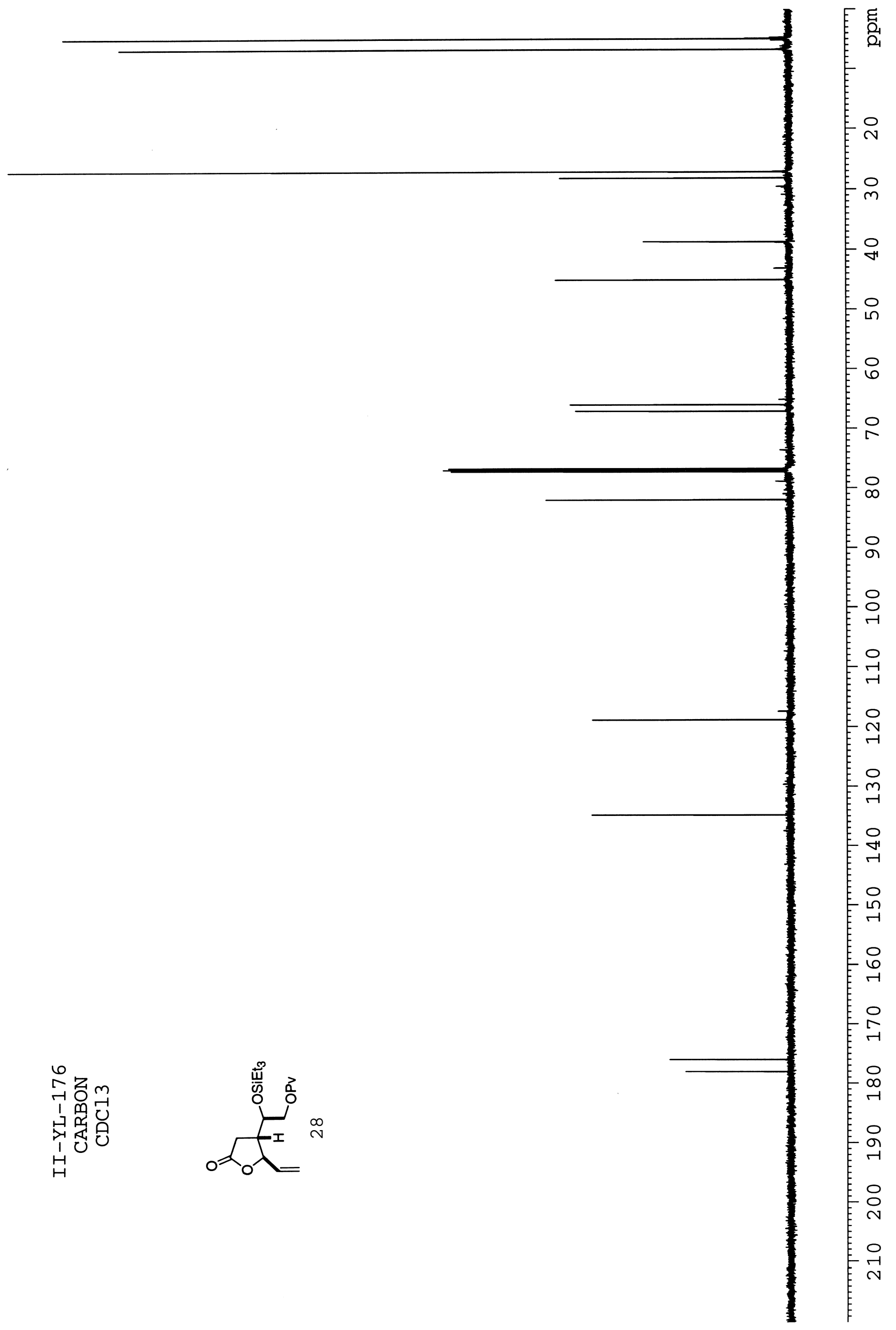




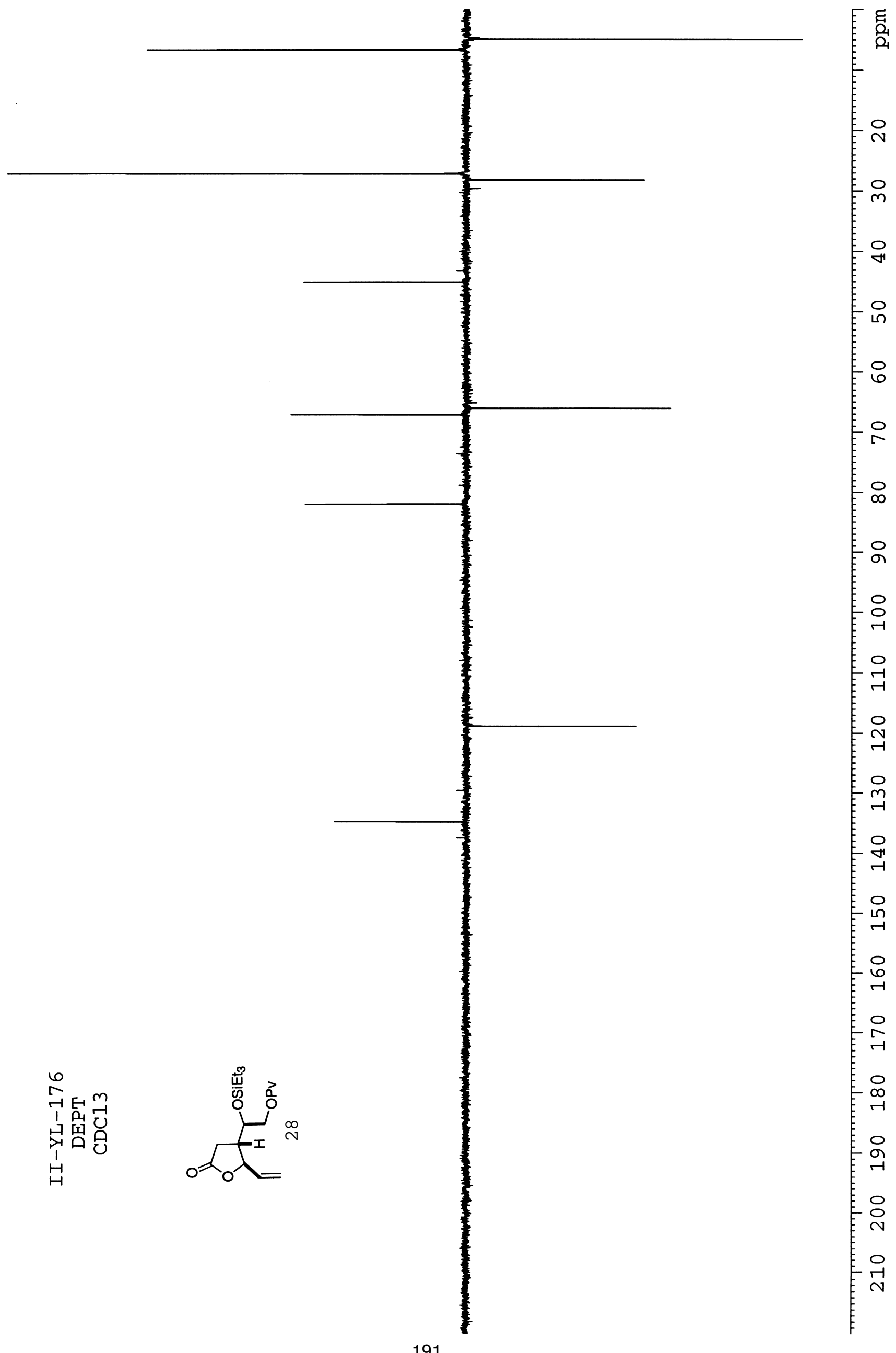



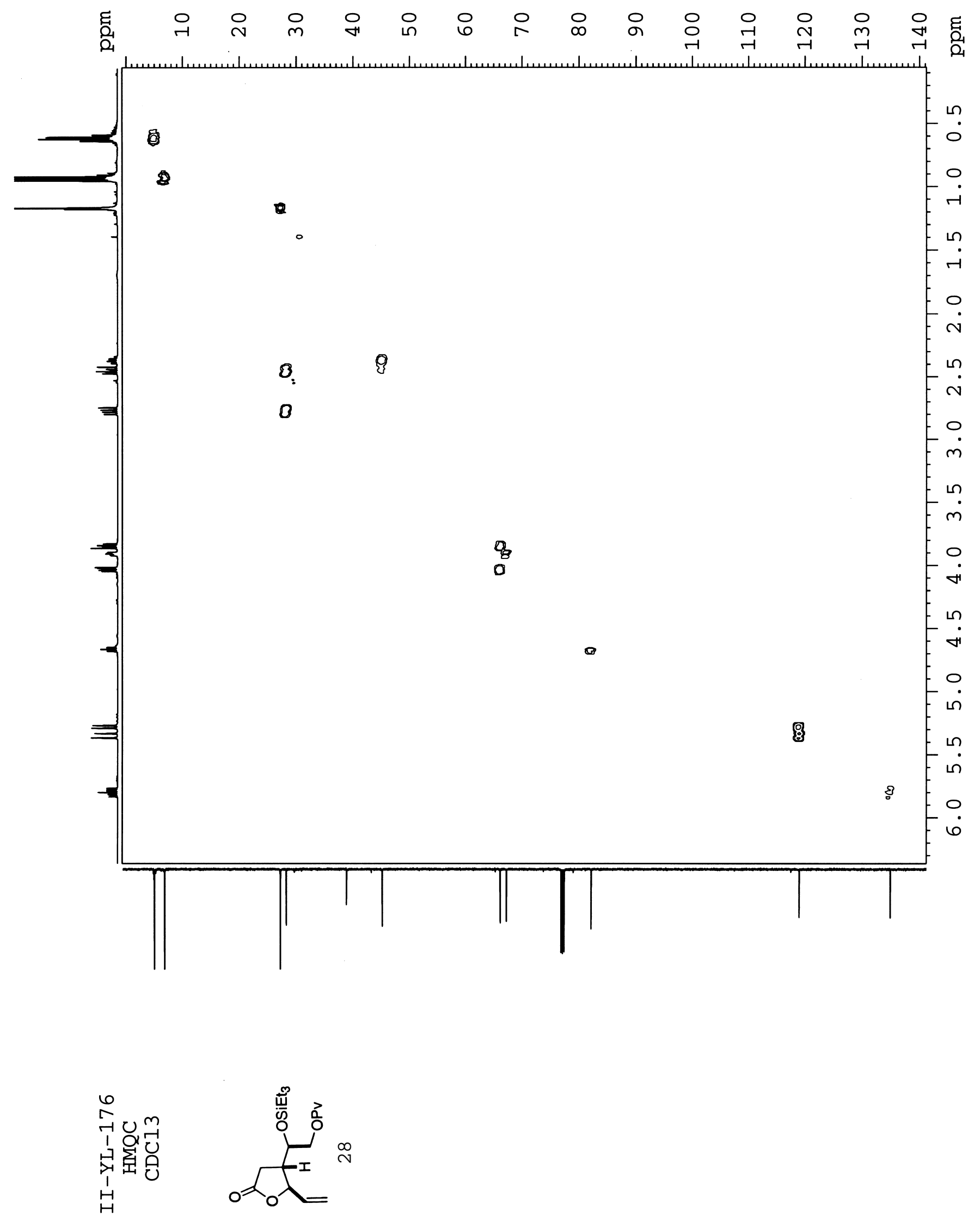


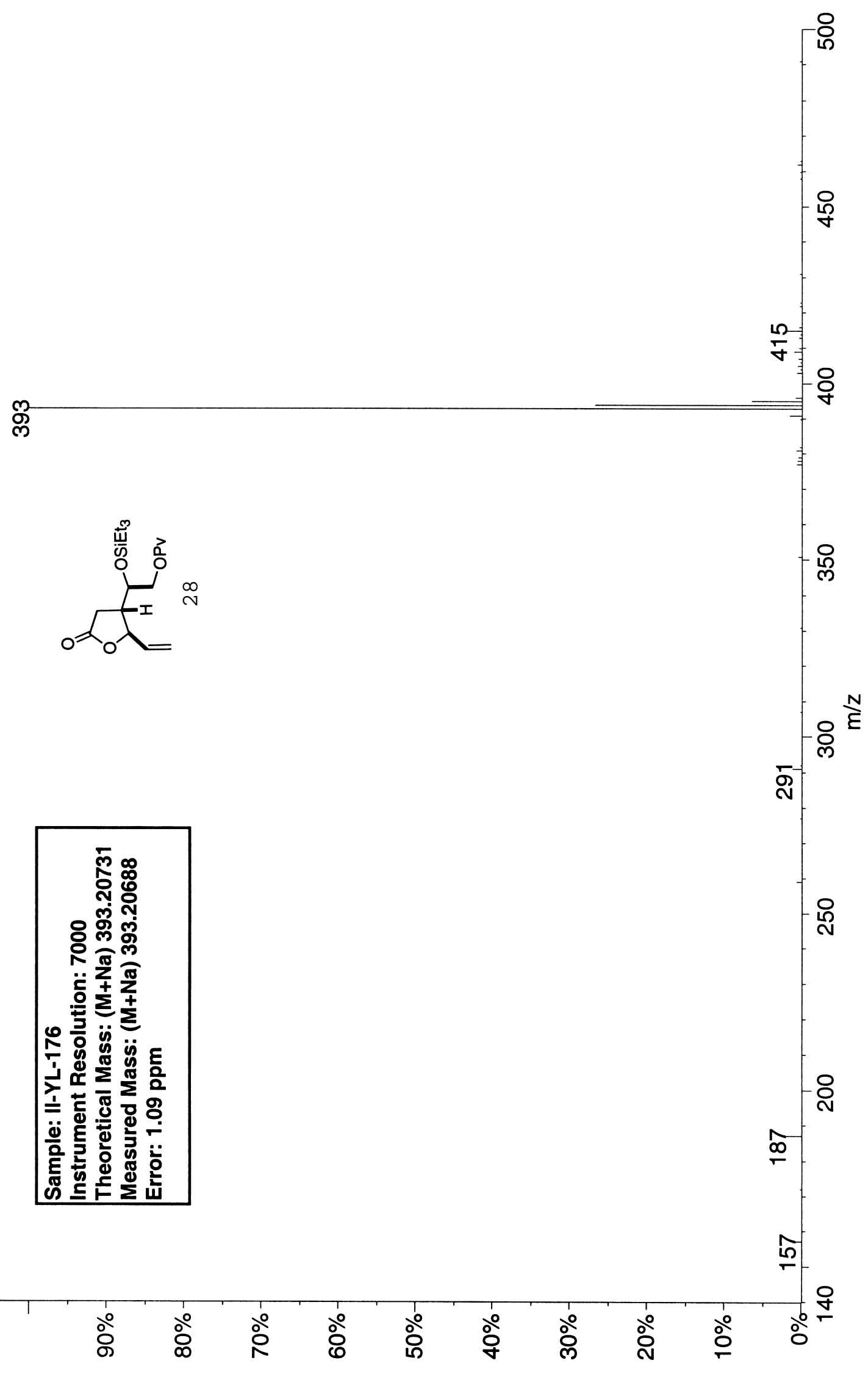




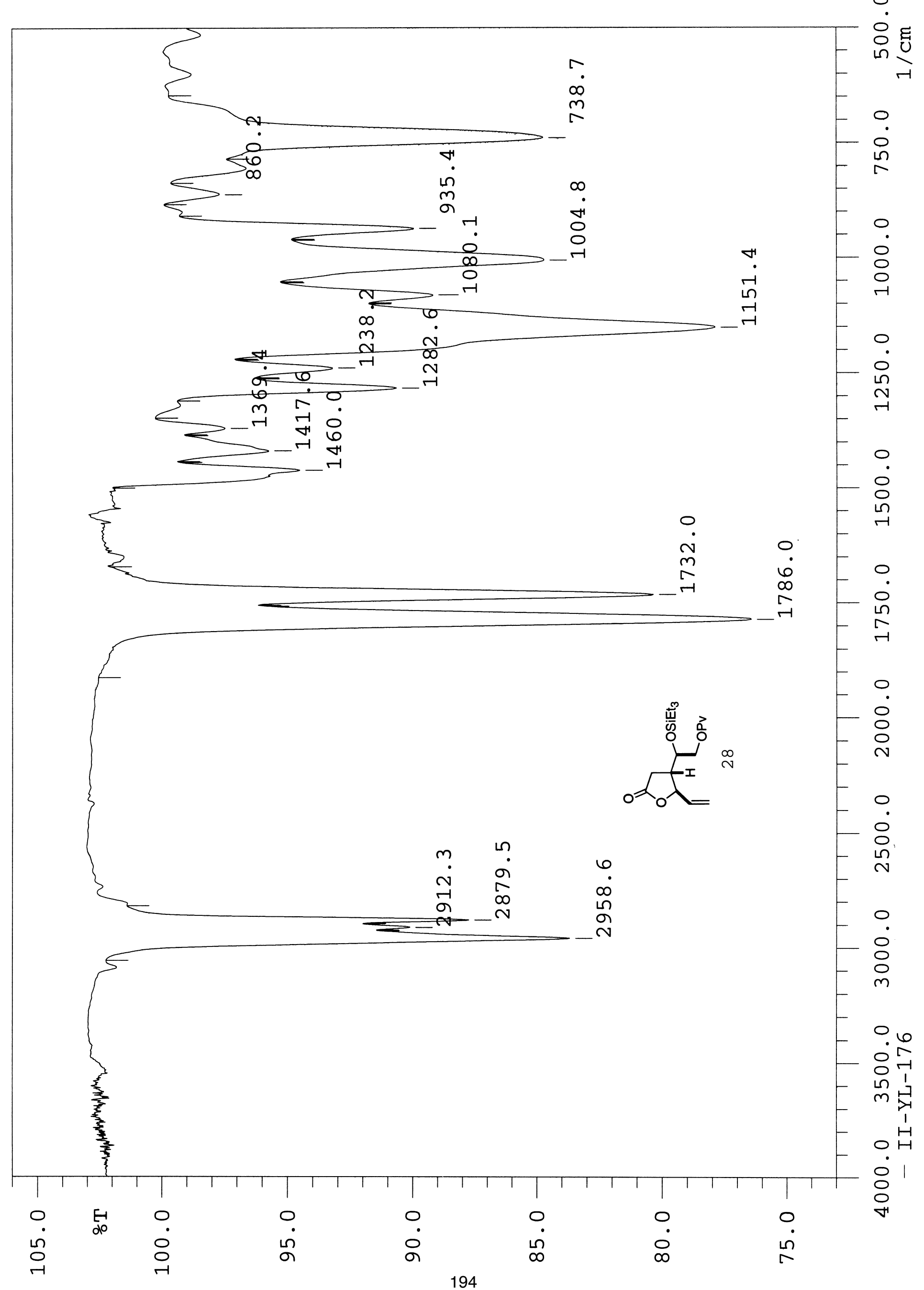




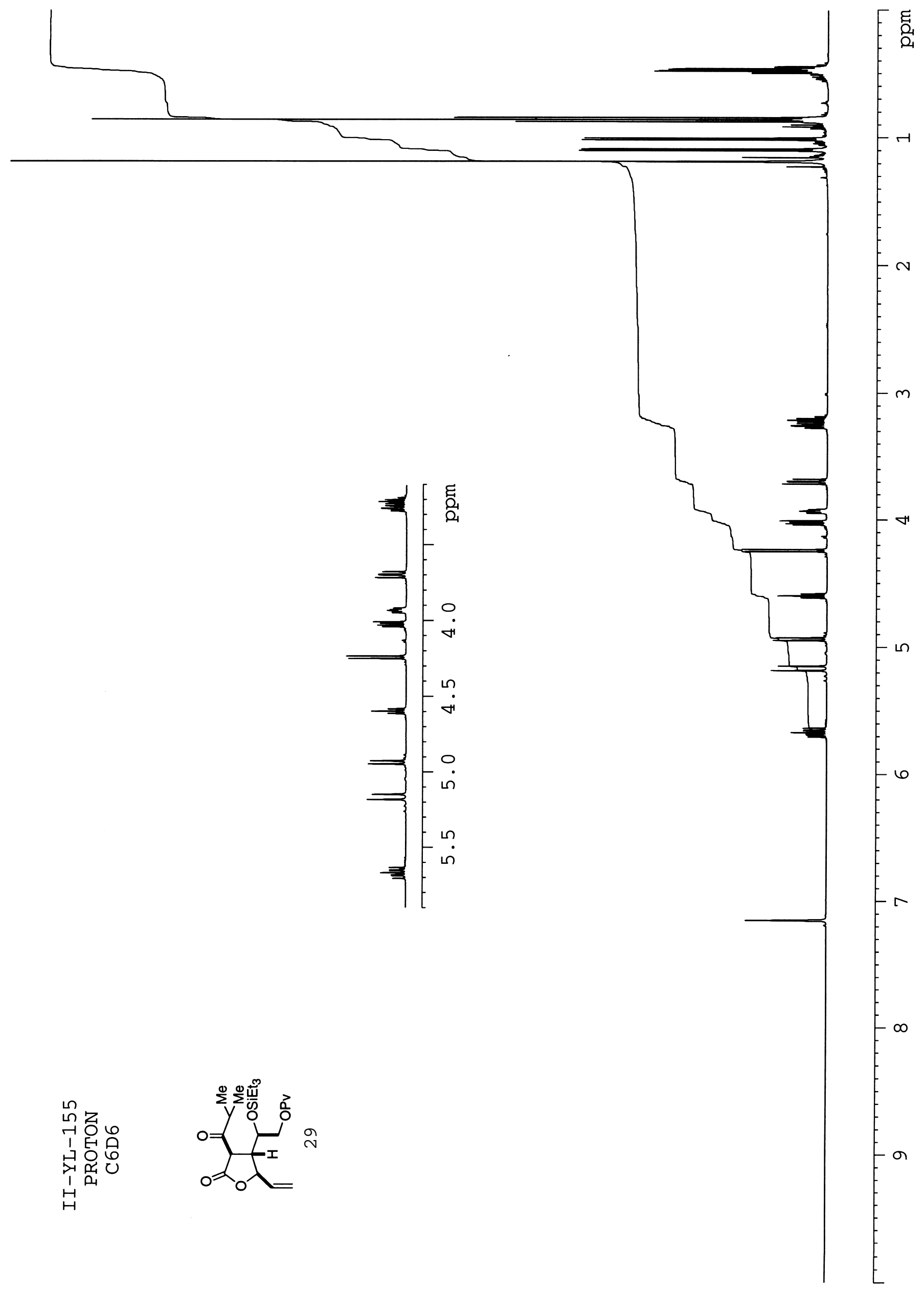



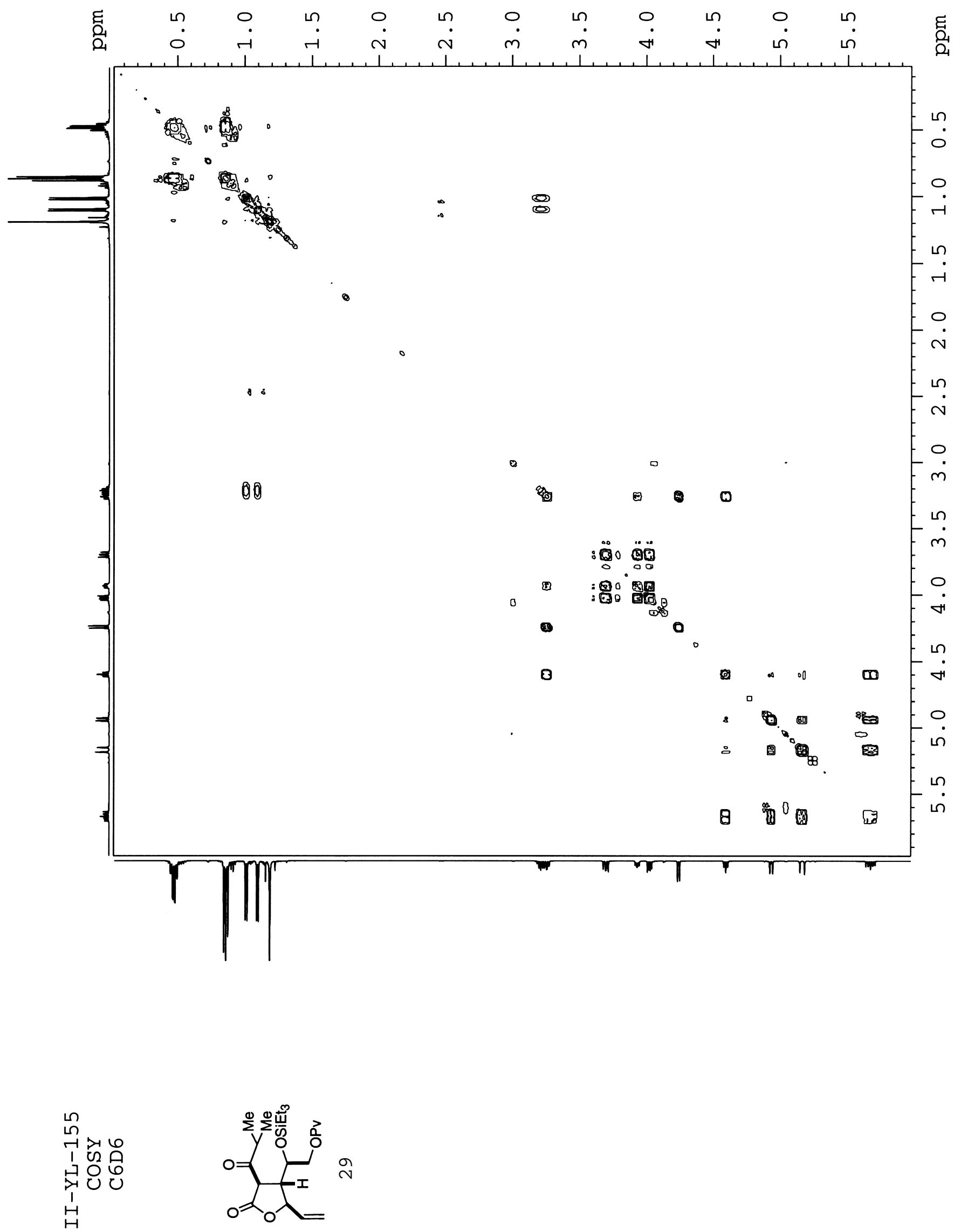

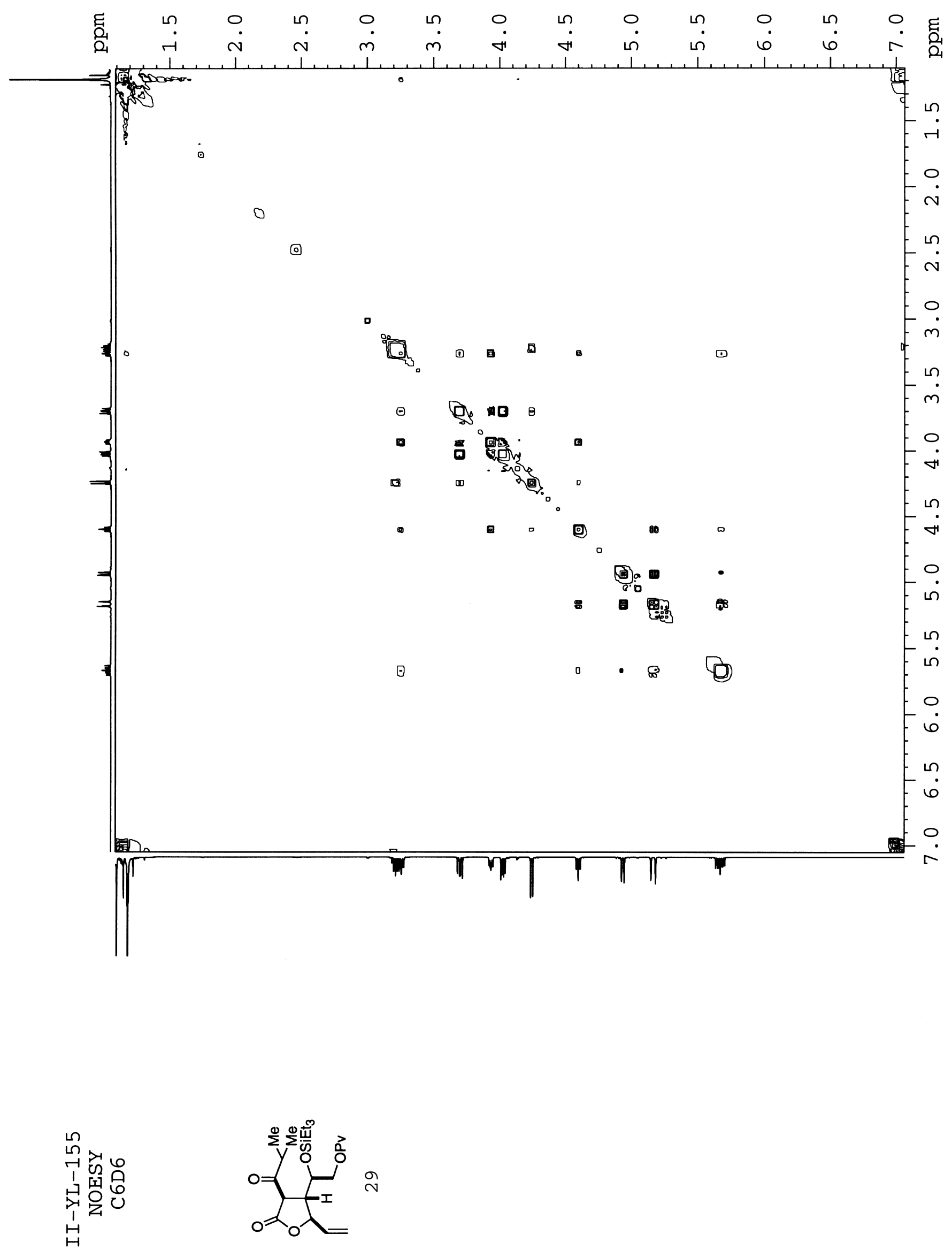


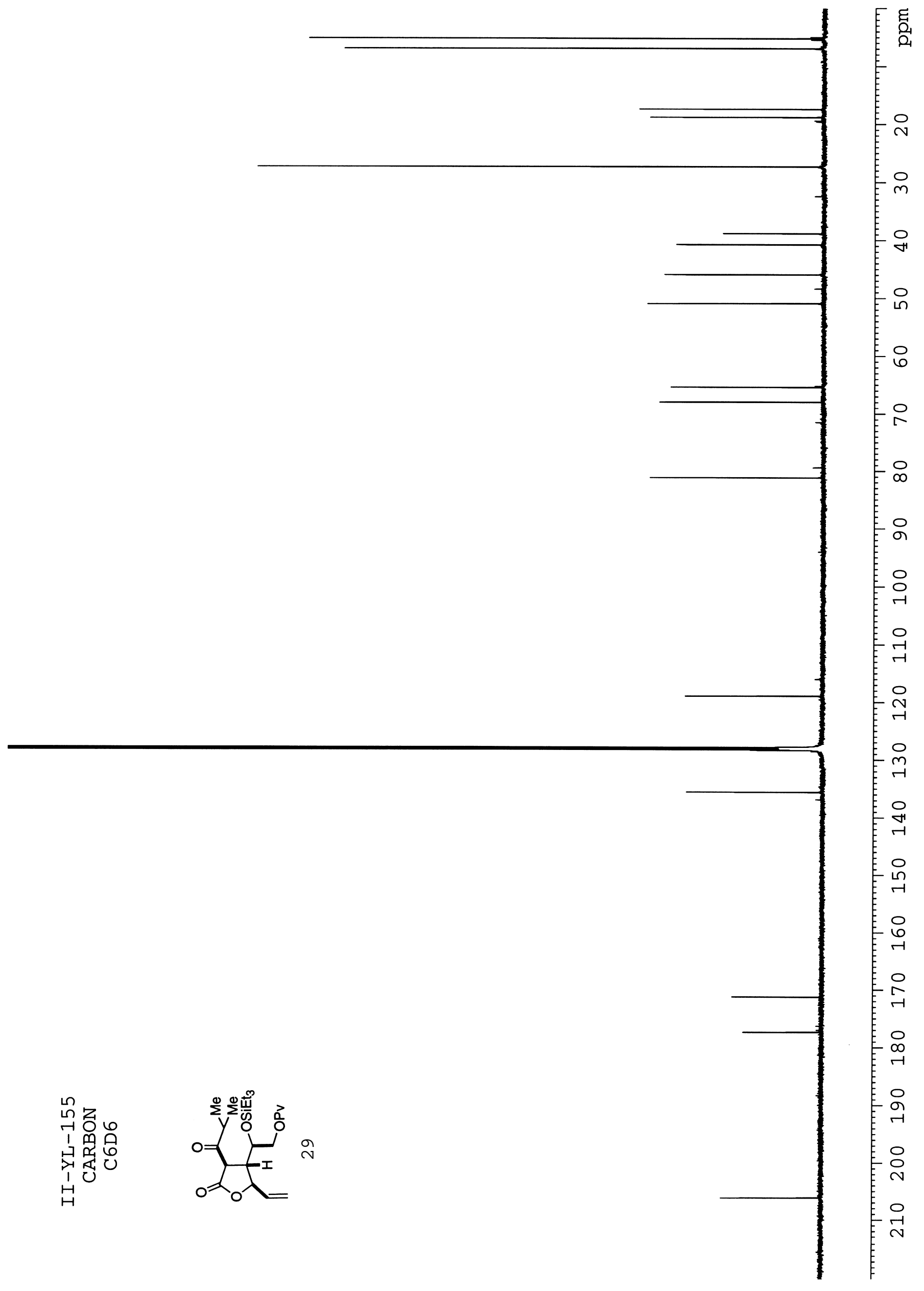




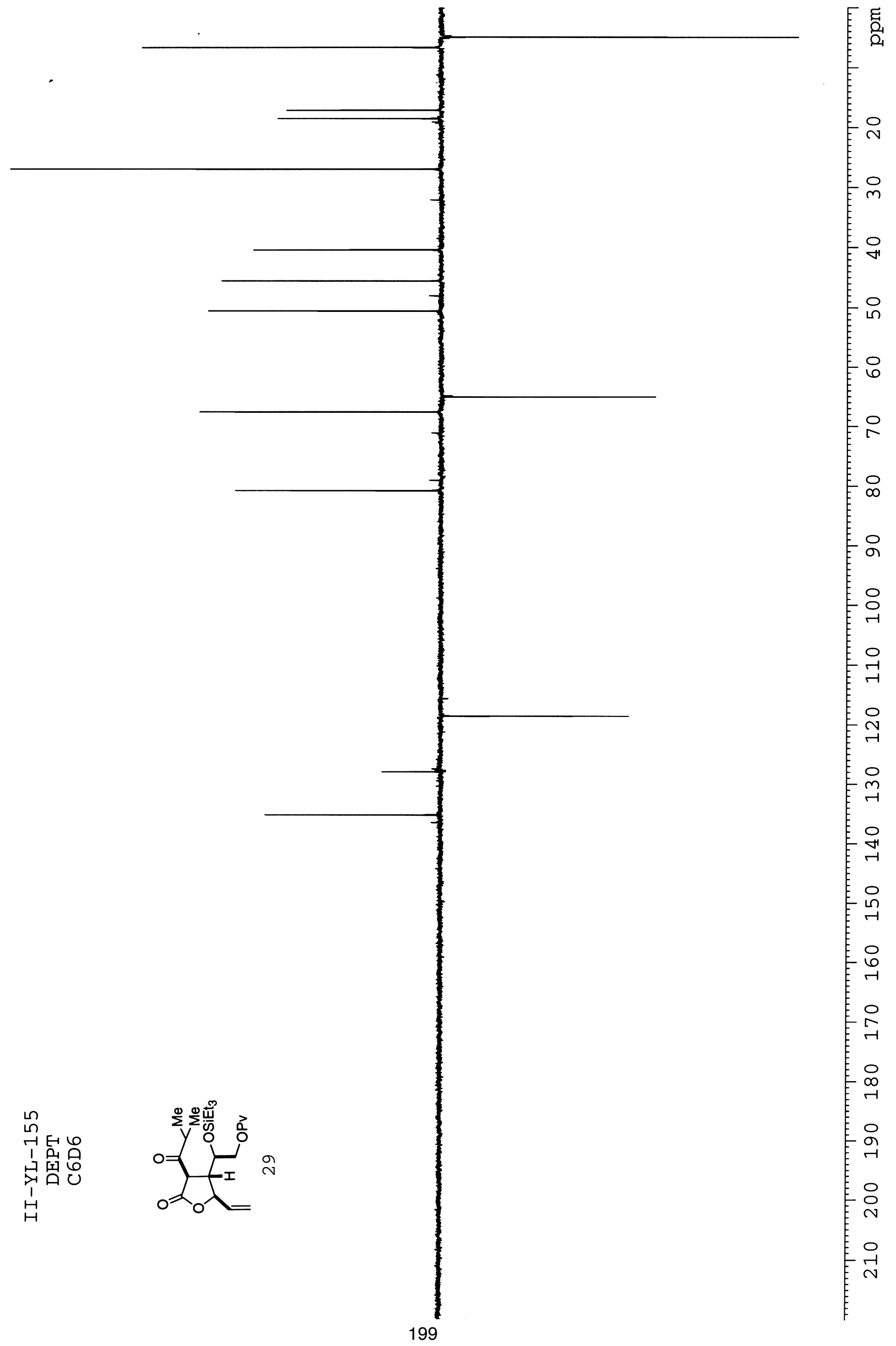



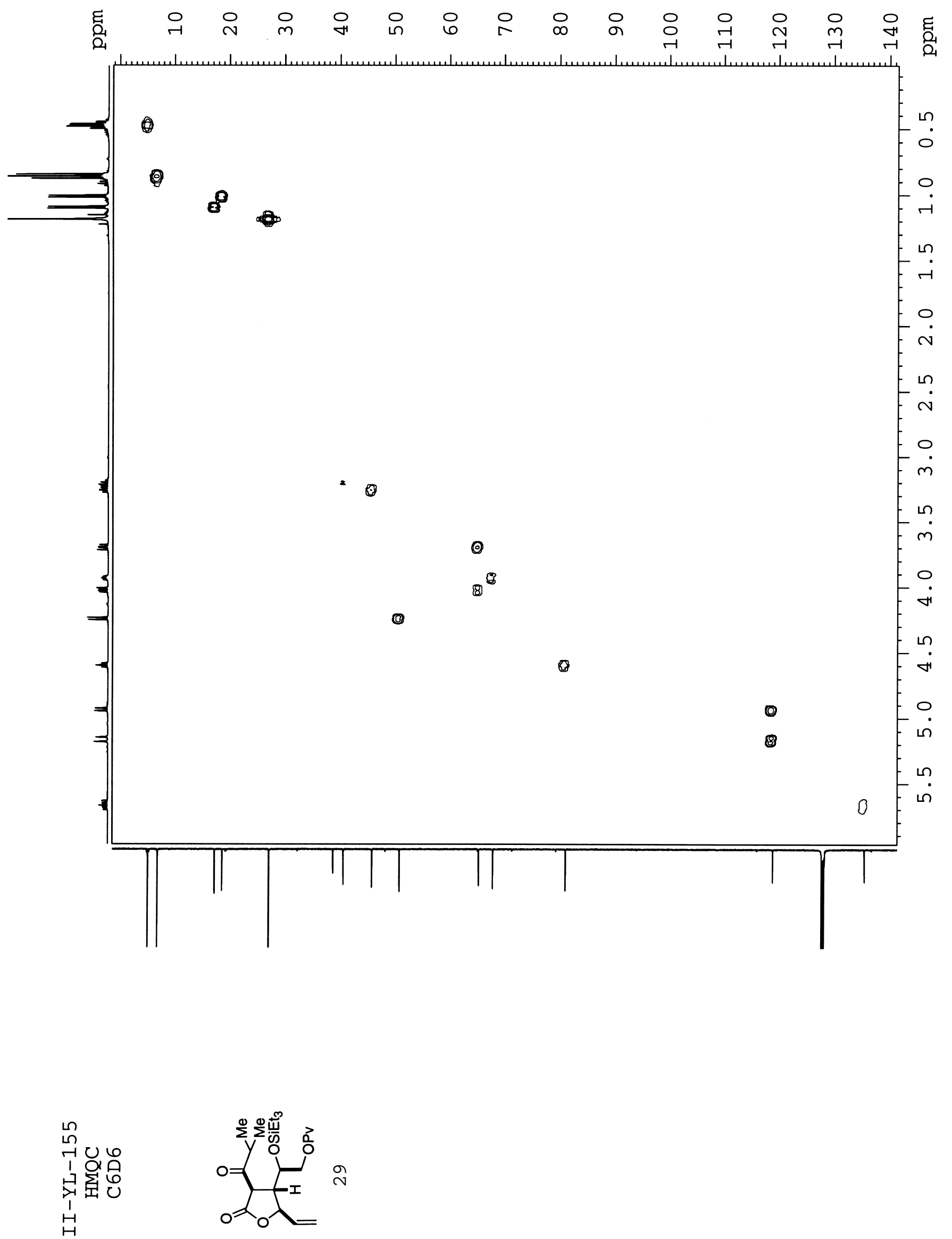


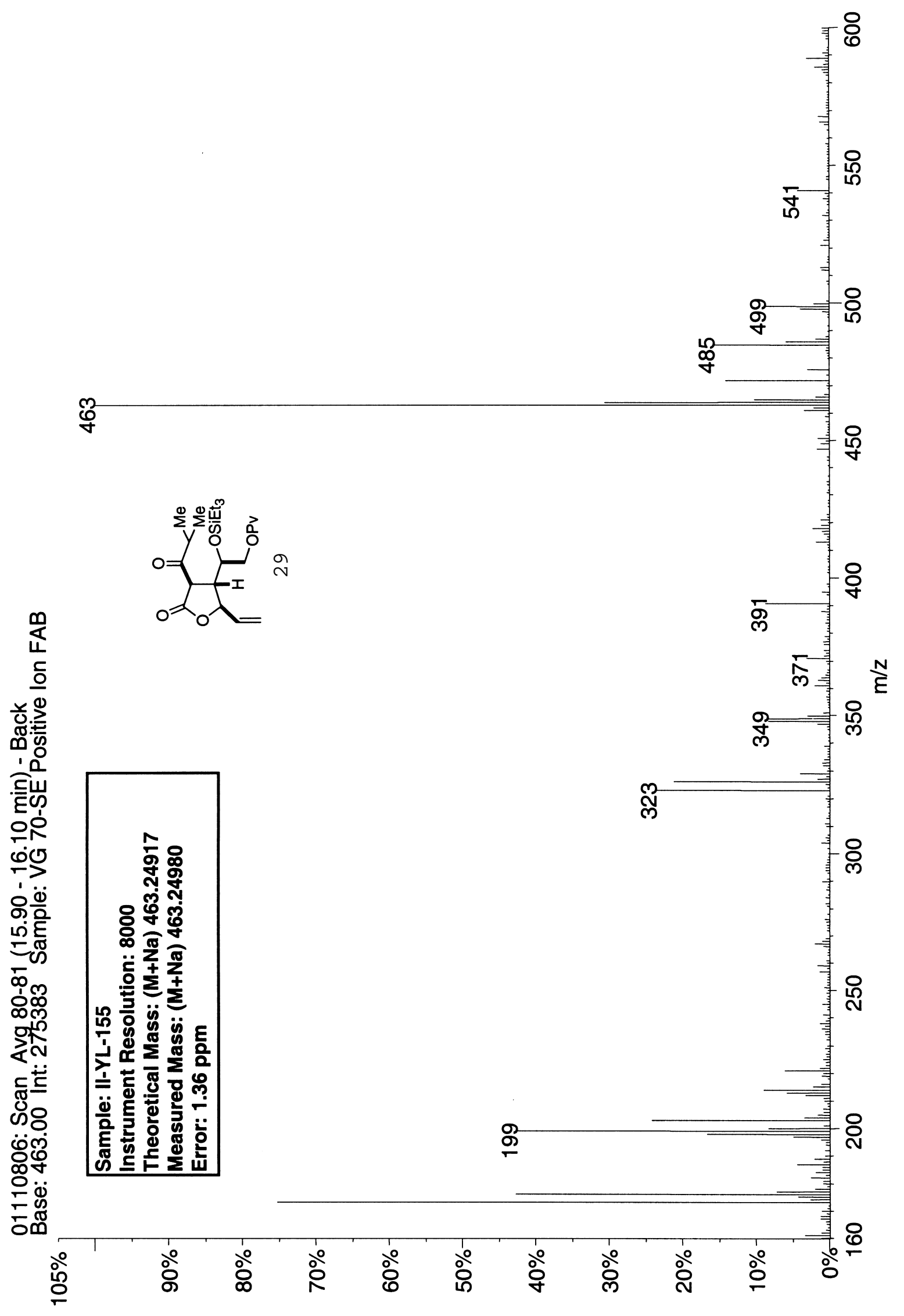




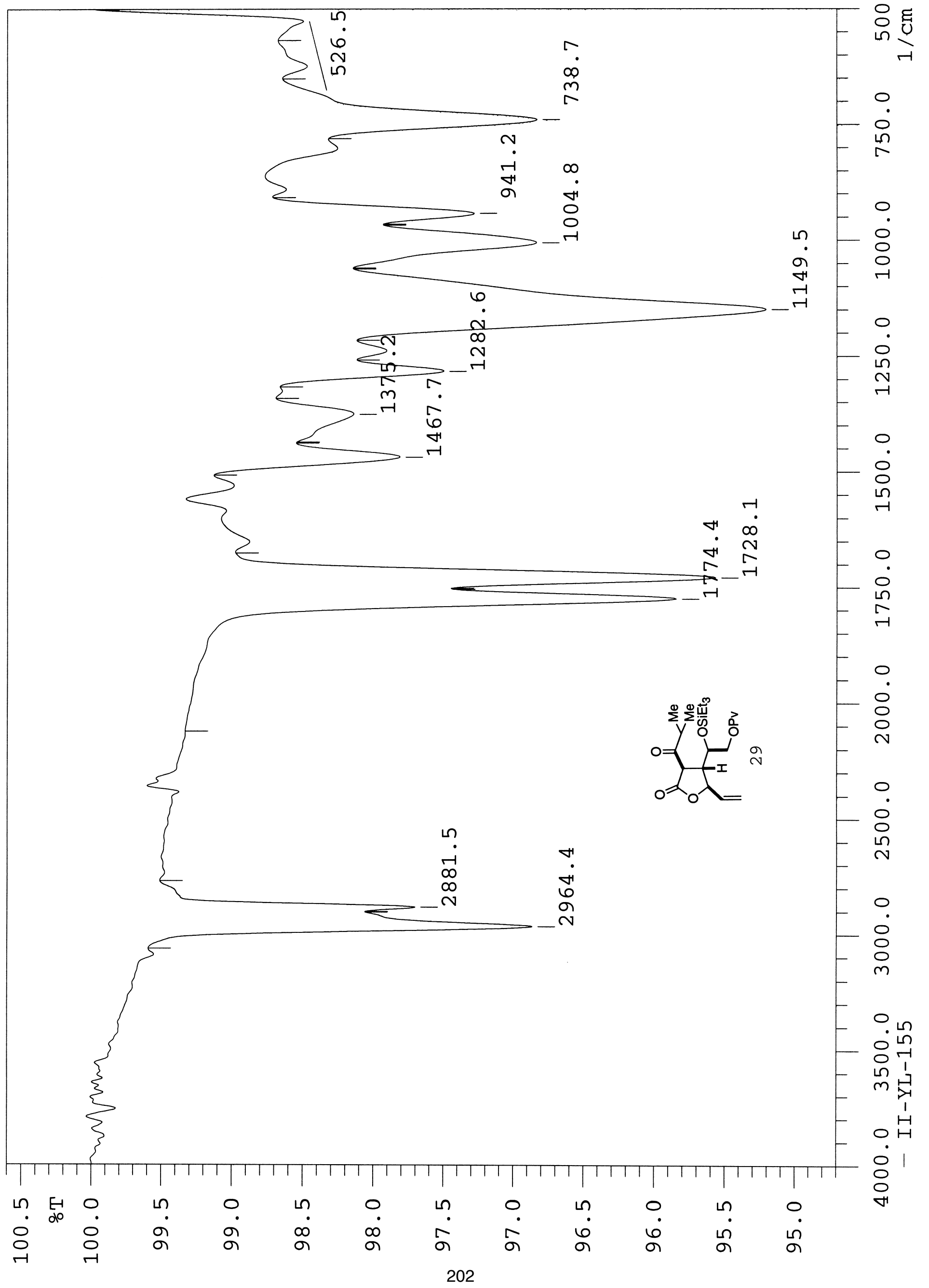




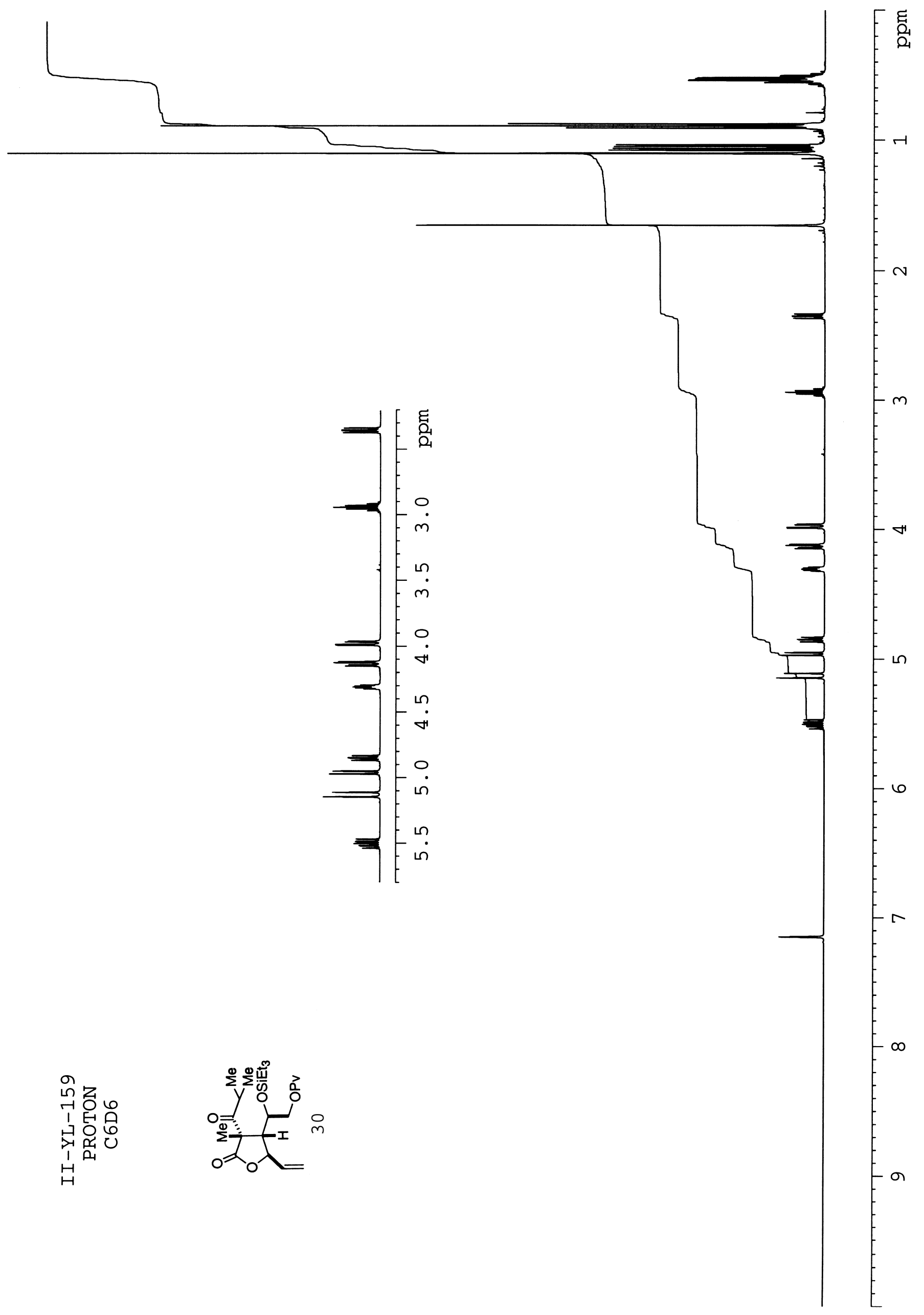




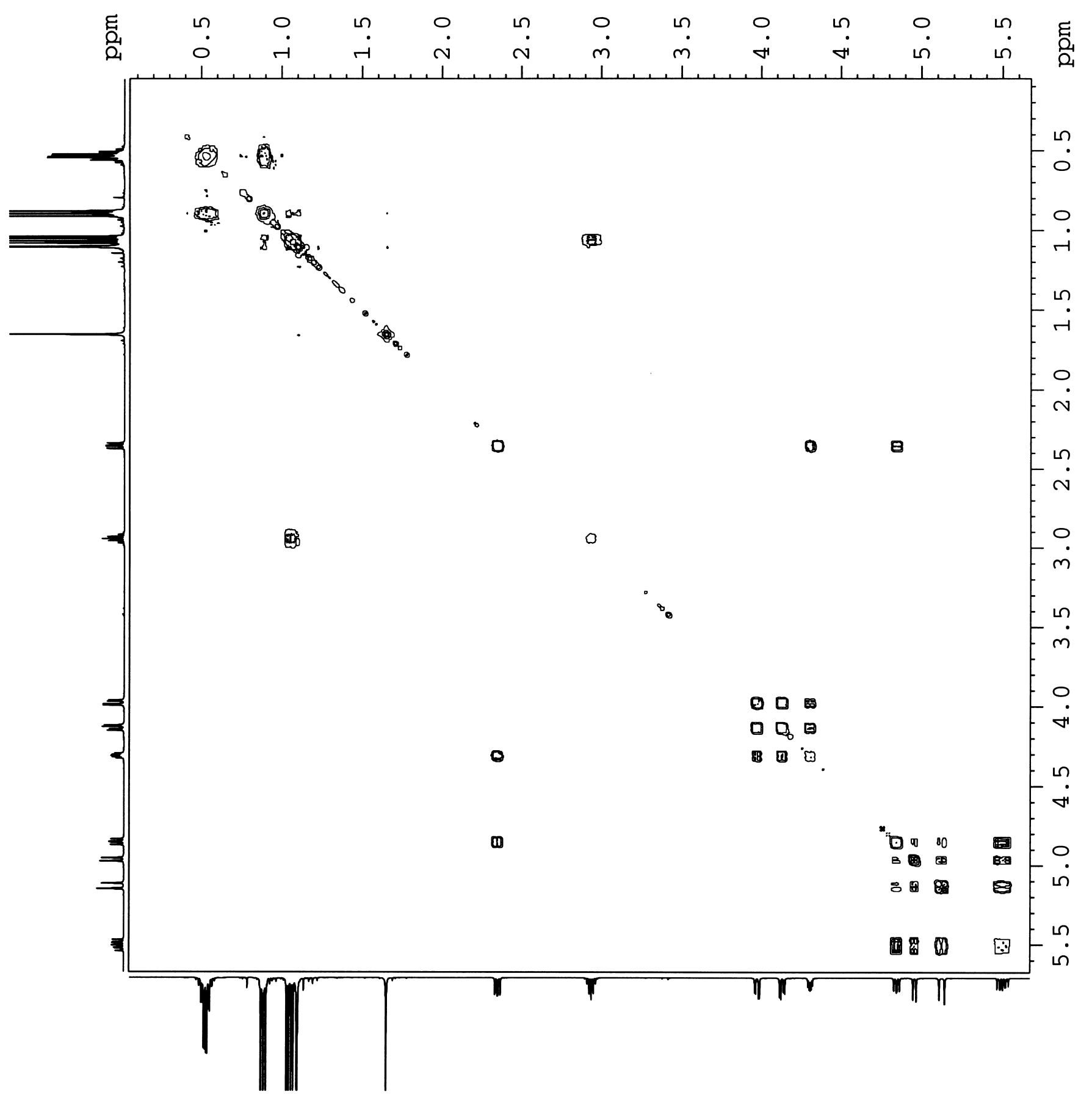

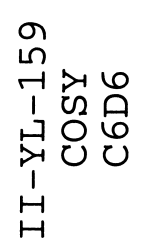

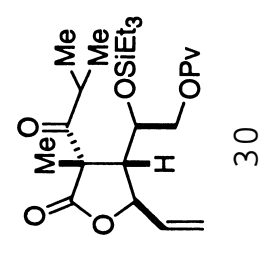




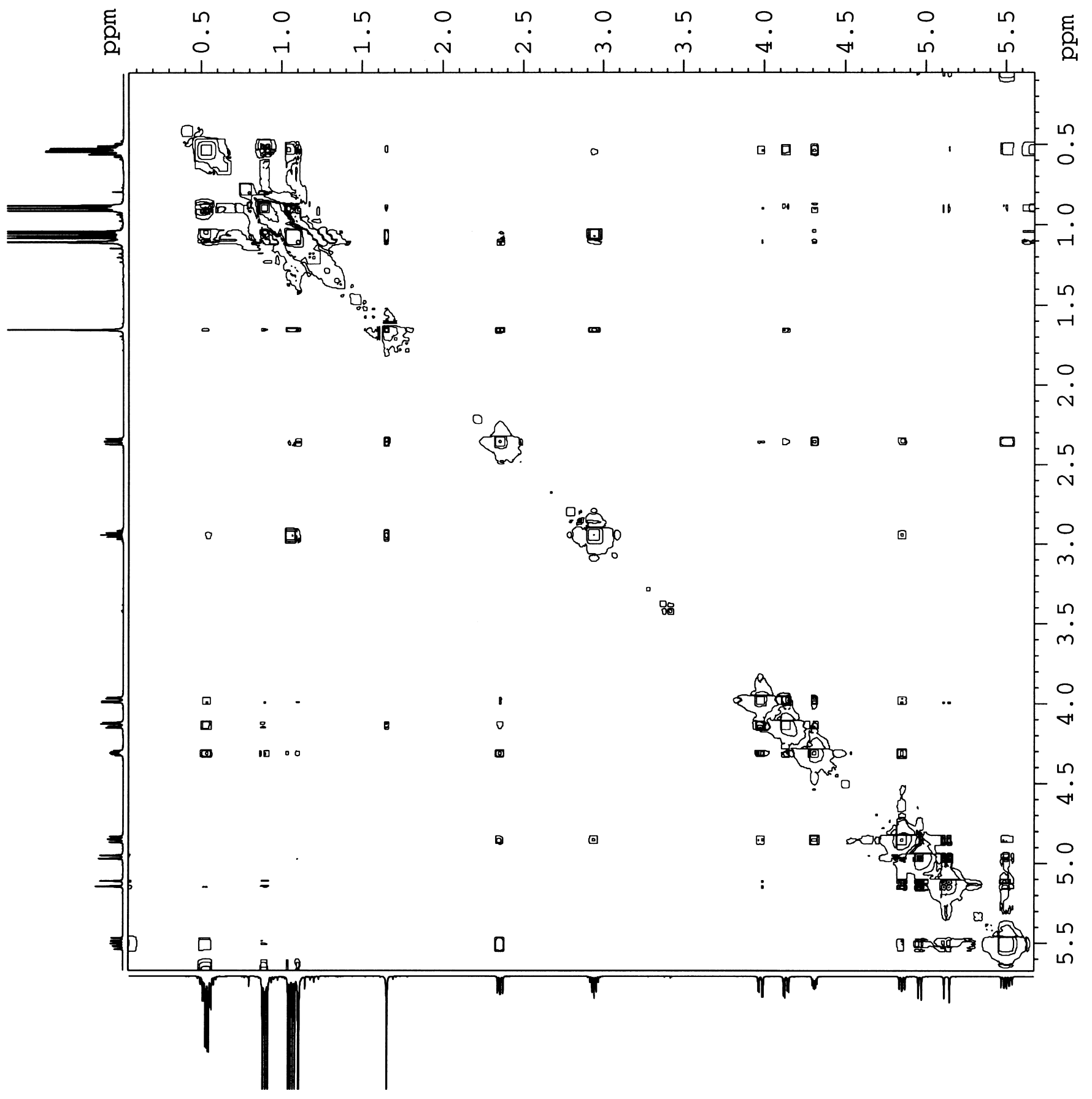

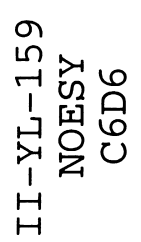

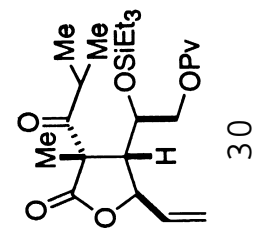




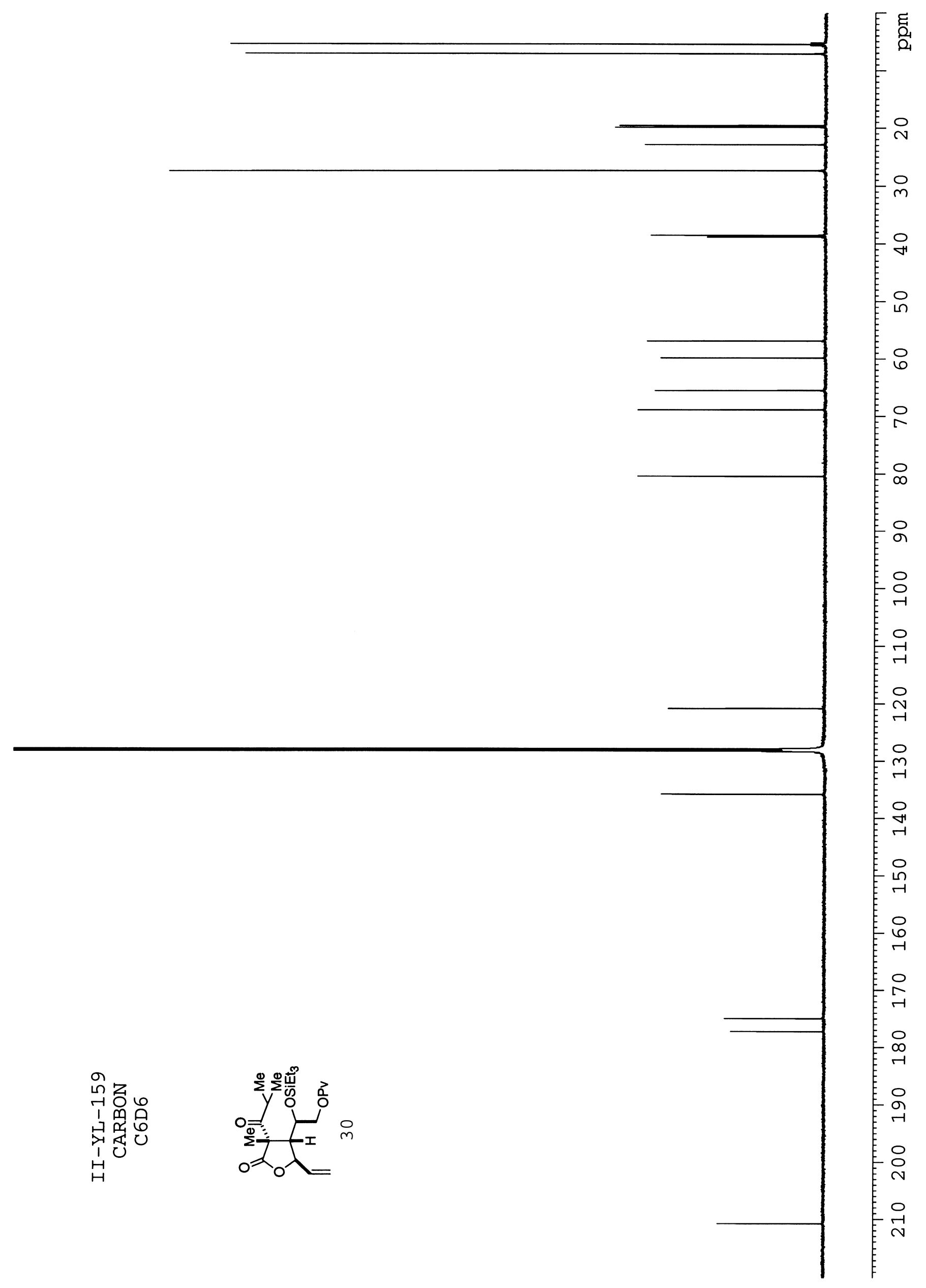




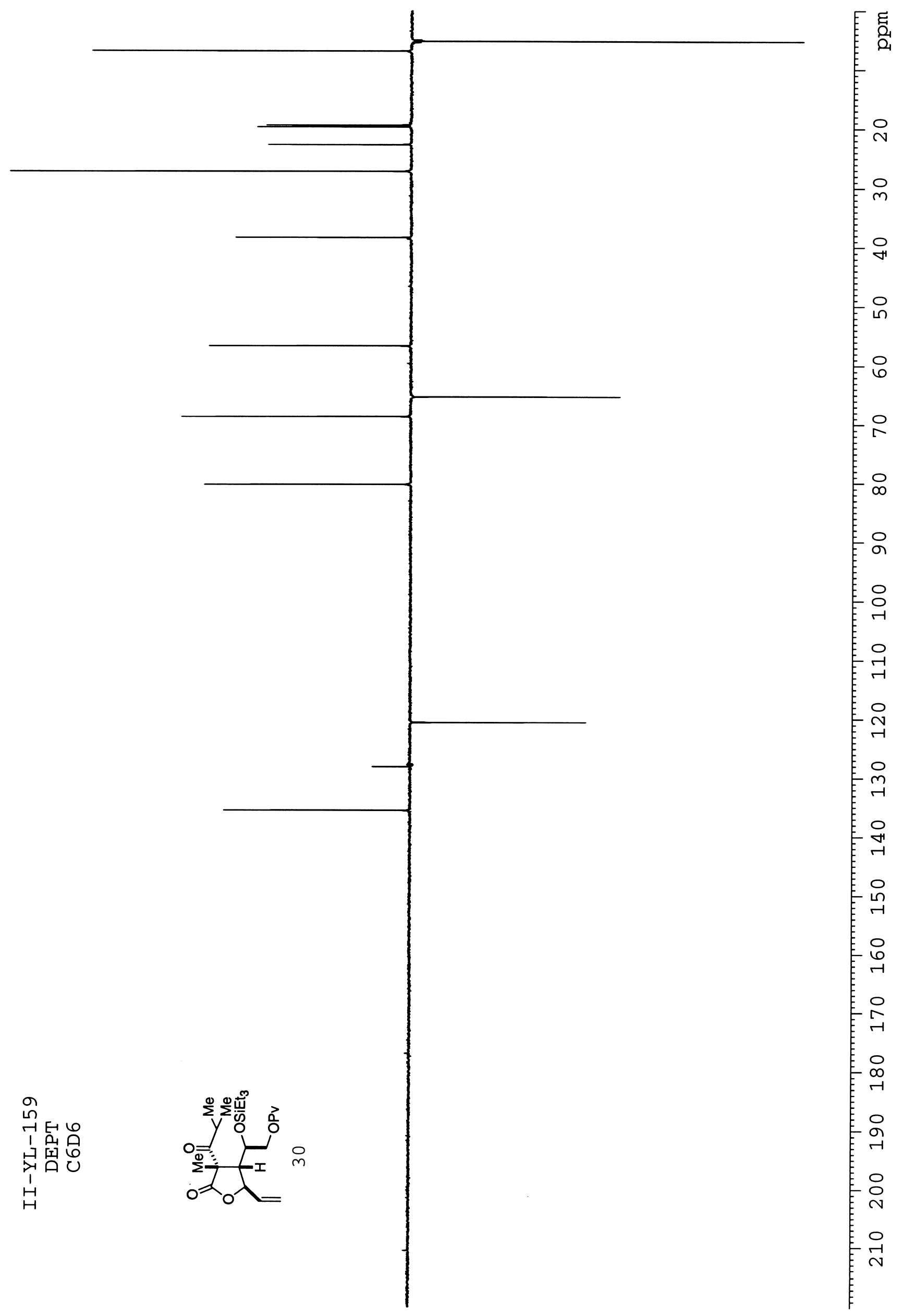



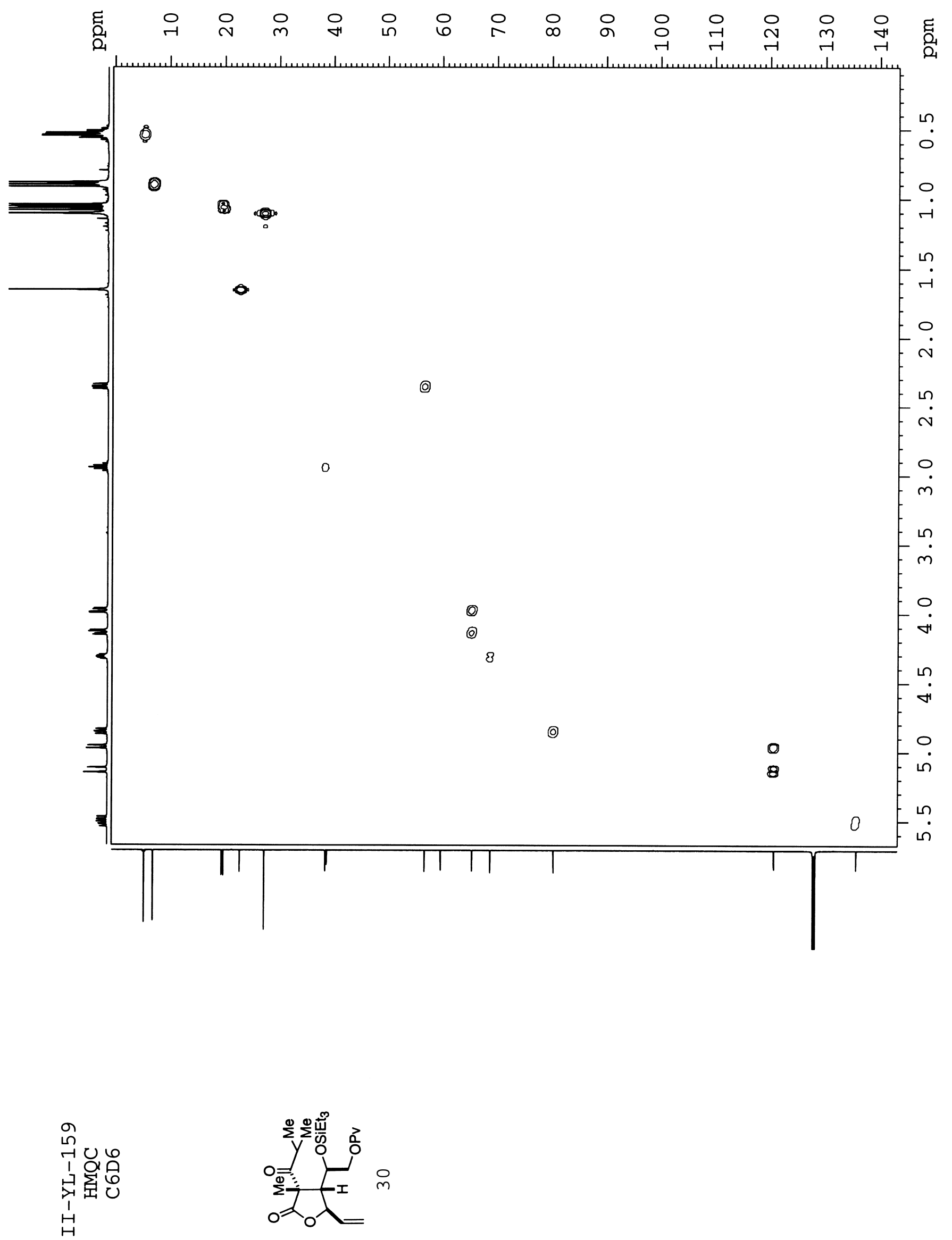


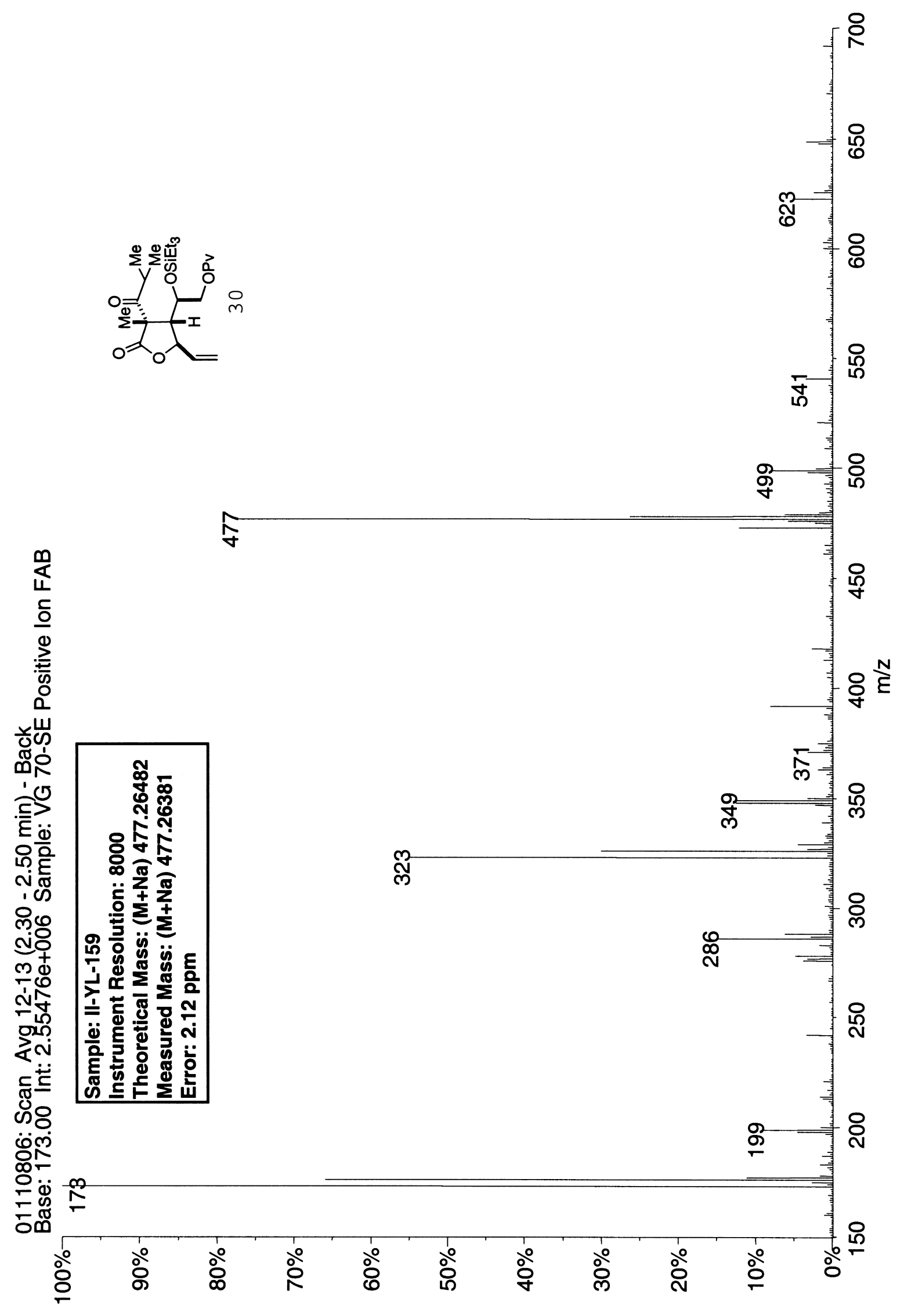




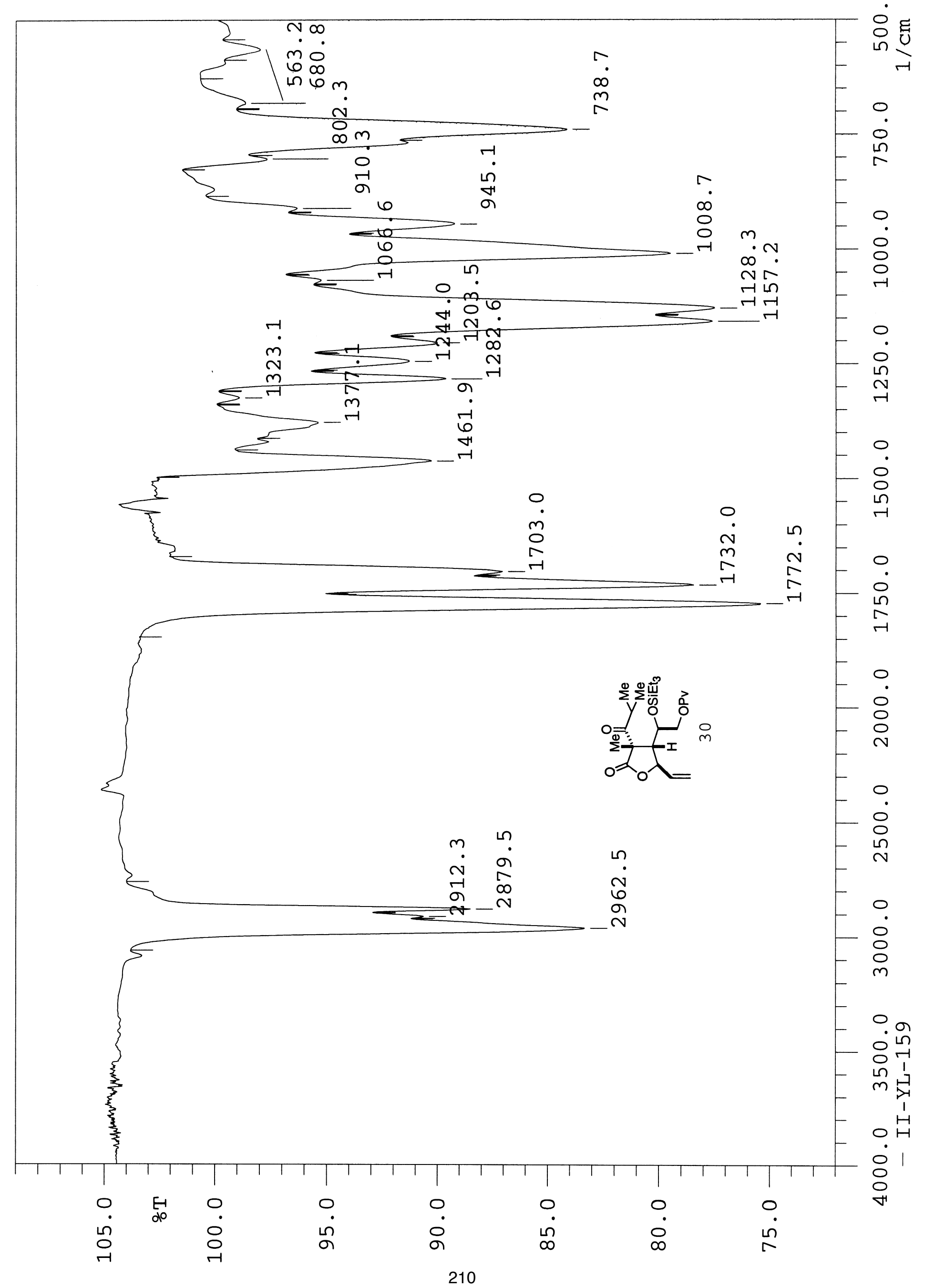




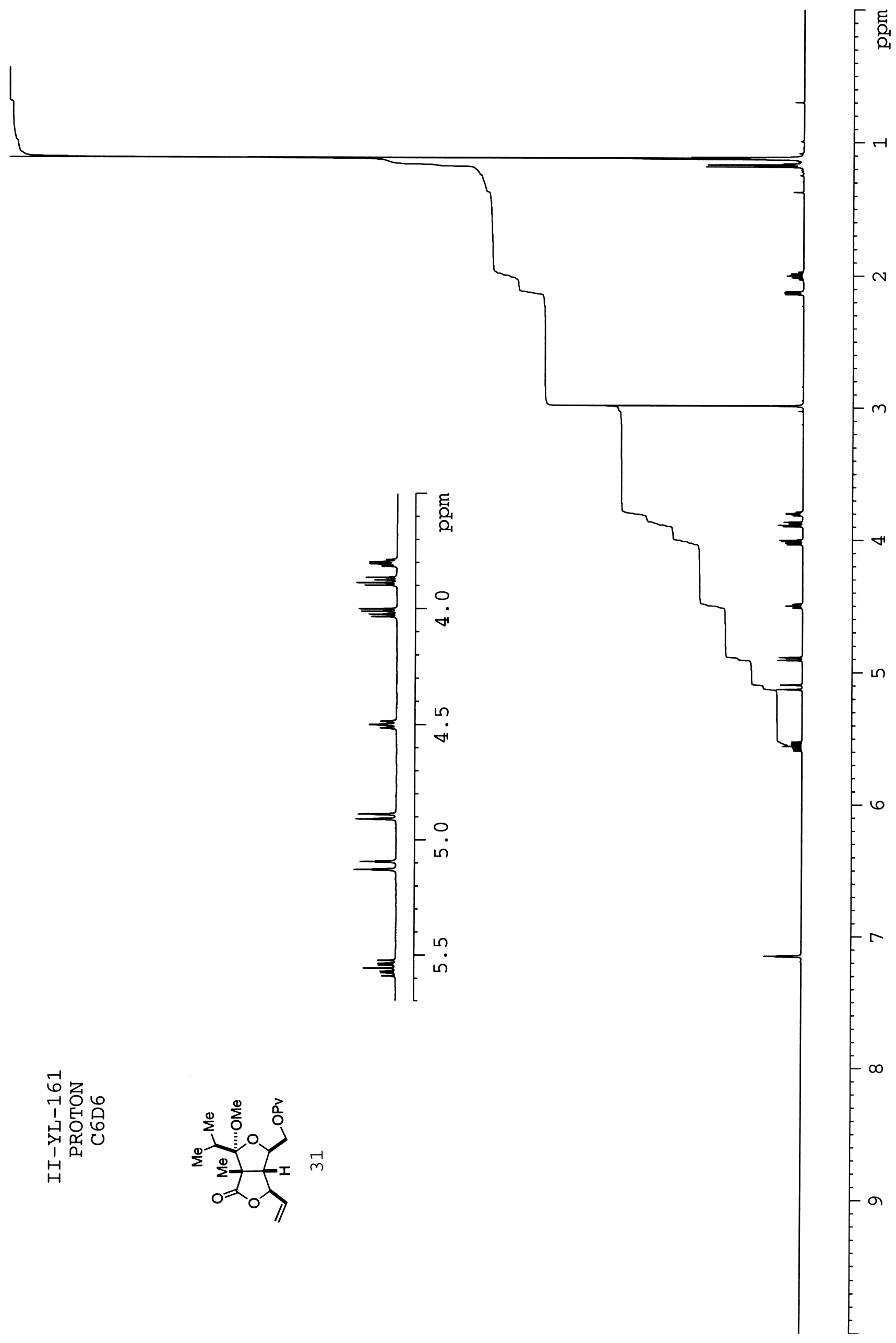




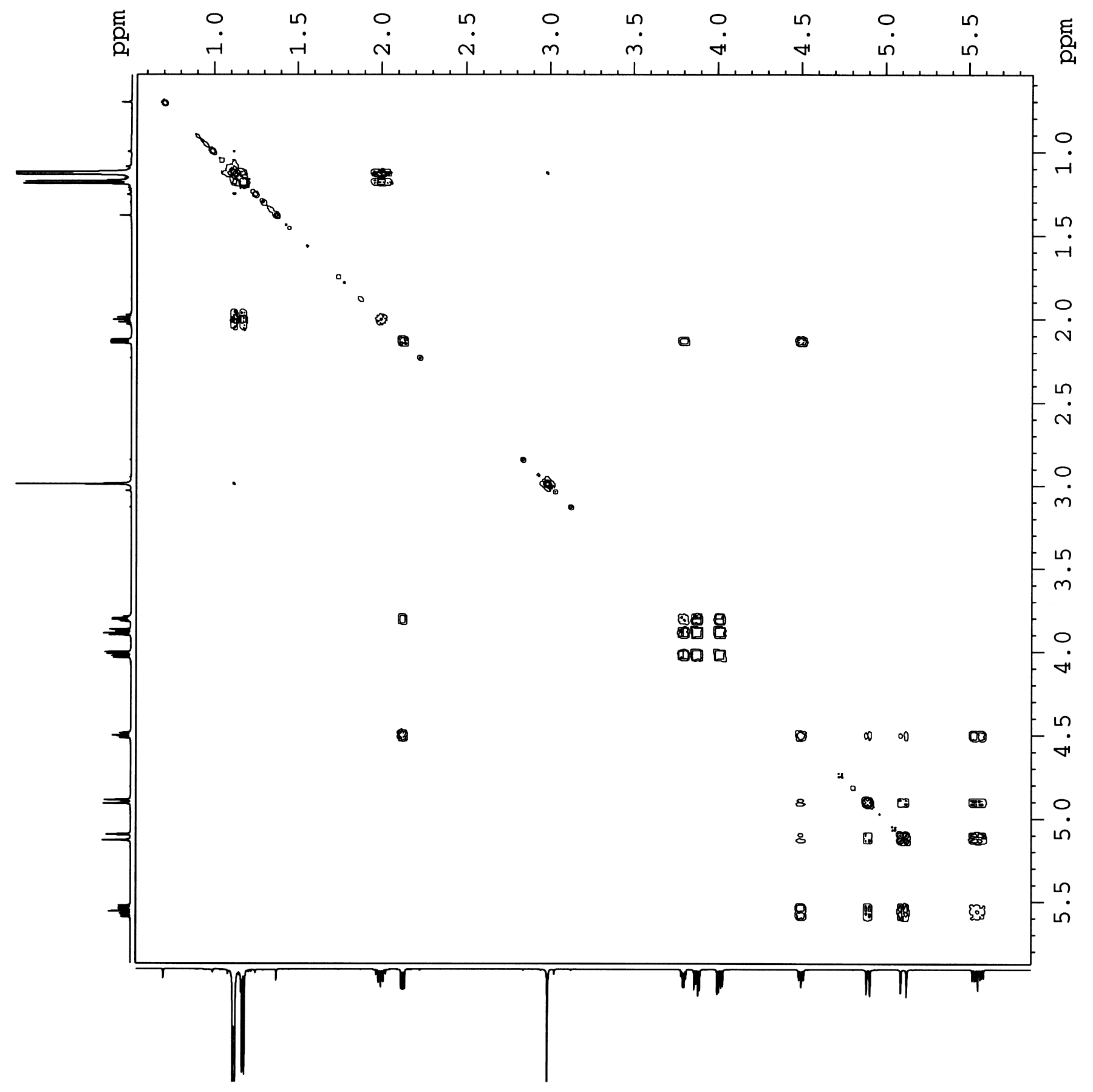

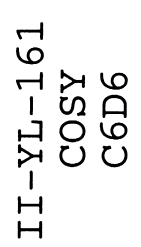

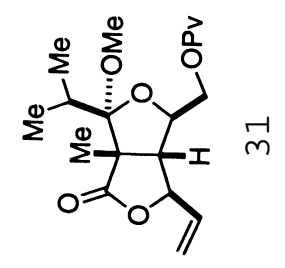




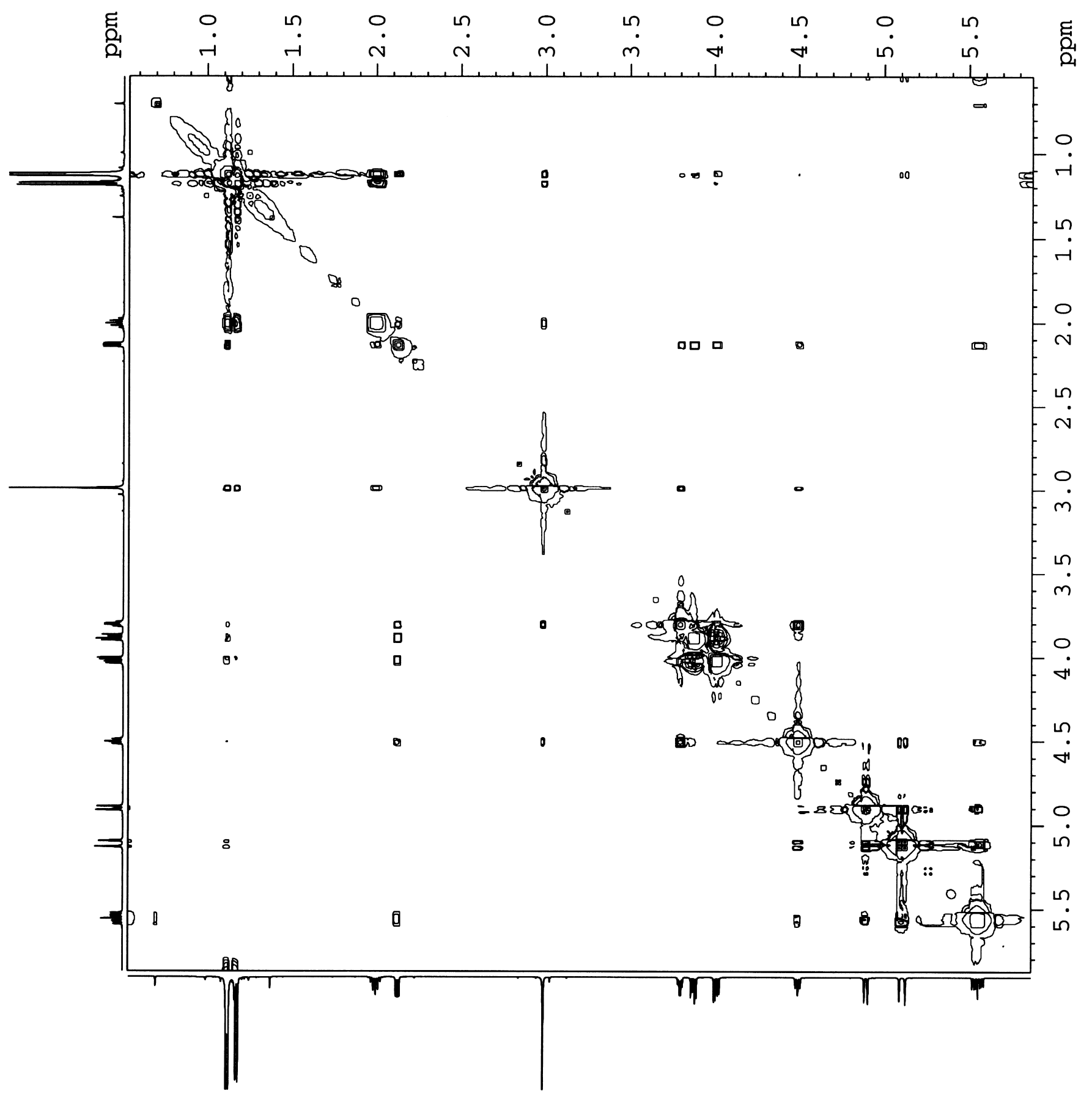

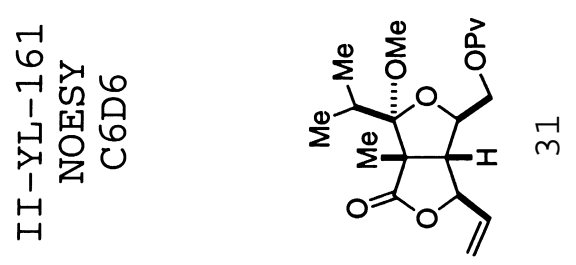




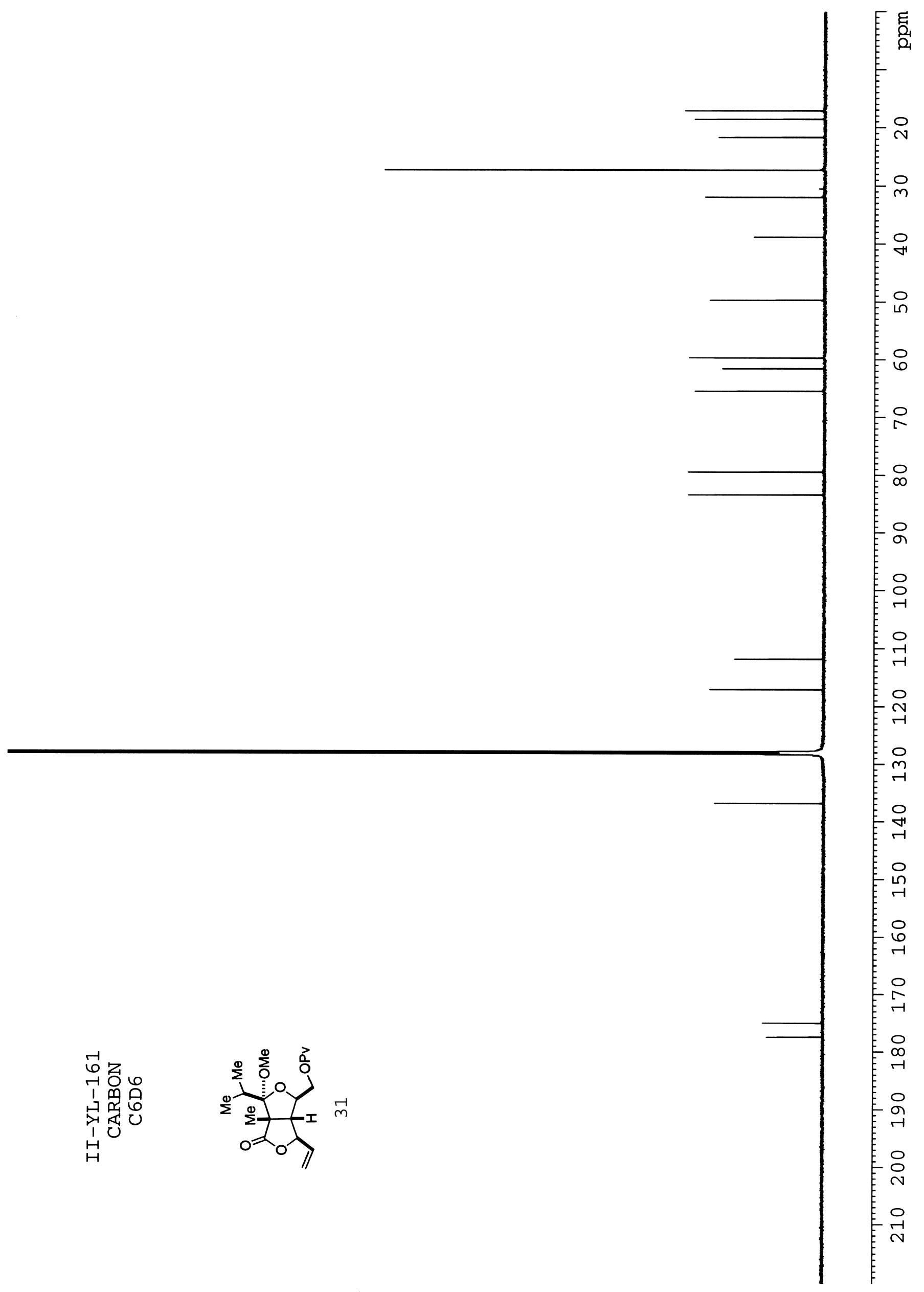




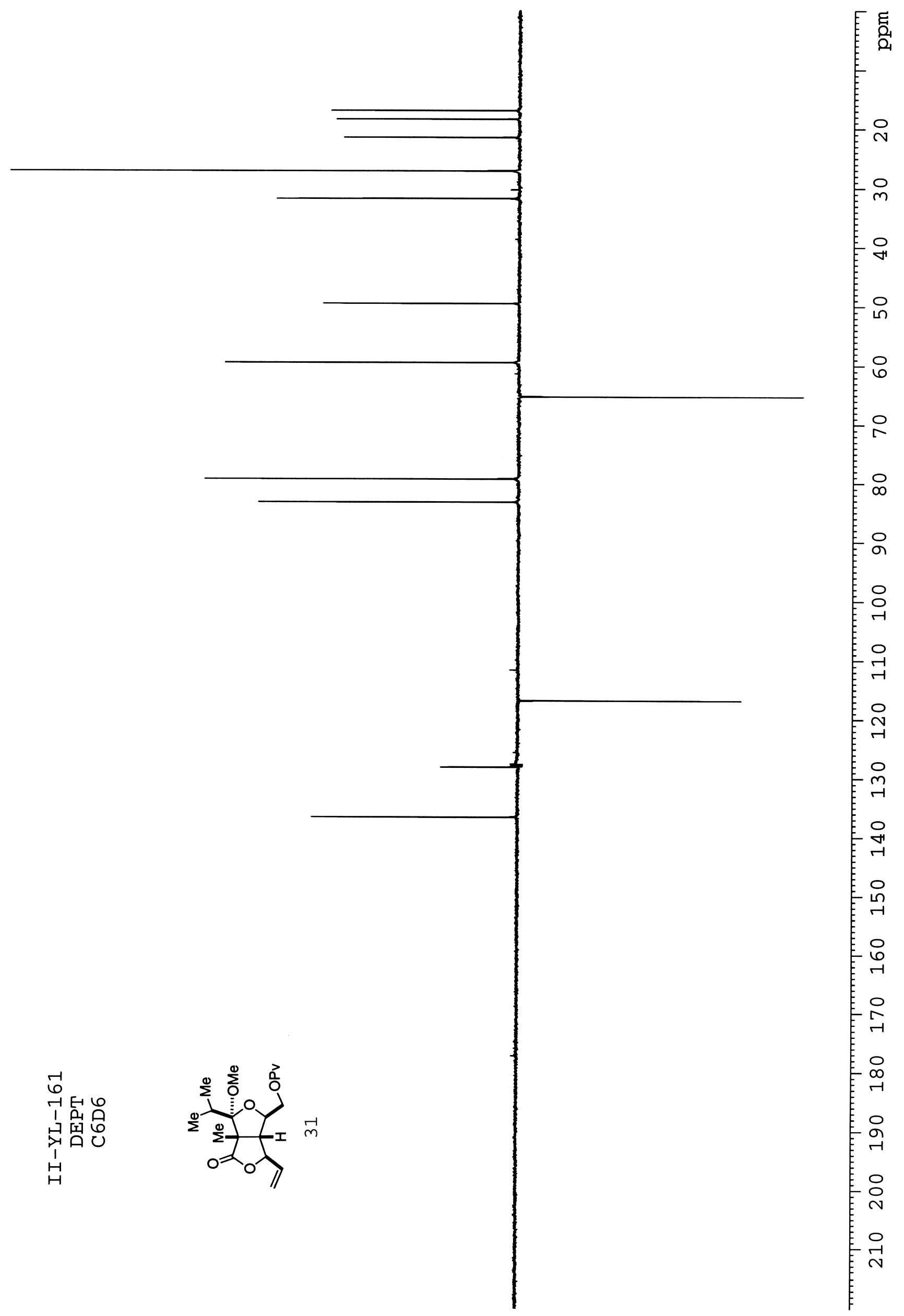




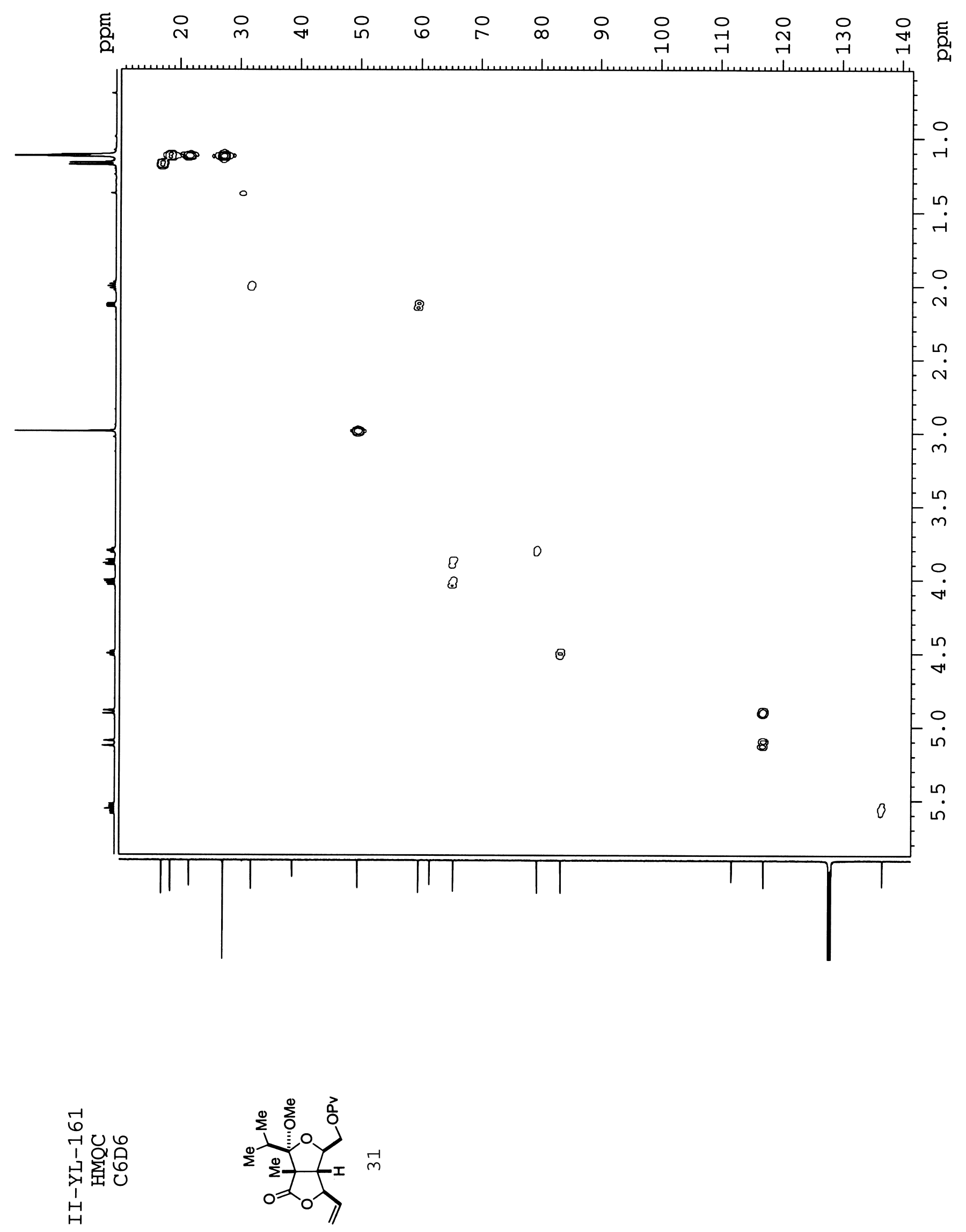



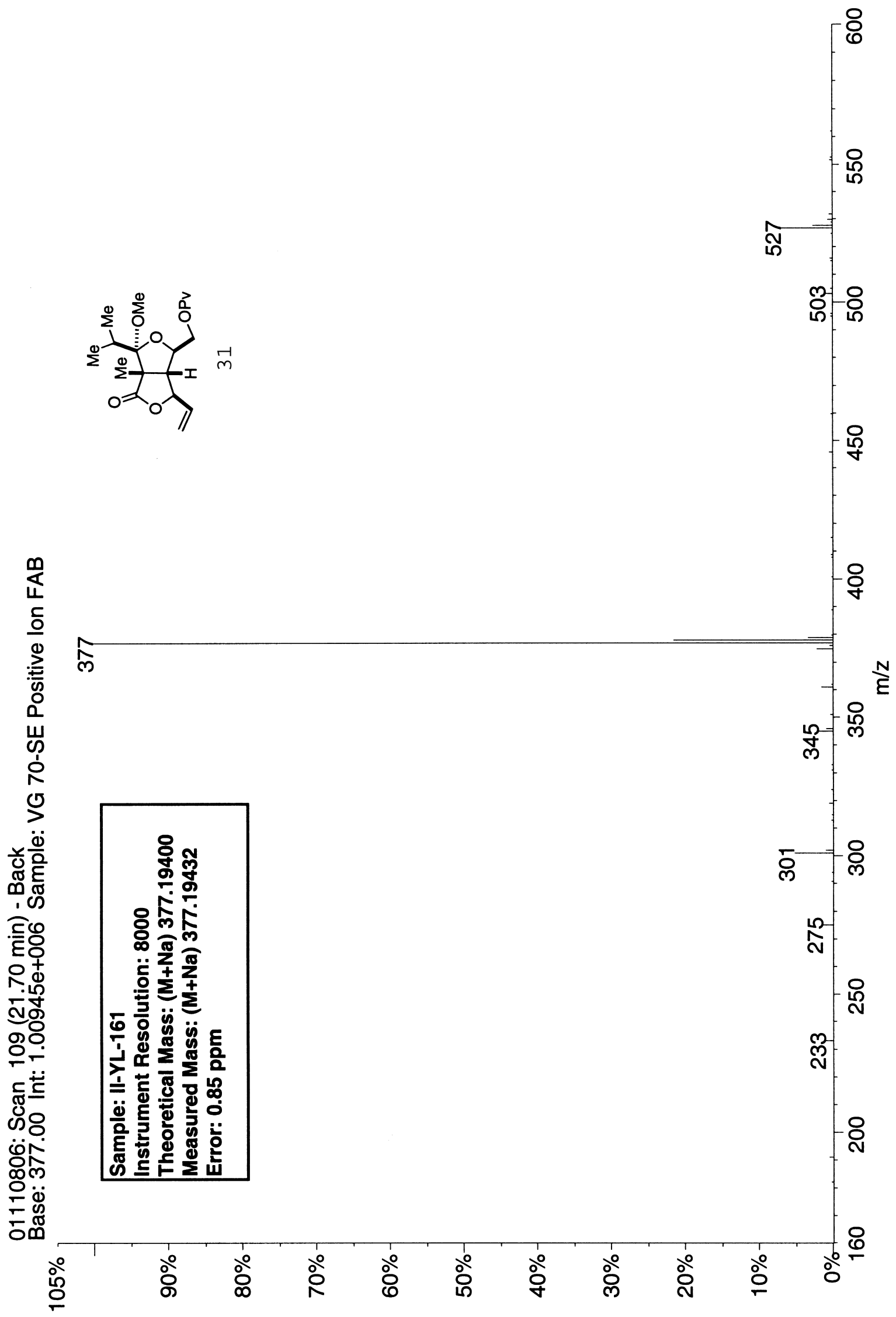


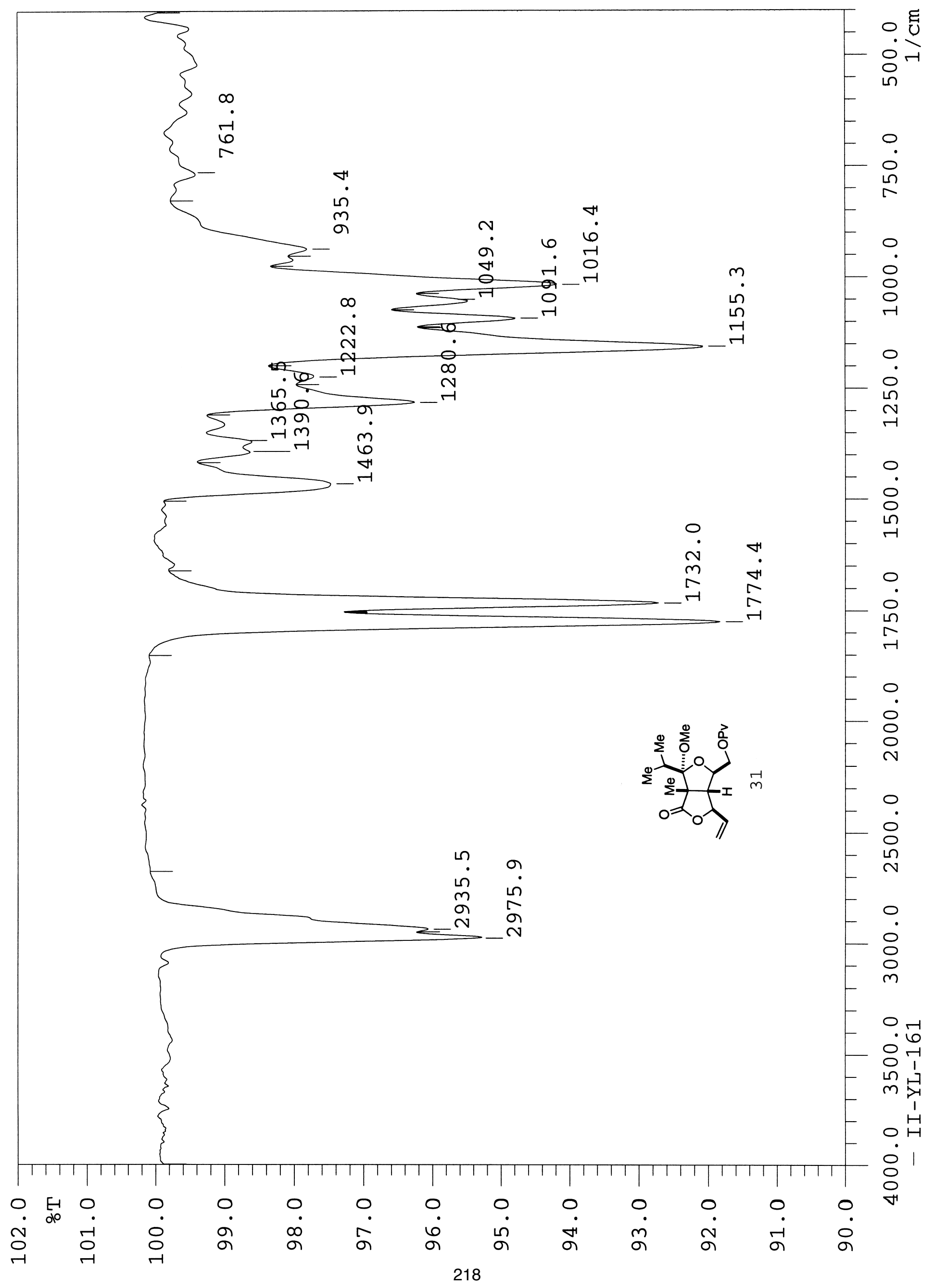




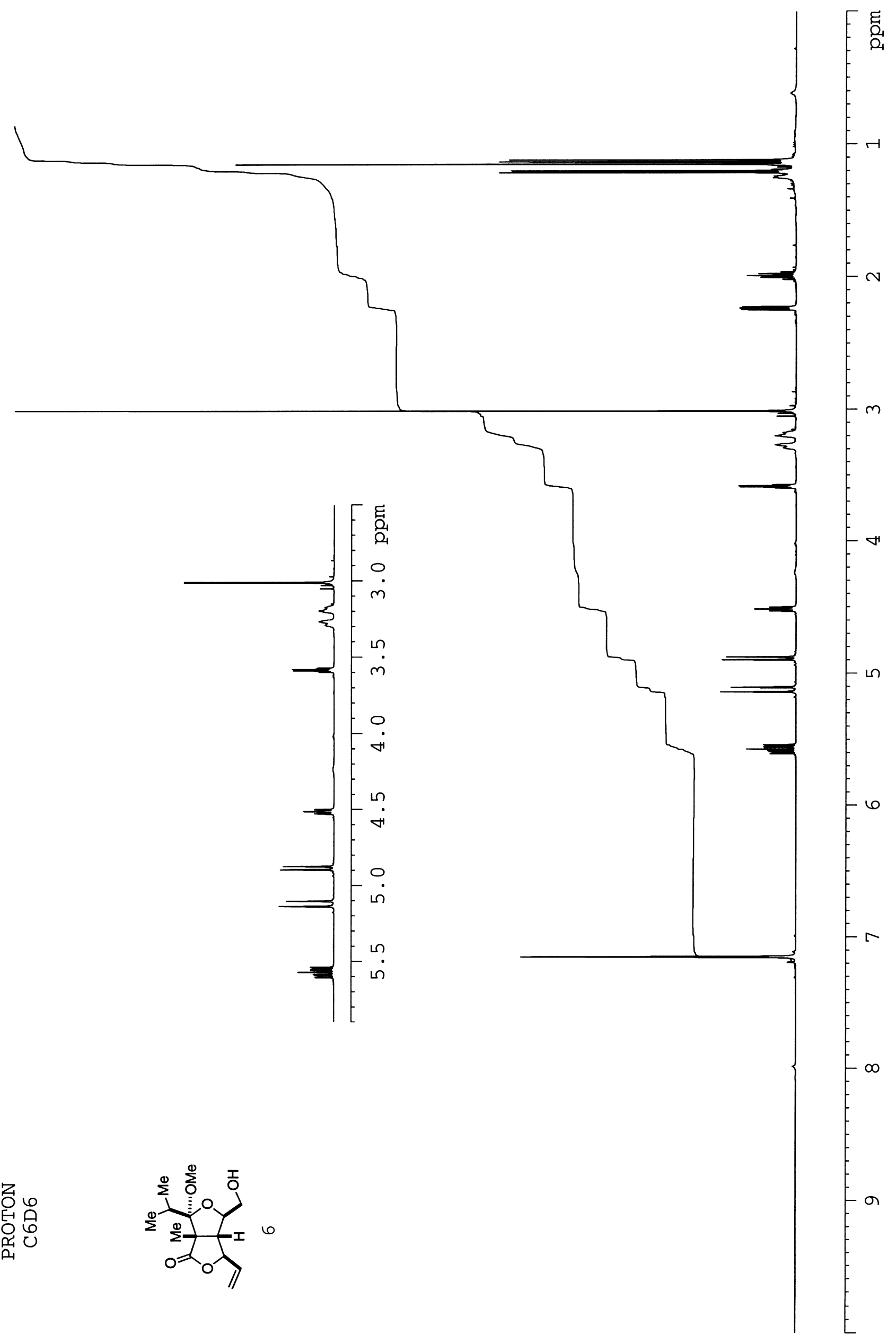



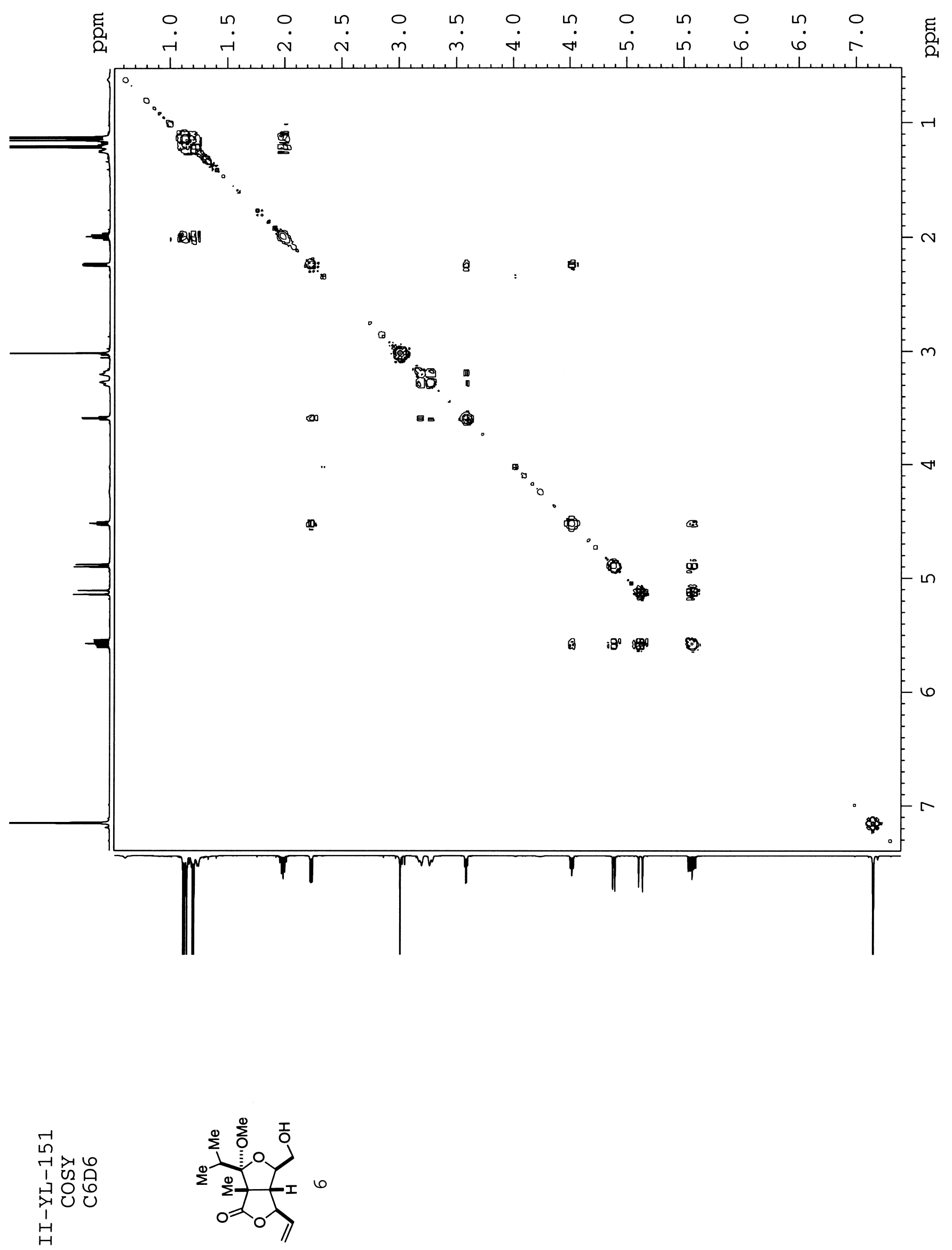


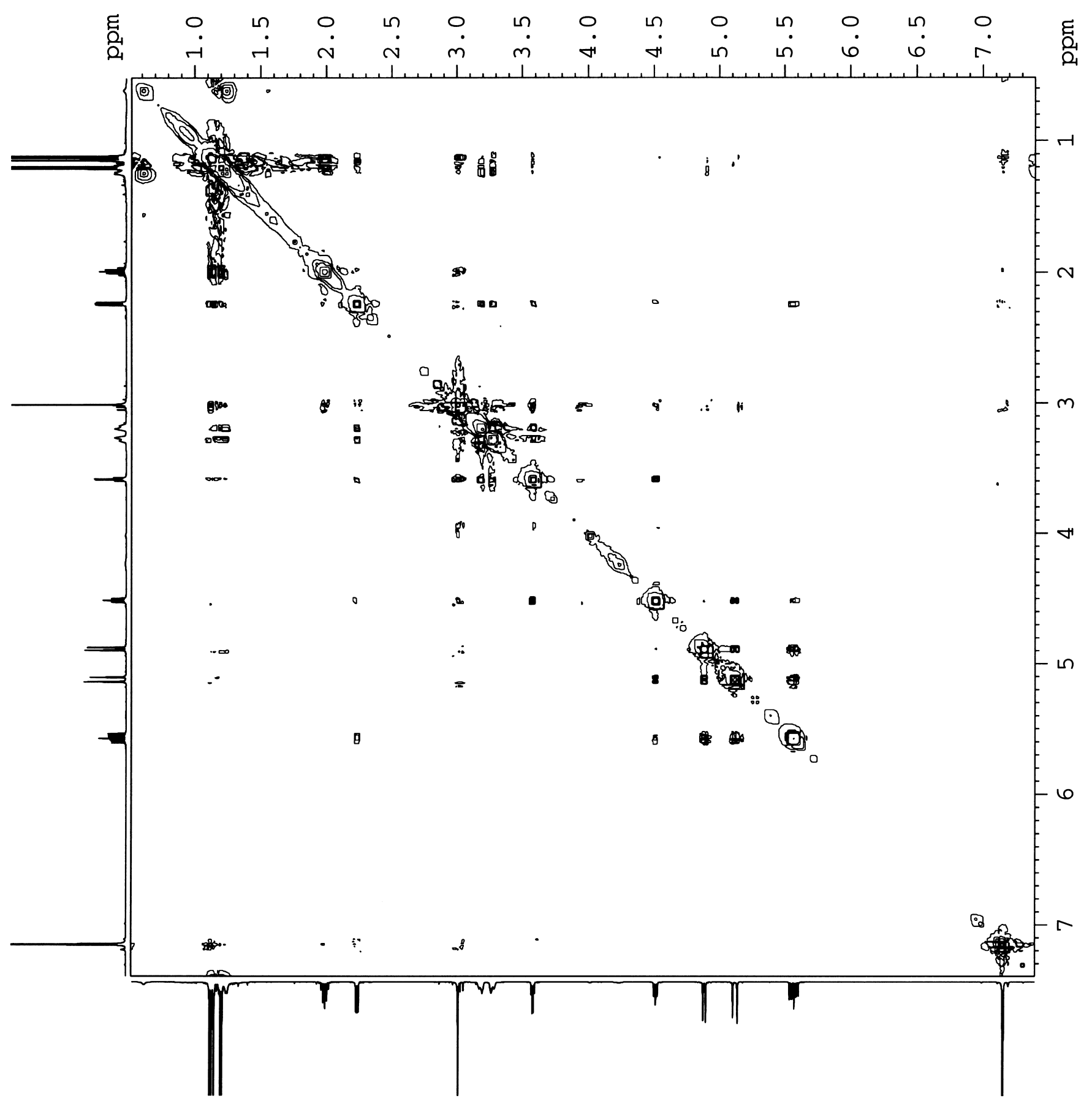

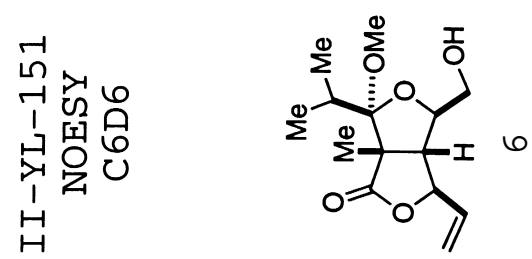




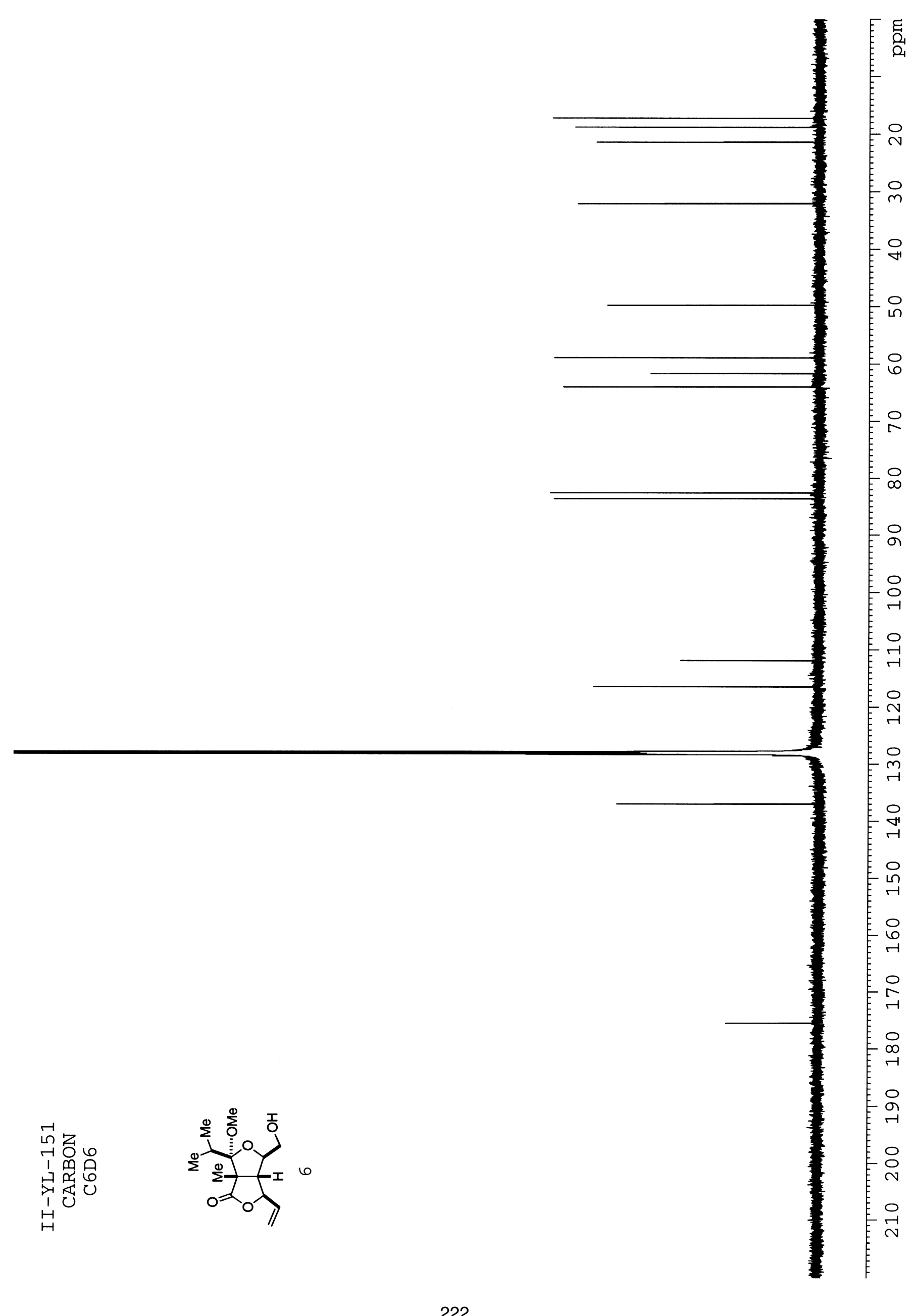



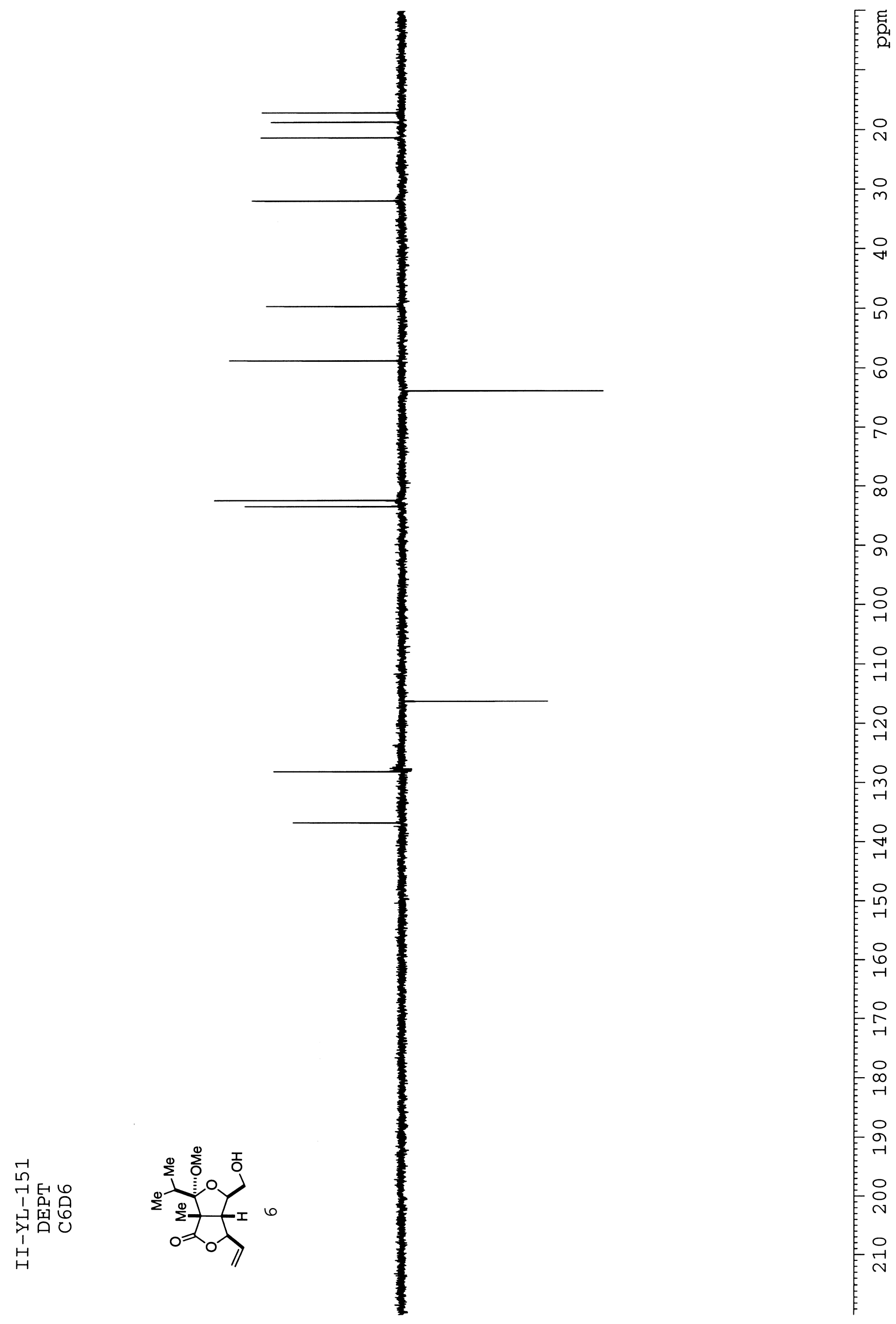


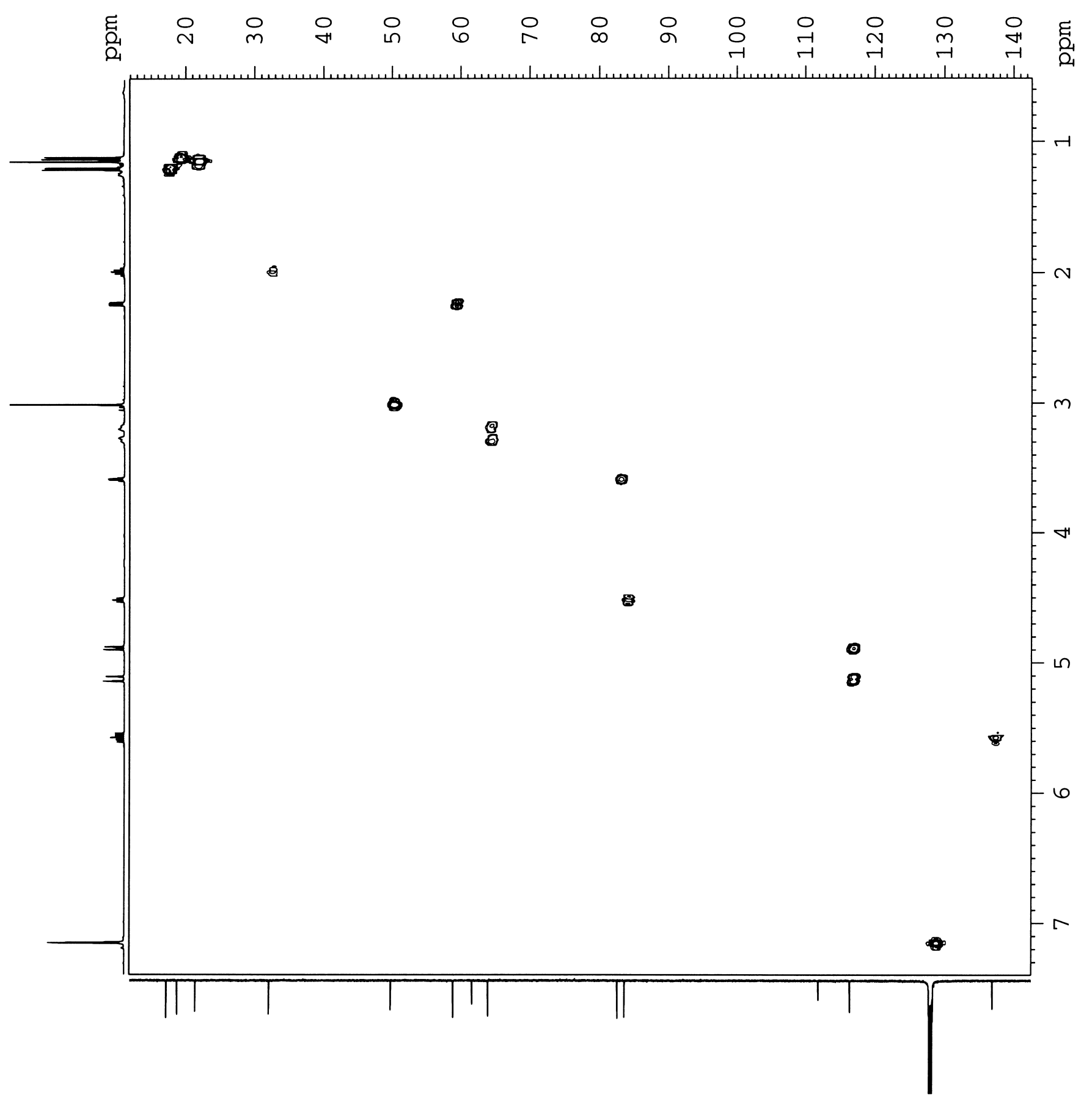

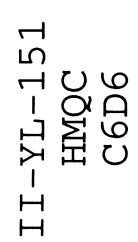

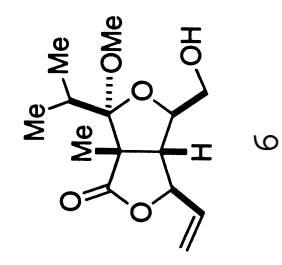




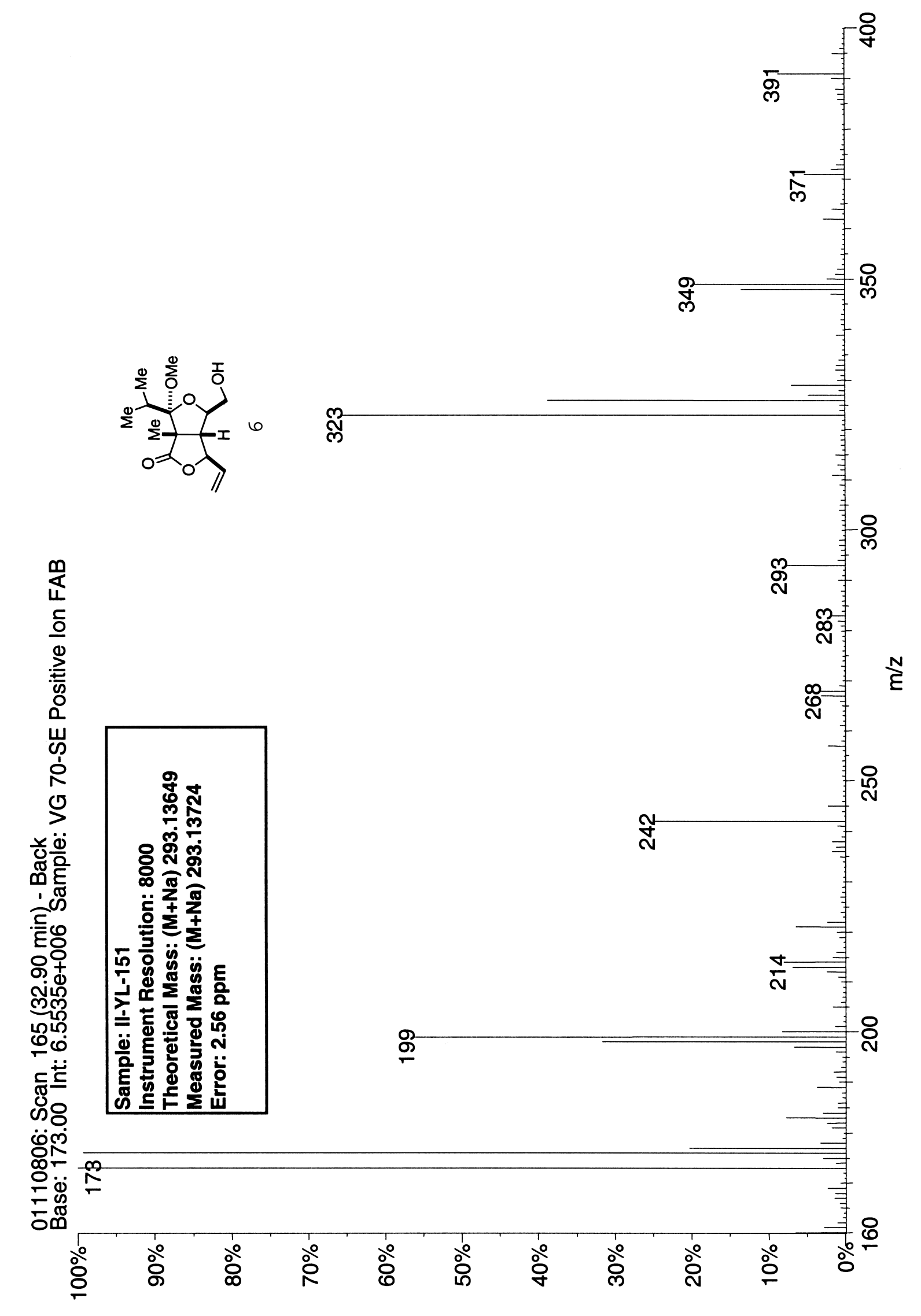




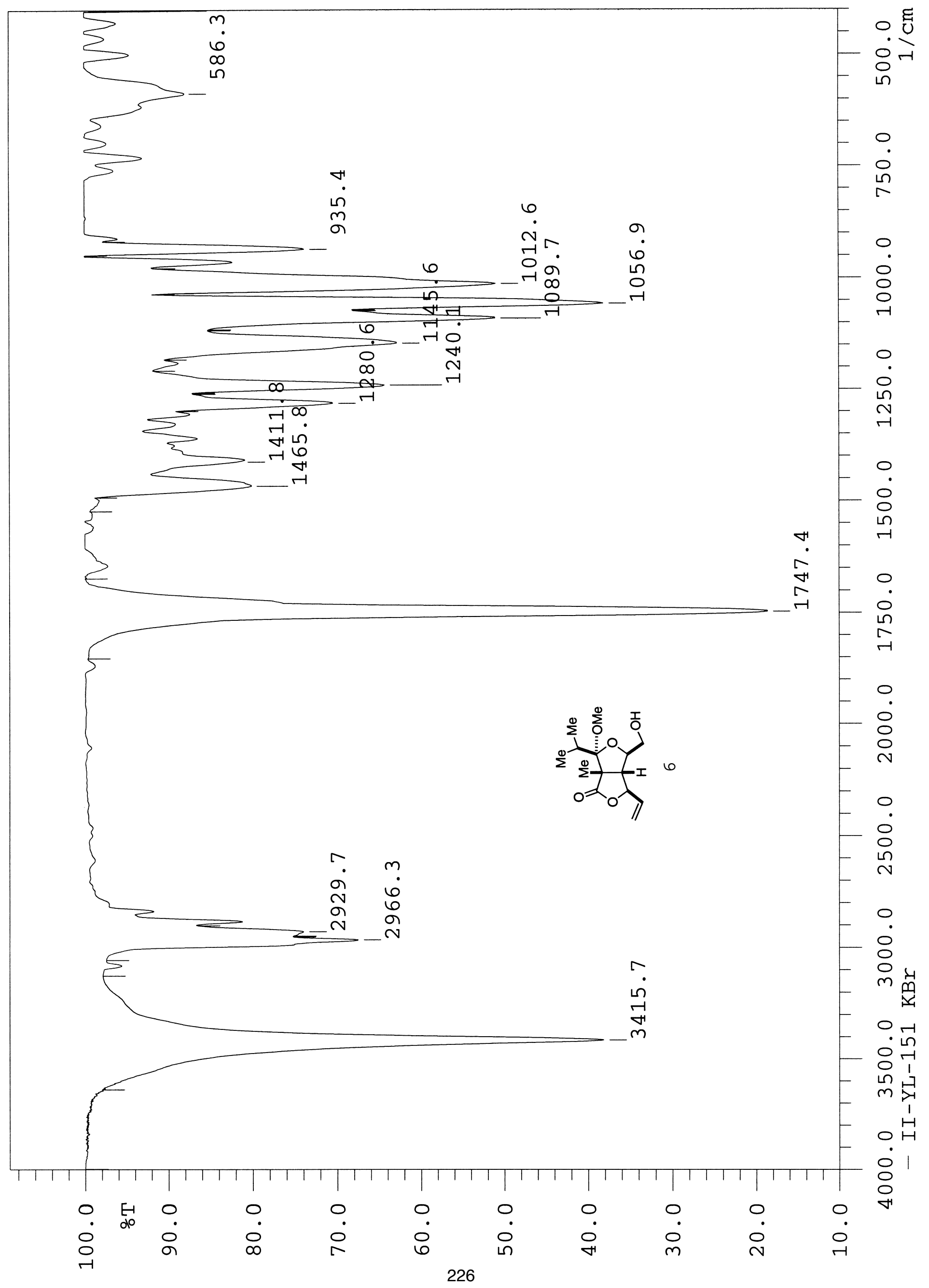




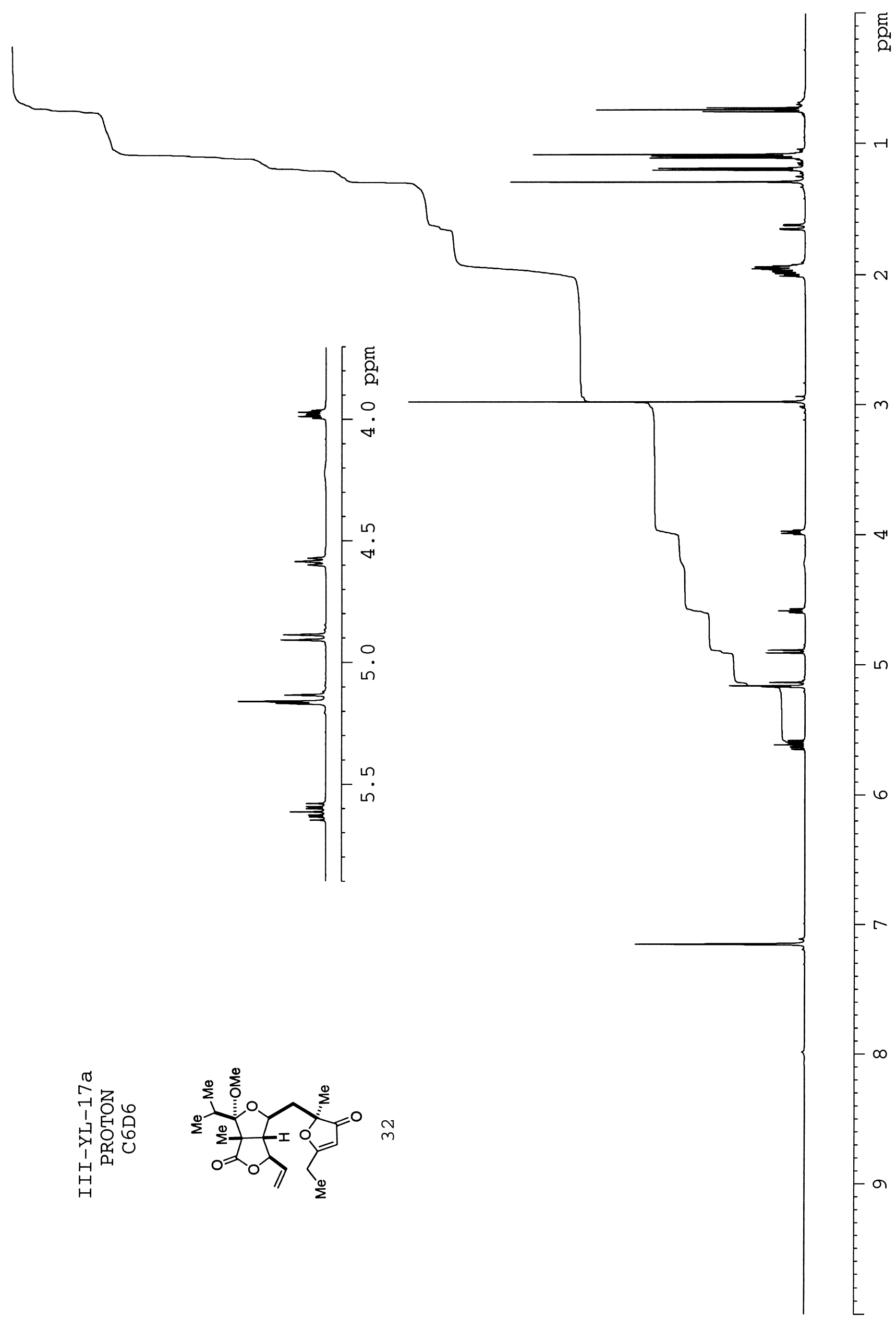




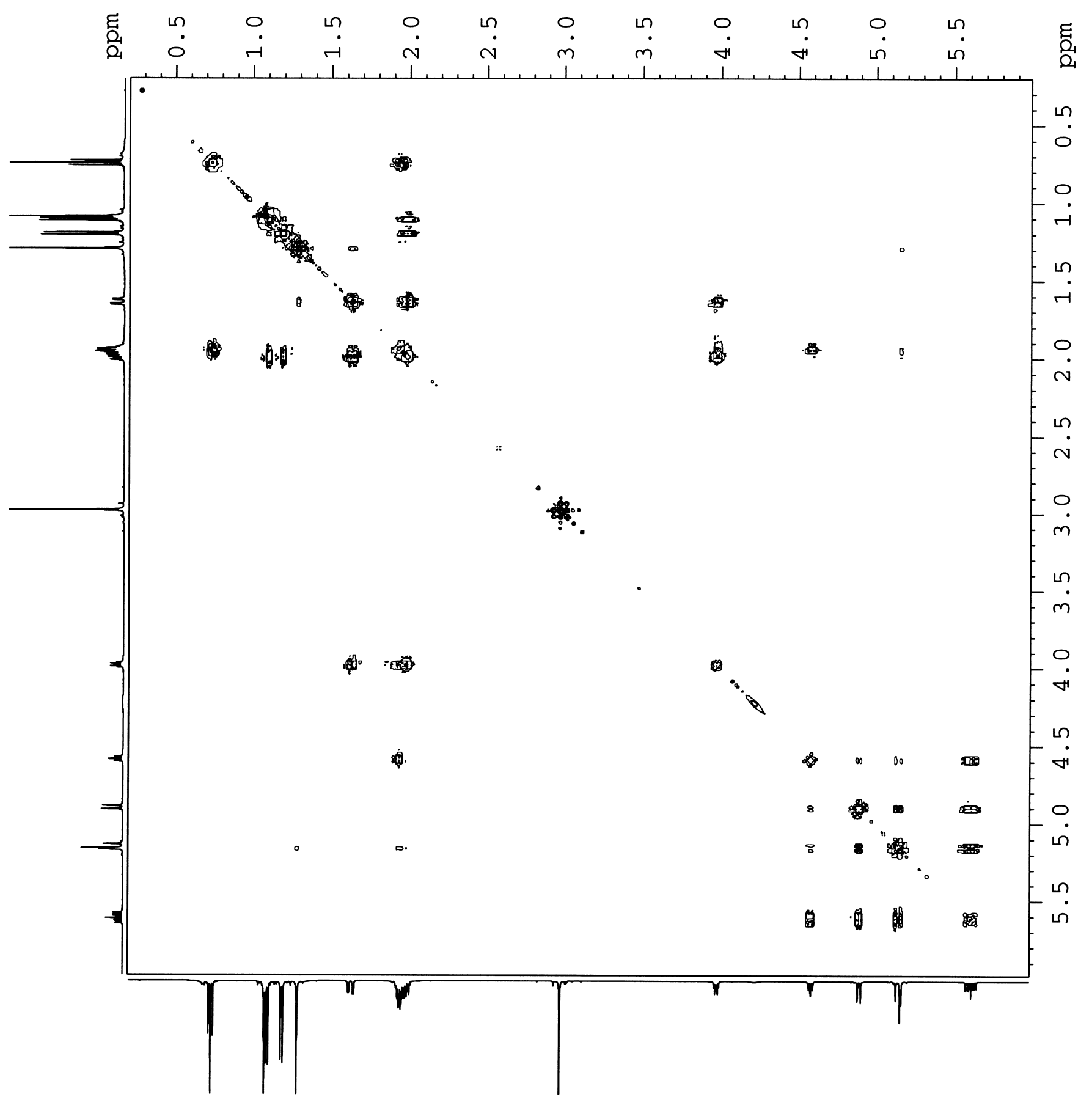

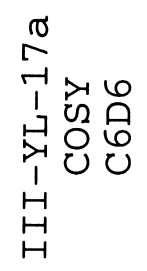

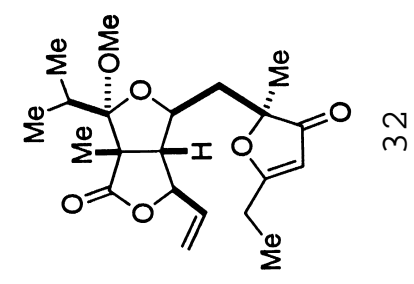




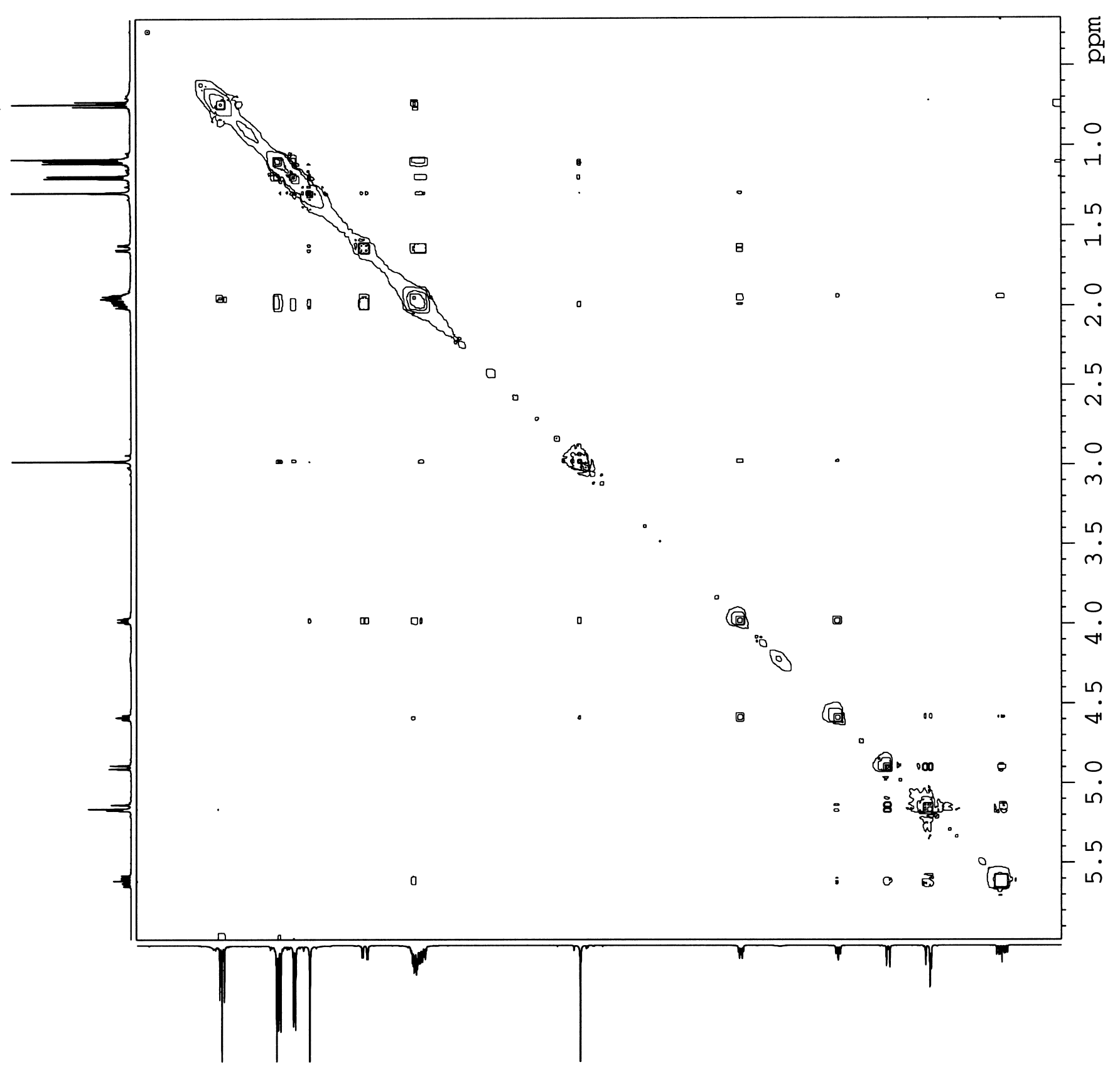

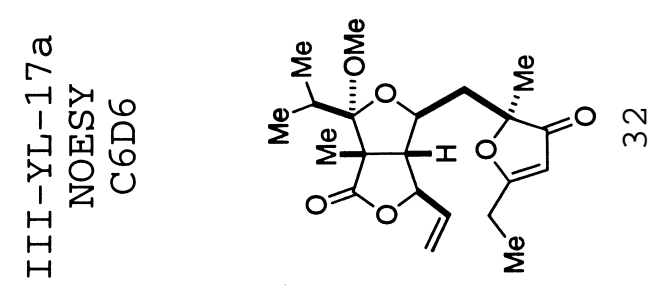




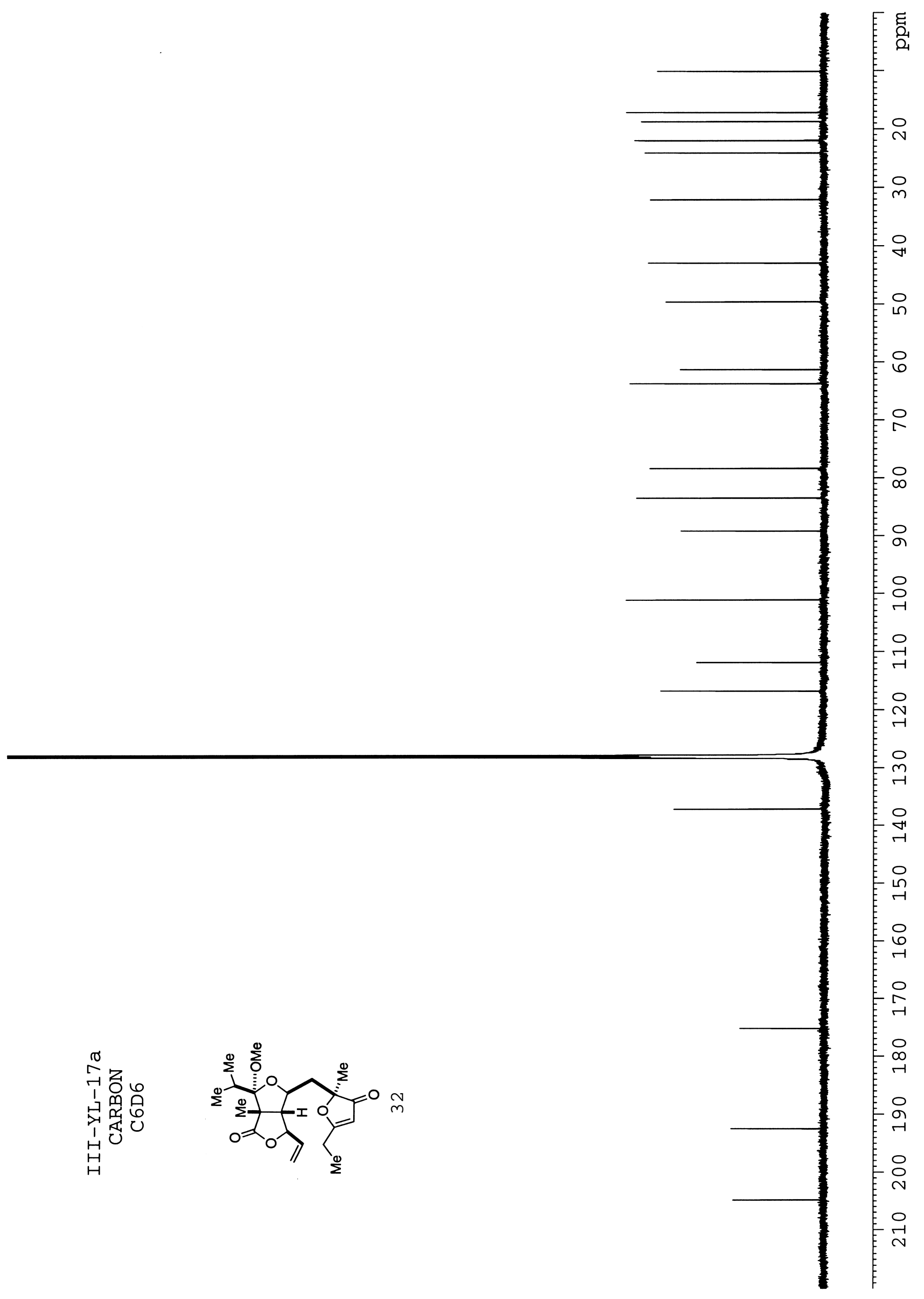




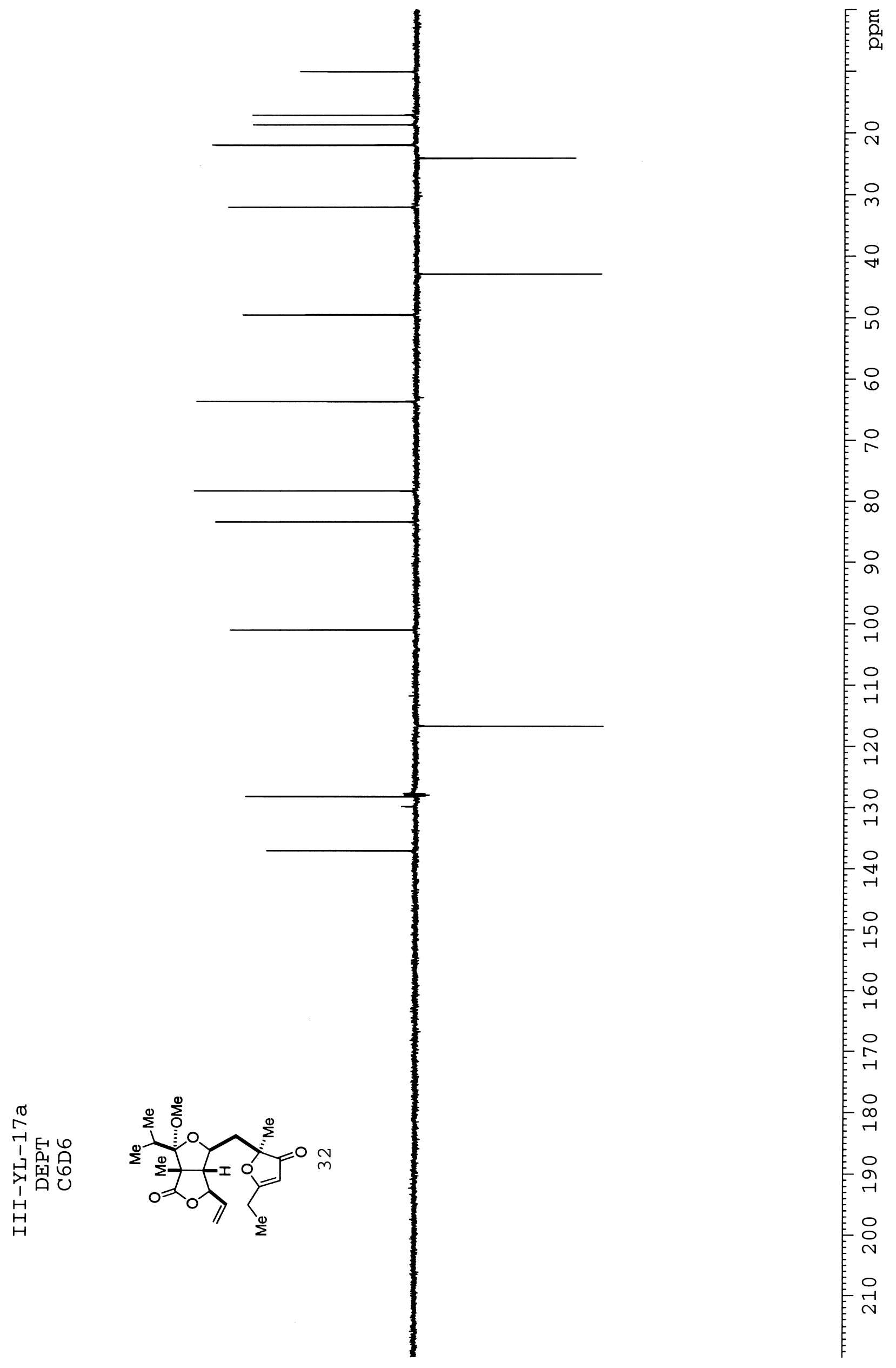




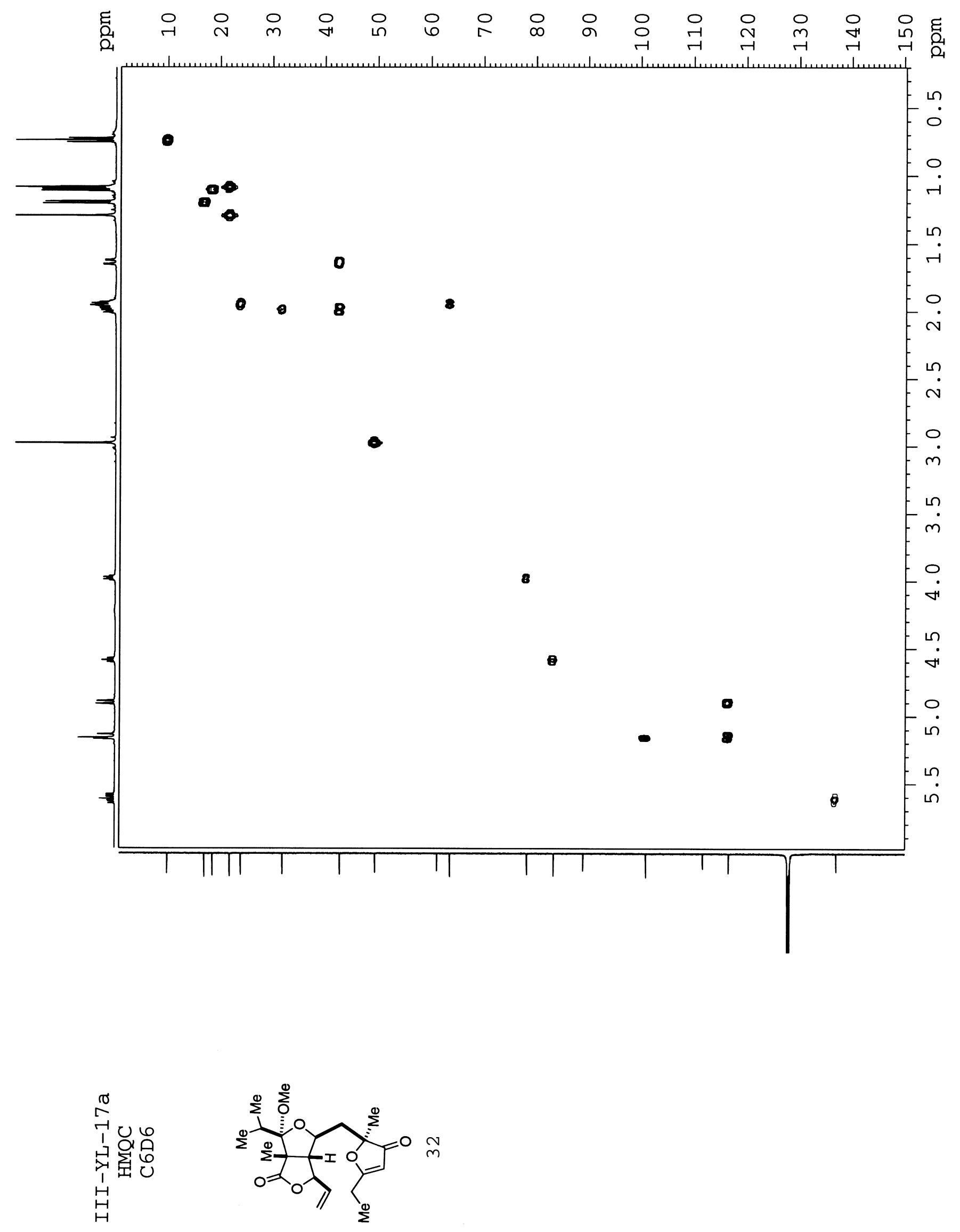




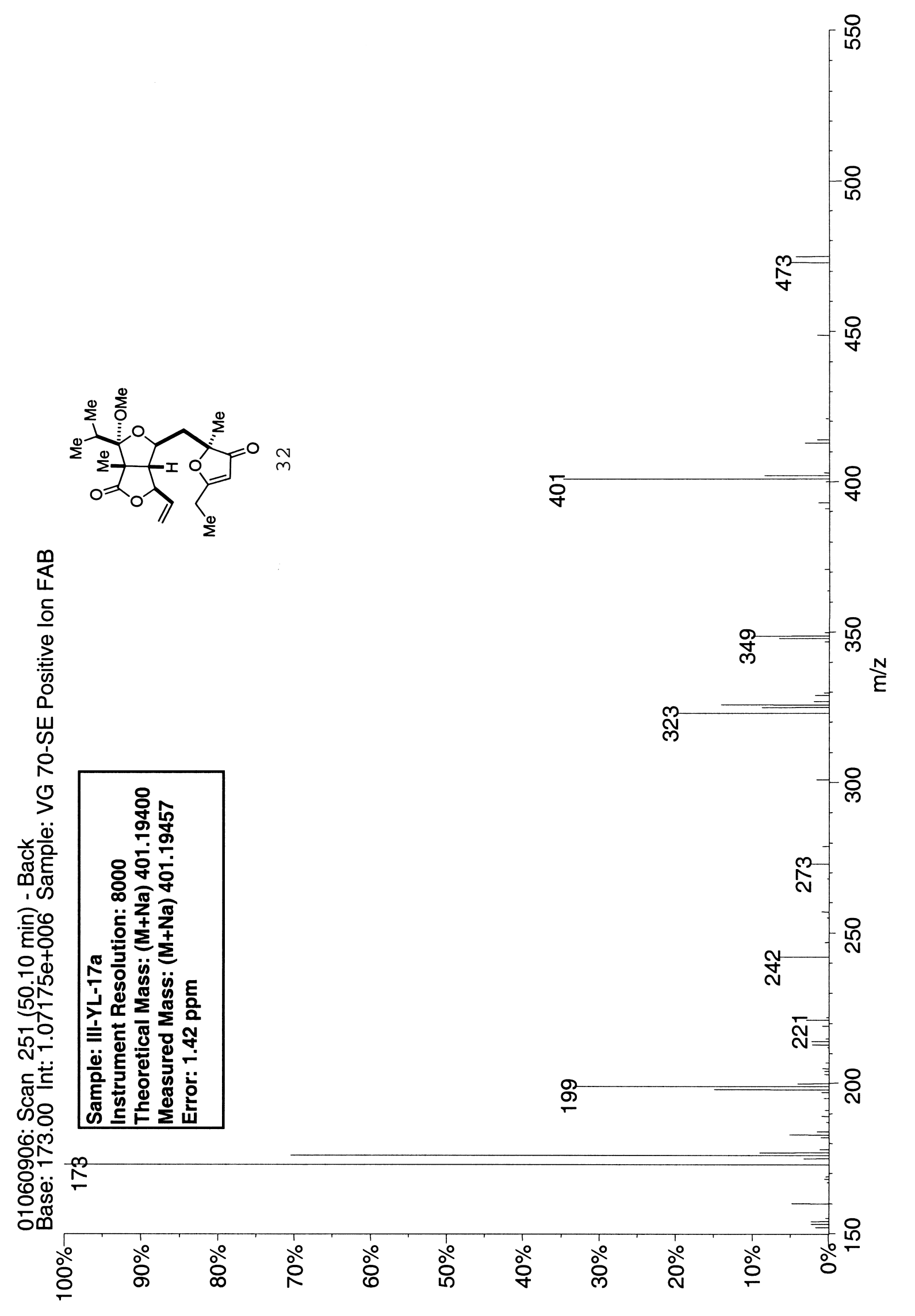




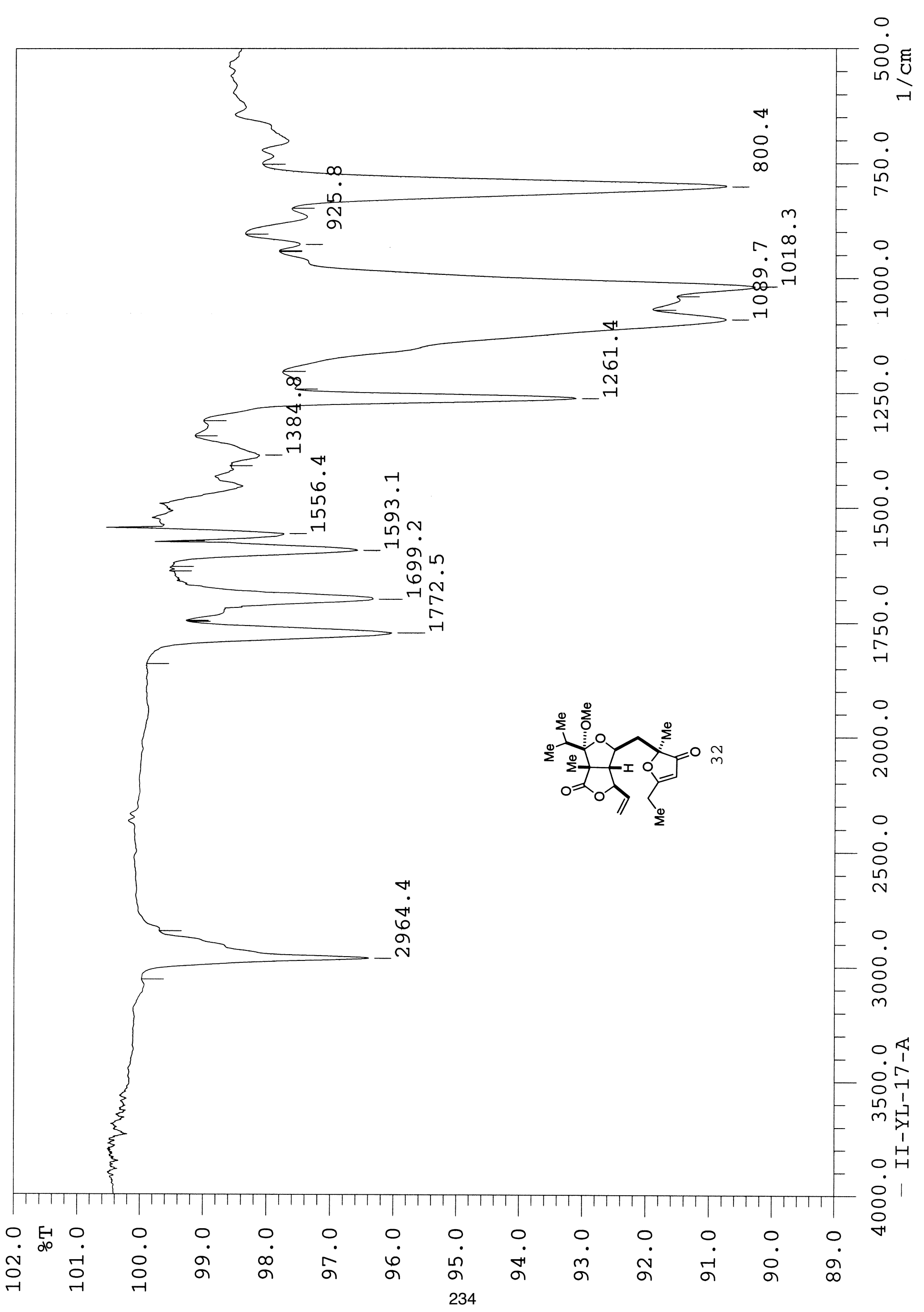




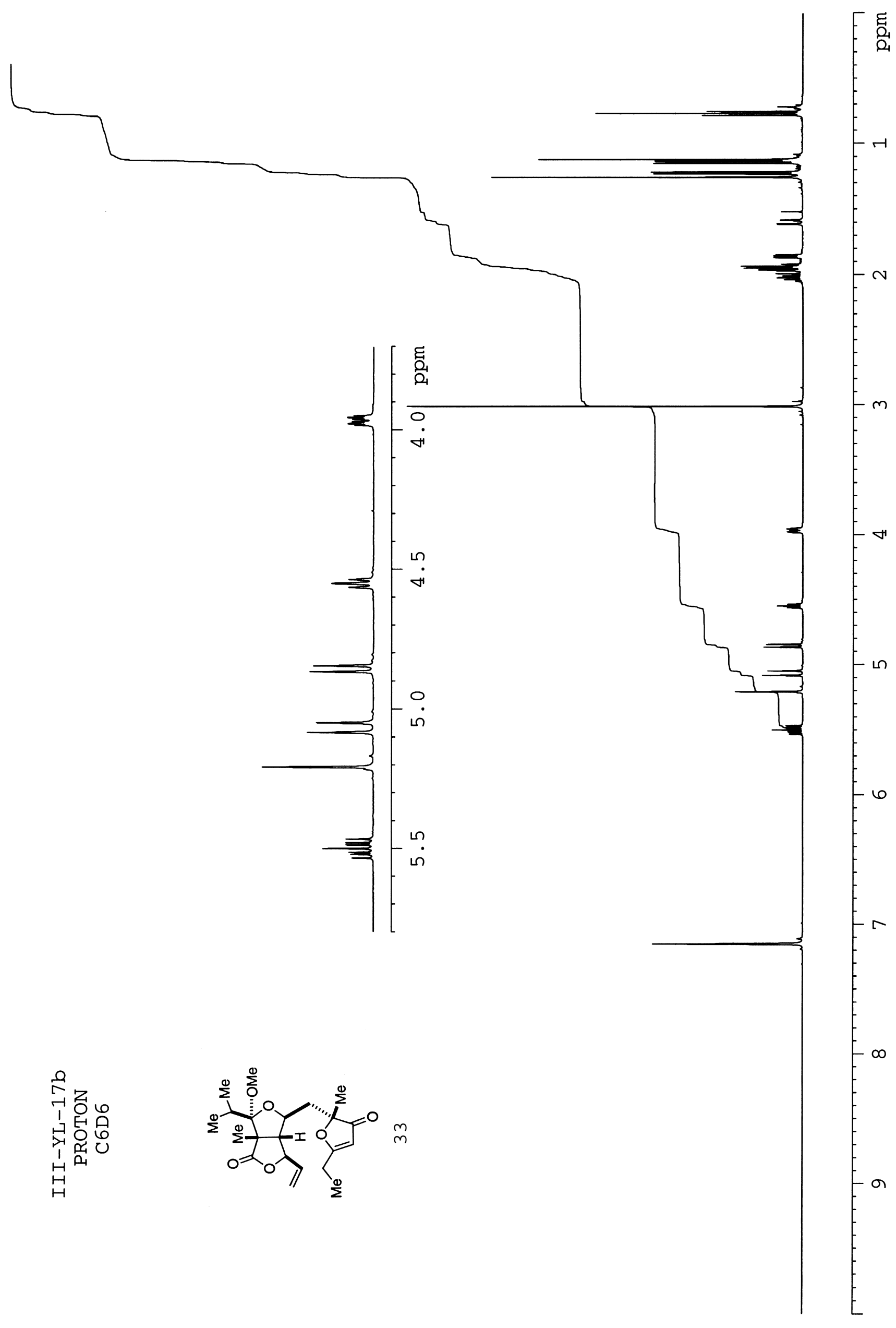



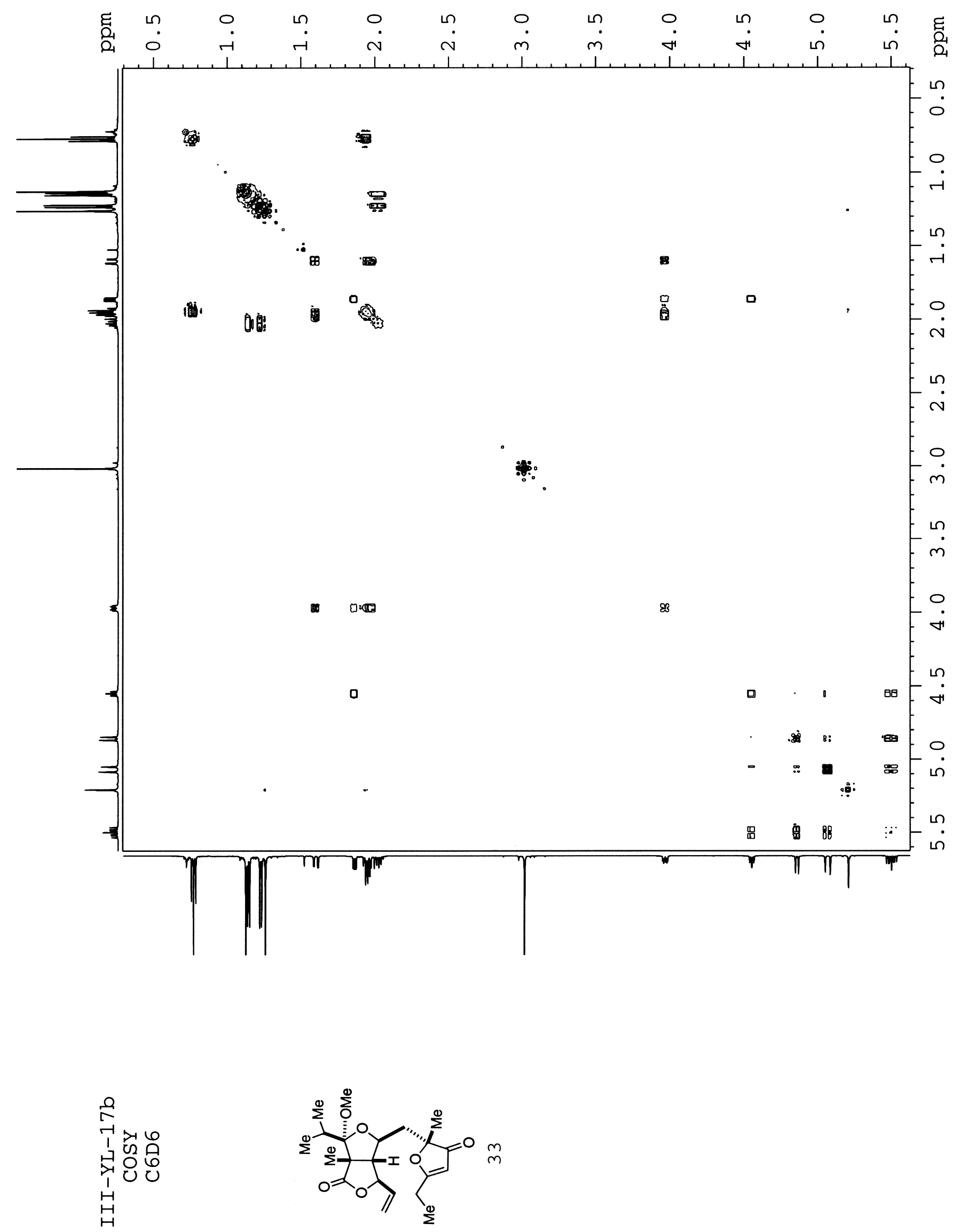

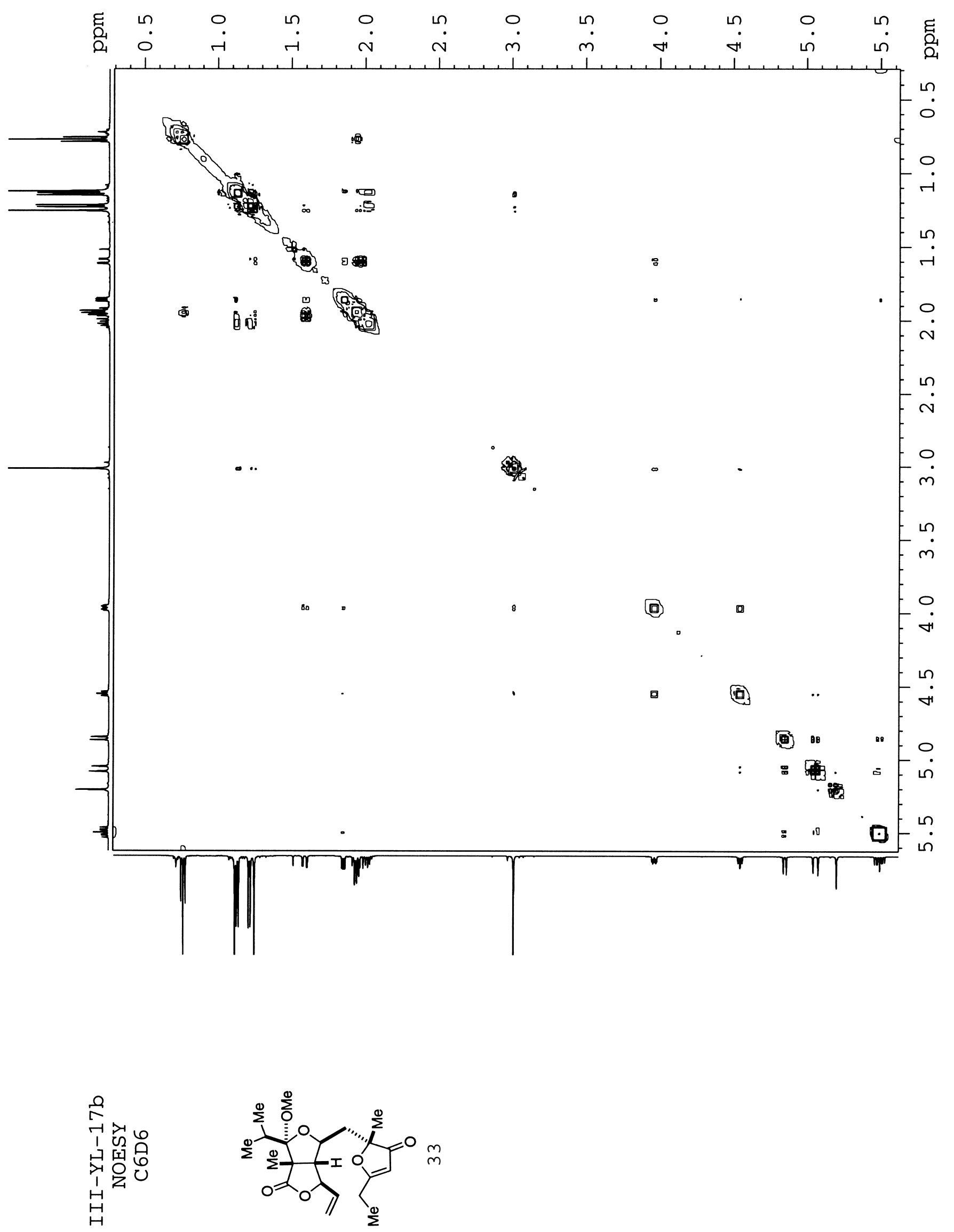


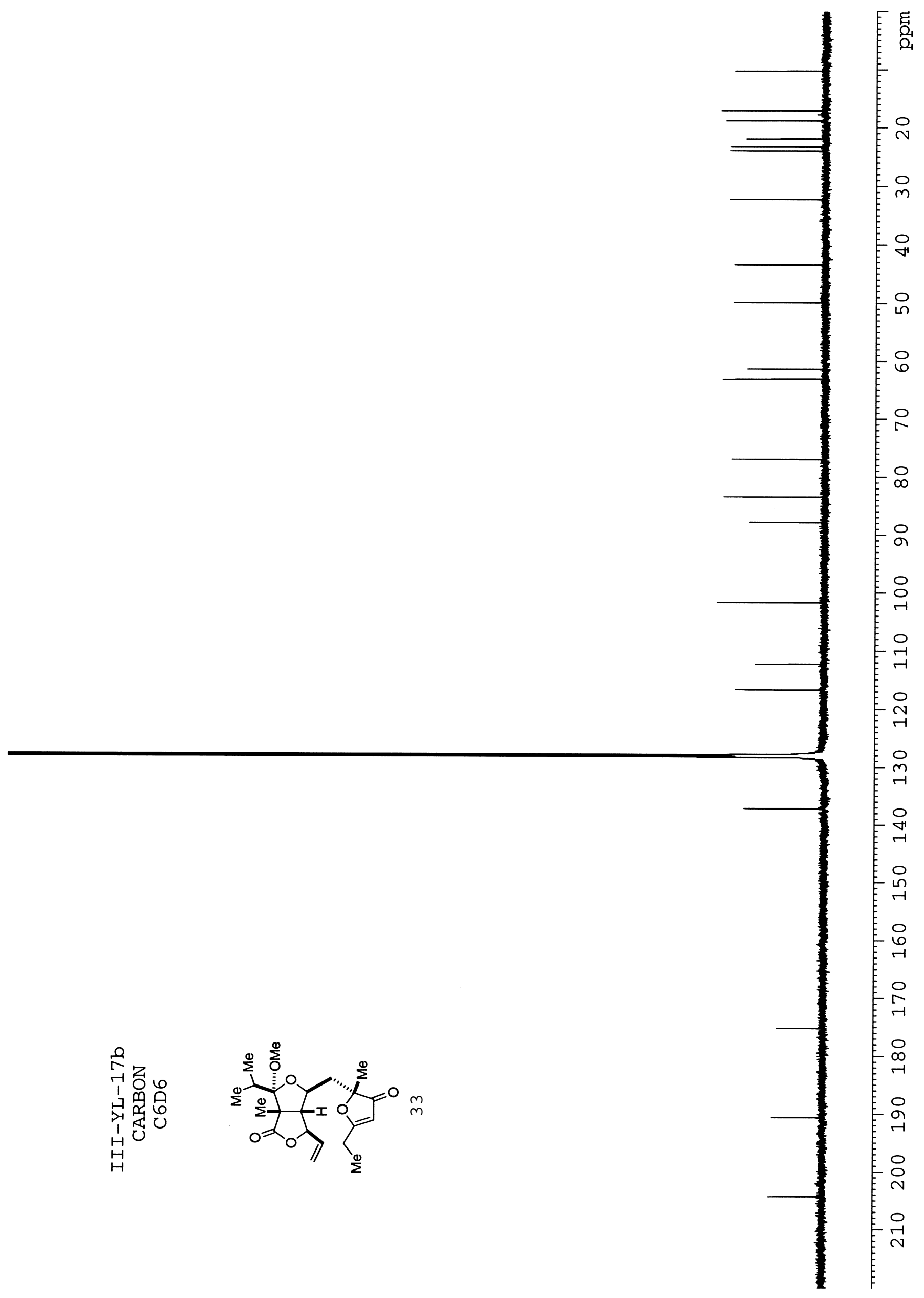



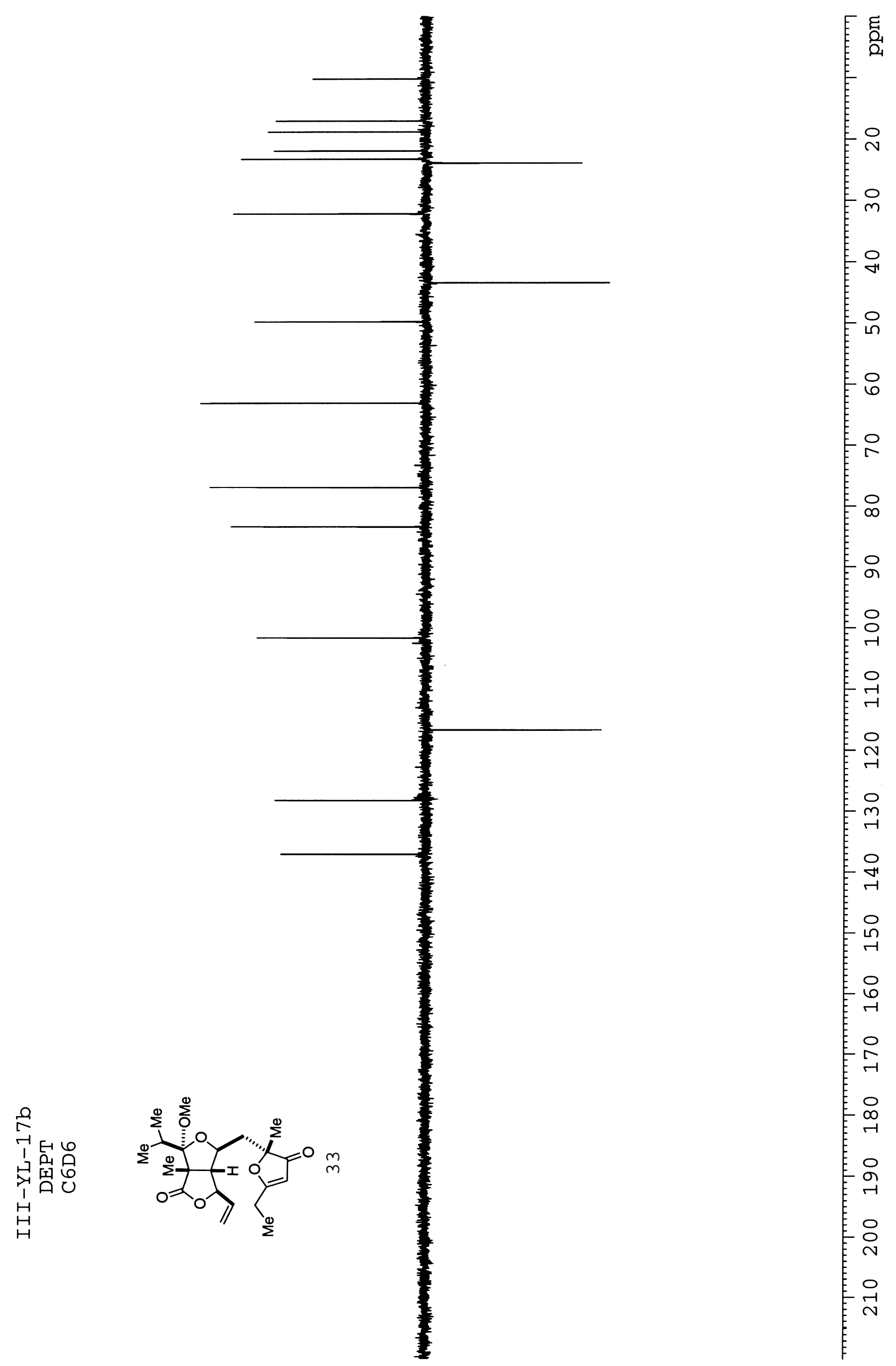


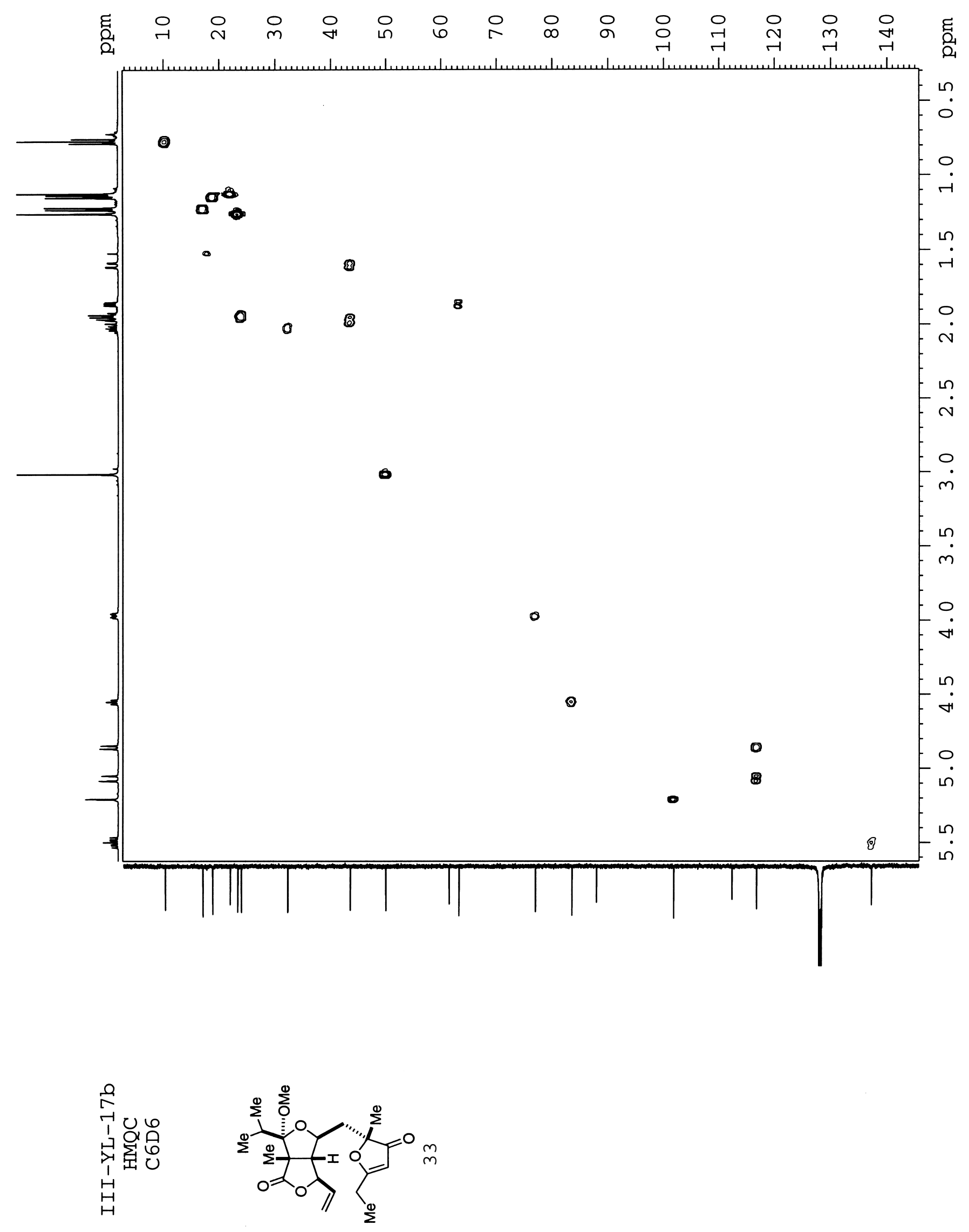



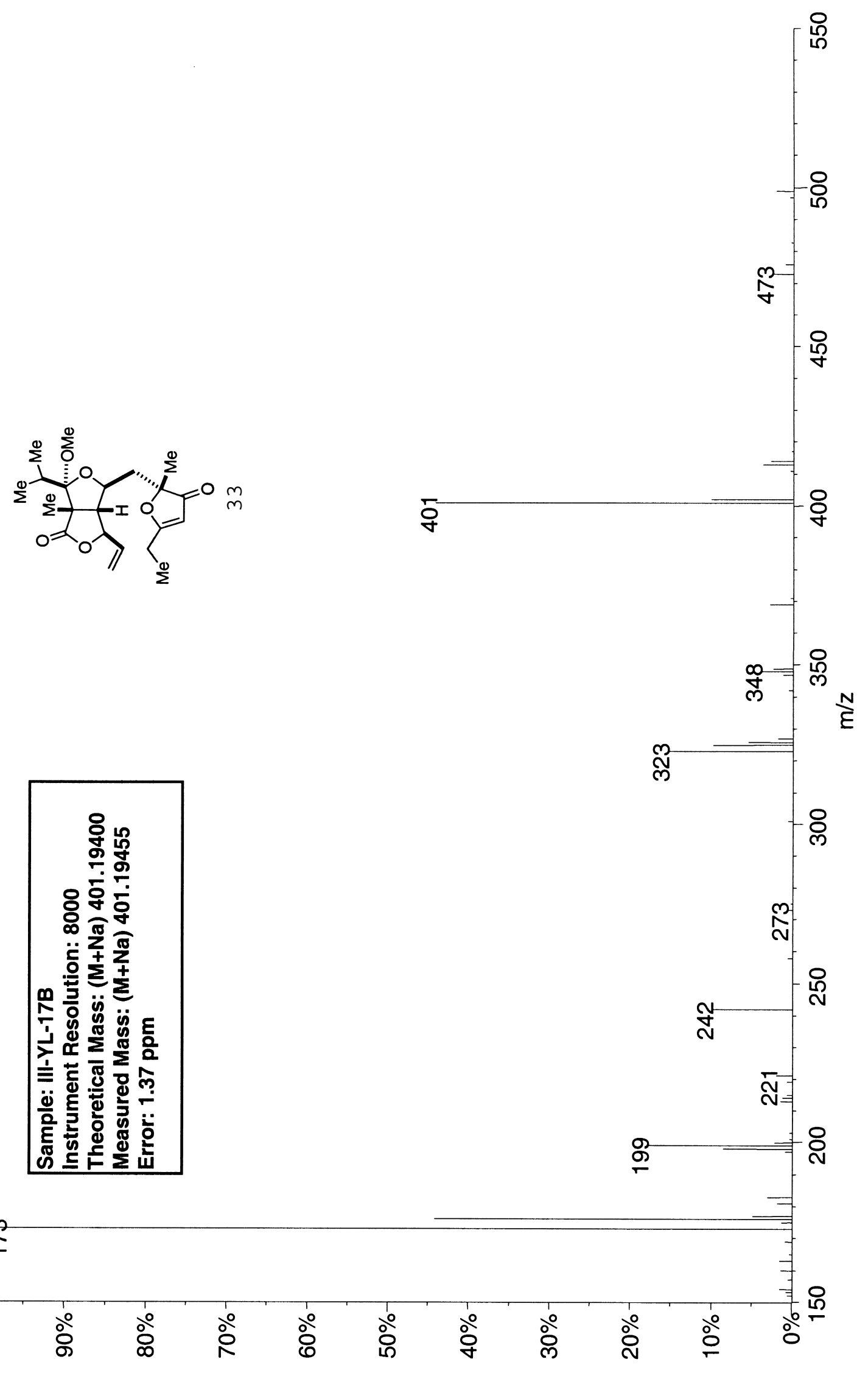


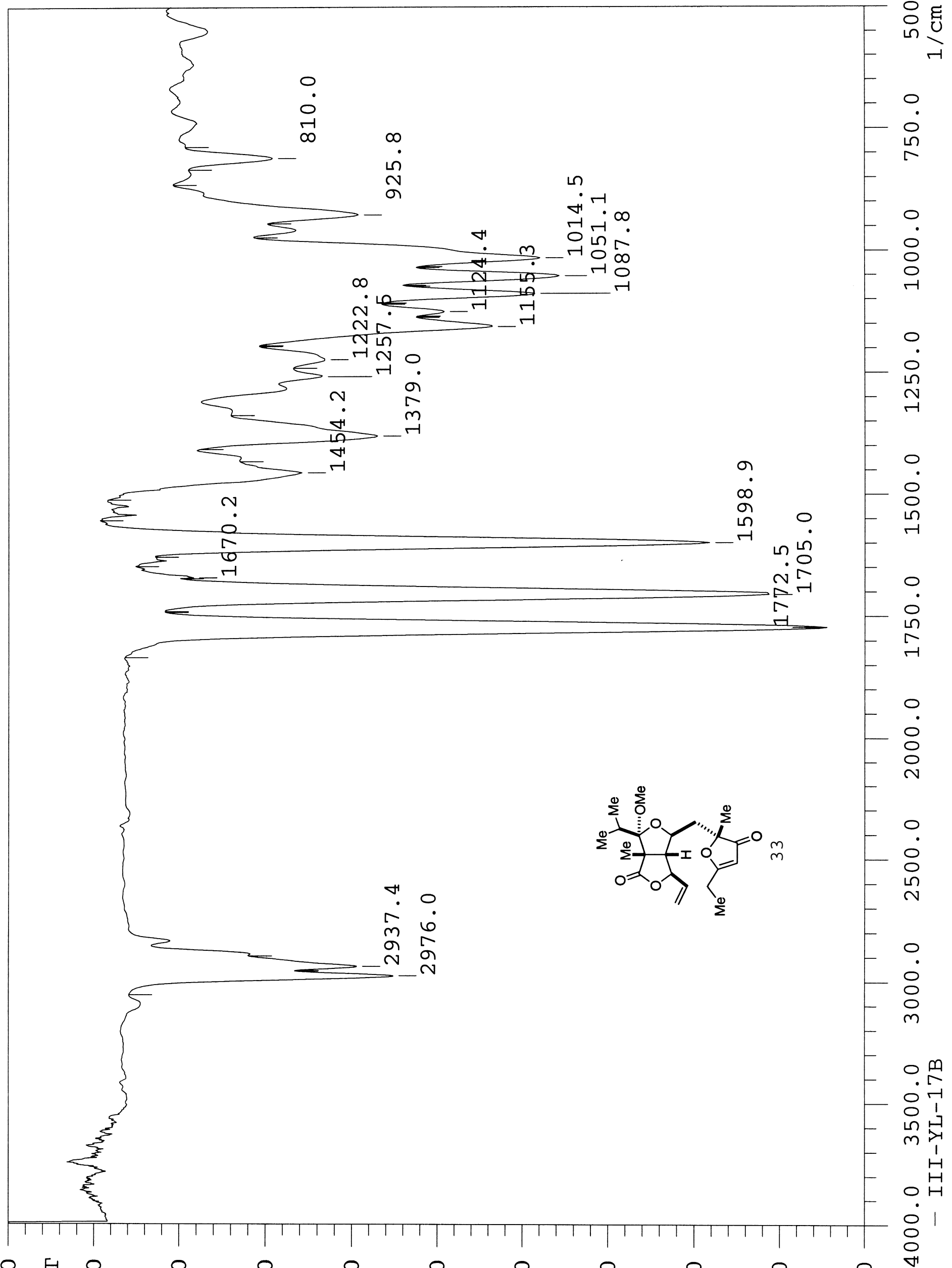

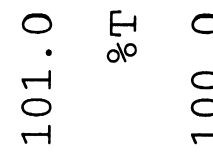

बे

0
$\infty$

๙ิ

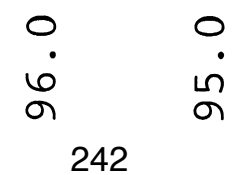

वं

0
ñ

ำ

ने 


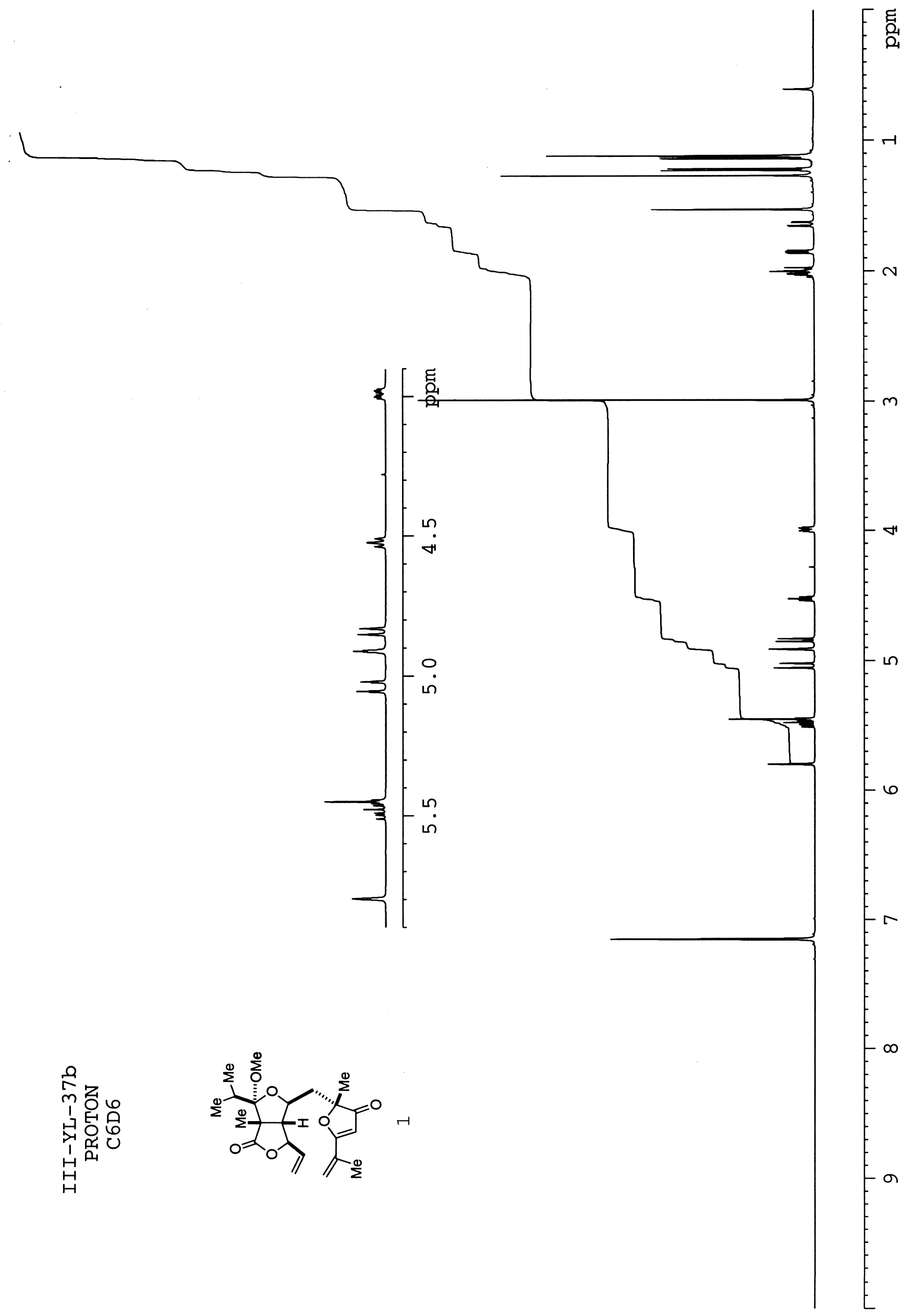



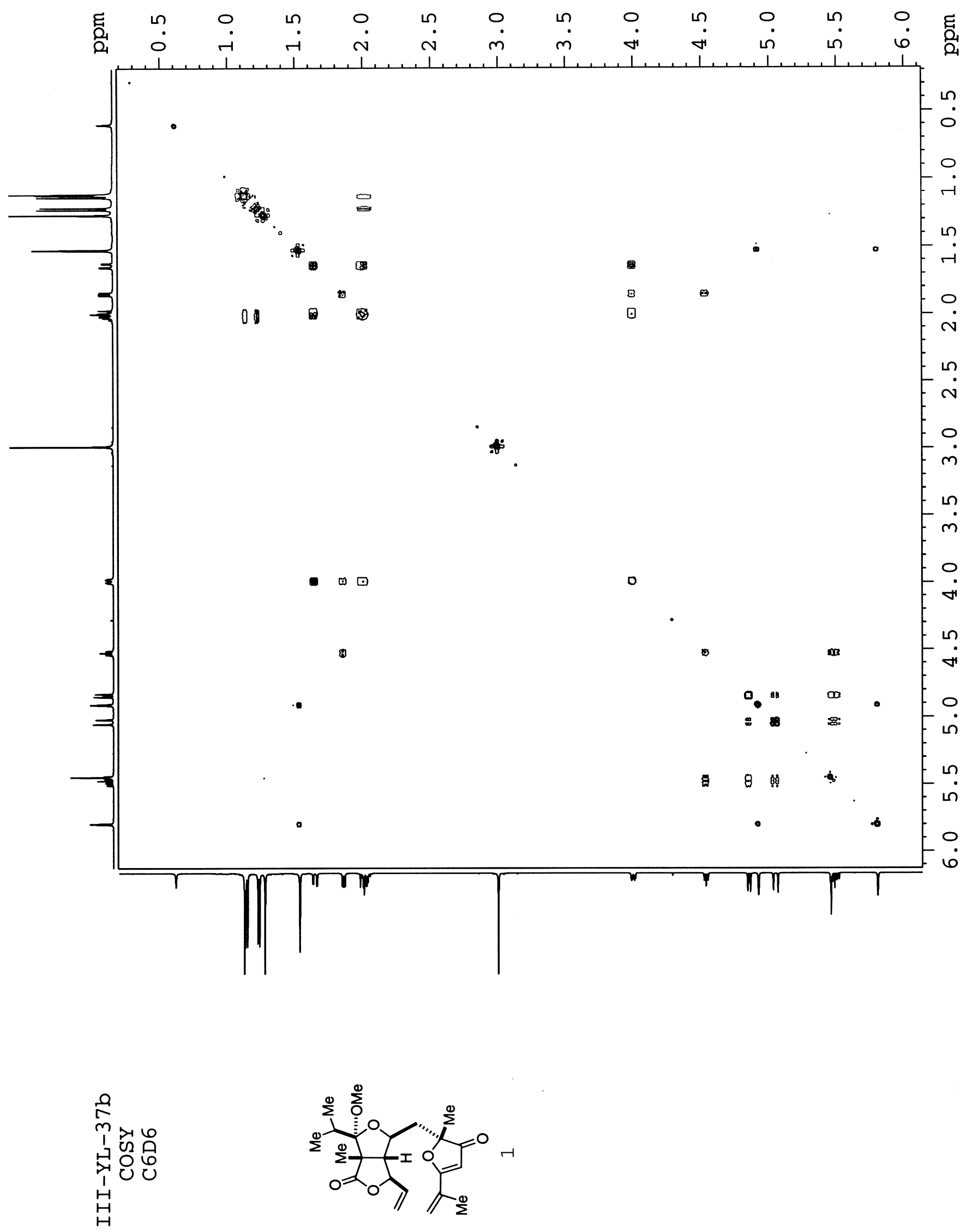

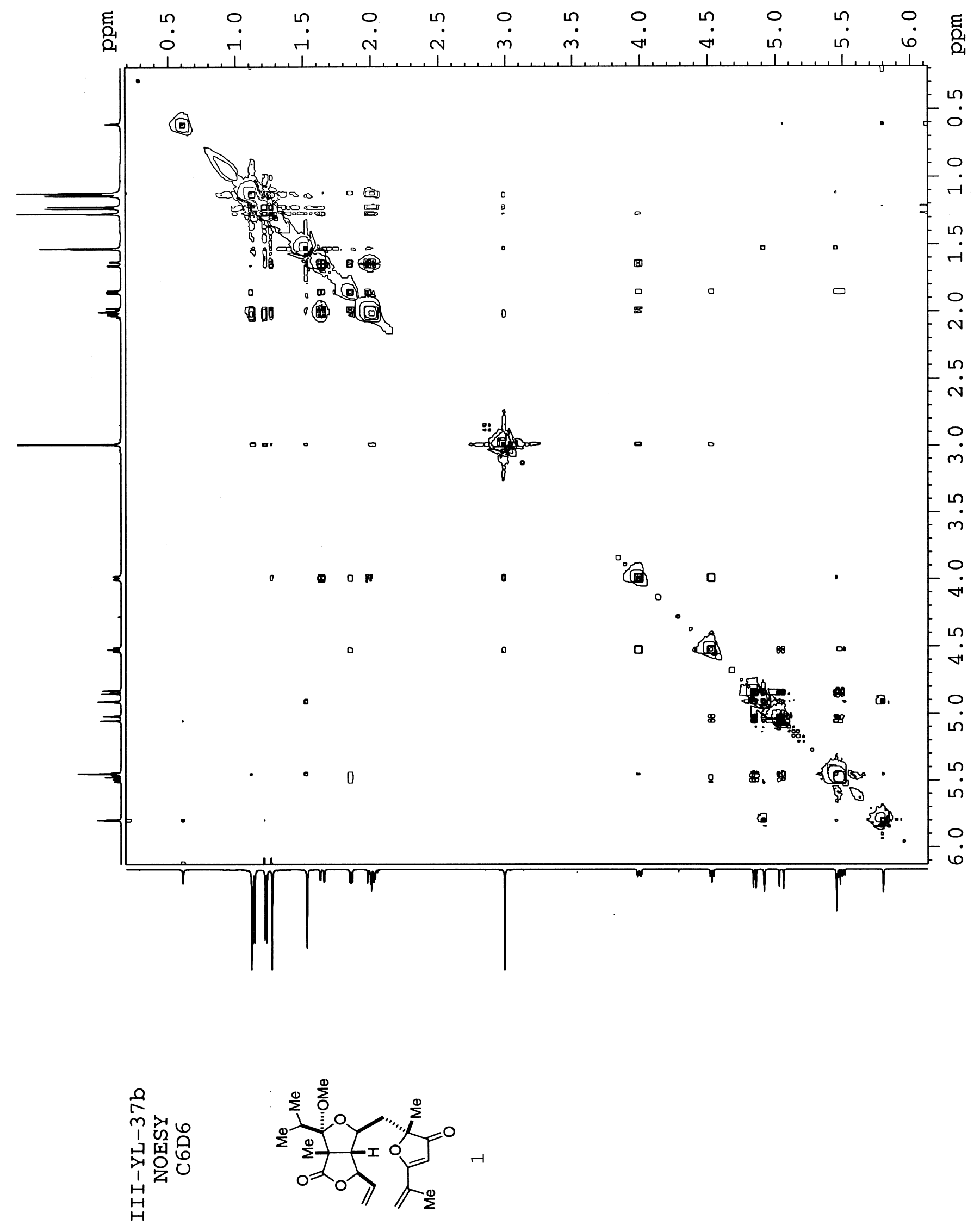


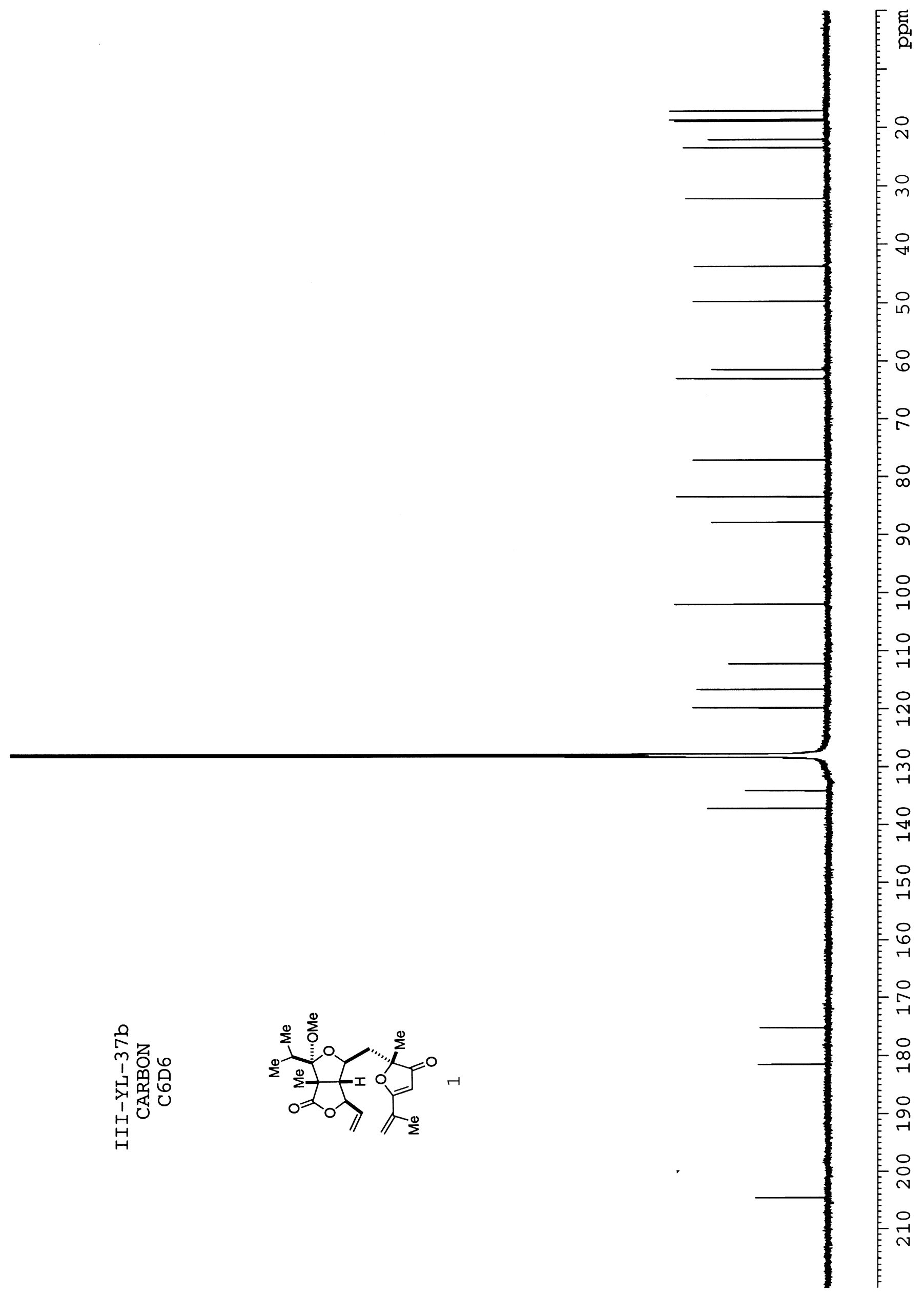




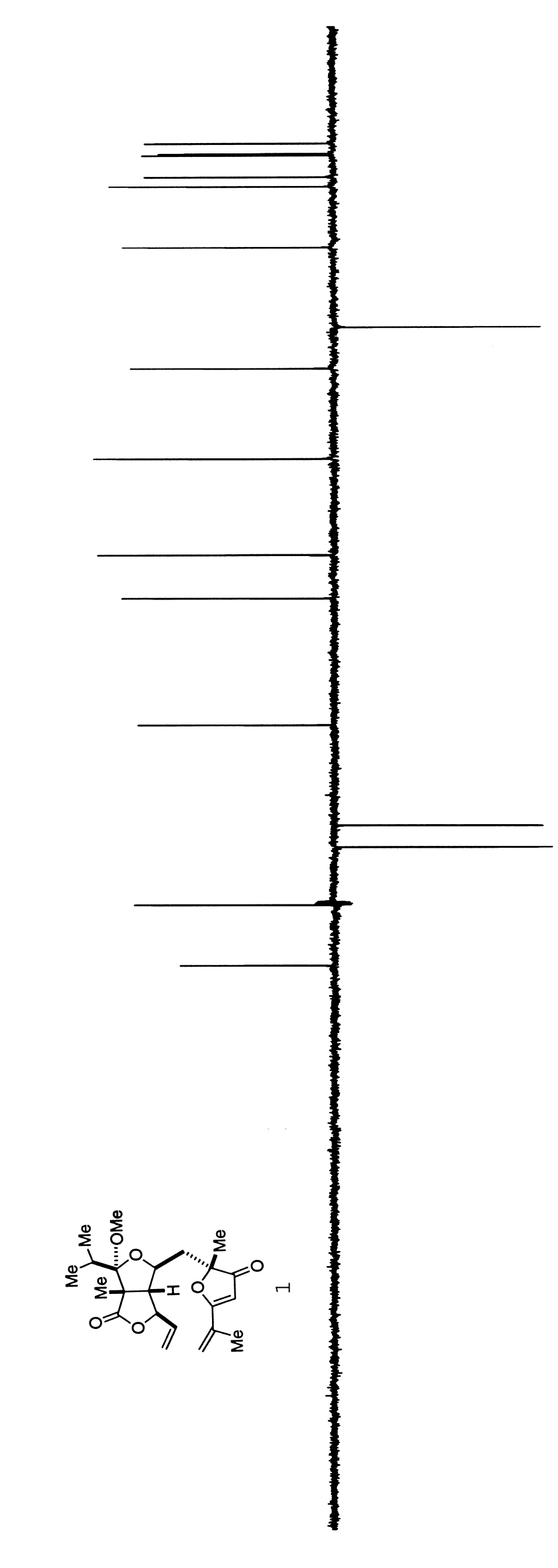



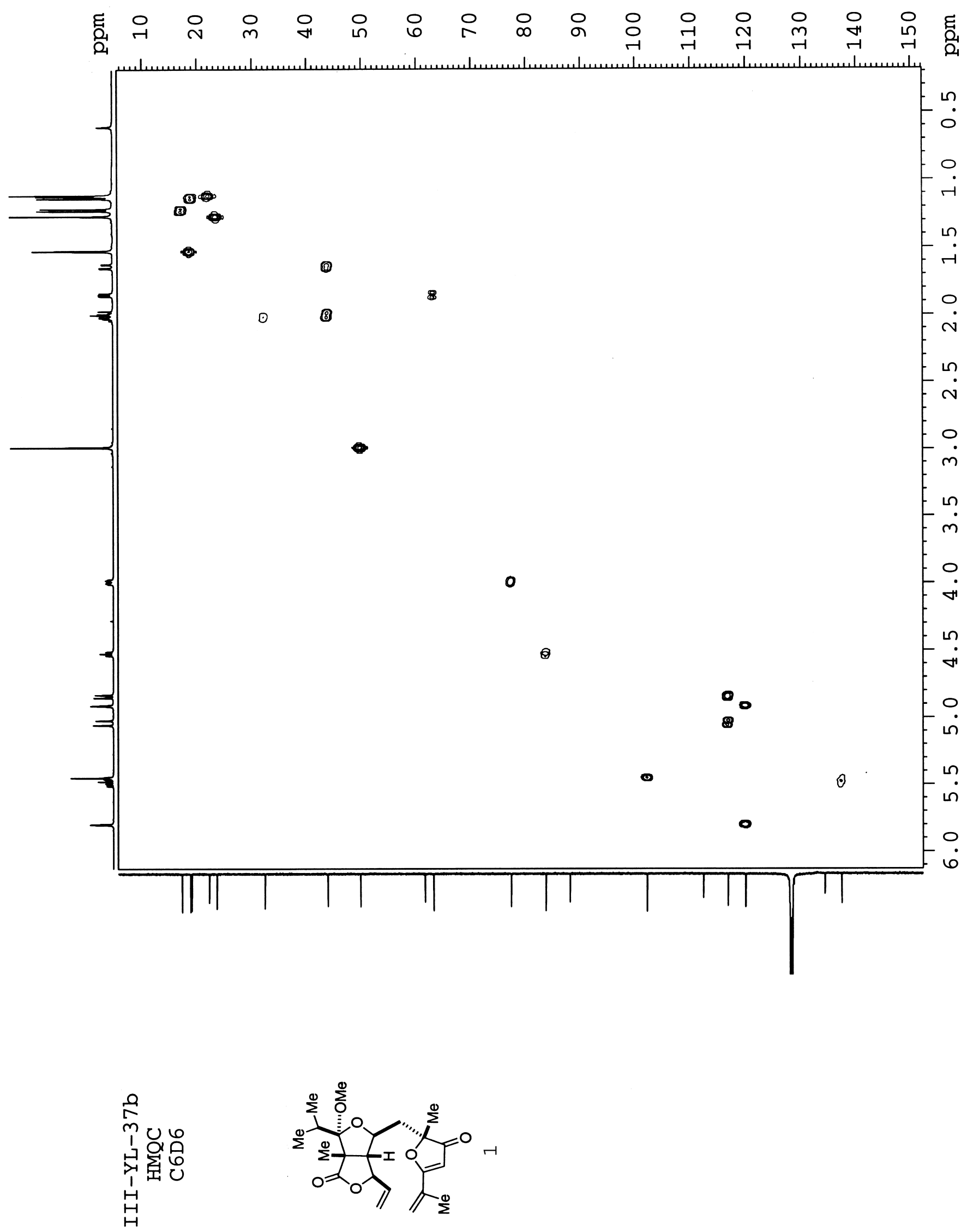


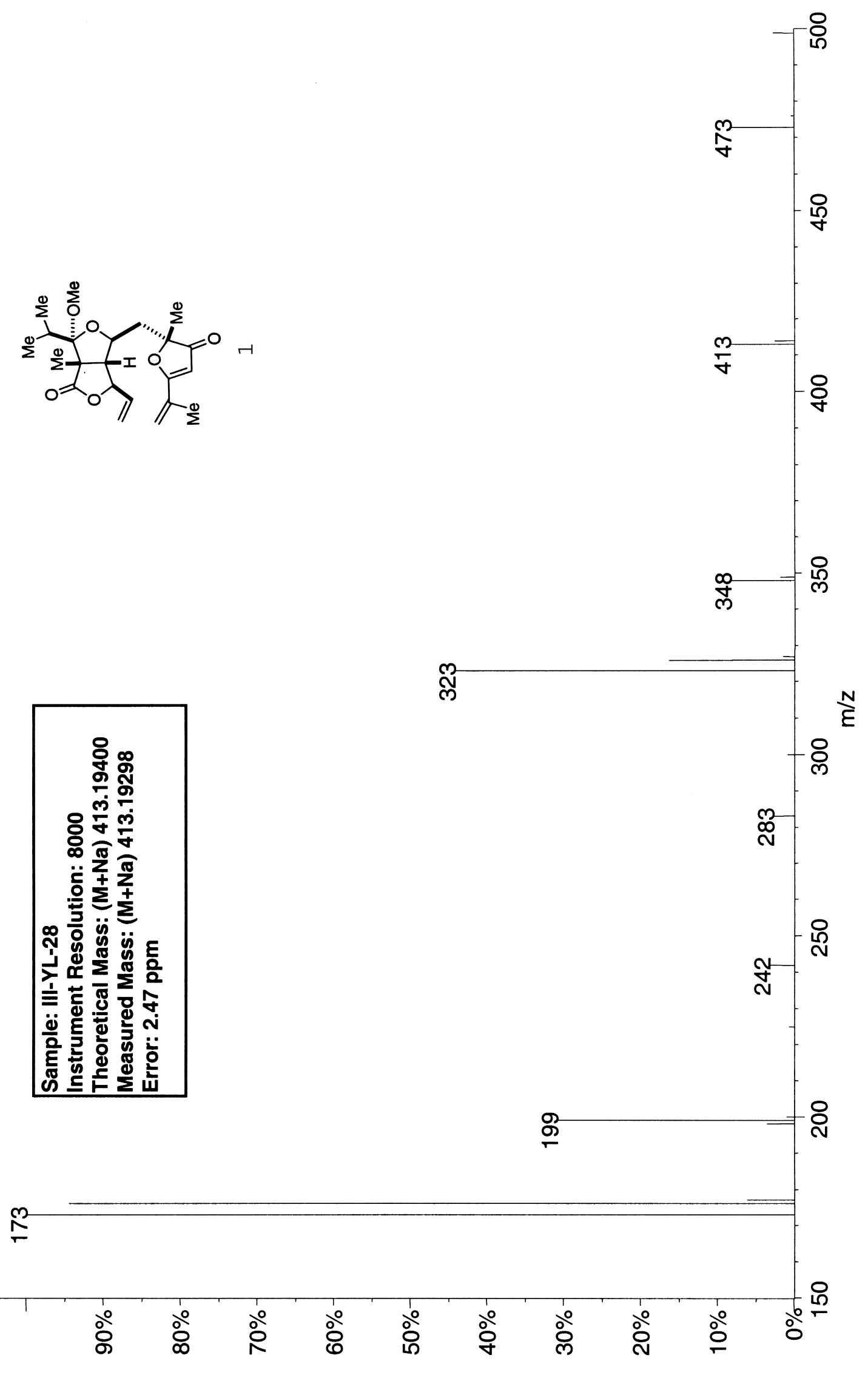




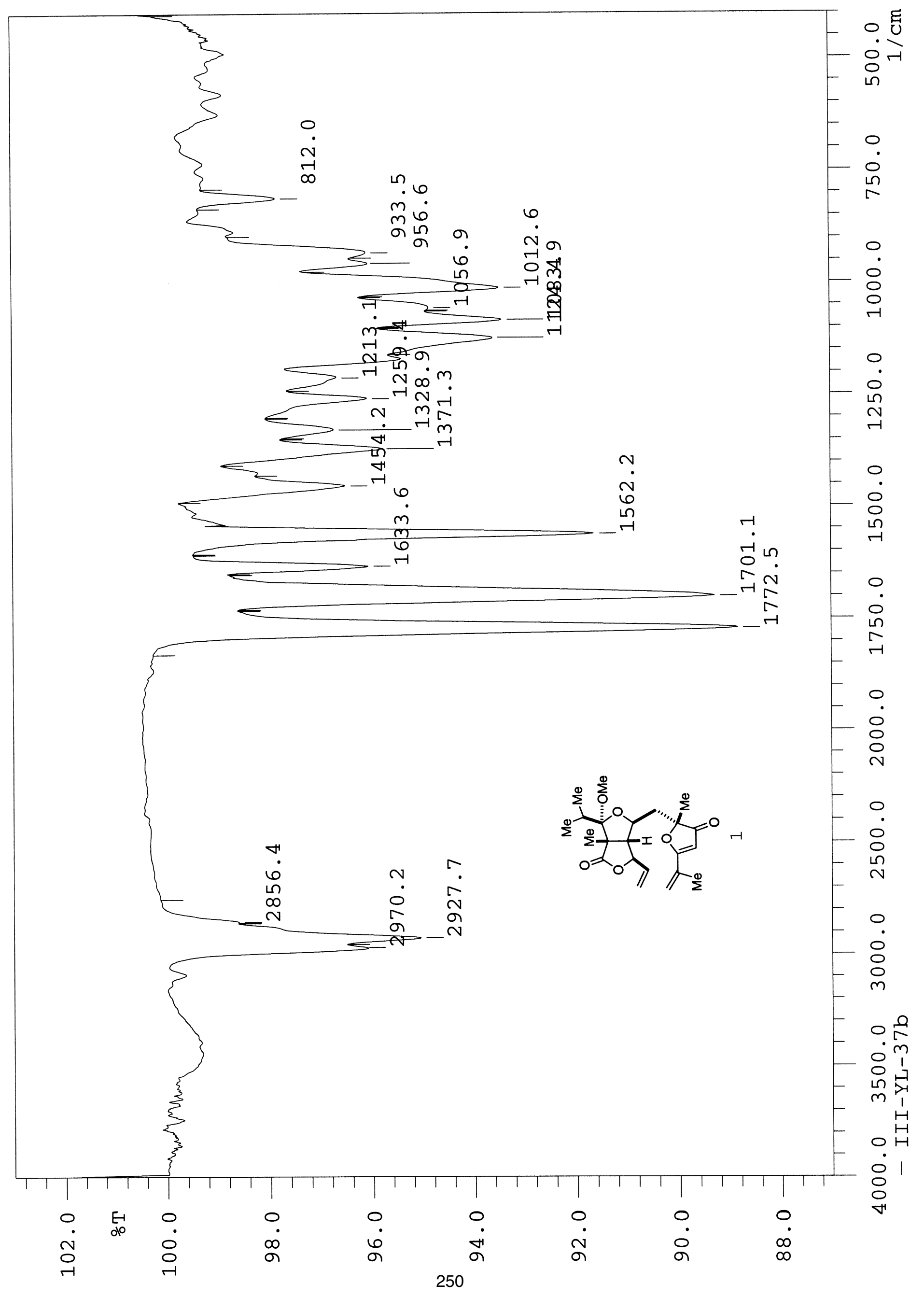




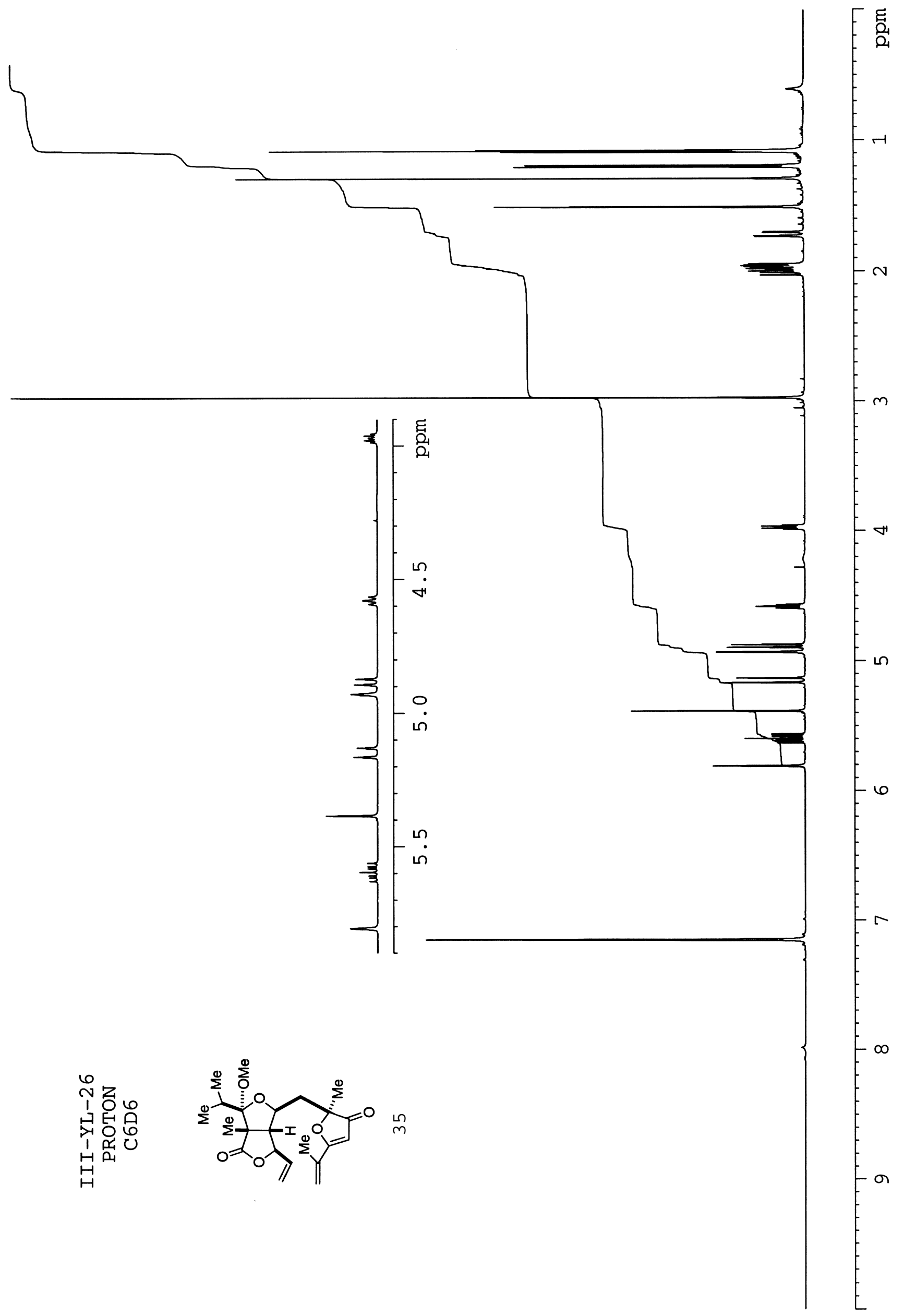




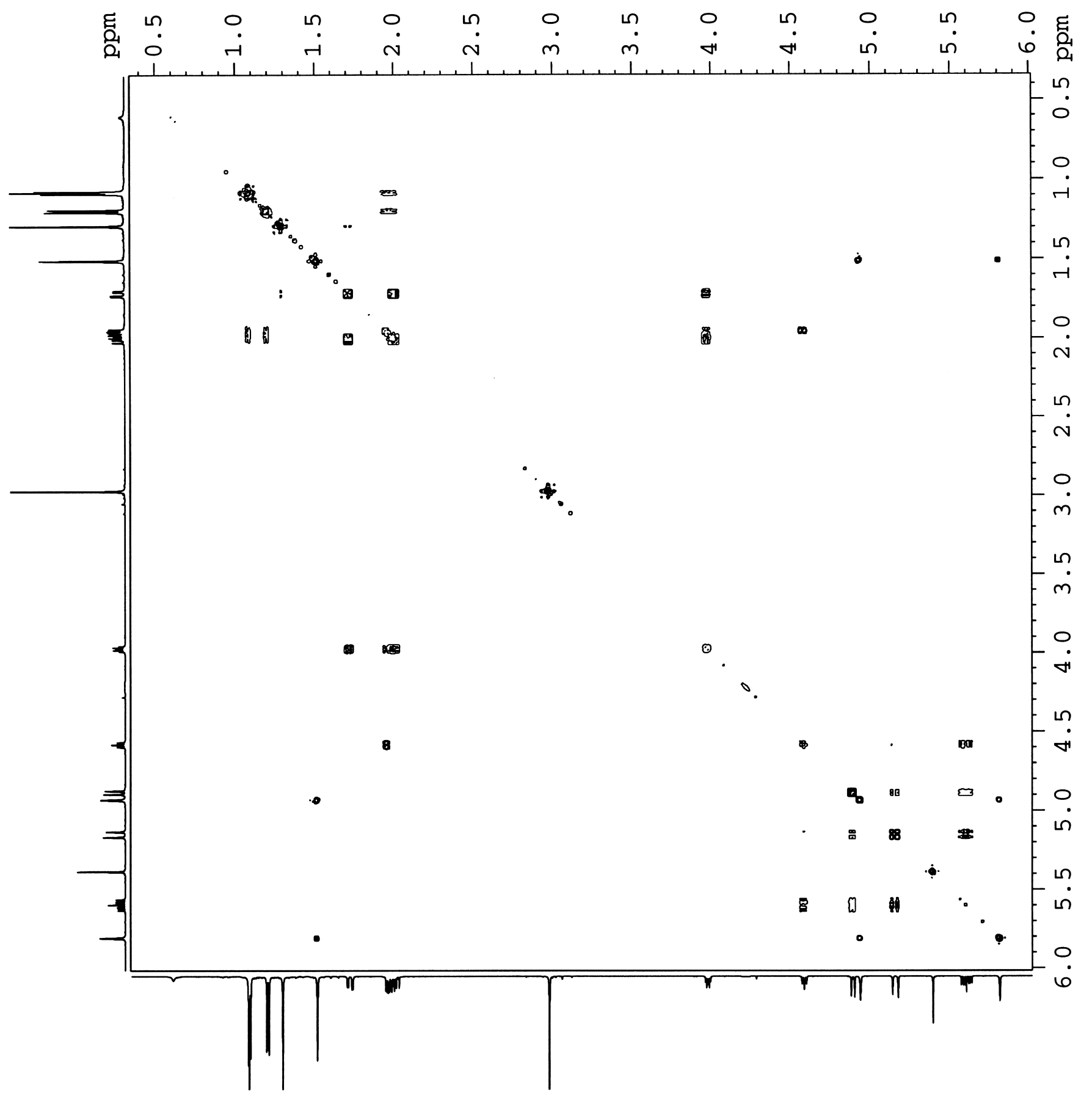

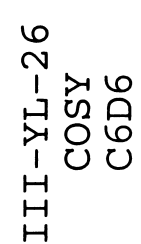

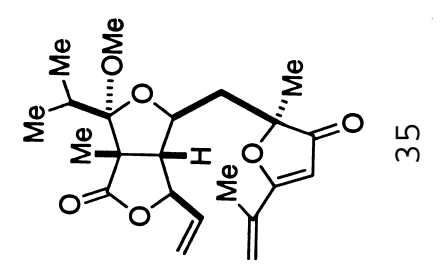




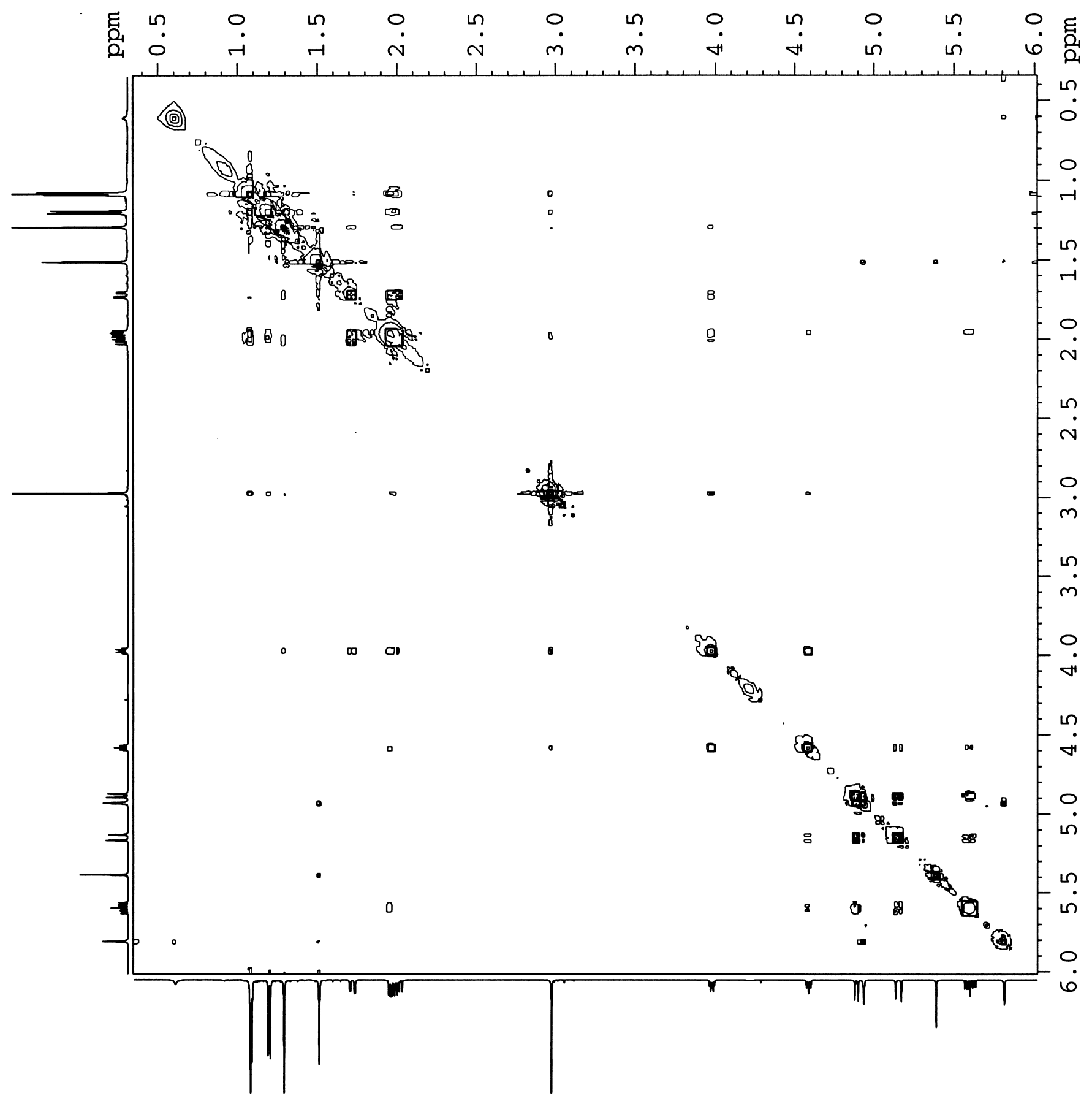

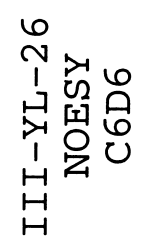

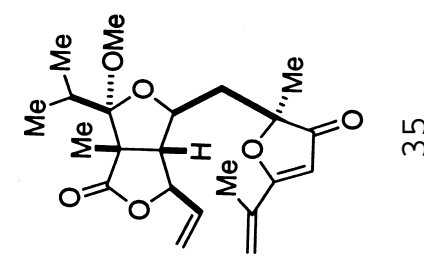




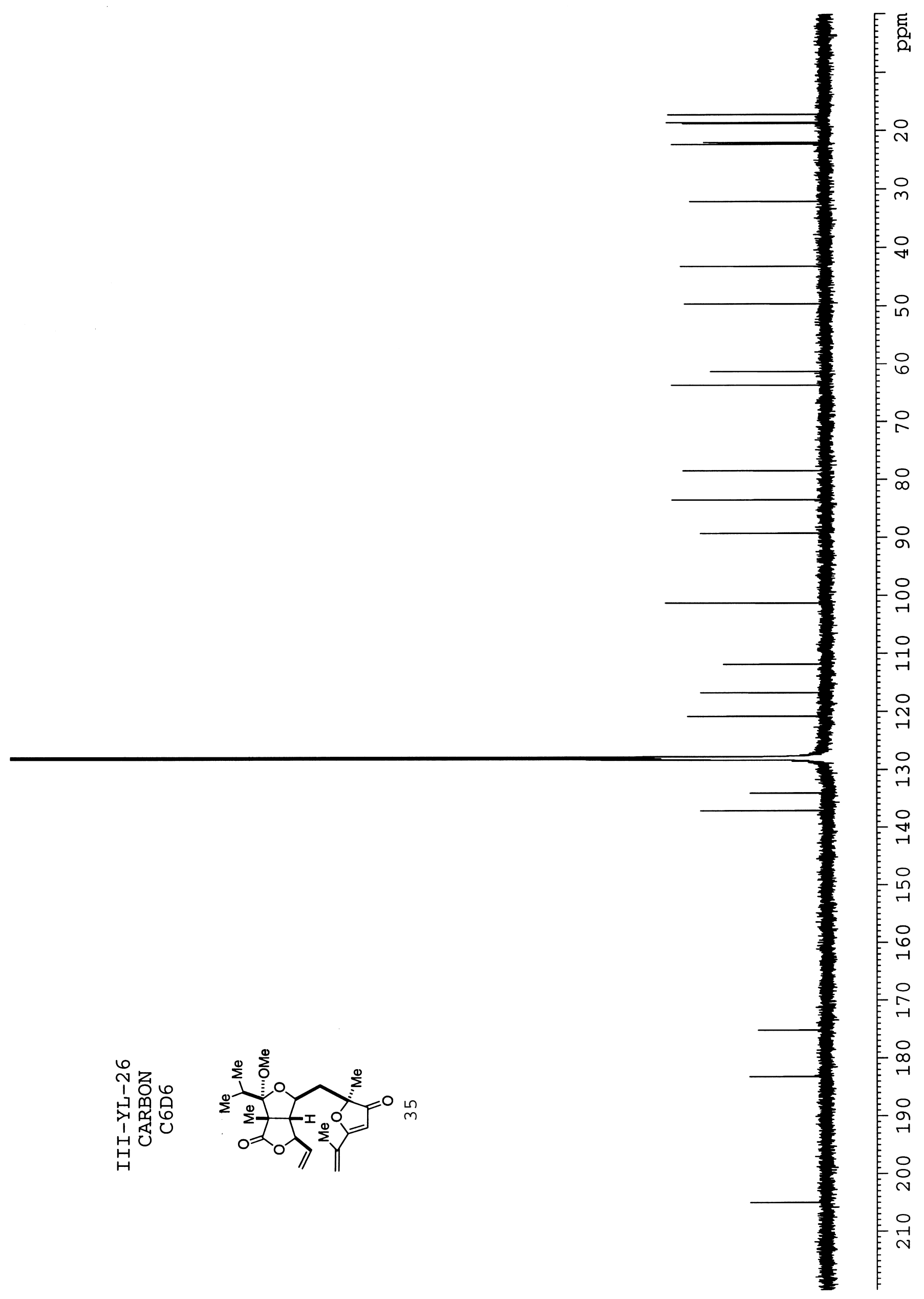




$$
\text { 青 }
$$




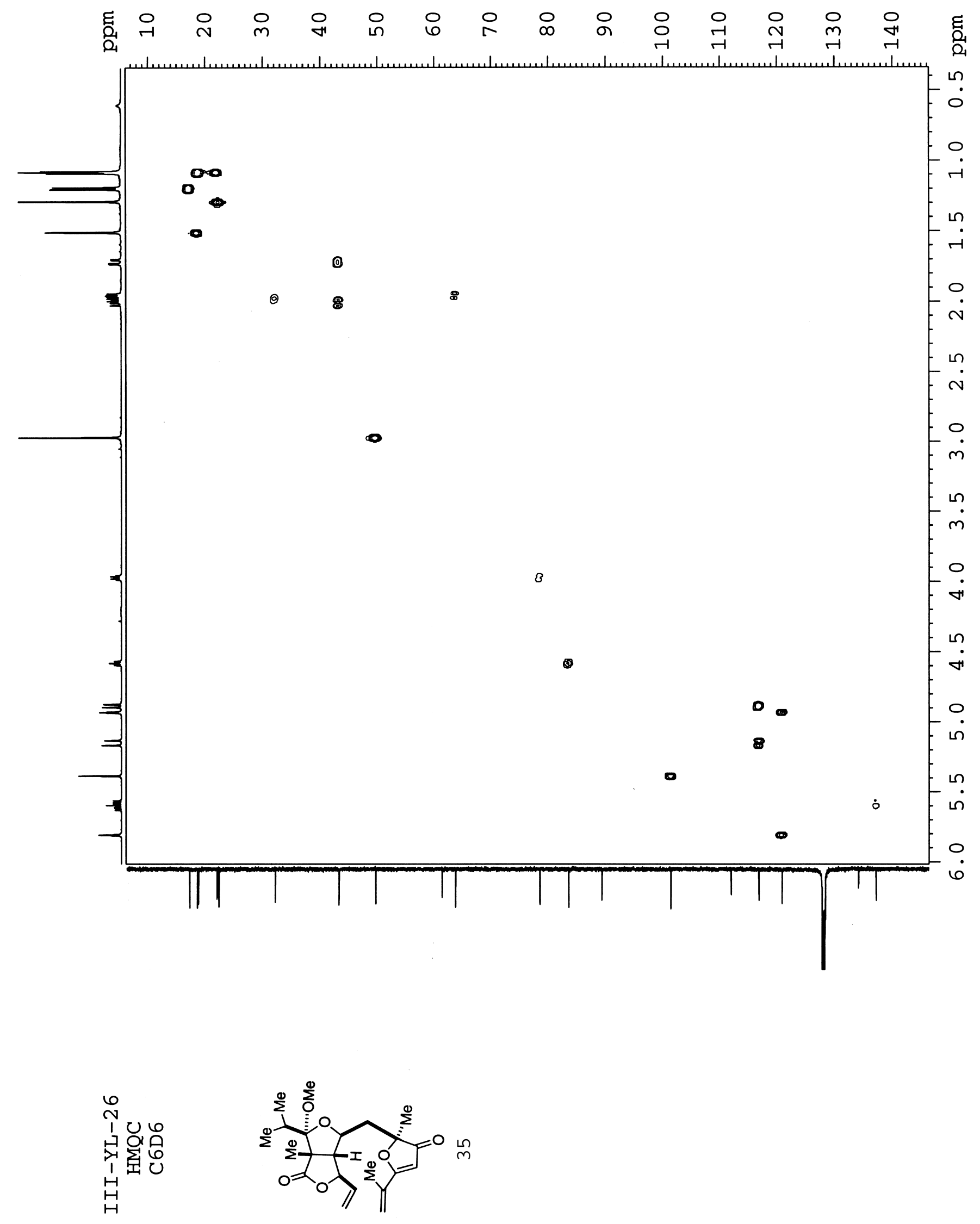




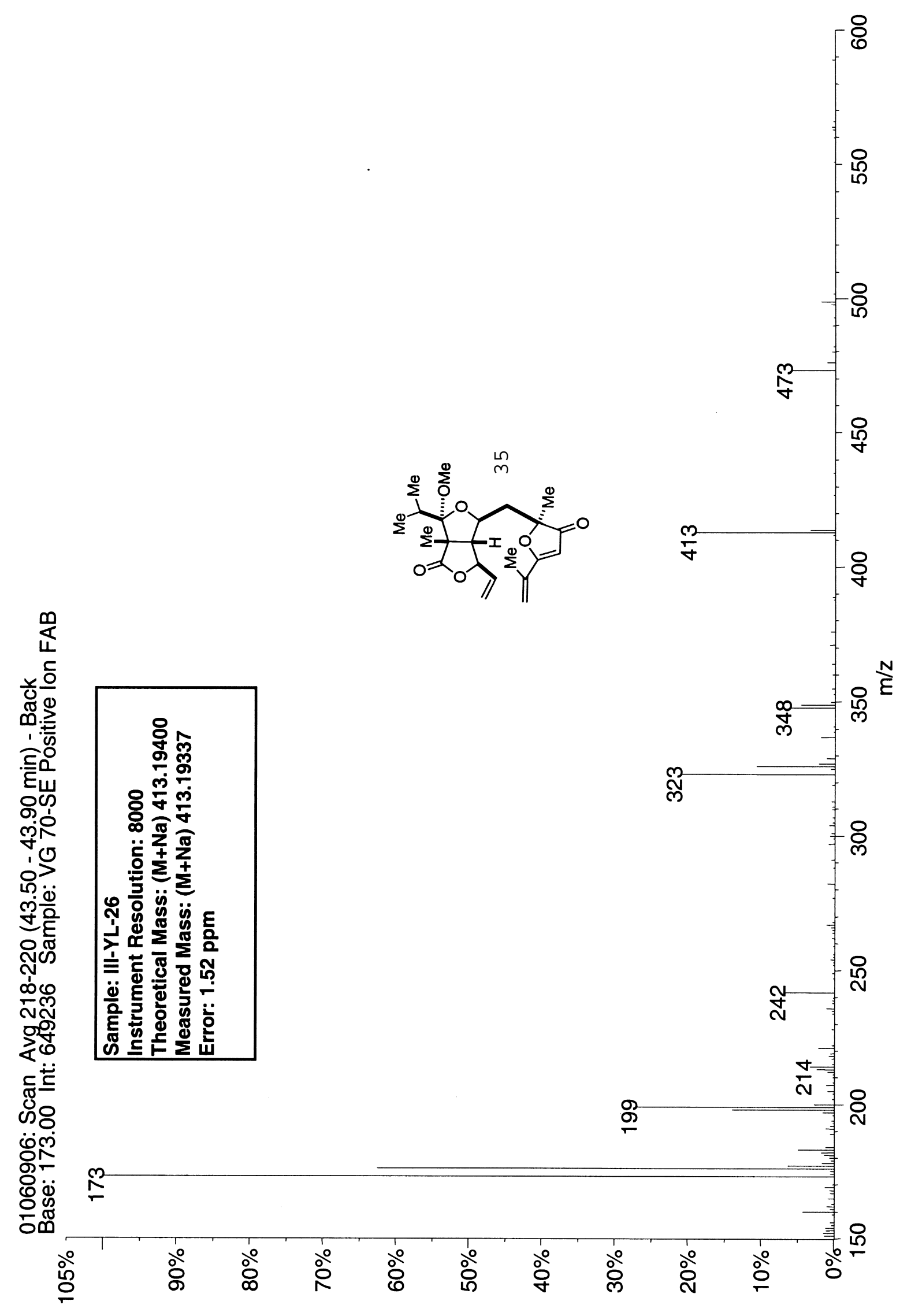




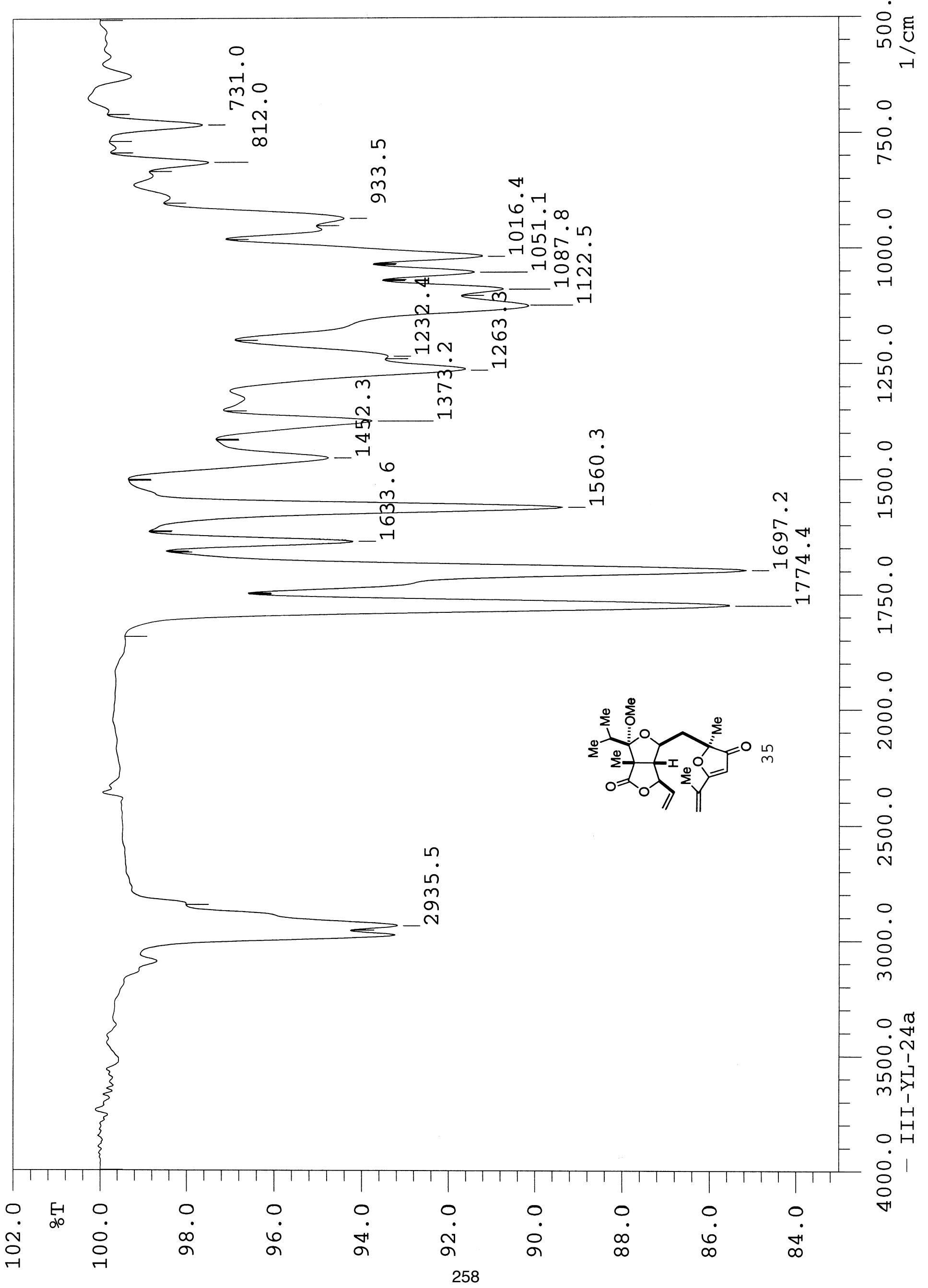




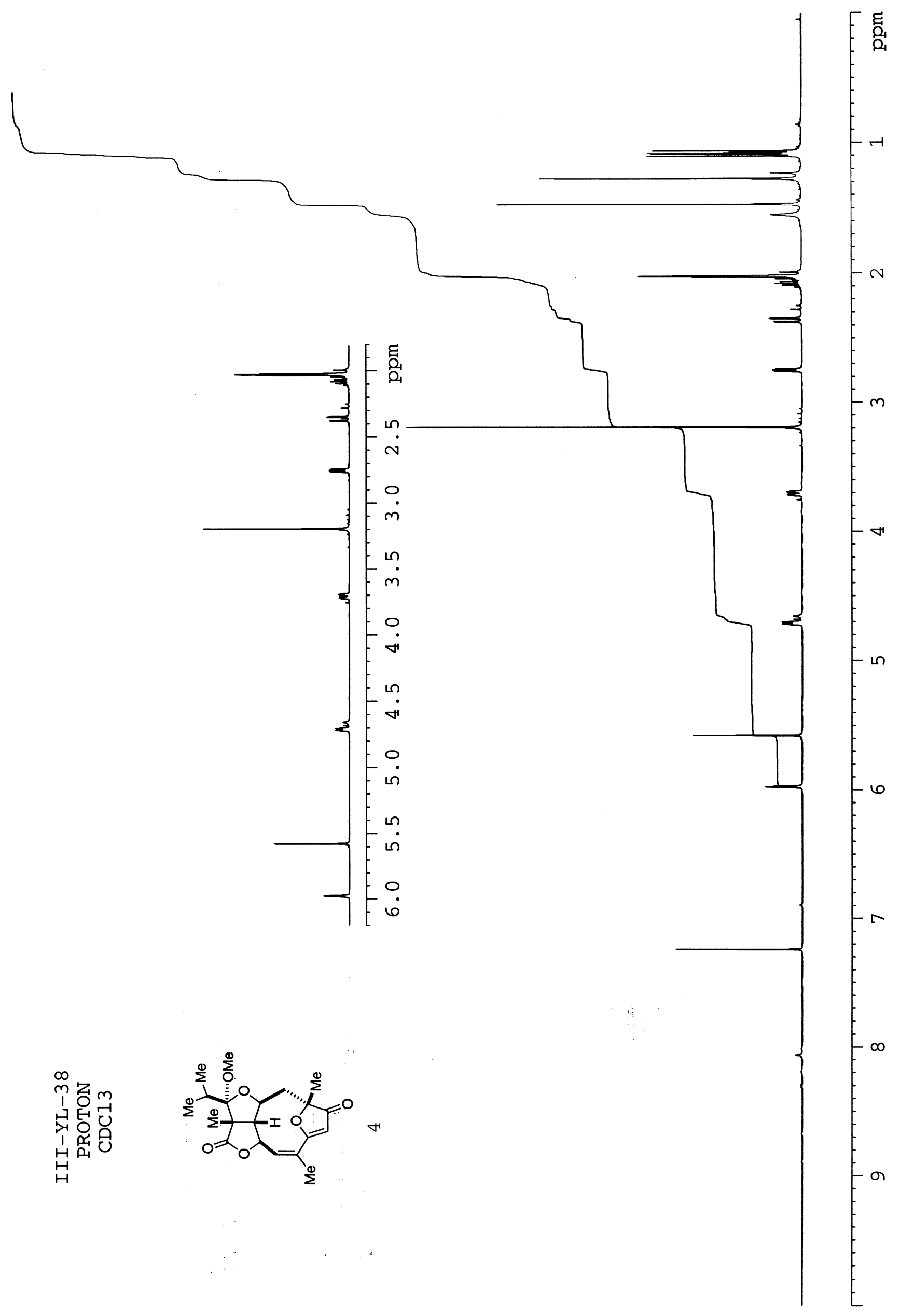




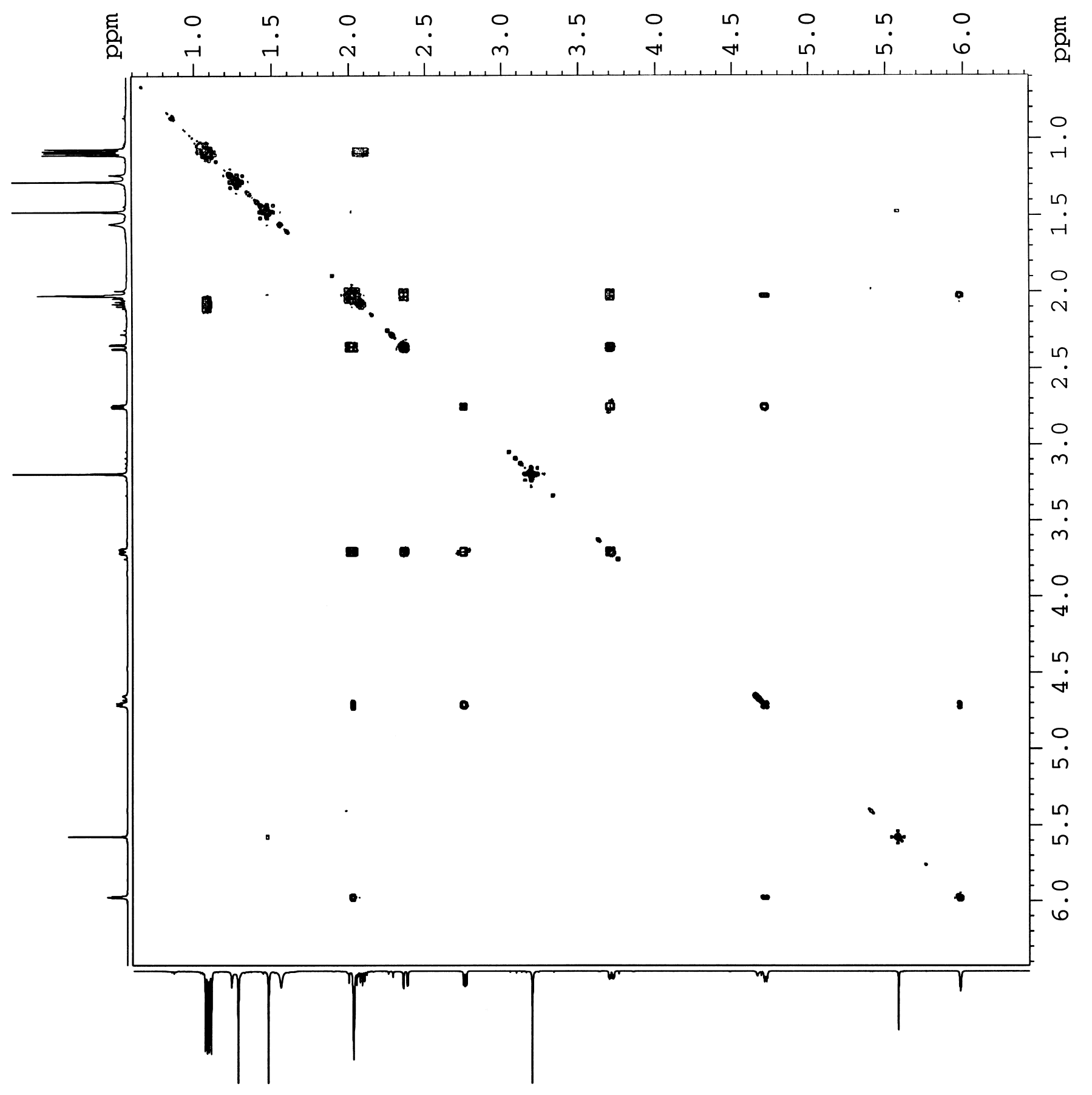

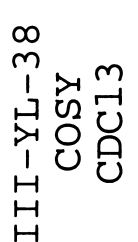

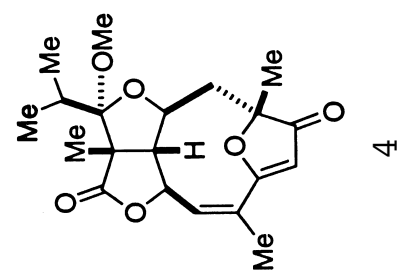




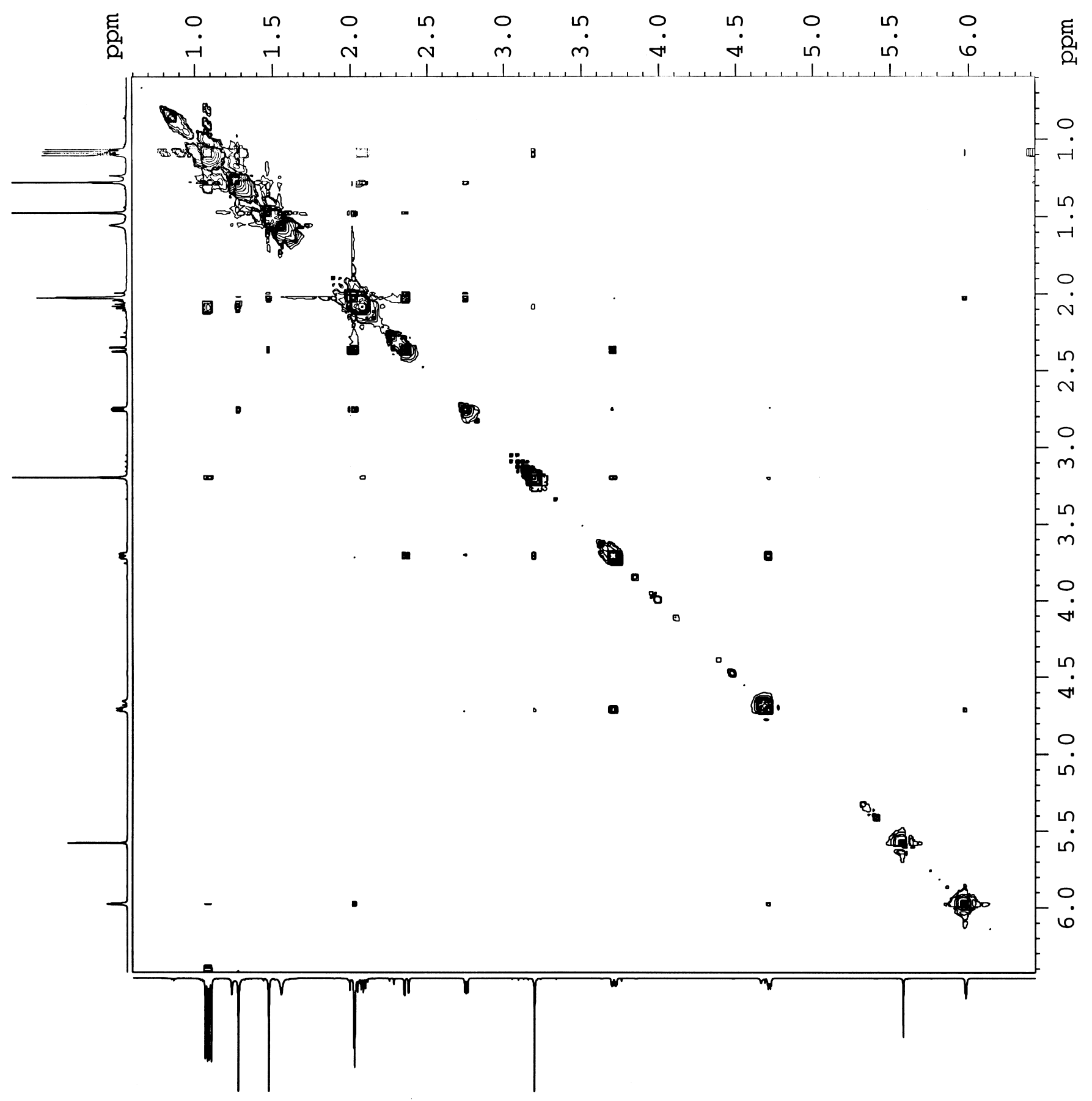

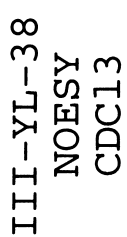

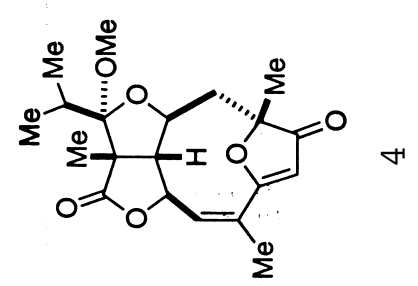




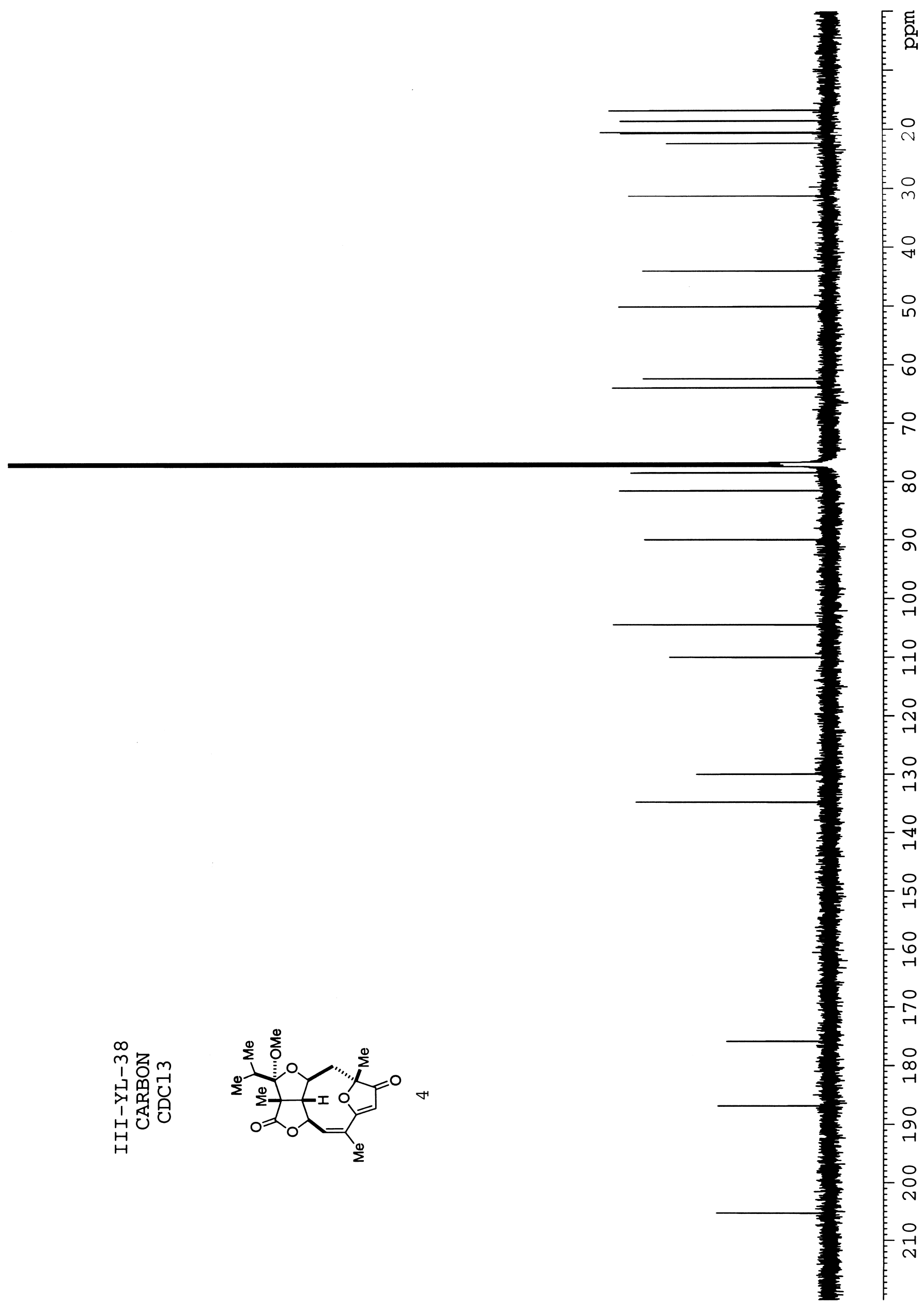




$$
.1
$$




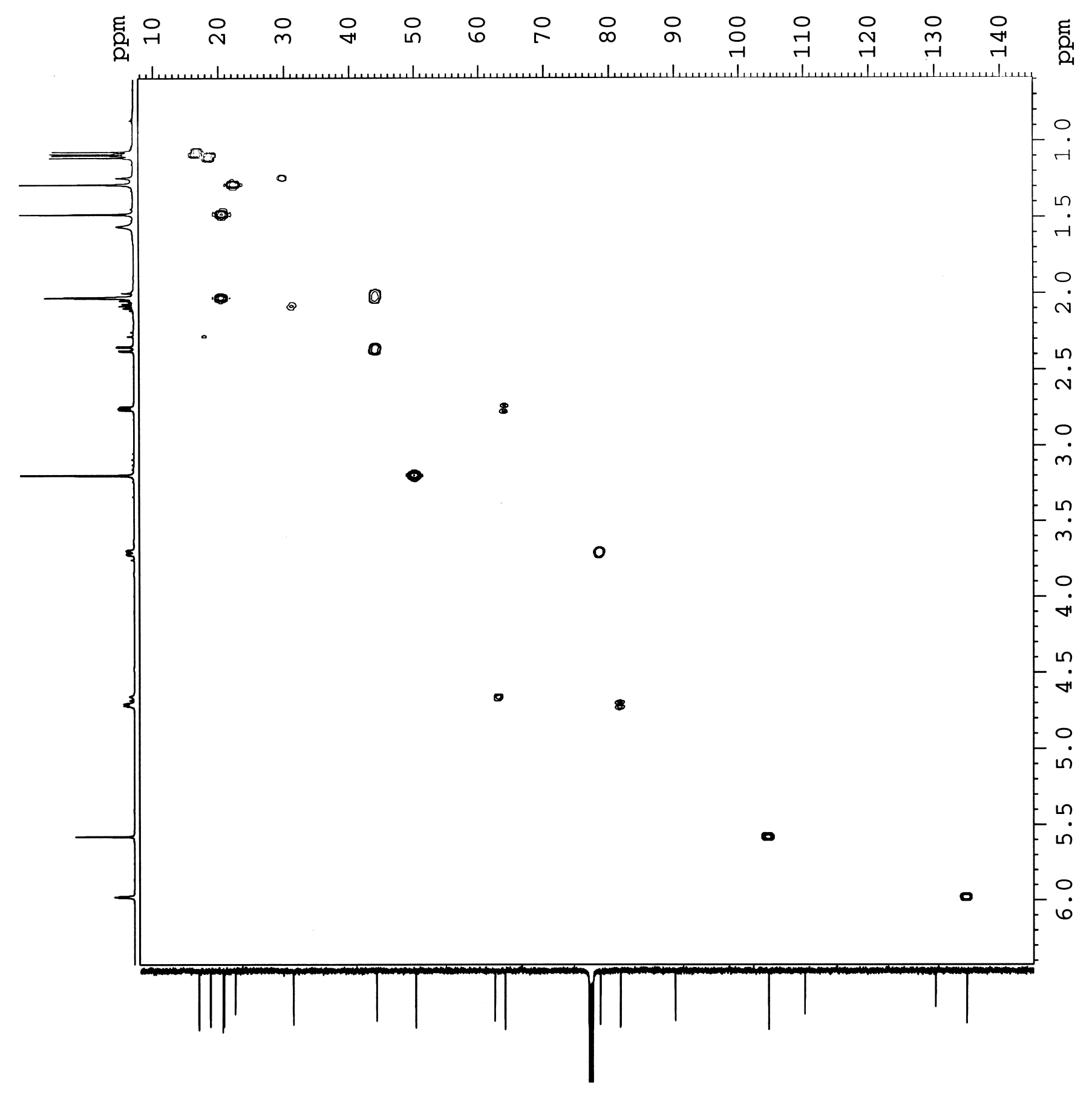

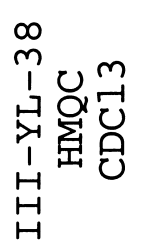

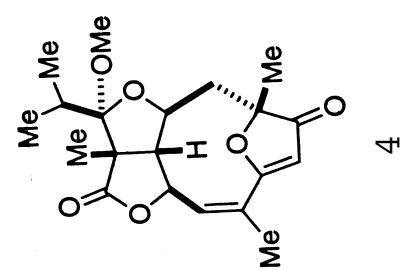




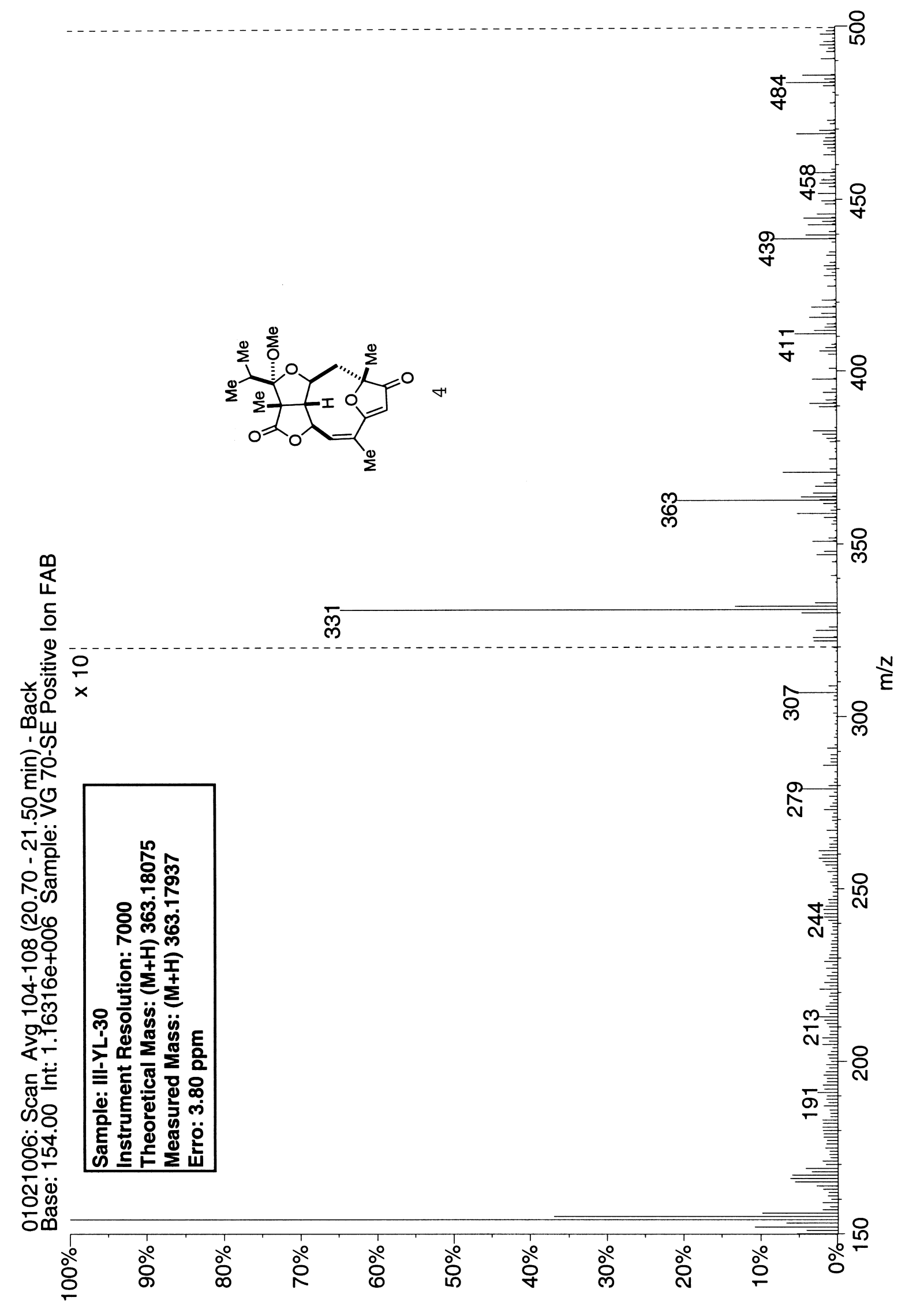




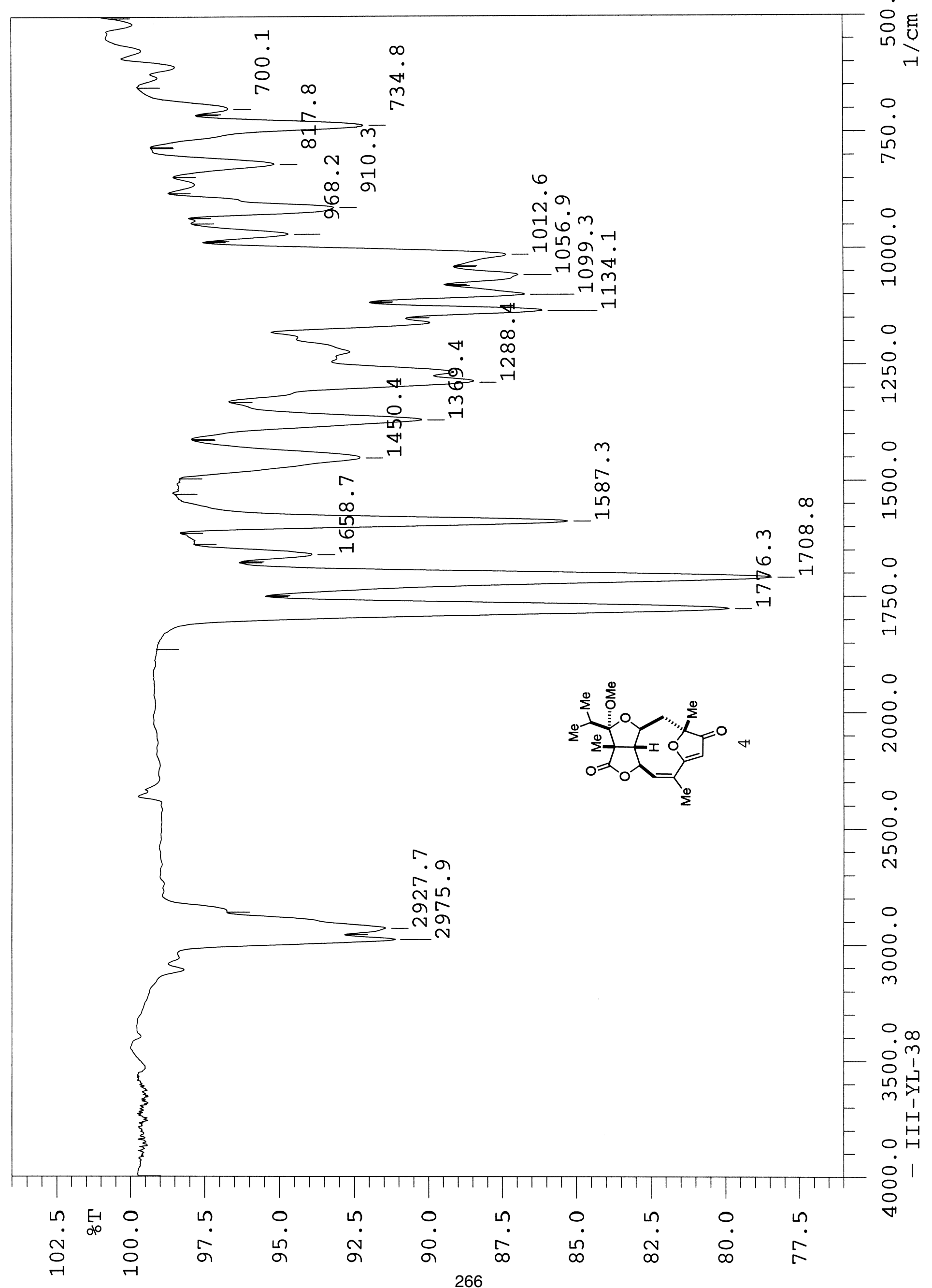




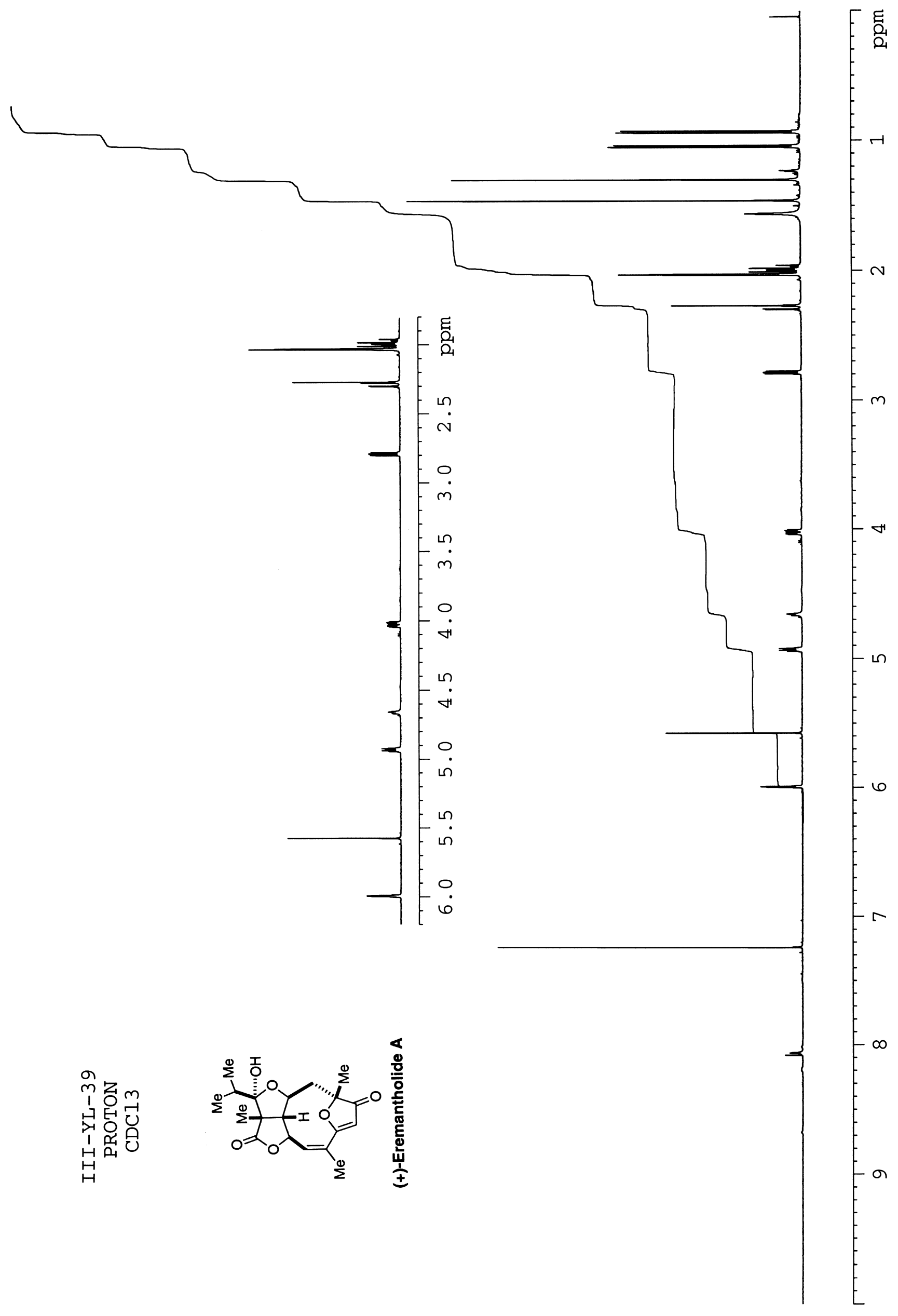




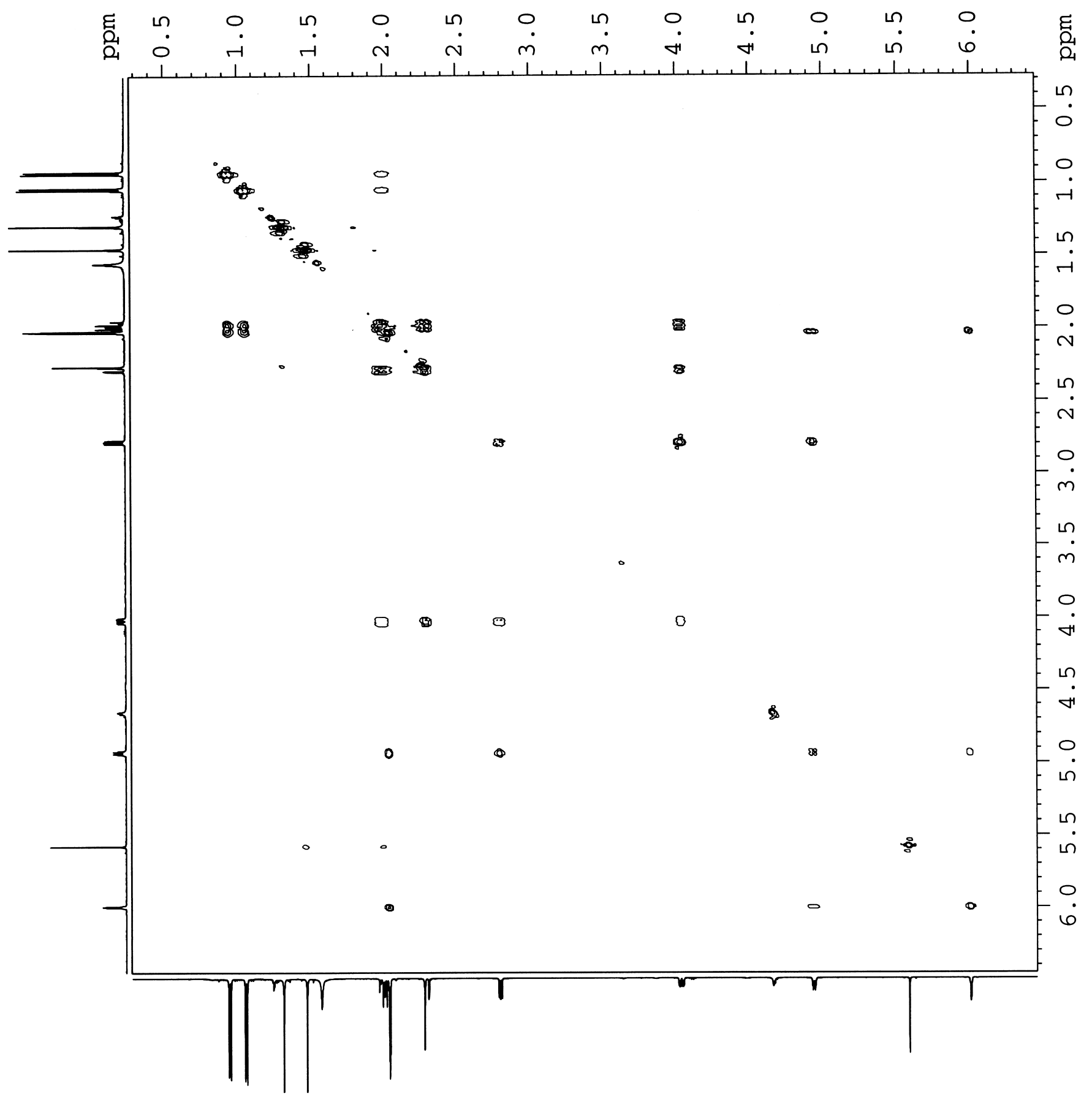

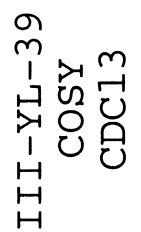

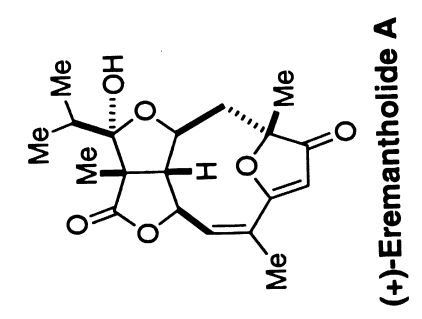




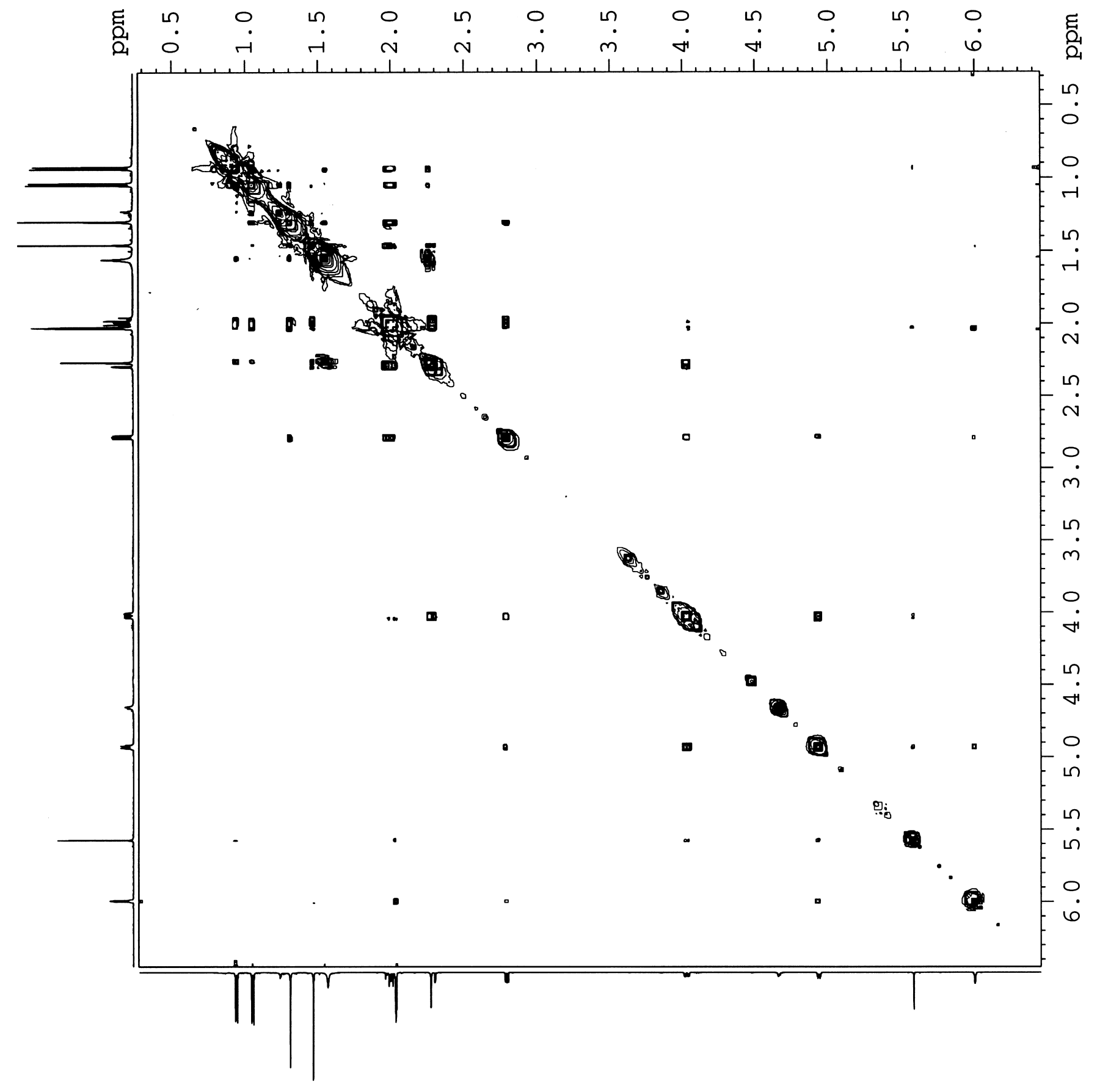

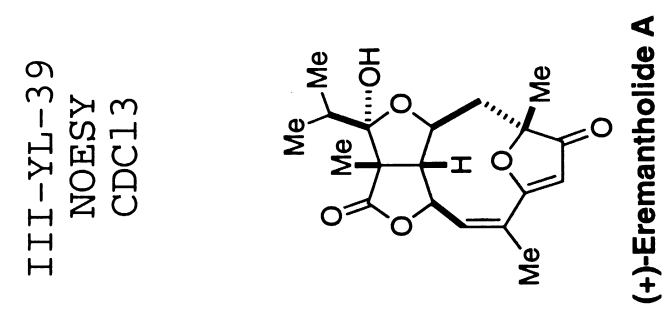




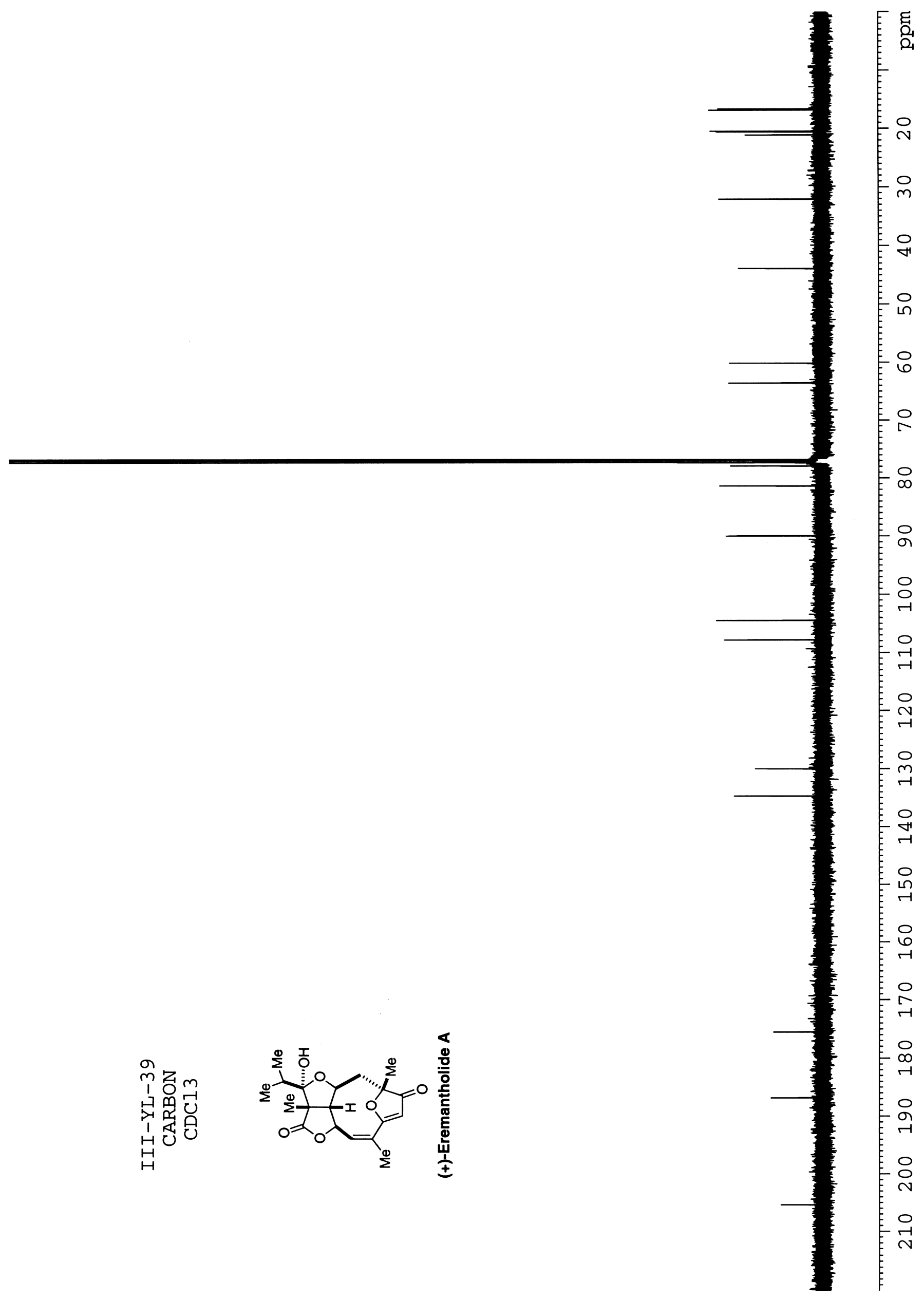




$$
1
$$



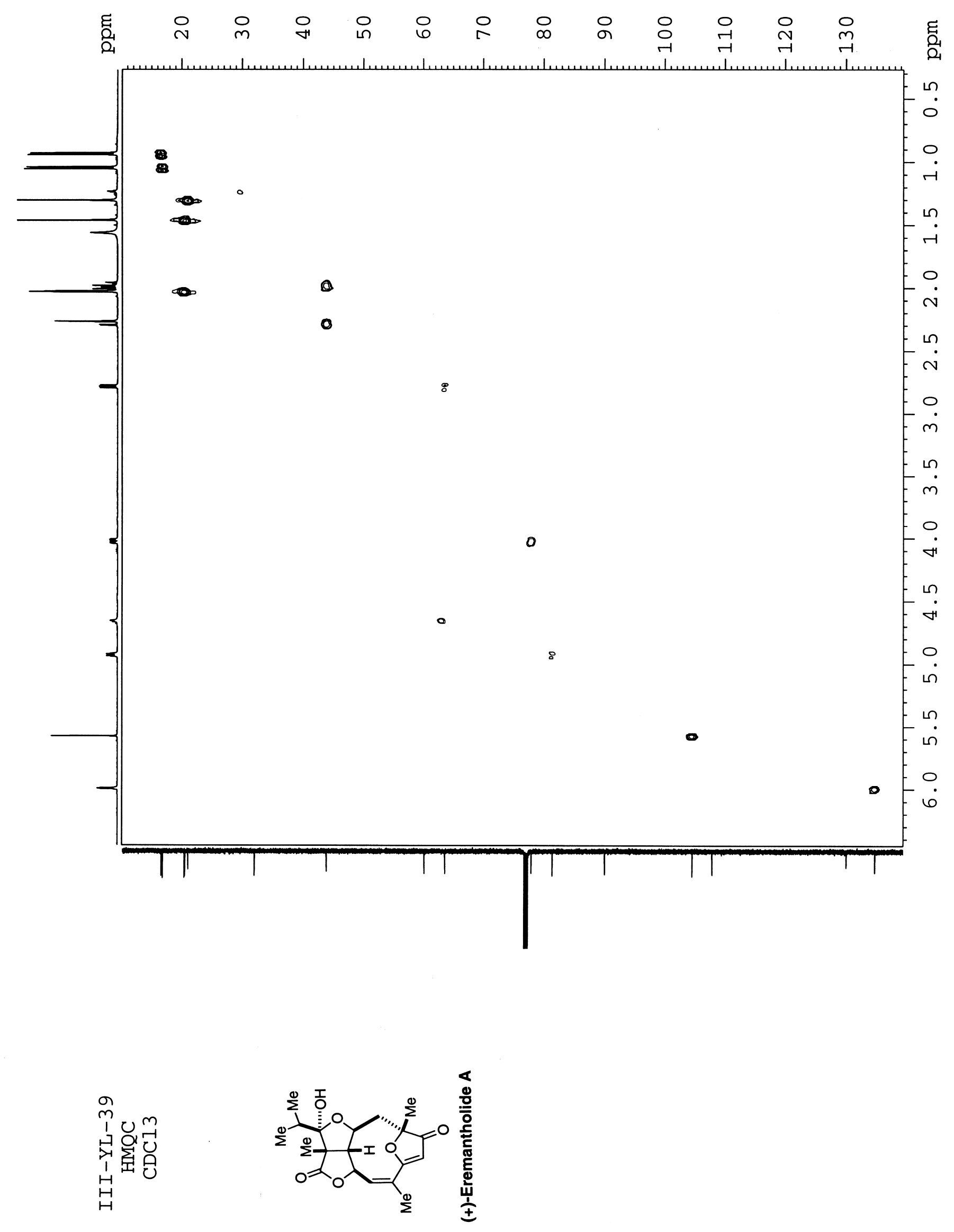


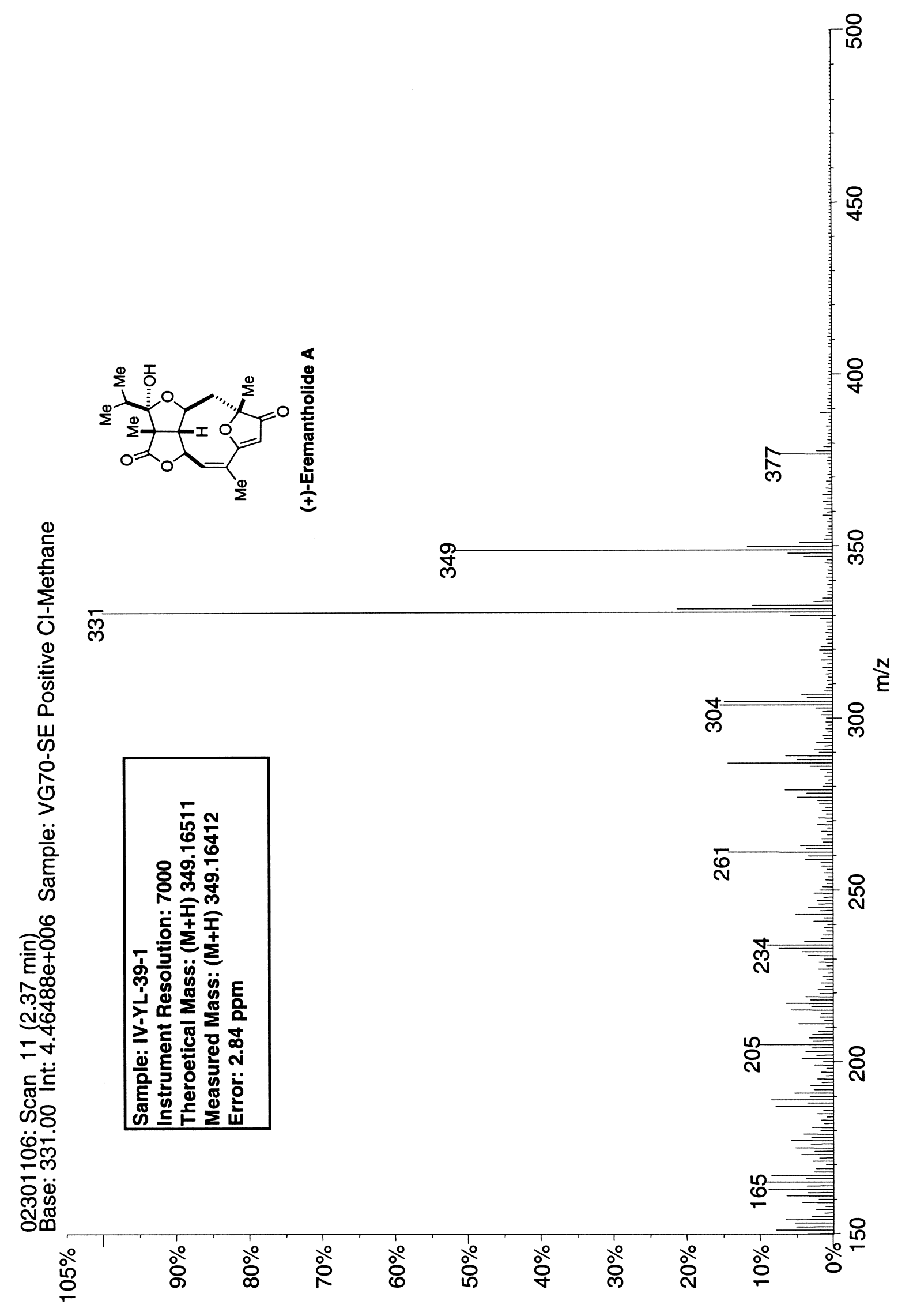




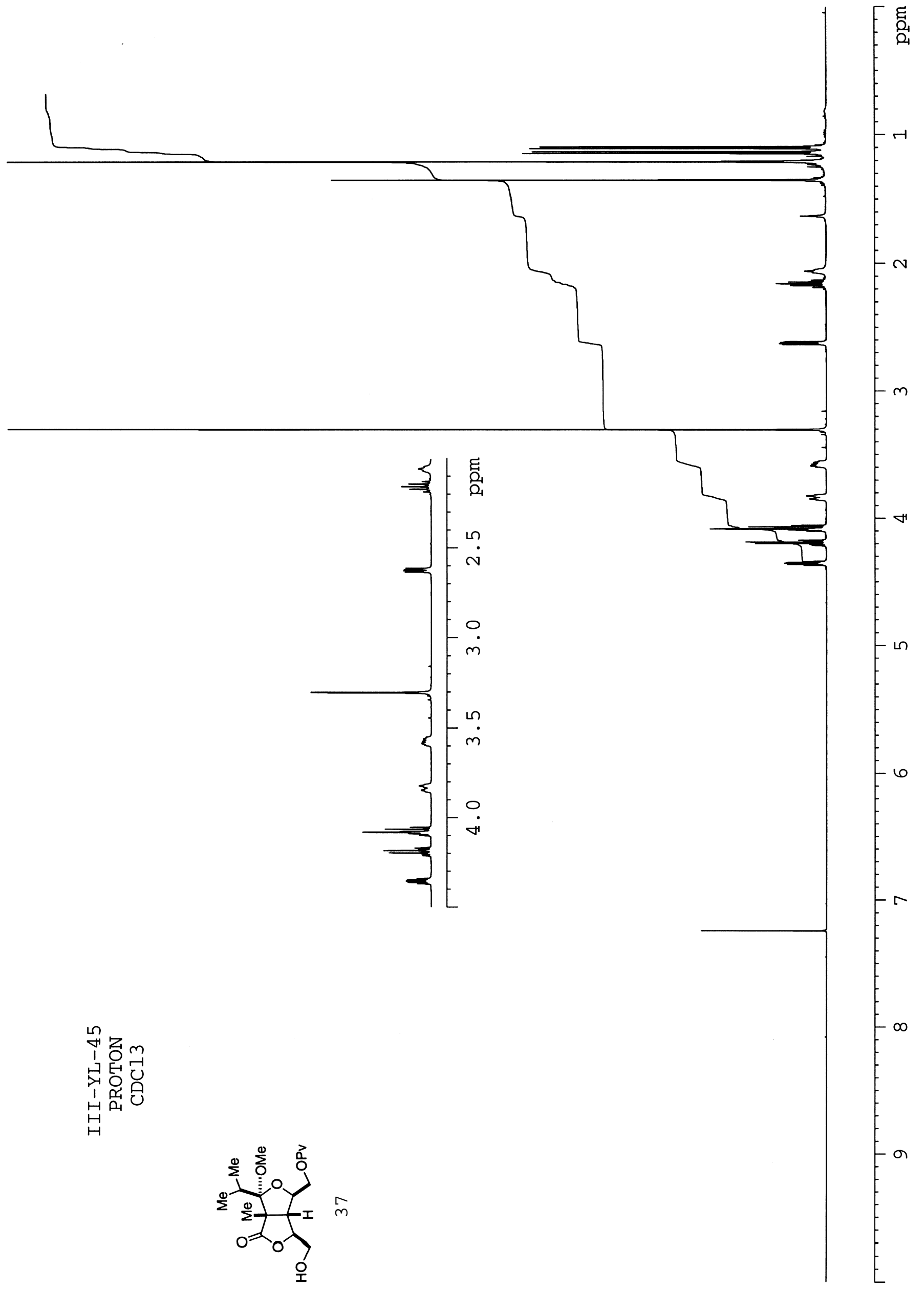



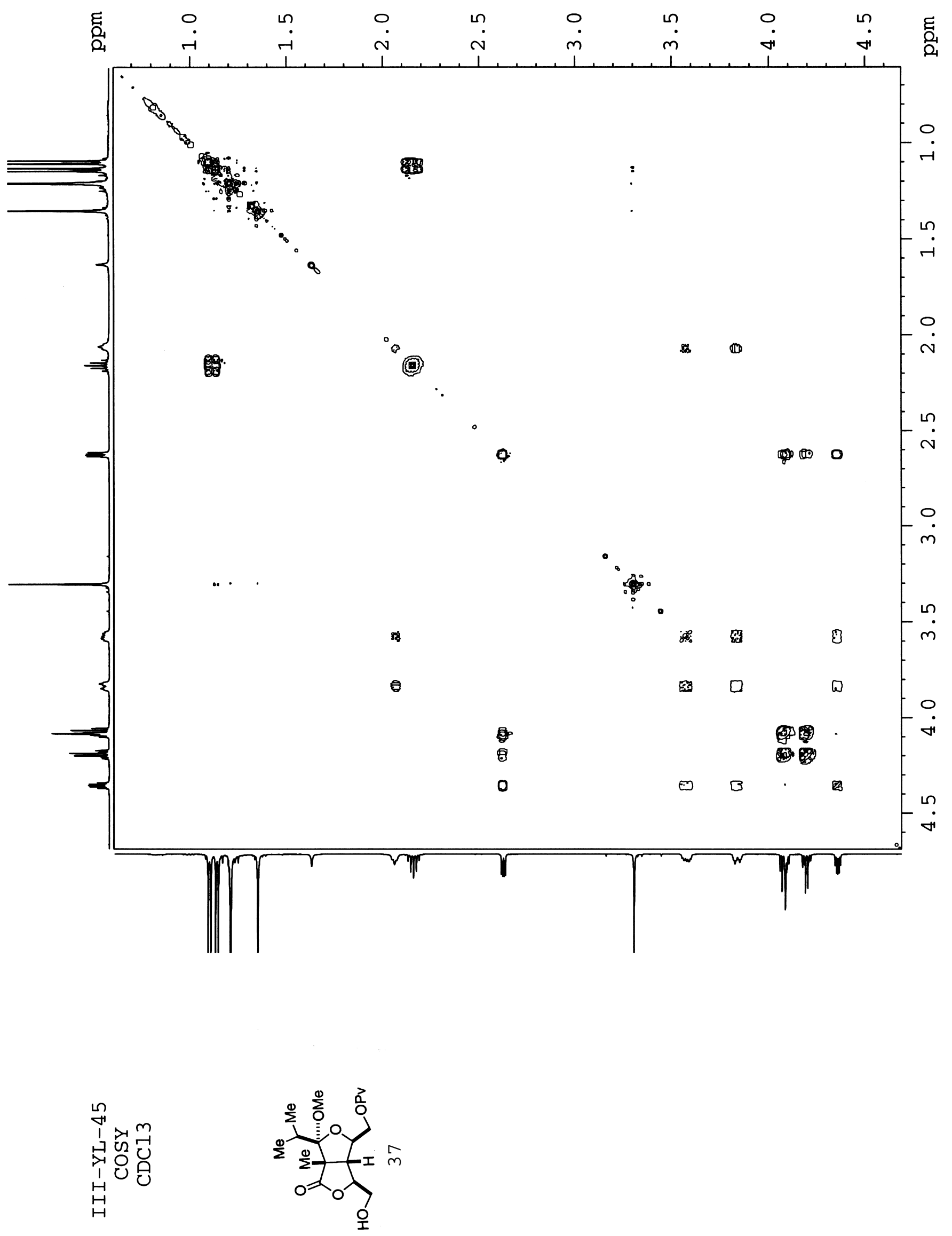

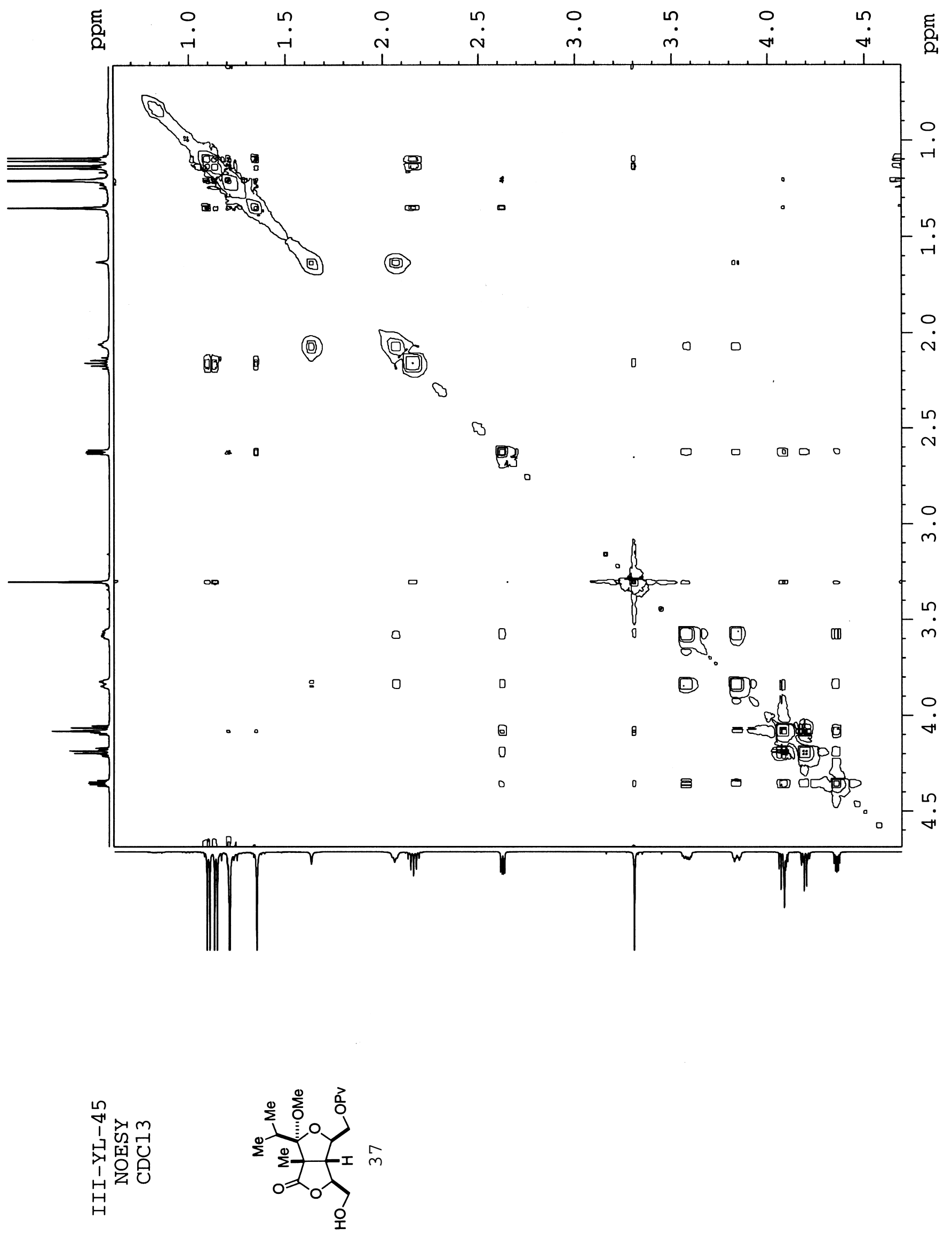


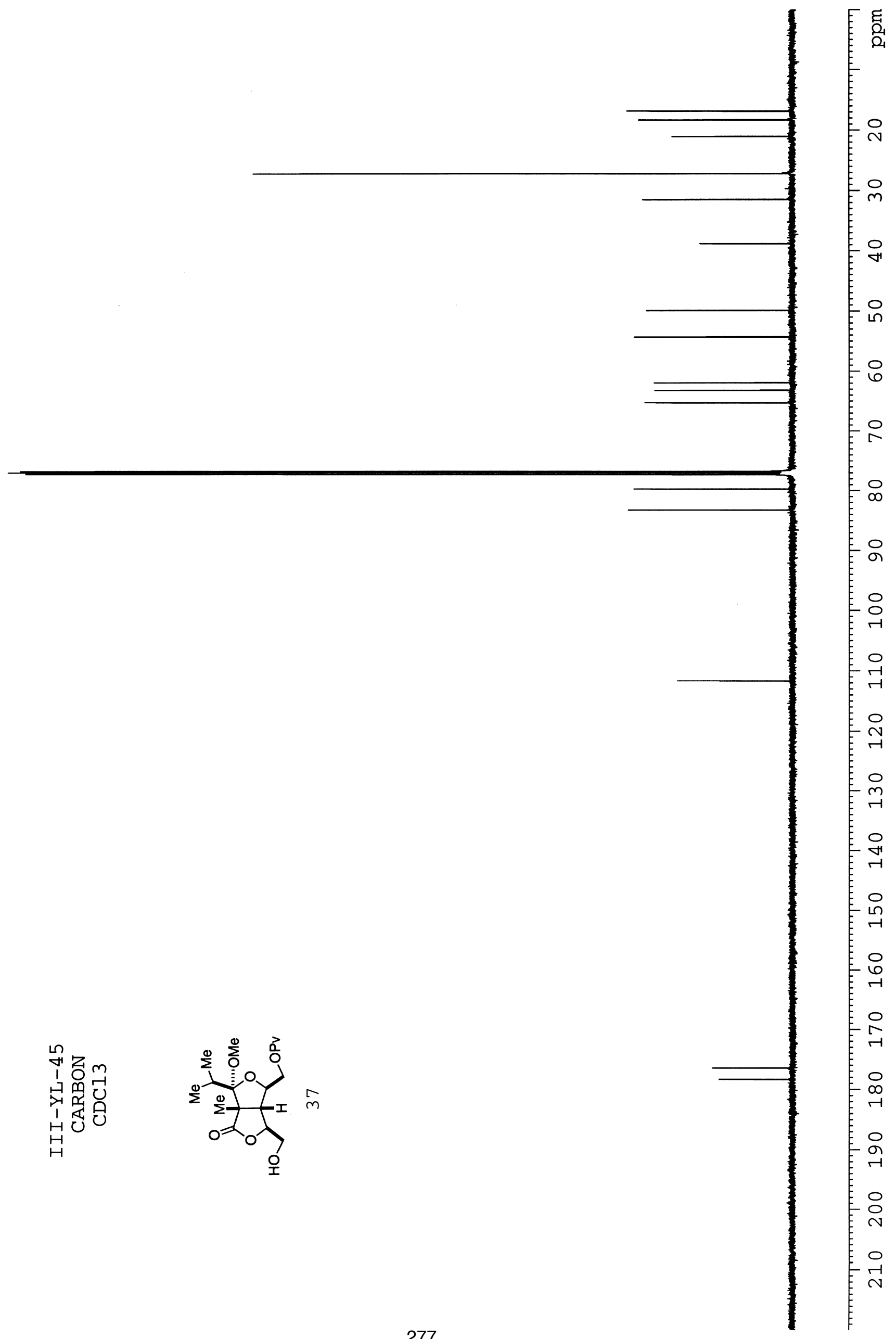




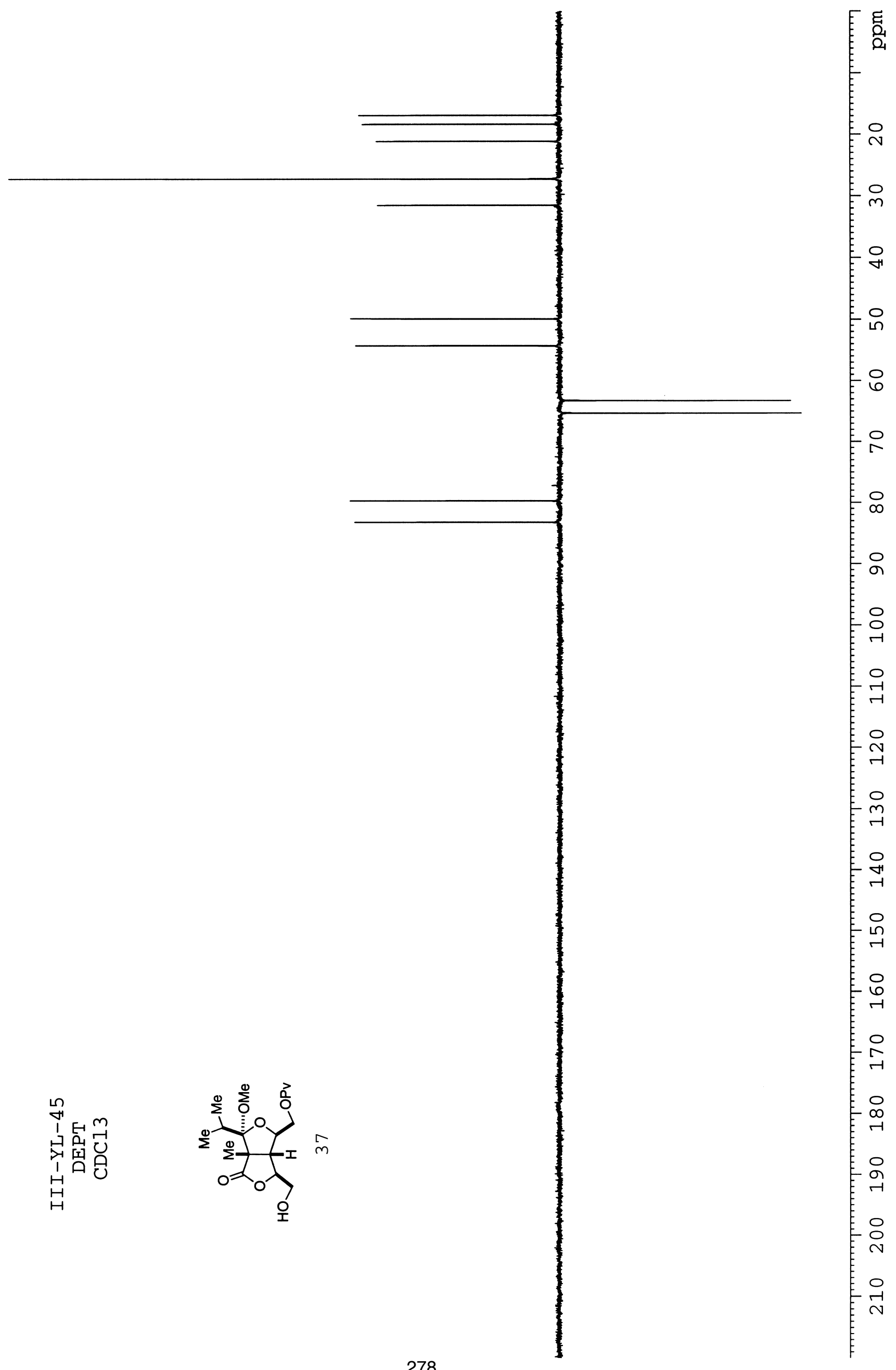



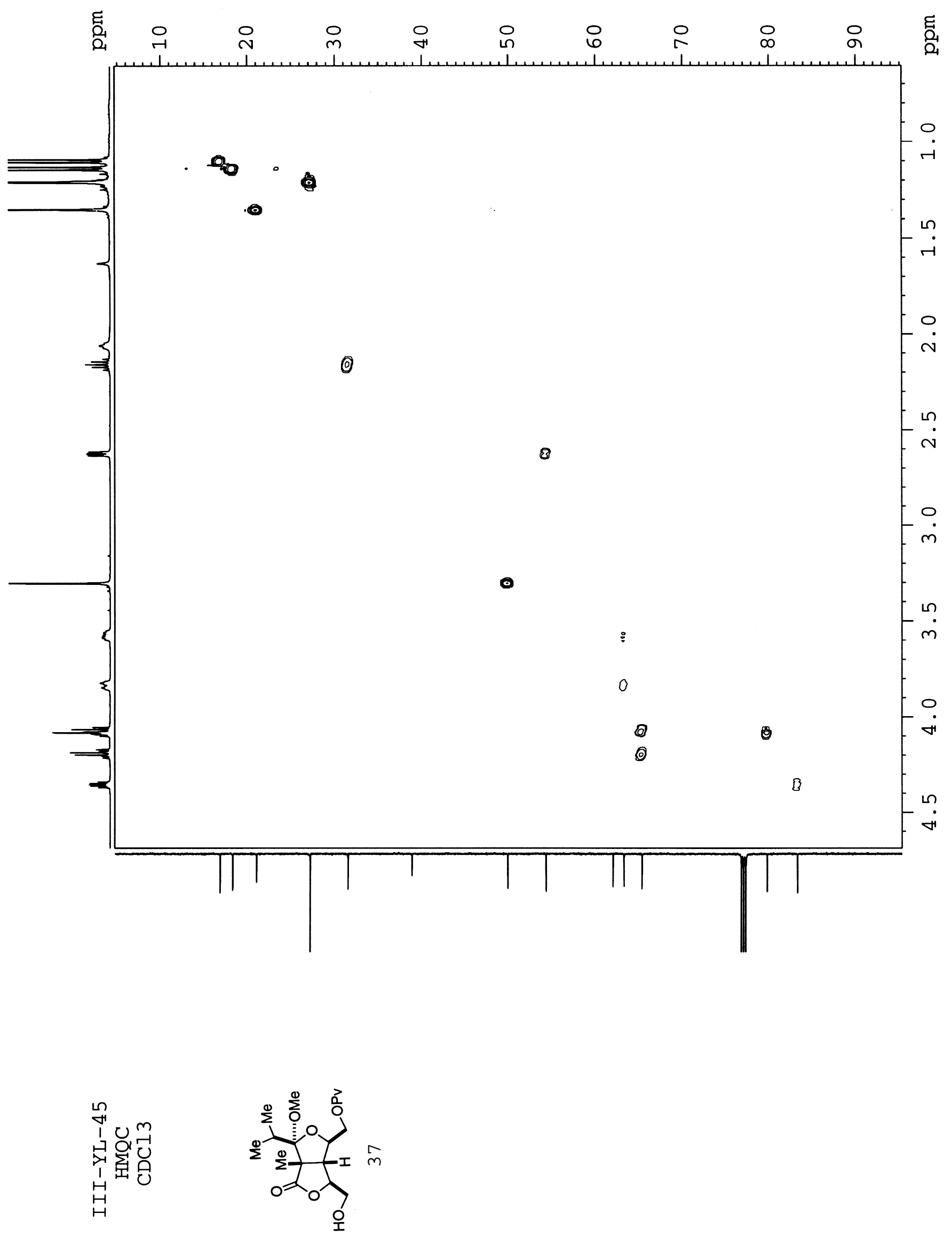


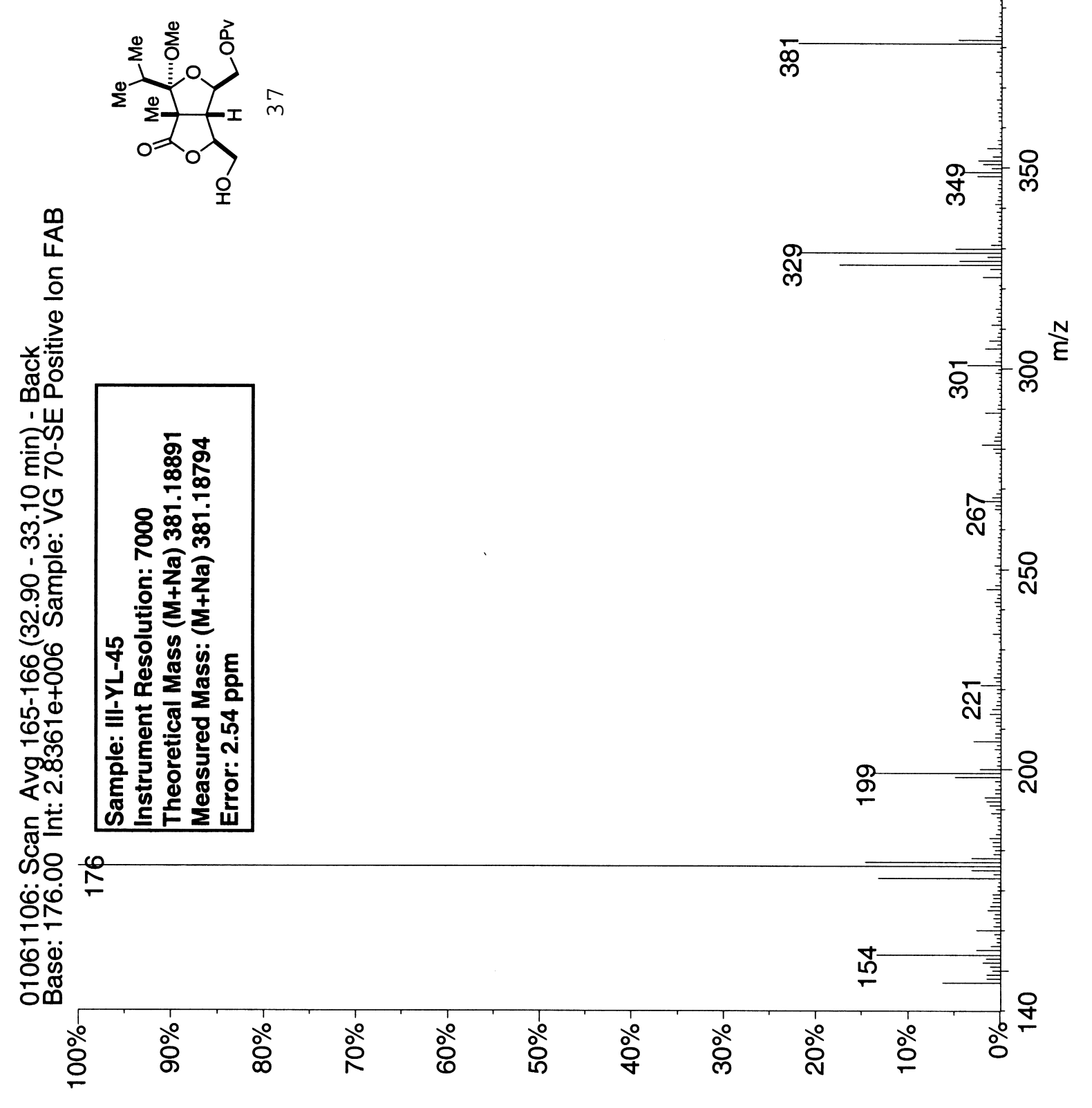




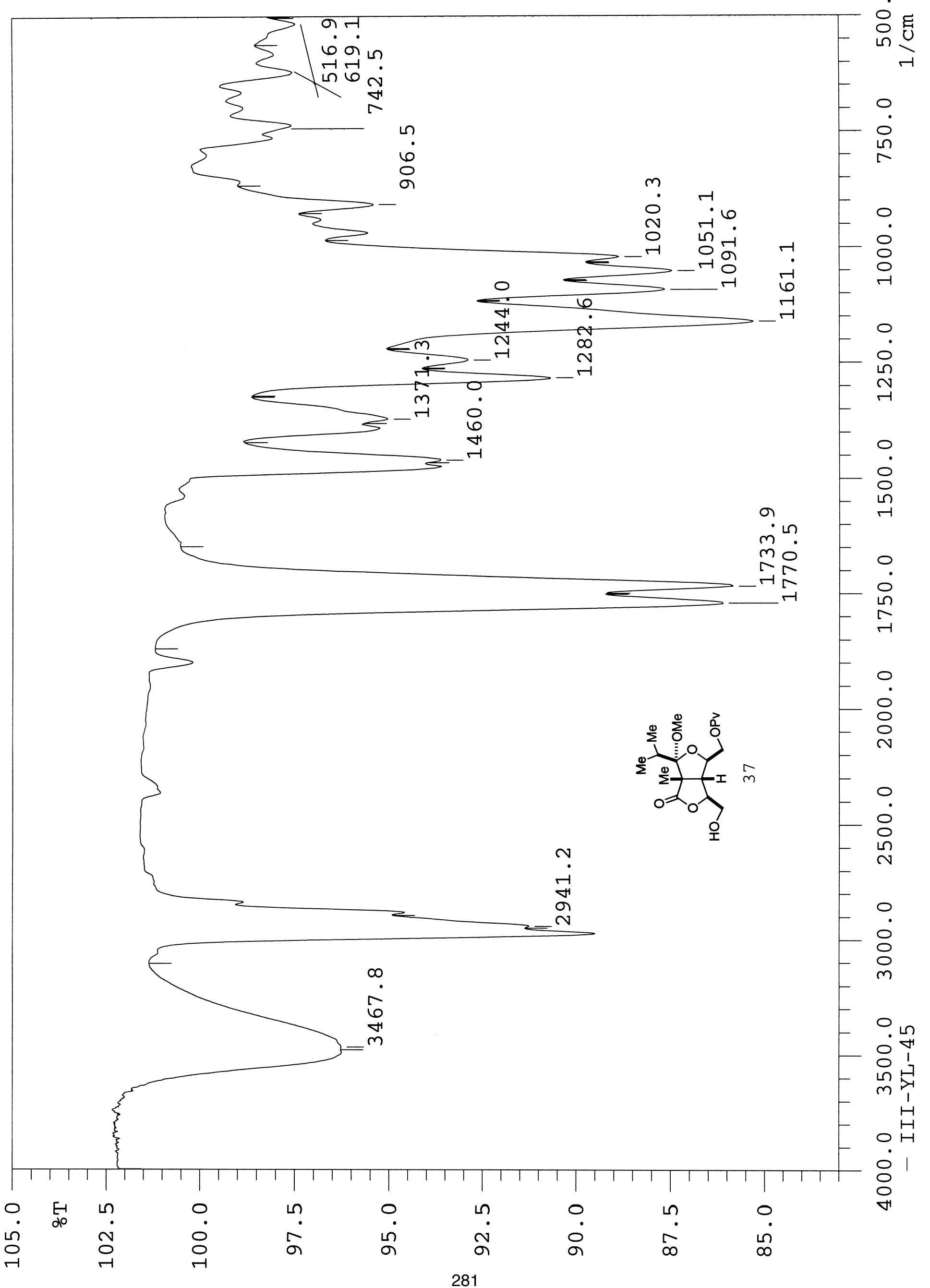




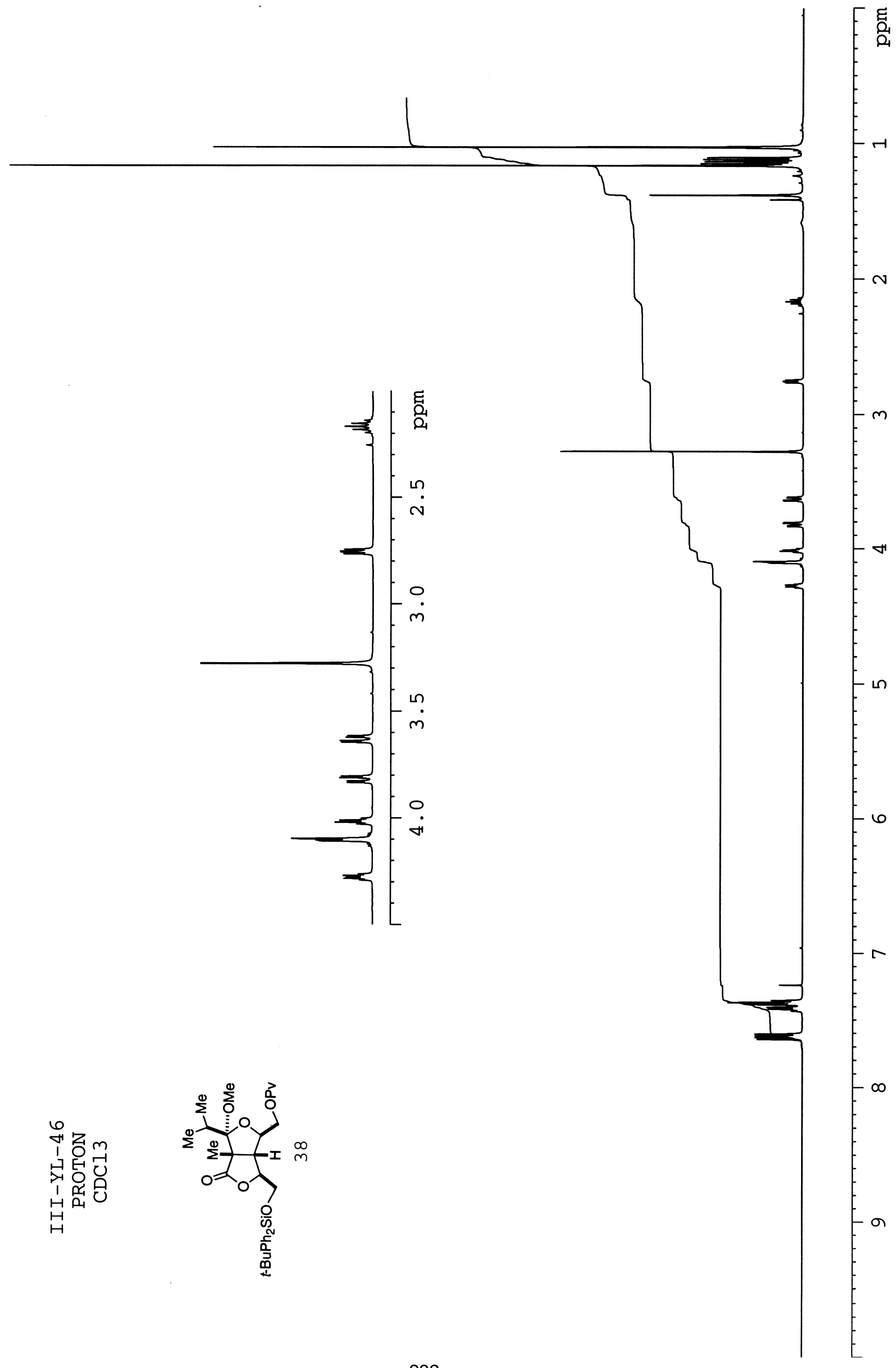




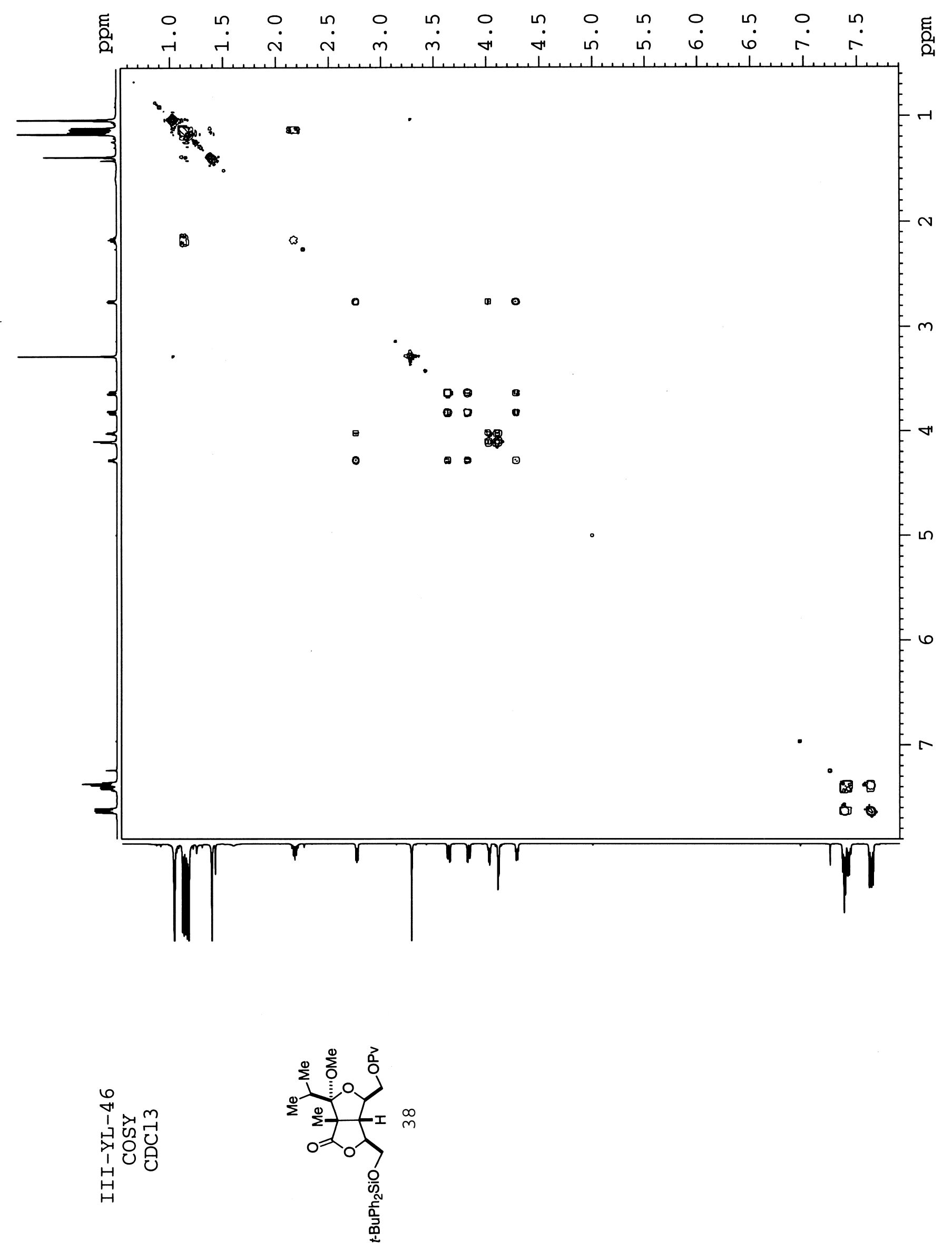




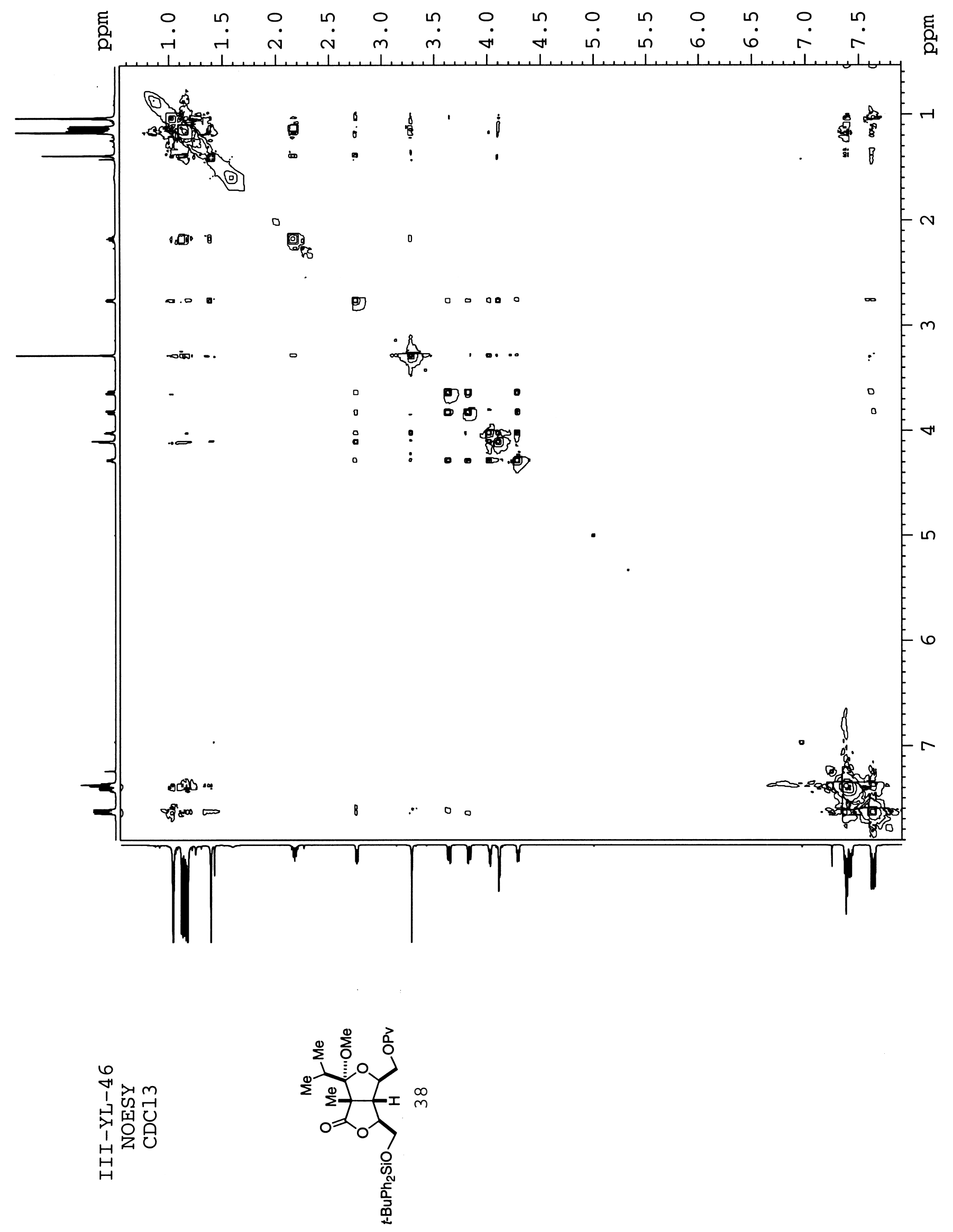




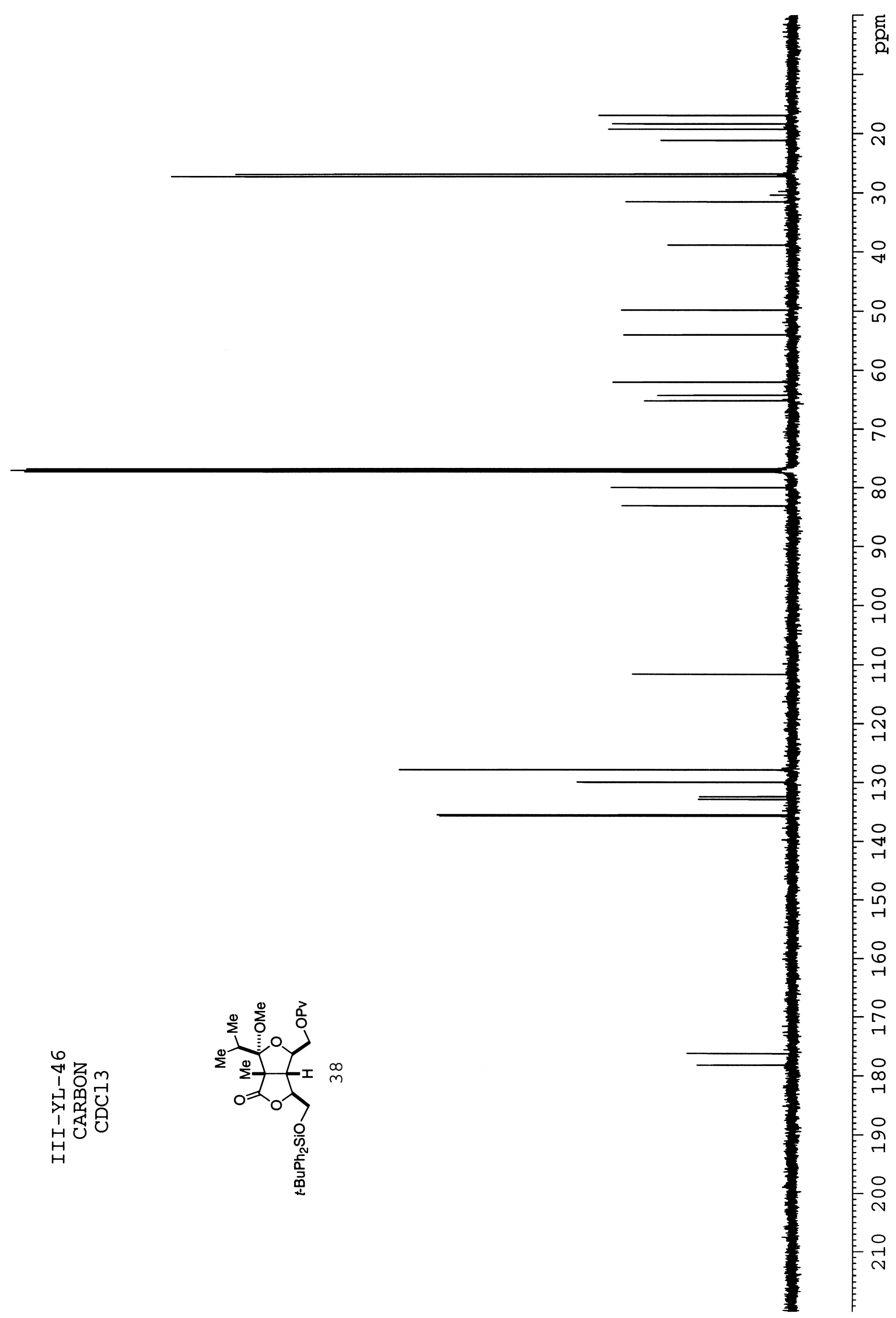




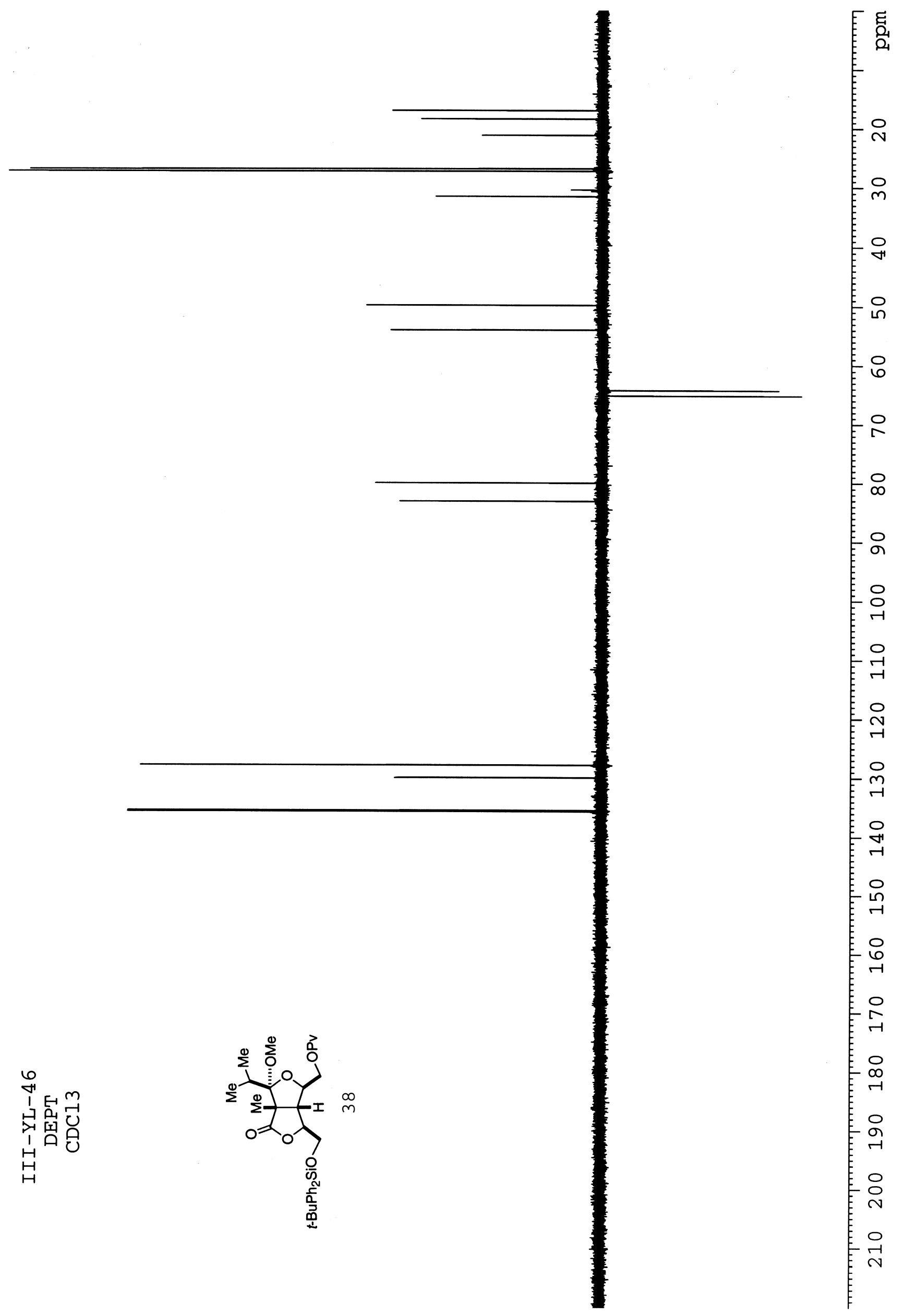




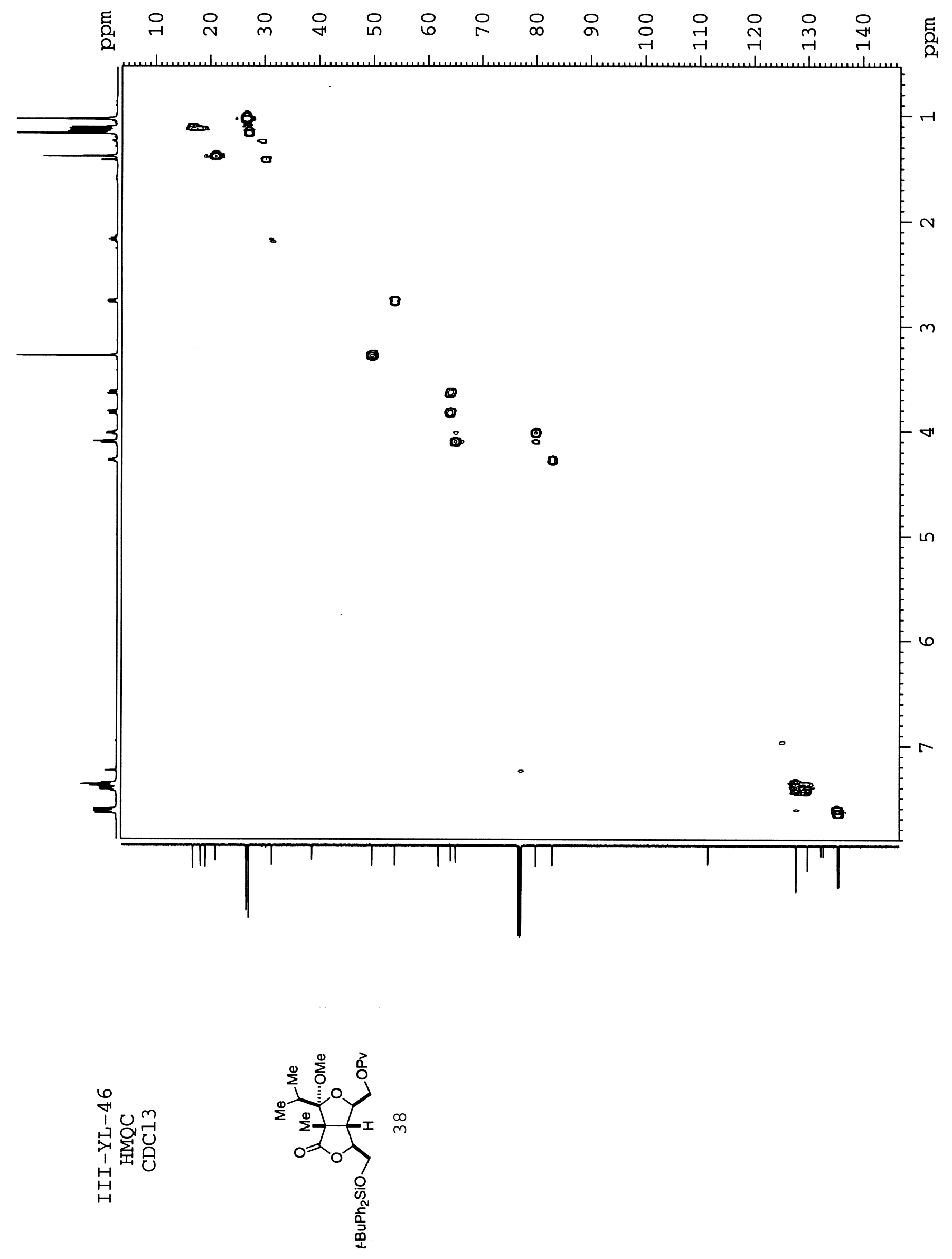




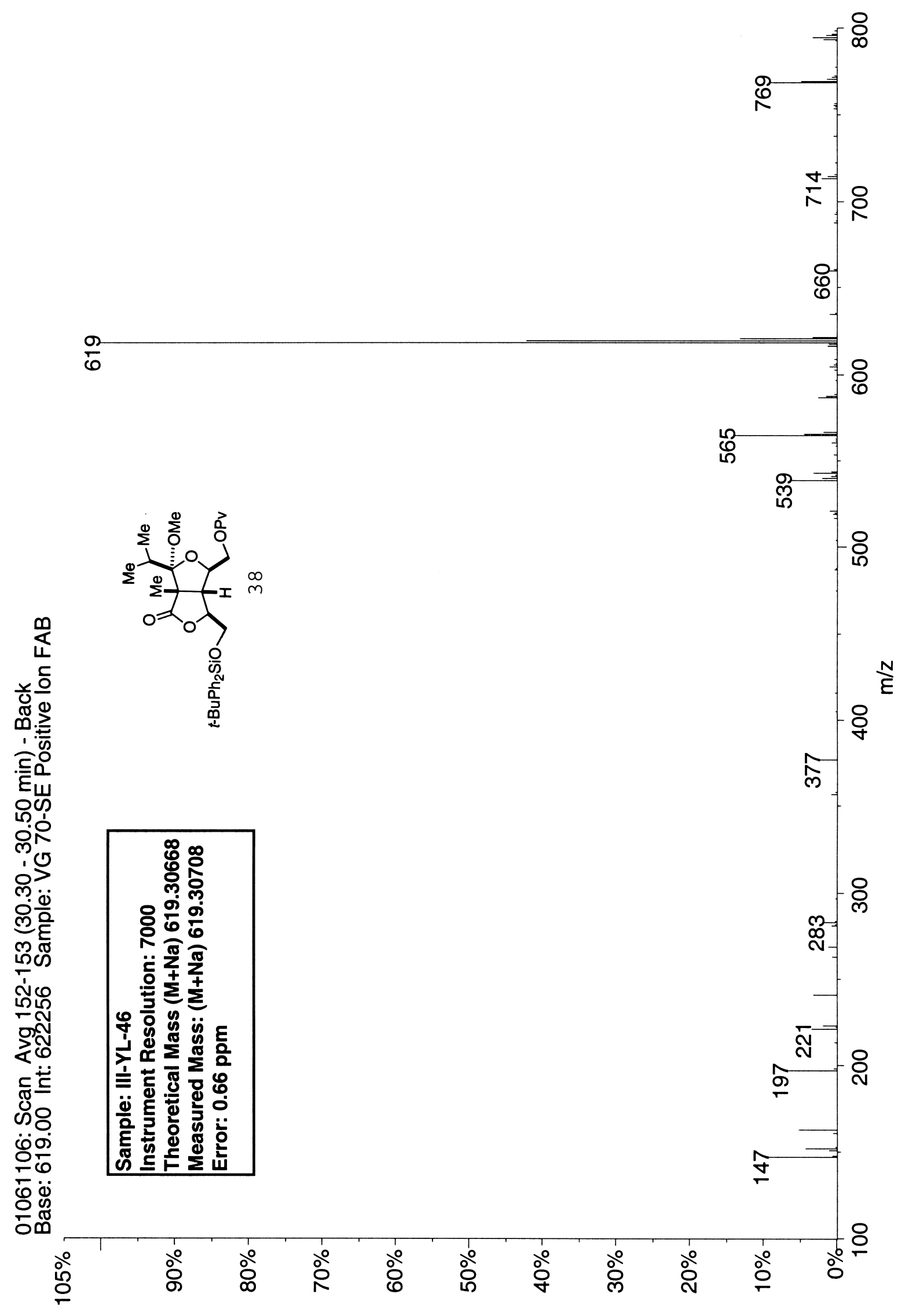




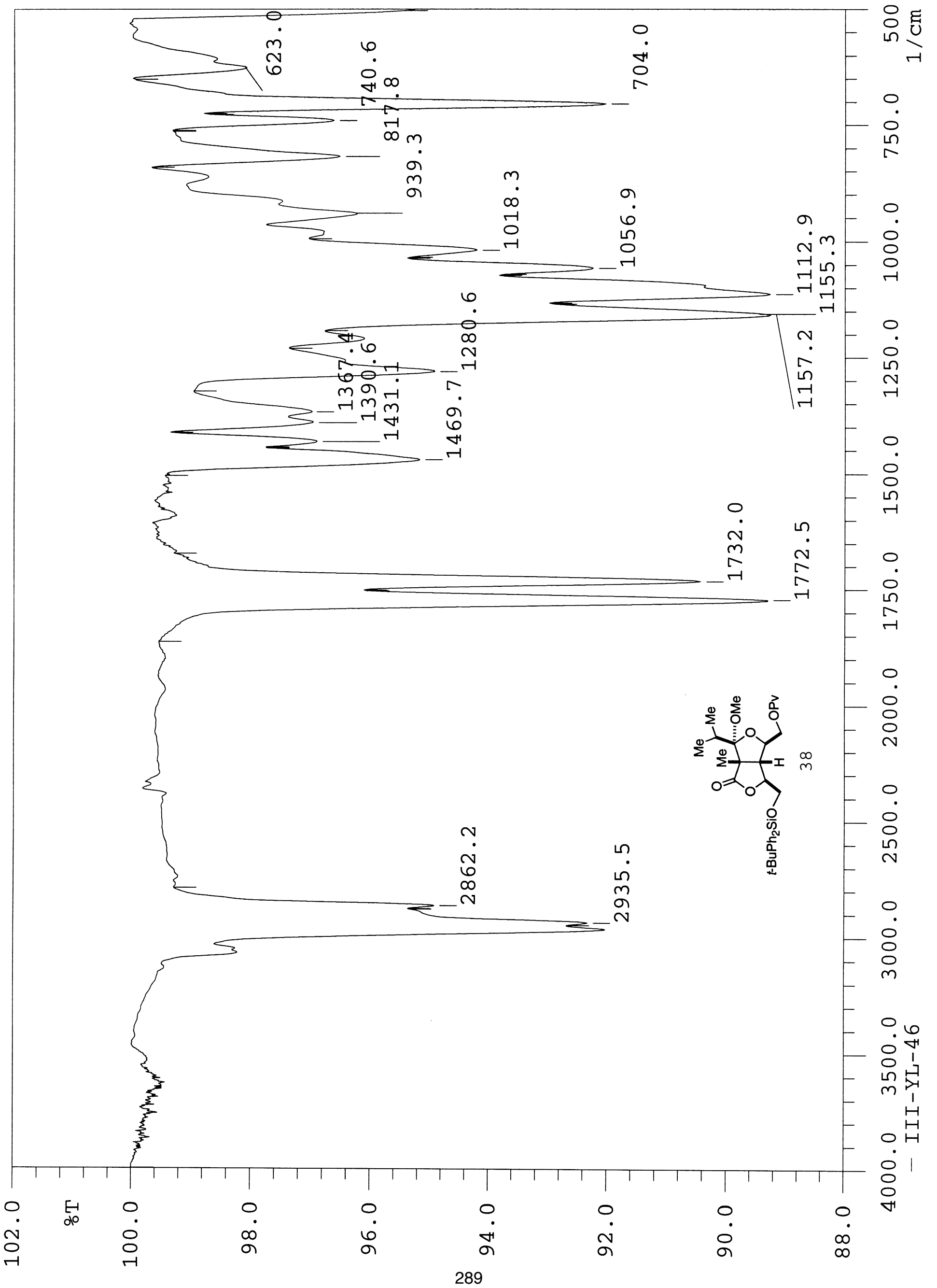




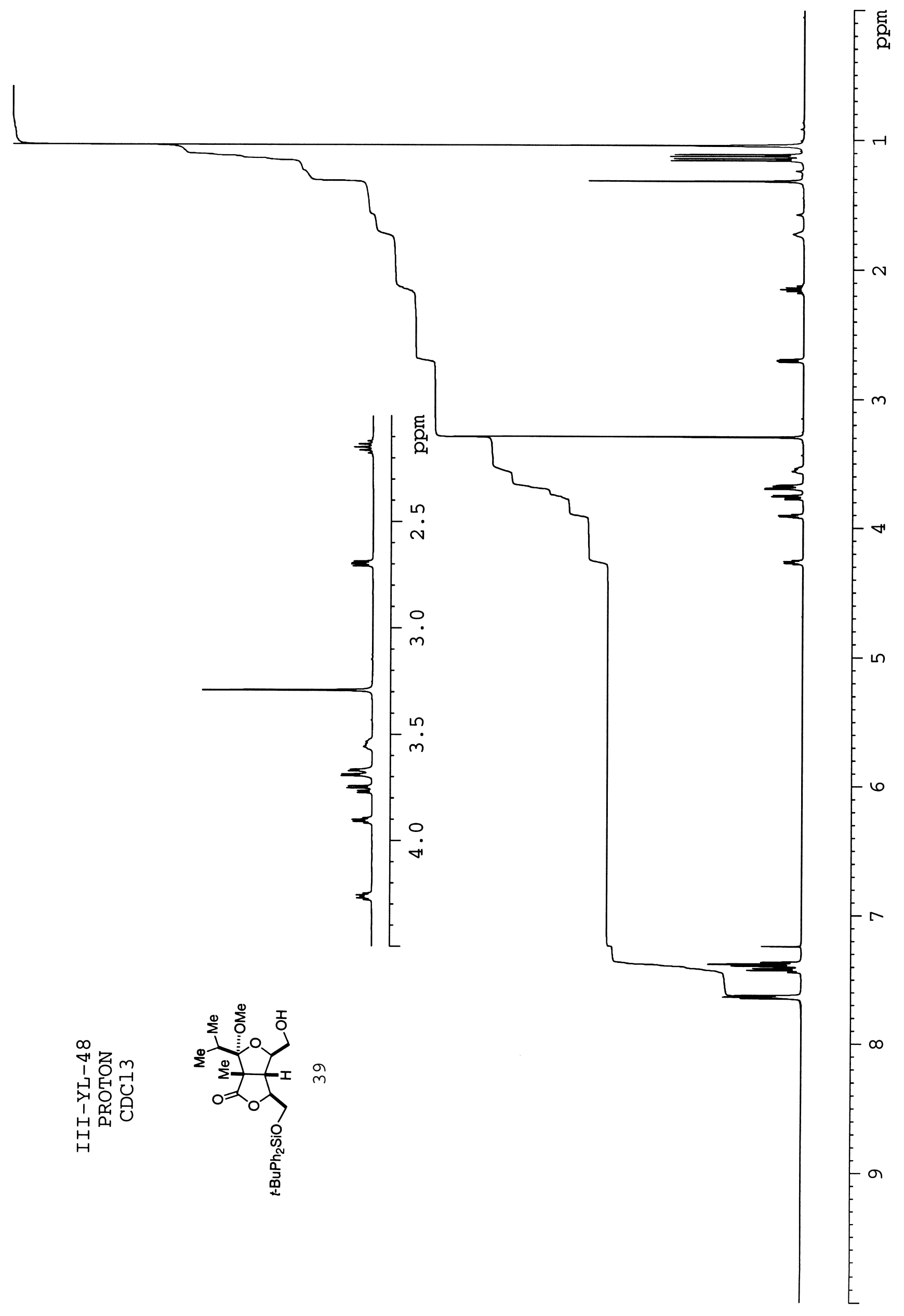




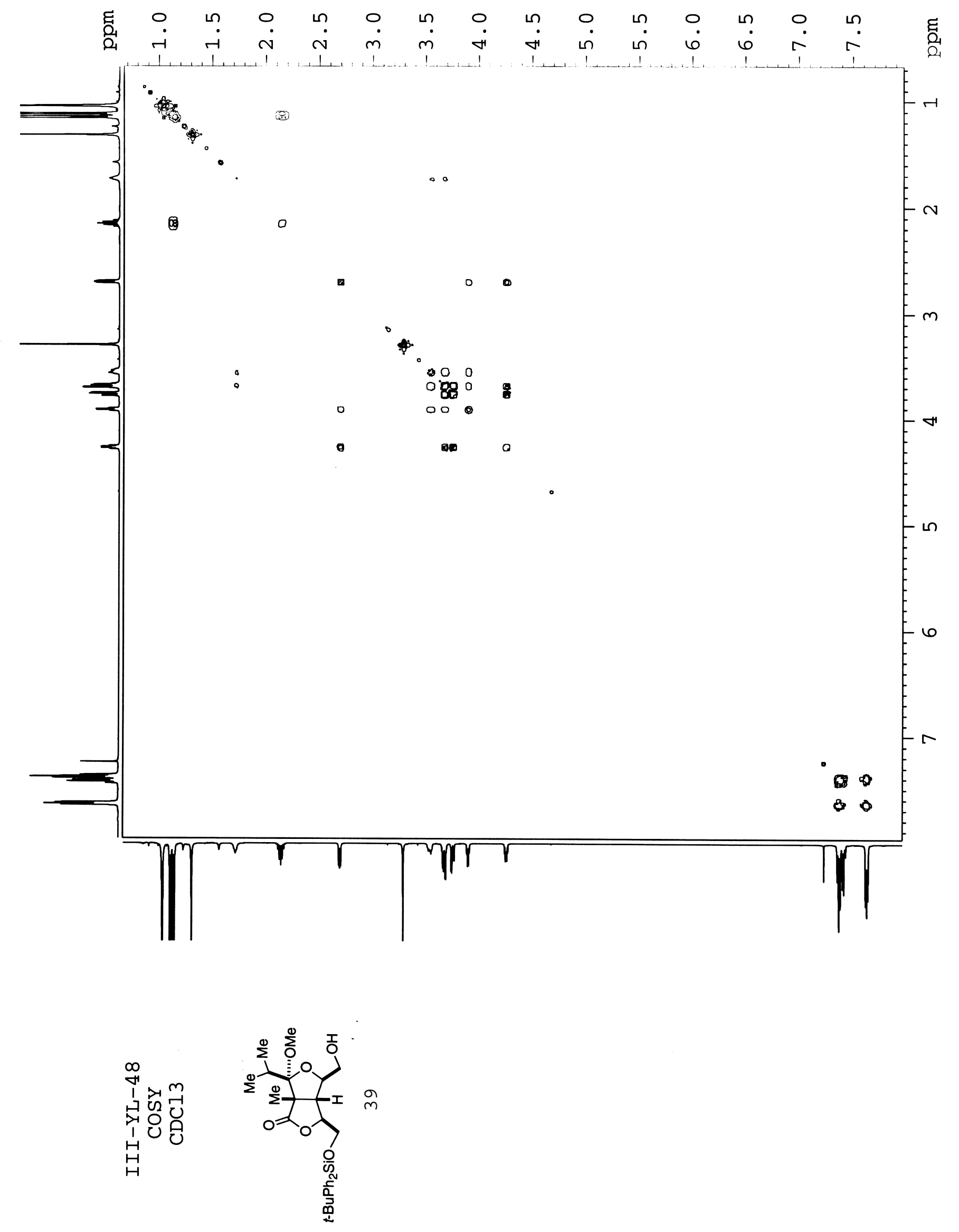




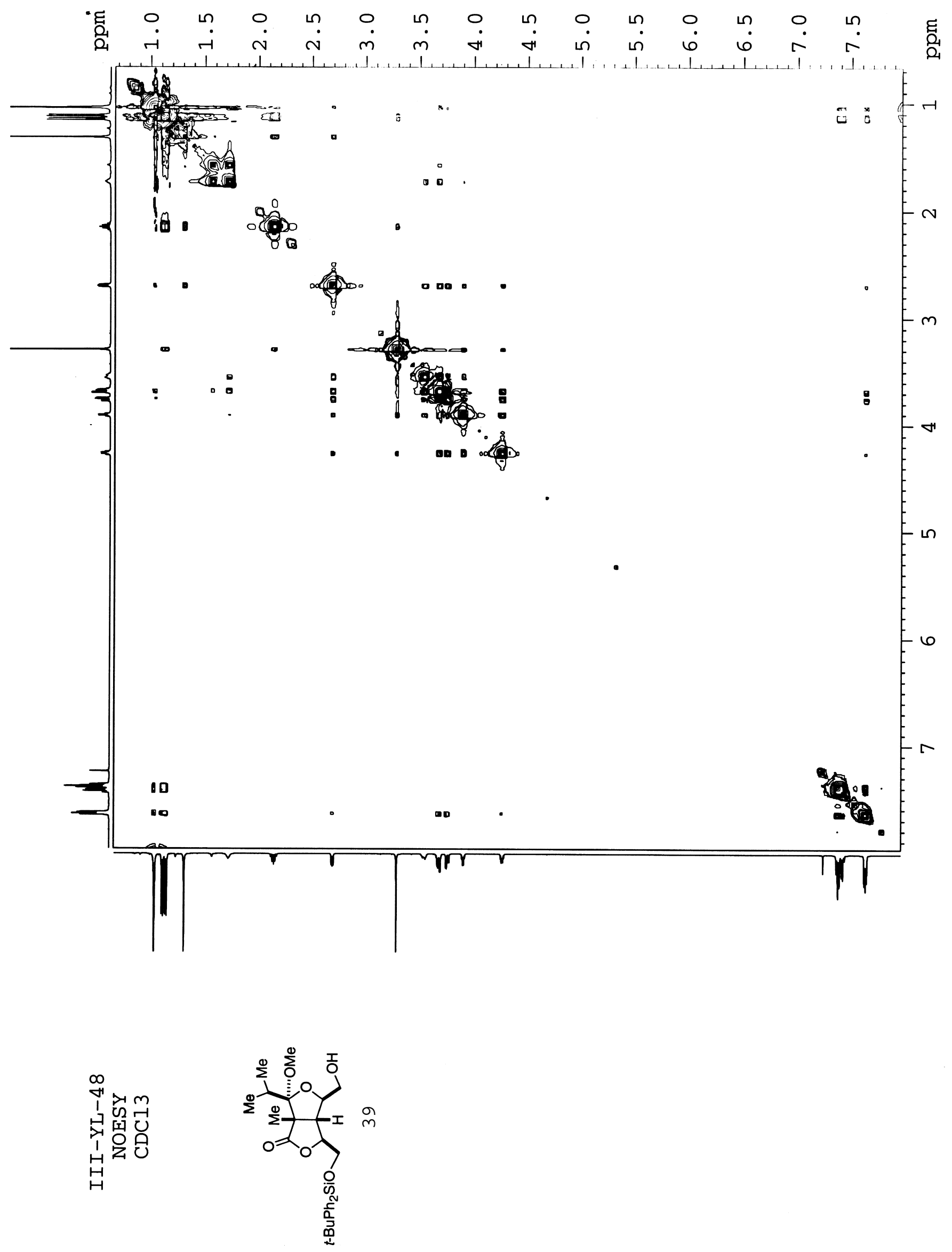




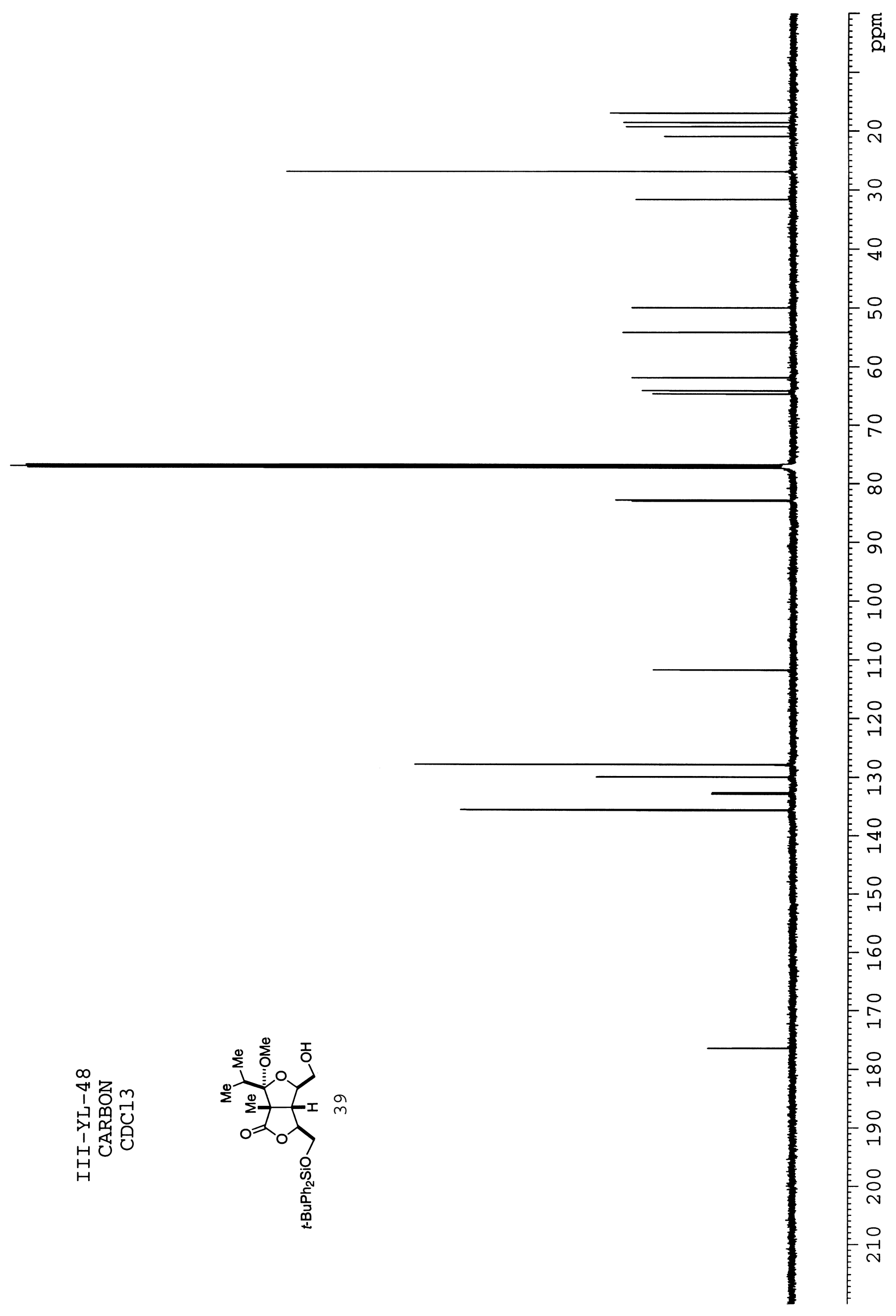




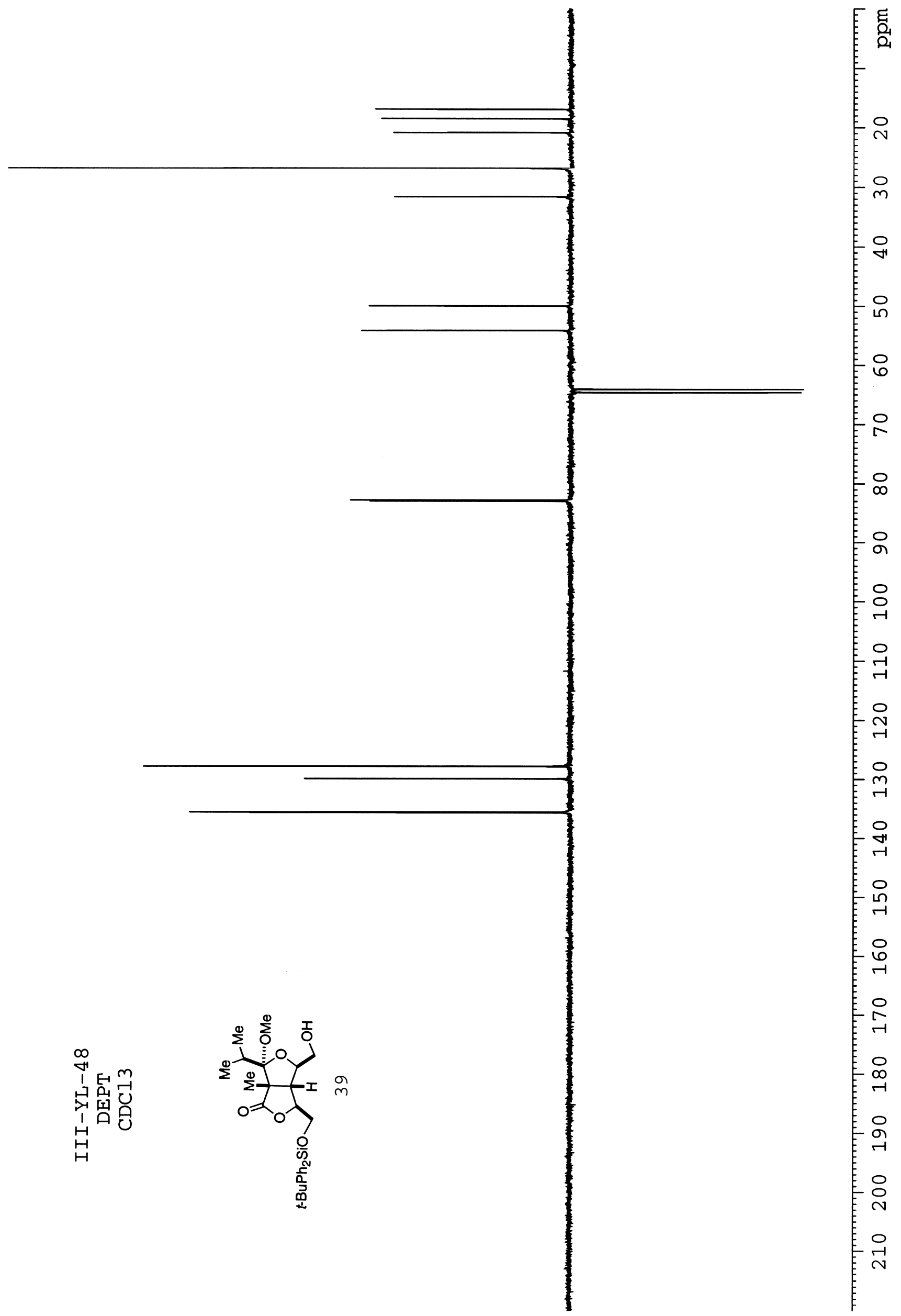




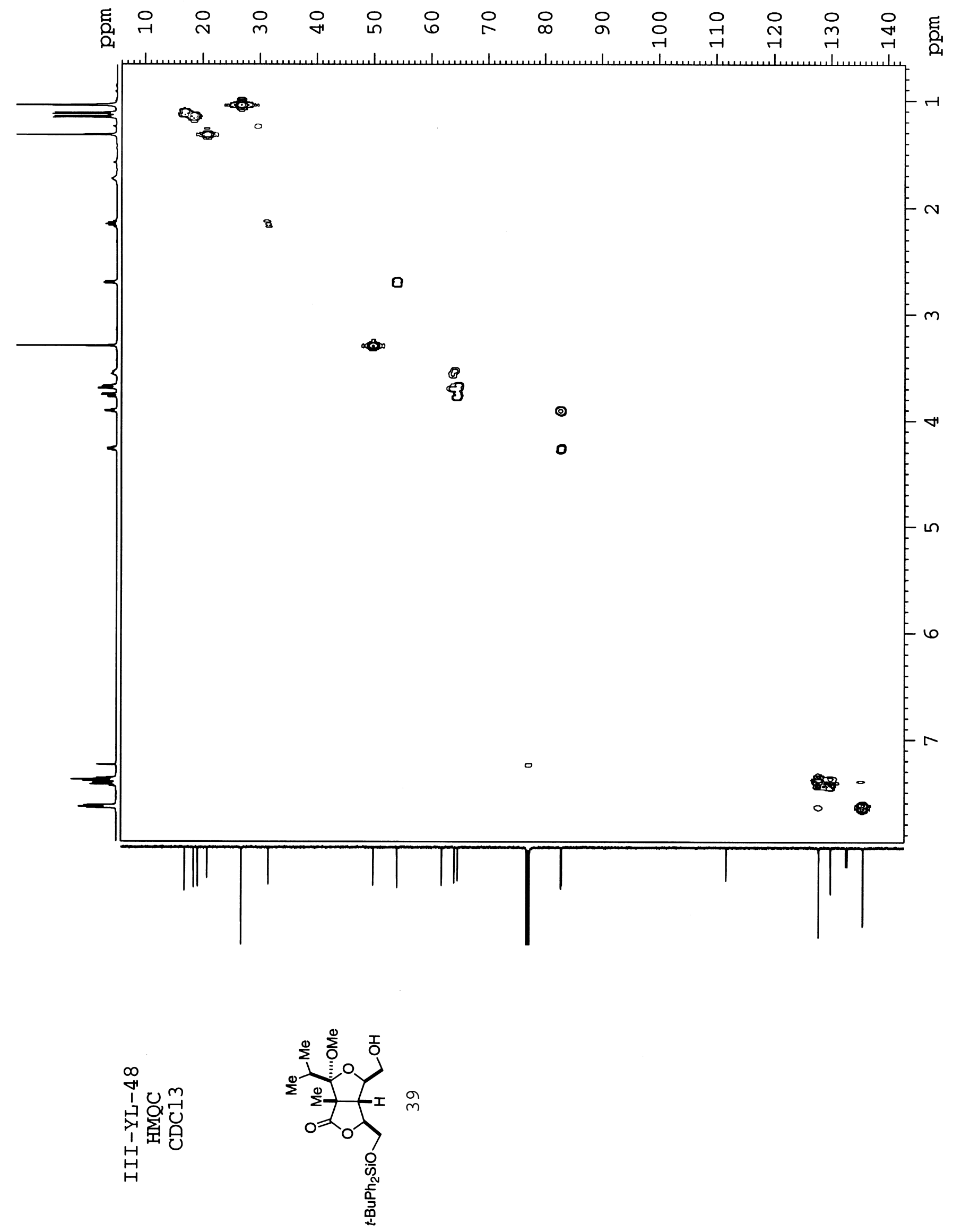




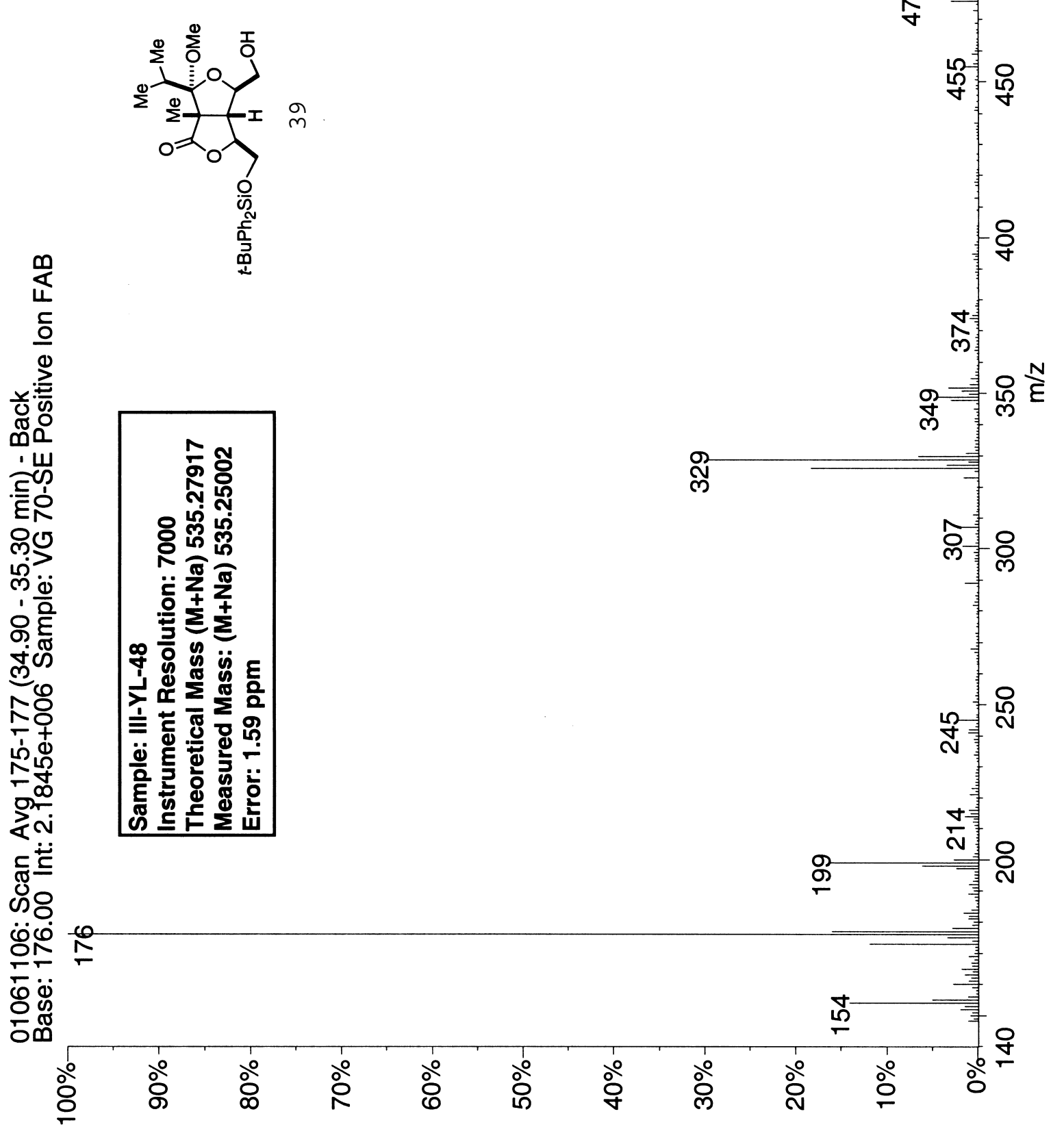




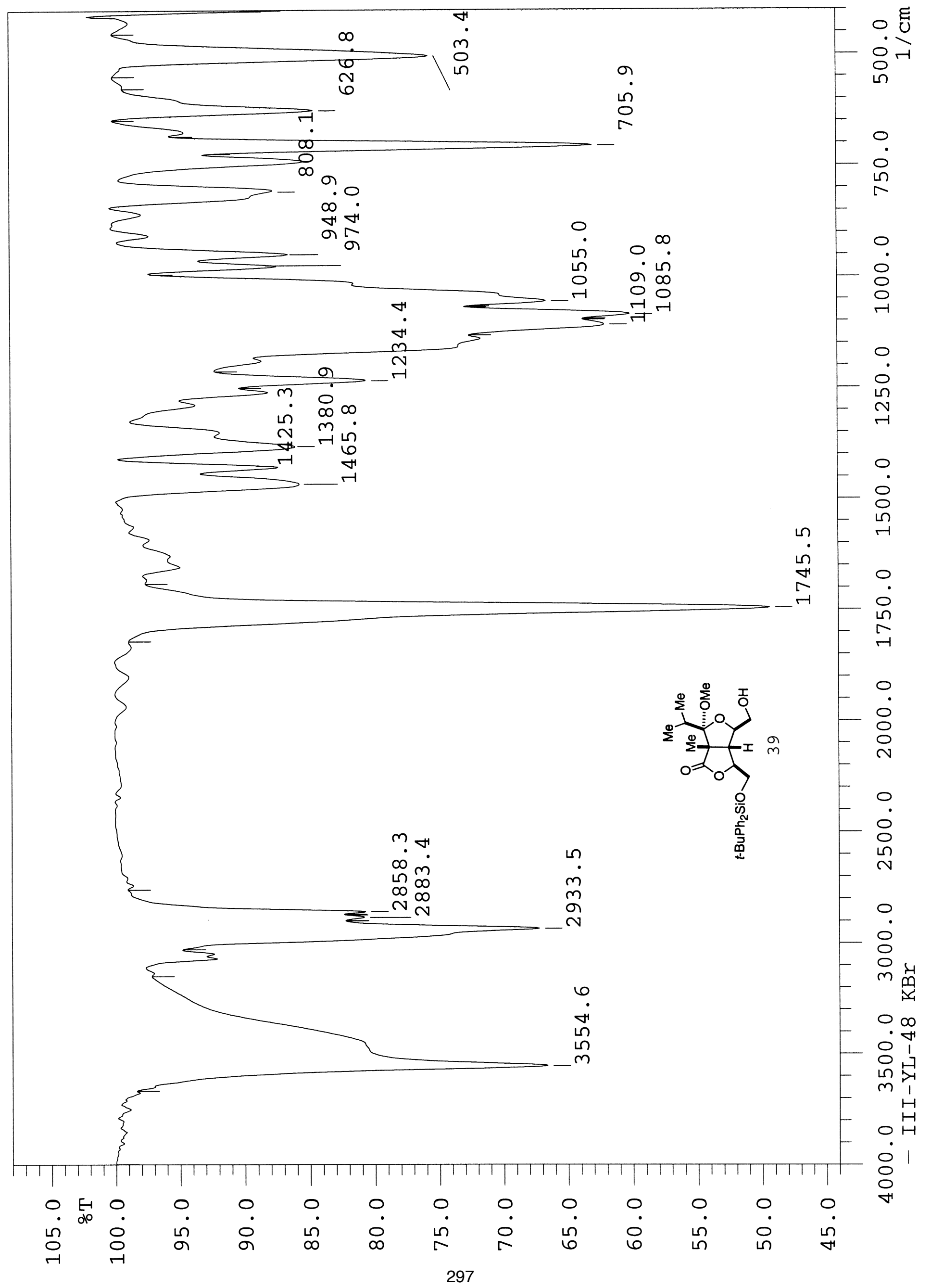




\title{
Asymmetric Total Synthesis and Formal Total \\ Synthesis of the Antitumor Sesquiterpenoid (+)- Eremantholide A
}

\author{
Yi Li and Karl J. Hale* \\ The UCL Center for Chemical Genomics, \\ The Christopher Ingold Laboratories, \\ The Chemistry Department, \\ University College London, \\ 20 Gordon Street, \\ London WC1HOAJ, UK.
}

Supporting Information

Part 3. Individual $500 \mathrm{MHz}{ }^{1} \mathrm{H}$ NMR Spectra of 32 and 33 in $\mathrm{CDCl}_{3}$. The $500 \mathrm{MHz}^{1} \mathrm{H}$ NMR Spectra of the

Crude Unseparated Mixtures of 32 and 33 Obtained

From the Alkylations of 2 with 3 Using Lithium bis[(S)-

1- $\alpha$-methylbenzylamide] and its $(R)$-Enantiomer in

PhMe $\left(-78{ }^{\circ} \mathrm{C}\right.$ to $0^{\circ} \mathrm{C}$ over $\left.2 \mathrm{~h}\right)$.

$400 \mathrm{MHz}{ }^{1} \mathrm{H}$ NMR Spectrum of 43 in $\mathrm{CDCl}_{3}$. 


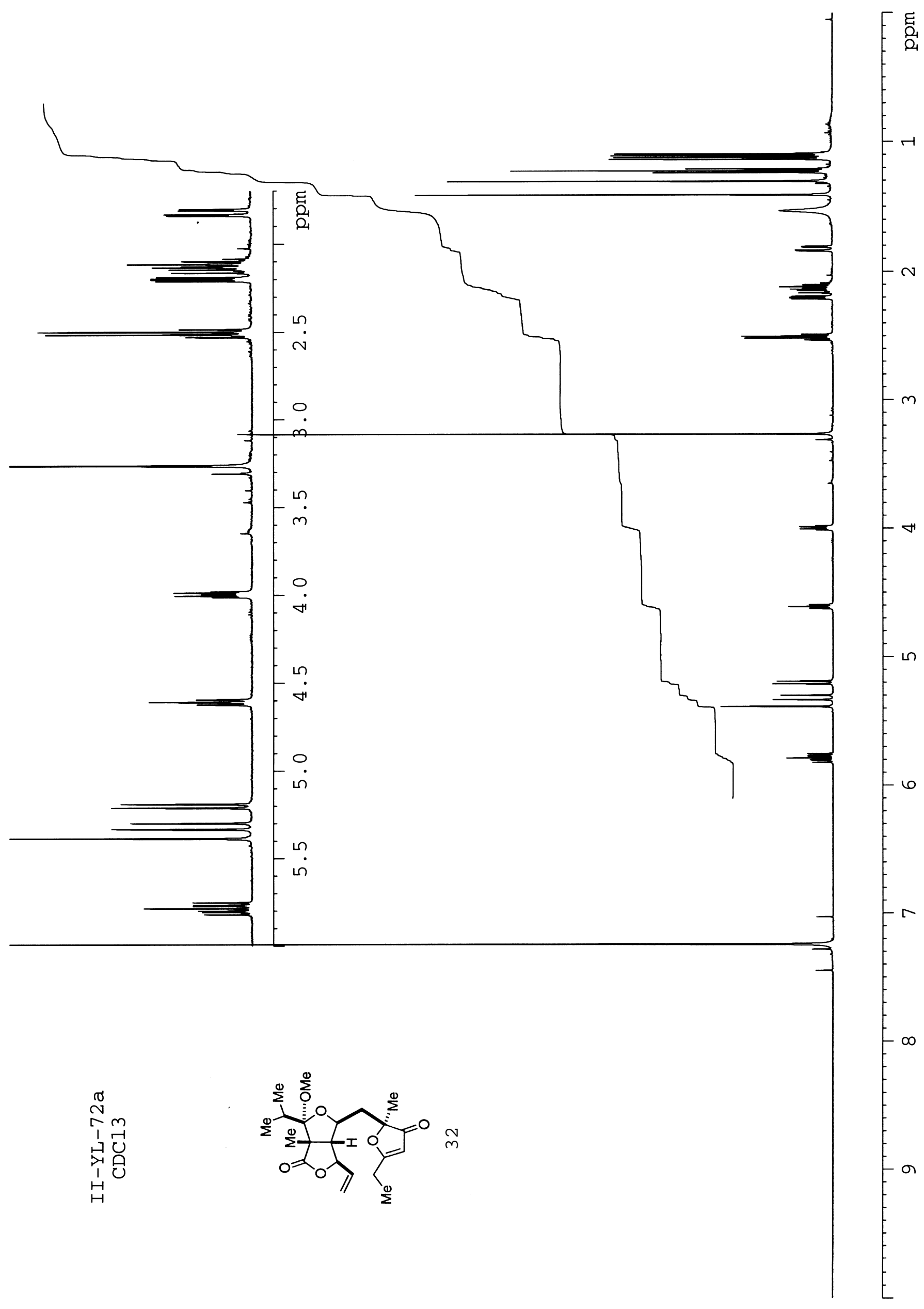




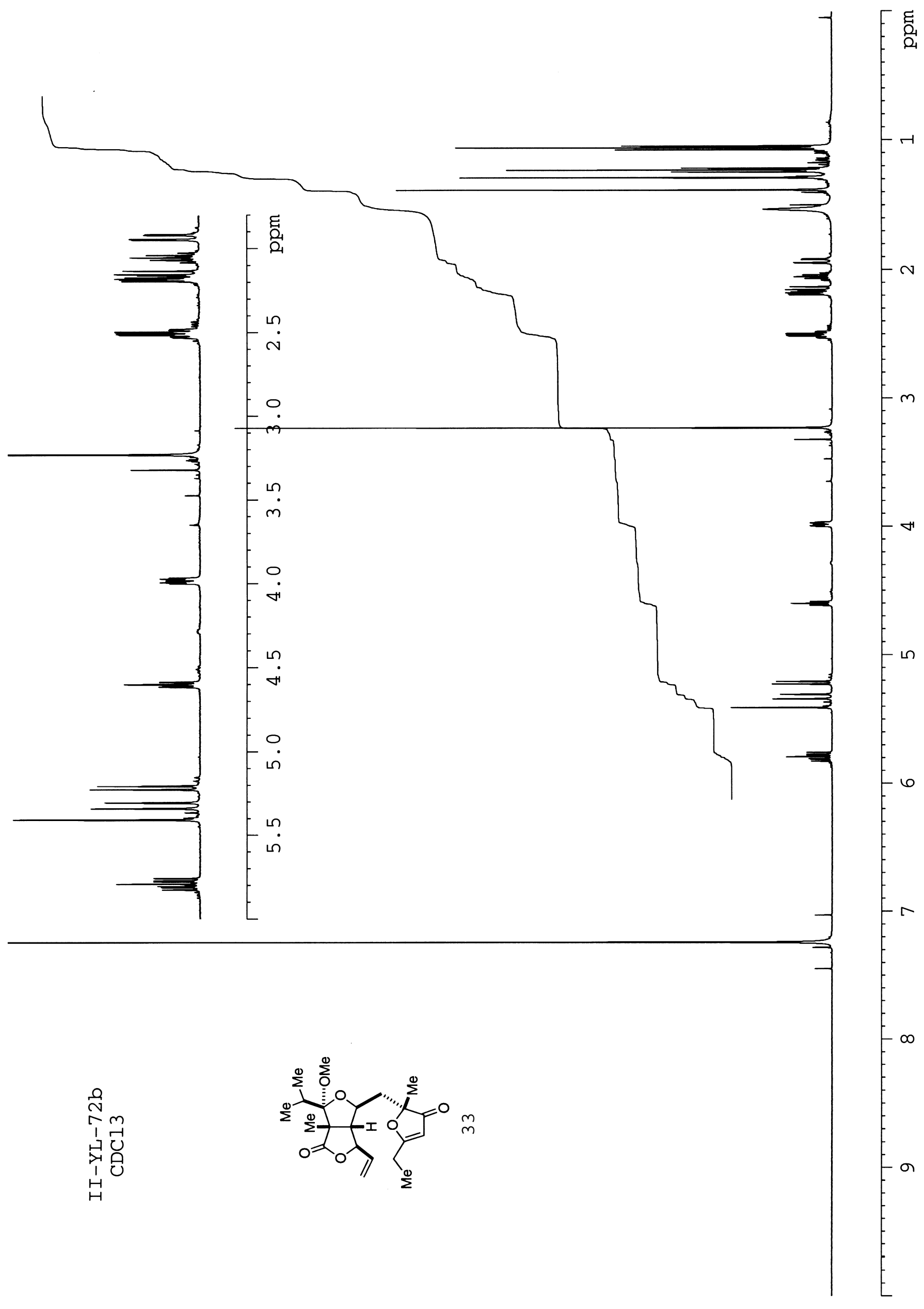




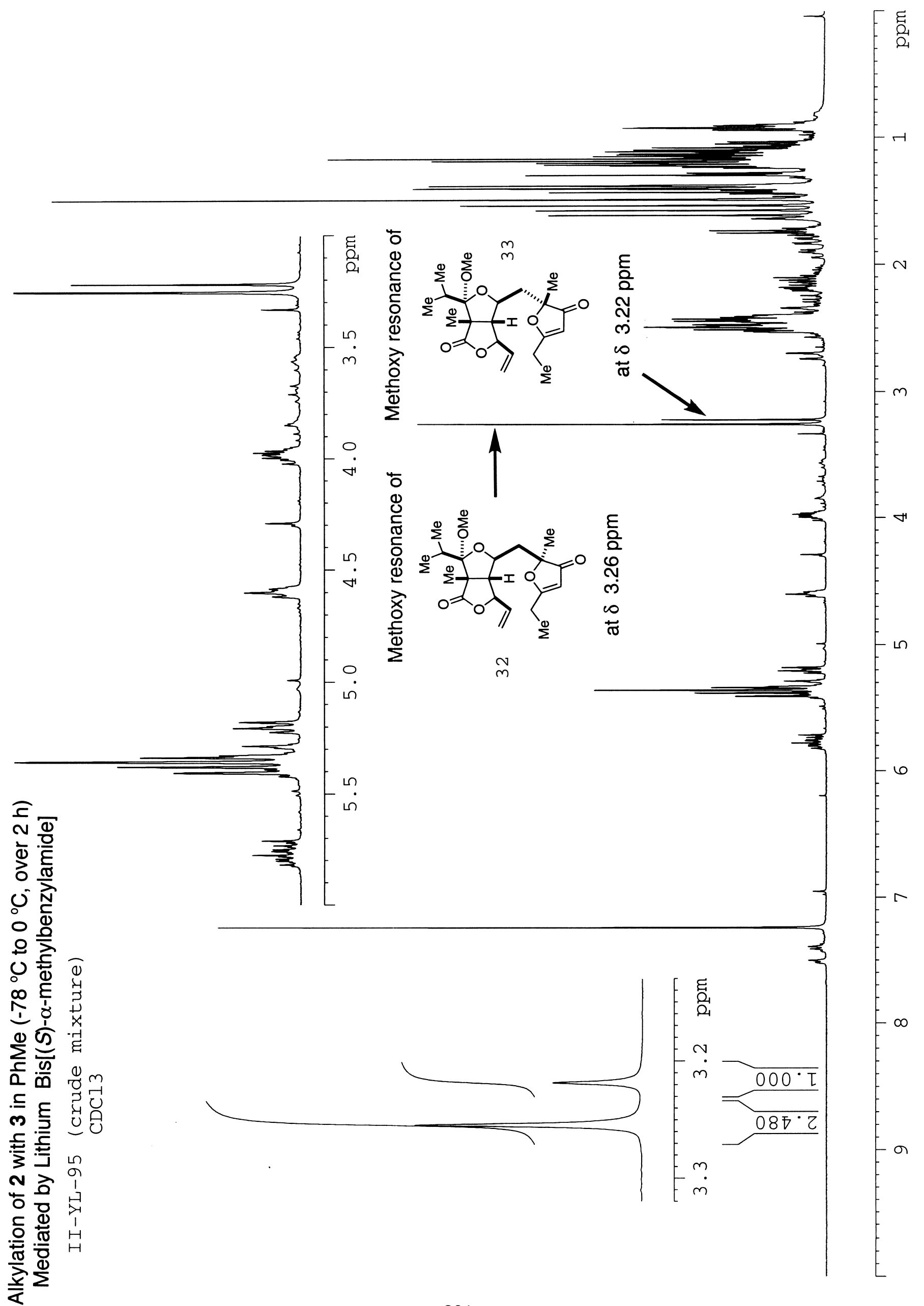




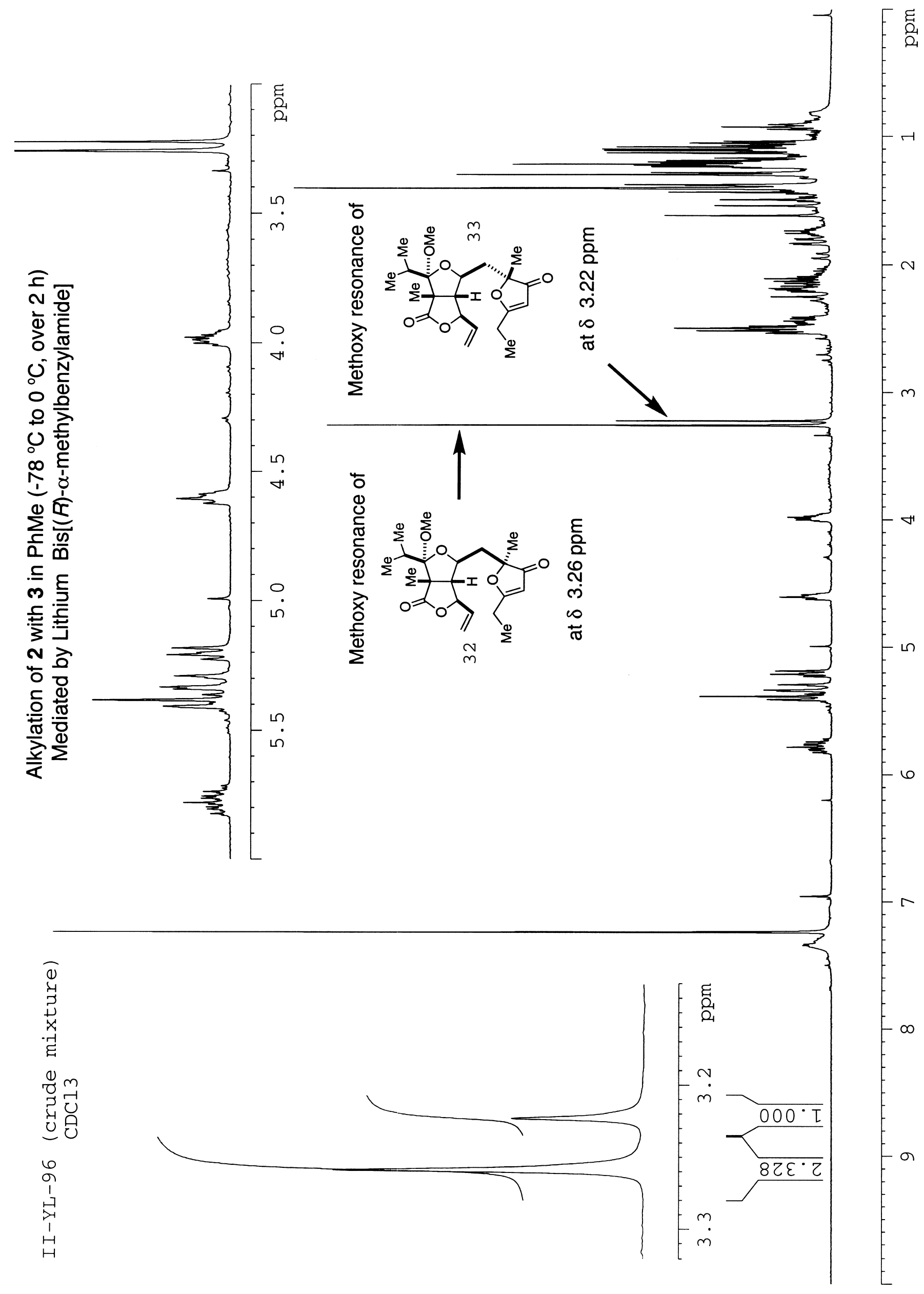




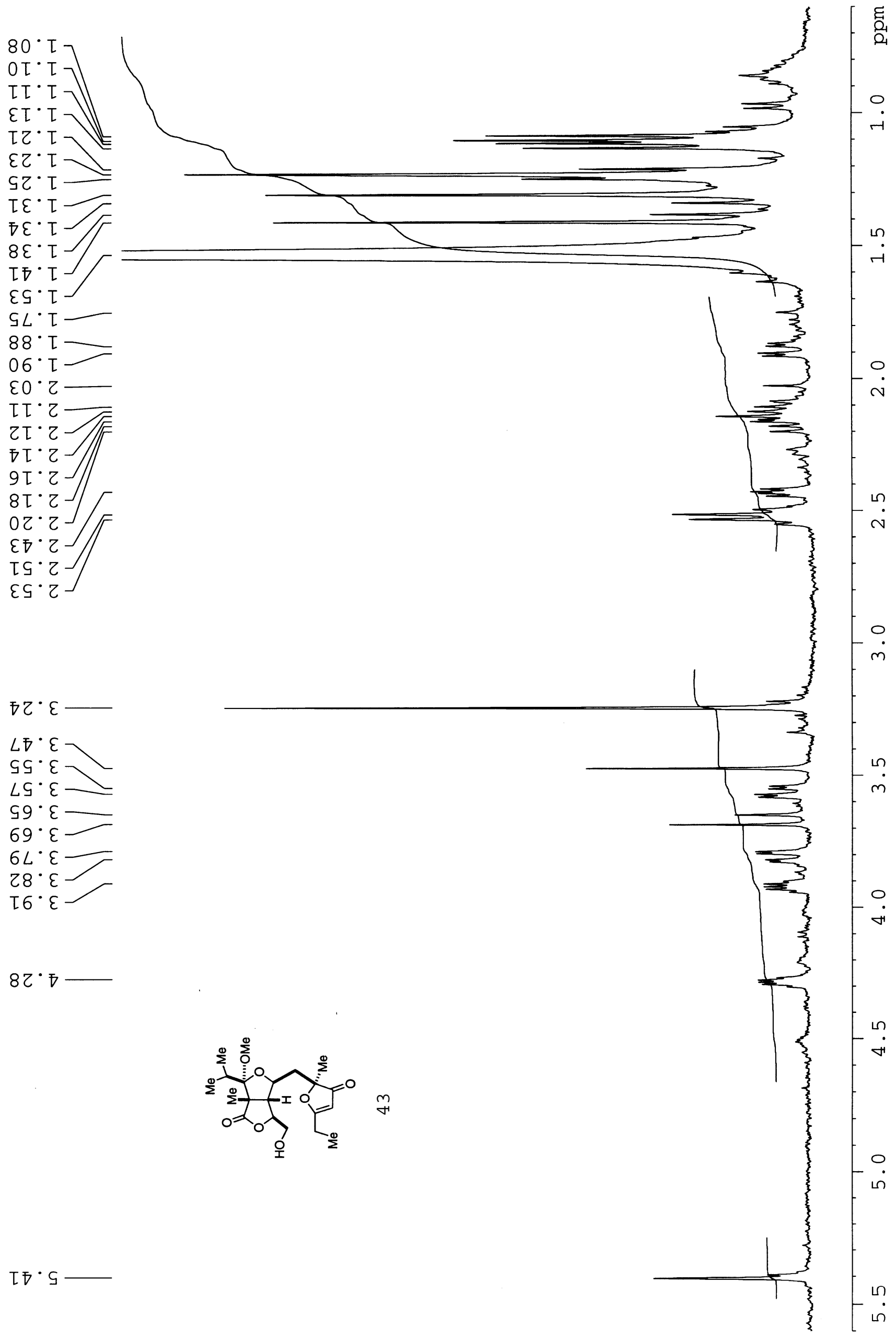

